COLUMBIA LIBRARIES OFFSITE

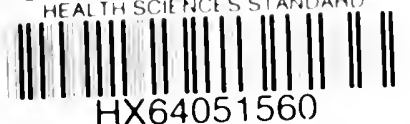

RD32 Y91 1898

Atlas and epitome of

RECAP 


\section{MEDICAL HAND-ATLASES.}

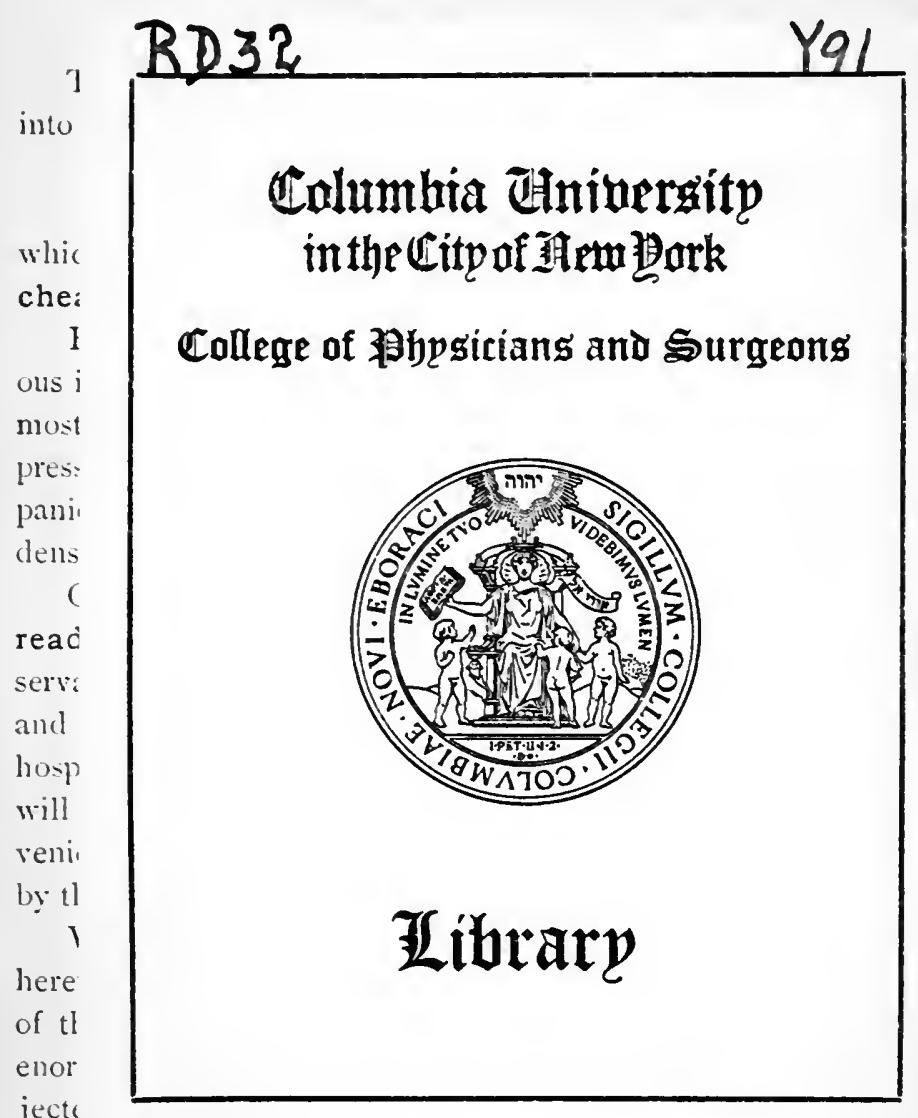

;anslations

ress, and

es numer-

ted by the

wenty im-

is accom.

ains a con-

ted.

ley offer a

Such ob-

al centers;

of routine

tese books

and con-

nterpreted

ession has

is because

le and an

their protional dis-

tribu

atlases the

best artistic and professional talent, to produce them in the most elegant style, and yet to offer them at a price heretofore unapproached in cheapness. The great success of the undertaking is clemonstrated ly the fact that the rolumes have already appeared in nine different languages-German, English, French, Italian, Russian, Spanish, I)anish, Swedish, and IImgarian.

The same careful and competent editorial supervision has been secured in the English cdition as in the originals. The translations have been edited by the leading American specialists in the different subjects. The volumes are of a uniform and convenient size ( $5 \times 7 / 2$ inches), and are substantially bound in cloth.

(For List of Books, Prices, etc, see back cover.) 
have $n^{\text {ad }}$ ed. 
Digitized by the Internet Archive in 2010 with funding from Columbia University Libraries 


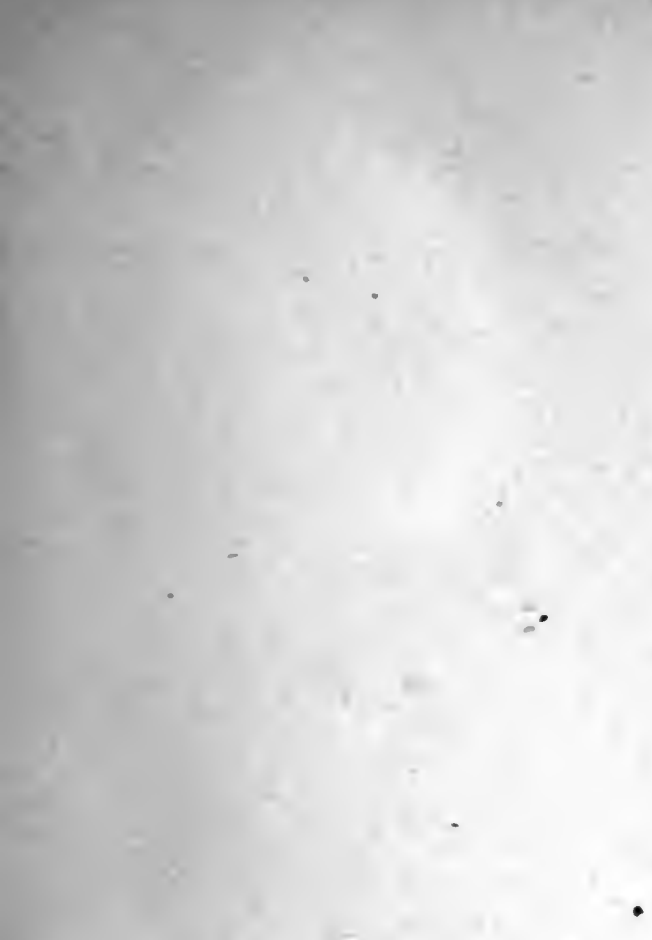

$=$

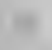

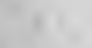





\section{ATLAS AND EPITOME}

UF

\section{OPERATIVE SURGERY}

BY

DR. OTTO ZUCKERKANDL

Privat-docent in the University of Vienna

AUTHORIZED TRANSLATION FROM THE GERMAN

EDITED B:

J. CHALMERS DACOSTA, M.D.

Clinical Professor of Surgery in Jefferson Medical College, Philadelphia ;

Surgeon to the Philadelphia Hospital, etc.

With 24 Colored Plates and 217 Illustrations in the Text

PIILADELPHIA

W. B. SAUNDFRS

925 Walnut Street

I $S_{9} 8$. 


$$
\begin{gathered}
R D 32 \\
y 91
\end{gathered}
$$

COPYRIGHT, 1898 ,

BY W. B. SAUNDERS.

Duplicate Dr. Eugene A. Sinith 7-20-29 D

WESTCOTT \& THOMSON B

PRESS OF W. B. SAUNDERS,

$4_{3 / 36}$ 


\section{EDITOR'S NOTE.}

Dr. Zuckerkaxdr reguires no introduction to Englishspeaking readers. In this Atlas he has laid down the rules and methods of surgical procedure with the clearness that springs from definite knowledge and the emphasis that is born of conviction. He describes lucidly and tersely the operations of modern surgery, and in a manner that fits the book to be a guide to the surgeon who operates upon the living or to the student who works upon the cadaver, the verbal descriptions being reinforced and illuminated by a large number of excellent original cuts.

The aim of the translator has been to portray the spirit rather than the exact words of the author.

In a very few places the editor has added notes that are explanatory in their nature, and are not to be considered as portions of the text. 


\section{PREFACE.}

THIs epitome of operative surgery is intended as an elementary work for students in this subject. For this reason those groups of operations whose practice upon the cadaver forms the basis of practical instruction are described in detail and illustrated in their most conspicuous aspects. Other operations, whose performance falls largely to the lot of the skilled surgeon, and whose practice upon the cadaver appears less important, are described concisely. I am indebted to my collaborators, Mr. B. Keilitz, artist, and Mr. M. Silbermark, student of medicine, for the care that they have given to the illustrative portion of the book. The former has supplied the colored plates and the autotypes in the text; the latter has rendered material assistance in the execution of photographs, which have been reproduced as wood-cuts, as well as in the preparation of anatomic specimens.

The AUthor. 


\section{DESCRIPTION OF PLATES.}

Plate 1. Intestinal suture.

Plate 2. Exposure of brachial and axillary arteries.

Plate 3. Exposure of cubital, ralial, and other arteries.

Plate 4. Exposure of the femoral artery beneath Poupart's liganent, and also at the middle of the thigh.

Plate 5. Exposure of the femoral artery in the adductor canal.

Plate 6. Exposure of the popliteal artery.

Plate 7. Exposure of the anterior and posterior tibial arteries.

Plate 8. Transverse division of the leg.

Plate 9. Transverse division of the thigh.

Plate 10. Transverse division of the forearm.

Plate 11. Transverse division of the arm.

Plate 12. Temporary resection of the lower jaw by the method of Langenbeck.

Plate 13. Laryngofissure.

Plate 14. Inferior tracheotomy.

Plate 15. Infrahyoid pharyugotomy.

Plate 16. Exposure of the common carotid artery and of the subclavian artery below the clavicle.

Plate 17. Exposure of the lingual artery.

Plate 18. Topography of the supraclavicular triangle.

Plate 19. Formation of a gastric fistula through the abdominal wall (gastrostomy); establishment of a preternatural anus (colostomy).

Plate 20. Union of divided intestine by means of the Murphy button.

Plate 21. View of the interior of the bladder after suprapubic cystotomy in Trendelenburg's position.

Plates 22, 23. Radical operation for inguinal heruia by the method of Bassini.

Plate 24. Exposure of the external iliac artery. 
.

. 


\section{DESCRIPTION OF FIGURES.}

Figs. 1-7. Knives of varions kinds.

Fig. 8. Pen-like node of holding the knife.

Fig. 9. Violin-low mode of holding the knife.

Fig. 10. Division of a raised fold of skin with a sharp-pointed knife.

Fig. 11. Mole of holding and nsing the resection-knife.

Figs. 12, 13. Node of holding and using the tenotome.

Fig. 14. Incision upon the grooved director.

Fig. 15. Mode of using the blunt-pointed knife.

Fig. 16. 1)issection between two pairs of forceps.

Fig. 17. Division with seissors.

Fig. 18. Mode of holding the trocar.

Fig. 19. The thermocantery.

Fig. 20. The wire snare.

Fig. 21. Écraseur.

Fig. 22. The arched saw.

Fig. 23. The chain-saw.

Fig. 24. The circular saw.

Figs. 25-28. Suture of wounds.

Fig. 29. Surgical needles.

Fig. 30. Purse-string suture.

Fig. 31. Mode of introducing the needle.

Figs. 32-35. Various kinds of tendon-suture.

Fig. 36. Bone-suture.

Fig. 37. Bone-clamp.

Fig. 38. Metallic nails.

Fig. 39. Artery divided between two ligatures.

Figs. 40-42. Varieties of knots.

Fig. 43. Cutaneous incision for ligating the axillary and brachial arteries.

Fig. 44. Musculature of the upper extremity.

Fig. 45. Cutaneous incisions for ligating the cubital, radial, and ulnar arteries.

Fig. 16. Phlebotomy. 
Figs. 47, 48. Musculature of the thigh.

Fig. 49. Cutaneous incisions for ligating the femoral artery.

Fig. 50.

II scculature of the leg, posterior aspect.

Fig. 51. Cutaneous incisions for ligating the popliteal artery.

Fig. 52. - Musculature of the leg, anterior aspect.

Figs. 53, 54. Cutaneous incision for ligating the anterior and posterior tıbial arteries.

Figs. 55-5s. Amputation br the circular incision in two stages.

Figs. 59, 60. Formation of a musculotegumentary flap by transfixion.

Fig. 61. Formation of tegumentars flaps.

Fig. 62. Circular incision after formation of two musculotegumeutary flaps.

Fig. 63. Oral incision.

Figs. 64, 65. Suture of the womnd after amputation.

Fig. 66. Circular incision, flap-incision. for amputation of the leg.

Figs. 67, 65. Incision for amputation of the leg by the method of Heine.

Fig. 69. Diagrammatic representation of amputation of the leg bs the method of Bier.

Fig. 70. Amputation by the nethod of Syme.

Figs. 71-7. Amputation br the method of Pirogotf.

Figs. 76-s0. Direction of the sawed surfaces in Pirogoff's operation and its modificatious.

Figs. 81, 82. Achillotenotoms.

Fig. 83. Amputation of all the trues through the metatarsus.

Figs. 81, 85. Lisfrane's articular line.

Figs. s6-8s. Exarticulation by the method of Lisfranc.

Fig. 89. Chopart's articular line.

Figs. 90-92. Exarticulation of the foot below the astragalus.

Fig. 93. Cutaneous incisions for exarticulation at the knee-joint.

Fig. 94. Diagrammatic representation of Gritty's operation.

Fig. 95. Diagrammatic representation of Ssabauajeff's operation.

Figs. 96-98. Amputation of the thigh bs the method of Gritty.

Fig. 99. Wound left by Ssabanajef's operation.

Fig. 100. Exarticulation at the hip-joint. Cutaneous incision.

Figs. 101-104. Enucleation of the fingers.

Fig. 105. Exarticulation of the hand. Circular iucision.

Figs. 106, 10\%. Exarticulation of the hand. Flap-incision.

Fig. 108. Enucleation of the hand. Opening of the mrist-joint.

Fig. 109. Enucleation of the hand. Formation of a plantar flap.

Fig. 110. Amputation of the forearm. Cutaneous incision.

Fig. 111. Enucleation at the elbow-joint. Cutaneous incision.

Fig. 112. Amputation of the arm. Circular incision. 
Figs. 113, 114. "Flap-ineisions for amputation and enucleation of the arm. Fig. 115. Fxarticulation of the shoulder. Formation of an axillary musculueutaneous tiap.

Figs. 116, 11\%. Exarticulation of the shoulder by the method of Esmarch. Fig. 118. Resection of the shoulder. Cutaneous incision.

Figs. 119, 120. Reseetion of the shoulder by the methed of Iangenbeck. Fig. 121 . Resection of the elbow-joint. Situation of the ulnar nerve. Figs. 122-124. Incisions for resection of the elbow-joint.

Fig. 125. Resection of the elbow-joint. Exposure of the constituents of the joint.

Figs. 126. 127. Resection of the wrist-joint. Dorsoradial incision.

Fig. 125. Resection of the hip-joint. Cutaueous ineision.

Figs. 129, 130. Resection of the knee-joint. Anterior arched incision by the method of Textor.

Fig. 131. Suprapatellar ineision by the method of Hahn.

Fig. 132. Resection of the ankle-joint. Bilateral longitudinal incision, br the method of König.

Figs. 133-135. Resection of the ankle-joint bs the method of ReverdinKocher.

Figs. 136-13s. Reseetion of the foot br the method of WladimiroffMikulicz.

Figs. 139, 140. Temporary resection of the skull.

Fig. 141. Trephine-situation for exposure of the meningeal artery.

Figs. 142-144. Resection of the upper jaw.

Figs. 145, 146. Resection of the lower jaw.

Fig. 14i. Temporary resection of the lower jaw by the method of Sedillot.

Fig. 148. Submental extirpation of the tongue by the method of Billroth.

Fig. 149. Cutaneous incision for extirpation of the tongue by the method of Kocher.

Figs. 150, 151. Wedge-shaped excision of the lower lip. Suture.

Figs. 152, 153. Cheiloplasty bs the method of Dieffenbach.

Figs. 154, 155. Cheiloplasty by the method of Langenbeck.

Figs. 156-153. Rhinoplasty with the skin of the forehead.

Figs. 159, 160. Partial rhinoplastr.

Figs. 161-163. Nélaton's operation for cleft palate.

Figs. 164-166. Malgaigne's operation for cleft palate.

Figs. 16\%-169. Mirault-Langenbeek's operation for cleft palate.

Figs. 170-172. Operation for bilateral cleft palate.

Fig. 173. Incisions for the relief of tension after extensive division of the lip. 
Fig. 174. Exposure of the frontal nerve.

Fig. 175. Exposure of the infra-orbital nerve.

Fig. 176. Exposure of the mental nerve.

Fig. 17. Cutaneous incisions for operations upon the air-passages.

Figs. 178, 179. Tracheal cannulæ.

Figs. 180-184. O'Dwyer's outfit for intubation.

Fig. 185. Esophagotomy.

Fig. 186. Cutaneous incisions for ligation of the vessels of the neck.

Fig. 18\%. Scalene opening.

Fig. 188. Amputation of the mammary gland.

Fig. 139. Elevation of the pelvis.

Fig. 190. Incisions in the abdominal wall.

Figs. 191-196. Varieties of catheters and sounds.

Fig. 19\%. Introduction of a partially rigid instrument into the bladder.

Figs. 198, 199. Introduction of a rigid instrument through the urethra.

Figs. 200-203. Catheterization.

Fig. 204.

Attachment of retention-catheter.

Figs. 205-20\%. Puncture of the bladder.

Fig. 208. Suprapubic cystotomy.

Figs. 209, 210. Prostatotomy.

Figs. 211, 212. Operation for shortened frenulum.

Figs. 213, 214. Plastic operation for urethral fistula.

Fig. 215. Exposure of the constricting ring of an inguinal hernia.

Fig. 216. Exposure of the kidney.

Fig. 217. Invagination-suture of the stumps of the ureters. 


\section{CONTENTS.}

\section{A. Division of the Tissues.}

Mode of holding the knife . . . . . . . . . . . 15

Dissection of the tissues . . . . . . . . . . 2t;

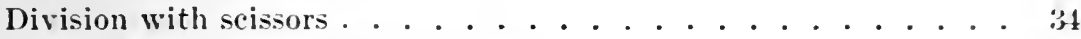

Division of tissues by puncture . . . . . . . . . . . 33

Bloodless method of dividing tissues . . . . . . . . . . . 39

Division of bone . . . . . . . . . . . . . . . 41

B. Reunion of the Tissues.

Suture of wounds . . . . . . . . . . . . . . 44 4

Suture of muscles . . . . . . . . . . . . . . . . . . . . 49

Suture of tendons . . . . . . . . . . . . . . . . . . . 49

Suture of nerves . . . . . . . . . . . . . . . 51

Suture of bones. . . . . . . . . . . . . 52

Intestinal suture . . . . . . . . . . . . 53

Suture of the bladder. . . . . . . . . . . 55

\section{Operations on the Extremities.}

1. Ligation of Vessels in Continuity.

Indications . . . . . . . . . . . . . . . 5i

General considerations upon performance of ligations . . . . . 55

Ligations in the upper extremities . . . . . . . . . 62

Ligation of the axillars artery . . . . . . . . . 633

Ligation of the brachial artery . . . . . . . . . . 65

Ligation of the cubital artery . . . . . . . . . 66

Ligation of the radial and ulnar arteries . . . . . . . . 70

Ligations in the lower extremities . . . . . . . . . . it

Ligation of the femoral artery . . . . . . . . . it

1. Under Poupart's ligament . . . . . . . . . 75

2. In the middle of the thigh . . . . . . . . . 75

3. In Hunter's canal . . . . . . . . . . . . . . 
Ligation of the saphenous vein . . . . . . . . . . . 77

Ligation of the popliteal arters . . . . . . . . . . . . 77

Ligation of the anterior and posterior tibial arteries . . . . . 80

\section{Amputations and Enucleations.}

Indications . . . . . . . . . . . . . . . . . . . 86

General considerations upon amputations . . . . . . . . 87

Circular incision . . . . . . . . . . . . . . . 88

Flap-amputations . . . . . . . . . . . . . . 96

Oral incision... . . . . . . . . . . . . . . 103

Division of the bone ............... 108

Amputations AND Exarticulatioxs of THE Lower Fxtrenity. Amputation of the leg . . . . . . . . . . . . . . 112

Srme's amputation . . . . . . . . . . . . . . . . 113

Pirogoff's amputation of the foot . . . . . . . . . . . . 120

Achillotenotomy . . . . . . . . . . . . . . . . . 132

Exarticllations Axp Aypltations ix the Regios of the Foot.

Exarticulations through the interphalangeal or metatarsophalangeal

joints . . . . . . . . . . . . . . . . . 132

Amputation of all toes through the metatarsus . . . . . . . . 137

Exarticulation of the great toe with the metatursus . . . . . . 138

Exarticulation through the tarsometatarsal joints by the method of

Lisfranc . . . . . . . . . . . . . . . . . 139

Intertarsal amputation . . . . . . . . . . . . . . . . 146

Intertarsal exarticulation (Chopart) . . . . . . . . . . . . . 149

Subastragaloid enucleation (Malgaigne) . . . . . . . . . 150

Exarticulation of the leg at the knee-joint . . . . . . . . 153

Amputation of the thigh . . . . . . . . . . . . . 154

Circular incision in two stages. . . . . . . . . . . . . 155

Flap-formation . . . . . . . . . . . . . . . . 156

Osteoplastic supracondylar amputation of the thigh by the method

of Gritty . . . . . . . . . . . . . . . . . . . . . . 161

Osteoplastic supracondryar amputation of the thigh by the method of Ssabanajeff . . . . . . . . . . . . . . . 162

Exarticulation at the hip-joint . . . . . . . . . . . . 163

Exarticulation at the hip-joint by the method of Esmarch . . . 163

Exarticulation at the hip-joint, with the formation of two musculo-

tegumentary flaps by transfixion 
Amputations and Exarticulations of the Upper Exthemity.

PAGE

Exarticulations of the fingers through the interphalangeal joints;

through the metacarpophalangeal joints . . . . . . . . 167

Exarticulation of the little finger through the metacarpus by the

method of Walther . . . . . . . . . . . . . . . . 199

Amputation of a finger through the metacarpus . . . . . . . . 174

Exarticulations at the wrist . . . . . . . . . . . . . . . 175

Enucleation of the hand by means of a circular incision and the formation of a cuff . . . . . . . . . . . . . . . . . . . 176

Enucleation of the hand by means of anterior and posterior cutaneous flaps . . . . . . . . . . . . . . . . . . 181

Amputation of the forearm . . . . . . . . . . . . . . 181

Enucleation at the elbow-joint . . . . . . . . . . . 182

Amputation of the arm . . . . . . . . . . . . . . 181

Exarticulation of the humerus . . . . . . . . . . . 185

Deltoid musculotegumentary flaps . . . . . . . . . . . . 186

Oval incision by the method of Esmarch . . . . . . . . . . 188

\section{Resections at the Joints of the Extremities.}

General considerations upon resection of the joints . . . . . . 191

Indications . . . . . . . . . . . . . . . . 192

Resections of THE JoInts of THE UPPER EXTREMITY.

Resection of the shoulder-joint by the method of Langenbeck . . 193

Resection of the elbow-joint by the method of Langenbeck . . . 199

Resection of the elbow-joint by the method of Kocher . . . . . 205

RESECTION OF THE WRIST-JOINT.

Resection of the joints of the hand by the method of Langenbeck . 205

Resections of the JoINts of the Fingers.

Resection at the metacarpophalangeal joint . . . . . . . . 206

\section{Resections of THE JoINTS OF THE LOWER Extrenity.}

Resection of the hip-joint . . . . . . . . . . . . . 207

Resection of the hip-joint by the method of Langenbeck . . . . 207

Resection of the hip-joint by the method of Velpean . . . . . . 207

Resection of the knec-joint . . . . . . . . . . . . . . . 209

Resection of the ankle-joint by the method of Langenbeck . . : . 211

Resection of the ankle-joint by the method of König . . . . . . . 213

Resection of the ankle-joint by the method of Reverdin-Kocher . 214 
Resection of the aukle-joint by the method of Wladimiroff-Mriku-

licz . . . . . . . . . . . . . . 214

Osteotomy . . . . . . . . . . . . . . . 218

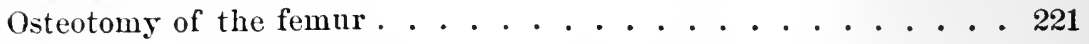

\section{Operations on the Head and Neck.}

Trephining.

Indications for trephining . . . . . . . . . . . . . . 223

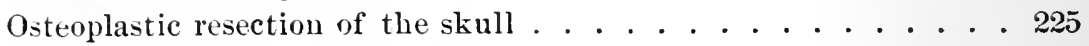

RESECTIONS OF THE JAWS.

Resection of the upper jaw . . . . . . . . . . . . 229

Temporary resection of the upper jaw . . . . . . . . 233

Resection of the lower jaw . . . . . . . . . . . 234

Temporary resection of the lower law . . . . . . . . 239

Median division of the lower jaw . . . . . . . . 240

Lateral division of the lower jaw . . . . . . . . . 240

Operations ox the Tongue.

Submental extirnation of the tongue . . . . . . . . 251

Extirpation by the method of Kocher . . . . . . . 2 251

Plastic Operations.

General considerations . . . . . . . . . . . 252

Rhinoplasty . . . . . . . . . . . . . . . 254

Operation for saddle-nose . . . . . . . . . . 256

Plastic correction of defects of the cheek . . . . . . . . 259

Operations for harelip . . . . . . . . . . . . . . 260

Staphylorrhaphy and uranoplasty . . . . . . . . 265

Operations on the Nerves of the Head.

First (ophthalmic) division of the trigeminus . . . . . . . 267

Second (superior maxillary) division of the trigeminus . . . . 267

Third (inferior maxillary) division of the trigeminns . . . . . . 269

Exposure of the superior and inferior maxillary branches by the method of Krönlein . . . . . . . . . . . . 272

Exposure of the inferior maxillary division at the base of the skull . $2 \pi 3$

Intracranial operations on the trigeminus . . . . . . . 274

Operations on the Air-passages.

Larringotomy

Cricothy rotomy . . . . . . . . . . . 276 
Extirpation of the larynx . . . . . . . . . . . . . 27\%

Tracheotomy . . . . . . . . . . . . . . . 278

Intubation . . . . . . . . . . . . . . . . . 281

Pharyngotomy . . . . . . . . . . . . . . . 28t

Esophagotomy . . . . . . . . . . . . . 288

Ligations OF THE Vessels in THE REgiON OF THE NECK.

Ligation of the imnominate artery . . . . . . . . . . . . . 291

Ligation of the common arotid artery . . . . . . . . . 292

Ijigation of the external carotid artery . . . . . . . . . . 292

Ligation of the superior thyroid artery . . . . . . . . . . 294

Ligation of the lingual artery . . . . . . . . . . . . 295

Ligation of the subclavian artery . . . . . . . . . . . 295

(a) Above the clavicle. . . . . . . . . . . . . . 297

(b) Below the clavicle. . . . . . . . . . . . . . 298

Ligation of the inferior thyroid artery . . . . . . . . . . 299

Operations for goiter . . . . . . . . . . . . . . 299

\section{Operations upon the Trunk and the Pelvis.}

Paracentesis thoracis . . . . . . . . . . . . . . 302

Thoracotomy . . . . . . . . . . . . . . . 303

Ligation of the interual mammary artery . . . . . . . . . . 304

Amputation of the breast . . . . . . . . . . . . . 304

Abdominal puneture .. . . . . . . . . . . . . 307

Celiotomy .................. . . 30s

Operations upon the STOMACH AND THE INTESTINes.

Formation of a gastric fistula ; gastrostomy . . . . . . . . 311

Formation of an intestinal fistula; enterostomy . . . . . . . 313

Formation of a preternatural anus . . . . . . . . . . 313

Resection of the bowel . . . . . . . . . . . . . . 315

Exclusion of the bowel . . . . . . . . . . . . 317

Gastro-enterostomy . . . . . . . . . . . 318

Operations upoN THE Biliary APIARATUs.

Operations upon the gall-bladder . . . . . . . . . . . . . 319

Operations upon the cecum and upon the vermiform appendix . . 321

OPERATIONS UPON THE URINARY ORgANS.

Catheterization . . . . . . . . . . . . . . 3:2

Puncture of the bladder . . . . . . . . . . . . . 336

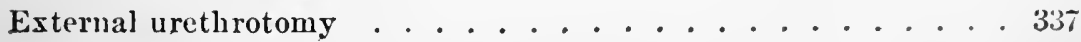


External urethrotoms with a guide . . . . . . . . . . 340

External urethrotoms without a guide . . . . . . . . . 340

External urethrotoms in the presence of rupture of the urethra . . 342

Interual urethrotomy . . . . . . . . . . . . . 343

Lateral section . . . . . . . . . . . . . . . 344

Yedian section . . . . . . . . . . . . . 344

Urethrostomy . . . . . . . . . . . . . 345

Litholapasy . . . . . . . . . . . . . . . 345

Suprapubic section . . . . . . . . . . . . 347

1. High incision for stone . . . . . . . . . . . . . 348

2. High incision for the performance of endovesical operations . 349

3. High cystostomy . . . . . . . . . . . . . . 350

Operations tpon the Prostate, the Semixal Vesicles, axd THE VAS DEFerexs.

Prostatotomy . . . . . . . . . . . . . 355

Extirpation of the seminal vesicles . . . . . . . 356

Prostatectomy . . . . . . . . . . . . 356

Resection and excision of the vas deferens . . . . . . . 35ז

Estirpation of the testicle . . . . . . . . 358

Operation for hrdrocele. . . . . . . . . . . 359

Operation for phimosis . . . . . . . . . . 360

Amputation of the penis . . . . . . . . . 362

Urethral fistulæ ............... . . 363

OPERATIONS FOR HERNiA.

Herniotomy . . . . . . . . . . . . . . . 364

Radical operation . . . . . . . . . . . . . . . . . 367

(a) For inguinal hernia . . . . . . . . . . 367

(b) For femoral hernia . . . . . . . . . . . 371

(c) For umbilical hernia ... . . . . . . . . . 371

Ligation of the iliac arters . . . . . . . . 372

Operatioss upox the KipNeYs.

Nephrotomy and Nephrectoms . . . . . . . . . . 374

Operations npon the ureters . . . . . . . . . . . 377

Operations upor the Rectum axd the ANus.

Amputation and resection of the rectum . . . . . . . . 379

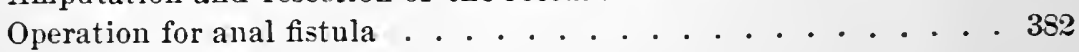

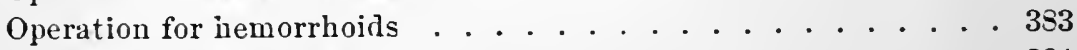

Operation fcr atresia ani ................ 384 


\section{OPERATIVE SURGERY.}

\section{GENERAL CONSIDERATIONS.}

\section{(A) Division of the Tissues.}

The soft parts may be divided by either bloody or bloodless means : by bloody means, with the linije or the scissors or by puncture; by bloodless means, with the actual cautery, the glowing or the cold wire-loop, the elastic ligature, and the écraseur.

Bones may be divided either with chisel and mallet, with the sair, with bone-shears, or bone-forceps, or with appropriate apparatus in special situations (osteoclasis).

The most important and the most generally used instrument of the surgeon, the linife, consists of a blade and a handle, which are either made of one piece or are articulated by means of an adjustable joint.

In accordance with the form of the blade the following varieties of knives are recognized:

The bellied scalpel;

The sharp-pointed knife ;

The blunt-pointed knife.

In the bellied scalpel (Fig. 3) the cutting-edge is convex, and the back straight and continuous with the handle of the knife.

The sharp-pointed linife is convex upon its back as well as upon its cutting-surface, terminating in a sharp point (Fig. 2).

The blunt-pointed linife is provided with a peripheral button-like extremity (Fig. 1). 
FIG. 1.-Blunt-pointed knife.

FIG. 2.--Sharp-pointed knife (sharp-pointed bistoury).

Fig. 3.-Simple bellied scalpel.

FIG. 4.-Resection-knife.

FIG. 5.-Amputation-knife.

FIG. 6.-Tenotomes, with convex and concave cutting-edges.

FIG. 7.-Blunt-pointed bistoury.

In accordance with the size and construction of knives further distinctions are made of amputation-knives (Fig. 5), resection-knives (short, strong knives, Fig. 4), tenotomes (Fig. 6), etc.

Division of tissues with the knife is effected by a combination of pressure and traction, the proper amount of each force employed being a matter of experience. Beginners often err in exereising only pressure with the cutting-edge of the knife.

For the dirision of the skin from the surface a simple bellied scalpel is used. This is grasped between the thumb, the index and the middle finger like a pen, the ulnar border of the little finger resting upon the operative surface, while the tissues to be divided are made tense with the fingers of the left hand (Fig. 8). This is the mode of holding the knife in making small cutaneous incisions and in free dissection of the tissues.

In making ineisions of greater length the knife is held between the thumb and the pulps of the four fingers like a violin-bow, the tissues to be divided being made tense with the left hand. The operating hand is held free, without any support whatever (Fig. 9).

If the knife is to be carried, with a single stroke, through a thick layer of soft structures down to the bone, as, for instance, in exposing a joint, it is held like a tableknife and pushed through the soft tissues (Fig. 11). The division is effected by a sawing movement of the knife.

For the subcutaneous division of tendons the tenotome is grasped either between the thumb, the index and the middle finger (Fig. 12), or as an ordinary knife is held 
DIVISION OF THE TISSLES.

19
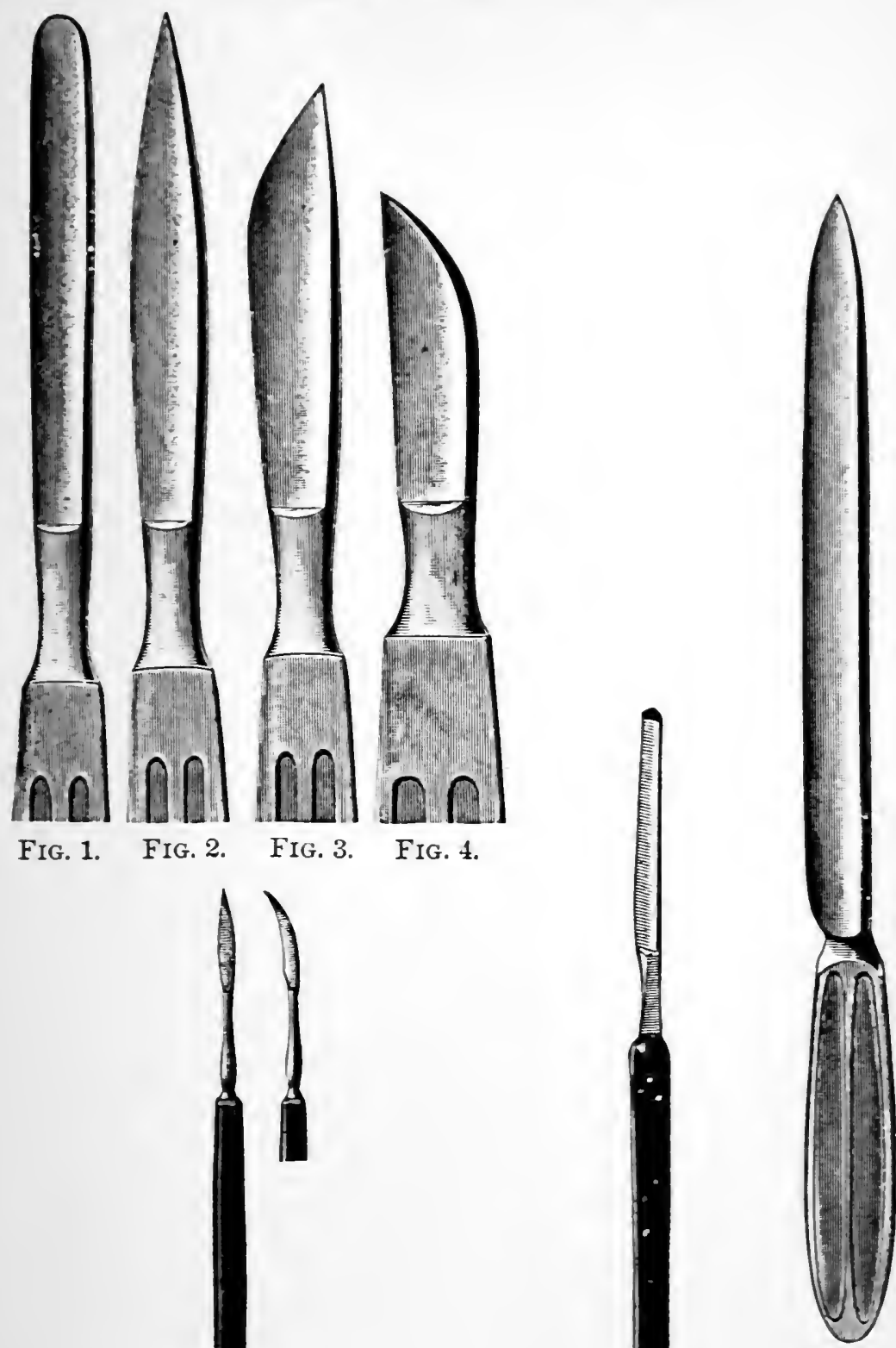

FIG. 5.

FIG. 6.

FIG. 7. 



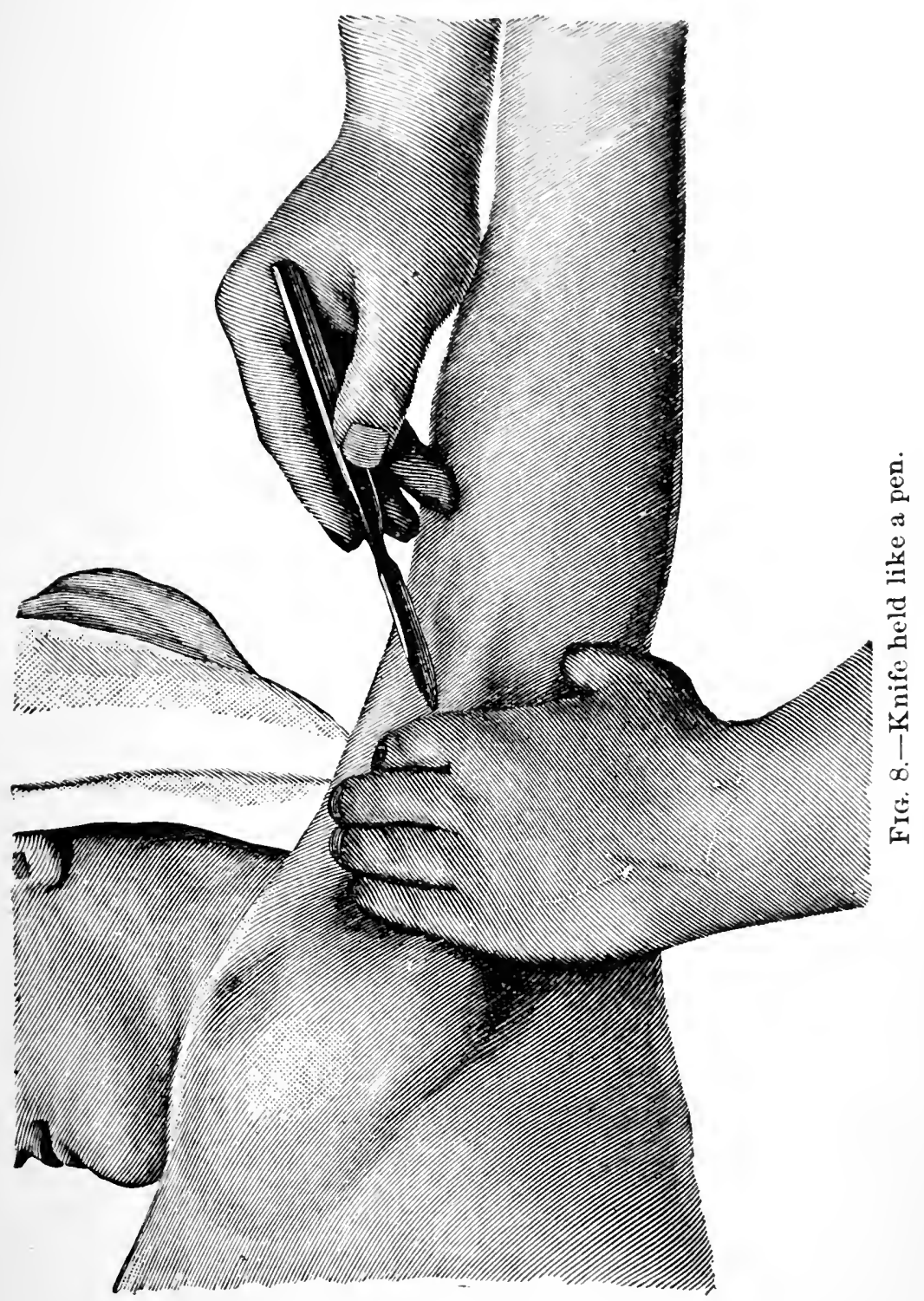





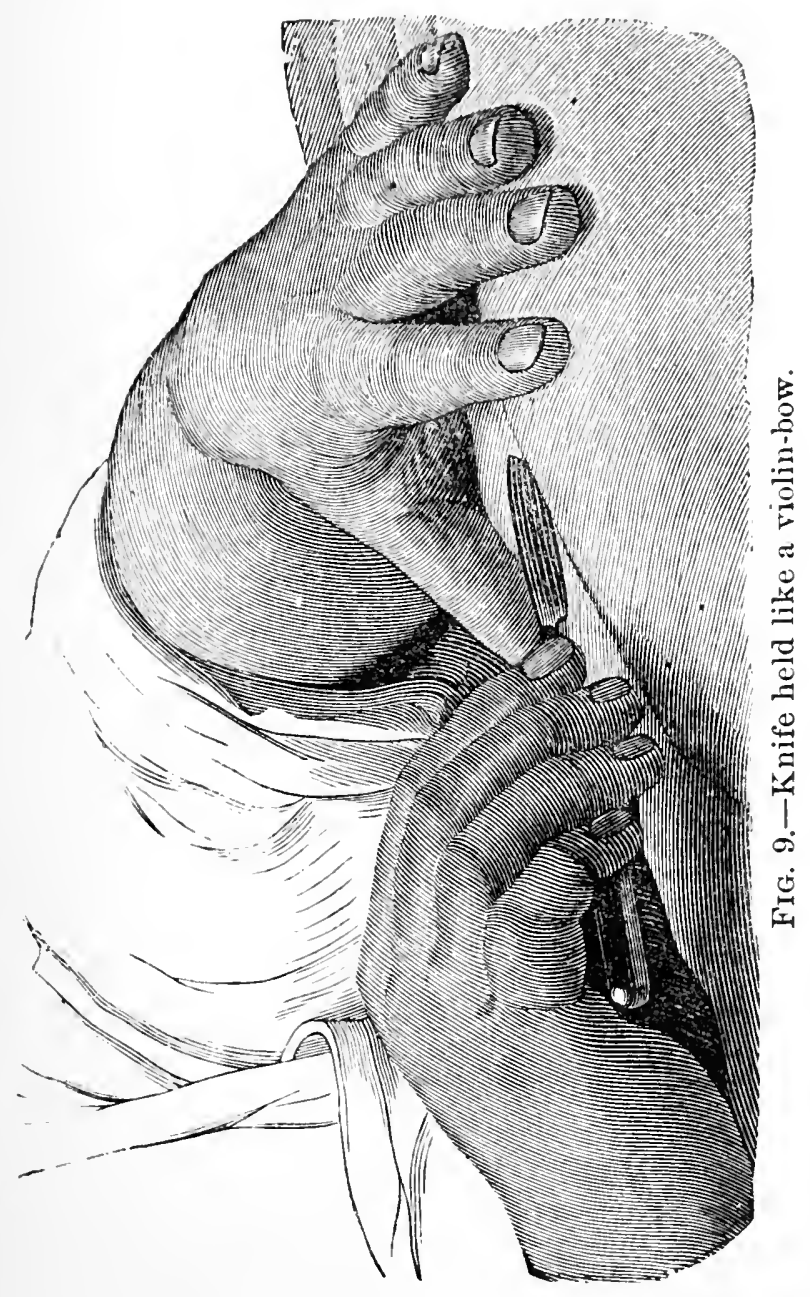



in paring fruit, within four fingers, while the thumb is supported upon the operative surface (Fig. 13).

If the skin is to be divided from within outward, a sharp-pointed knife is used, which is passed at right angles through the base of a raised fold of skin and carried toward the skin (Fig. 10).

If a circular incision through the skin in the entire periphery of a part is to be made-for example, around the ankle-the knife is grasped within the whole fist, with the cutting-edge applied at right angles upon the skin covering the surface of the extremity opposite to the side upon which the operator stands, being earried around

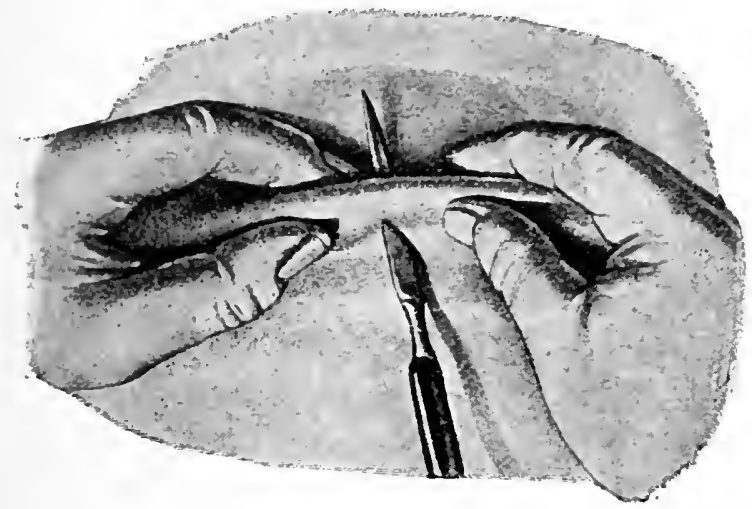

Fig. 10.-Division of a raised fold of skin from base to surface.

the extremity according to rules that will receive discussion later (see page 88).

In general, cutaneous incisions should sever the skin vertieally. Oblique division of the skin is indicated only in certain cases.

Cutaneous incisions, in accordance with their form, are either linear, semilunar, tongue-shaped (flap), T-shaped, $\mathrm{H}$-shaped, or I-shaped, trapdoor-like, anchor-shaped (I), ete.

The length of the incision varies in accordance with the depth to which access is desired. In general, cutaneous incisions should not be too small, as longer inci- 
sions permit careful inspection of deep-seated structures, and are thus more convenient and also more conservative.

Deep Dissection.-Accurate surgical dissection consists in attaining the desired end, the exposure or the enucleation of a structure, with most complete protection possible of arljacent tissues. Structures that obstruct the

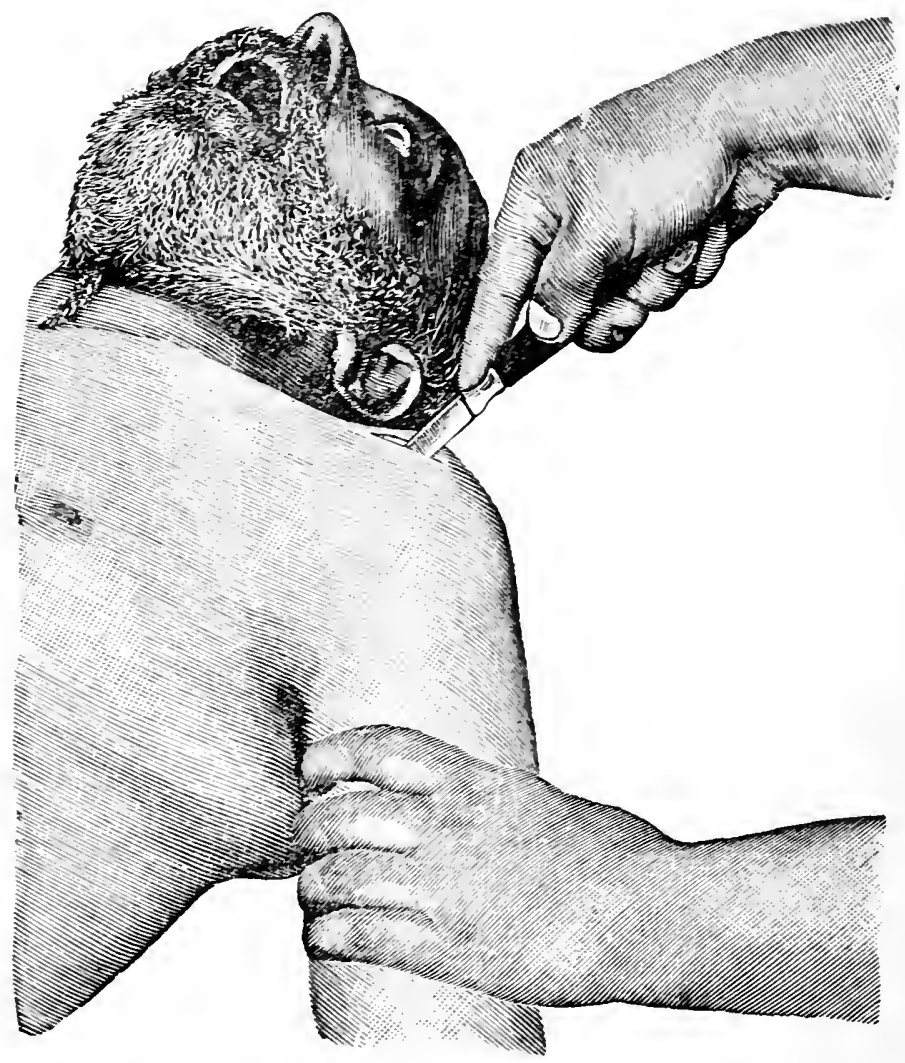

FIG. 11.-Mode of using the resection-knife.

field of operation may be displaced by means of retractors or tenacula (blunt, sharp, with one or more teeth); nerves and vessels should never be held between the blades of forceps, but should always be carefully displaced with the aid of blunt retractors. If a blood-vessel be in the way, it may be secured with two ligatures and divided 
DIVISION OF THE TISSUES.

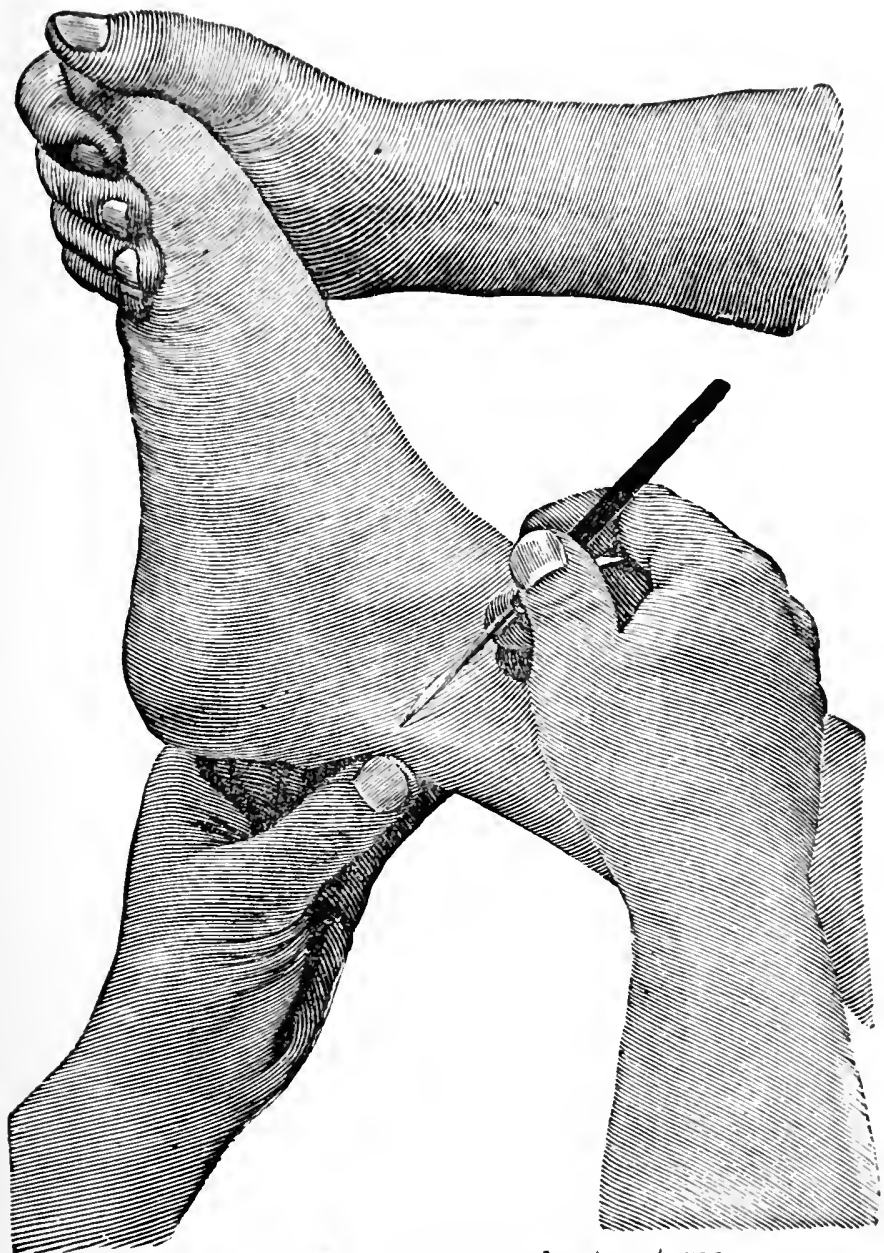

FIG. 12.-Mode of using the tenotome. 



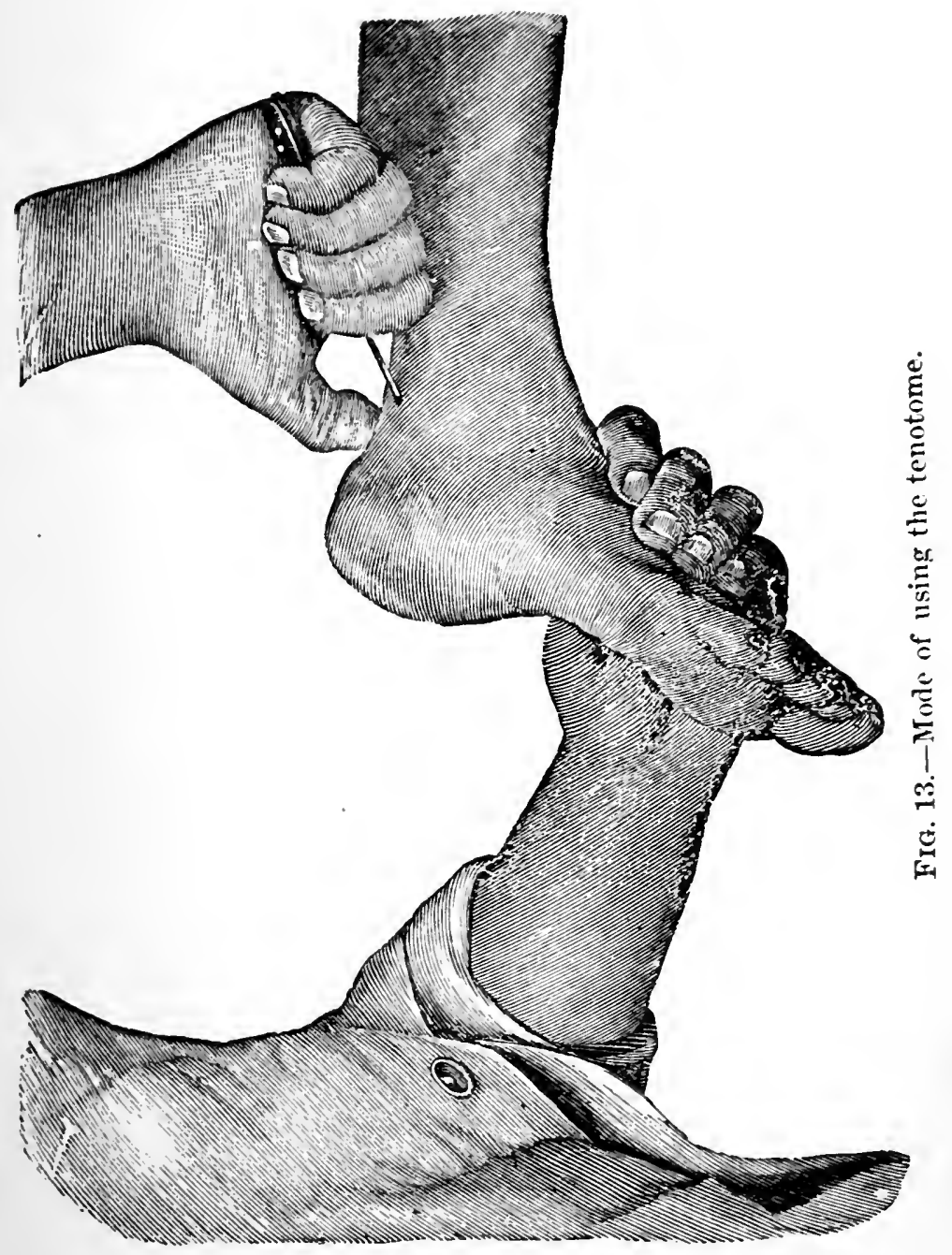




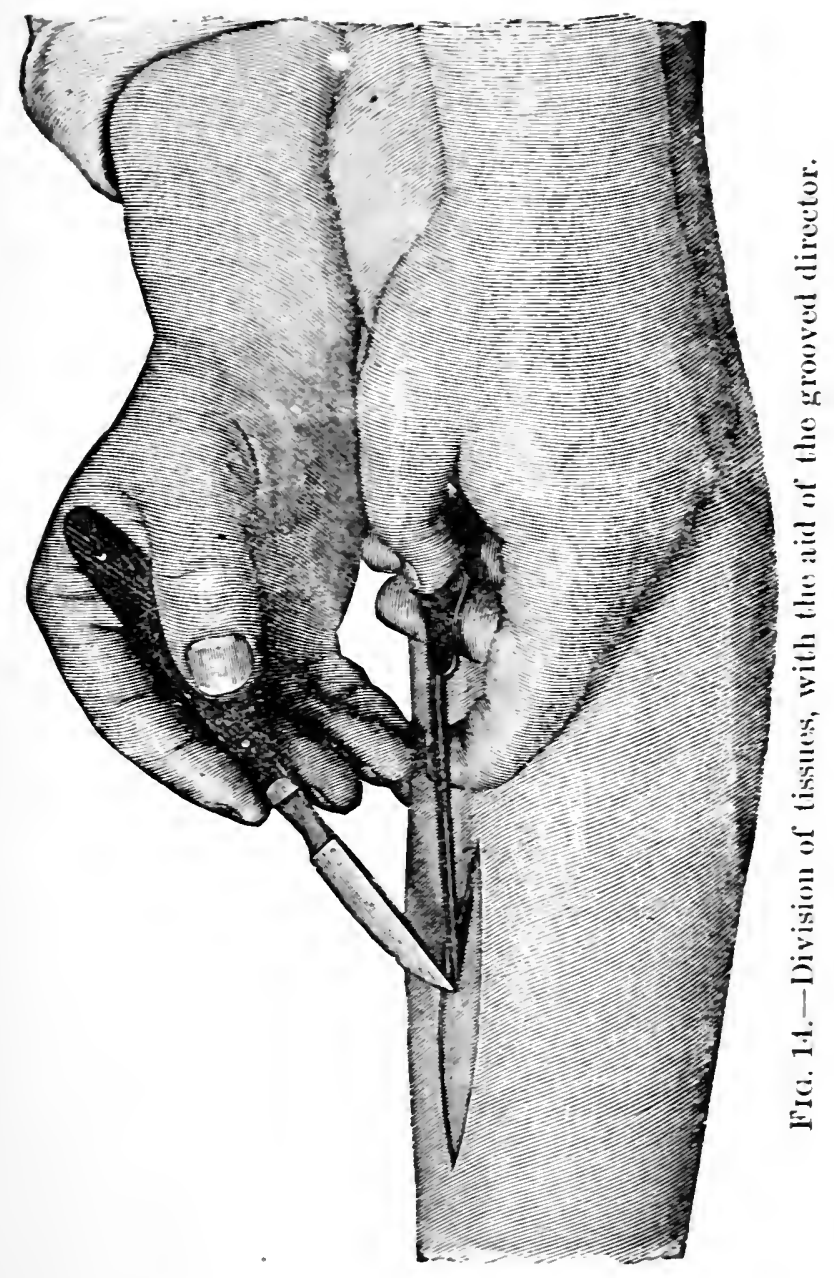


between these. In order to practise deep disseetion safely several methods may be pursted :

(1) Free dissection,

(2) Dissection with the aid of the grooved director,

(3) Dissection between two pairs of foreeps,

(4) Blunt dissection.

Free dissection with the sealpel requires anatomical certainty and skill in the use of the knife, which is held like a pen.

Dissection with the aid of the grooved director is to be recommended when in the course of operation the anatomical relations permit the tissues to be separated layer by layer. It is applicable in the performance of herniotomy, in the open operation for hydrocele, in division of the abdominal walls, etc. With a pair of anatomic forceps held in the left hand a small cone of the tissue to be divided is raised up and slit open at its base with a knife. Through the opening thus made a grooved director is introduced, with its groove directed upward, and pointed in the direction of the proposed incision. The groove of the director serves as a guide for the back of the knife in making the incision (Fig. 14). ${ }^{1}$

In operating between two pairs of forceps the operator and his assistant pick up successively the tissues to be divided at opposite points, and the fold of skin thus raised is incised between the two instruments (Fig. 16). This mode of procedure is applicable in opening the abdominal cavity and in the performance of herniotomy.

Blunt dissection for the isolation of structures in loose cellular tissue is accomplished by pushing the tissues back with two pairs of anatomic forceps and thus avoiding hemorrhage. This method is especially indispensable in the exposure of vessels and nerves and of the trachea. ${ }^{2}$

If a considerable layer of muscular tissue is to be

1 It is rarely advisable to use a grooved director. It lacerates parts, gives irregular incisions, and hence militates against primary union. A surgeon rarely finds the instrument necessary.-ED.

${ }^{2}$ In accomplishing blunt dissection the Allis dissector is of the greatest value.-ED. 
divided with the first incision, a strong, short, resectionknife is used. This is grasped like a table-knife, and pushed vigorously through the soft structures down to the bone, when with sawing movements thick layers of muscle may be divided (Fig. 11).

For circilar division of masses of muscle the knife is held within the whole fist and used according to certain rules (see page 95).

If a band in the depth of the wound and not accessible to the eye, or a constricting ring, is to be divided, this is usually effected by means of the bhunt knife or herniotome under guidance of the finger. The blunt point protects the tissues from injury when the knife is introduced, as

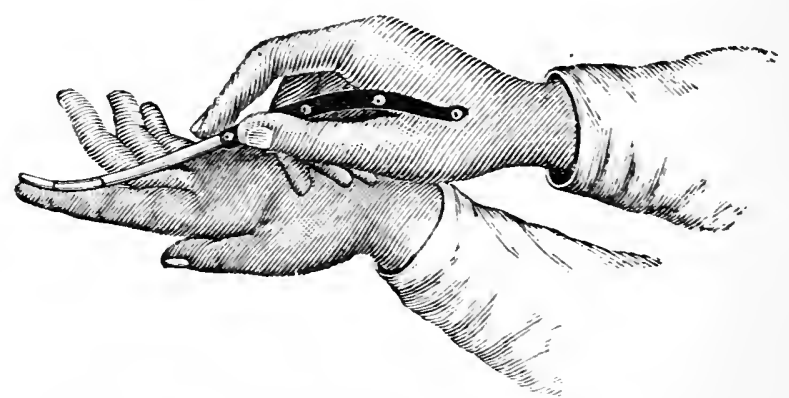

Fig. 15.-Mode of using the blunt-pointed knife.

well as the pulp of the left index-finger, upon which the knife is supported. After the precise point at which the incision should be made has been determined by means of the introduced index-finger, the bhunt-pointed knife is grasped like a pen and, with its back supported upon the palmar surface of the index-finger, is introduced into the depth of the wound. The division is effected through the pressure exerted by the finger upon which the knife is resting (Fig. 15).

Division with Scissors. -The scissors is used for the division of strand-like structures, tendons, muscles, vessels; also certain structures that on account of their consistency are unsuitable for division with the knife, as, 


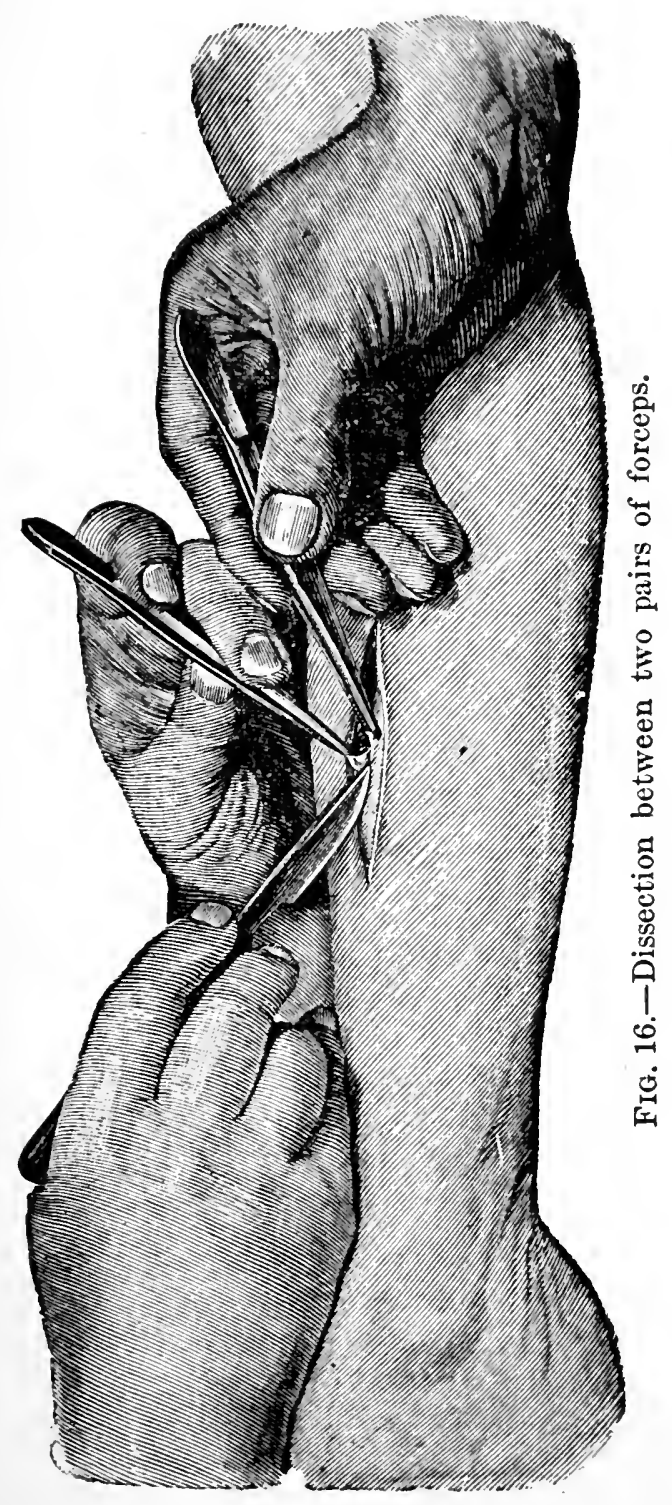



for instance, the yielding intestinal tissues when resected are divided by the scissors. The seissors may also be used alternately with a knife in the dissection of tissnes in the process of enucleating structures. ${ }^{1}$ The blunt blade of the seissors is introduced beneath the layer of tissues to be divided.

Seissors with straight blades or scissors eurved upon the flat are employed. In using the scissors the thumb)

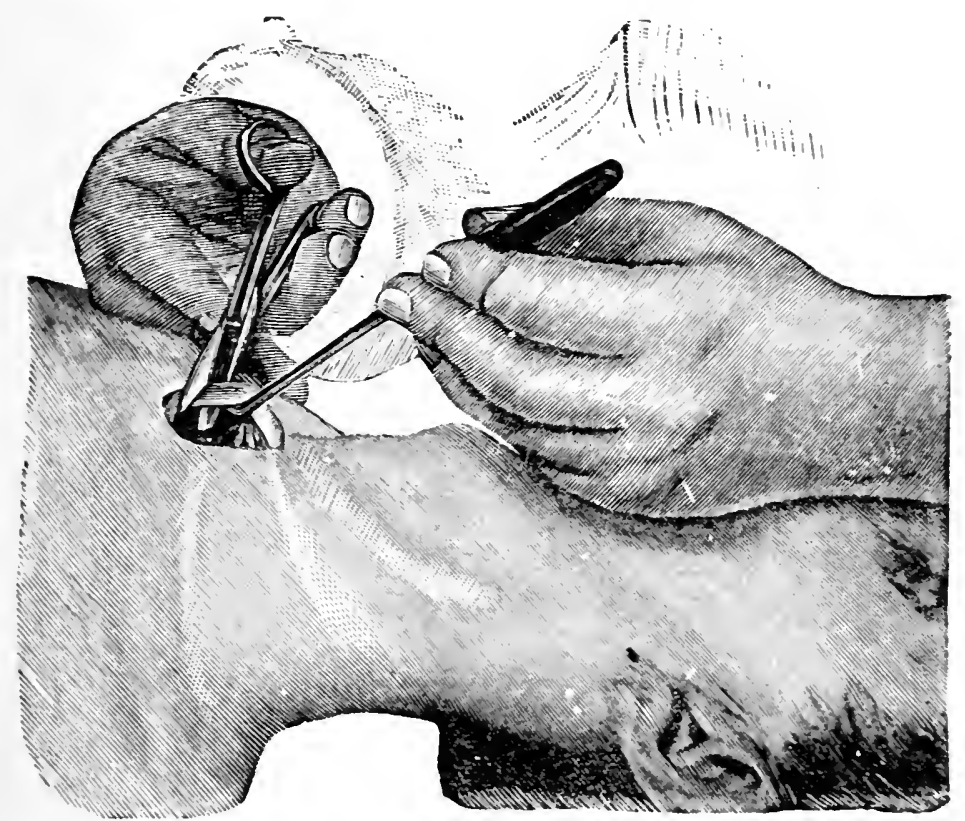

FIG. 17.-Division with scissors.

and the middle finger are introdueed into the rings of the handles and the index-finger is placed upon the lock (Fig. 17).

The tissues to be divided are caught between the blades of the sciscors, division being effected by the movement of the blade held by the thumb against the other, which is grasped firmly.

1 The scissors are very useful in enucleating dermoid cysts, sebaceous cysts, adherent fatty tumors, burse, and fibromata.-ED. 
Division of Tissues by Puncture.-Division of tissues by puncture is practised when fluid is to be evacuated through a cannula introduced into pathological or physiological cavities (puncture of abscesses, of hydrocele); or when a sharp, hollow needle is employed to introduce fluid into the cellular tissue or into the parenchyma of organs (subcutaneous, parenchymatous injections). Exploration with the introduced needle is at times necessary for diagnostic purposes in the depth of the tissues. Finally, in the introduction of sutures, puncture-canals are necessary.

For puncture, straight or curved tubular instruments (trocars) are required, which are provided with a stilet, whose sharp extremity projects a slight distance beyond

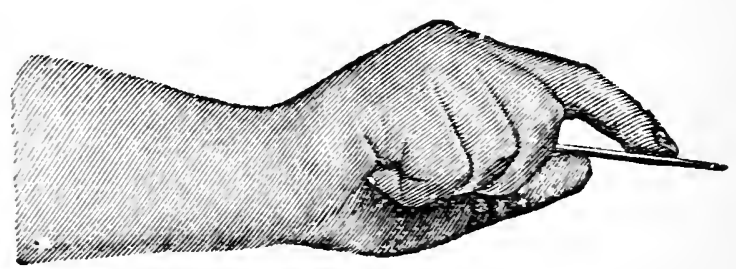

FIG. 18.-Mode of using a trocar.

the end of the tube. In making a puncture the trocar is so held in the full fist that the instrument rests in the hollow of the hand, the index-finger marking the point upon the shaft to which it may enter (Fig. 18).

With a vigorous push the instrument is forced rertically through the skin in the selected situation and into the cavity. The entrance of the trocar into the cavity is indicated by a change in the sense of resistance. The cannula is now grasped at its extremity with the thumb and the index-finger of the left hand and the trocar is withdrawn.

Puncture made with slender instruments for diagnostic purposes is known as exploratory puncture. The escape of the contents through the slender tube of the cannula 
must often be aided by aspiration by means of an attached syringe. ${ }^{1}$

For the purpose of making subentaneons injections a fold of skin is raised and the needle of the syringe is introduced horizontally through the skin into the subentaneous cellular tissue, into which the fluid is foreed by pressure upon the piston of the syringe." In making parenehymatous injections the needle of the syringe is introduced directly through the skin into the interior of the organ (thyroid gland, lymphatic glands).
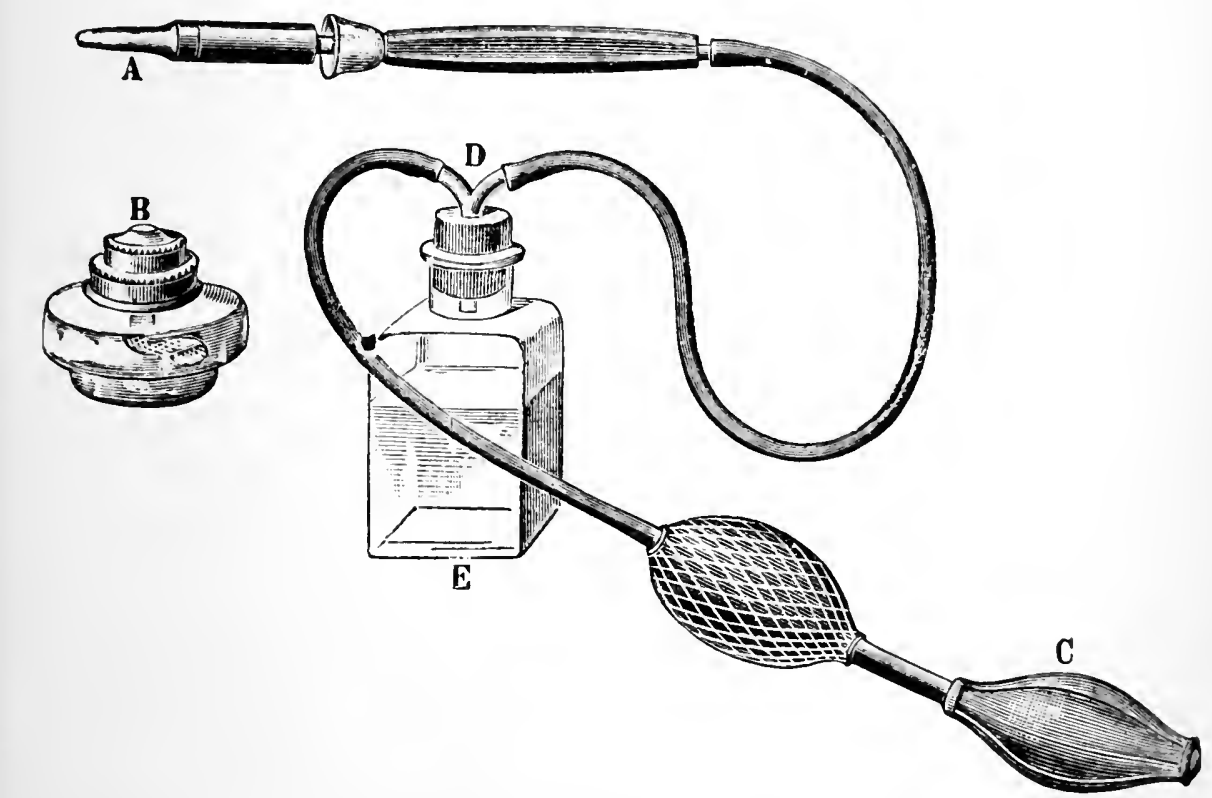

Fig. 19.-The thermocautery of Paquelin: $A$, tip ; $B$, spirit-lamp; $C$, rubber air-bulb; $D E$, reservoir for benzine.

\section{Bloodless Methods of Dividing Tissues.-(1)} The actual cautery, consisting of an iron rod brought to at

1 The old grooved exploring-needle has been generally abandoned in favor of the hollow needle. 'The former instrument is unsafe if infective material is withdrawn by it, as the fluid eomes in contact with the canal of puneture and will probably infect it.-ED.

2 After introducing the needle move it from side to side to see that it is free and to be certain it has not ejtered a blood-vessel.-ED. 
glow in the fire, finds no application in this crude form in

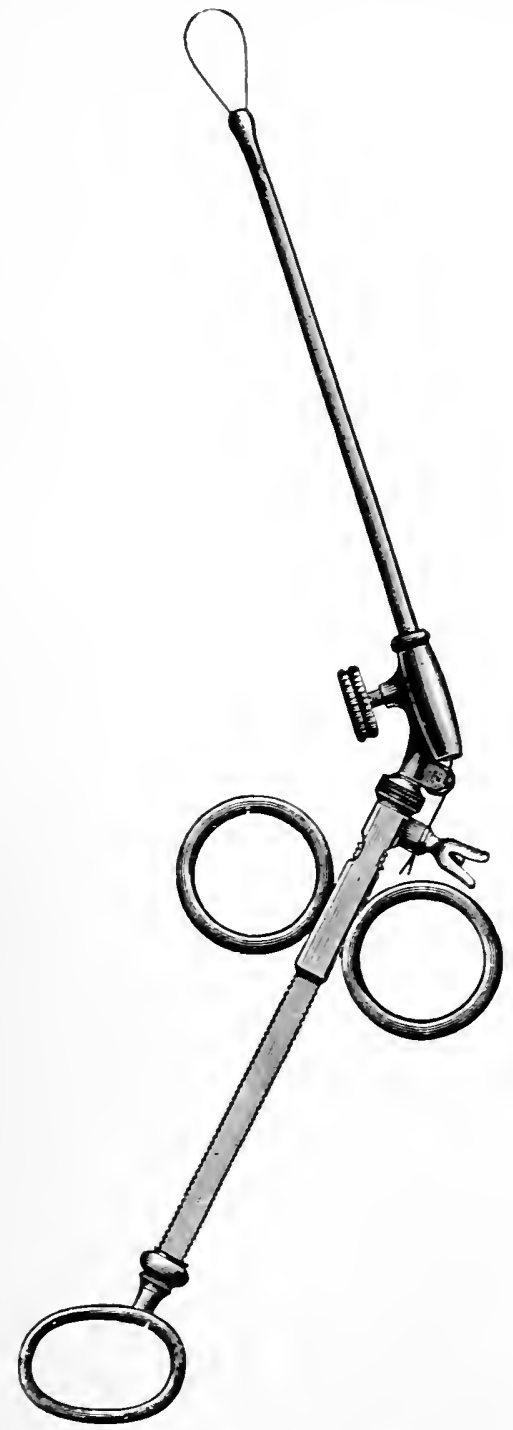

FIG. 20.-Wire loop (snare).

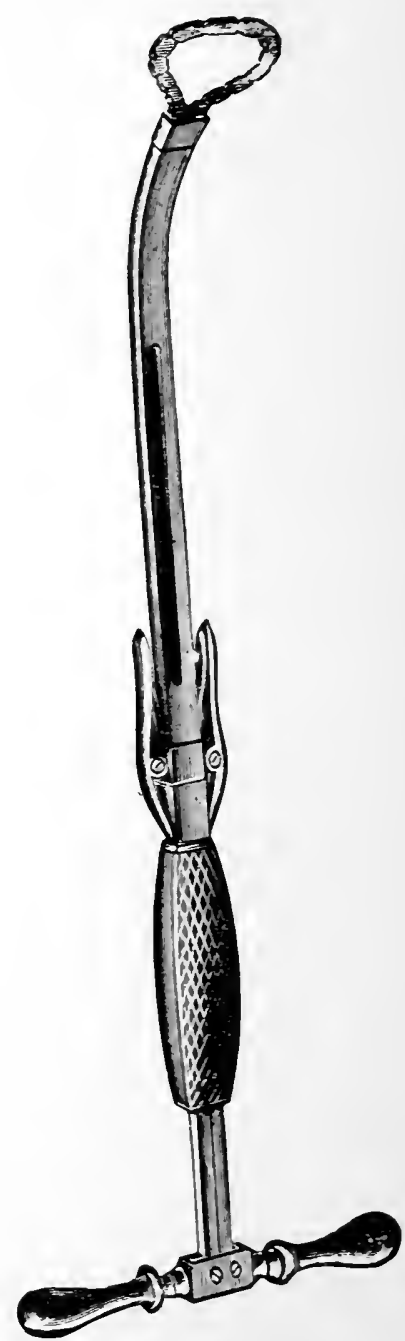

Fig. 21.-Écraseur.

modern surgery, although tissues may be divided without loss of blood by means of the glowing extremity of the 
thermocautery of Paquelin (Fig. 19), or with a wire brought to a glow by means of an electric current (galranocautery). The extremity of the thermocautery brought to a red heat in the flame of an alcohol-lamp may be kept in a continuous glow by means of a fine spray of petroleum-ether. By regulating the stream of petroleum-ether vapor-that is, by more or less energetic manipulation of the air-loulb attached to the apparatusall degrees of incandescence up to white heat may be secured.

$\mathrm{By}$ means of the various linds of loops or snares tissues may be separated at their base if they be so constituted that the loop may be made to surround the line of division. The galuanocaustic snare brought to a glow by means of an electric current and gradually tightened divides the tissues without loss of blood.

The simple wire snare, which crushes directly through the tissues, can only be employed in the removal of structures of slight resistance-for instance, nasal polypi. The tightening of the loop is effected by means of a suitable screw-attachment (Fig. 20).

The elastic ligature (Dittel), an India-rubber band tightly wrapped around the tissues to be divided, operates through the continuous pressure exerted and cuts its way through gradually in the course of days or weeks. The process is so gradual that the surface exposed after separation represents a simple granulating wound. The elastic ligature may also be employed successfully in the severance of dense fibrous pedicles of considerable extent, as in the removal of some uterine myomata.

Écrasement, the crushing of tissues with the aid of a linked chain, was emplosed in large numbers of cases during the middle of the present century. The écraseur of Chassaignac (Fig. 21) permits of quite gradual tightening of the chain, which is withdrawn link by link within the shaft of the instrument. Écrasement has been almost totally displaced by the galvanocautery and the thermocautery.

Division of Bone.-This is effected with the aid of the saw, chisel and mallet, bone-shears, and bone-forceps. 
Bones are broken subeutaneously either manually, or with the aid of special apparatus (osteoclasts).

Bloody division of bone should always be preeded by detachment and division of the periosteum in the line of intended operation.

The arched saw (Butcher's) (Fig. 22) is used whenever the conditions permit of free movement of the instrument,

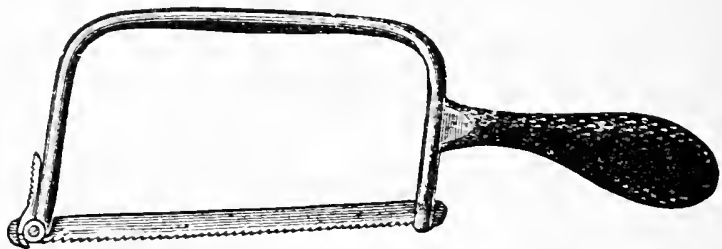

Fig. 22.-Arched saw (Butcher's).

as in division of the bones of the extremities in the course of amputations and of the articular extremities in the course of resections.

In the use of the arehed saw eare should be taken that the instrument is held accurately in the plane of intended division. The saw is first applied with slight, almost with no, pressure, greater foree being employed after a
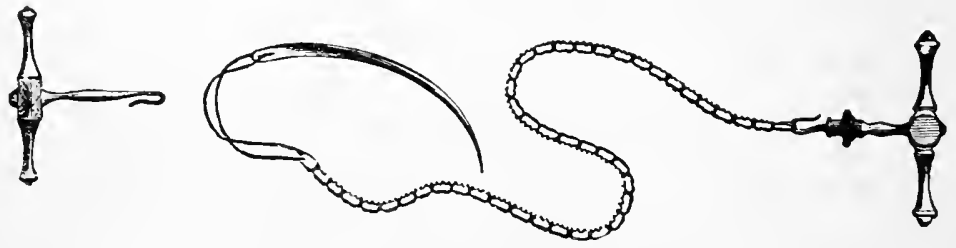

FIG. 23.-Chain-saw.

groove has been formed for the blade of the instrument.

If the bone to be divided is so situated that free movement of the arehed saw is likely to be interfered with, as in division of the lower jaw, of the zygomatic process of the upper jaw, ete., the chain-saw (Fig. 23) or the wire saw may be employed with advantage. The former eonsists of a series of toothed links united by joints, the termi- 
nal links being provided with openings through which appropriate handles may be attached. The wire saw consists of a wire provided with a regular spiral thread like a serew, and it is used in the same mamner as the chain-saw.

Of late it has become customary to use forms of circular saws in operating upon the bones of the skull. 'These may be driven by means of foot-power, or the electric current, or hand-motors (Fig. 24). It is thonght that in employing the circular saw in operations upon the skull the con-

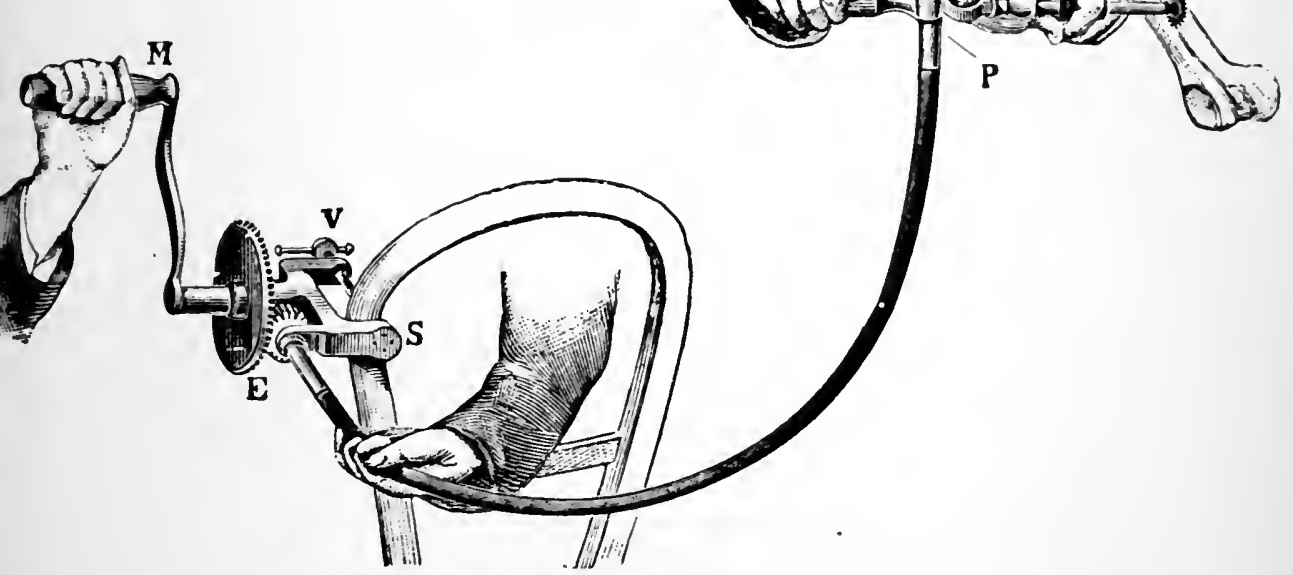

FIG. 24.-Circular saw with hand-motor.

cussion resulting from the use of the chisel and mallet is avoided.

Chisel and mallet likewise may be employed in operations upon the bones of the extremities, as in linear osteotomy, and in chiselling of neeroses, althongh resections with the chisel are also practised upon the bones of the skull.

Thin bony structures, as the ribs, phalanges, projecting splinters upon sawed surfaces, may be divided by means of bone-shears or bone-forceps. 
To freshen bone-surfaces the sharp spoon and strong knices may be used with advantage.

Osteoclasis, the breaking of bone, may be undertaken for orthopedic reasons in cases of badly united fractures and of deformities of the extremities, either manually or with the aid of apparatus (osteoclasts). Manual osteoclasis suffers from the disad vantage that the fracture may fail to take place in the desired situation. Useful forms of osteoclasts have been derised by Rizzoli and by Robin. That of the latter is constructed upon the principle of the onearm lever, and permits the breaking of the bone at preeisely the point seleeted, with the slightest possible injury of the orerlying soft structures.

\section{(B) Reunion of the Tissues.}

The reunion of tissues has for its object the firm and unyielding approximation of the several layers of an existing wound throughout the period of healing. Such approximation may be effected shortly after the reception of the injury (primary suture), or at a time when the wound has entered upon the stage of granulation (secondary suture).

Bloodless approximation of divided tissnes, skin-wounds, by means of adhesive agents, such as eollodion, adhesive

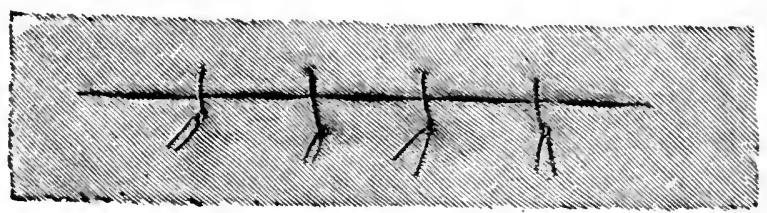

FIG. 25.--Simple knotted (interrupted) suture.

plaster, is only applicable in eases of exceedingly slight injury, without eonsiderable separation of the margins of the wound. Wounds of greater extent, if capable of primary union, should always be closed by bloody suture. 
By means of curved needles (Fig. 29, $a$ and $b$ ) threads are passed through the lips of the wound and tied. The suture is introduced at right angles to the direction of the wound, passing through corresponding points on opposite sides. The needle's are passed either with the free hand or are grasped and directed by forceps-like instruments, needle-holders (Fig. 31). In so-called pedunculated

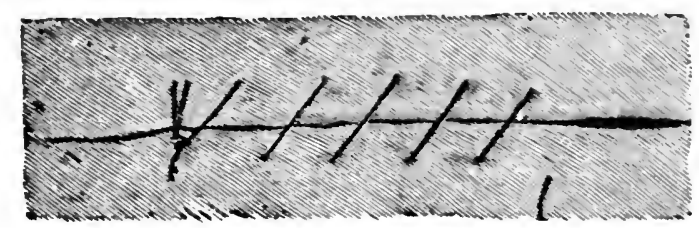

FIG. :6.-. Simple continuous suture.

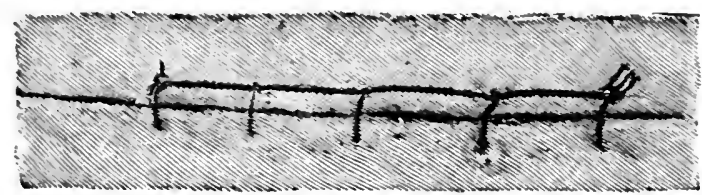

FIG. 27.-Continuous glorer's suture.

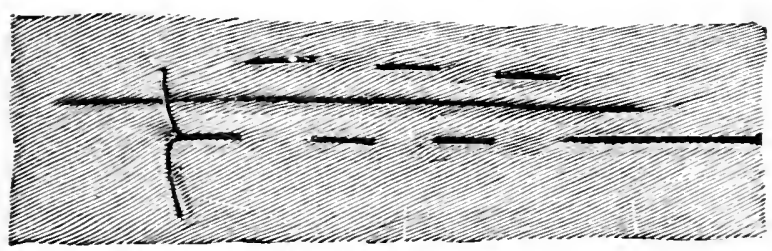

Fig. 28.-Continuous mattress-suture.

needles the needle and its holder form a continuous instrument. $^{1}$

Silk, catgut (absorbable), and metallic wire (silver, lead) are used as suture-material.

If the wound be but superficial, approximation of the margins of the skin with sutures will be sufficient, the needle being introduced in general at a greater or lesser

1 In the United States many surgeons use Hagedorn needles. For intestinal work small, sharp, round sewing-needles are employed, preferably calyx-eyed, which are easily threaded.-ED. 
distance from the margin of the wound, in accordance with its depth.

In order to avoid the formation of cavities in the closure of sinuous wounds it is wise either to unite the tissues in
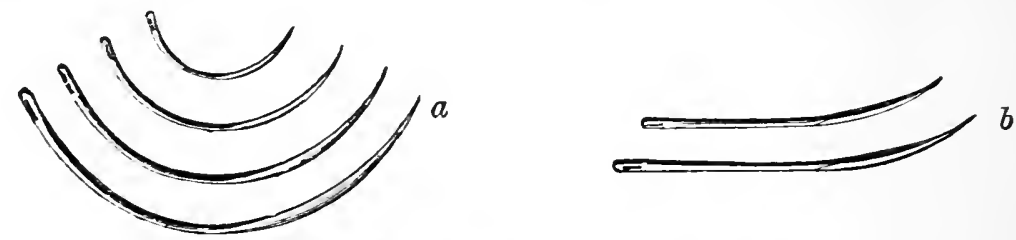

FIG. 29.-Tarieties of surgical needles.

the depth of the wound (buried suture), or to approximate extensive surfaces of the wound.

If the wound be a complicated one, muscles, tendons, and nerves being injured, these structures must be sever-

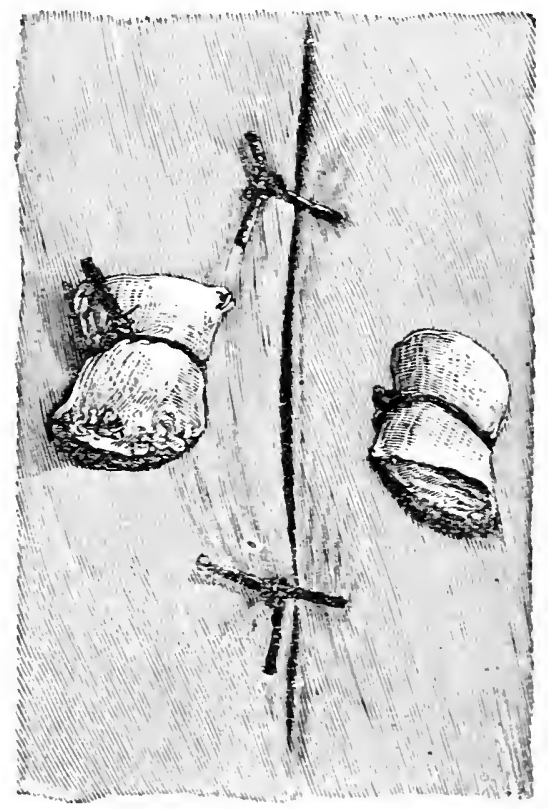

FIG. 30.-Gauze-pad suture.

ally isolated and united before closure of the wound is proceeded with. 
REUNION OF THE TISSUES.

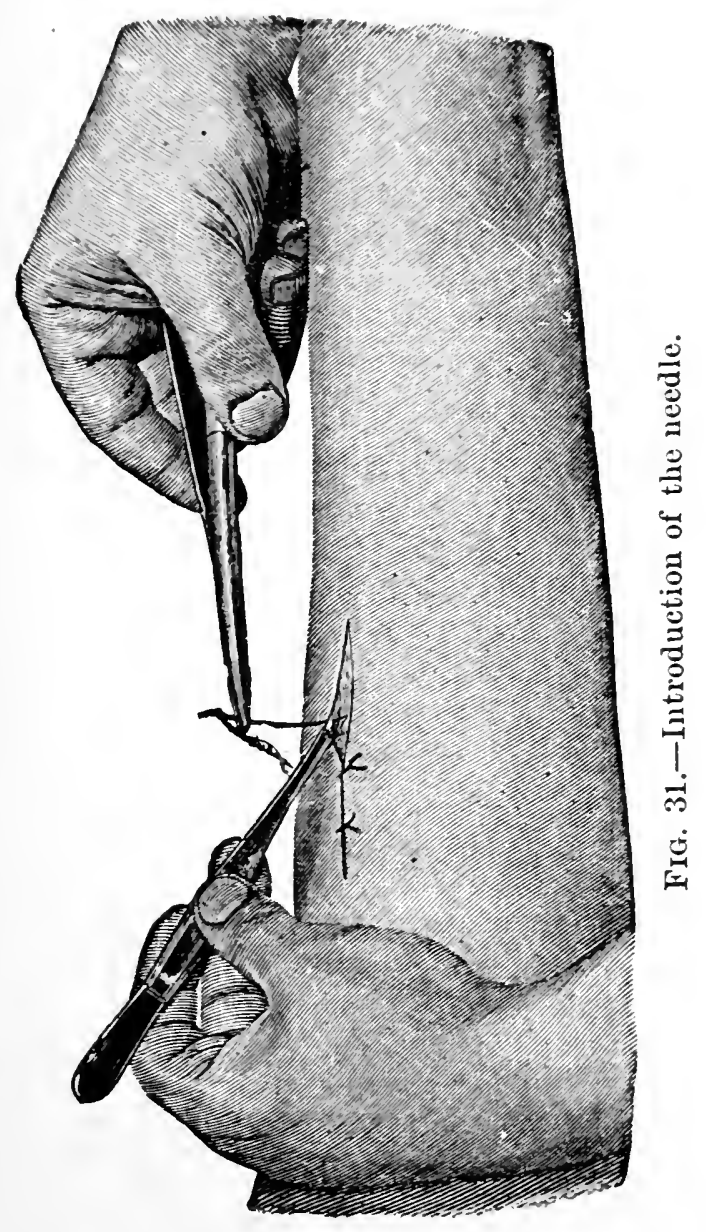



In the elosure of cutancous wounds the simple linotted sills (intermpted) suture is sulficient (1ig. 25). By the introduction of deep and superficial sutures the endeavor is made to secure as perfect approximation as possible of the surfaces of the wound and margins of the skin. Inversion of the latter is to be avoided by andurate apperoximation of the margins of the womnd.

The continuous suture, a single threal being employed withont interruption, is also available in the mion of "altaneous wounds. It is applied in various ways, as in the single rmuning suture (Fig. 26), as the glover's suture (each loop being tied separately, but not cut apart-Fig. 27 ), and as the mattress-suture (rumning back and forth through both lips of the wound-Fig. 28). The mode of introduction is illustrated in the aceompanying figures.

When extensive areas of wound-surface are to be brought in approximation deep sutures are emploved, both extremities of the thread being armed with either lead plates (Lister) or small parls of gäuze (Wölfler) (Fig. 30). As the suture, thus applied, may be drawn firmly togrether and be securely fixed, it is possible to bring the margins of the wound in contact throughout a considerable extent. Interealated knotted sutures will insure exact approximation of the margins of the wound. In using Lister's lead-plate suture wire is employed as the suturematerial, the fixation being effected by means of small perforated lead buttons, which are drawn over the wire, then pressed flat with a pair of forceps and thus securely fixed.

Divided muscles are united with knotted catgut or silk sutures in such a manner that the cut surfaces are brought together in accurate apposition. Kangaroo-tendon is often used as material for buried sutures.

In uniting divided tendons also the cut ends should so far as possible be brought in apposition. In the union of flat, band-like tendons-for instance, the extensors on the dorsum of the hand and on the extensor aspect of the fingers-the divided ends may be overlapped and thus 
sutured. Hüter recommended in all instances the employment of this so-called paratendinous suture (Fig. 32).

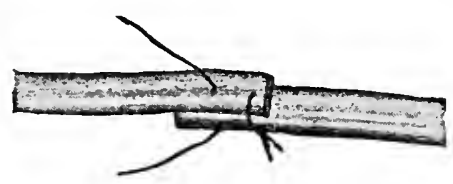

FIG. 32.-Paratendinous suture.

Strong, thick tendons, such as the flexors of the fingers, the tibialis anticus and posticus, the peronei, etc., may, when divided, be united by simple knotted fine silk sutures applied in a longitudinal direction. To prevent cutting through of the sutures these may be advantageously passed at right angles through the divided extremities (Fig. 33).

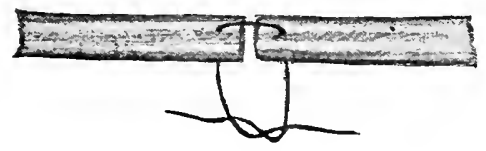

Fig. 33.-Transfixion of divided tendon by suture.

The application of supporting loops of thread, which are subsequently tied together, is also a useful procedure (Fig. 34).

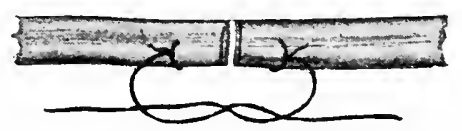

FIG. 34.-Ends of divided tendon held by supporting loops.

Before suture of a tendon is undertaken preliminary operative procedures, such as division of the skin and of the sheath of the tendon, are sometimes necessary in order to secure the central extremity of the tendon. The same end may often be attained by centrifugal bandaging of the part, or with the aid of a sharp tenaculum. After the tendon has been united by suture it is important so to 
place the extremity operated upon in its dressing that the sendon is mantained in a position of greatest possible relaxation until firm mon has taken place.

If by reason of separation of the extremities of a divided tendon approximation cammot be etlected in the usmal manner, the continuity of the tendon may be established by means of an auxiliary operation, tenoplasty. From the side of one of the extremities of the divided tendon a small portion is so freed that it can be turned over toward the other extremity, with which it is united by suture (Fig. 35).!

Nerve-suture, as first practised by Robert and Nélaton, may be employed in eases of recent injuries attended with division of nerves, as well as a secondary procedure after isolation and freshening of the divided extremities. The object of nerve-suture is the approximation of the

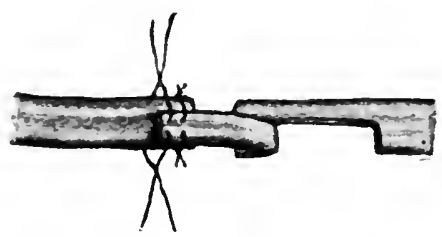

FiG. 35.-Hüter's tenoplasty.

transverse edges of the divided nerve-strand. To this end fine threads are passed either directly through the substance of the nerve, and the transverse surfaces of the divided extremities are brought in apposition, or the extremities are so united that they overlap one another upon their lateral surfaces (paraneuiral suture).

The extremities of the divided nerve may be so united that the sutures are not passed through the substance of the nerve, but through the surrounding connective tissue. In this way the extremities are brought indirectly in approximation (perineural suture).

Neuroplasty, based upon the same principle as the tenoplasty of Hiiter, may also be employed successfully in the union of divided nerves. ED.

${ }^{1}$ Instead of silk, we can employ kangaroo-tendon or chromic gut.- 
The position and fixation of the extremity operated upon should prevent all disturbanee of the nerve after the operation.

The union of bones is effected by suture in a manner analogous to that in which the soft tissues are united (Fig. 36), except that wire is employed with especial advantage as suture-material. The eliannels for the pas-

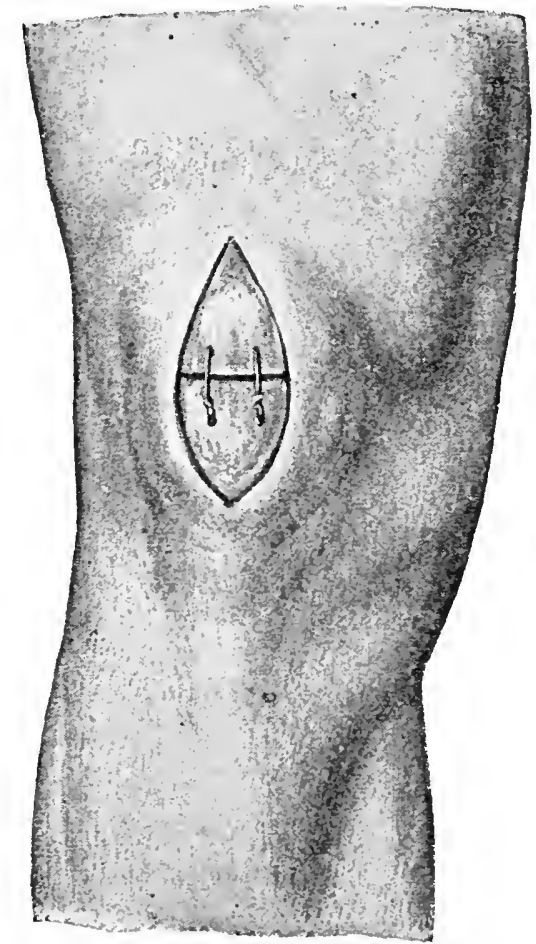

FIG. 36.-Suture of the patella with wire.

sage of the sutures must be made by means of a drill or an awl. The wires are fastened by twisting their extremities together. Braces or clamps also may be employed in the same way as they are used in securing boards in scaffolding (Gussenbauer's clamp, Fig. 37).

Severed bones may further be united by means of nails or ivory pegs (Fig. 38). Nails may also be driven into 
the bone through the overlying soft tissues in oreler to maintain the fiagnents in apposition (percutaneons nailing of bones).

Metallic stitures, pegs, and mails are only to be removed after the lanse of weeks, when firm mion of the divided bone has talien place.

Special Forms of Suture.-Suture of the Bowel.It may be necessaly to mite either a penctriting or at non-penetrating wound of the intestine, of the completely divided bowel throughont its whole cirenmferenere. Thie suture should in general be so applied that the resistant layers of the intestinal wall, such as the muscularis, are brought in aceurate apposition by means of elosely applied

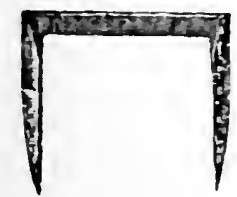

FIG. 37.--Bone-brace.

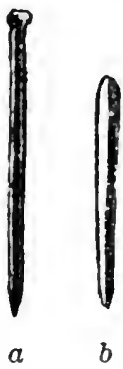

FIG. 33.-a, metallic nail ; $b$, ivory peg.

knotted sutures. Over these broad folds of serosa should be approximated by suture, to facilitate rapid primary union.

In the introduction of intestinal sutures the affected portion of bowel is brought out through the abdominal wound and suitably protected against cold and infection. The escape of intestinal contents should be prevented. To this end the intestine is grasped, centrad and peripherad of the wound to be mited, with suitable clanns. (Gussenbauer's, Rydygier's, Hahn's intestinal clamps), with the fingers, or with bands of sterilized gauze.

Linear wounds of the intestine or the stomach, as well as of the gall-bladder, are united by two rows of closely 


\section{Plate 1.-Suture of the Bowel.}

Fig. 1.-Intestinal sutures introduced from the serous surface. $a$, involving all layers with the exception of the mucosa; $b$, Lembert's seroserous suture.

Fig. 2.-Circular intestinal suture, applied from the lumen of the bowel and including all layers.

placed knotted sutures of silk. In the suture of wounds of the intraperitoneal portion of the urinary bladder catgut is to be preferred. The first row of sutures includes all of the divided layers. It is unimportant whether the mucous membrane be included or not, while it is highly important that a thick layer of the muscularis is included. The sutures are applied closely together and from either the serous surface or the lumen of the bowel. In the first instance the sutures are to be tied externally, in the latter internally. When the suture has been thus applied throughout the whole extent of the wound and slosture has been effected, a seroserous suture (Lembert) is finally introduced. Surfaces of serous membrane from 4 to $5 \mathrm{~mm}$. wide are brought in apposition by introducing the needle a short distance from the margin of the wound and bringing it out close to the margin, and repeating the process in reversed order upon the other side. When the ends of the suture are tied the serons layer on either side is raised in folds, so that two broad surfaces of peritoneum are brought in approximation. In order to give greater stability to the scroserous suture the subserous issues and even a portion of the muscularis are taken up with the serous layer. The folds thus formed are thicker, while a considerable extent of surface is brought in apposition (Plate 1, Fig. 1).

Both rows of intestinal suture may be applied in the form of knotted sutures, or as a continuous suture.

If a divided portion of bowel is to be united throughout its circumference, similar principles may be followed. Two rows of elosely applied sutures will suffice. The tirst includes all of the layers of the bowel, and is in- 
Tab. 1.

Fing 1

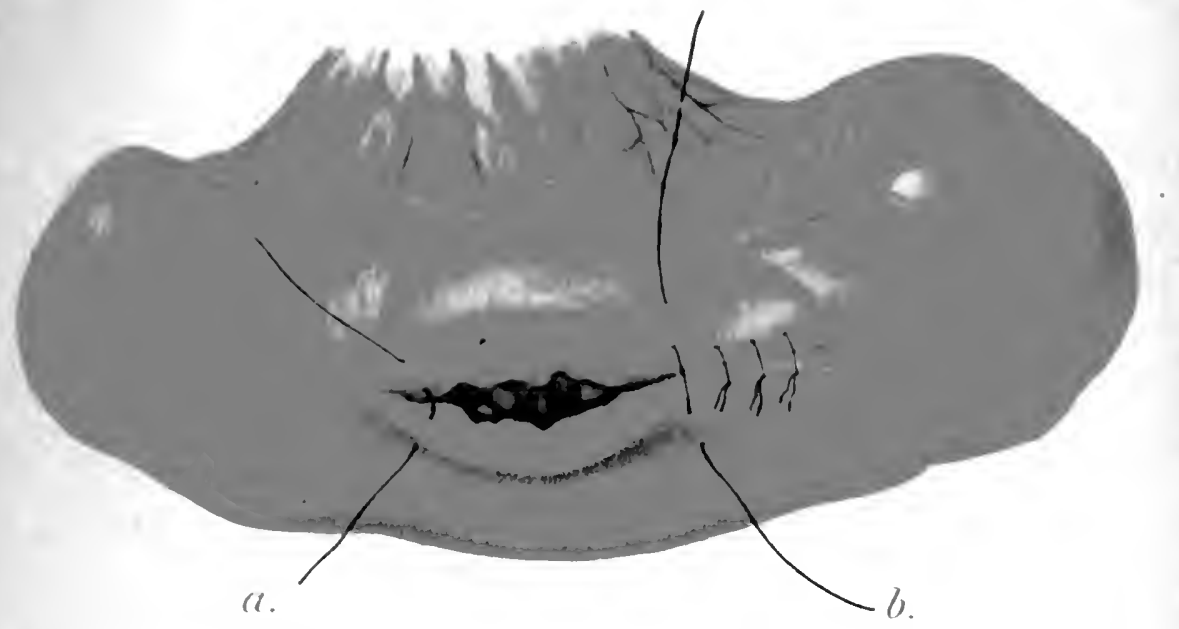

Figg.

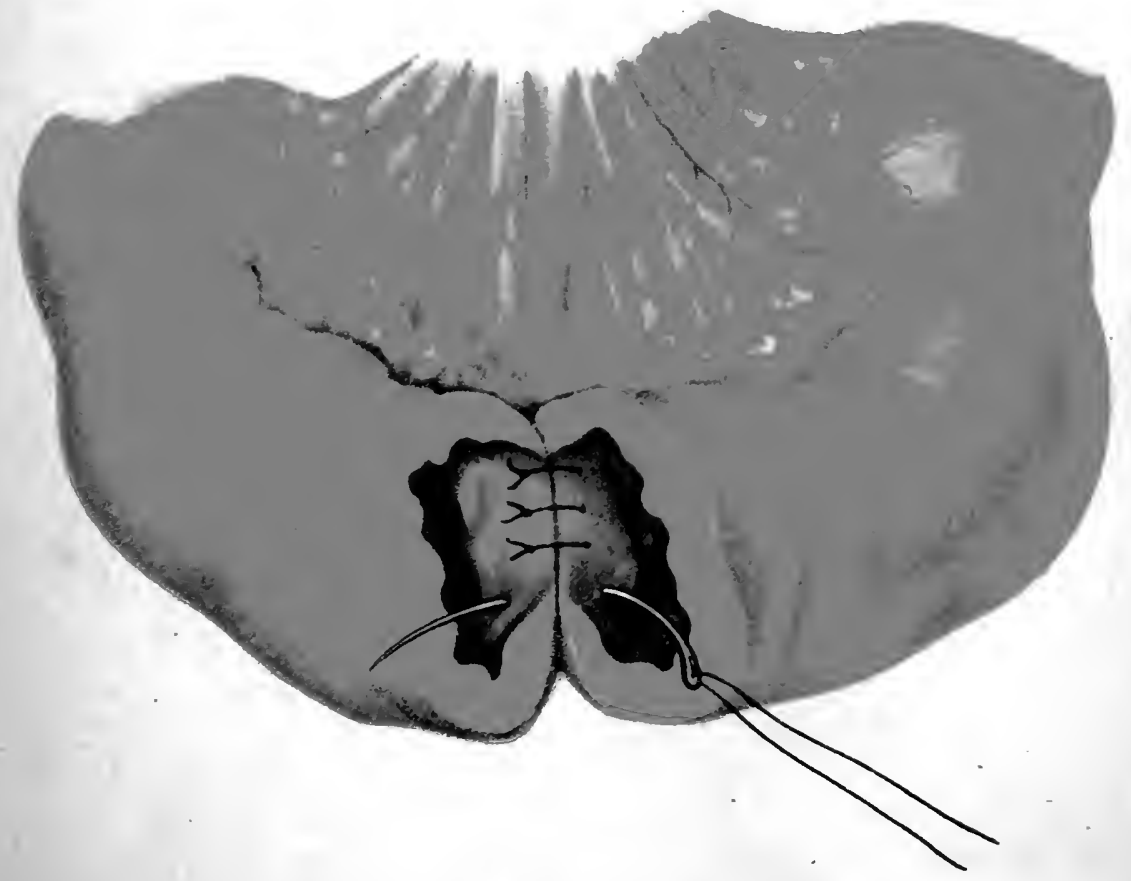

Lith..Anst F: Reishhold, Mïnshen. 
tended to fix the cut surfaces in apposition; the second brings the serous surfaces together. The operator should see that corresponding portions of the circumference are brought accurately in contact with one another. 'To this end a suture is applied at the mesenteric attachmont of the bowel and also one at a corresponding point on the opposite side of the periphery, hoth involving all of the layers of bowel. In this way the entire circumference is divided into two equal parts. 'The margins of that portion most removed from the operator are first united either by closely applied knotted sutures, or a continuous suture, and it is advisable under these circumstances to apply and tie the sutures from the lumen of the bowel. "This suture should include all of the layers of the bowel, and care should be taken that thick lavers of the muscularis are bromght into intimate apposition. The margins of the wound closest to the operator are first united by sutures passed from and tied upon the serous surface. When this suture, including all the layers of the howel, has been applied to the entire periphery of the bowel closure will have been substantially effected. In order to secure primary union of the intestinal wound within a short time a second row of seroserous (Iembert) sutures is applied over the first. Under these circumstances, also, it is arvisable to include with the serous layer a portion of the musenlar layer, as in this way the serous folds are made firmer and can be brought in closer approximation.

Suture of the bladder, when the injury involves the intraperitoneal portion of this viscus, is effected upon prineiples similar to those that have been just laid down. except that in this event it is advisable, in order to avoid incrustations, to use eatgut instead of silk as the suturematerial. One row of sutures will aceurately approximate the muscular layer of the bladder, which is often from one-half to one centimeter thick. It is customary to exelude the mucous membrane from the suture. $\dot{A}$ second row of I embert's sutures unites the serosa. In 
the closure of wounds of the bladder, as in closure of those of the intestine, the continuous suture or closely applied knotted sutures may be employed.

Wounds of the extraperitoneal portion of the bladder are to be so united that the thick layer of muscular tissue is brought in firm apposition by means of catgut sutures, with exclusion of the mucosa.

The various methods of suture for closure of wounds of the bladder have been largely supplanted by the simple method just described.

Wounds of the gall-bladder may be closed by suture in a manner analogous to those of the bowel. 


\section{OPERATIONS ON THE EXTREMITIES.}

\section{Ligation of Vessels in Continuity.}

An injured and bleeding ressel may be seized directly within a wound and the hemorrhage controlled by ligation. Another mode of procedure consists in exposure of the central extremity of the divided ressel for the purpose of its ligation. This variety of ligation of ressels in eontinuity will now be considered.

Indications. - (1) Injuries. - (a) stab-loounds, gnnshot-rounds, contused ucounds, and laverated wounds of the large arteries. If possible, ligation is to be undertaken at the site of injury. This is difficult in lacerated tissues suffused with blood, when the wound is unfarorably situated, or when the artery is injured directly at its origin from a main branch.

(b) Subcutaneous Inceration of large vessels; also when ligation at the site of the lesion is impossible.

(2) Hemorrhages from suppurating wounds through erosion of large vessels; further arterial hemorrhage from gangrenous tissues or disintegrating new-growths.

(3) In order to render an operation bloodless the main arterial branch of the operative area mar be previously ligated-e. g., the lingual artery preceding extirpation of the tongue, the femoral artery preceding enucleation of the hip-joint, and preceding extirpation of carernous tumors.

(4) In order to induce retrogression of morbidly altered organs or neoplasms the blood-supply is cut off by ligation of the main artery-e. g., of the thyroid artery in the presence of goiter, of the spermatic artery in the presence of tumors of the testicle, of the internal iliae artery in the presence of hypertrophy of the prostate, etc. In the same category belongs the treatment of elephantinsis of the leg by ligation of the external iliac or the femoral 
artery, with which some surgeons have secured good results. ${ }^{1}$

(5) Aneurysms.-According to the method of Hunter, the afferent artery is ligated in the treatment of aneurysm, but on account of the supply of blood to the aneurymal sac through the collateral circulation this method is not so reliable as that of Antyllus.

In cases of trigeminal neuralgia resisting other operative measures, ligation of the carotid artery has been repeatedly practised (Pátruban).

The treatment of epilepsy by ligation of the vertebral artery has also been proposed.

Method of Ligation.-At definitely determined points upon the surface of the body the slin is divided and with eareful protection of important structures the sheath of the ressel is exposed. This is then opened, and the artery, separated from the accompanying veins for a small portion of its extent, is raised fiom its bed for the purpose of ligation. Two ligatures are now applied, and the artery is divided transversely between them with a single crit of the seissors. The wound in the skin is closed by suture.

The cutcneous incisions are made in selected situations in a definite direction so as to render possible aceess to the artery by the shortest route. Usually the incision in the skin corresponds with the eourse of the artery. Thus, in the extremities this incision, with a few minor exeeptions, coincides with the longitudinal direction of the vessel.

In order that the incision in the slin may be placed in the proper situation, it is necessary to make eareful scrutiny of the surface of the body. To facilitate this, prominent, readily palpable points of the skeleton, ats well as muscular prominenees, and the intervening depressions, are selected as landmarks.

1 In some regions extirpation of a vessel is better than ligation, if we wish to arrest the growth of a tumor. Dawbarn has recently pointed out that ligation of the external carotid is of slight value in sareoma of the tonsil, as the anastomotic cireulation is so quickly established, but extirpation of this vessel causes great shrinking of the growth.-Ev. 
The length of the cutaneous incision will be regulated by the depth to which aceess is desired: the deeper the wound the larger must be the opening in the skin. The incision for exposure of the internal iliac artery will measure from 15 to $20 \mathrm{~cm}$. ( 6 to $8 \mathrm{in}$.), while that for the ratdial artery at the wrist-joint need not be more than 1 or $1.5 \mathrm{~cm} .\left(\frac{\mathrm{i}}{3}\right.$ or $\left.\frac{1}{2} \mathrm{in}.\right){ }^{1}$

When the skin and the subcutancous connective tissue have been penetrated (the base of the wound is no longer moved with movements of the margins of the skin), the sheath of the vessel is carefully approached by dissection either with the free hand, between two pairs of forceps, or with the aid of the groosed director. Muscles, nerves, and veins that obstruet the way are displaced with blunt hooks. If a vessel prevents access to the artery, it may be ligated in two places and divided between.

The sheath of the vessel is detached from the artery for a short distance by blunt dissection with anatomic forceps, or it is divided upon the grooved director, in accordance with the character of the tissues. After the sheath has been opened, either a single vesscl will be exposed or both artery and vein will come into view. The most reliable guides are furnished by the topographic-anatomic relations. The artery must be recognized as such from its situation, as all other means of identification may under eireumstances fail. The color of the ressel is as little distinctive as is its thickness, as the walls of both arteries and veins possess the same color in the dead subject in consequence of imbibition, while the thickness of the wall of the vessel is subject to wide individual variations. As a rule, however, the walls of the arteries are thieker than those of the veins, althongh not rarely in enfeebled individuals, and particularly in females, the arteries are small and thin-walled. It might be supposed that during life pulsation of the vessel would be an infallible means of recognizing the arteries; but this is not always so, as

1 Operation through a very small incision is often possible, but it is a needless effort and is uusafe for a beginner.-Ev. 
arteries at times exhibit no pulsation after free loss of blood, while on the other hand reins may apparently pulsate through transmission of movement in the arteries. The anatomical relations therefore are the only reliable guides for distinguishing between artery and vein.

When the sheath of the ressel has heen opened and the artery brought into vicw, this must be isolated with two

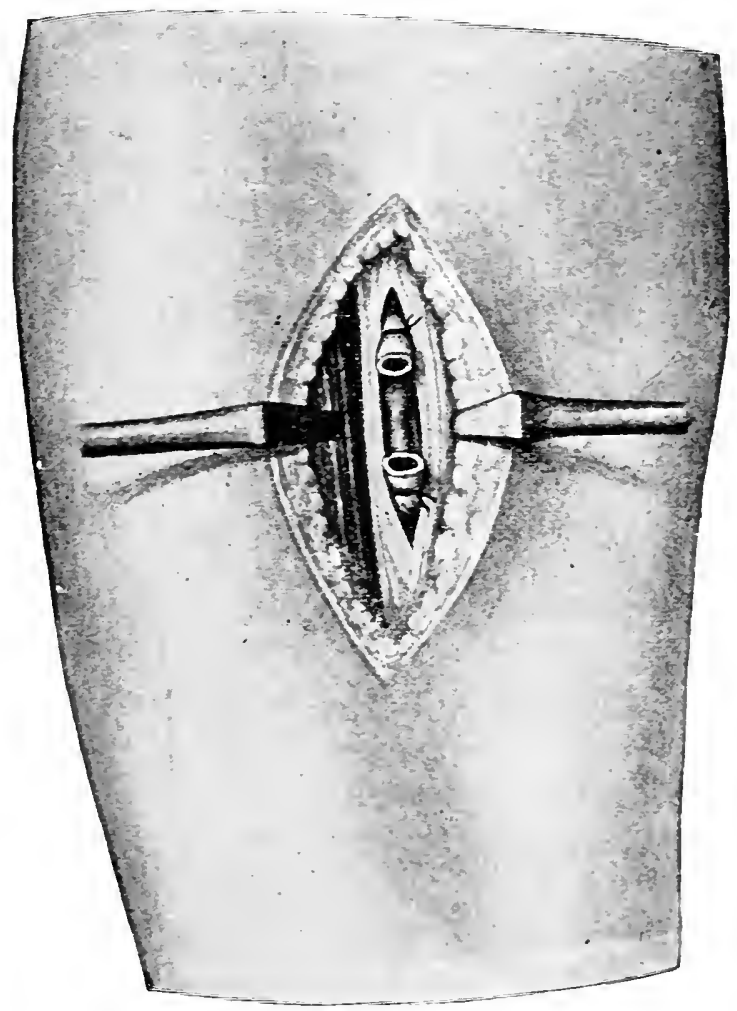

FIG. 39.-Artery divided between two ligatures.

pairs of forceps for a distance of 1 or $1 \frac{1}{2} \mathrm{~cm}$. ( $\frac{1}{3}-\frac{1}{2}$ in.) and raised from its bed for the application of the ligature. To this end one lip of the opened sheath is grasped with a pair of forceps held in the left hand and traction made, while with the blades of the other pair of forceps held in the left hand the loose tissue surrounding the artery is 
detached in its longitudinal direction. From time to time the fixing and the dissecting hand are alternated, care being taken to avoid inchusion of the artery, of a vein, or of a nerve between the blades of the forceps. From the side of the artery next which lies the vein [or rather the most dangerous neighbor, for in some situations the nerve must be avoided rather than the vein.-ED.], the ligating instrument, armed with a ligature, is passed beneath the artery, the ligature wound about the vessel and tied. A second ligature is applied in similar manner at about a distance of $1 \mathrm{~cm}$. ( $\frac{1}{3} \mathrm{in}$.) from the first, and, between the two, the artery, raised from its bed, is ent squarely with a single stroke of the seissors. The divided ends of the vessel retract somewhat in either direction (Fig. 39). The application of two ligatures and eutting between possess some adrantages, but are not always necessary.

The retraction and the relaxation of the extremities of the divided vessel afford more farorable conditions for thrombus-formation, through narrowing of the lumen, than simple occlusion of the lumen of the vessel. Beside, by division of the ressel between the ligatures a view is obtained of the posterior wall of the artery, and in this way ligature of the artery just in advanee or just beyond the origin of a lateral branch can be avoided. Either contingency is equally unfivorable to thrombus-formation. The small wound made is closed with knotted sutures.

The knot of the ligature is tied as follows: after the ligature has been made to surround the artery the free end on either side is grasped with the finger's of the corresponding hand. Before the knot is tied the ends are so crossed that the right passes behind the left and is received into the left hand, while the left passes in front of the right and is received into the right land. When the extremities have been thus crosed a simple knot is tied. Before the second knot is placed upon the first the free extremities must again be changed and in such a manner that that upon the left is passed in front of that upon the 
right. The sailor's knot (Fig. 40) thus formed is more secure than the ordinary gramny's knot (Fig. 41).

If in the first part of the knot the extremities are twisted twice, instead of once, there results the so-called surgical linot (Fig. 42).

The sailor's knot is employed not only in the application of ligatures, but whenever it is desired to tie a secure knot.

Ligations in the Upper Extremity.-The artery supplying the arm, the forearm, and the hand may be

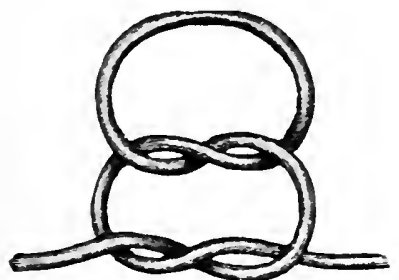

FIG. 40.-Sailor's knot [reef-knot].

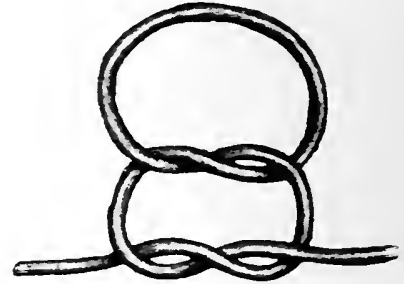

FIG. 41.-Granny's knot.

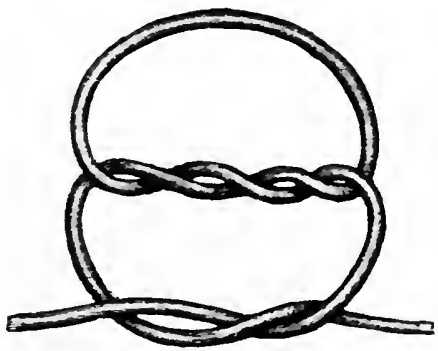

FIG. 42.-Surgical knot.

exposed for purposes of ligation in the axilla, upon the upper arm, at the flexure of the clbow, and upon the forearm.

The axillary artery is the continuation of the subclavian from the lower border of the fourth rib to the surgical neck of the humerus. When the arm is abducted it lies in intimate relation with the bone; below the pectoralis minor the ressel lies in contact with the coraco-brachialis. Of the brachial plexus only the median nerve is situated 


\section{Plate 2.-Exposure of the Axillary Artery and the Brachial Artery.}

$M C b$, coracobrachialis; $f$, inner portion of the fascia of the upper arm; $M$, median nerve; $C i$, lesser internal cutaneous nerve; $C a$, greater internal cutaneous nerve; $A x$, axillary artery; $B$, biceps; $F b$, brachial fascia. $A$, brachial artery. $I^{r} b$, brachial vein.

of the vessels and nerves of the arm. It does not extend quite to the anterior axillary fold, as the belly of the biceps diminishes in size at this point before passing over into the tendon. The border of the pectoralis major and the upper extremity of the biceps form here two sides of a triangle whose base is constituted by the coracobrachialis (Fig. 43).

This triangle, formed by the elerations of three muscles, is the situation in which the artery is to be exposed.

It would be a mistake to look for the vessel in the axillary cavity, which forms a space filled with fat, connective tissue, and lymphatic glands next to the lateral wall of the chest. The artery, which lies close to the humerus, is therefore to be looked for in relation with this bone at the apex of the axillary cavity.

The incision is made along the line of the coracobrachialis muscle in the continuation of the internal bicipital sulcus (Fig. 43). After the subcutaneous connective tissue has been passed the thin fascia of the arm will be exposed, through which the fibers of the coracobrachial muscle will be visible. The fascia is divided upon the grooved director and the lower (inner) lip of the wound in skin and fascia retracted with tenacula. There now comes into view the median nerve embedded in loose cellular tissue, and this is drawn out of the way with a simple blunt hook. The artery is now exposed and can be readily isolated and ligated (Plate 2). Care must be taken that the nerve alone is grasped and drawn out of the way, as otherwise the artery, which lies directly behind it, may also be displaced and removed from the field of view. 
Tab. 2.

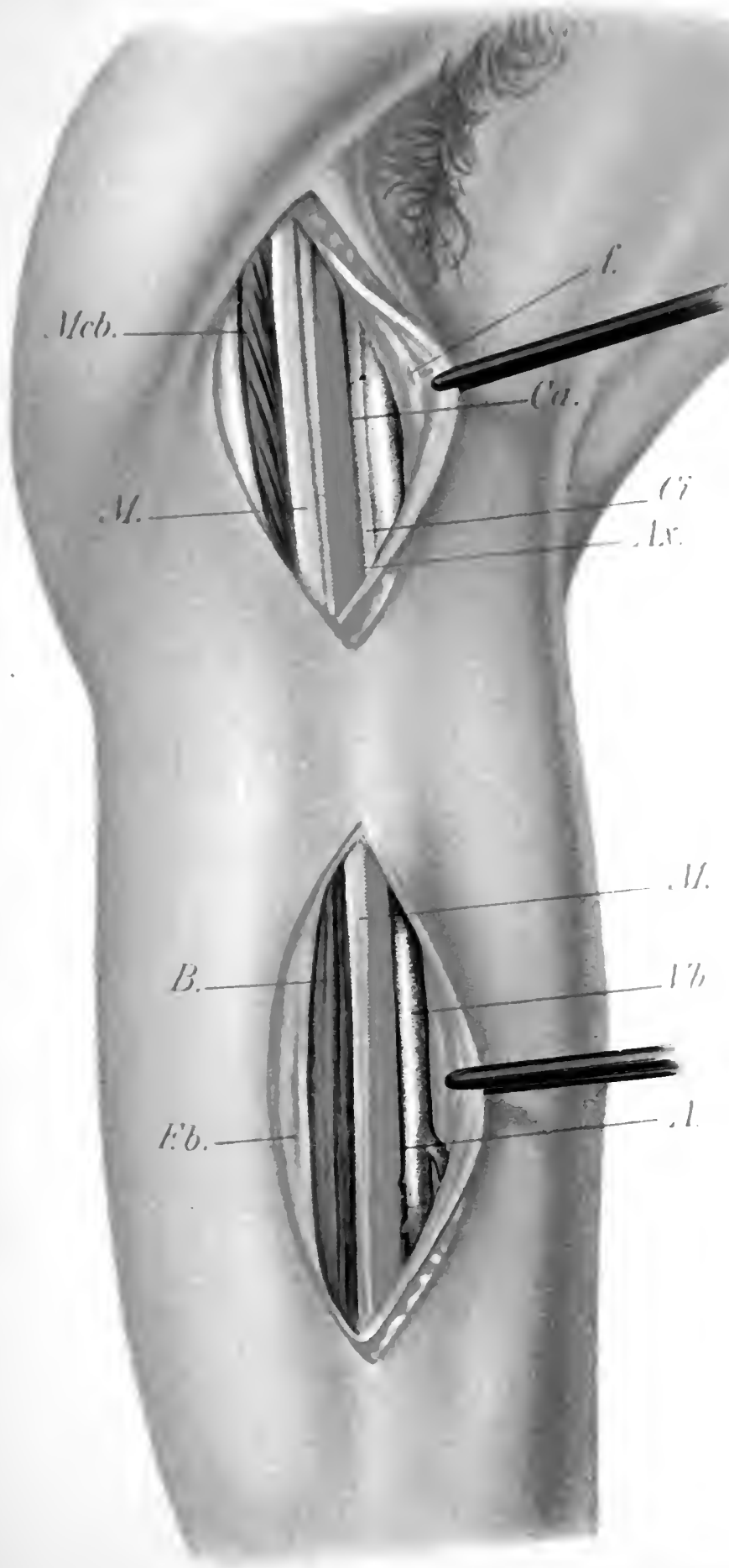

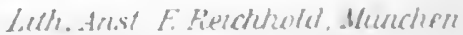



After division of the fascia of the coracobrachial muscle the greater internal cutaneons nerve sometimes presents itself. 'This small nerve ean scarcely be confounded with the median nerve, which comes into view after further retraction of the lower (inner) margin of the womd.

The artery is accompanied by one or several, sometimes by a whole plexus of veins.

Ligation of the Brachial Artery.-The continuation of the axillary artery from the surgieal neck of the humerus to its point of division at the flexure of the elbow is known as the brachial artery. The ressel lies in the internal bicipital suleus, and it is often accompanied by a network of veins. The median nerve lies over the upper half of the artery, which it conceals, while in their further course the nerve lies upon the ulnar side of the vessel. The basilic vein, which likewise lies in the internal bicipital sulens, is separated from the group of large vessels and nerves by the fascia. The sheath of the vessel consists of loose cellular tissue.

The patient oceupies the same position as in ligation of the axillary artery. The incision is made at about the middle of the arm, slightly over (external to) and parallel with the internal bicipital sulcus (Fig. 43). Skin and subcutaneons connective tissue are divided and the fascia of the biecps muscle is opened in the same direction and throughout the same extent. The fibers of this muscle must be clearly exposed to view. The lower (inner) lip of the wound in the fascia is drawn downward (inward) with a tenaculum and the median nerve thus exposed. The nerve is lifted from its bed and drawn aside with a blunt hook, when the brachial artery is exposed accompanied by veins. The artery is isolated by means of two pairs of forceps and is ready for ligature (Plate 2).

The rule to make the incision somewhat above (external to) the bicipital sulens in order to reach the median nerve below is to be recommended on account of the difficulties encountered in reaching the artery 
through a mass of structures, including the greater internal cutaneous nerve, the median nerve, and the basilic vein, when the incision is made directly over the vessels and nerves. If the ineision is male below (internal to) the bicipital sulens, an inexperienced person may err by failing to recognize the exposed uhar nerve and looking in rain for the artery behind it.

The relations between the median nerve and the brachial artery are variable within certain limits. In rare instances the artery lies in front of the nerve. In cases of high division of the radial and uhnar arteries one of the ressels lies in front of and the other behind the nerve. The presence behind the median nerve of an artery proportionately small, as compared with the rest of the body, is suggestive of such high division of the brachial artery.

Ligation of the Cubital Artery.-The continuation of the brachial artery in the flexure of the elbow is known as the cubitul artery. The ressel lies in the internal cubital sulcus, and, covered by the aponeurosis of the biceps muscle, is embeded in the depression between the pronator radii teres and the biceps. The artery is in this situation aceompanicd by two symmetrically placed reins. The median nerve does not oecupy the same intimate relation with the artery as it does higher up, but lies at some distance upon the uhnar aspect of the artery. Separated from the artery by the bicipital fascia and situated subeutaneonsly is the cubital plexus of reins (median basilic, median cephalic), which commmicate in the flexure of the elbow with the reins accompanying the artery.

The simplicity of the relations existing in the arm in consequence of the prominences formed by the biceps and the triceps and the presence of the interinal and external bicipital sulci, is replaced at the flexure of the elbowjoint by complexity resulting from the presence of the two large groups of forearm-muscles. The spindle-shaped belly of the biceps, which gradually diminishes in size, is separited by a sulcus upon the right and the left respectively from the muscular prominences of the extensors 


\section{LIGATHON OF TESSELS IN CONTTNUITY.}

and flexors of the forearm, which originate in this situat-

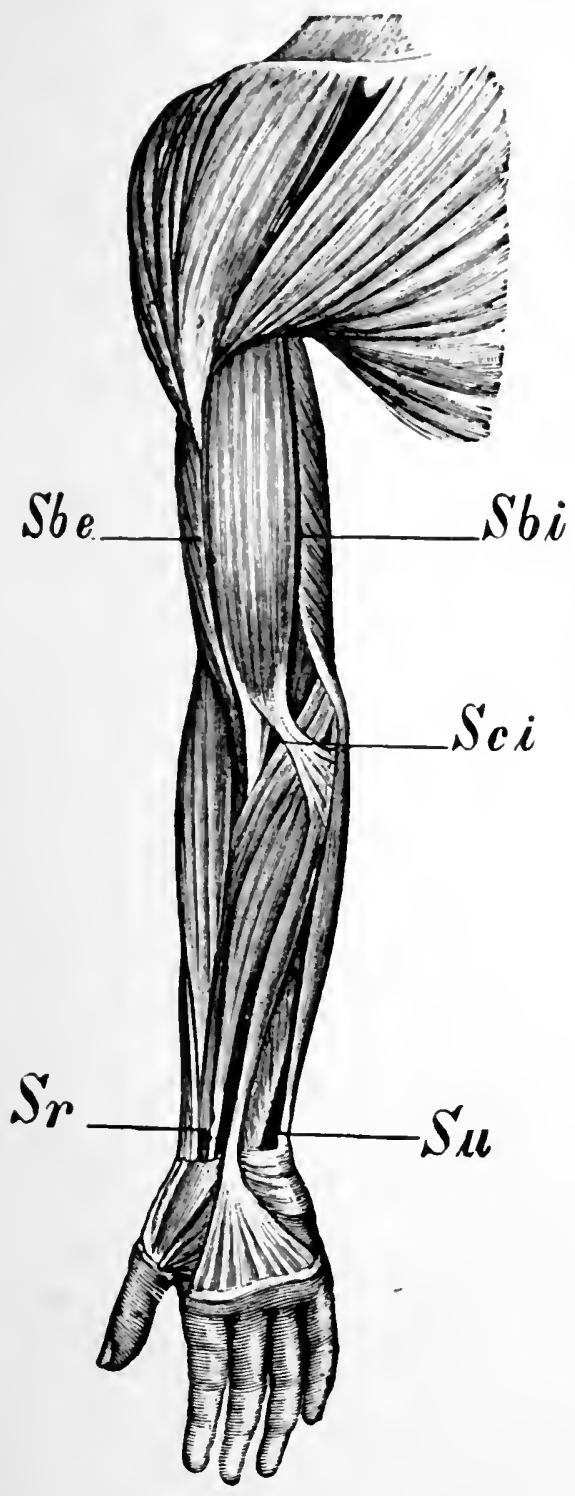

FIG. 44--Arrangement of the muscles in the upper extremity: Sbi, internal bicipital suleus; slbe, external bieipital sulcus; sci, internal cubital sulcus; sin, radial sulcus; Su, ulnar suleus.

tion. There results thus a $Y$-shaped formation, of which the two limbs, in some degree the continuations of the 


\section{Plate 3.-Exposure of the Cubital Artery.}

$L$, transverse section of the aponeurosis of the biceps muscle; $A$, cubital artery accompanied by veins; $\boldsymbol{M}$, median nerve; $\boldsymbol{V}$, cubital veins.

\section{Exposure of the Radial and Ulnar Arteries.}

$A r$, radial artery; $A u$, ulnar artery at the inner side of the tendon of the internal ulnar muscle $(U)$.

bicipital sulei of the arm, are designated the internal and external cubital sulci. The inner fiurow of the flexure of the elbow is bounded by the biceps or the brachialis internus and the pronator radii teres, the outer by the biceps and the supinator longus. The internal bicipital sulcus is covered by the radiating aponeurosis of the biceps (Fig. 44).

To ligate the bieipital artery the forearm is extended at the elbow-joint and held in a position of maximum supination. Information as to the direction and situation of the intermal bicipital suleus is sought through palpation. The incision is made in the continuation of the internal bicipital sulcus and passes from within and above downward and outward, corresponding to the direction of the internal cubital sulcus (Fig. 45). After division 'of the skin eonsideration should be given to the network of veins at the flexure of the elbow. When possible the way is eleared by retraction of the veins with blunt hooks. The shining aponeurosis of the biceps muscle now appears in the wound and it is divided upon the grooved director in the direction of the cutaneous wound. The artery lies immediately beneath the aponeurosis, accompanied by two veins, in a bed of loose connective tissue. The median nerve lies to the ulnar side of the artery (Plate 3 ).

In case of high division of the brachial artery one or both branches may lie upon the bicipital fascia. This possibility is to be thought of when bleeding from the reins at the flexure of the elloow is to be undertaken, or when one of the median veins in this situation is to be exposed and opened for infusion with a saline solution or for transfusion of blood. 
'Tab. 3.

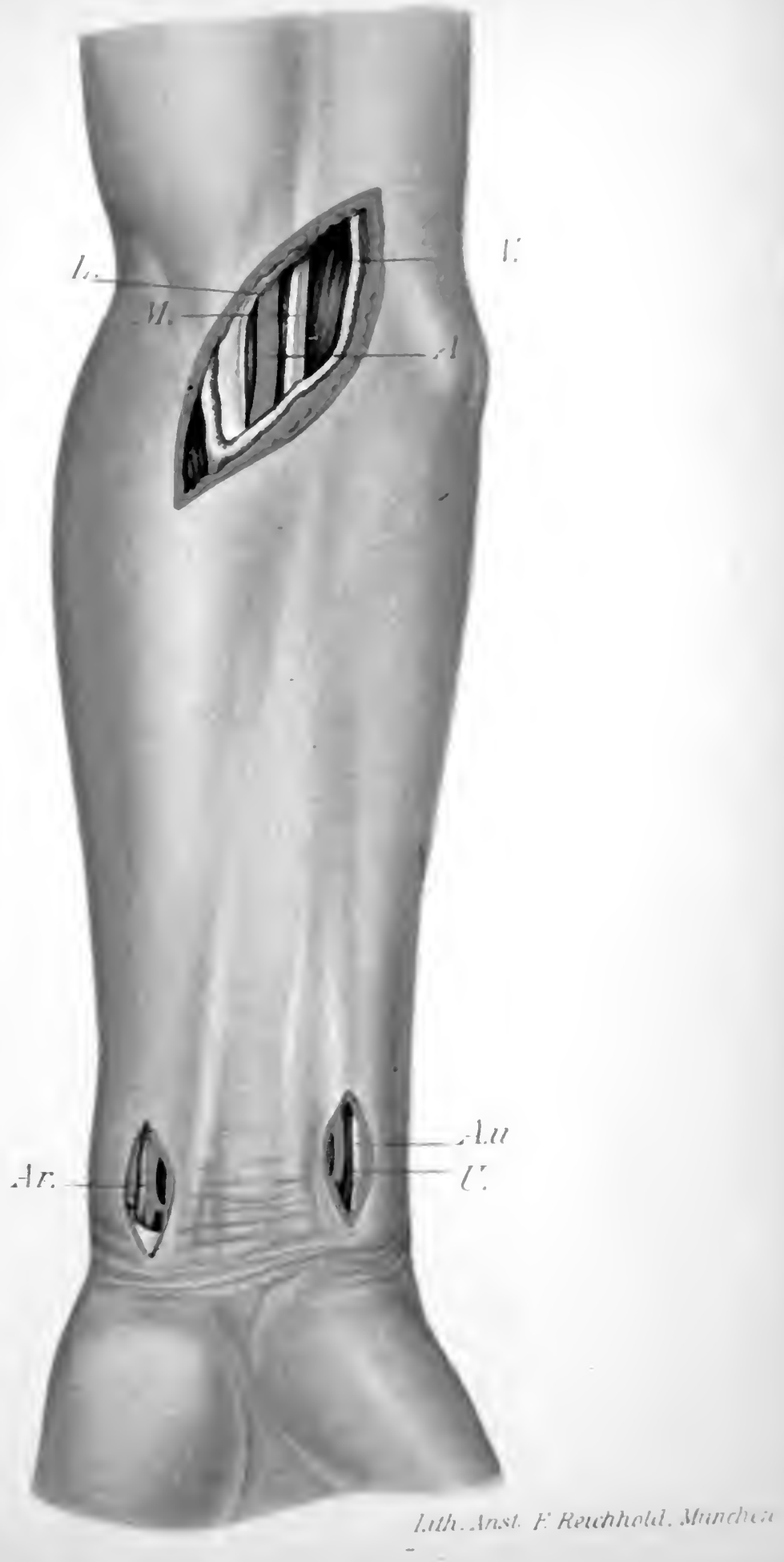




\section{$-$}


In the practice of phlebotomy a cloth or handage is bound circularly about the middle of the arm in such a mamner that the retmin of blood through the veins form the foream is prevented wathout obliteration of the radial pulse. With the furearm extended, a sharp-pointed knife is introduced oblique!y into one of the tensely distended nordian veins (Fig. 46), so that the blood spurts from the wound in a stream. When the

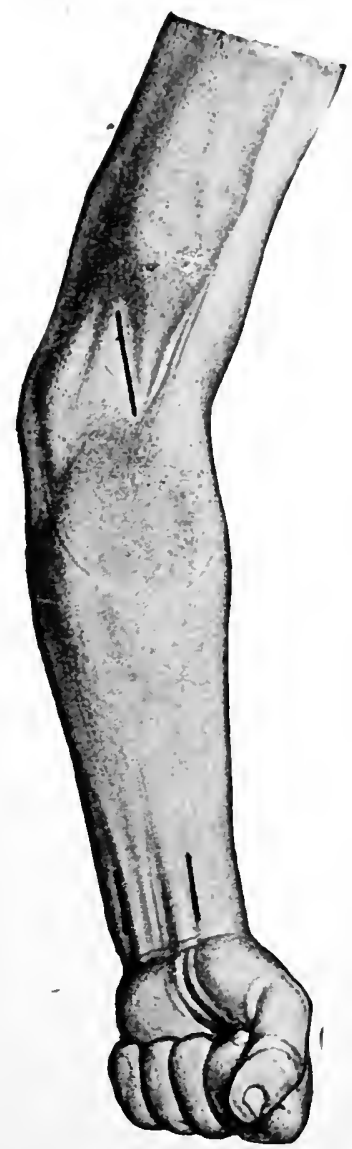

FIG. 45.-Incisions for exposure of the cubital, radial, and ulnar arteries.

desired amount of blood has escaped the compress is released, and the small wound is covered with a dressing and a bandage.

Transfusion of blood or venous infusion of saline solution also is practised through the median basilic vein. Through an incision analogous to that made for ligation of the cubital artery the vein, lying subcutaneously. is exposed for a distance of several centimeters and isolated by blunt 
dissection. The ressel is grasped with a pair of anatomic forceps and snipped with the scissors. without being totally divided. The rein is now closed by ligature on the peripheral side of the incision. Through the opening thus made the cannula is introduced into the vein in a centripetal direction and fixed. To make the infusion a sterilized rubber tube armed with a funel is most advantageously employed, attached to the cannula. From a half-liter to a liter and a half of fluid are per-

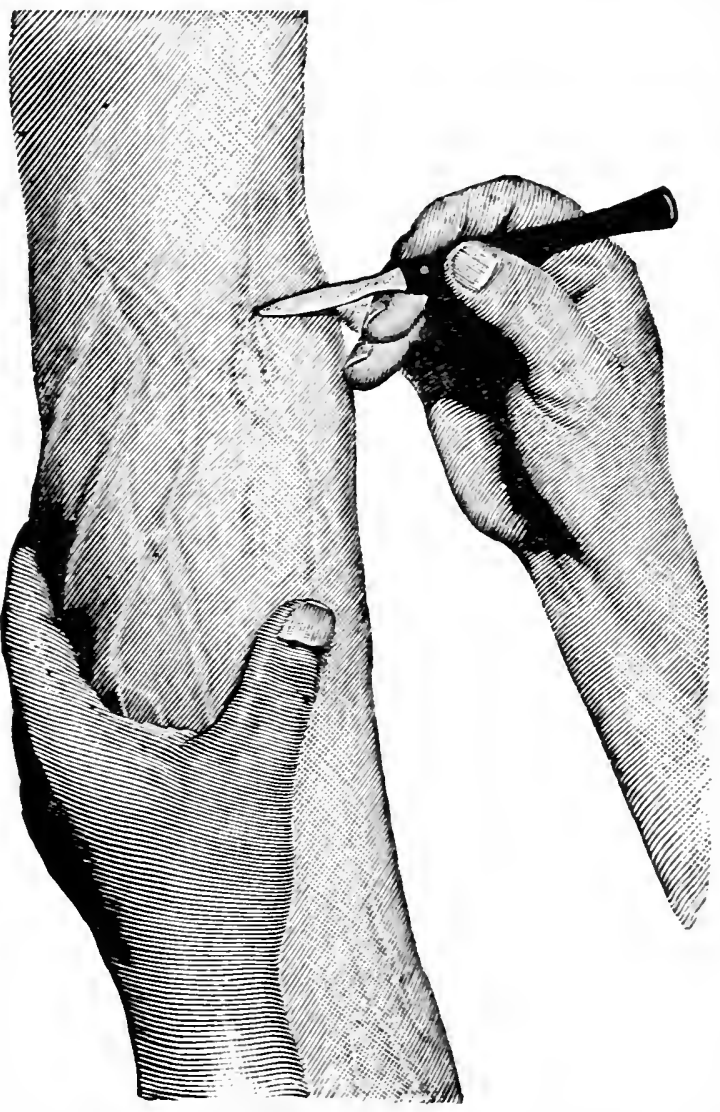

Fig. 46.-Phlebotomy at the flexure of the elbow: opening of the median basilic rein by puncture with a sharp-pointed knife.

mitted slowly to flow into the vein under a low degree of pressure. For purposes of infusion sterilized physiologic ( 0.6 per cent.) solution of sodium chlorid or defibrinated human blood may be employed. After the infusion has been completed the vein is ligated upon the central side of the opening and the small wound is closed by suture.

\section{Ligation of the Radial and Ulnar Arteries.-The}


muscles upon the palmar aspect of the forearm are divisible into three grompls. The main mass is firmed by the flexors of the fingers, whieh arise by a common head from the inner aspect of the lower extromity of the humerus. The forearm is bomeded nom the nilnar side by the flexor carpi ulnaris, upon the radial side by the supinator longus. Between the tenclons of these museles and the mass of the flexors of the fingers there is thus formed in the lower third of the forearm upon either side a longitudinal furrow or depression which is used as a guide in finding the radial and the ulnar artery respectively (Fig. 45).

The radial artery corresponds in its course with the direction of the radius. In the ripper third of the forearm the artery lies in close relation with the supinator longus muscle and is deeply situated. In the lower third it lies more superfieially in the sulcus between the tendons of the flexors and that of the supinator longus. Just above the wrist-joint the artery, with its accompanying two veins, lies upon the lower extremity of the radius, covered only by skin and the thin fascia.

The ulnar artery, after its origin from the brachial, crosses the common head of the flexors, among which it pursues its course, until it reaches the tendon of the flexor carpi ulnaris, along the inner side of which it reaches the wrist-joint.

The typical situation for the ligation of both arteries is just above the wrist-joint. The forearm is placed in a position of maximum supination, with the hand in slight dorsal flexion. To expose the radial artery an incision is made just above the wrist-joint corresponding to the depression between the tendon of the supinator longus and those of the flexors (Fig. 45). After division of the skin, the artery, covered by delicate translucent fascia, is seen, situated between two veins. After division of the fascia the artery can be isolated and ligated (Plate 2).

The ulnar artery is reached through a short incision in the ulnar sulcus just above the wrist-joint somewhat to 
the radial side of the readily palpable tendon of the flexor carpi ulnaris. The radial margin of this tendon is exposed and drawn to one side with a tenaculum. The deep

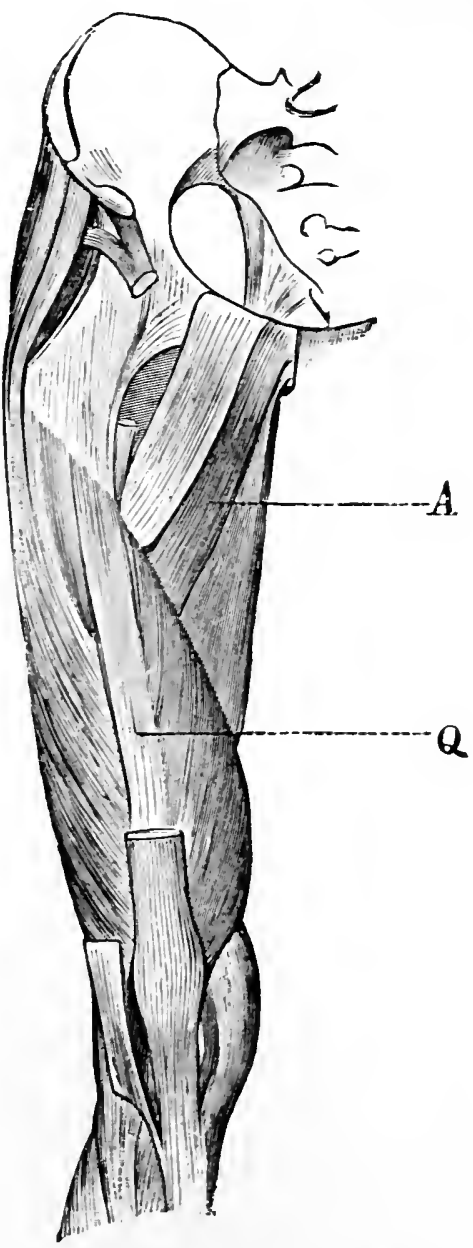

FIG. 47.-Arrangement of the nuscles of the thigh: $A$, abductor group; $Q$, quadriceps femoris.

layer of fascia enclosing the flexors is brought into view and divided upon a grooved director. The artery, accompanied by two veins, is now disclosed. In close relation with the artery upon its ulnar side lies the ulnar nerve. 
The radial artery, in its further course, is conveniently accessible upon the dorsum of the hand between the tendon of the Hexor longus pollicis and that of the extensor brevis pollicis, in the su-called tabatiere.

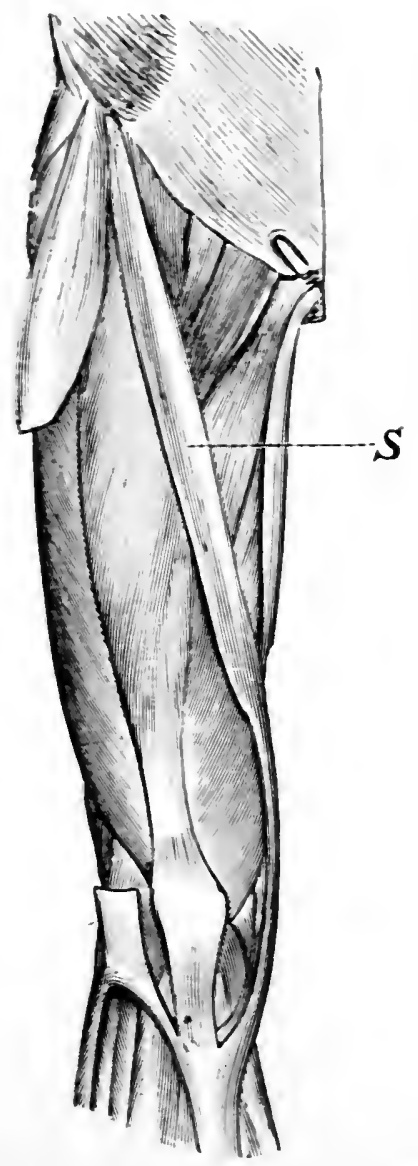

FIG. 48.-Course of the sartorius muscle (S).

In the palm of the hand the superficial palmar arch of the ulnar artery can be exposed after division of the tough palmar aponeurosis. The cutaneous incision in the palm passes from the middle of the root of the hand toward the base of the little finger. 


\section{Plate 4.-Exposure of the Femoral Artery.}

Below Poupart's ligament. in the opened sheath of the vessels, are to be seen upon the median side the femoral vein, and upon its outer side the femoral artery. In the middle of the thigh the sartorius muscle (S) is drawn outward, the deep layer of the fascia being divided, and the artery is exposed, with the vein behind it.

\section{Ligations in the Lower Extremity.-The mus-} clés of the thigh are so grouped that between the extensors and flexors, which are arranged srmmetrically upon the anterior and posterior aspects of the femur, on the median side one group of muscles passes from the pelvis to the inner aspect of the femur, separating the quadriceps from the flexors. The depression thus formed between the extensors and the abductors (Fig. 47) serves as a pathway for the ressels passing orer the margin of the pelvis and corresponds in its direction with the course of the ressels. The sartorius muscle bridges over this gutter (Fig. 48) and constitutes thus an important landmark in locating the ressels.

The femoral artery, the continuation of the external iliac, emerges from the pelvis under Poupart's ligament at a point midway between the srmphysis pubis and the anterior superior iliac spine. The femoral vein at its entrance into the pelvis lies to the median side of the artery. The artery passes downward and inward, and in its course follows the depression between the extensors and the adductors. From the middle third of the thigh onward the sartorius muscle lies in front of the artery, which in this situation is covered by the tense fibers of the deep layer of the fascia lata. The artery, with its accompanying vein, enters the popliteal space through an opening in the adductor magnus (Hunter's canal), at the junction of the middle and lower thirds of the thigh. The femoral vein below Poupart's ligament lies to the median side of the artery. In the further course of the ressels they cross in such a way that the rein comes to lie behind the artery. This relation is attained in the middle 
l'ab 4 .

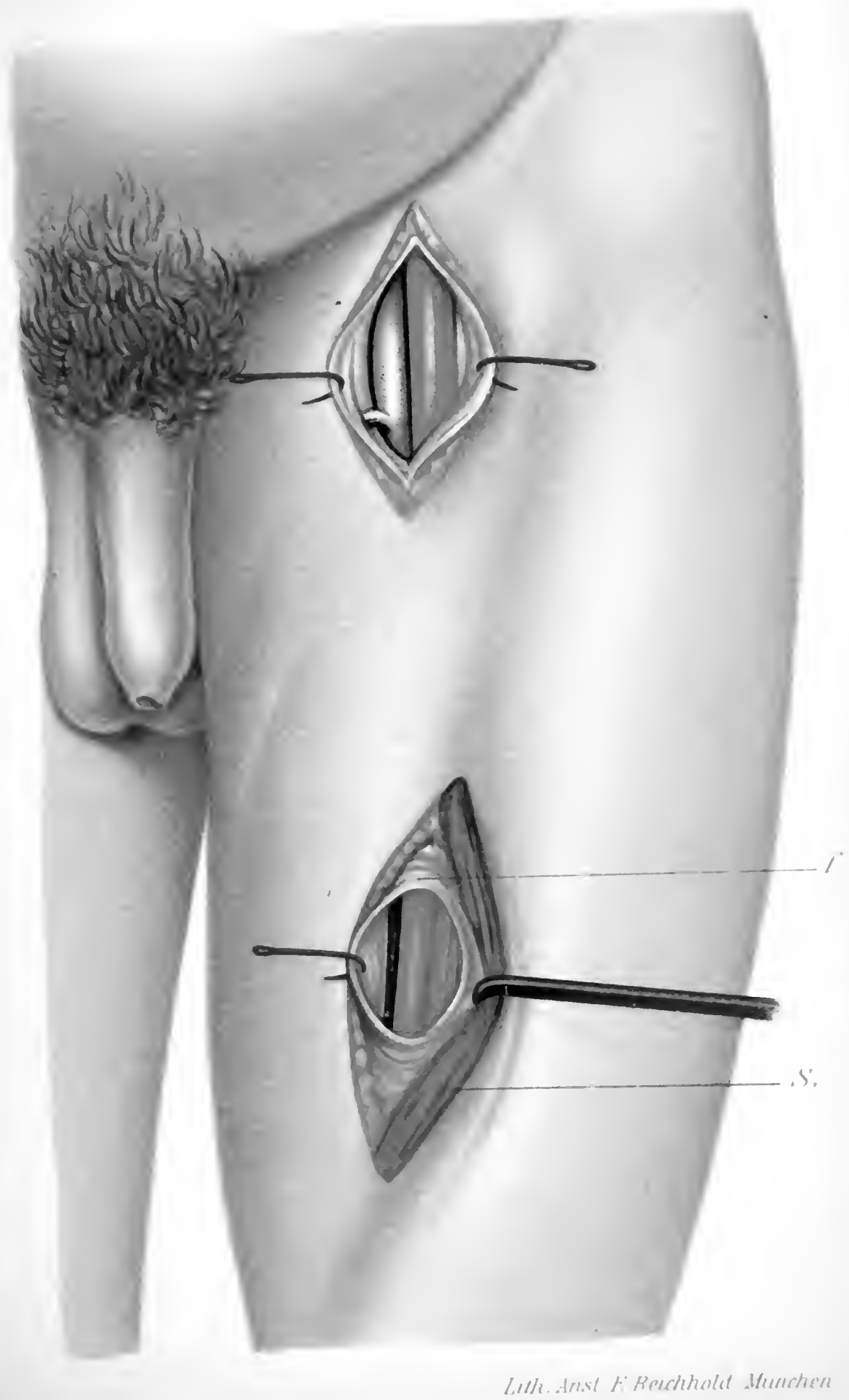


of the thigh, and the ressels thus pass thromgh Hunter's eanal. In entering the popliteal spare from the posteriol aspect the rein comes first into view, while in front of it, in intimate relation, lies the artery.

'The femoral artery may be ligated:

(1) In the subinguinal depression, directly below Poupart's ligament.

(2) In its course behind the sartorius musele, at the junction of the middle and upper thirds of the thigh.

(3) In Hunter's canal.

I. Ligation of the Femoral Artery below Poupart's Ligament.-The incision into the skin is made parallel with the axis of the thigh from Poupart's ligament downwar for a distance of from 5 to $8 \mathrm{~cm} .(2-3 \mathrm{in.})$. 'The upper' extremity of the incision corresponds with a point midway between the symphysis pubis and the anterior superior iliac spine (Fig. 49, a). After division of the skin and the fatty connective tissue careful dissection is made downward in a vertical direction until the sheath of the vessels is recognized by its fibrous structure and whitish appearance. The sheath is divided upon a groored director and the artery isolated for a short distance with two pairs of forceps and raised from its bed. The femoral vein can be brought into view upon the median side of the artery. The crural nerve is some distance to the outer side of the vessels, covered by the deep layer of the fascia lata.

II. Ligation of the Femoral Artery at the Junction of the Middle and Upper Thirds of the Thigh.-By inwarl rotation of the thigh the depression following the course of the sartorius muscle, from above and without downward and inward, upon the inner aspect of the femur, can be brought into view. 'The incision in the skin is begun at the junction of the middle and upper thirds of the femur, and follows the line of this depression along the inner border of the sartorius muscle (Fig. 49, b). After the subeutaneous connective tissue has been passed the delicate fascia of the thigh comes into view and should 


\section{Plate 5.-Exposure of the Femoral Artery in the Adductor Canal.}

$\mathrm{Vi}$, vastus internus; $S$, sartorius. The fibrous covering of Hunter's canal $\left(f^{\prime}\right)$ is divided, with exposure of the femoral artery and vein.

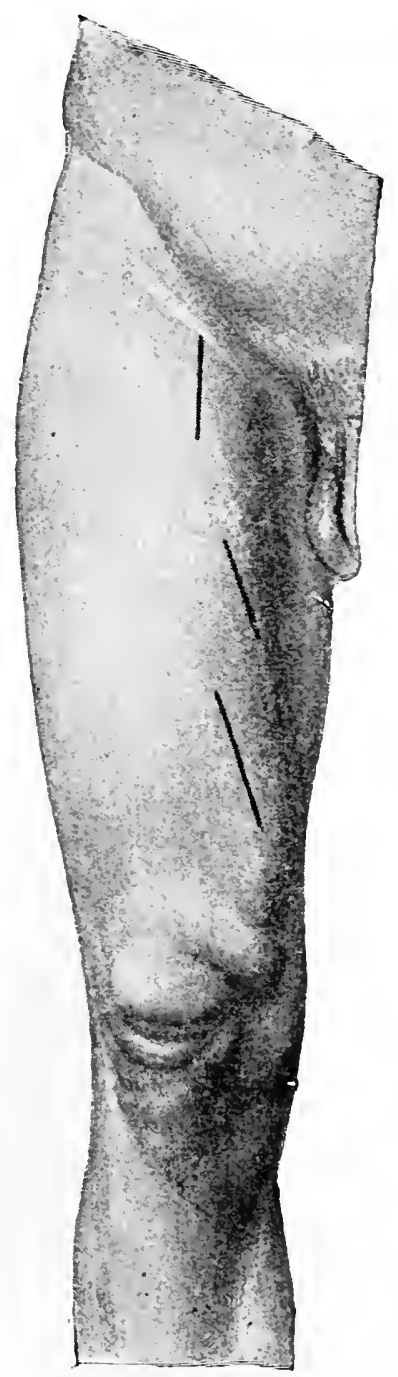

FIG. 49.-Cutaneous incision for ligation of the femoral artery : $a$, below Poupart's ligament; $b$, below the sartorius muscle; $c$, in the adductor canal. 

be divided in the direction of the entancons incision. If the incision be properly placed, the sartorius muscle comes into view after division of the fiscial, being recognized by its muscular fibers rumning paralled with the cutaneonis incision. If the fibers of the exposed muscle pass from within and above outward and downward, or the reverse, it may be known that the incision has been made too far inward or ontwand, and that the musele discloced is the adductor magnus or the vastus internus. The median border of the silltorius musele is exposed by disisection with the knife, and the muscle raised from its bed and drawn outward, when the deep layer of the fascia lata will be seen stretched tightly over the resseds. After division of the fitscia upon the grooved director, the artery, which in this situation lies in front of the vein, is isolated by blunt dissection and ligated (Plate 4).

\section{Ligation of the Femoral Artery in the Adductor} Canal.-The extremity is flexerl at the knee and the hip and slightly abducted and rotated outward at the hipjoint. The eutaneous incision is begun at the junction between the middle and lower thirds of the thigh along the lateral border of the sartorius muscle (Fig. 49, c). The dissection advances between the vastus internus and the sartorius until the silvery, fibrous sheath stretehed between the adductor magnus and the vastus internus comes elearly into view. The fibers of this sheath, which form the covering of the adductor canal, are divided, when the artery is disclosed. The rein lies behind the artery, with which it is intimately related by connective tissue (Plate 5).

Ligation of the internal saphenous vein has heen reeommender by Trendelenburg in the tratment of varicose veius of the lower extremities. $\Lambda$ cutaneons incision about:3 cm. (1 in.) long is made on the inner aspect of the thigh at the junction of its middle and upper thirds. A catgut ligature is thrown around the isolated rein and the extremity elevated in order that the bloor may make its exit. Two ligatures are applied to the rein and the ressel is divided between them. The small wound in the skin is closerl by sutures.

Ligation of the Popliteal Artery.-The popliteal space 
Plate 6.-Exposure of the Popliteal Artery. Left Lower Extremity, Flexor Aspect.

$S$, semimembranosus; $B$, biceps femoris; $T$, triceps; $I$, branches of the sciatic nerve; $A$, popliteal artery; $V$, popliteal vein.

is bounded above by the biceps femoris and the semimembranosus muscles, and below by the two heads of the

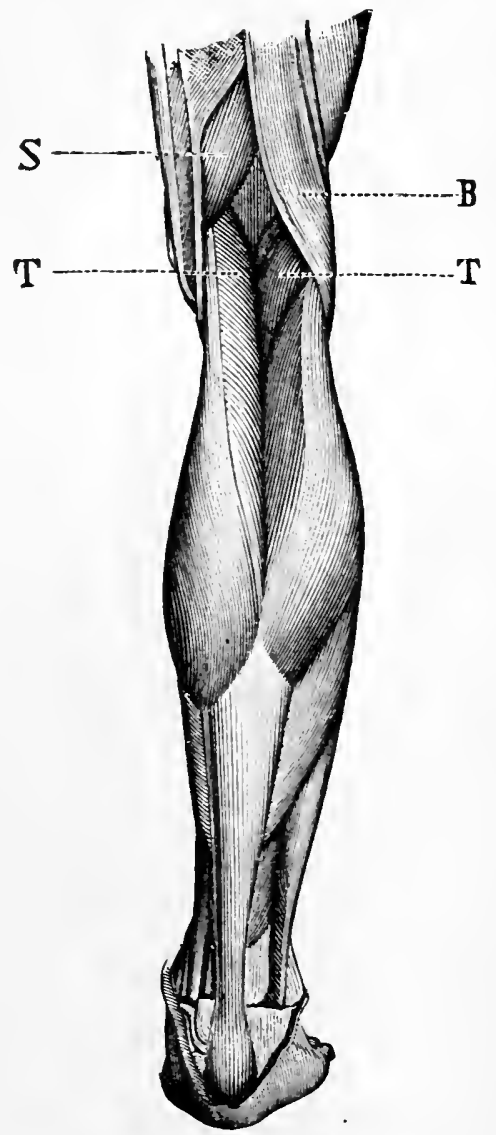

FIG. 50.-The muscles of the popliteal space and of the calf of the leg: $S$, semimembranosus; $B$, biceps femoris; $T, T$, heads of the gastrosnemius.

gastrocnemius (Fig. 50). The popliteal artery and vein pass in the longitudinal axis of this lozenge-shaped space, 

the artery more deeply than the vein and almost in contact with the eapsule of the linee-joint. The continuation of the seiatic nerve lies over the ressels and almost immediately beneath the superficial fisciat.

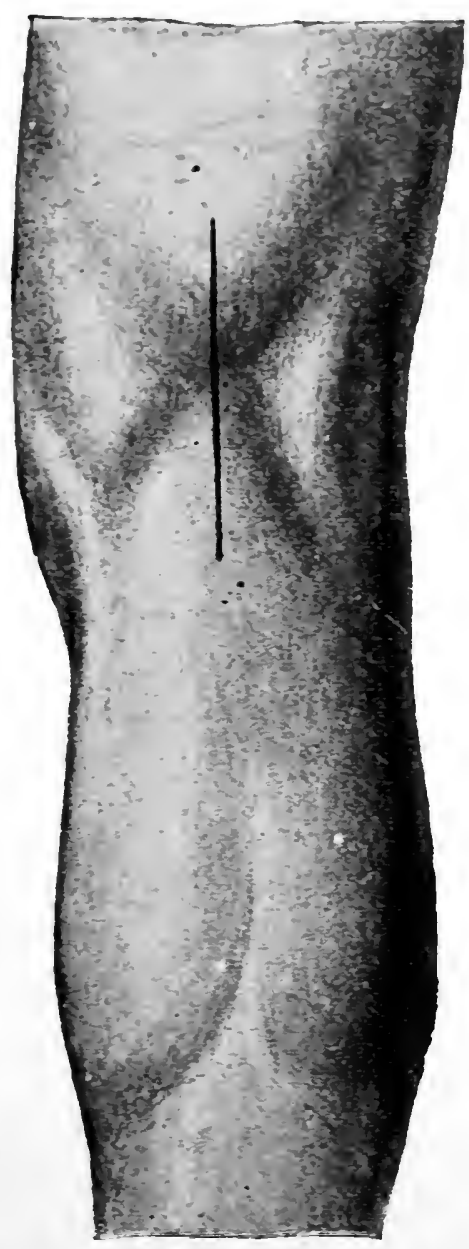

FIG. 51.-Cutaneous incision for ligation of the popliteal arters.

For the ligation of the popliteal artery the patient is placed in the prone position, and a longitudinal incision is made in the middle line of the popliteal space (Fig. 51). After division of the skin and the fascia the tibial 
nerve, which is superficial, is exposed. This nerve serves as a guide to the ressels, which will be found by advancing more deeply into the fatty connective tissue of the popliteal space at the side of the nerve. 'The rein lies behind the artery, with which it is intimately connected by cellular tissue.

Another way of reaching the popliteal ressels consists in entering the popliteal space from the inner aspect of the lowermost extremity of the thigh behind the tendons of the adductors, betreen these and the tendons of the gracilis and the sartorius. The extremity is flexed at the knee-joint, adducted, and rotated outward at the hip-joint. The adductor tendon can be felt on the inner side above the inner condrle, behind which the skin forms a slight depression. An incision is made in this situation in the long axis of the thigh, and after division of the fascia the adductor tendon can be separated from the sartorius and gracilis muscles by blunt dissection. The popliteal mass of fat exposed is entered from the side, and the sheath of the vessels is found upon the floor of the popliteal space.

\section{Ligation of the Anterior and Posterior Tibial Arteries.-} The muscles of the leg are so arranged that the flexors lie upon the anterior surface of the interosseous ligament, while upon the posterior surface lie the flexors. The latter are covered by the calf-muscles. The peronei muscles are grouped about the fibula. Upon the anterior aspect of the leg the ressels lie directly upon the interosseous ligament and pass among the muscles toward the dorsum of the foot. Upon the postcrior aspect the ressels and the nerves pass in the interval between the calfmuscles and the flexors.

The popliteal artery divides at the lower border of the popliteus muscle into the tibioperoneal trunk and the anterior tibial artery. The posterior tibial artery and the peroneal artery, branches of the tibioperoneal trunk, pass downward between the superficial and deep layers of muscles upon the flexor aspect of the leg, separated from the calf-muscles by a layer of fascia.

The posterior tibial artery runs close to the tibia and comes to lie behind the internal malleolus, in which situation, corresponding to the origin of the abductor hallucis, it divides into the internal and external plantar arteries. 
The peroneal artery runs in the same plane along the fibula toward the exterual malleolus, where it terminates in several branches.

The anterior tibial artery penetrates the interosseous ligament from the flexor aspect of the leg and thus comes

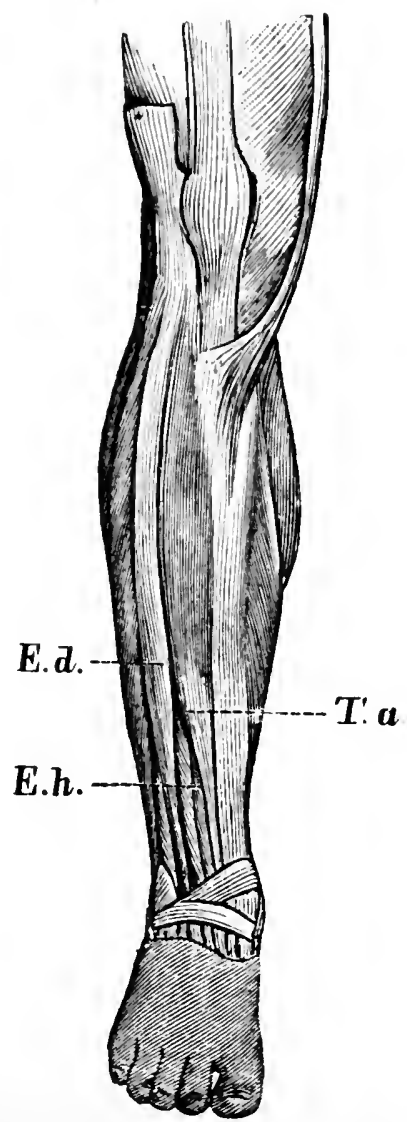

Fig. 52.-A Arrangement of the muscles of the leg, anterior aspect: the arteries lie upon the interosseous ligament and pass between the tibialis anticus muscle (T.a.) and the extensor digitorum (E.d.), or the extensor hallucis (E.h.).

to lie upon the anterior surface of the ligament beneath the muscles. In this situation the vessel runs along the outer border of the tibialis antieus musele toward the foot (Fig. 52). Arrived at the dorsum of the foot, the artery, 


\section{Plate 7.}

FIG. 1.-Exposure of the anterior tibial artery of the left leg. The fascia is opened, and the tibialis antieus muscle $(J a)$ is retracted toward the median line, and the extensor hallucis $(E . h$.$) toward the outer$ side. In the interval between the two museles the deep perineal nerve (P.p.) comes first into view and behind it the artery surrounded by reins.

FIG. 2.-Exposure of the posterior tibial artery behind the internal malleolus. The tortuous artery accompanied by two veins is visible beneath the dirided fascia $(F)$.

here known as the dorsalis pedis, lies upon the onter side of the tendon of the extensor hallucis longus. In the space between the metatarsal bone of the great toe and the second metatarsal bone the artery descends toward the sole of the foot, where it unites with the external plantar artery, one of the two terminal bramches of the posterior tibial artery.

The anterior tibial artery is accompanied by the deep peroneal nerve and the posterior tibial artery by the posterior tibial nerve.

The anterior tibial artery is ligated typically in its course at a point corresponding to the junction of the middle and lower thirds of the leg, and as the dorsalis pedis upon the dorsum of the foot. The posterior tibial artery is exposed for ligation in its course upon the leg and behind the internal malleolus.

In ligating the anterior tibial artery an incision is marle upon the anterior aspect of the leg in a situation corresponding with the junction of its middle and lower thirds, a finger's breadth external to the crest of the tibia (Fig. 53). After division of the tense and tough fascia in the line of the cutaneous incision, the dissection is proceeded with from the outer side of the tendon of the tibialis anticus muscle between this and the adjacent extensor hallucis longus down to the interosseous ligament. Before the vessel is reached the deep peroneal nerve eomes into view. Behind this lies the artery, surrounded by a network of veins (Plate 7, Fig. 1). To facilitate isolation of the vessel it is well to separate the tibialis anticus and 

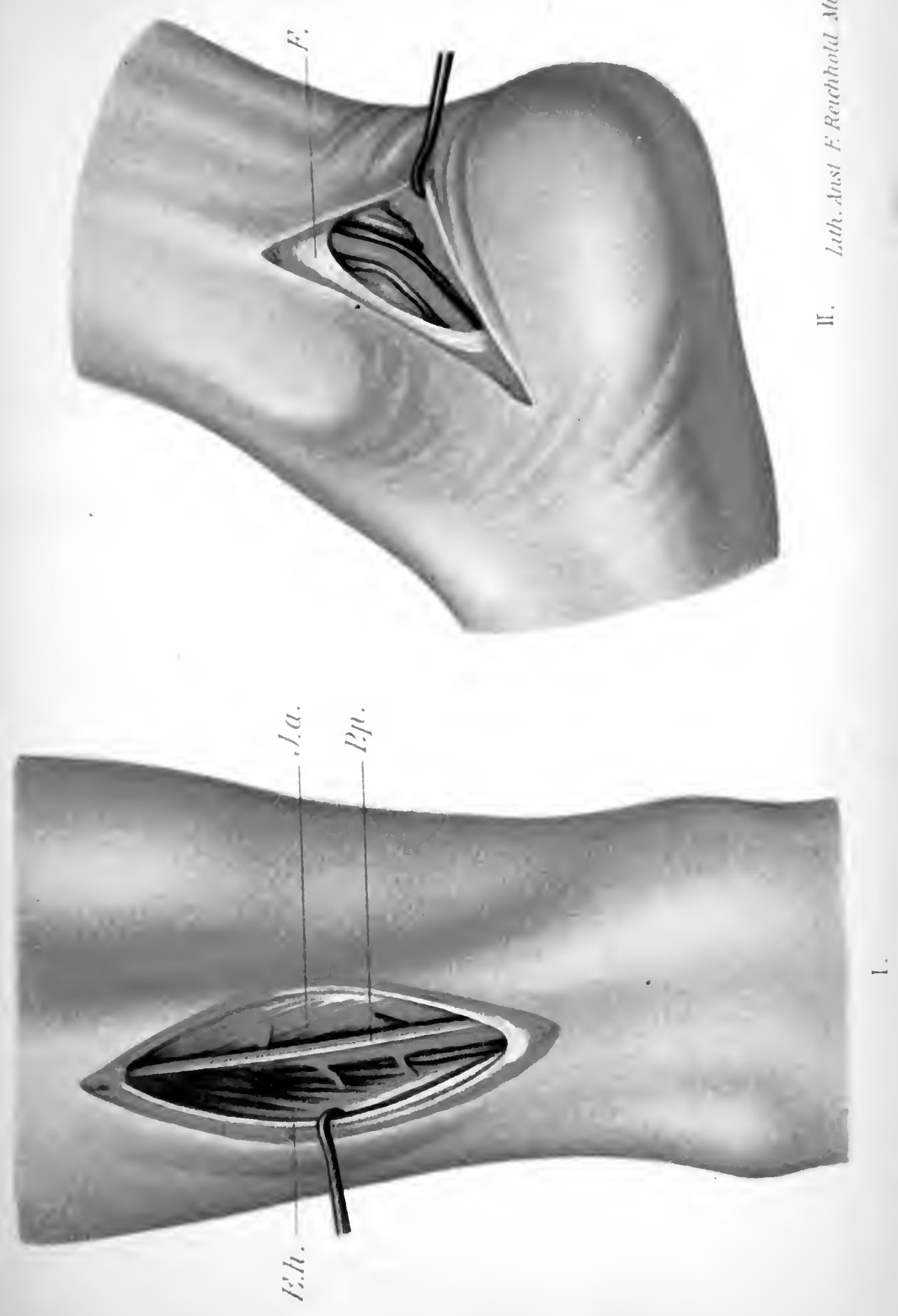

the extensor hallucis museles vigorously with tenacula. In order to reach the artery readily it is important to follow accurately the interval between the muscles, as otherwise aceess to the vessels will be attended with difficulty.

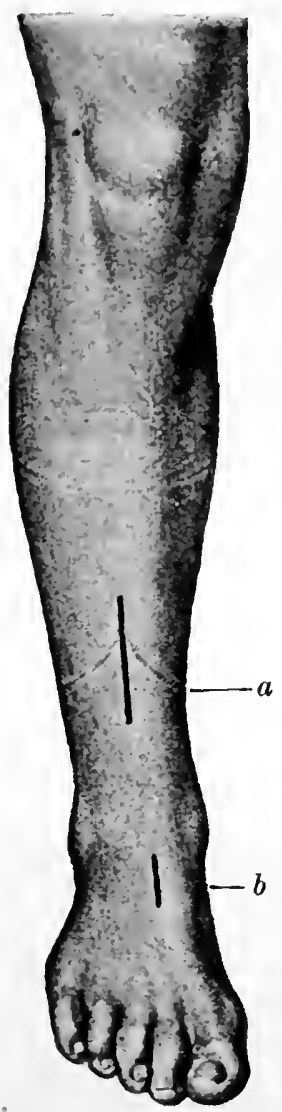

FIG. 53.-Cutaneous incisions for ligation of the anterior tibial artery (a) and the dorsalis pedis artery $(b)$.

Entrance is secured from the outer border of the tibialis posticus muscle.

To expose the posterior tibial artery in its course along the leg, a longitudinal incision is made in the middle third of the leg, a finger's breadth internal to the median border 
of the tibia (Fig. 54, a). After division of the skin and fascia the fibers of the soleus muscle are freed from their attachment to the bone, and the interval between the superficial and the deep group of calf-muscles occupied by loose connective tissue comes into view. If the muscles of the calf in the extent of the wound are raised from their bed

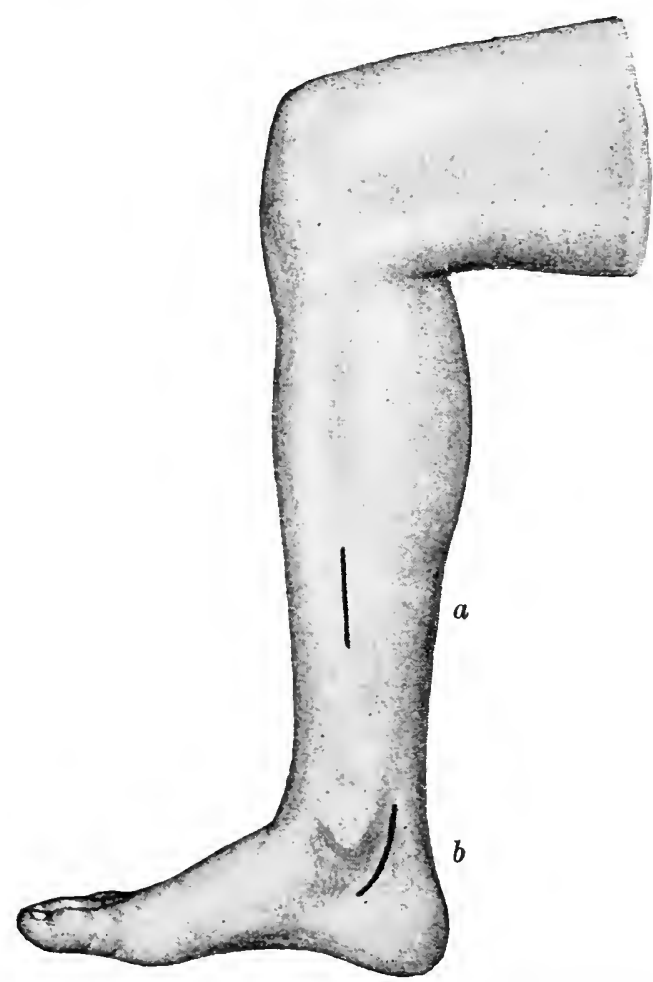

FIG. 54.-Incisions for ligating the posterior tibial artery in the leg (a) and behind the internal malleolus $(b)$.

with a blunt hook, the posterior tibial artery, accompanied by the nerve of the same name and sereral veins, will be found beneath the layer of fascia covering the deep group of muscles between the tibialis posticus and the flexor hallucis longus muscle.

In the ligation of the posterior tibial artery behind the internal malleolus the foot is rotated outward in maximum 
degree and a curved incision encircling the malleolus is made at a point midway between the extrenity of the internal malleolus and the median border of the tendo Achillis (Fig. 54, $b$ ). If the fibrous fiscia is exposed, the artery comes into view, accompanied by two veins. After division of the fascia the artery can be isolated by blunt dissection. If the procedure be properly carried sut, the muscular sheath of the tibialis posticus, the flexor hallucis longus, and the flexor digitorum is not opened. The tibial nerve lies to the outside of the ressels (Plate $i$, Fig. 2).

The dorsalis perlis artery is ligated upon the dorsum of the foot in front of the ankle-joint, the foot being held in strong plantar flexion. The tendon of the extensor hallucis is thus made palpable and an incision is made to its outer side and parallel with it. The fascia is now divided, and in close relation with the bone, the artery, accompanied by two veins, is found to the outer side of the tendon of the extensor hallucis longus. 


\section{Amputations and Enucleations.}

The surgical removal of an extremity or portion of an extremity is designated an amputation when the separation is made in the continuity of the bone, and an enucleation if the separation is made at an articulation. The soft parts are variously divided in order that they may adequately cover the wound left after division or separation of the bone.

Indications.-(1) Derelopmental anomalies, supernumerary fingers, or acquired incorrigible deformities (contractures, atrophic extremities).

(2) Injuries, such as rending or crushing of the extremities, destruction of the skin over a large area; in general, when restoration of the function of the part seems to be excluded by the degree and the character of the injury.

(3) Caries of joints in adults, when conservative operations are no longer possible, or when the articular disease is superadded to a pre-existing tuberculosis of the lungs or of other organs.

(4) Infections, phlegmonous inflammations of the tissues, gangrenous destruction of joints, osteomyelitis with epiphyseal separation and suppuration of joints, always when the morbid process is not to be localized.

(5) Neoplasms, malignant disease of bones and soft parts; rarely benign growths also, such as enchondromata of the fingers, furnish an indication for amputation or enucleation.

(6) Gangrene of the extremities when a line of demarcation has formed.

(7) Incurable circumferential leg-ulcers and elephantiasis.

(8) Incurable pseudarthroses.

Reamputation is indicated in the presence of-

(1) Neuralgia of the stump, due to the presence of neuromata ;

(2) Conical amputation-stumps. 
In the performance of amputation the patient is so placed that the member to be amputated is conveniently accessible. The upper extremities are raised to a horizontal position and there maintained by assistants. In removal of portions of the lower extremities the member, which is held horizontally, extends beyond the edge of the table. An assistant grasps the extremity at its peripheral end, and another assistant fixes it on the central side of the point of removal.

Provisional control of hemorrhage is effected during the operation either through digital compression of the artery or by surrounding the extremity with the elastic bandage of Esmarch.

Digital compression is only available when the artery can be compressed against a firm base, as a bone. Thus, the femoral artery is compressed against the horizontal branch of the pubic bone at the point where it passes under Poupart's ligament; the subelavian artery is compressed in the supraclavicular fossa against the first rib.

In controlling hemorrhage by means of the elastic bandage of Esmareh the extremity is held vertically for a short time to facilitate the eseape of blood by gravitation, and is then surrounded by an elastic bandage or an elastic tube above the point of proposed operation.

Position of the Operator.-If the operator be righthanded, he takes such a position that the member to be amputated is upon his right. In amputations of the right upper extremity the member is abducted and raised to a horizontal position. The operator stands upon the outer side, with his face direeted toward the feet of the patient. In amputations of the left upper extremity the operator stands between the trunk of the patient and the abducted arm, with his face direeted toward the patient's head. In amputations of the right lower extremity the operator stands upon the outer side, and in operating upon the left side between the abducted lower extremities of the patient. The operator stands with his right foot slightly in advance 
and supports himself with his left hand upon the stump while making the incisions with his right.

Steps of the Operation.-The operation includes the following four steps:

(1) Division of the skin and the muscles;

(2) Removal of the part, sawing through the bone; and

(3) Control of hemorrhage by ligation of the vessels;

(4) Treatment of the wound.

The division of the parts is so effected that after removal of the amputated part union of the wound by suture is possible, the stump is adequately covered by the soft parts, and the cicatrix, especially following amputations of the lower extremity, is farorably situated. The soft parts are divided more or less transiersely to the long axis of the member (circular incision), or flaps are formed from the soft tissues, which, after completion of the amputation, may be brought together and united. An intermediate mode of procedure between the two is known as racket or oral incision.

Circular Incision.-In accordance as the soft parts are to be divided transversely or obliquely to the long axis of the part to be amputated a distinction is made between the transverse and the oblique circular incision. The division of the soft tissues down to the bone may be effected by a single incision, or the skin and the muscles may be divided at different layers by two incisions. The first method, which has been attributed to Celsus, has only a limited field of application. The second (J. L. Petit, Cheselden) was originally practised in such a way that the skin was first divided in a circular manner. The muscles were then divided by a second more proximal incision, while retraction was practised. This form of incision in two steps is thus but a modification of the procedure of Celsus.

A better purpose is served by dividing the skin circularly at some distance toward the periphery from the point at which the amputation is to be made. To this 


$$
\text { AMPETATIONS AND ENLCLEATIONS. }
$$

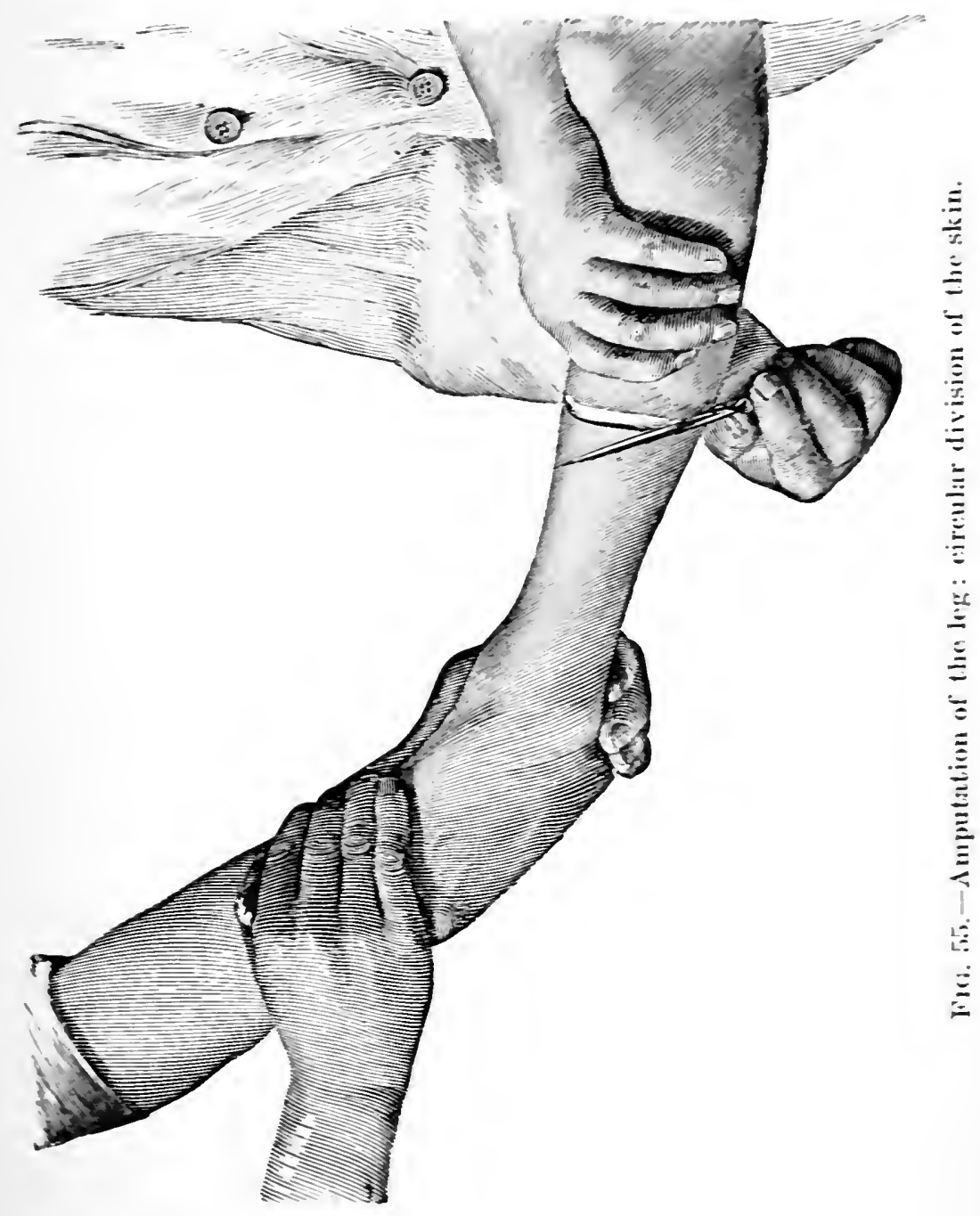


- 
AMPUTATIONS AND ENUCLEATIONS.

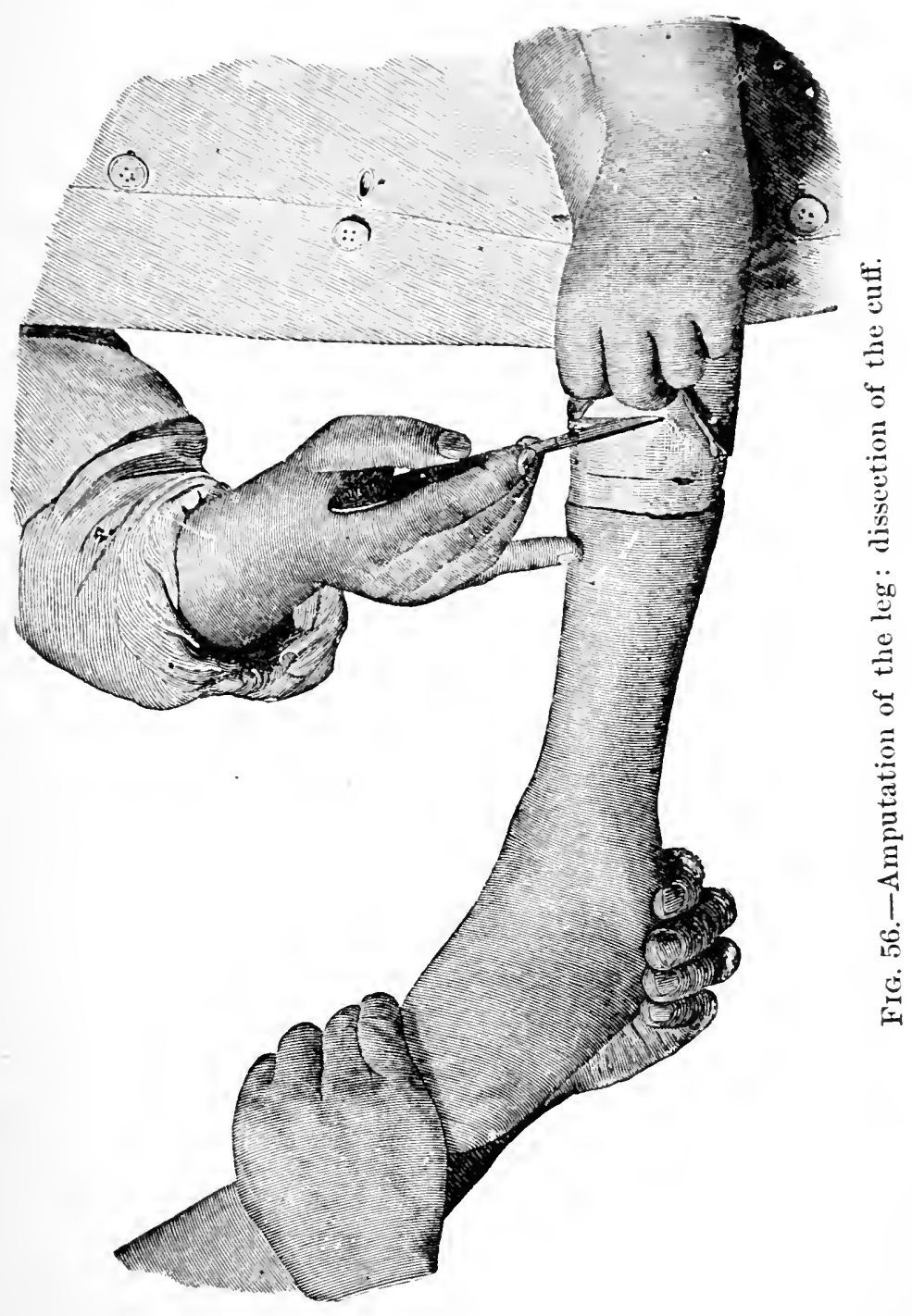




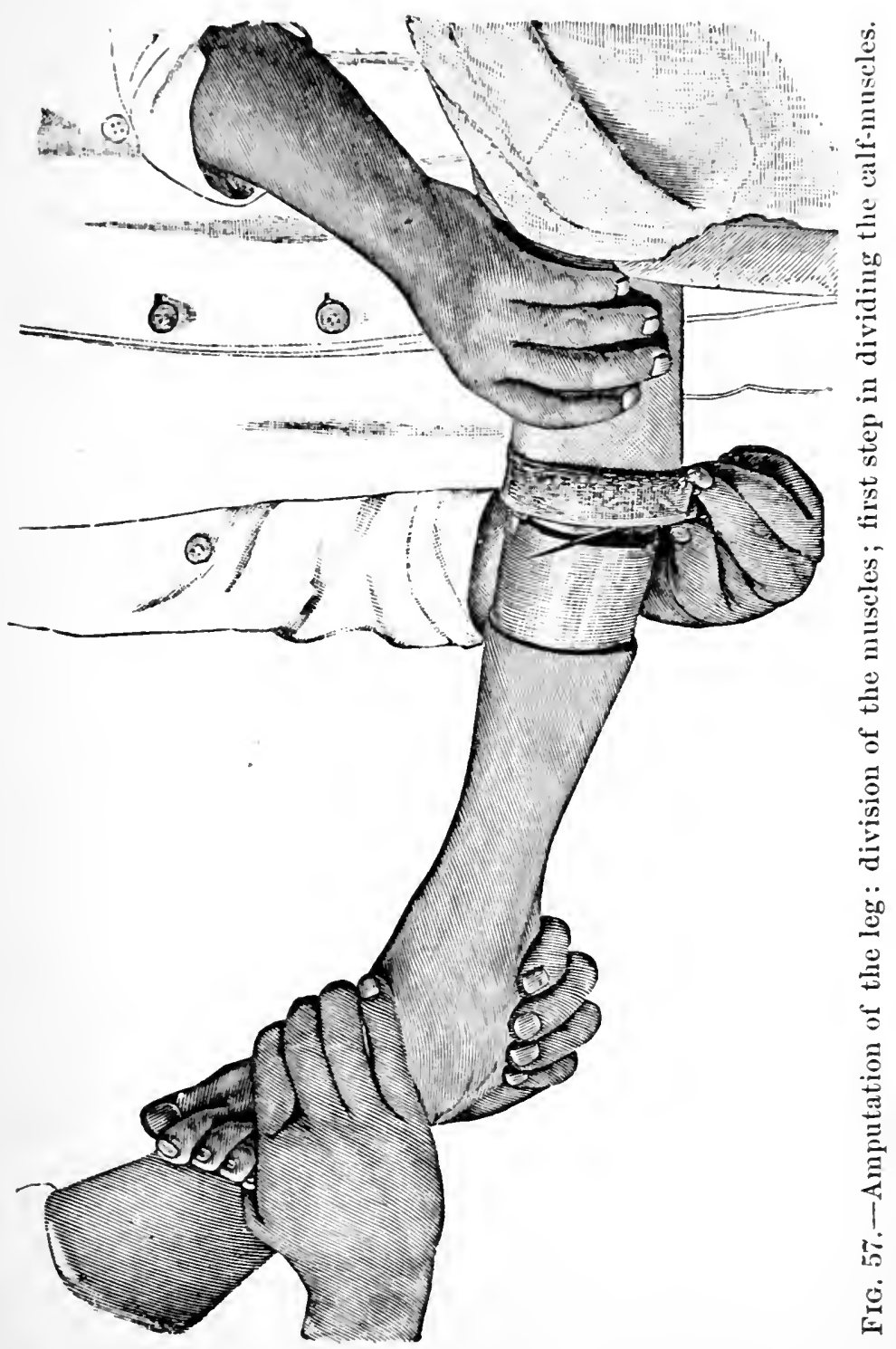



end the knife is applied upon its heel, with its point directed toward the operator, and carried around the lower cireumferenee of the member (Fig. 55). The knife is now applied with its heel at the beginning of the incision already made, and its point directed from the operator and carried around the upper cireumference of the member in an opposite direction. 'There thus results a circular incision formed of two conjoined semicircular ones. When the skin throughout the entire circmuference has been evenly divided down to the muscular fascia it is dissected all around from the subjacent tissues in a central direction, so that there results a hollow eylinder or cuff constituted of skin and subentancous connective and fatty tissue. In the dissection of this cuff the operator grasps the margin of the wound from above with thumb and index-finger at a point most remote from him and proceeds to separate it from the subjacent tissues. The fibers uniting the skin and the fascia are divided by strokes of the knife held vertically to the underlying structures (Fig. 56). The adjacent skin is now grasped and separated from the subjacent tissues in the same manner. The operator in this way gradually encircles the entire circumference of the extremity. When the starting-point has been reached the process of detachment is renewed, and this is repeated until a cuff of sufficient size has been obtained that can be everted and turned on itself.

It is important that the line at which the cuff is folded over should occupy a plane at right angles to the longitudinal axis of the member. The size of the cuff will be governed by the diameter of the member to be amputated. In general the entaneous incision will lie toward the periphery at a distance from the plane of amputation equalling about two-thirds of the diameter of the extremity at the level of this plime.

The muscles are divided with long strokes of the amputation-knife at the level where the cuff of skin has been folded over in such a way that the eut surfaces of the muscles form an even plane. In the division of the 
muscles the knife is held in the same manner as in making the cutaneous incision (Figs. 55 and 57). Experience will teach the proper degree of pressure and of traction to be exercised. The linife is applied upon its heel and pressure exerted against the resisting mass of museles, the operator at the same time making traction toward himself. In this way the muscles are divided with a single stroke at the margin of the cuff, the knife being drawn from heel to toe in a horizontal plane toward the operator. Without changing the position of the knife the direction of pressure is reversed, the blade being applied with full force and passing from toe to heel, while the hand is raised, the remainder of the muscular tissue upon the lower portion of the circumference and upon that portion most distant from the operator being divided. Next the muscles of the upper portion are divided. To this end the knife is applied from above with its heel in the beginning of the incision and its point directed from the operator, and the muscles are cut through.

In amputations of the leg and the forearm the division of the structures between the two bones requires a special procedure. After the muscles have been divided circularly in the manner described, a small knife is introduced into the interosseous space, with its edge at right angles to the bone, and the contained structures are severed. The details of this procedure are described at length under Amputations of the Leg.

Flap-incision.-In this method of operating the soft parts are incised in the form of flaps, which are used to eover the stump. Flaps may be made in various shapese.g., semicircular, tongue-shaped with parallel margins and rounded corners, etc. They consist of either skin or skin and muscular tissue, or skin and periostenm, and are accordingly designated tegumentary flaps, musculotegumentary flaps, tegumentary-periosteal flaps, etc. Portions of bone may also be included with the skin in flaps and be so adapted to the wound-surface as to contribute to the formation of a satisfactory stump. Flaps are always con- 
A.MUTATIONS ANU EYCCLEATIONS.

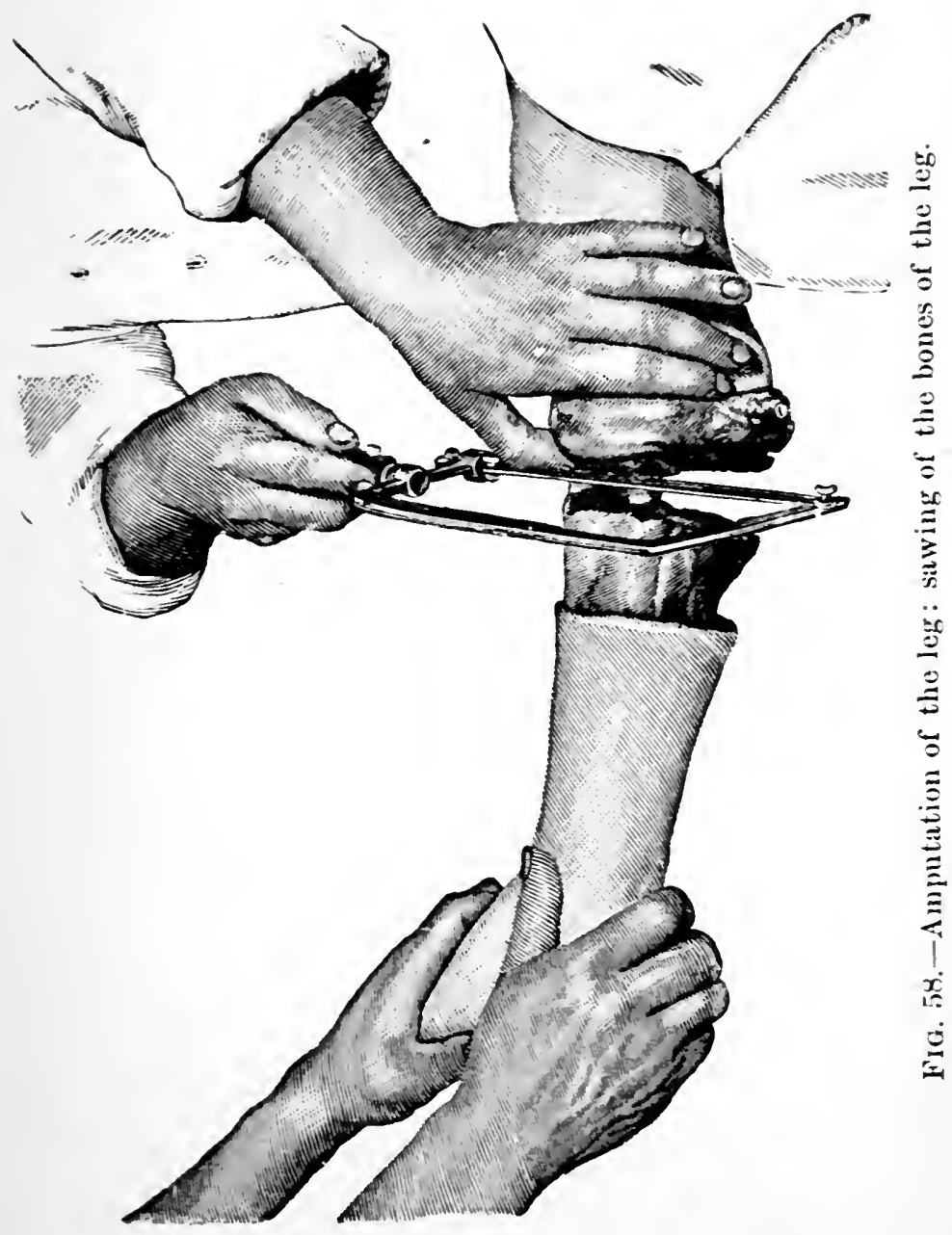


AMPLTATIONS AND ENLCLEATIONS.

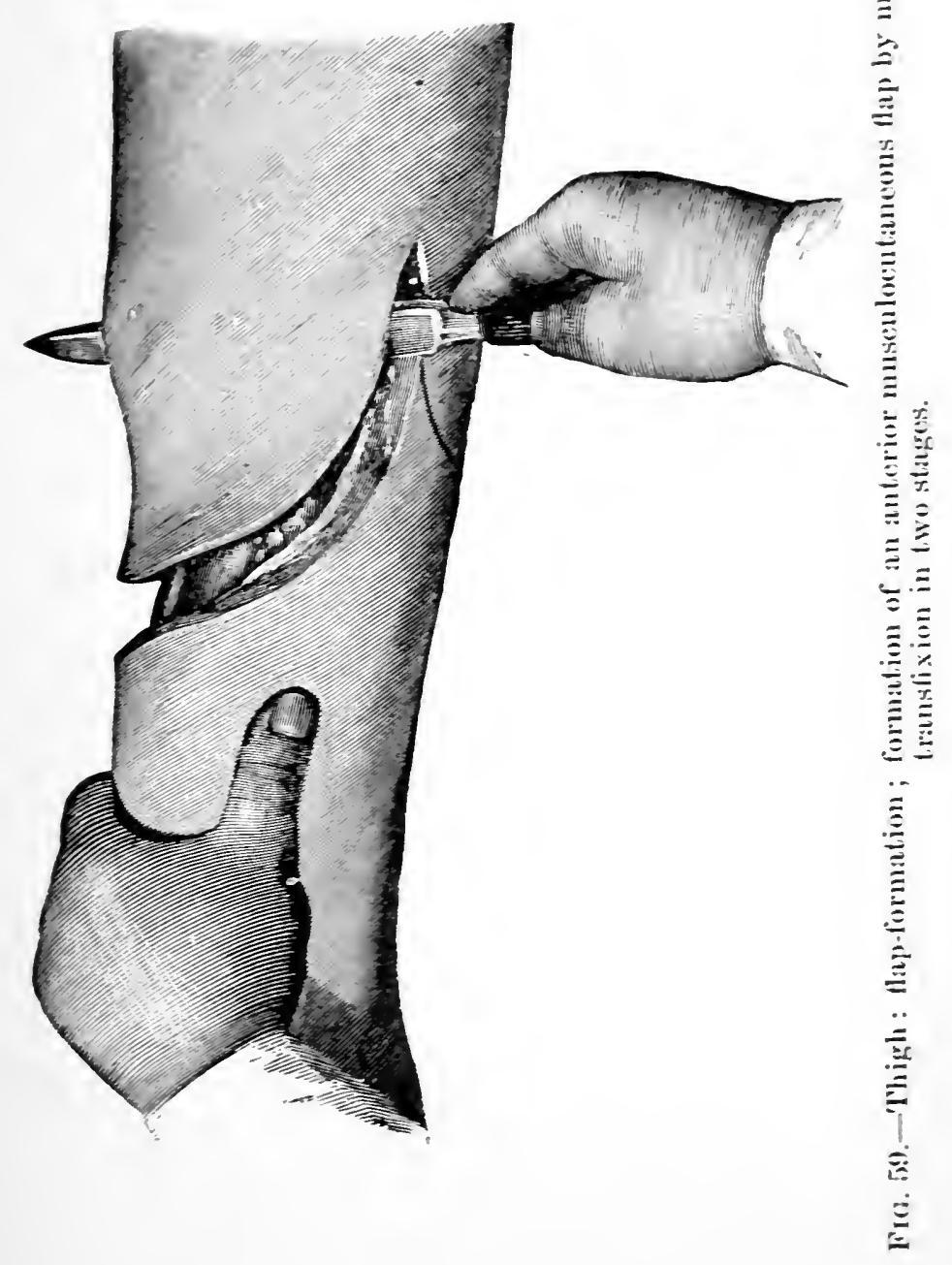





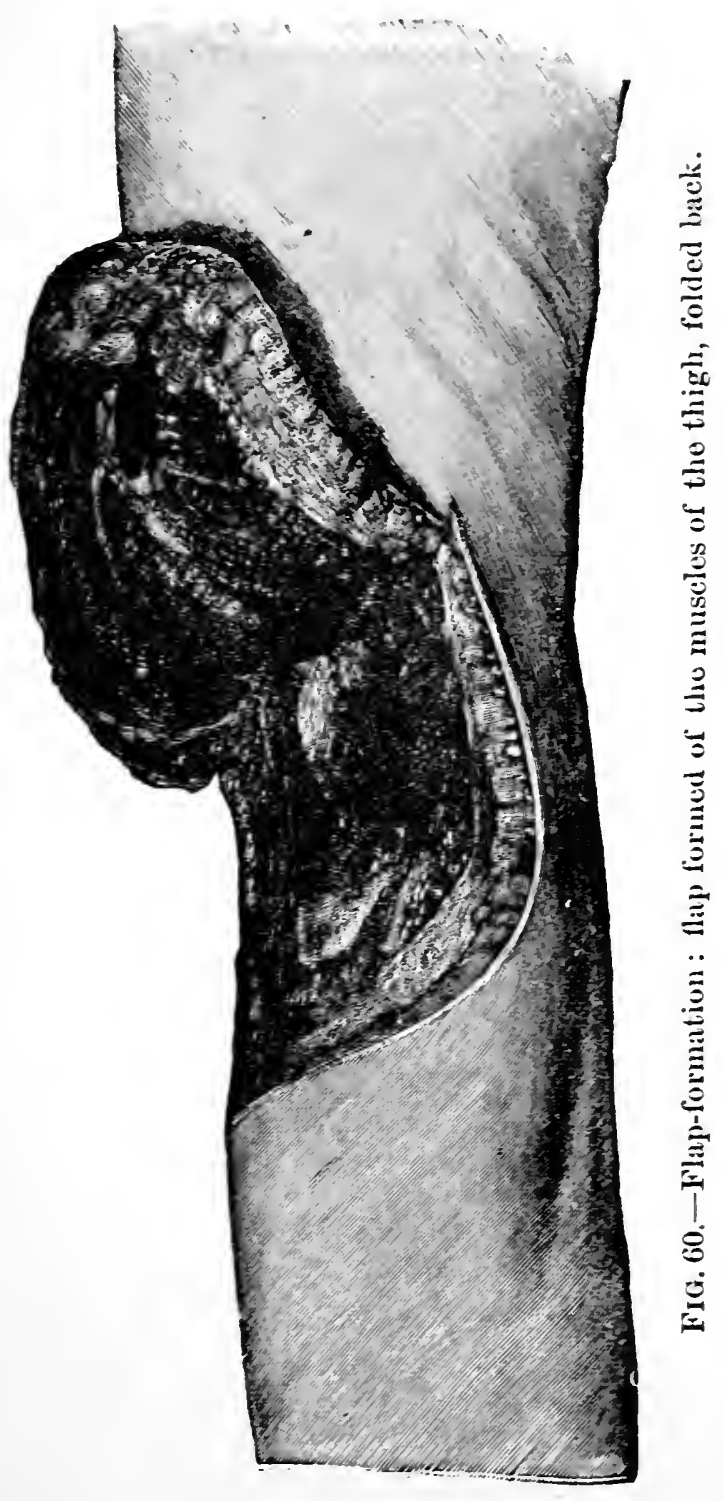




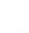




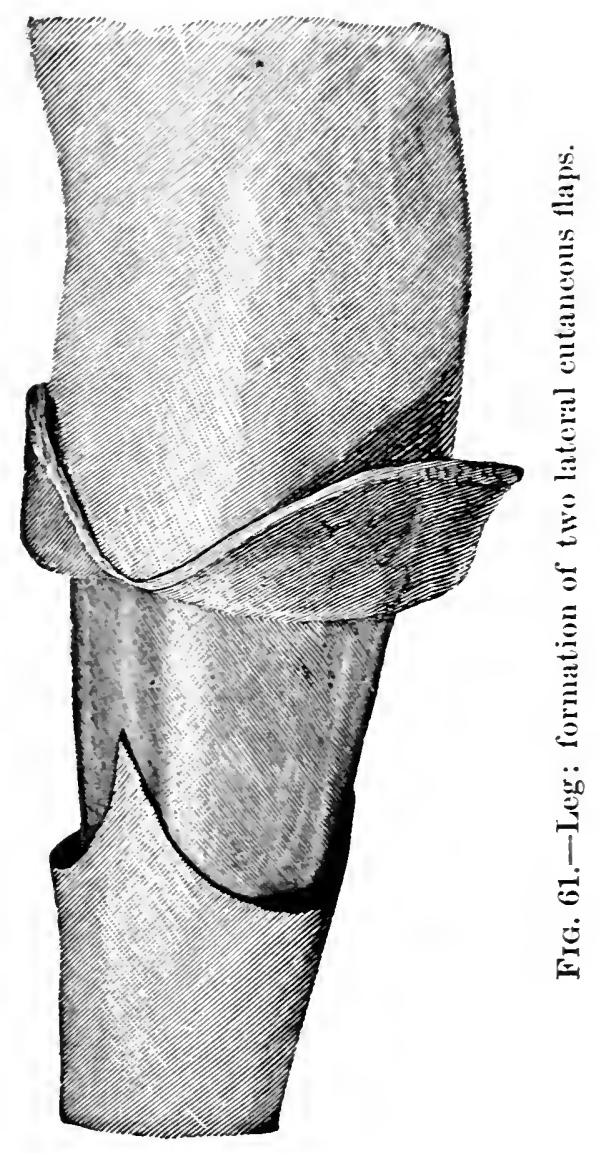




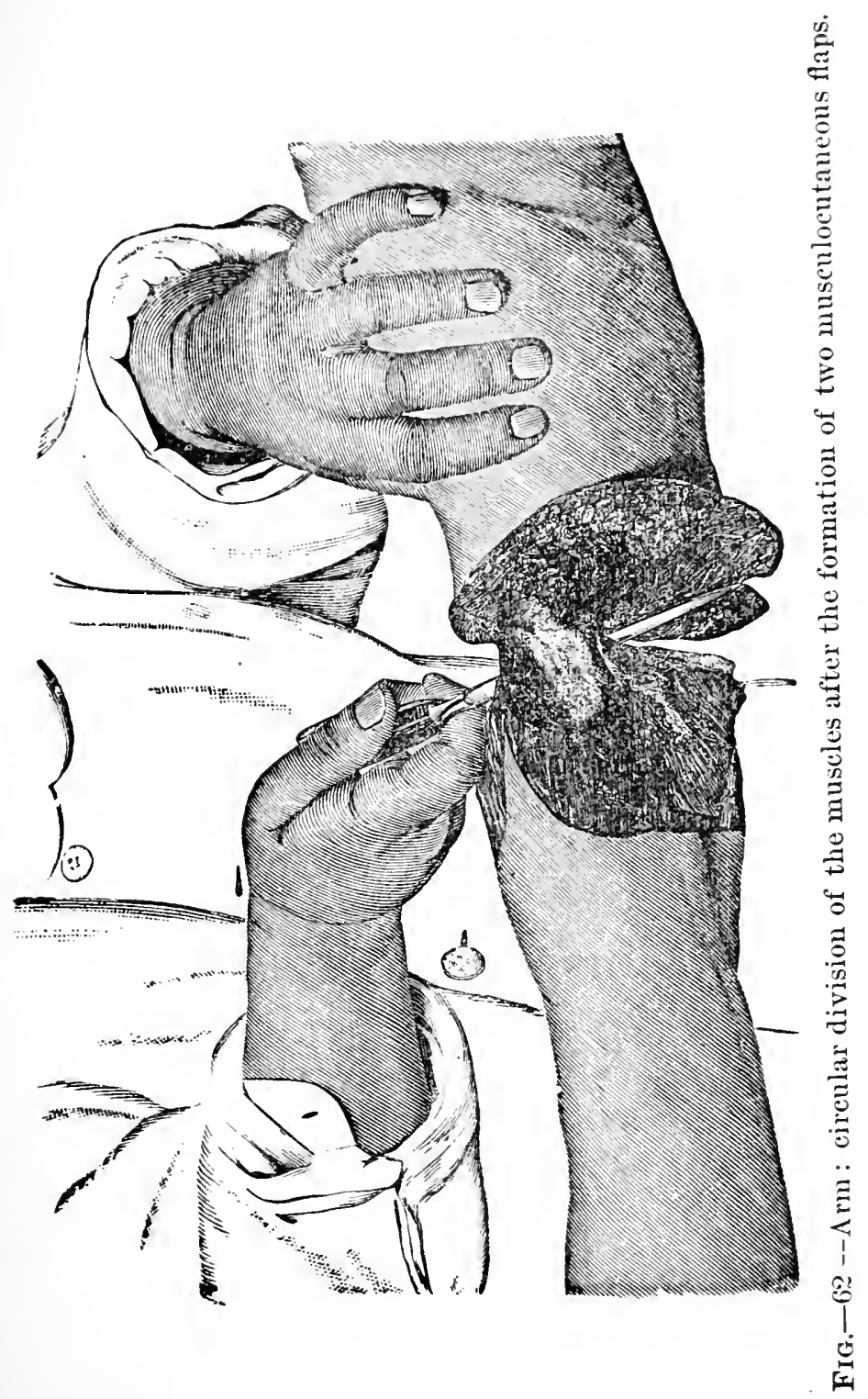


stituted of a greater amount of structure at their base than at their fiee extremity. 'The stmmp is covered with a single flap (Lowdham, Verduin) or with two flaps (Ravaton). Flaps may be made of equal or of unequal size, and in accordance with their situation they are designated anterior, lateral, or posterior.

The formation of flaps may be effected by incisions from without inward (Langenbeck), or the reverse by cutting from the base toward the periphery (transfixion). Under the conditions first indicated the shape of the flap is outlined with the knife, and the skin and fascia in this distribution divided down to the muscular laver, when the flap retracts somewhat. From the line of retraction the muscular layer is divided with long strokes of the knife down to the bone, when the whole flap can be turned back. When a tegumentary flap is to be formed the skin, after the outline of the flap has been made with the knife, is grasped with a pair of toothed forceps or a tenaculum, and raised, being progressively separated from the subjacent structures.

In making flaps by transfixion smooth wound-surfaces are afforded. One of two modes of procedure may be followed. The flap may be formed by direct transfixion, the knife being thrust transversely through the muscles close to the bone and cutting toward the skin. In the other mode of procedure the operator outlines the shape of the flap with the knife, at the same time dividing the fascia so that the flap retracts somewhat. The knife is now introduced at the base of the flap in the manner already described and carried through the muscles to the end of the flap, where it makes its exit. By this means is attained the most adrantageous distribution of the muscles in the flap, which at its periphery will consist only of skin and fat (Figs. 59 and 62 ).

When two flaps have been formed in one of the ways described, they are folded orer and the muscular tissues yet attached to the bone are divided by a circular incision (Fig. 62). In amputations of the leg or of the 
forearm the muscular tissue in the interosseous space should be divided according to established rules before the bones are sawed through.

The oval incision results when the circular incision is joined at some point of its periphery by a longitudinal incision, which, like the circular incision, passes through the periosteum down to the bone. The apex of the oval is placed either upon the extensor aspect of the joint or upon one of its lateral aspects. From it pass in a peripheral direction two diverging incisions, which meet trans-

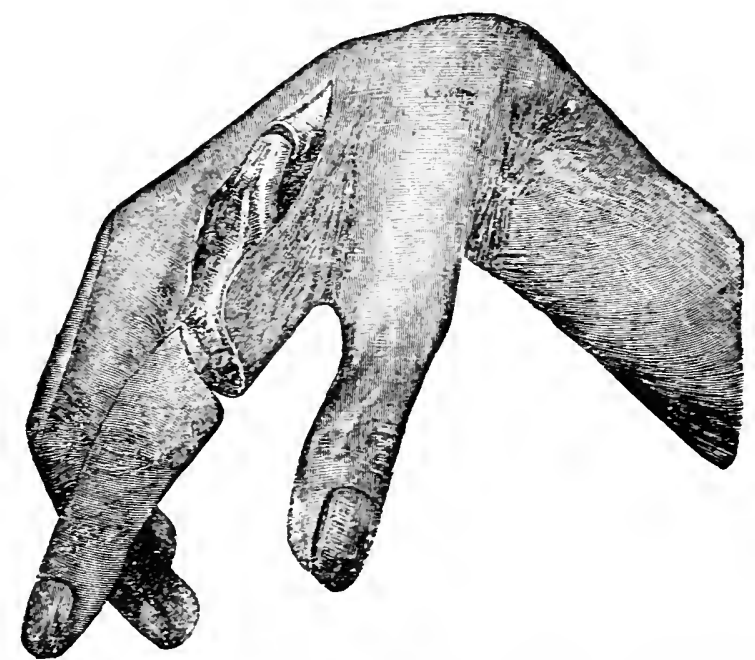

FIG. 63.-Oval incision for amputation of the index-finger at the metacarpophalangeal articulation.

versely at an opposite point of the periphery (method of Scoutetten). The form of the wound corresponds with that of a heart, the apex of the oval being the most central portion. The bone is removed at this level (Fig. 63). After union by suture the wound is linear. The oval incision is employed especially in enucleation of fingers and toes; less commonly in enucleation of the shoulder-joint or the hip-joint.

Division of the Bone.-After complete division of the soft parts and incision of the periosteum the latter, in the 
area of the incision, is carefully detached from the bone for a short distance in a proximal direction with a raspatory, in order that a sufficient surface be exposed for the application of the saw and the periostem is not crushed or torn from its attachments, and primary union be thereby prevented. In sawing through the bone care should be taken that the division is effected as far from the periphery as possible; that the sawed surface is at right angles to the longitudinal axis of the bone; and that the soft parts are protected from all injury. The muscles are drawn with tenacula out of reach of the saw or the stump is wrapped in suitable compresses and thus protected.

In applying the saw the nail of the thumb of the left hand is placed rertically upon the denuded bone (Fig. 58), and the support thus afforded is used as a guide for the blade of the saw. At first the saw is manipulated without pressure. Only after a groove has been formed in the bone may the sawing be proceeded with more rapidly, with the application of a certain degree of pressure. An assistant, stationed at the periphery, holds the extremity in a position of extension and aims to keep the sawed surfaces apart in order that the blade of the instrument shall not become impacted. The irregular edges of the divided bone are trimmed by means of bone-forceps, and small projections are cut off."

After complete removal of the amputated part attention is directed to the definite contiol of hemorihage. The main arterial and venous trunks, recognizable by their position, are isolated in the stump with the aid of two pairs of dissecting-forceps, clamped in sliding-forceps, and ligated. In addition to the main arterial branches all vessels that can be seen running in the connective-tissue interstices of the muscles are also ligated. The Esmarch bandage can now be freed, and it may be necessary to apply additional ligatures. Parenchymatous hemorrhage is controlled by compression.

The care of the wound has for its object accurate ap- 
proximation of the wound-surfaces, with the avoidance of dead spaces, as well as exact approximation of the margins of the skin. The muscles maly be separately united by buried sutures or laree areas of surface by means of ganze-pad sutures, while the skin is closed by superficial

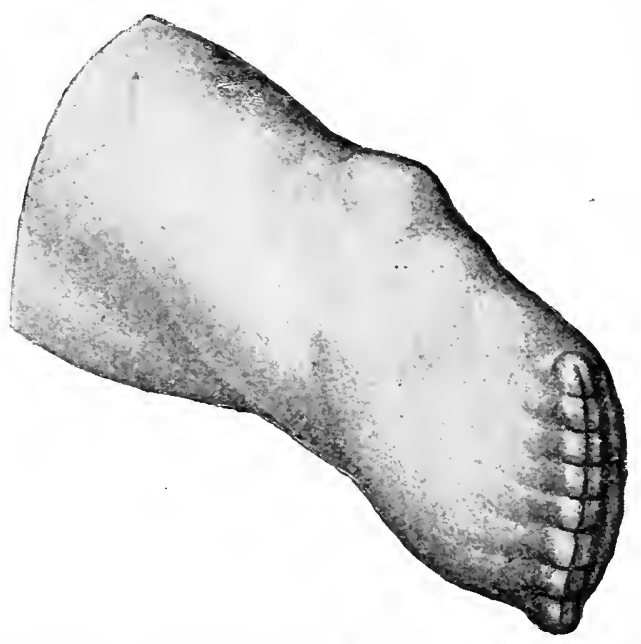

FrG. 64.-Cutaneous suture after amputation of the leg through a circular incision.

knotted sutures, or by a continuous suture (Figs. 64 and $65)$.

If it has been possible to effect the amputation under complete aseptic conditions, the wound may be closed entirely by suture; otherwise drains may be brought to the surface out of the depth of the wound. A like purpose may be served by the introduction of strips of sterile or antiseptic gauze.

In the performance of exarticulation the same general principles may be observed as in the performance of amputation. The operator stands at the periphery of the extremity, holding the part to be removed in the left hand, while the exarticulation is effected with the right. In the majority of cases the joint is opened from its extensor aspect. Flap and oval incisions are generally employed, less commonly circular incisions, with the 
formation of a cuff: The flaps are so formed that their base corresponds with the plane of the joint at which the separation is to be made. As a rule, flaps of "umusual size are made. Upon that side of the joint on which the capsule is first opened the flap may be marle by transfixion or by dissection from without inward. When the exarticulation is completed the soft parts upon the opposite side of the joint are diyided from within outward. To

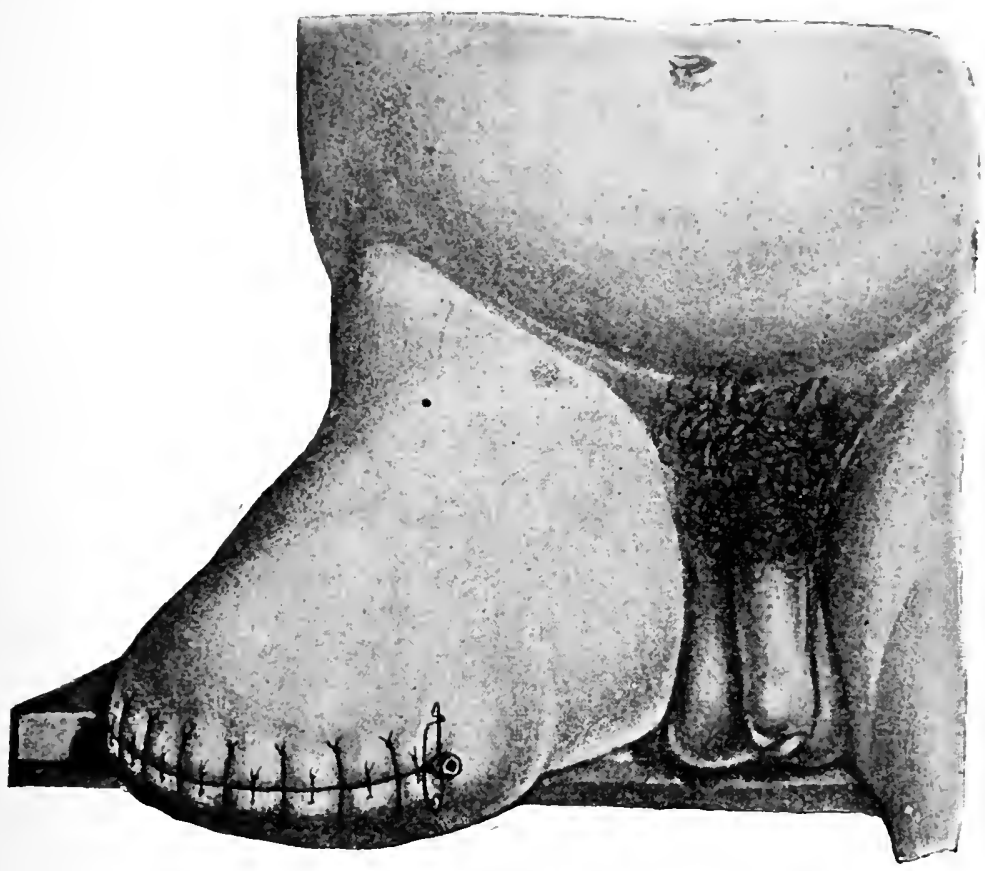

FIG. 65.-Cutaneous suture after flap-amputation of the thigh.

this end the operator draws upon the extremity, grasped with the left hand and already freed at the articulation, in such a way that the bridge of skin still uniting the part with its central attachment is made smooth and tense. The knife is introduced into the wound and divides the tissues transversely. In making the division care should be taken that the muscles are first divided and then, somewhat further toward the periphery, the 
skin. The methods of performing exarticulation are in part so carried out that with the last incision of the knife, which passes from the wound and forms the flap, the main vessels are severed. During the process of division the artery may be closed by pressure with the finger in the wound. A method of exartieulation (Esmarch) often employed with large joints consists in cireular division of the soft parts in the upper third of the extremity down to the bone after applieation of the Esmarch bandage. The bone also is divided at the level of the incision through the muscles. After ligation of the vessels the constricting band is removed. A longitudinal ineision is made through the soft parts down to the bone from the joint to the primary wound in such a way that large vessels and nerves are not divided. With the wound thus made held open by means of hooks, the joint is opened and the remainder of the bone removed with the utmost care. This combination of eircular and longitudinal incisions constitutes a variety known as the racket-incision.

\section{Amputations and Exarticulations of the Lower} Extremity.-Amputation of the Leg.-The removal of the leg may be undertaken at varying levels. It was formerly the custom to amputate the leg under all circumstances in its upper third at the site of election. This method had for its object the use of a wooden leg, upon which the flexed knee was comfortably received after the wound had healed. At present, however, the principle is followed to be as conservative as possible in amputations of the leg, and in the removal to take the greatest care of the healthy portion of the extremity. For this reason amputations are no longer performed at the site of election, but at the site of necessity.

Among the methods of amputation of the leg employed are the circular incision in two steps, with the formation of a cuff; and various forms of flap-operations: two lateral tegumentary flaps; two lateral musculotegumentary flaps; one anterior tegumentary periosteal flap; and a posterior short musculotegumentary flap from the 
ealf (Heine). I single large musculotegumentary flap from the substance of the calt also may serve to cover the stump.

Amputation of the Leg with a Circular Incision in Two Steps. - In assistant rotates the leg towarl the operator and a circular incision is made through the skin, beginning at a point most remote from the operator, and progressing toward himselt, , until the entire circumference of the part is dissected and a cutf is formed. When this has been separated for a sufficient distance all aromel and folded back the laver of muscles is divided. The incision through the muscles of the calf is made in three steps. Finally the muscles upon either side of the interosseous ligament are divided. This complex incision should be made exactly in the same plane, so that the ressels are not divided at rarring levels.

In making the figure-of-eight incision the knife is placed horizontally, with its heel upon the upper surface of the tibia, so that its pointed extremity is directed toward the operator. It is steadily held in a horizontal position and drawn from heel to toe, introduced into the interosseous space close to the tibia up to its handle, and the soft tissues between the two bones divided. The fibula being reacher, the knife is drawn from heel to toe around this bone and passed horizontally again into the interosseous space, with its point directed from the operator and its blade upward, dividing any remaining muscular fibers from the fibula toward the tibia. The operator now introduces the index-finger and the thumb of his right hand into the wound and grasps the interosseous ligaments to assure himself that all of the muscles have been transversely divided. Before the saw is used the assistant rotates the member inward. The periosteum is detached from the bone with the raspatory at the line of division. The saw is applied upon the tibia in such a manner that the fibula also is brought within the range of its action. A groove is first carefully sawed in the tibia, and when the blade of the saw has thus secured a good 


\section{Plate 8.-Transverse Division of the Right Leg in its Middle Third.}

$t$, tibia; $f$, fibula; $E$, group of extensors (tibialis anticus, extensor digitorum communis, extensor hallucis) $S$, soleus; $G$, gastrocnemius; $T p$., tibialis posticus; Pr., peronei; Trt., anterior tibial artery, with the corresponding vein and the deep peroneal nerve; $T$, posterior tibial artery, with the corresponding veins and the posterior tibial nerve; $P$, peroneal artery and vein.

grasp the fibula also is brought within the sphere of its activity and both bones are divided simultaneonsly (Fig. $58)$.

The stump thus made shows the cross-section of the two bones, with the interosseous ligaments stretched between them. Anteriorly, lying upon the ligament, is the group of extensors, while upon the opposite side lie the flexors. Surrounding the fibula the peroneal group of muscles is visible. The powerful mass of the calf-muscles forms the most superficial layer upon the flexor aspect. Between this and the flexors pass the posterior tibial and peroneal arteries. Lying upon the anterior aspect of the interosseous ligament is the anterior tibial artery. The center of the field is occupied by the tibialis posticus muscle, which is a useful landmark in looking for the ressels. In front of this, but separated by the interosseous ligament, is the anterior tibial artery, and elosely behind it are the posterior tibial and peroneal arteries to the fibular and tibial sides respectively.

Flap-amputations of the Leg.-Two Lateral Tegumentary Flaps of Equal Size.-The base of the flaps corresponds with the level at which the bones are to be divided. Anteriorly the margins of the flaps meet in the line of the crest of the tibia. The shape of the flaps is ontlined with the knife introduced down to the fascia, when the flaps are dissected from the subjacent structures and turned back (Fig. 61). The incisions through the muscles are to be made in the typical manner described at right angles to the axis of the extremity. The muscles of the calf are divided in three steps; then those of the 

interosseous space by the figure-of-eight incision ; finally the bone is divided in the manner described. To prevent the projection of the sharp anterior crest of the tibia after division with the saw this prominence is either broken off with forceps or salwed off. To this end the crest is sawed into in an oblique direction, from above downward

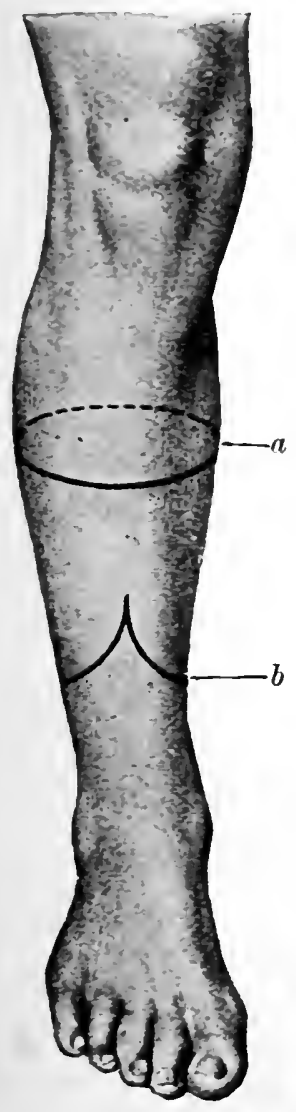

FIG. 66.-Incisions for amputation of the leg: $a$, circular incision for amputation at the site of election; $b$, lateral flap-incisions.

and backward, for some distance, before the bone is removed. When the tibia is now divided transversely a portion of the bone at the crest falls ont and the previous prominence is removed.

Two Lateral Musculotegumentary Flaps.-The shape of the flaps is the same as that just described. An incision 
is made through the skin and the fascia down to the muscle. After the skin has been retracted the flaps are formed either by transfixion or by incision from without inward. When the flaps are folded back the muscles are divided

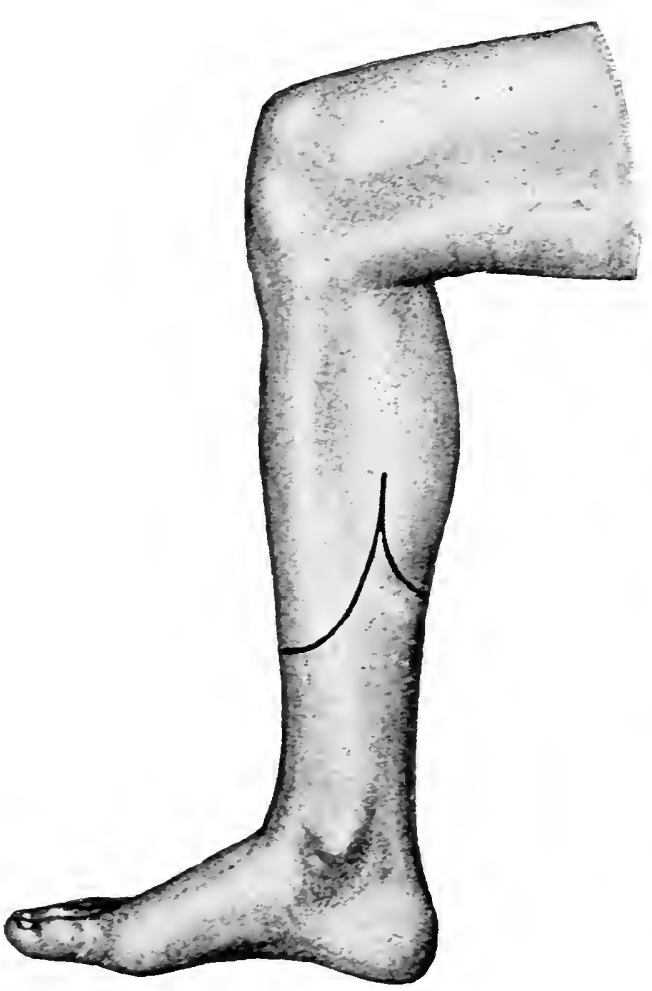

FIG. 6\%.

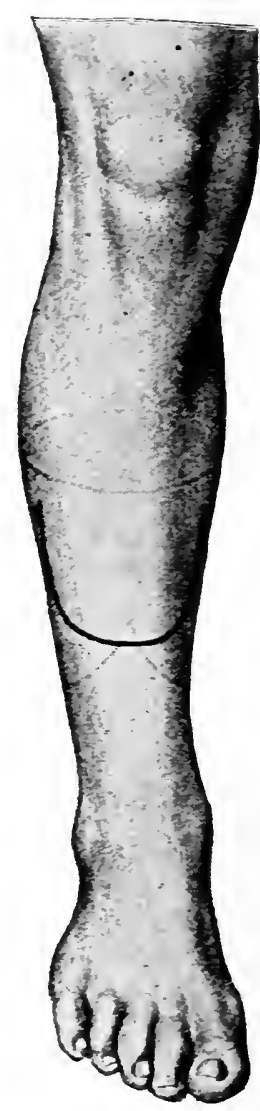

FIG. 68.

FIGS. 6\%, 68.-Cutaneous incisions for amputation of the leg, after Heine: showing anterior and lateral aspects.

by a figure-of-eight incision and the bone is sawed through at the level of the base of the flaps.

Anterior Long Tegumentary Periosteal Flap, with a Posterior Short Irusculotegumentary Flap (Heine).-A broad quadrangular flap with rounded corners is made upon the anterior aspect of the leg (Figs. 67 and 68). In the situa- 
tion to which the flap, after division of the -kin, is retrated the periostem of the anterion surface of the tibia is inci-ed transversely, parallel with the lower boneler of the flap. The skin upon either side of the tlatp is di-sected toward the tibia from the subjacent tissues, and the periostemm of the tibia corresponding to the latemal bomndaries af the flatp is divieled longitudinally. In the proees- at dissecting the flap the periosteum is detached from the bone hy neans of a raspatory and thus retains its comnection with the freed skin. After the anterior flap has been dissected to its base the bone is raised and a shorter arched flap consisting of the skin and the muscles of the calf is formed upon the posterior aspect of the leg by an incision fiom without inward. The muscles of the interosseous space are then divided and the bones are silwed through in the usual manner.

A single lateral flap is made correspondingly longer and with a broader base. It mar be constituted of skin, on the inner side of skin and periosteum, or finally of skin and muscle. When the fiap has been dissected a circular incision through the skin is made upon the opposite portion of the circumference of the leg miting the extremities of the flap and after retraction of this the muscles are divided in the usual manner.

To increase the supporting power of the stump following amputation of the leg Bier, after healing of the wound, remores a wedge-shaped portion of bone abore the level of the stump, so that the lower extremity of the latter can be turned forward and upward through an are of ninety degrees and be permitted to mite in this position. Br this means closure of the medullary cavity is effected and the supporting surface is formed of healthr, well-padded skin, free from cieatrices, whose muscles do not undergo atrophy by reason of preserving their natural attachment to the bone. The principle upon which the nethod is based is illustrated by the accompanving diagrammatic representation (Fig. 69). The medullary cavity of the divided bone may, according to Bier, be closed also with 
a loose piece of periosteum or with a foreign body, such as stanniol-paper.

Supramalleolar Amputation of the Leg by Syme's Method.-The ankle-joint is opened and the bones of the leg divided just above the malleoli and the wound covered with a cutaneous flap obtained from the heel. The patient occupies the dorsal decubitus. The foot is raised above the horizontal and the operator stands to its peripheral side. Grasping and fixing the foot by the heel with his left hand the operator makes an incision, always beginning on the left side, from the apex of the malleolus vertically toward the sole of the foot, then transversely
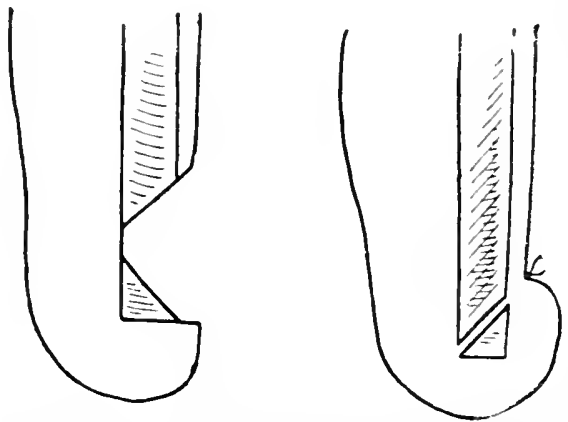

FIG. 69.-Diagrammatic representation of amputation of the leg after the method of Bier.

through the sole and again vertically upward to the other malleolus, dividing the tisstes doin to the calcaneum (stirrup-incision). A second incision, made over the anterior aspect of the ankle-joint unites the extremities of the first, with which it makes a right angle and it also extends down to the bone. This incision should open the joint between the trochlear surfice of the astragalus and the lower extremity of the tibia and the fibula. In order to expose the joint fully the lateral ligaments must be divided on either side. The incision through the capsule has the following form: $\square$, the short limbs passing through the lateral ligaments. Only after the lateral ligaments (at the outer malleolus, the anterior and posterior astragalo- 
fibular, and the calcancotibular; at the internal malleolus, the deltoid ligament) have heen divided will the head of the astragalus be free, even with slight plintar flexion of the joint. If the posterior wall of the calpoule be divided, the upper surface of the calcanem comes inturiew. The tuberosity of the calcaneus is freed from its coverings by vigorous incisions made vertically upon the bone, with the foot bent in maximum plantar flexion (Fig. 70). When

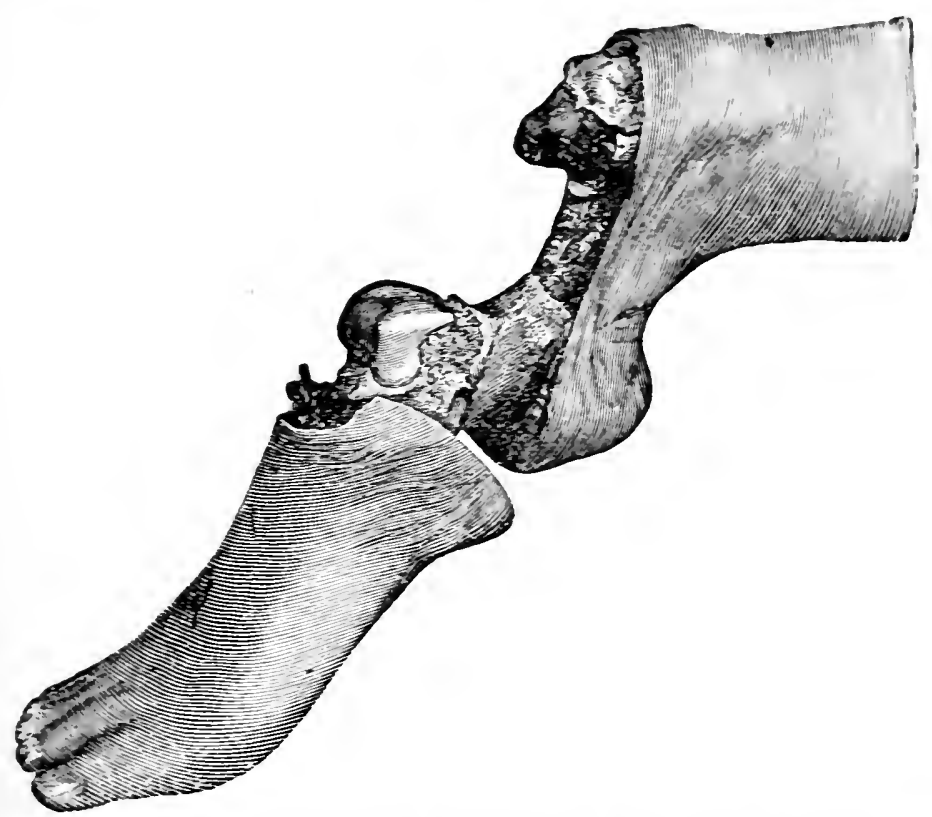

FIG. 70.-Amputation of the foot br the method of Srme: enucleation of the tuberosity of the calcaneum from its coverings.

the foot is thus freed and detached the lower extremities of the tibia and the fibula are freed from the soft tissues, surrounded by a cireular incision, and sawed through transversely.

The operation is attended with certain disadrantages, the excarated heel-flap not being properly adapted to approximation with the leg. while the excaration is further farorable to the accumulation of considerable quantities of secretion. Although srme's operation no longer receives the recognition which was formerly accorded it, it still deserves con- 
sideration, as it represents the predecessor of a number of admirable operations (Pirogotf, (iritty, etc.).

Amputation of the Foot by the Method of Pirogoff.Pirogoff's operation consists in osteoplastic supranalleolar

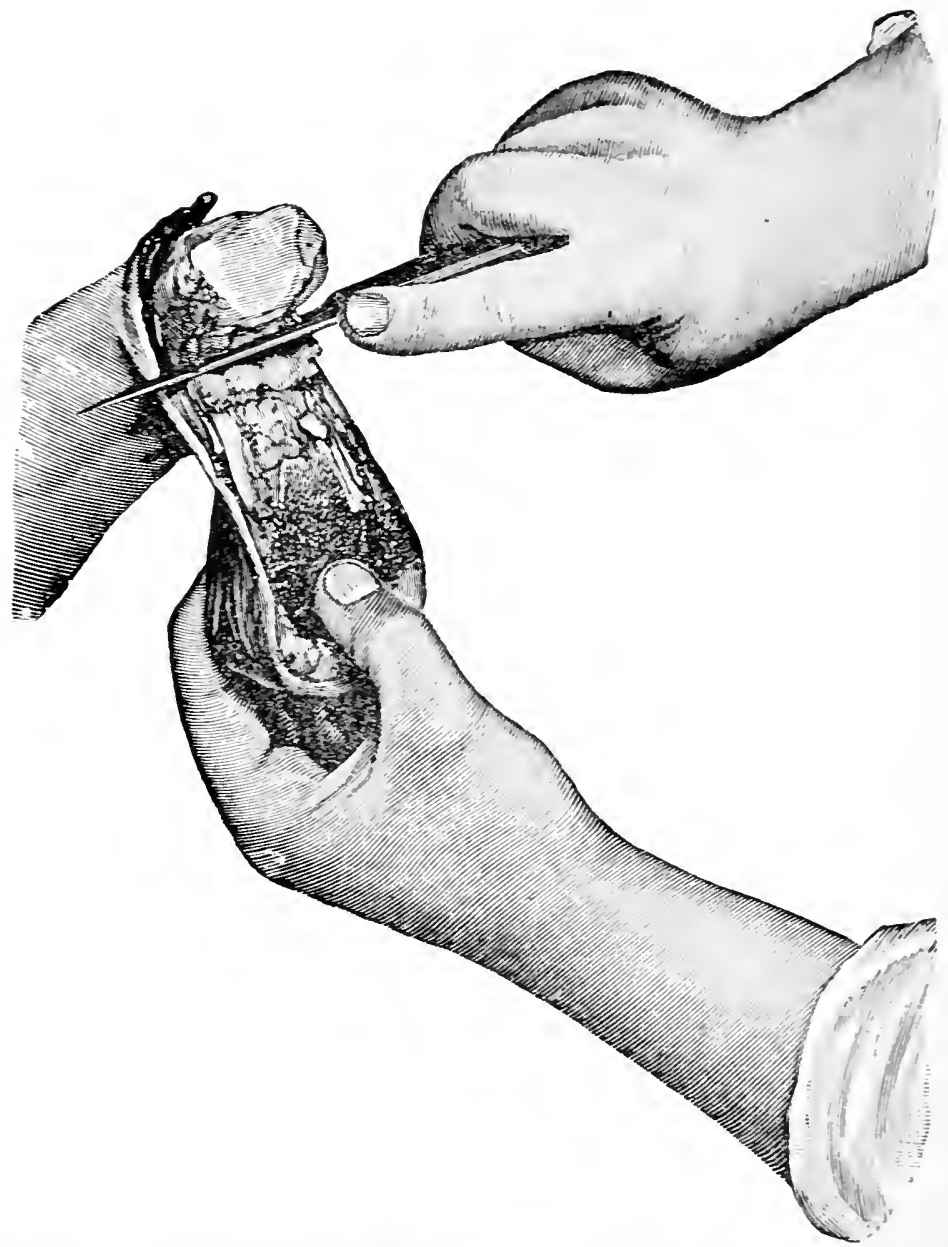

FIG. 71.-Pirogoff's amputation: detachment of the soft parts from the posterior aspeet of the lower extremity of the leg: the blade of the knife is direrted against the bones.

amputation of the leg, with the formation of an osseoustegumentary flap from the heel. This procedure over- 
eomes the disadrantages and difficulties of Syme's operation by not separating the tuberosity of the calcanemm, but sawing through the bone so that its posterior segment retains its connection with the skin and enters into the formation of the flap. The operator occupies the same position as in the operation of sime and the incisions through the skin are mate in a similar manner. The operator fixes the foot with his left hand and, begimning on the left side, cuts from the apex of one malleolus vertieally toward the sole of the foot (Fig. 72), then trans-

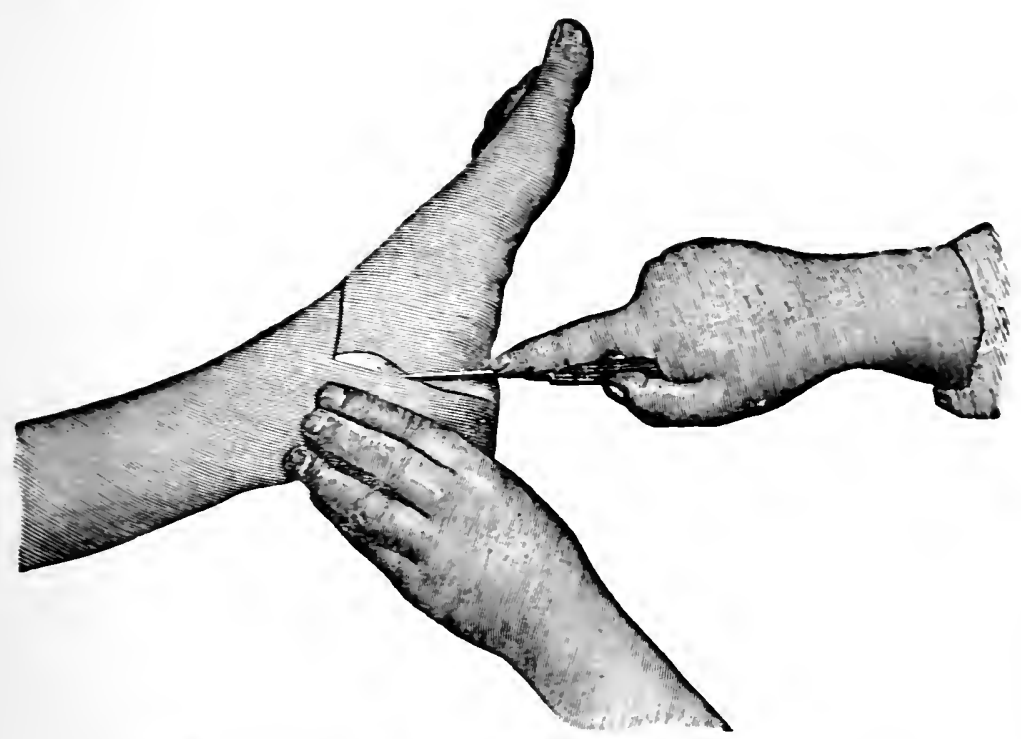

FIG. 72--Pirogoff's amputation: cutaneous incisions.

versely throngh the sole, and again vertically upward to the apex of the other malleolus, dividing the soft tissues down to the bone (stirrup-incision). An anterior transverse incision mites the extremities of the primary stirrup-incision. This incision divides the tendons of the extremities transversely and opens the eapsule of the ankle-joint. In order to open widely the joint between the heald of the astragalus and the lower extremities of the tibia and the fibula the lateral ligaments must first be 
divided. In effecting this division, especially upon the inner aspect, the incision should be made close to the astragalus, in order to avoid injuring the posterior tibial artery. After the joint has been frecly exposed the posterior wall of the eapsule comes into view, after division of which the upper surface of the tuberosity of the calcaneum is exposed. The operator, who until now has stood at the periphery of the foot, changes his

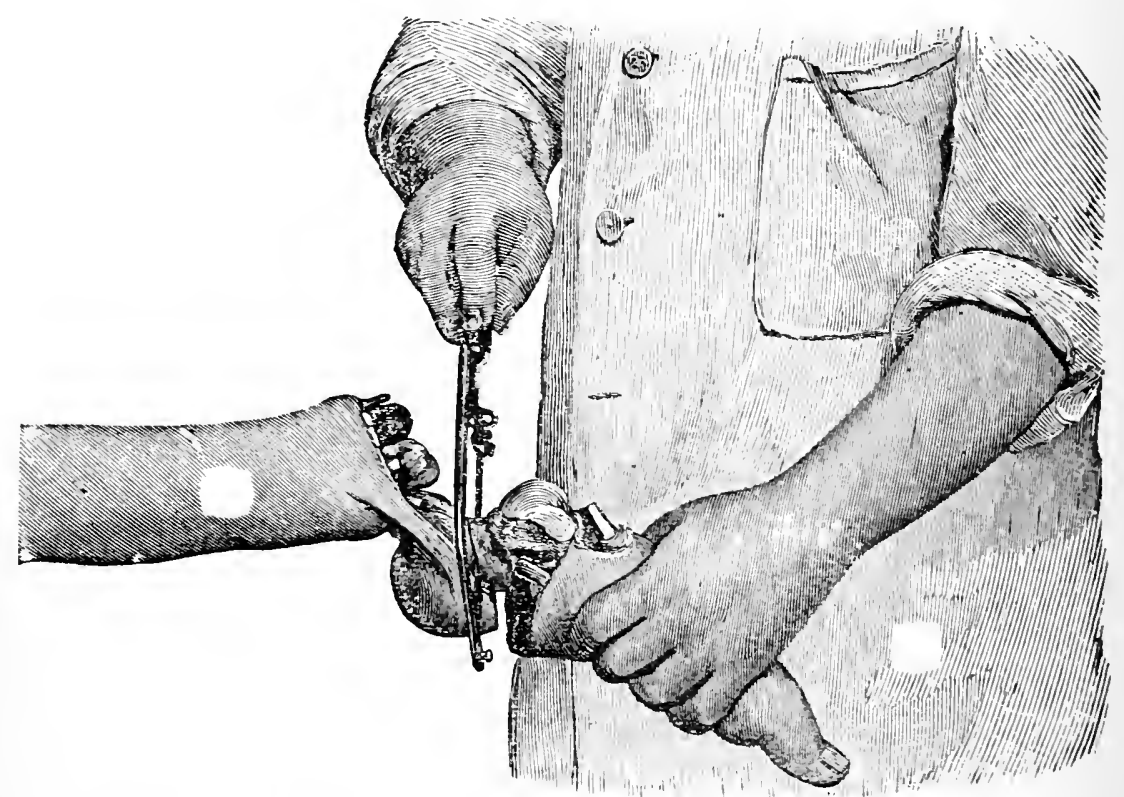

EIG. 73.-Pirogoff's amputation: division of the calcaneum with the saw.

position, grasps the foot with his left hand and applies the saw to the upper surface of the tuberosity of the caleaneum (Fig. 73). This process is divided in a plane corresponding to that of the stirrup-incision, when the foot appears to be separated. The lower extremities of the tibia and the fibula are prepared for division with the saw, the soft parts upon the posterior aspect being first dissected close to the bone. This incision demands especial care in order to aroid division of the posterior 


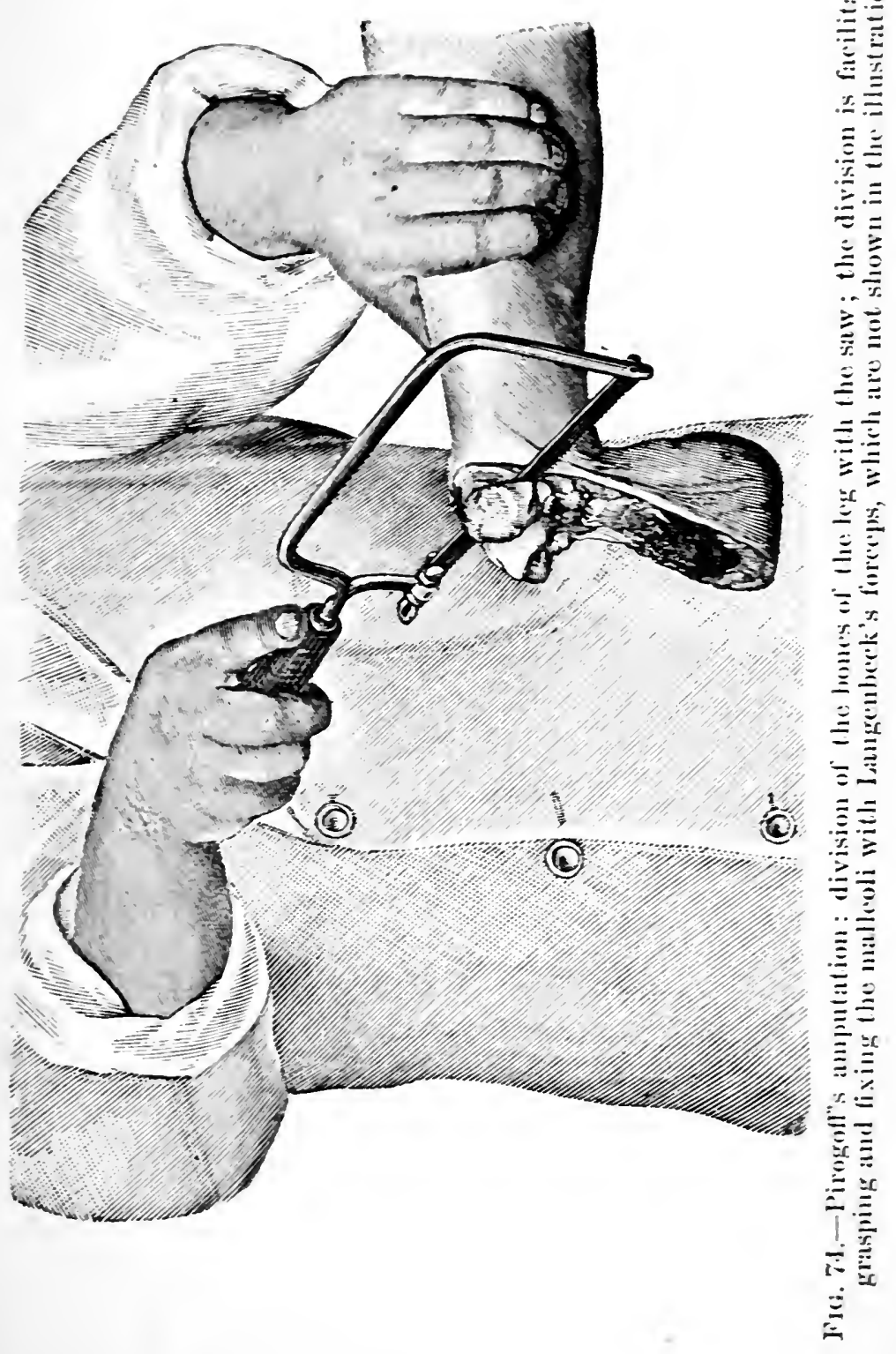



tibial artery, which is escential for the nutrition of the heel-portion of the flap. The surgeon grasps the flap between the thumb and the index-finger of his left hand, flexes it backward in maximum degree and separates the

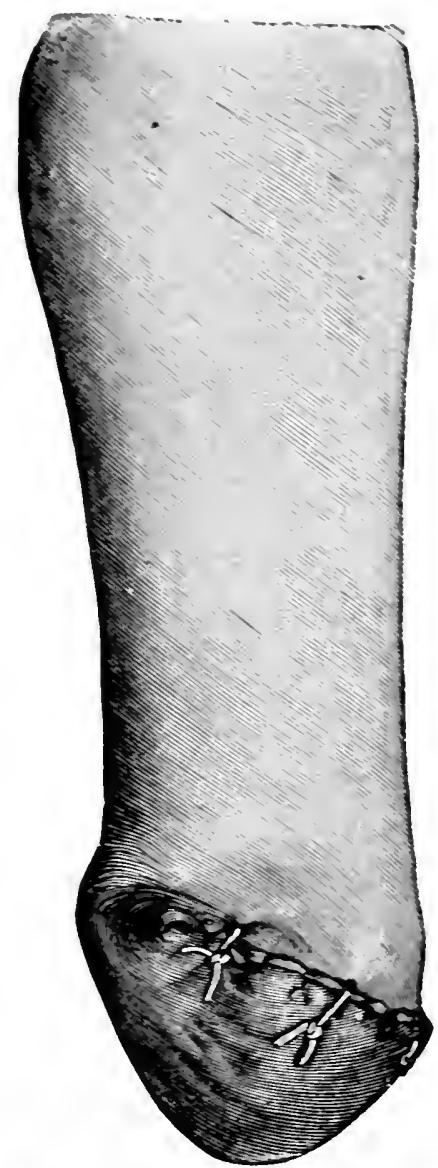

FIG. 75.-Stump left after Pirogoff's amputation.

soft parts from the posterior aspect of the tibia in such a way that the blade of the knife is brought in direct contact with the bone (Fig. 71).

Upon the anterior surface it suffices to displace the tendons, when the bones of the leg may be divided circularly 
just above the malleoli. In sawing through the bones the leg is held horizontally, the operator standing as if amputating, with the member to be amputated to his right. An assistant grasps one of the malleoli with Langenbeck's forceps, when the division of the bone transrersely to the longitudinal axis of the leg may be proceeded with (Fig. T4). The stumps of the tendons are grasped with forceps and divided with scissors at the level of the wound. The ressels are secured by ligatures. The only vessels concerned are the anterior and posterior tibial arteries. The first is readily found upon the anterior surface of the tibia at the side of the tendon of the extensor hallucis. If the successive steps of the operation have been properly followed, the posterior tibial artery will be found to have been divided on the inner aspect of the heelflap, somewhere about the middle of the vertical limb of the stirrup-incision. In closing the wound the heel is moved through an are of $90^{\circ}$ and the sawed surface of the calcaneum brought in simple apposition with that of the tibia or fixed by bone-sutures or perentaneous pegs. The cutaneous wound is united transversely (Fig. 75).

The operation of Pirogoff has undeniable advantages over deep amputation of the leg, as exemplified by Syme's operation. The shortening is reduced to a minimum by the preservation of the posterior extremity of the calcaneum, which forms a prolongation of the bones of the leg. The strong, well-cushioned skin of the heel makes an admirable walking-surface, while the cicatrix does not lie within this area.

Pirogoff in his first communication upon the subject had already called attention to the fact that after making the first incision into the sole of the foot the division of the calcaneus could be proceeded with immediately from the sole (Figs. 76 and 77), and exarticulation at the anklejoint next effected. He also had made the suggestion to give an oblique direction to the heel-flap in order to bring a larger portion of the calcanem and the skin of the 


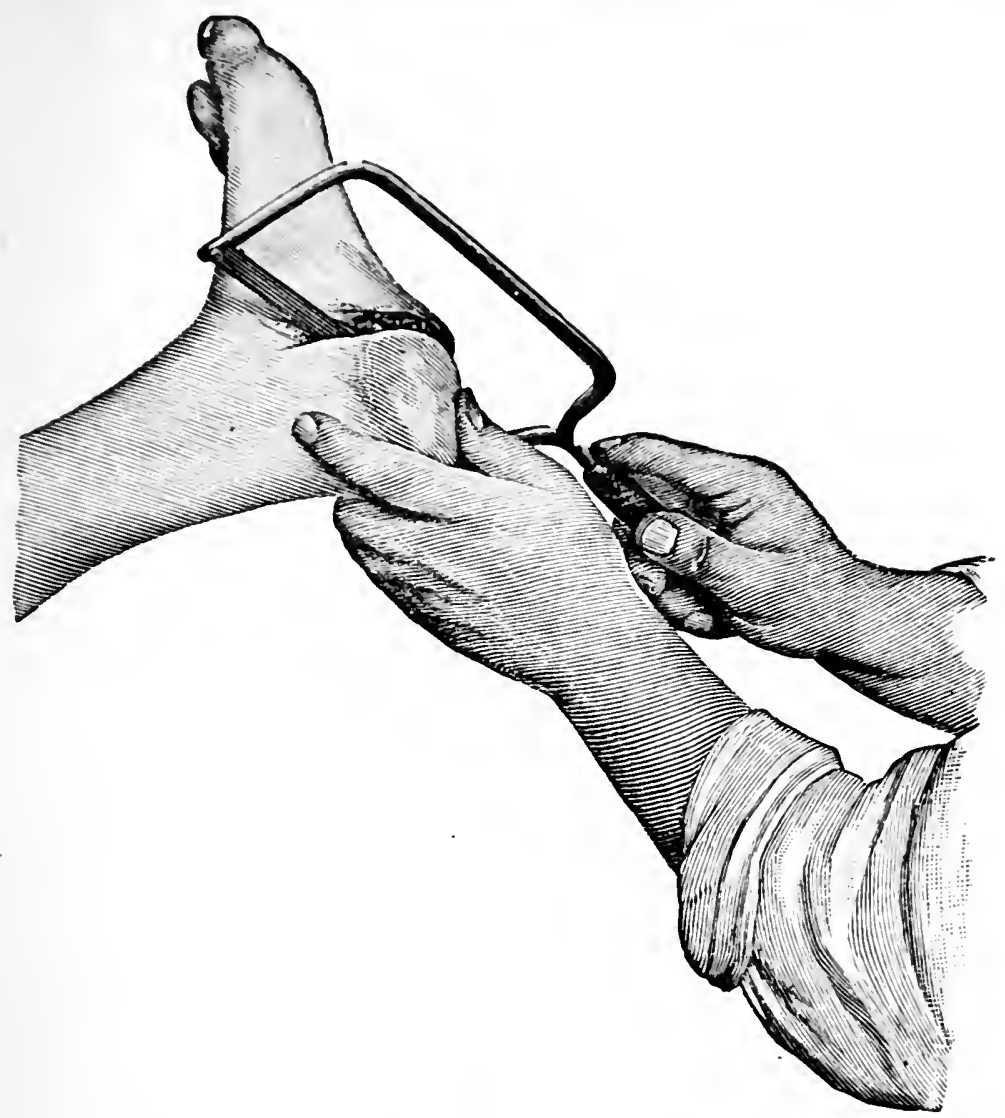

FIG. 76.-Günther's modification of Pirogoff's amputation : division of the calcancum from the sole of the foot.

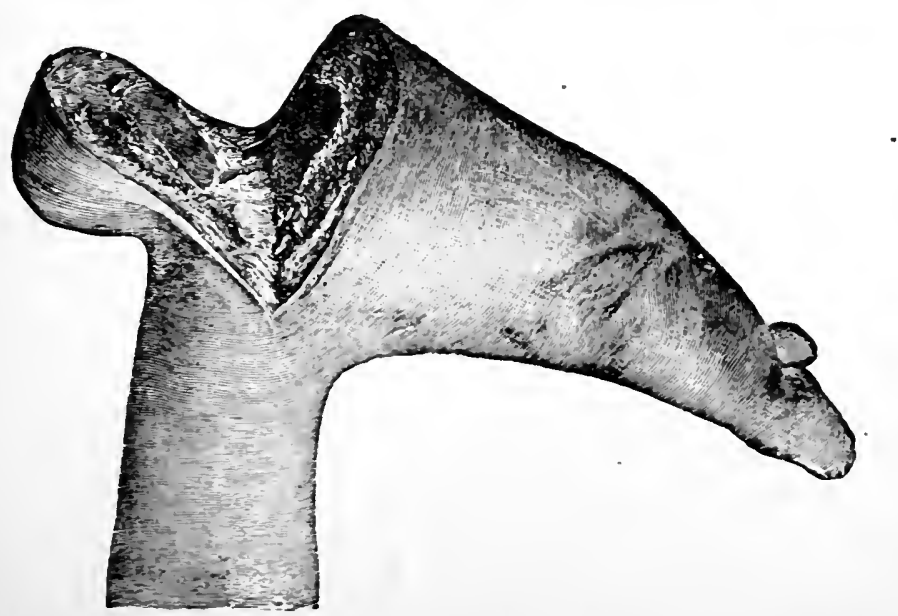

FIG. 77.-Configuration of the foot after division of the calcaneum. 


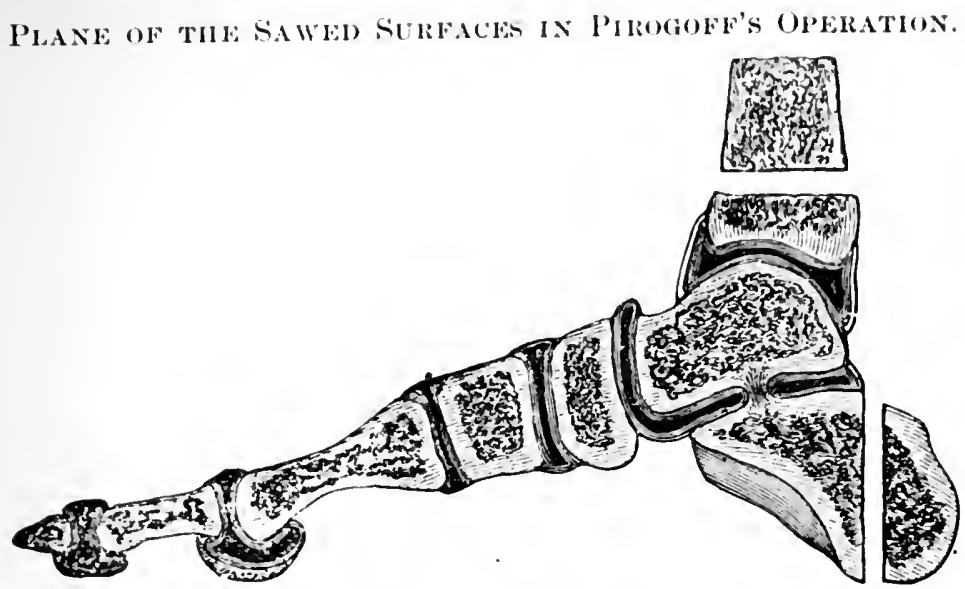

FIG. 78.-Origiual method.

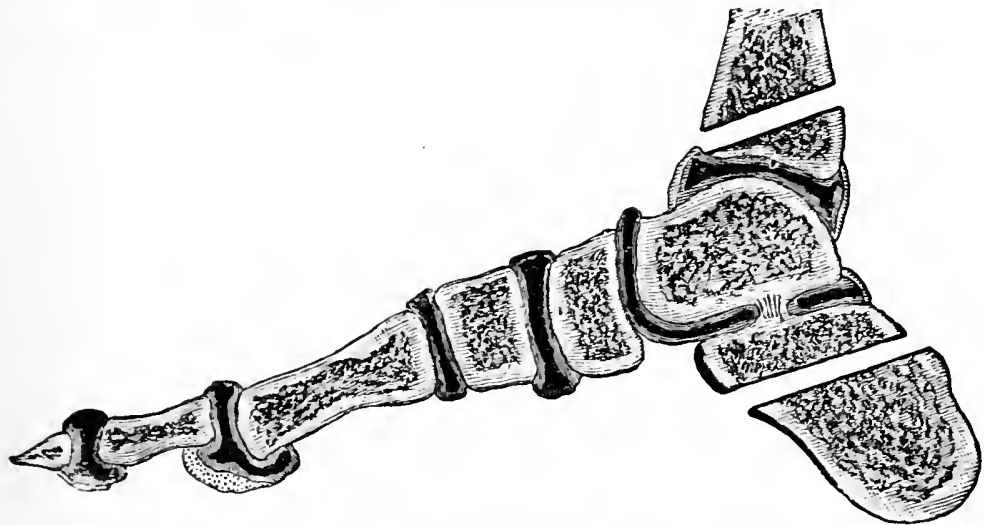

FIG. '99.-Günther's modification.

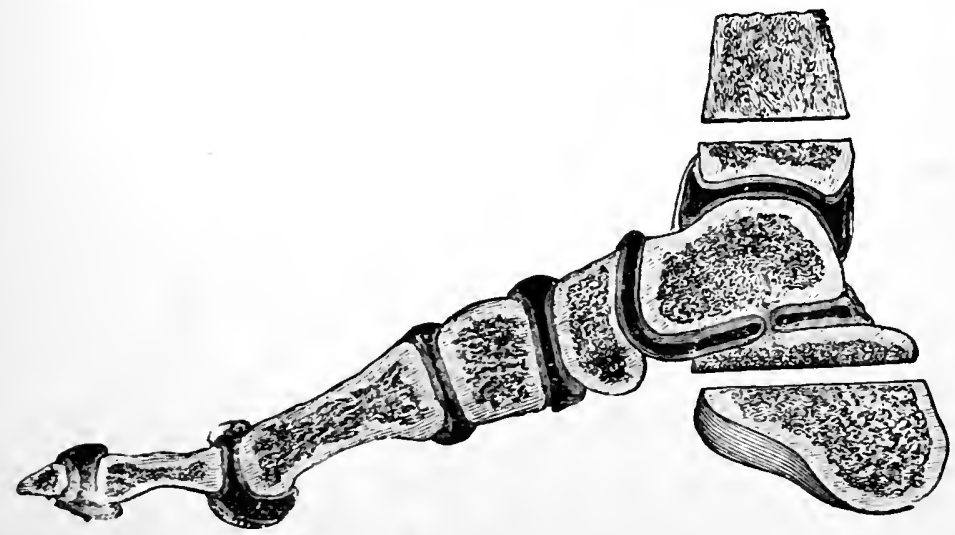

Frg. 80.-Lefort's modification. 


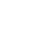


sole into the posterior flip. These modifications have been elaborated by Gïnther and Lefort.

While the eutancons incision and the planes of the two incisions through the bones are at right angles to each other in Pirogotf's operation (Fig. Ts), the tuberosity of the ealeaneum is divided obliquely from behind above forward and downward in (iünther's modifieation; also, the lower extremities of the tibia and the fibula are not separated in the form of a plate, but in that of a wedge, and in such a mammer that the base of the wedge corresponds with the posterior and the apex with the anterior boundary of the tibia. As a matter of course, the cutaneous incisions are to be modified accordingly. The stirrup-incision will pass from the posterior boundary of the malleoli, not vertically downward, but obliquely" forward toward the sole, so that a larger portion of the slin of the sole is eontained in the heel-flap. The stump is thus changed in so far that not only does the tuberosity of the ealcancum form the walking-surface, but also a portion of the sole, the natural walking-surfice, serves as a support for the stump.

The walking-surface becomes still broader if the calcaneus is divided almost horizontally in accordance with the modification of Lefort (Fig. 80). The stirrupincision passes obliquely forward to Chopart's joint, while the dorsal incision forms a flap with its convexity directed forward, and it extends also to the line of Chopart's joint. The dorsal flap is dissected back, the ankle-joint opened, and the ealeaneum sawed through from its tuberosity forward in the direction of the cutaneous incision into the ealcaneocuboid joint, the foot being held in a position of maximum plantar flexion. The foot is now separated at Chopart's joint and the lower extremities of the tibia and fibula are divided in the manner described.

Bruns reeommends horizontal division of the calcaneum, though in an arehed direction. The sawed surface of the calcaneum is thus concave, while that of the tibia presents a corresponding convexity. 
The limitations of Lefort's modification are naturally narrower than those of the typical operation of Pirogoff. If the calcaneum is perfectly healthy, and this is a necessary condition for the employment of Lefort's operation, it would seem preferable to select the less serious procedure of exarticulation at Chopart's joint.

As a preliminars step in the performance of Pirogoff's operation division of the tendo Achillis (Achillotenotomy) is undertaken. The tendon is dirided, either through an open wound or subcutaneously, in a transierse direction, a fiuger's breadth above its attachment to the tuberosity of the os calcis.

In performing subcutaneous tenotoms the knife (tenotome) is passed throush the skin and the tendon is divided by cutting either toward or from the skin. Lnder the condition first named the operator grasps the tenotome, as a table-knife is held in paring fruit, with the flexed four fingers of the riuht hand, while the thumb is supported on the heel (Fig. (1); the knife is passed from right to left in front of the tendon. The latter is subjected to passive tension and is divided by short rocking morements of the knife toward the thumb, which is placed upon the tendon as a guide and to afford resistance (Fig. 82). The jerk with which the extremities of the divided tendon separate indicates the completion of the operation.

When, on the other hand, the tendon is to be divided from without inward the foot is so adjusted that the tendon is completels relaxed. The tenotome is grasped between the thumb, the index, and the iniddle finger and introduced upon the flat, from right to left, between the skin and the tendon (Fig. 12). The tendon is thus brought beneath the blade of the knife and is gradually divided by rocking movements, while an assistant subjects it to maximum tension by corresponding morement of the joint.

Exarticulations and Amputations of the Foot.-Exarticulations of the Toes in the Interphalangeal or Metatarsophalangeal Joints.-Dorsal Opening of the Joint, with the formation of a Plantar Tegumentary Flap by Incision from within Outurd.-The operator grasps with the indexfinger and the thumb of the left hand the toe flexed at the joint of separation, and the joint is opened by a transverse incision upon the extensor aspect, somewhat toward the periphery from the highest prominence of the joint. Then the lateral ligaments are completely severed until the joint is fully opened. Next a flap is formed from the plantar tissues by an incision from the wound outward. The length of the flap will be governed by the transverse extent of the exposed bone. 


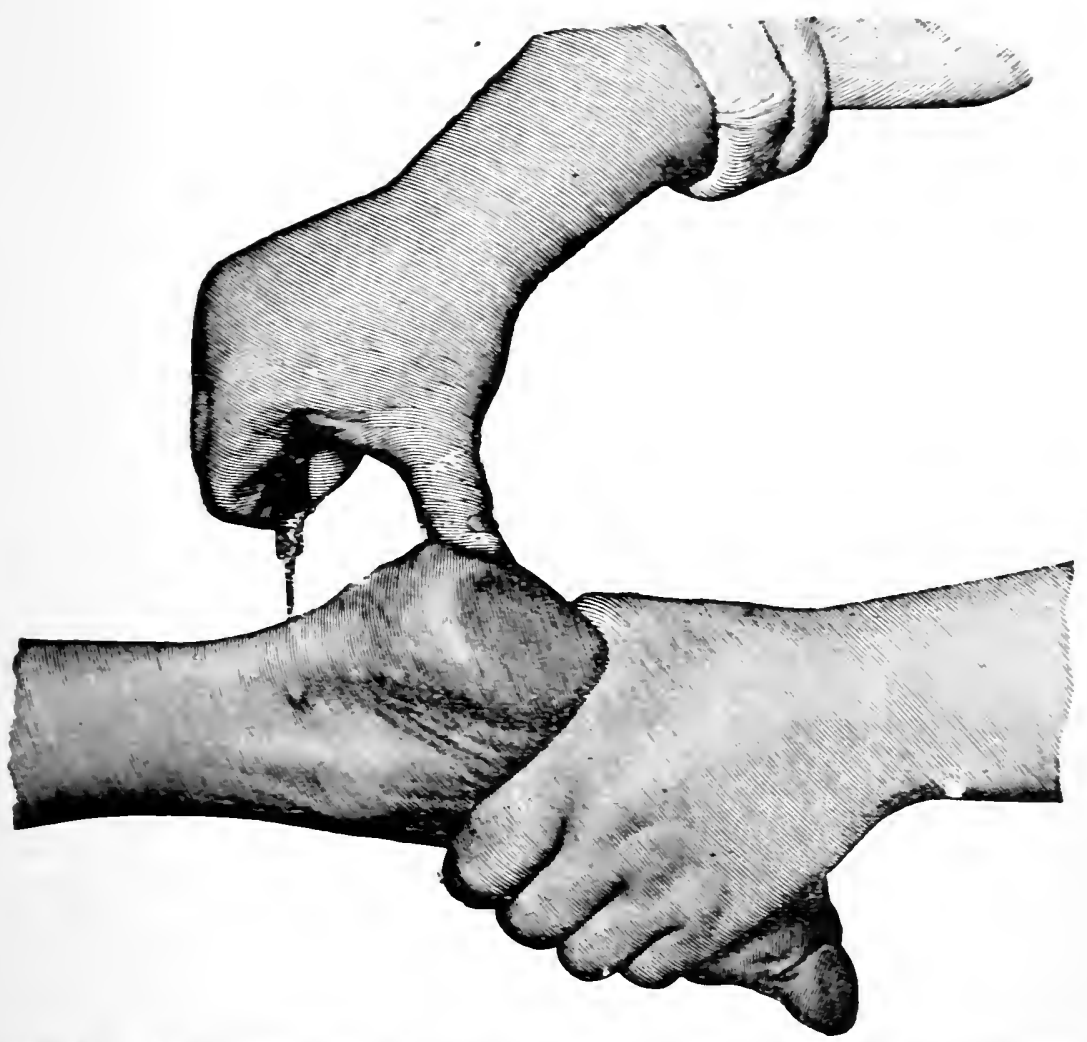

FIG. 81.-Achillotenotomy: the tenotome is passed beneath the relaxed tendon. 



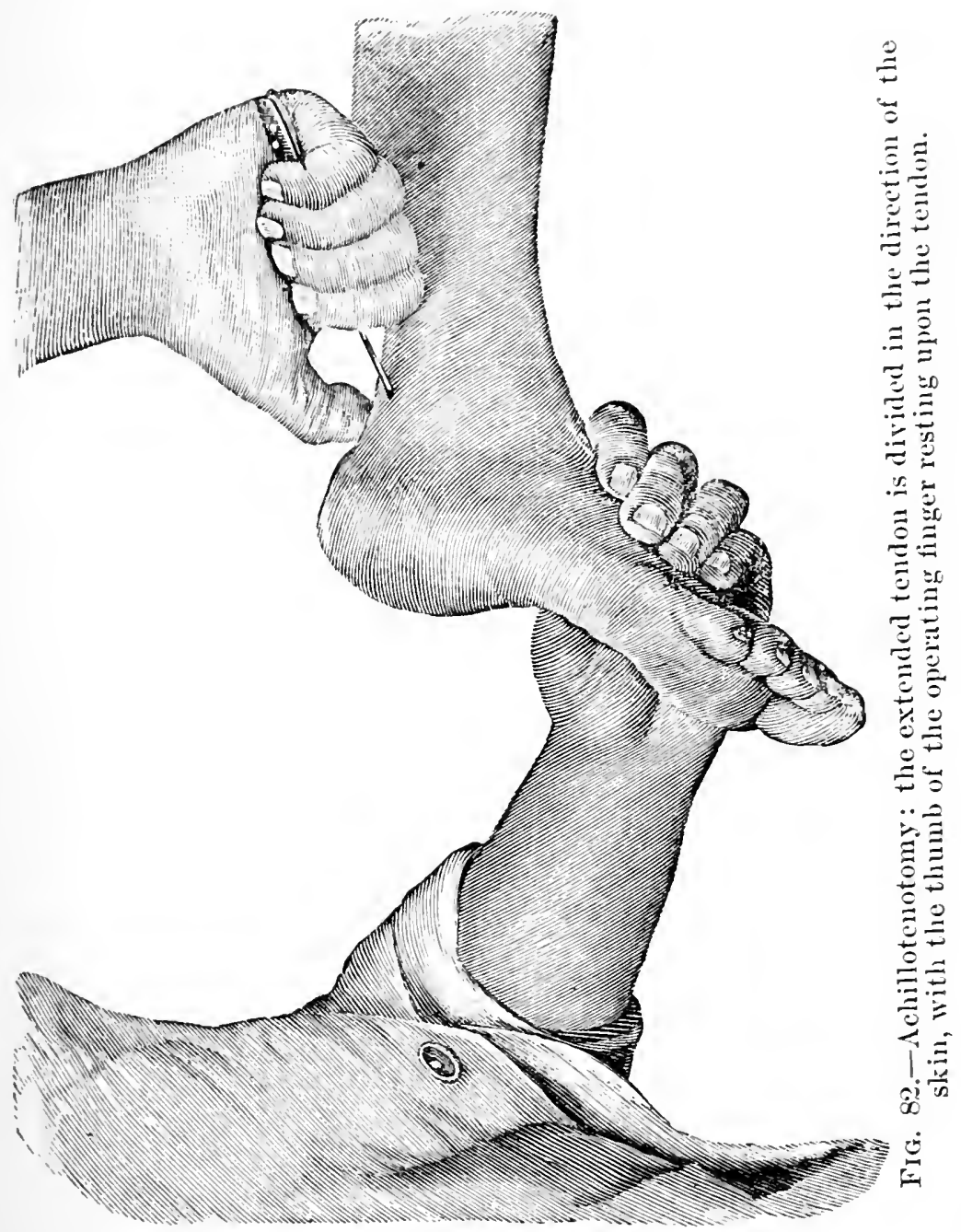



A large dorsal and a short planter tegumentary flap may also be employed advantageously in the removal of toes; as well as twroluteral flapss of equal size; or a single lateral tegumentury flop. In all cases the flaps are carefully outlined with the knife, separated from the subjacent structures, and the joint opened transversely from the extensor aspect. The flaps shonld always be so situated that their base corresponls with the joint-line at which the removal is to be effecterl.

In making an oval incision the knife is applied upon the dorsal anperet somewhat behind the line of the joint. The incision then runs parallel with the longitudinal axis of the digit in the middle line until it has passed the joint, on the distal side of which it deviates toward the right, in order to mun transversely upon the flexor aspect. From here it is continued around the bone until it reaches the dorsal aspect again, where it is completed by being carried to its point of origin. The joint is exposed upon its dorsal aspect by dissecting the two sides of the oral, and opened transversely, when removal may be effected after division of the plantar tendons.

Amputation of a Toe through the Metatarsal Bone.-An oval incision is made, its apex corresponding with the point at which the metatarsal bone is to be sawed through. The incision passes longitudinally over the metatarsus beyond the metatarsophalangeal joint. It then passes around to the flexor aspect and back again on the opposite side, to return to its point of origin. The metatarsus is dissected free in the course of the longitudinal incision and divided with the phalangeal saw or the chain-saw. The distal extremity is grasped and enucleated, and if this procedure has been carried out to the transierse incision in the flexor fold of the metatarsophalangeal joint the separation will have been completed.

Amputation of all Toes through the Metatarsus.-A large semicircular plantar and a short dorsal cutaneous flap are made. The operator stands at the periphery of the extremity, with the foot held in maximum dorsal flexion, 
and a plantar flap is cut and separated from the metatarsal bones. The dorsal tegumentary flap is then outlined and likewise disseeted free. The metatarsal bones are surrounded totally by a circular incision, the museles in the interosseous spaces divided, the periosteum of each bone individually pushed back at the place at which it is to be sawed through, and the saw finally applied from the dorsum of the foot to all of the bones simultaneously.

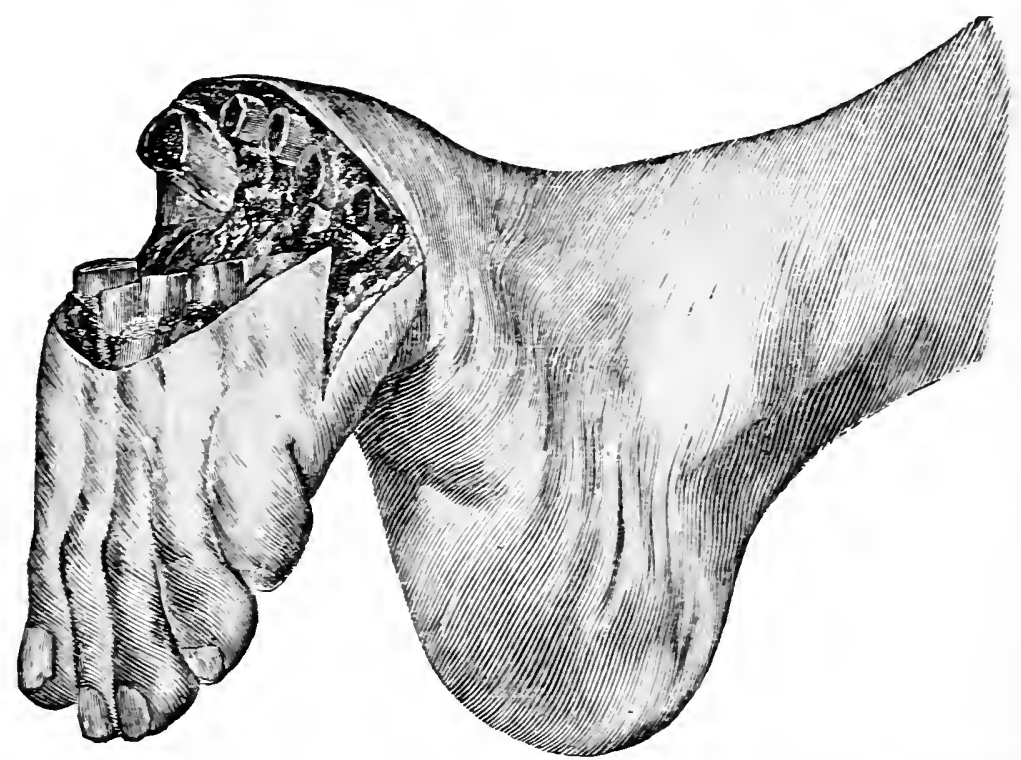

FIG. 83. - Amputation of the toes through the metatarsus: plantar musculo-tegumentary flap.

The plantar flap may also be formed by cutting outward from the wound after the bones have been sawed through. The dorsalis pedis artery and the digital arteries will require ligation.

Exarticulation of the Grieat Toe, torgether with the ILetatarsal Bone.-An oral incision is made with a prolonged apex. The dorsal incision begins on the extensor aspect over the metatarsophalangeal joint and passes in the longitudinal axis of the metatarsus to the head of this bone, where it deviates to one side, then surrounds the entire 
base of the toe, and thus again reaches the dorsal aspect of the toe, joining the original longitudinal incision. The incision at all points is mide down to the bone. Hooks are introduced on either side of the longitudinal incision, and the museles are separated fiom the metatarsus. While the toe is raised the metatarsal bone can also be freed from the muscles upon its under surfice, when the joint between the internal cunciform bone and the base of the metatarsus can be opened on its dorsal aspect. Linear closure of the wound is finally effected.

Exarticulation of the Little Toe, together with the Metatarsal Bone.-A lateral flap is formed according to the method of Walther. The operator grasps the abducted little toe and applies the blade of the knife vertically in the interdigital fold between this and the adjacent toe, when the soft parts of the interspace are divided, by sawing movements of the knife held close to the metatarsal bone of the little toe, to the tarsus. From this point, with abduction of the toe and the metatarsus, entrance is gained into the joint between the fifth metatarsus and the cuboid bone, when the toe is bent outward at the joint at a right angle or more. The operator incises the tissues around the tuberosity of the metatarsal bone close to the bone, and with sawing-movements detaches the soft parts from the outer side of the metatarsal bone until a flap has been secured of sufficient extent to cover the wound made. The flap is cut transversely from the wound outward.

The little toe may also be exarticulated at the tarsometatarsal joint by means of an oval incision, the apex of which is made upon the dorsum of the foot, as in removal of the great toe. The apex of the oval, as well as the longitudinal incision, may also be placed advantageously upon the lateral border of the toot.

Removal of the Foot at the Tarsometatarsal Joint. Lisfranc's Operation.-It is important to determine the situation of the ends of the line of the tarsometatarsal joint upon the inner and outer borders of the foot. The outer extremity corresponds with a point just behind the readily 
palpable tuberosity of the fifth metatarsal bone, while the inner extremity is about a thumb's breadth in advance of the prominent tuberosity of the scaphoid bone. The line of the tarsometatarsal joint is not a directly transverse line between the two points named, but it pursues a complex course. From the fifth metatarsal bone it passes forward at an angle of $45^{\circ}$; then it passes inward along the base of the fourth metatarsal bone. The next joint, between the external cunciform and the base of the metatarsal bone of the middle toe, is directly transverse, but projects somewhat forward. The articulation between the metatarsal bone of the second toe and the middle cuneiform bone is also transverse, somewhat behind that of the third metatarsal bone and in the line of that between the fourth metatarsal and the cuboid bone. The internal cuneiform bone projects forward (Figs. 84 and 85).

Steps of the Operation.- The principle of the operation consists in dorsal opening of the joint and the formation of a musculotegumentary flap from the sole by an incision from within the wound ontward. The operator stands at the periphery of the foot to be amputated, which he grasps from the sole, and with thumb and middle finger he marks the extremities of Lisfranc's articular line. Then an incision through skin and faseia is made on either margin of the foot down to the muscles between these two points - a thumb's breadth in advance of the scaphoid tuberosity on the inner side and just behind the tuberosity of the fifth metatarsal bone-on the outer side and carried forward beyond the heads of the metatarsal bones. An incision convex anteriorly over the dorsum of the foot then unites the posterior extremities of these lateral incisions. After division of the skin and the subcutaneous connective tissue the dorsal flap is somewhat retracted. At the point of retraction the tendons and muscles of the dorsum of the foot are divided accurately in the direction of the cutaneous incision. There are thus exposed upon the dorsum of the foot the bones and the ligaments of the joints throughout a small extent. The delicate dorsal cap- 


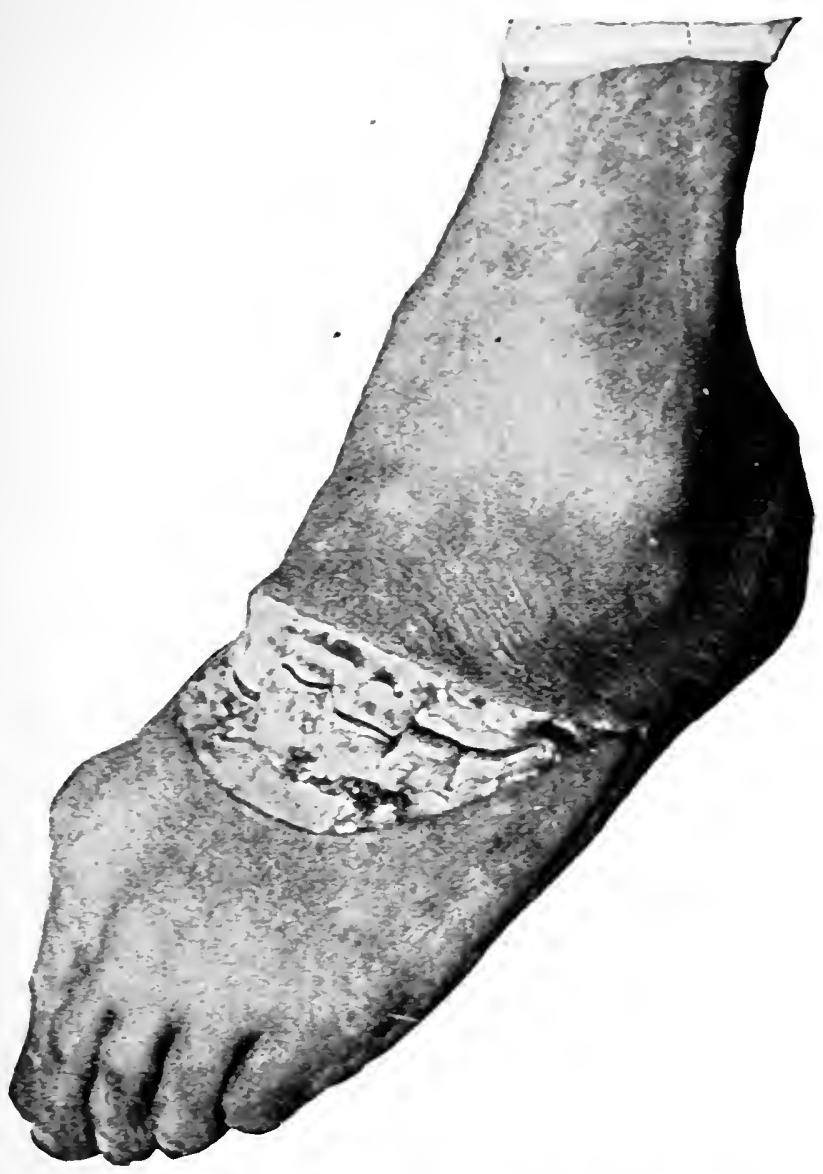

FIG. 84.-Lisfranc's articular line: exposed articular line upon the foot.

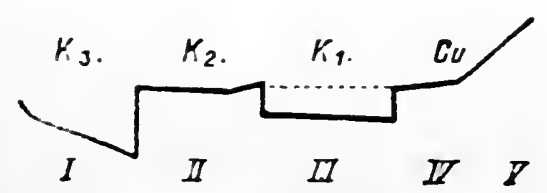

Fig. 85.-Course of Lisfranc's articular line (after van Walsen): $C u$, cuboid bone; $K_{1}, K_{2} . K_{3}$, external, middle, and internal cuneiform bones. $I, I I, I I I, I V, V$, articular surfaces of the corresponding metatarsal bones. $I$, metatarsal bone of the great toe. 
. 
Exarticulation of the Foot by Lisfrasc's Method.

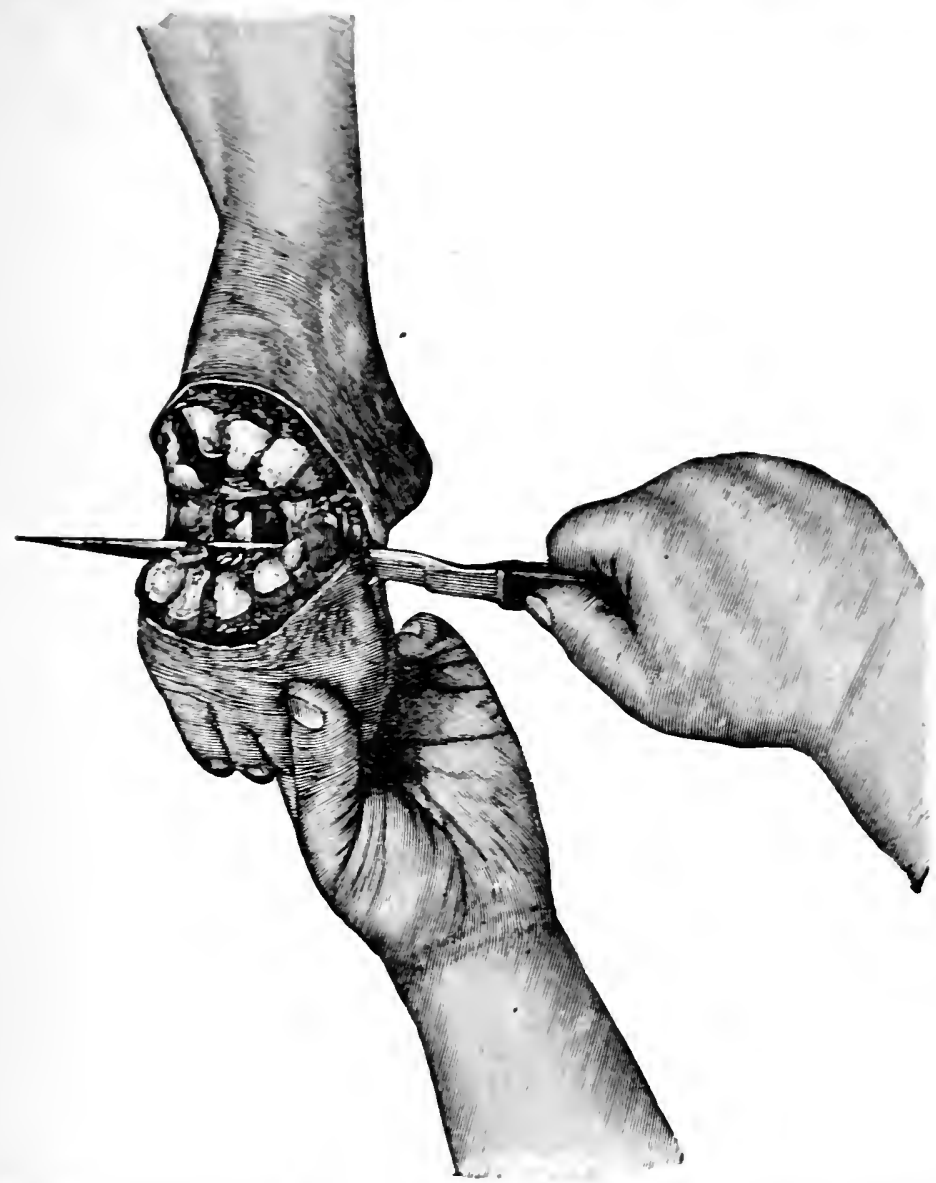

FIG. Sf. - The foot is flexed on the sole at Lisfranc's jrint: formation of the plantar musculotegumentary flap by incision from within outward.

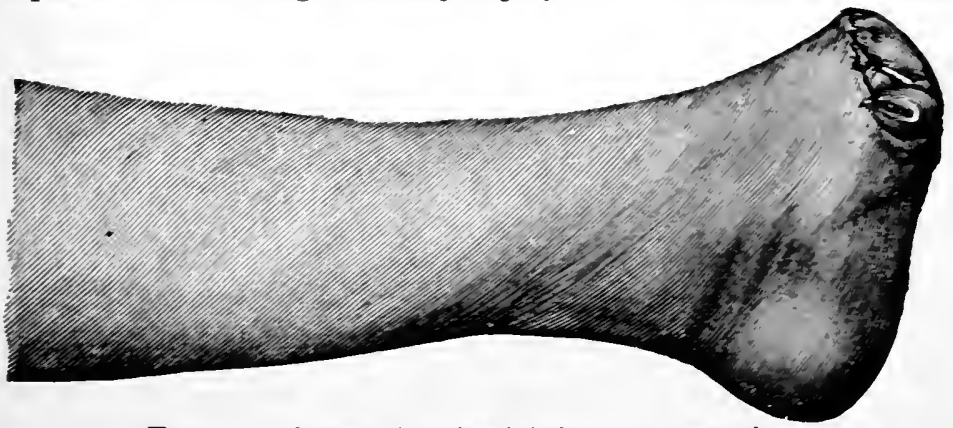

FIG. 87.-Stump left by Lisfranc's operation. 

sules of the joints are now slit open, with the foot held in slight plantar flexion. 'The opening of the joints is always begun at the external border, at the articulation between the fifth metatarsal bone and the lateral facet of the cuboid bone, as this can always be readily found if the knife is introduced behind the prominence of the fifth metatarsal bone and the incision is directed obliquely forward and inward. According to Bergmann, this joint coincides with the direction of a line passing from the tuberosity of the fifth metatarsal bone to the head of the first metatarsal bone. After this first joint has been opened the course of the remainder of the complex articular line can be made out from the landmarks mentioned. The operator therefore directs his knife more toward the middle line of the foot and opens the almost transverse joint between the cuboid bone and the fourth metatarsal.

The next also transverse joint is situated somewhat further forward. The transverse line of the joint between the second metatarsal bone and the middle cuneiform bone is readily found, corresponding with a prolongation inward of the articular line of the fourth metatarsal bone (Fig. 85). The joint between the first metatarsal bone and the internal cuneiform bone also is situated further forward. The articular surfaces must be exposed by short incisions directed against the bone. 'The longitudinal articular surfaces are likewise exposed, and with increasing plantar flexion of the foot the short ligaments that unite the bones in the depth, as well as the ligaments of the sole, in so far as these fall within the range of the incision, are divided, until the whole series of joints is opened to a maximum degree. It is now still necessary to form a plantar flap. This must be so constituted as to include at its base the soft parts of the entire sole. The substance of the flap gradually diminishes in amount toward its periphery, so that at its extremity it consists only of slin and subcutaneous connective and fatty tissue, and it is thus readily united with the delicate skin of the dorsum of the foot (Fig. 87). With a long knife an incision is 
made horizontally through the sole around the sesamoid bones at the head of the metatarsal bone of the great toe, the knife being brought out of the wound transversely beyond the head of the metatarsal bone (Fig. 88). The

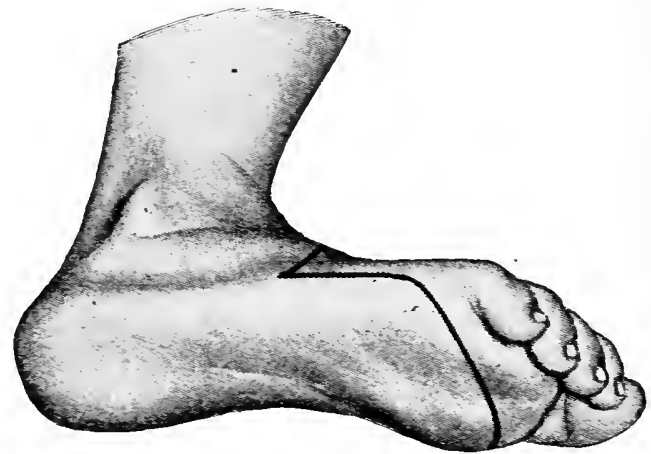

FIG. 83.-Form and extent of the plantar flap in Lisfranc's operation.

dorsalis pedis and the internal plantar artery are to be ligated in the stump.

'The stump left by Lisfranc's operation yields good functional results. The flap is firm and well padded, and the cicatrix lies upon the dorsal aspect quite out of the area of the walking-surface. The extensors of the anklejoint (tibialis anticus, peroneus brevis), important as antagonists of the triceps, are maintained in their attachments.

Intertarsal Amputation. - The joint between the anterior surface of the scaphoid bone and the three cuneiform bones is opened, and the cuboid bone is sawed through transversely in the lateral prolongation of the joint named. The cutaneous incisions are the same as in Lisfranc's operation. The joint in front of the scaphoid bone, recognizable by its articular surface with three facets, is opened from the dorsum of the foot and made to gape widely. The periosteum upon the dorsal surface of the cuboiri bone is incised transversely and the bone divided accordingly with the phalangeal saw. The bone is held in plantar flexion and the plantar flap is made as in Lisfranc's operation. 


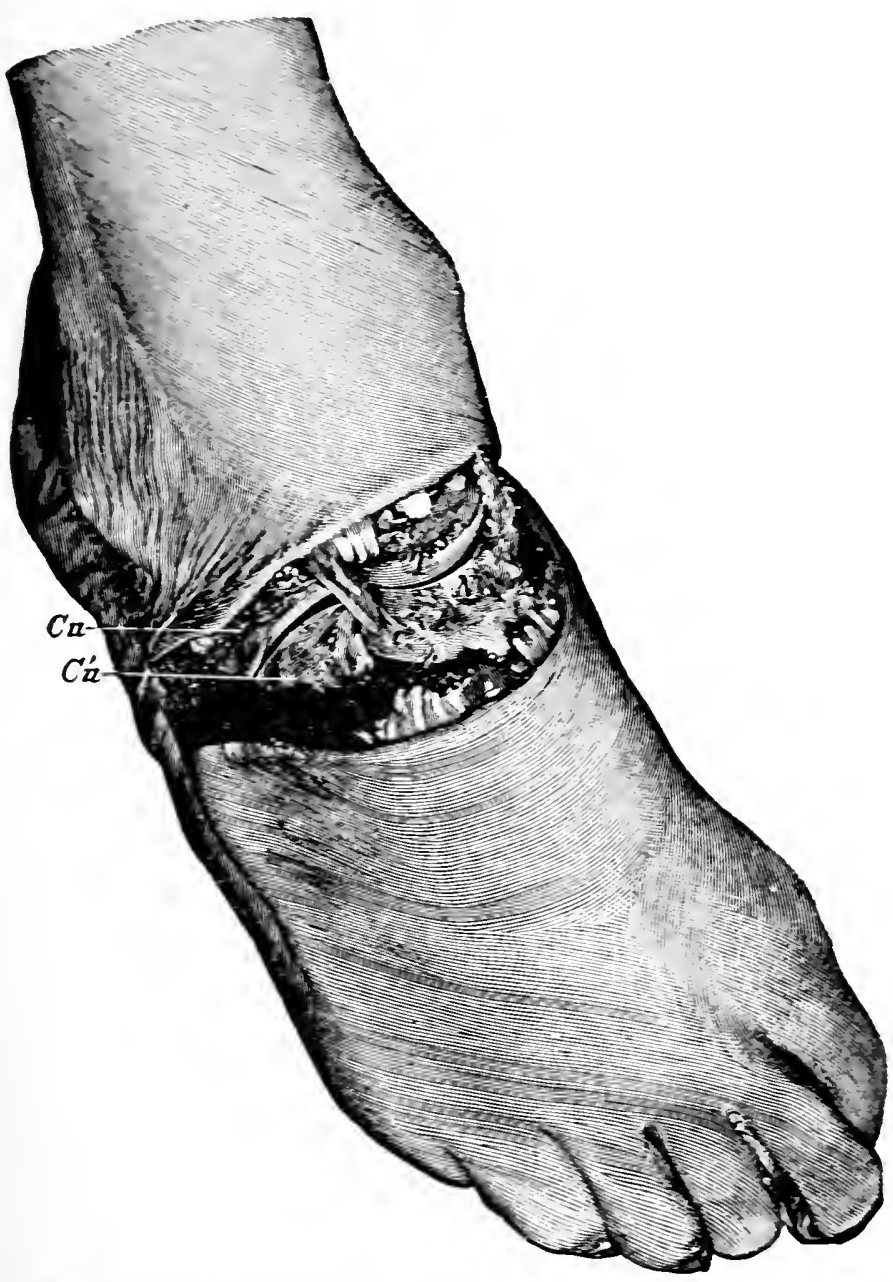

FIG. 89.-Chopart's articnlar line: $T$. head of the astragalus: $N$. scaphoid bone: $C u$, os calcis; $C u$. cuboid bone. The calcaneoscaphoid interosseous ligament is exposed bs dissection. 

Intertarsal Exarticulation. Chopart's Operation.-The astragaloscaphoid articulation upon the one hand and the calcaneocuboid articulation mpon the other hand constitute apploximately a transverse line in which the foot maly be divided within the tarsus. 'The extremity of this articular line upon the inner border of the foot lies just behind the tuberosity of the seaphoid bone; while wpon the outer side the alcancocuboid articulation will be entered if the incision is made a thumb's breadth behind the tuberosity of the fifth metatarsal bone. Chopart's joint is not represented by a directly transverse line between these two points, but is eurved somewhat as follows: the hear of the astragalus is directed with its convexity forward, while the anterior articular surface of the os ealcis is on the contrary excavated (Fig. 89). The calcaneoseaphoid interosseons ligament maintains the bones in apposition after division of the articular capsule.

The operator occupies the same position as in Lisfranc's operation and lateral incisions are made along the borders of the foot outlining the plantar flap, the posterior extremities of which are united by an incision passing transversely over the dorsum of the foot. The joint between the head of the astragalus and the scaphoid bone-is always opened first. It is not to be mistaken, as its situation is indicated by the prominent head of the astragalus, in advance of which the incision is made, as well as by the tuberosity of the scaphoid bone, behind which the incision passes. In order to divide the calcaneoscaphoid interosseous ligament and to open the calcaneocuboid articulation, the point of the knife is inserted in the outer extremity of the already opened astragaloscaphoid articulation, and the blade is directed toward the middle of the small toe, and the tensely stretehed ligament is divided with a slight degree of pressure. The foot is flexed in the line of the open joint; the ligaments of the sole of the foot are divided in the line of the incision, and the plantar flap is made similar to that in Lisfranc's operation, though correspondingly smaller, a finger's breadth 
behind the head of the metatarsal bone. In the stump the dorsalis pedis and the internal and external plantar arteries will require ligation.

If the directions given be not strictly followed, it is possible that instead of entering the astragaloeuboid joint the operator may enter that between the anterior surface of the seaphoid and the three cuneiform bones. The retention of the scaphoid bone would scarcely be a disadvantage, inismuch as the posterior tibial muscle is

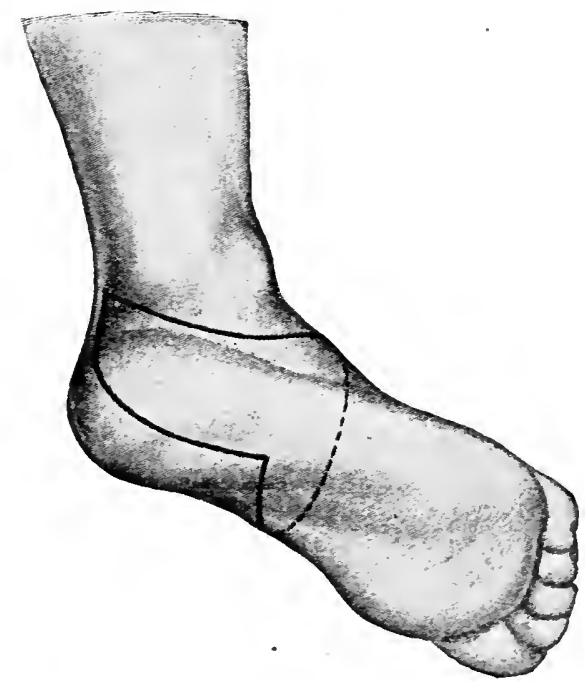

FIG. 90.-Subastragaloid enucleation of the foot: tegumentary incision.

attached to it. Jobert has recommended this method of preseaphoid enueleation as a regular procerlure.

The stump left by Chopart's exarticulation has a tendeney to fix itself in a position of club-foot. 'This defect, it is thought, ean be overeome by certain modifications, such as Jobert's prescaphoid exarticulation, as well as intertarsal amputation, inasmuch as the attachment of the tibialis anticus muscle to the scaphoid bone is preserved and dorsal flexion is rendered possible.

Subastragaloid Enucleation of the Foot.-(Textor, Gun- 
ther, Malgaigne.) - If after cuncleation of the foot at Chopart's joint the calcanemm is adrlitionally removed, the astragalus alone of the talsus is left in connection with the legr. This form of enuclation of the foot, which is known as exarticulatio pedis sub talo, was performed and introduced

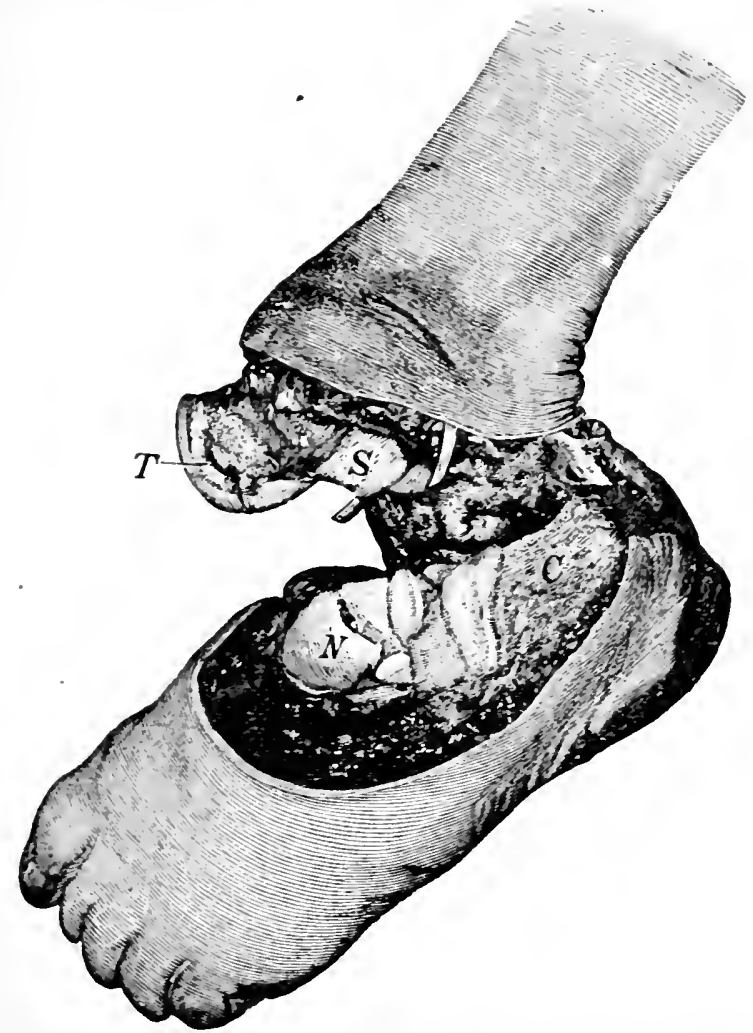

FIG. 91.-Subastragaloid enucleation of the foot: dissection of the flap from the inner surface of the calcaneum: $T$, astragalus; $C$, calcaneum.

by Malgaigne as a regnlar procedure. In the original operation the incisions were like those in Syme's operation, although the dorsal tegumentary flap extended beyond Chopart's joint. The foot was removed at this joint, and then the os calcis was extirpated. The best incision for amputation of the foot at the calcaneo-astragaloid and 
astragaloscaphoid joints is that of Günther, who employs an intornal flap extending to the sole to eover the wound.

The incision (Fig. 90) begins over the tuberosity of the os calcis in the midlle line of the heel, passes thence in

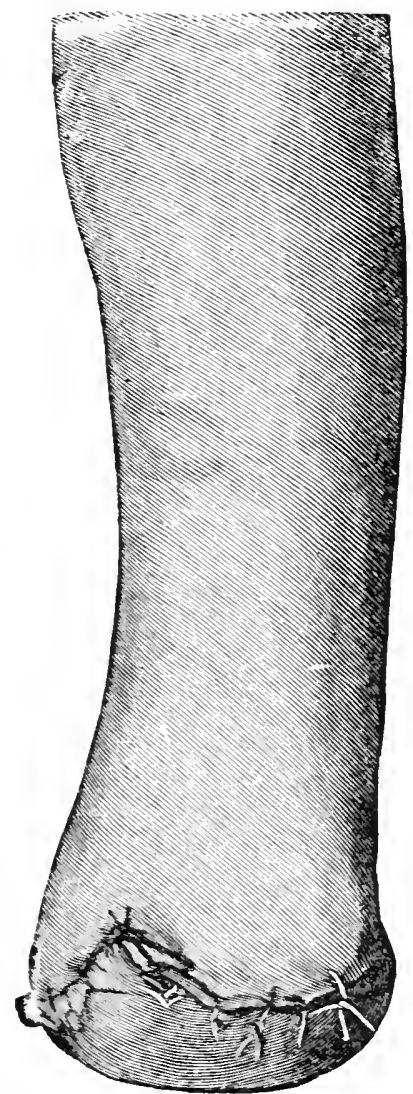

FIG. 92.-Stump following Malgaigne's operation.

an arched direction below the external malleolus, and turns at the level of Chopart's joint toward the median line, to continue transversely across the dorsum of the foot to its inner border, whence it courses over the sole of the foot to the middle line. From this point the incision is continued backward at an oblique angle, 
through the skin of the sole, to reach the point of origin over the tuberosity of the os calcis. The ineision everywhere reaches down to the bone.

The articulation between the head of the astragalus and the scaphoid bone is first opened, and then the connections between the astragalus and the os calcis are divided in the tarsal sinus. If the under surface of the astragalus is thus freed, the flap outlined is separated

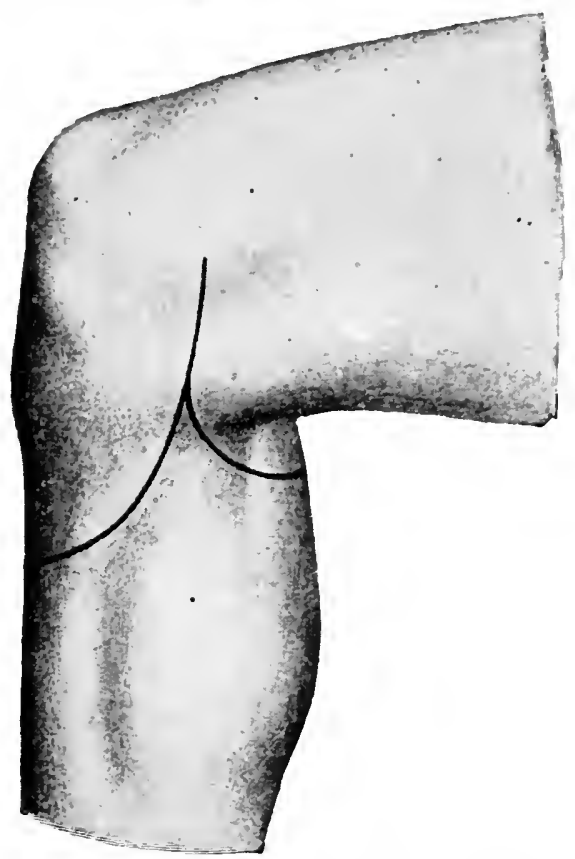

FIG. 93.-Enucleation at the knee-joint: outline of the flaps.

close to the bone from the inner surface of the os calcis, while the foot is rotated outward upon its longitudinal axis (Fig. 91). In opening the joint between the head of the astragalus and the concavity of the scaphoid bone the calcaneocuboid articulation should not be included within the range of the incision. The articulation between the trochlear surface of the astragalus and the bones of the leg should also be protected.

Exarticulation of the Leg at the Knee-joint.-An anterior 
tegumentary flap is made upon the extensor aspect of the leg, and the joint opened from this surface. A short musculotegumentary flap is formed upon the flexor aspect by an incision from the wound outward. The tegumentary flap has a broad base, and its lower extremity extends below the tuberosity of the tibia.

The operator stands at the periphery of the menber. The anterior flap is outlined by incision with the knife. The incisions, passing vertically downward from the most prominent points of the external and internal condyles of the femur, extend three or four fingers' breadth below the tuberosity of the tibia, at which level they are united by a transverse incision. The corners of the flap thus formed are rounded. The flap is now dissected from the subjacent structures to the level of the patellar ligament. With the extremity flexed at the knee the operator grasps the leg with his left hand, divides the patellar ligament with a single transverse incision, and enters the joint. The lateral ligaments and the crucial ligaments of the kneejoint are next divided, so that the leg is attached to the thigh by only the posterior wall of the capsule of the joint and the soft parts of the popliteal space. A long knife is introduced into the wound behind the tibia, and its edge is directed toward the periphery of the extremity, avoiding the head of the fibula, a short museulotegumentary flap being formed from the soft parts of the flexor aspect by incision from within outward. By these means the popliteal artery is not divided till the last stage of the operation. The patella remains connected with the anterior flap.

Pollosson recommends that the operation be so performed that the capsule of the joint is opened close to the tibia in order that after separation of this bone the capsule may be again closed by suture. In this way a carity is formed above the stump. Recovery is said to take place promptly and the stump is believed to gain in usefulness.

Amputation of the Thigh.-Among methods of amputation of the thigh that may be employed advantageously are: the circular incision in two steps, with the formation 
of a cuff; and of flap-incisions an anterior and a posterior musculotegumentary flap of equal si\%, or a lomer anterior and a short posterior musculotegumentary flap.

Amputation of the Thigh by Means of a Circular Incision in 'Two Steps.-The pelvis of the subject is brought to the edge of the table. In amputating the right thigh the operator stands upon the outer side; and in amputation of the left thigh, upon the inner side of the extremity, which is held securely in a horizontal position. At a suifficient distance towird the periphery fiom the point at which the bone is to be divided a circular incision is made through the skin down to the fascia, and a cuff turned back. At the point of reflection of this cuff the muscles are divided down to the bone in fom steps with vigorous strokes of a long knife. The bone is divided with the saw at a point somewhat proximal to the incisions through the muscles. For this purpose the operator with the indexfinger and thumb of his left hand pushes back the muscles upon the bone and divides the latter somewhat further from the periphery. The periostemm in the path of the incision is detached from the bone by means of a raspatory in the area to be sawed through, and the bone is divided with a saw, while the muscles are retracted by means of either tenacula or a divided bandage. In the center of the stump (Plate 9) may be seen the transverse section of the femur, around which the muscles are so grouped that upon the anterior surface lies the quadriceps femoris, while upon the posterior surface lie the flexors. To the inner side the group of adductors lie wedged between the flexors and the extensors. The depression between the adductors and the extensors is covered by the sartorius musele. In the space enclosed by these muscles, which is triangular in cross-section, are to be found the femoral artery and vein, as well as the saphenous nerve. Between the flexors is the sciatic nerve, always accompanied by vessels. In the connective-tissue interstices of the muscles are small arterial vessels divided transverscly or visible in longitudinal sections. After control of hemorrhage 


\section{Plate 9.-Transverse Incision through the Left Thigh at its} Middle Third.

$Q$, quadriceps femoris muscle; s, sartorius; $A d$, group of alductors; $F$, group of flexors; $G$, gracilis; $A . C$., femoral artery in a common sheath with the profunda artery, the femoral veins, and the saphenous nerve; $\mathrm{Ni}$, seiatic nerve.

the muscles are to be so united by buried sutures that the
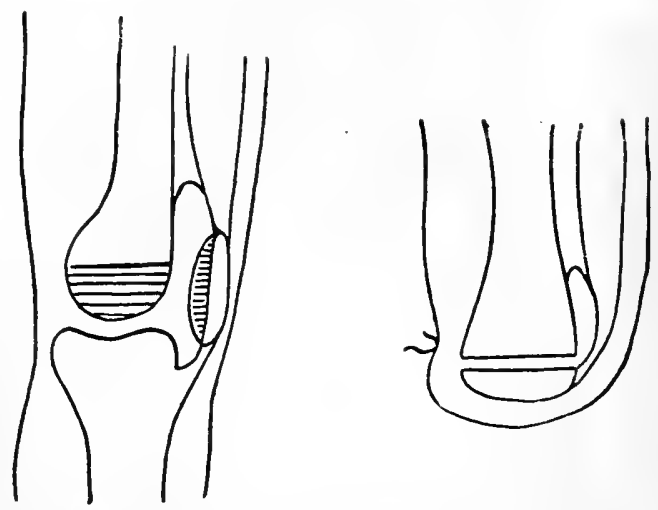

FIG. 94.-Diagrammatic representation of Gritty's operation.

formation of cavities and dead spaces is avoided. The skin is united by deep and superficial sutures.
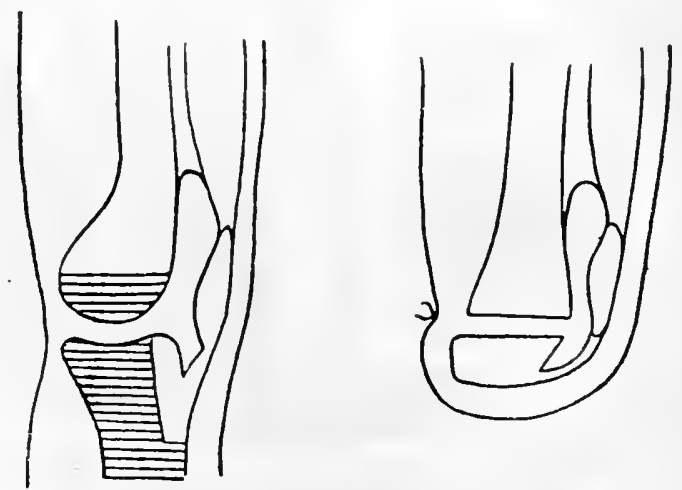

FIG. 95.-Diagrammatic representation of Ssabanajeff's operation. 
$=$

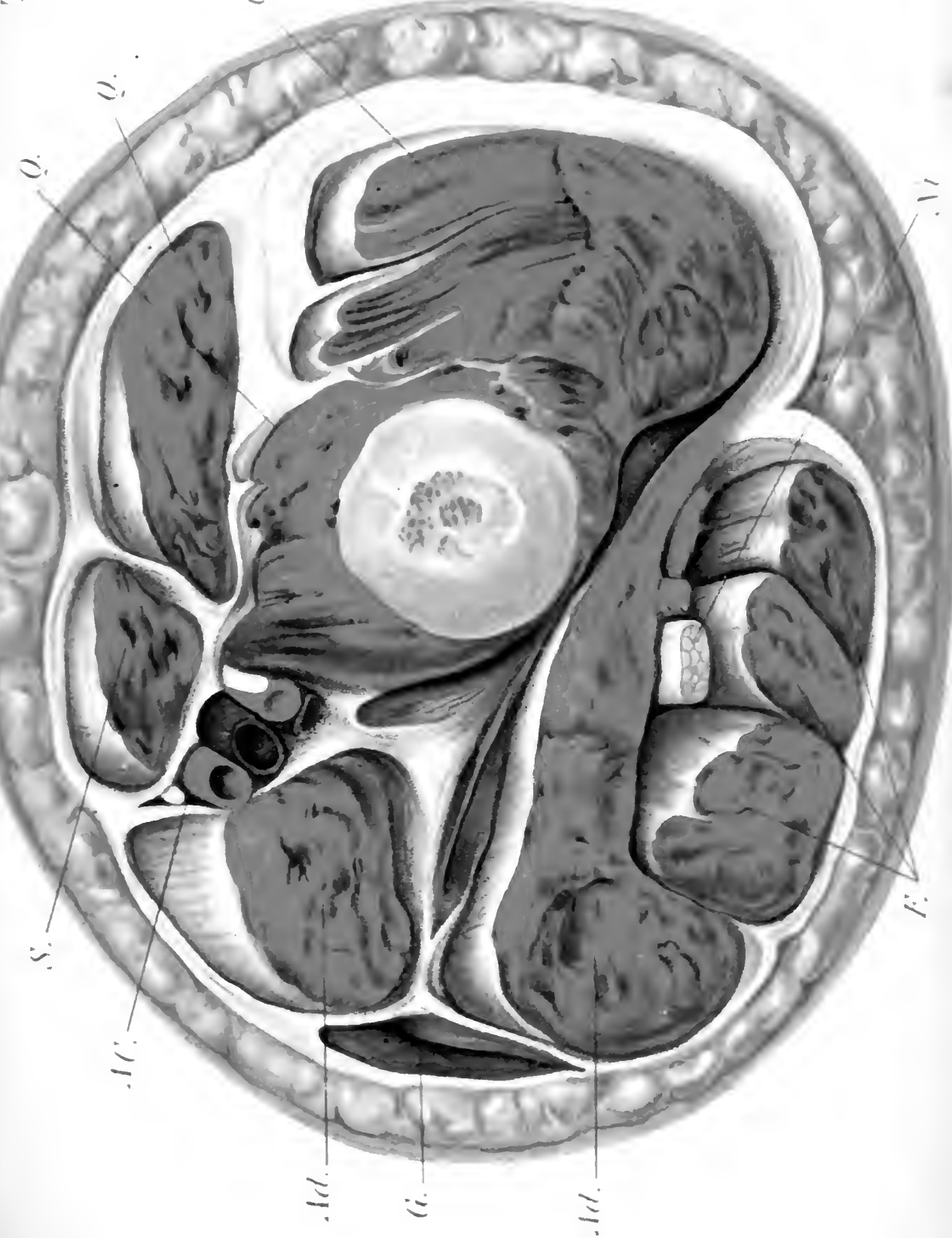


: 


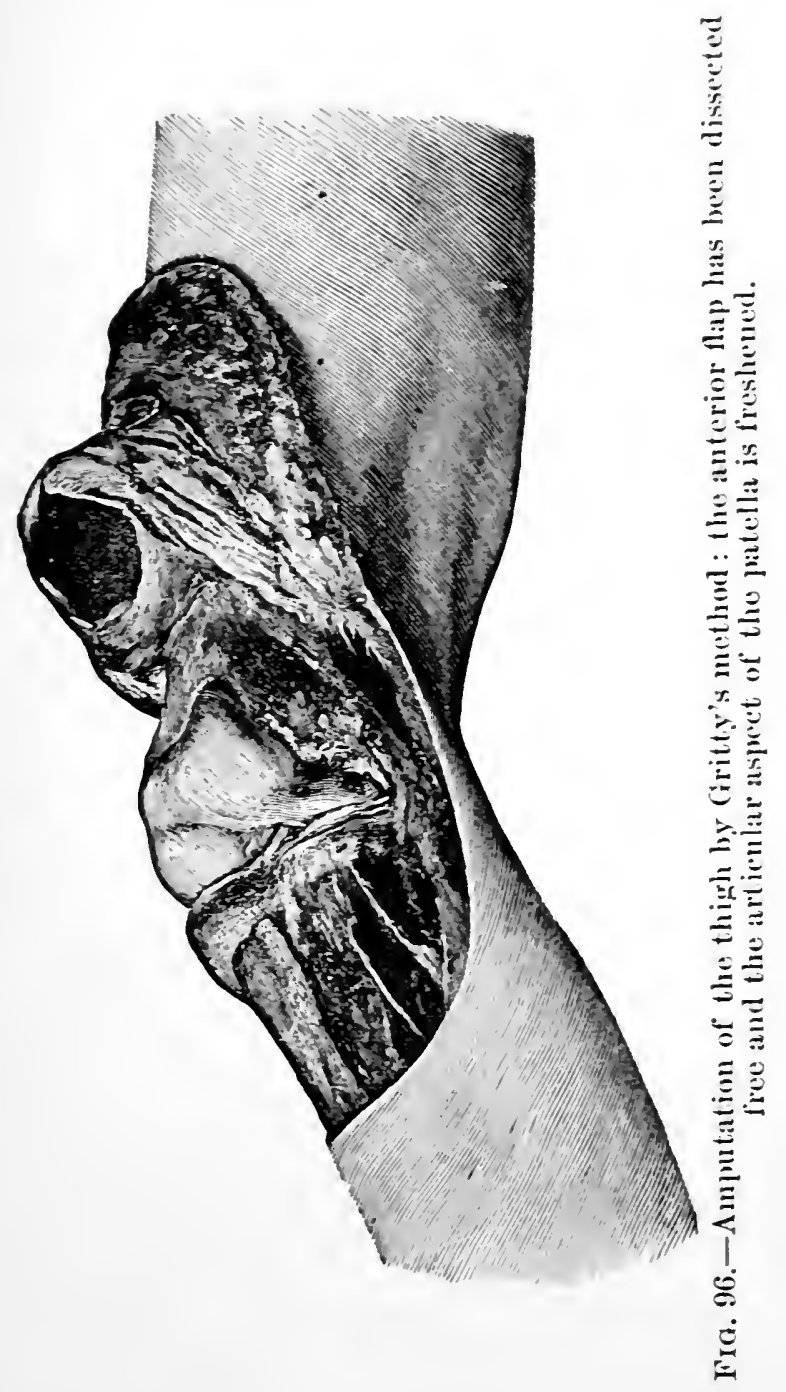




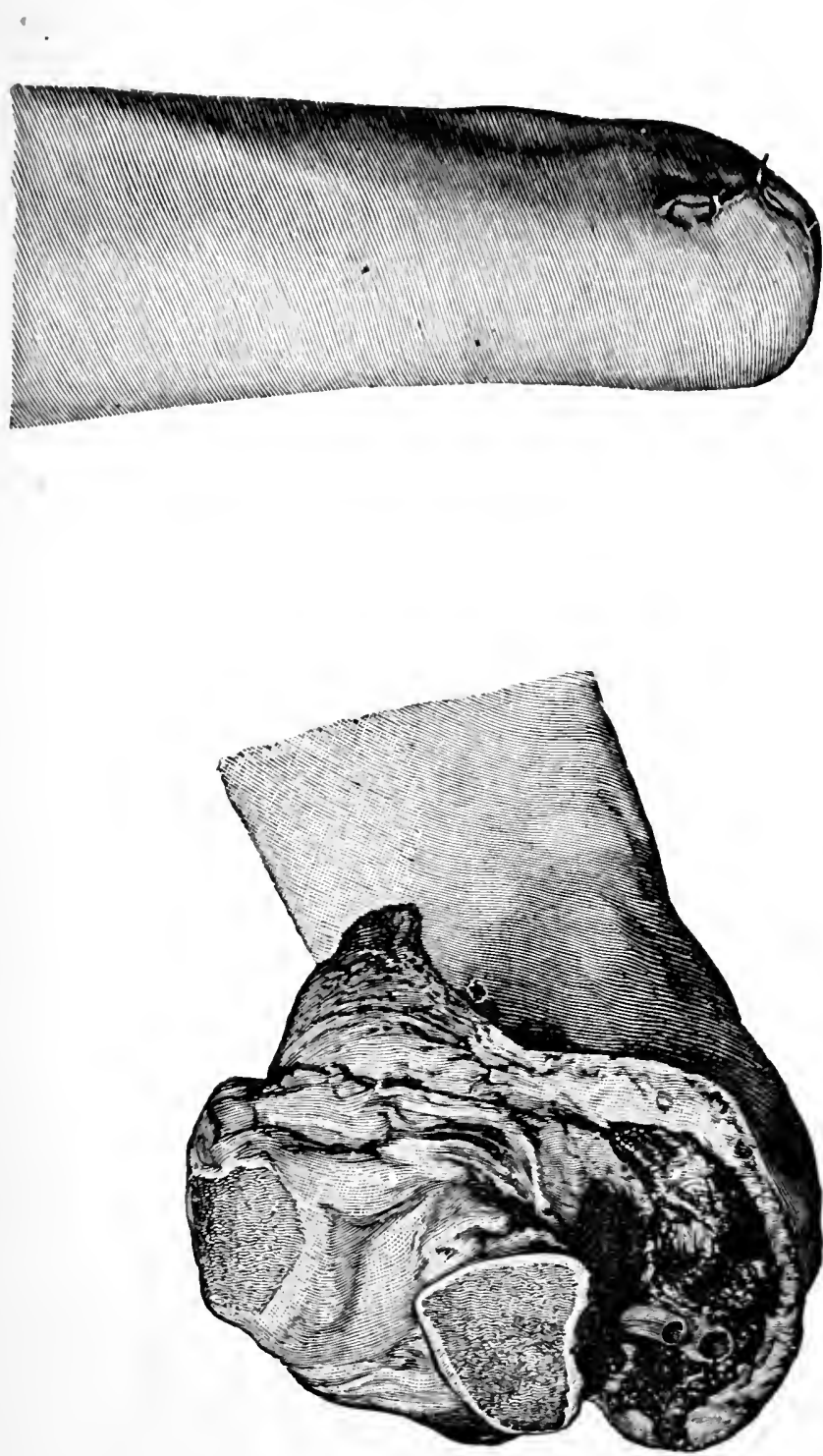

$\cong$

$\Xi$

15.

䤊

$\Xi$

$\mathcal{E}$

등

$=$

$\Xi$

$\stackrel{0}{\Xi}$

$\div$

$\Xi$

:

$\Xi$

引

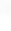

$\stackrel{\infty}{\sigma}$

$\Xi$

$\Xi$

: 

Musculotegumentary Flaps.-The flaps are semicircular and well rounded. Both flaps meet upon the lateral aspects of the thigh in such a mamner that the base of each corresponds to half the circumference of the part. The operator marks the outlines of the flaps by incisions patsing through skin, fatty connective tissue, and the fiscia latil, down to the muscles. Both flaps may be formed by transfixion or by incision from the periphery to the base. The flaps are reflected at their bases, and the muscles attached to the bone are divided by a circular incision. The bone is sawed through in the usual manner and the wound is closed by suture.

Osteoplastic Supracondylar Amputation of the Thigh by the Method of Gritty.-Gritty has ingeniously applied the osteoplastic principle of Pirogoff's operation to amputations at the knee-joint, the freshened surface of the patella being approximated to the sawed surface of the femur, and union taking place.

Mode of Procedure.-An anterior flap is made as for exarticulation of the leg. This is dissected free to the level of the patellar ligament and the joint is opened transversely in this situation. At the same time the lateral attachments of the capsule attached to the condyles of the femur are divided so that the flap, with the patella contained within it, can be reflected. The patella is surrounded by an incision on the synovial surface of the flap, and freshened by removal of its cartilaginous articular surface with the phalangeal saw (Fig. 96). The flap is now somewhat retracted, so that the supracondylar portion of the femur is exposed. An incision is made around the bone in this situation, the bone is sawed through, and a short tegumentary flap is formed from the soft parts of the popliteal space by an incision from within outward. The patella is approximated to the sawed surface of the femur and fixed in this situation by bone-sutures or percutaneous pegs. The stump (Fig. 98 ) yields a functionally good result by reason of closure 
of the medullary carity of the femur with bone and the favorable situation of the cicatrix.

Ssabanajeff has modified the method of amputating the leg at the knee by bringing a segment of the tibia in approximation with the sawed extremity of the femur, and the results are said to be superior to those of Gritty's operation. An incision is made through the skin on the anterior aspect of the leg, as in Gritty's operation. A

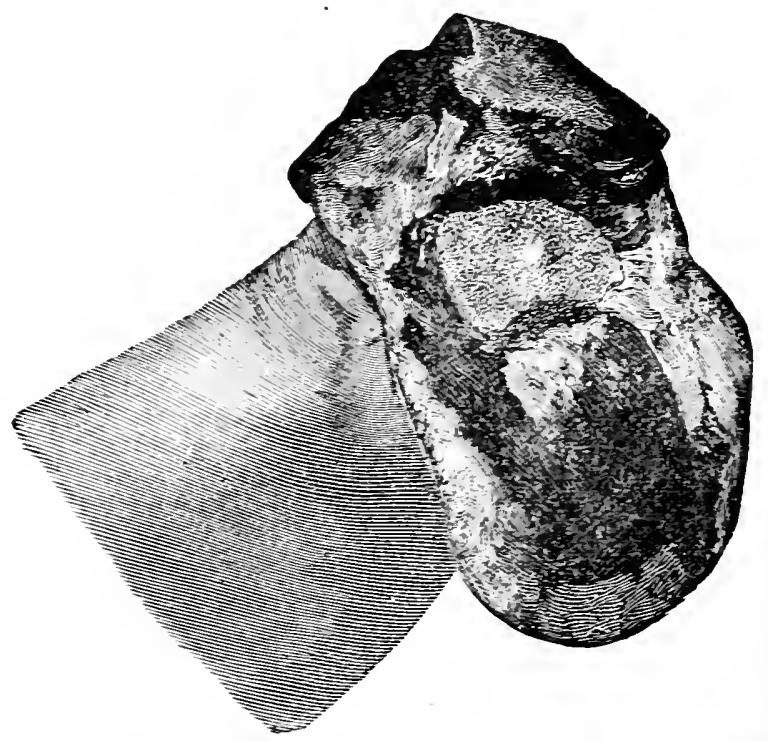

FIG. 99.-The mound made in Ssabanajeff's operation: $T$, sawed segment of the tibia for approximation to the femur; $F$, sawed surface of the femur.

short flap is outlined in the popliteal space with an arched incision and dissected free, the knee-joint being opened from the popliteal space. The leg is so bent at the kneejoint that the surface of the tibia is brought in apposition with the anterior aspect of the thigh. From the articular surface a transverse plate of bone is removed from the upper extrenity of the tibia as low down as its tuberosity, and this remains connected with the anterior flap. The thigh is further divided transversely through its condyles. 
The supporting surfice of the stump is thus formed by the tuberosity of the tibia, and clinical reports are in accord in the statement that it serres this purpose admirably (Figs. 95 and 99).

Exarticulation of the Femur at the Hip-joint by the Method of Esmarch.-The combination of circular amputation of the thigh with a longitudinal incision (Esmarch) permits the remoral of the fenur with a minimum loss of blood. The pelvis of the subject extends berond the border of the table and the operator stands as in the performance of amputation. After the application of an Esmarch bandage as close to the trunk as possible a circular incision is made through the skin down to the muscles in the upper third of the thigh. At the point of retraction of the shin the muscles are divided trpically in a circular manner down to the bone. The periosteum is likewise incised and the bone is sawed through. The next step consists in thorough ligation of the ressels in the transverse incision. After the lumen of all the visible vessels is closed by ligature the bandage is remored. Then a longitudinal incision is made upon the lateral aspect of the thigh, passing over the great trochanter, dividing the soft parts down to the bone, and extending to the level of the wound (Fig. 100). Hooks are introduced into the margins of the incision and the bone is freed from its attachments. When the separation has been effected throughout a sufficient extent, the operator grasps the bone with his left hand, opens the joint, dislocates the head of the femur, and severs the round ligament, when the central portion of the fenur can be removed. The whole operation can be performed without noteworthy loss of blood.

Exarticulation at the Hip-joint, with the Formation of an Anterior and a Posterior Musculotegumentary Flap by Transfixion.-The performance of enucleation, with the formation of toco large musculotegumentary flaps by transfixion, renders possible rapidity of exarticulation, although the control of hemorrhage is difficult. The mode of pro- 
cedure occupied a prominent position in surgery at a time when rapidity of operation, which had to be undertaken without anesthesia, was a primary consideration. At

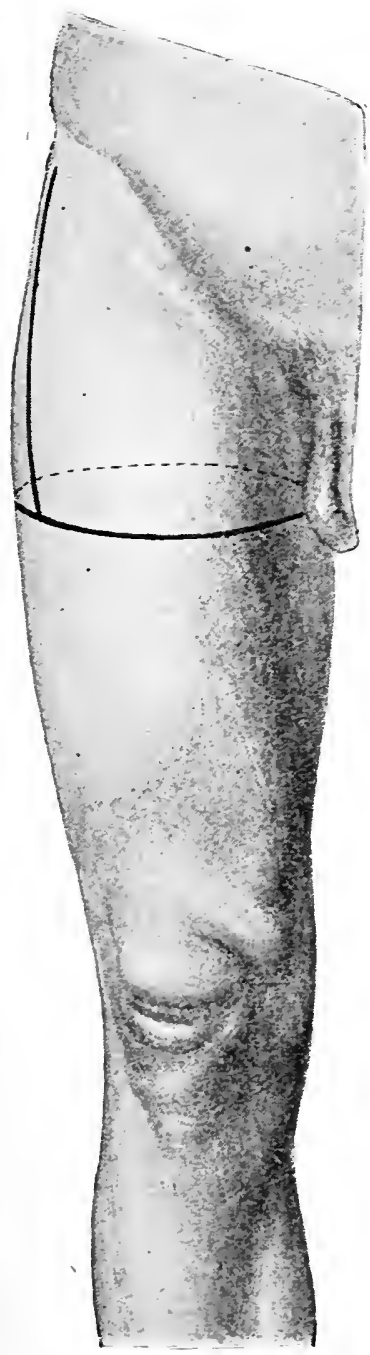

FIG. 100.-Exarticulation at the hip-joint: combination of circular incision and external longitudinal incision.

present, however, it is considered more important in an operation to reduce the loss of blood to a minimum. Thus, in exarticulation of the hip by the method of Verneuil the 


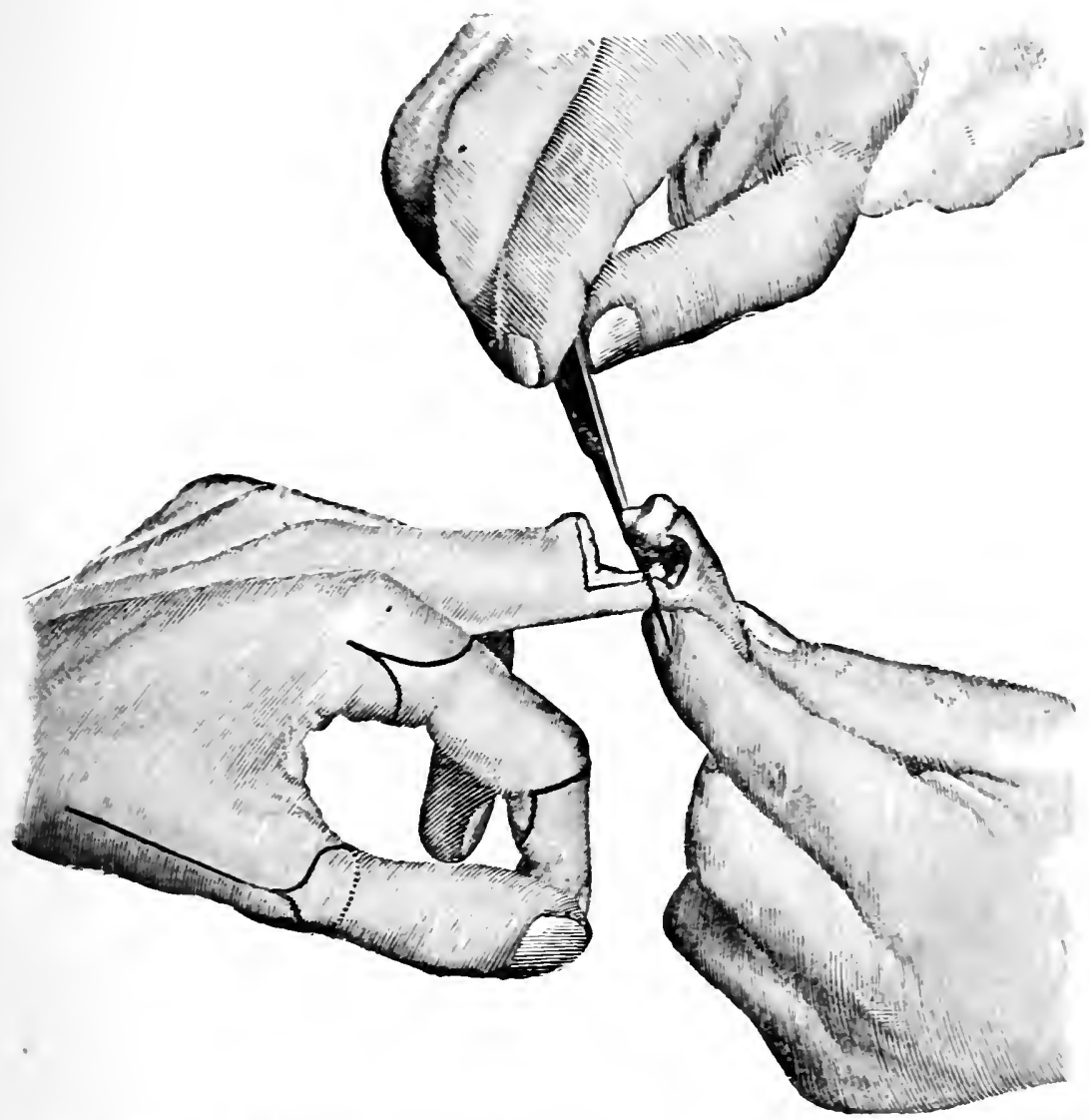

FIG. 101.-Enucleations of the fingers: enucleation of the middle finger at the interphalangeal joint; opening of the joint on its dorsal aspect. Formation of a palmar flap br incision from within outward. Upon the thumb: line of incision for remoral of the thumb at the carpometacarpal joint by means of an oval incision. Upon the index. finger : flap-incisions. 

musclès are divided, step by step, with an ordinary sealpel, divided vessels being immediately grasped, and larger vessels being secured in alvance of division by two ligattures. 'The operation under consideration, although it requires a longer time, is performed with the ntmost care and with least possible loss of blood. 'This method of' procedure, in which the separation of the tissues is effected according to the principles observed in the enucleation of tumors, is therefore designated extirpation.

Exarticulation at the Hip-joint by Means of Hlaptransfixion.-Two tongue-shaped flaps are made that reach to the junction of the middle and upper thirds of the femur, the knife being always introduced from right toward left. If, for instance, the left lower extremity is to be removed, the knife is introduced horizontally upon the onter side of the hip at a point midway botween the anterior iliac spine and the apex of the greater trochanter, and passed throngh the soft parts of the thigh just in front of the capsule of the hip-joint, and brought out in the genitocrural. fold. The flap is cut of snitable length and folded back. The operator grasps the thigh with his left hand, places the hip-joint in maximum extension, and opens the joint through an arched incision that penetrates the anterior wall of its capsule. The head of the femur is forced out of the wound and the round ligament is severed. The posterior wall of the capsule is next divided from the interior of the joint; the greater trochanter is exposed by a few incisions, and a musculotegumentary flap is formed from the soft parts of the flexor aspect by an incision from within outward. Care should be taken that the flaps are not too small, and it is further important in making the incision from within ontward that the muscles are properly divided, so as not to extend beyond the margins of the flap.

Amputations and Exarticulations of the Upper Extremity.-Exarticulation of the Fingers at the Interphalangeal Joints and at the Metacarpophalangeal Joints.- In the enucleation of part of a finger the inter- 
phalangeal joint is opened transversely upon its dorsal aspect and a flap is formed from the skin upon the flexor aspect by incision from within outward. The operator grasps with the thumb and index-finger of his left hand the finger flexed at the joint at which removal is to be effeeted. A transverse incision upon the dorsal aspect on

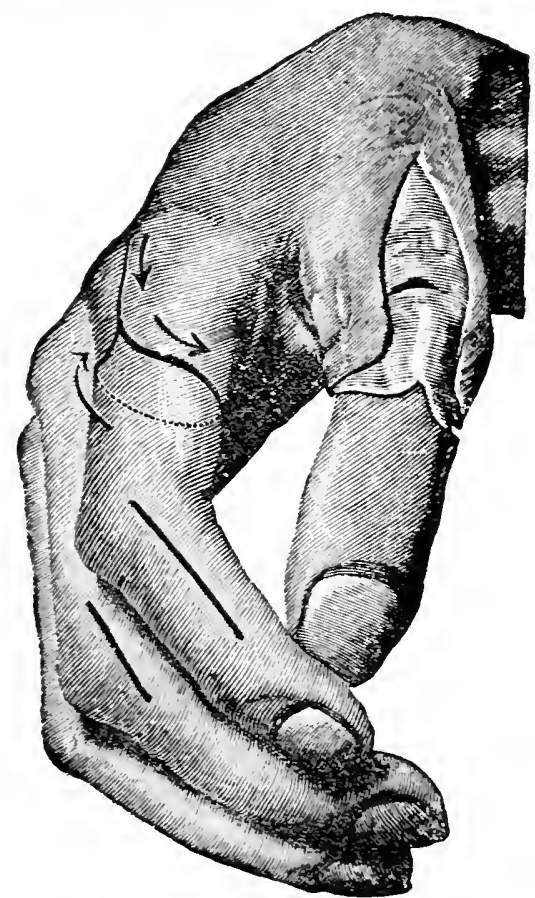

Fig. 102.-Enucleations of the fingers. On the index-finger : line of oval incision for enucleation at the metacarpophalangeal joint; cutaneous incision for resection of the middle phalanx. On the thumb: for the same operation the skin is alreadr freed and the joint has been opened on its dorsal aspect. On the middle finger: cutaneous incision for resection of the first interphalangeal joint.

the peripheral side of the most marked prominence of the joint opens this. The lateral ligaments of the capsule are divided, and, with the joint opened to its maximum extent, a short tegumentary flap is formed upon the palmar aspect by incision from within outward (Fig. 101). The length of the flap will be governed by the size of the wound to be eovered. 


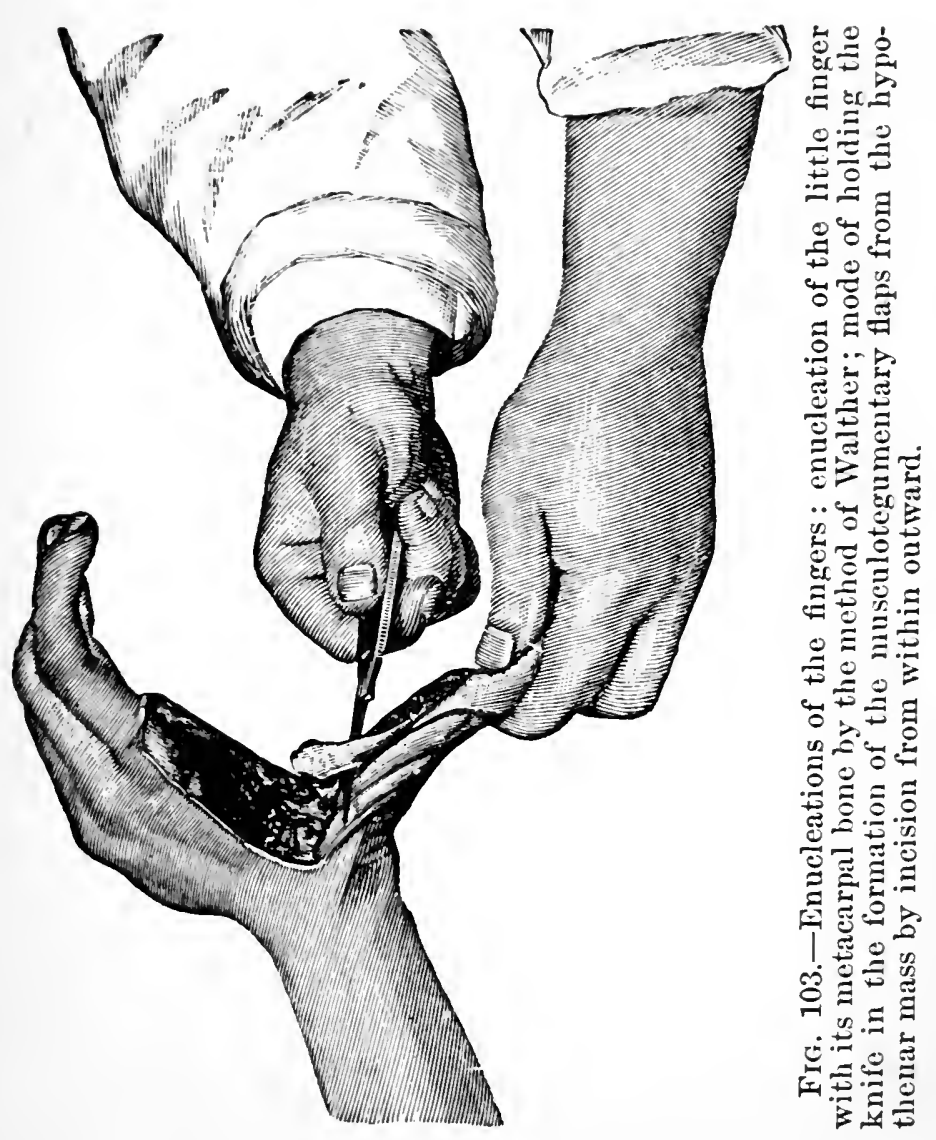



AMPUTATIONS AND ENUCLEATIONS.

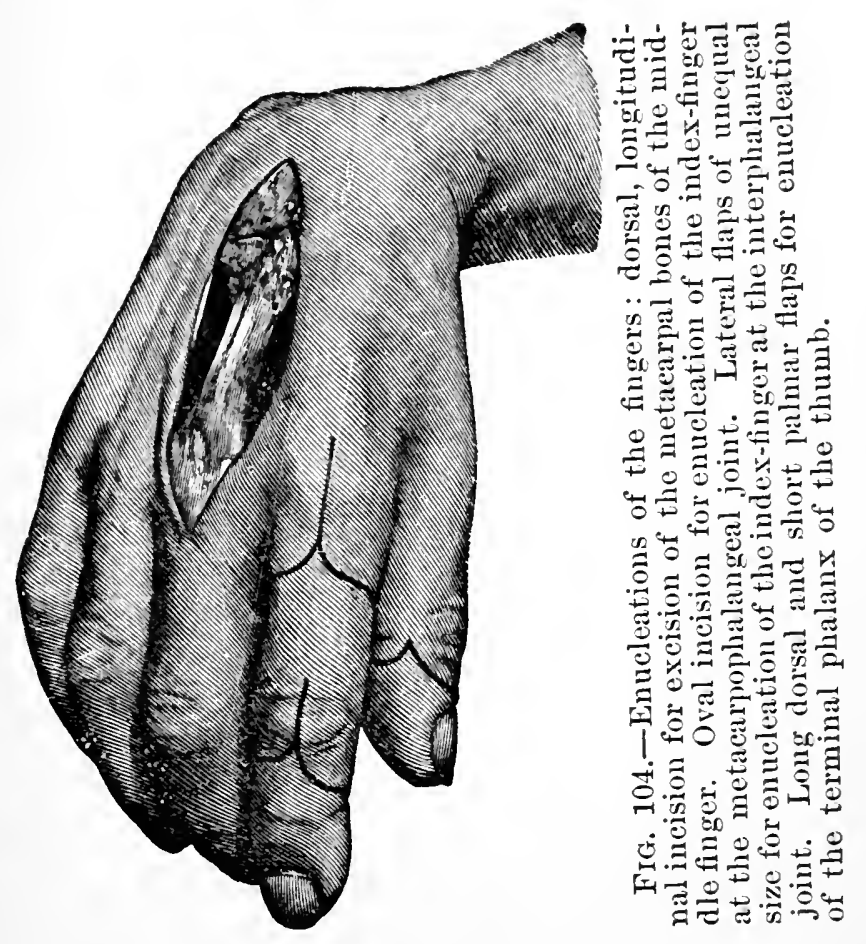


A large dorsal and a short plantar tegumentary flap may also be made advantageously, as well as two lateral flaps of equal size or a single lateral tegumentary flap.

The outline of the flip is always first carefully made with a knife; the flap is then dissected free from the subjacent tissues, and the joint is opened from the extensor aspect. The flaps should be so sitnated that their bases correspond with the line of the articulation at which removal is to be effected.

In making an oval incision the operator applies the knife upon the extensor aspect somewhat to the proximal side of the line of the artieulation and divides the tissues in the middle line parallel with the longitudinal direction of the finger until the joint has been passed. On the distal side of the joint the incision turns toward the right to run transversely through the flexor fold of the joint and it returns upon the opposite side of the finger, to terminate at its point of origin (Fig. 102). By detaching the tissues on either side of the oval from the subjacent struetures the joint is exposed upon its extensor aspect and the removal of the finger can be readily effeeted. Hemorrhage will be controlled by ligation of the digital arteries, which run on either side near the palmar surface. The wound left after oval incision is closed in a linear direction.

For enucleation of the thumb at the carpometacarpal joint an oval incision is best suited. The apex of the oval is situated upon the extensor aspect of the thumb at a point corresponding to that at which removal is to be effected. At the metacarpophalangeal joint the incision deviates toward the flexor aspect, passing transversely through the flexor fold of this joint and ascends upon the opposite side of the finger to join the longitudinal incision at an acute angle (Fig. 101). The incisions extend throughout down to the bone, from which the soft parts of the thenar eminence are carefully dissected. After the metacarpal bone has been freed the joint between the trapezium and the base of the metacarpal bone of the 
thumb is opened from the dorsal aspect and the finger is separated.

Exarticulation of the little finger together with its metacarpal bone by means of a flap from the integument of the ulnar border by the method of Walther. The fourth and fifth fingers are extended and held in a position of maximum abduction. With the dorsum of the hand directed toward the operator, the blade of the knife is introduced at the middle of the commissure between the fourth and fifth fingers and passed with sawing-movements through the soft parts of the interosseous space between the fourth and fifth metacarpal bones to the root of the hand. With the point of the knife, now directed toward the radial border, the ligaments uniting the bases of the two metacarpal bones are first divided, while the little finger is held in a position of marked abduction, after which, by traction in the direction of abduction, the finger can be bent outward in the joint between the unciform bone and the metacarpus. The operator now surrounds the base of the metacarpal bone and forms a flap from the soft parts of the hypothenar eminence by incision from within outward (Fig. 103). Often the flap is cut too short. The operation may also be performed with the aid of an oval incision. The apex of the oval, as well as its longitudinal incision, may be situated either upon the dorsal aspect or upon the ulnar border of the metacarpus.

Amputation of one finger through the metacarpus is effected, like amputation of a toe through the metatarsus, through an oval incision. The apex of the oval is placed upon the dorsal aspect at a point corresponding to the site of amputation. The longitudinal incision passes along the metacarpal bone somewhat beyond the metacarpophalangeal joint, where it encircles the finger through the flexor fold, to ascend on the opposite side and meet the longitudinal incision. The muscles are detached from the metacarpal bone, which is divided with the phalangeal or the arched saw. The peripheral extremity of the bone is enucleated and removed (Fig. 63). 
Enucleation of all four fingers through the metacarpal bones is best effected, like the analogons operation npon the foot, through a short dorsal and a long palmar tegnmentary flap. After the flaps have been formed the metaearpus is surrounded with a circular incision, the muscles in the interosseous spatees are divided with a knife, and division of the bones is effected with the saw.

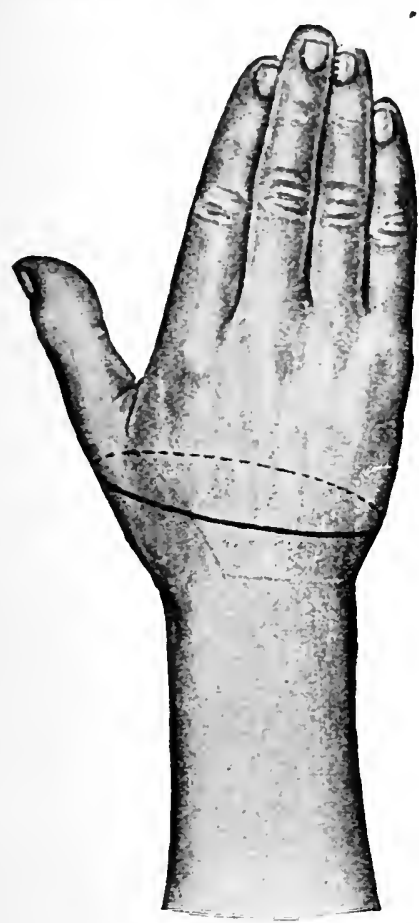

Frg. 105.-Exarticulation of the hand: circular incision in two steps.

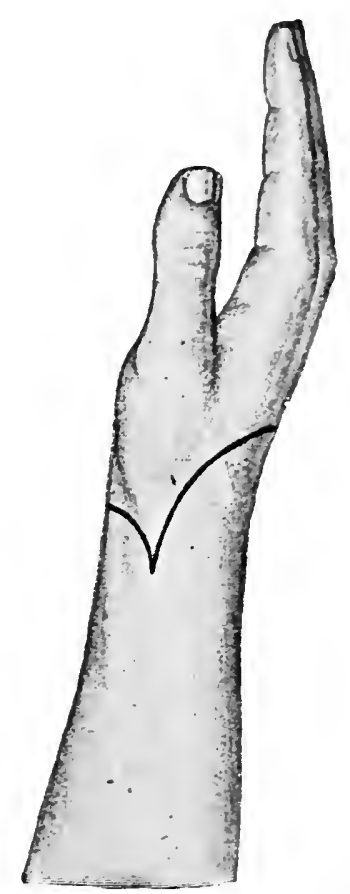

Fig. 106.-Exarticulation of the hand: dorsal and palmar flaps.

The oval incision also may be advantageously employed, the apex of the oval being situated upon one or other border of the hand.

Exarticulations at the Wrist.-The styloid processes of the radius and the ulna constitute the bony landmarks for locating the line of the wrist-joint. The radiocarpal joint, at which the hand is removed, corresponds aceurately with 
a transverse line upon the dorsum of the hand uniting the two styloid processes, when the hand is flexed upon the palm.

Enucleation of the Hand by Means of a Circular Incision and the Formation of a Cuff.-The forearm is placed in a position midway between pronation and supination. The operator occupies the same position as in amputation. A

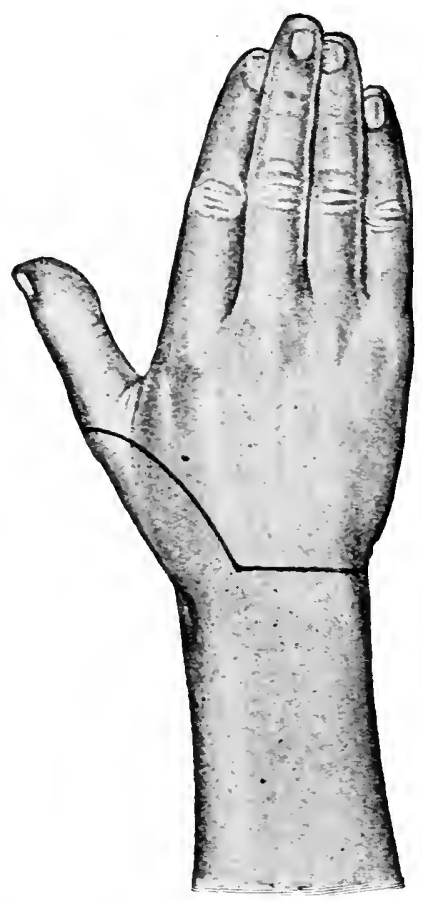

FIG. 10\%-Exarticulation of the hand: tegumentary flap formed from the thenar eminence.

circular incision is made through the skin two fingers' breadth beyond the apex of the styloid process of the radius (Fig. 105). After a cuff of the tissues has been dissected back the tendons are divided with long strokes of an amputation-knife. The operator stands at the periphery and grasps the member to be removed with his left hand, opening the wrist-joint upon its dorsal aspect, 

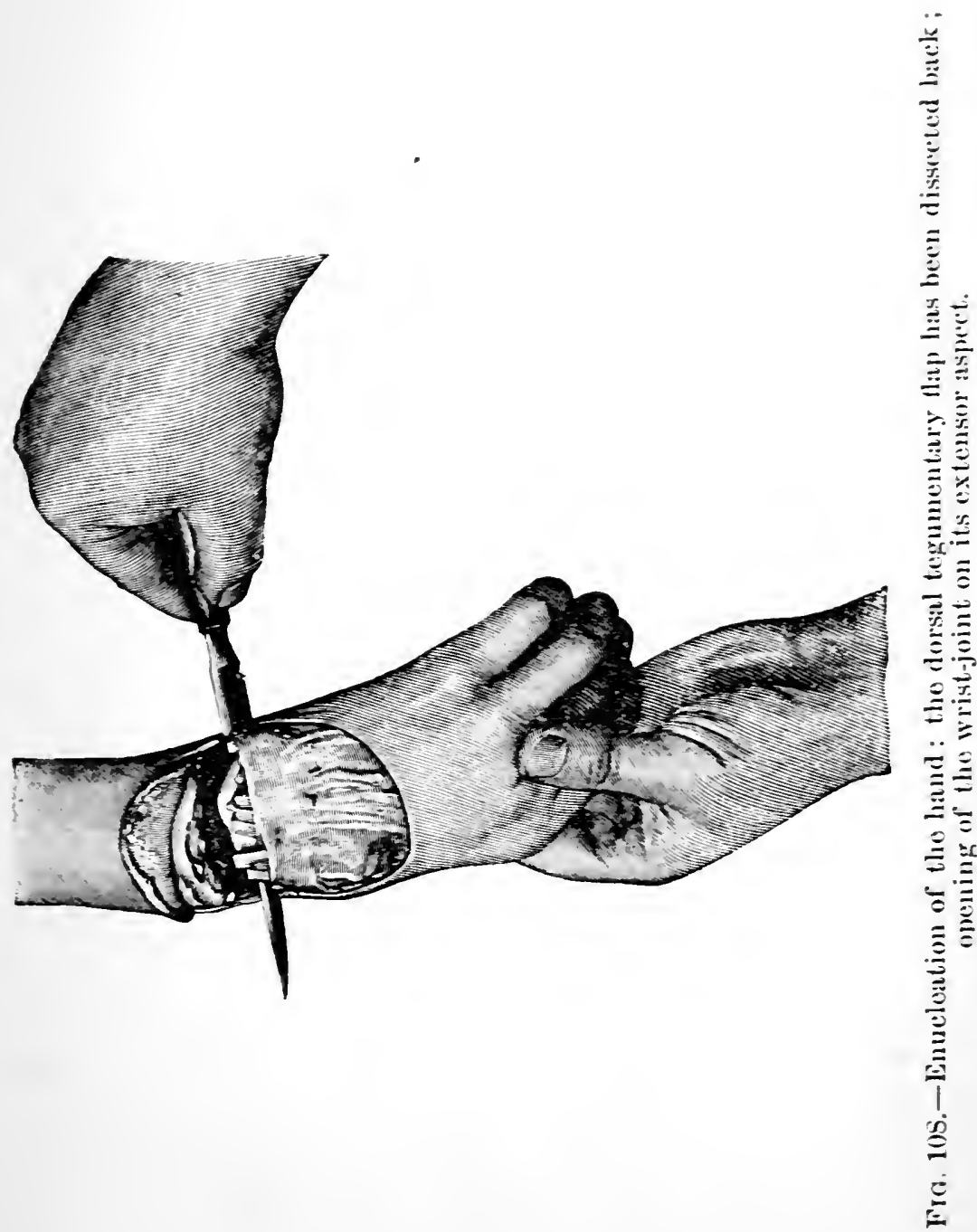
. 


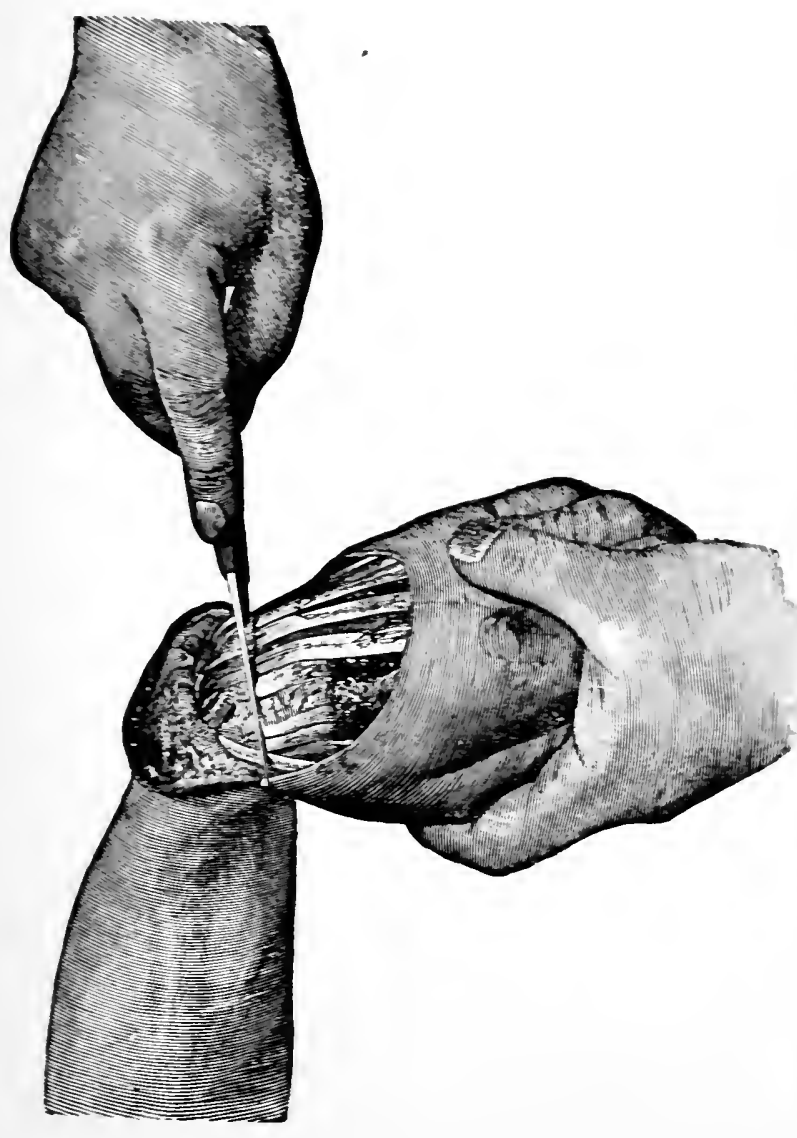

$\equiv$
$\equiv$

$\equiv$

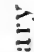

$\stackrel{\Xi}{\Xi}$

$\Xi$

In

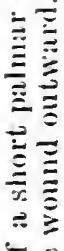

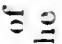

$\equiv \equiv$

主

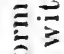

$=$

$\ddot{\Xi}$

三

政

$\div$.

$\equiv$

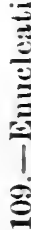

巳 
while the hand is held in a position of maximum palmar flexion, the eapsule being divided upon the palmatr aspect. The radial and unar arteries are divided transversoly on the palmar aspect of the foream in their respective sulei.

Emecleation of the Hend by ILecenss of Flap-incisions.The alpiees of the two styloid proesses form the extremities of the base of the flip. The dorsal tegumentary thap (Figs. 106 and 108) extends to the middle of the dorsmm of the hand. It is dissected free to the line of the joint, which is opened transversely upon its dorsal aspect. The lateral and palmar ligaments are divided, when the exposed flexor tentons of the fingers are matle tense by traction. The tendons are divided transversely and a short palmar flap is formed by incision from within the wound ontward (Figs. 108 and 109).

It may be necessary after the entcleation of the hand to use the integument of the thenar eminence to cover the wound (Fig. 107). The shape of the tegumentary flap is outlined by incision, the flap itself dissected free from the subjacent structures, and from its extremities a eireular incision is made around the wrist-joint. The division of the tendons and the disarticulation of the hand are effected in the manner described.

Amputation of the Forearm.-By means of a circular incision. The arm is held in horizontal abduction from the trunk in a position midway between pronation and supination in such a way that the thumb is directed upward. A circular incision is made, with the formation of a cuff in the usual manner. The muscles are divided, like those of the leg, by a figure-of-eight incision through the interosseous space. In applying the saw the forearm is placed in a position of eomplete supination, when both bones are divided simultaneously from the palmar aspect. In the transverse seetion thus exposed (Plate 10) are seen the bones, and they should lie parallel in a position of maximum supination. Between the ulna and the radius is stretched the interosseous ligament, with the interosseous artery and the accompanying vein and nerve 


\section{Plate 10.-Transverse Incision through the Middle Third of the Left Forearm.}

$r$, radius; $u$, ulna; F.s., flexor digitorum sublimis; F.p., flexor digitorum profundus; U.i., flexor carpi ulnaris; R.i., flexor carpi radialis; P.l., palmaris longus; S.l., supinator longus; Ext, group of extensor muscles; $U$, ulnar artery in a common sheath with the corresponding veins and the ulnar nerve; $R$, radial artery, with the corresponding veins and nerve; $M I$, median nerve; $J$, interosseous artery.

on its palmar aspect. The flexors are grouped upon the ulnar and palmar aspect, the extensors upon the radial and dorsal aspect of the stump. In the middle of the stump, between the superficial and deep groups of flexors, is the median nerve, divided transversely. In the connective-tissue interspace in which the nerve is contained lie also upon either side the ulnar and the radial artery.

Of flup-incisions suitable for anputation of the forearm the best consist of two musculotegumentary flaps of equal size upon the palmar and dorsal aspects. A single large palmar musculotegumentary flap may also be advantageously made to cover the wound (Fig. 110).

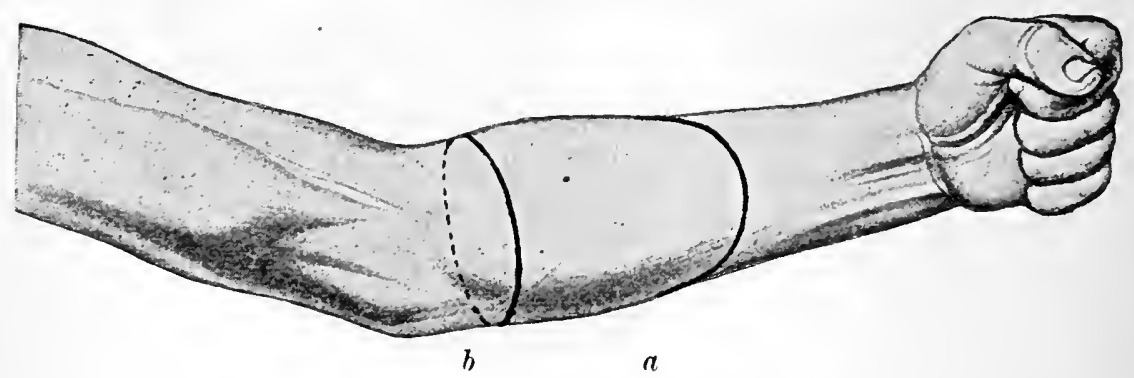

Fig. 110.- $a$, flap-ineision for amputation of the forearm ; $b$, exarticulation at the elbow-joint through a circular incision.

Enucleation at the Elbow-joint.-The two epicondyles are palpable on either side of the joint, and just below the external condyle the head of the radius can be felt upon pronation and supination of the forearm. The npper border of the radial head marks the situation of the articular line. 
l'ab. 10.

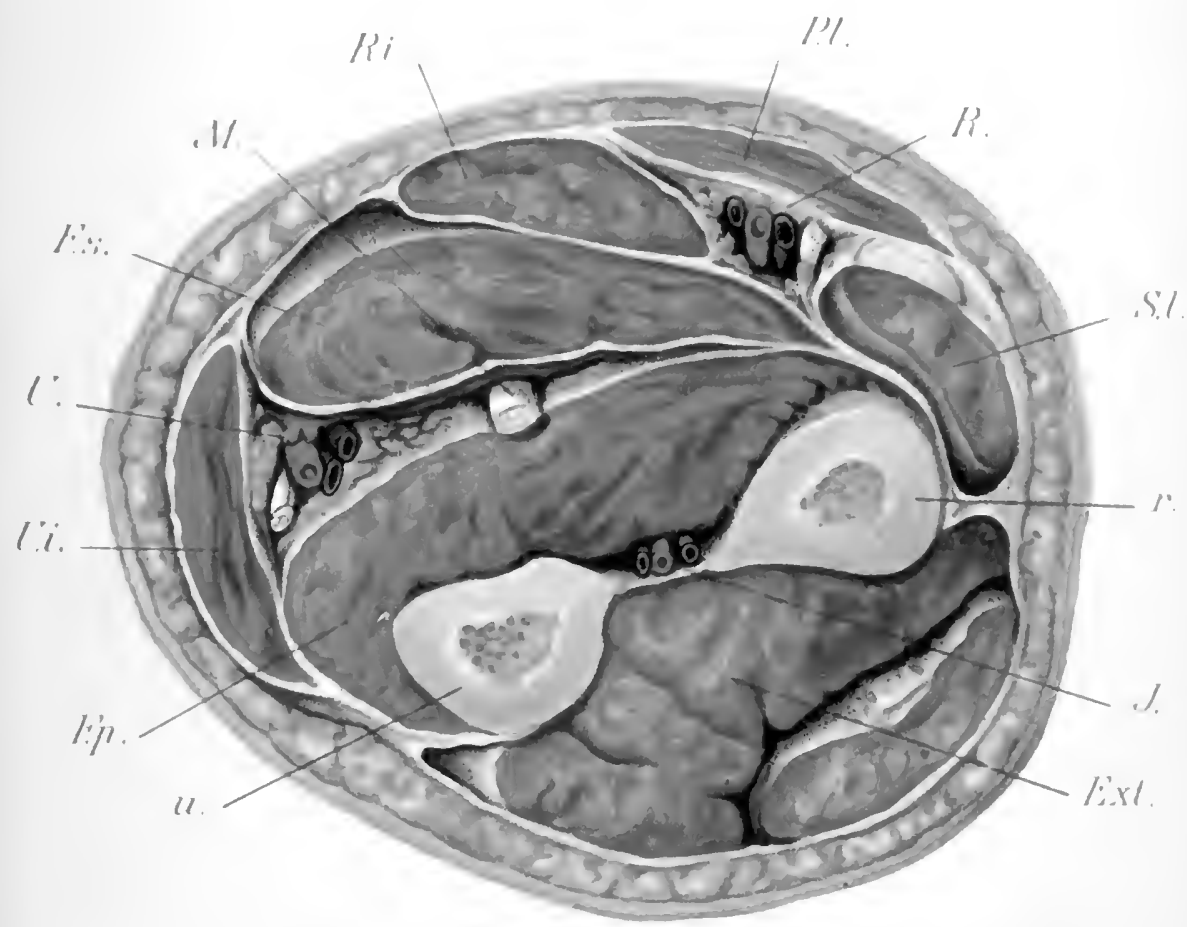


The enueleation is best effected through the formation of broad, well-pathed musculotegumentiar? flaps from the soft tissues of the flexor atspect of the forealm. The operator grasps with his lett hand the fincearm held in at position of maximum supination. A long, pointed knife is introduced from right to left below the epicondrles at the level of the artienlar line and passed transiersely. through the foream in ase proximity to the anterior surface of the joint. With sawing movements of the knife a flap is formed, which extends to the junction of the middle and upper thirds of the forearm (Fig. 111). The flap is reflected upward and with a sealpel the anterior portion of the aipsule of the ellow-joint is divided

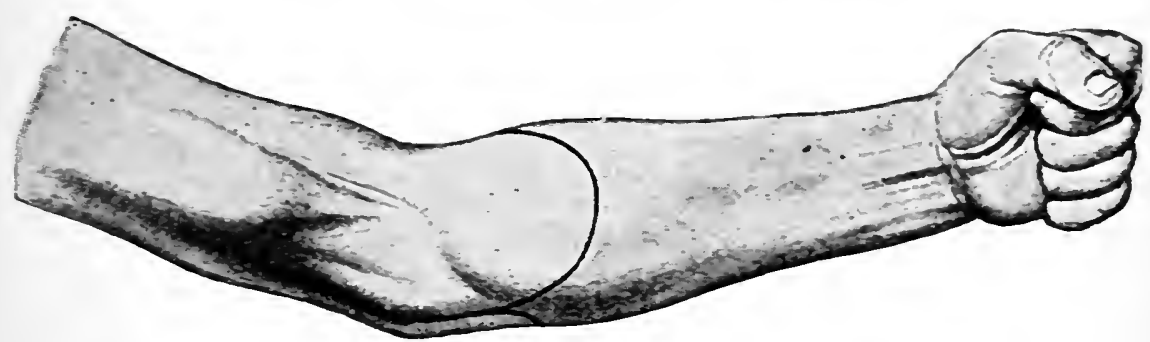

FIg. 111.-Exarticulation at the elbow-joint: Alap-incision.

transversely, so that the trochlea and the radial head of the humerus are exposed. With the elbow-joint overextended the operator divides the external and then the internal lateral ligament. Continuing the over-extension the olecranon is brought into the wound. The attachment of the triceps muscle is dissected close to the bone and a short tegumentary flap is formed upon the dorsal aspect by incision from within the wound outward. Upon the flexor aspect the two branches of the brachial artery are to be ligated.

The circular incision for exarticulation of the elbowjoint is made about three or four fingers' breadth below the line of the articulation, and a cuff is dissected in the usual manner to the level of this line and reflected up- 


\section{Plate Ir.- Transverse Incision through the Right Arm at its Middle Third.}

$B$, biceps muscle ; $B r . i$, brachialis anticus ; $T$, triceps ; $A$. b., brachial artery in a common sheath with the corresponding vein and the median nerre (IS); $U$., uluar nerve: $R$., radial nerve; $M$. . c., musculocutaneous nerve.

ward. The joint is opened and the exarticulation effected in the manner described.

Amputation of the Arm.-A cireular incision may be emploved, as well as the formation of two musculotegumentary flaps.

The circular incision (Fig. 112) is made either in the customary manner in two steps, with the formation of a

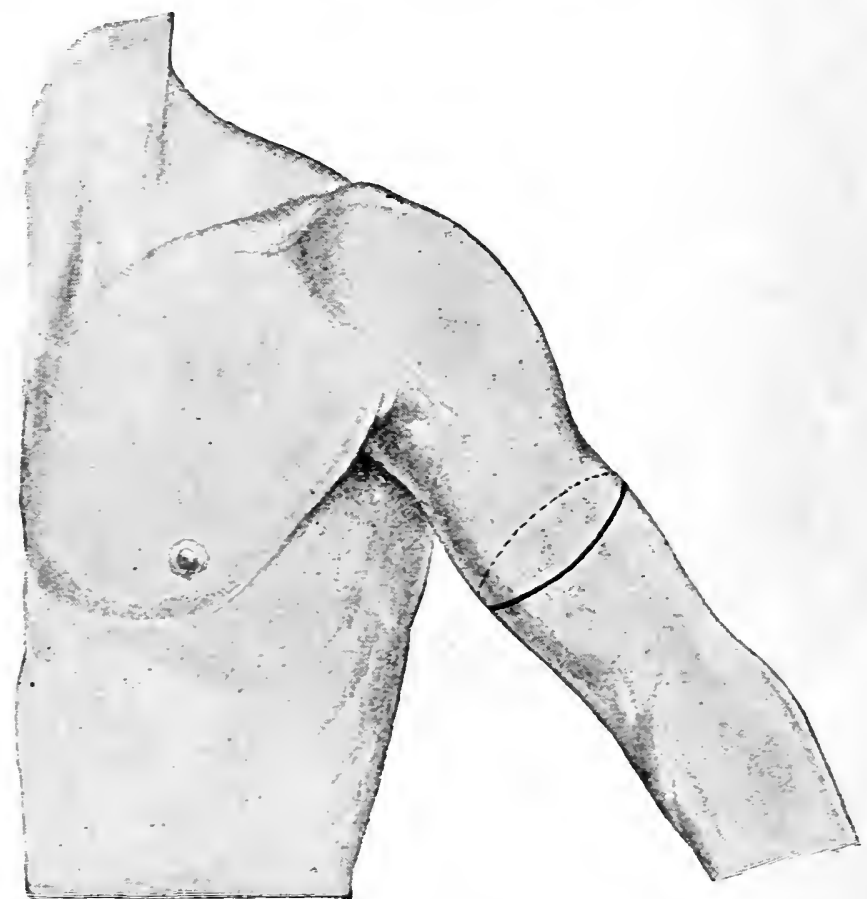

Frg. 112.-Amputation of the arm: circular incision.

cuff, or with a single stroke of the knife after the soft parts have been vigorously retracted. In the stump of 
Tab 11.

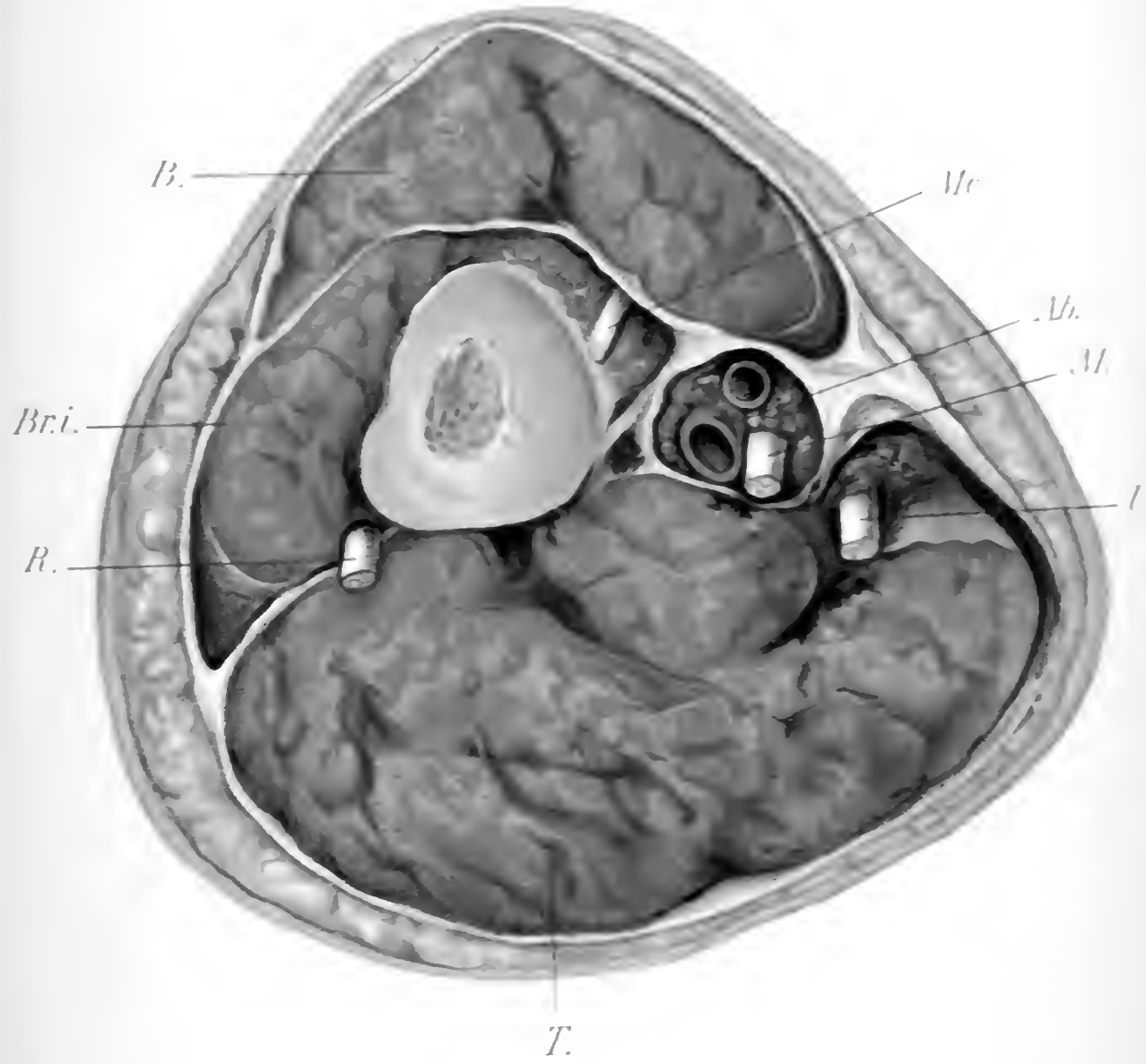




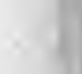


emploved are modified accordingly. The artery is either ligated in advance, or it is divided at the last stage of the operation while digital compression is made.

Exarticulation by Means of a Deltoid Musculotegumentary Flap.--The trunk of the subject is elevated and the operator outlines a flap in the deltoid region with a U-shaped incision whose upper extremities correspond

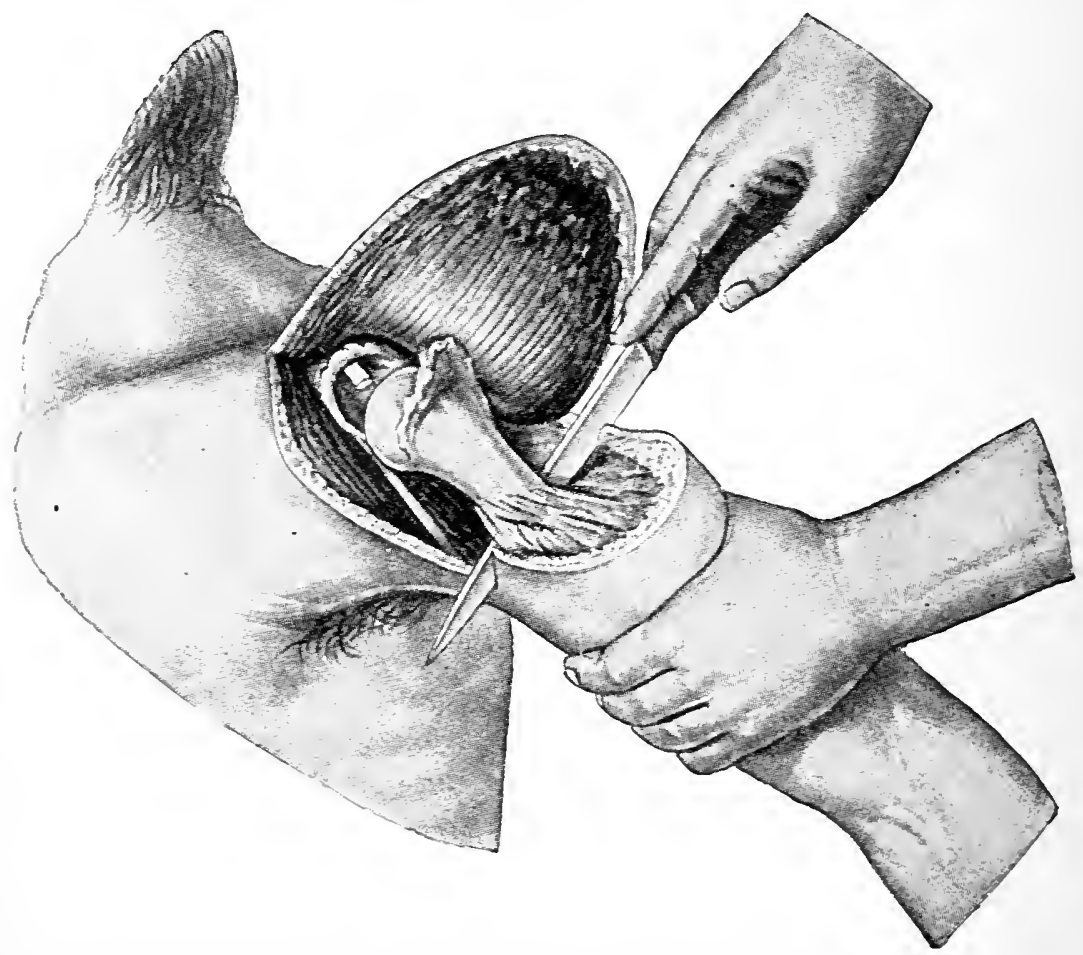

FIG. 115.-Exarticulation of the humerus : formation of an axillary musculotegumentary flap by incision from within the wound outward.

with the acromion and the apex of the coracoid process, and which extends as low as the insertion of the deltoid musele (Figs. 113 and 114). After the tissues are divided down to the muscles the flap retracts somewhat. The musculotegumentary flap is dissected from the bone by. long strokes of the knife. Br dissection of the flap the shoulder-joint is exposed. The operator grasps the arm 
with his left hand, and divides the eapsule of the joint by applying the knife vertically upon the head of the humerus and passing it in an arched direction over the most prominent convexity of the bone (Fig. 117). The heal of the humerus is forced out of the wound, the attachment of the posterior wall of the capsule separated from the bone, and the surgical neck of the humerus, as well as the upper

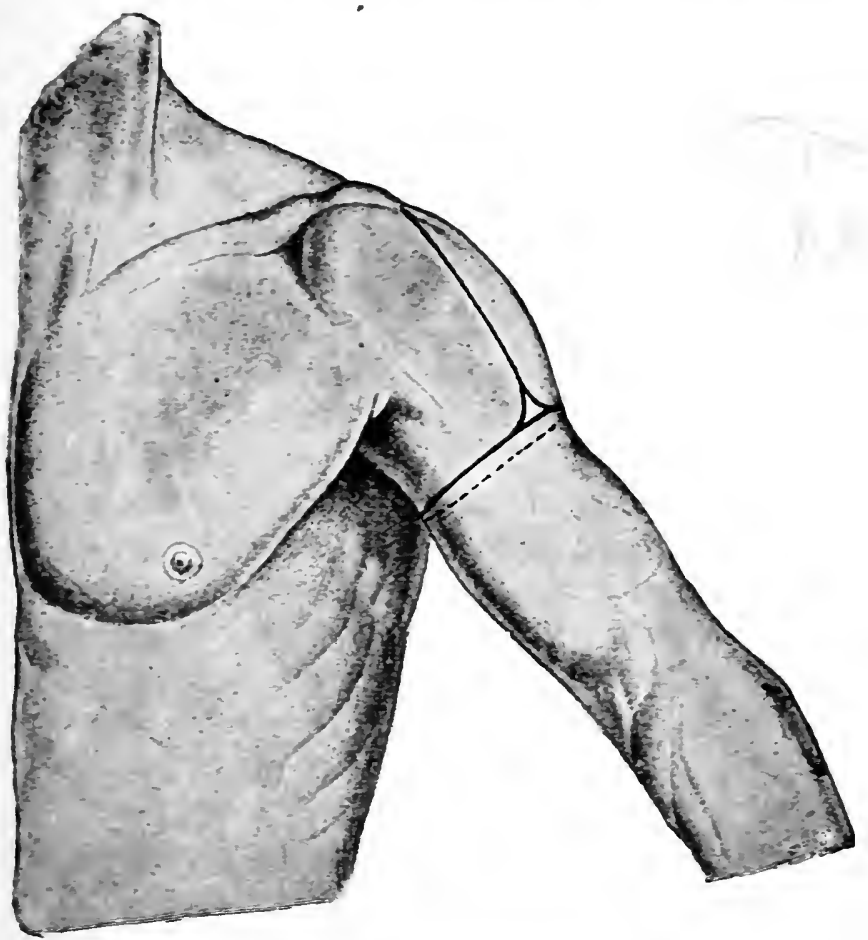

FIG. 116.-Exarticulation of the humerus by a combination of circular and longitudinal incisions.

extremity of the shaft of the bone, is freed from the soft tissues. In this way a bridge is formed of the soft tissues of the axilla in which the vessels are contained. While an assistant grasps this bridge between the thumb and index-finger of each hand in such a way as to compress the artery, a flap is cut from the soft tissues of the axilla at a level corresponding with the attachment of the pecto- 
ralis major muscle to the humerus from within the wound outward (Fig. 115). While the digital compression is continued, the axillary artery is ligated. At the inner side of the deltoid flap branches of the posterior circumflex artery are to be secured by ligature.

Exarticulation by Means of a Circular Incision in Conjunction with a Longitudinal Incision by Esmarch's Wethod.-The arm is surrounded at the highest point possible by an Esmarch tube. The patient occupies a similar position to that for amputation of the arm. A circular incision is made through the skin below the attachment of the deltoid muscle, and at the point of retraction the muscles are divided circularly and the bone is sawed through. Hemorrhage from the divided surface is controlled and the elastic tube is removed. The body of the subject is raised still higher and a longitudinal incision is made from the coraco-acromial furrow down to the wound (Figr. 116). The incision passes through the deltoid muscle and exposes the capsule of the joint. The margins of the incision are separated by tenacula and the muscle is detached from the shaft of the bone. The joint is opened in the manner described and the bone is separated carefully from its attachments (Fig. 117).

An oral incision may also be employed in operations at the shoulder-joint in such a way that the longitudinal portion of the incision is made from a point midway between the coracoid and acromion processes to the insertion of the deltoid, through the muscle down to the joint, while the transverse incision through the flexor aspect passes only through the skin. The margins of the longitudinal incision are separated by tenacula, the joint is opened, and the head and the neck of the humerus are exposed and forced out of the wound. While the artery is compressed. in the wound the operator cuts the base of the oral toward the axillary carity in the incision previously outlined. 


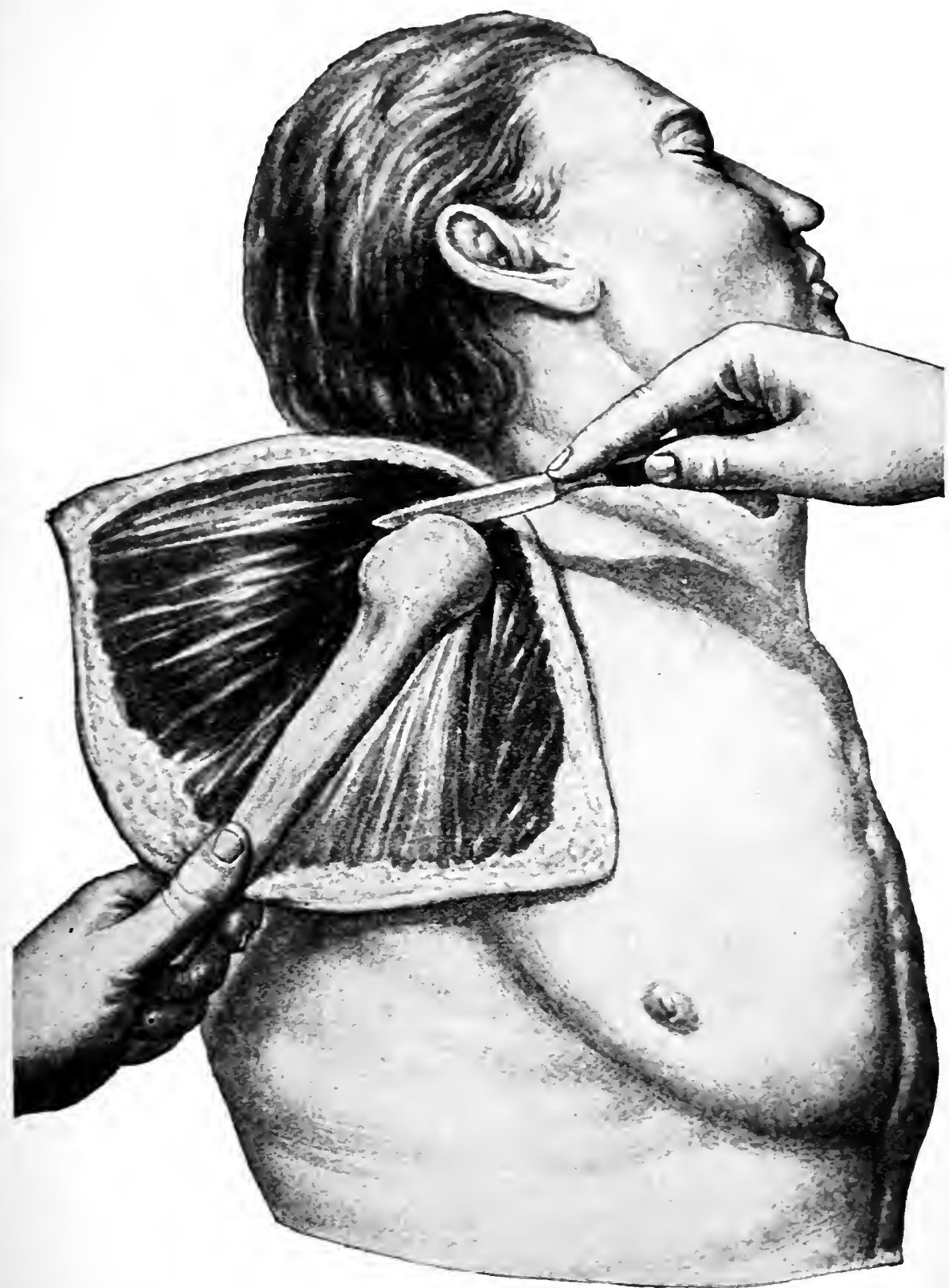

Fig. 117.-Exarticulation of the humerns by Esmarch's method: division of the articular capsule. 



\section{Resections at the Joints of the Extremities.}

By resection of a joint is understood the systematic removal of its constituent parts, with conservation of contiguous structures. In the presence of tuberculous disease, as well as of severe injuries about the large joints, resection promised to be a conservative substitute for amputation. It can thus be realized that this mode of procedure was expected to prove a great advance in surgery, in times of both war and peace. The methods of operation were so selected that compensation for the loss of the parts removed could reasonably be hoped for. With this thought Langenbeck devised operations for all of the joints in which the capsule was permitted to retain its connection with the periosteum, for whose osteoplastic capability experimental proof had been furnished (subperiosteal resection). Langenbeck's incisions are still largely used in the performance of resections. The introduction of asepsis, as well as a more precise knowledge concerning the nature and the distribution of the tuberculous process in joints, has changed our riews of these operations fundamentally. Antisepsis has rendered possible conservative treatment with success of injuries to joints, even without resection, for which previously amputation would have been undertaken. The nature of the tuberculous process and the extent of its distribution in the joints, further, make it undesirable to adopt the routine plan of procedure in every case of removing the bones cntering into the constitution of the joint, while the capsule as such is permitted to remain. Resection of tuberculous joints is no longer regarded as a typical procedure to be employed in every case, as, for instance, is the extirpation of tumors; nevertheless, opening of the joints of the dead subject in a typical manner is practised, as by this means the surgeon familiarizes himself with methods by which it is possible to open the joints with great care, and which render accessible throughout their whole extent 
the parts that enter into the formation of the joint, as well as the synovial surfaces of the capsule. Thus, in a certain sense, the preliminary operation is performed, with which clinically in the individual case the special operation of removal of tuberculous disease is conjoined. The capsule of the joint is widely opened (arthrotomy), the synovial sac freed throughout its whole extent, and in accordance with the extent of the morbid process the extirpation of the synovia (synorial arthrectomy), excochleation of areas of bone, possibly after exposure by means of the chisel and mallet, or resection of the articular extremities (osseous arthrectomy) is undertaken. When the disease is advanced the bones are sawed through. In some joints division of one of the bones entering into the articulation with a saw must be undertaken in order that the joint may be made accessible throughout its whole extent for the effectuation of the necessary operative measures.

\section{Indications :}

1. Injuries, complicated destruction of the constituents of the joint, especially if large portions of bone are completely severed from their attachments.

2. Tuberculosis of joints, if conservative measures (rest and fixation of the joint, treatment with iodoform, bloodstasis, minor local measures) have failed.

3. Deformities of the joints. Orthopedic resections for the correction of severe, otherwise irreparable alterations in form (contractures, ankyloses).

4. Luxations, if irreducible and attended with marked limitation of function.

5. Acute inflammatory processes in bones, osteomyelitis with epiphyseal separation and suppuration of the affected joint.

6. Flail-joints which it is desired to ankylose artificially (arthrodesis).

The incisions are made with short, strong knives, through the soft parts, down to the bone. After division of the capsule this, together with the periosteum, is separated from its attachment to the bone and the latter is 
divided with the saw. The incisions through the soft parts are so arranged that transerese division, especially of muscles, tendons, large nerres and ressels, is so fiar as possible aroided. Iangenbeck's incisions for resection correspond mostly with the longitudinal axis of the extremities. The artienlar capsule is opened as freely as possible in the direction of the cutaneons incision. "The margins of the wound in the capsule being separated widely by means of tenacula, the operator begins, by means of a series of closely approximated incisions with a resection-knife, which is alwars applied rertically upon the bone, to scparate the attachments of the capsinle together with the periosteum. The bones are forced out of the wound and divided by means of the arched saw, the metacarpal saw, or the wire saw, or the chain-saw. The plane of the sawed surface varies with the individual joints.

For the correction of angular contractures wedge-shaped excision of bones is necessary. In place of this, archshaped resection (Helferich) may be employed, in conjunction with which the shortening is slighter.

After resection of the bone has been effected the sawed surfaces are brought in apposition and fixed by means of nails or clamps, or even without these in a bandage. The division of the capsule, of the muscles, and of the skin is elosed by sutures. By the introduction of drainagetubes or of capillary drains escape of possible secretion is provided for.

Resections of the Joints of the Upper Extremity.-Resection of the Shoulder-joint by the Method of Langenbeck.- The patient is placed upon the operatingtable in a sitting posture in such a manner that the shoulder projects somewhat besond the border of the table. The operator stands upon the side of the trunk, with his face directed toward the shoulder. He grasps the upper arm at its middle with the left hand and with the arm hanging naturally he enters the resection-knife held almost vertically into the coraco-acromial trigone 
(Fig. 11). The incision is made in the longitudinal axis of the arm through the deltoid muscle almost to its insertion into the humerus and down to the capsule (Fig. 118). The upper extremity of the incision divides the tense band between the acromion and coracoid processes. After the margins of the wound have been widely separated by hooks the lateral wall of the capsule is exposed. With slight rotation outward of the arm the tuberosities of the

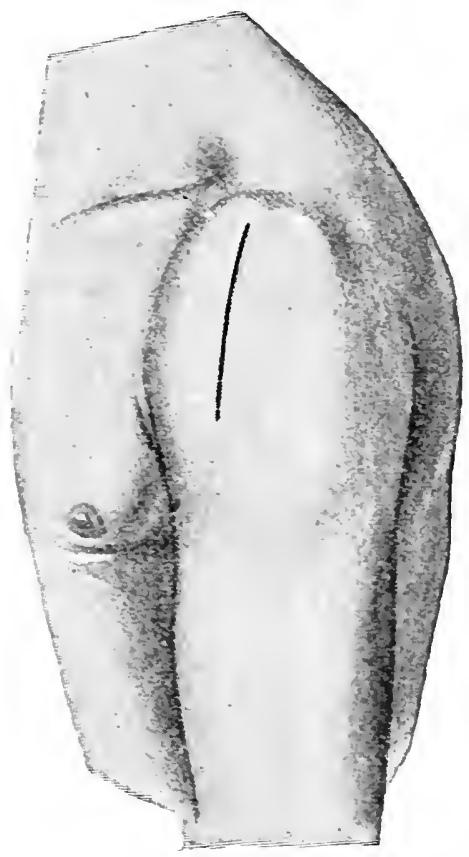

FIG. 11s.-Rescetion of the shoulder: longitudinal incision.

humerus and the bicipital groove are brought to the level of the wound. The capsule is incised and divided upon a grooved director in a line corresponding to this groove upward to the glenoid cavity, and downward to the surgieal neck of the humerus. The tendon of the biceps thus exposed is raised from its bed by means of blunt hooks and displaced inward over the head of the humerus. From the incision in the capsule made to free the tendon 
RESECTIONS AT JOINTS OF THE EXTREMITIES. 195

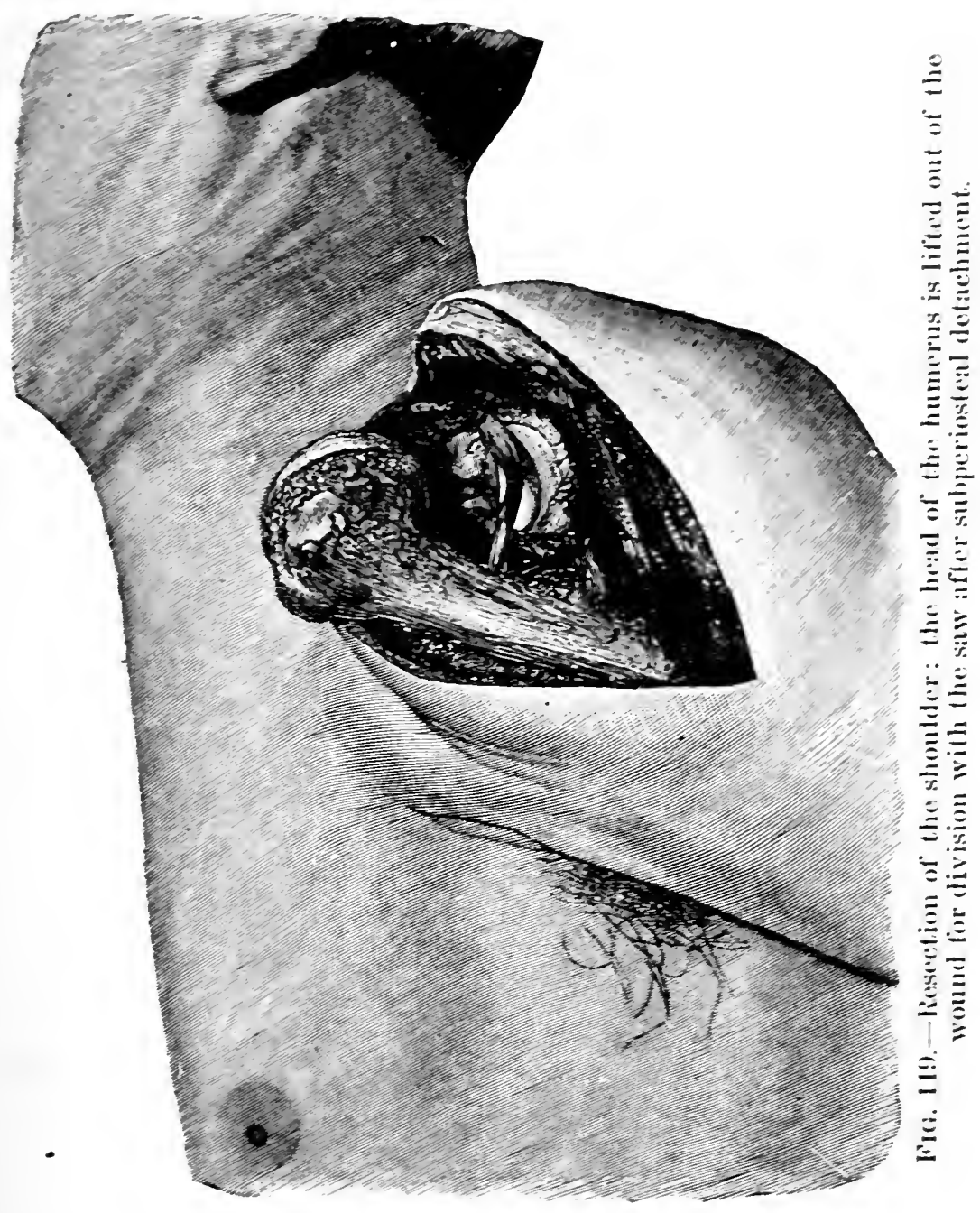





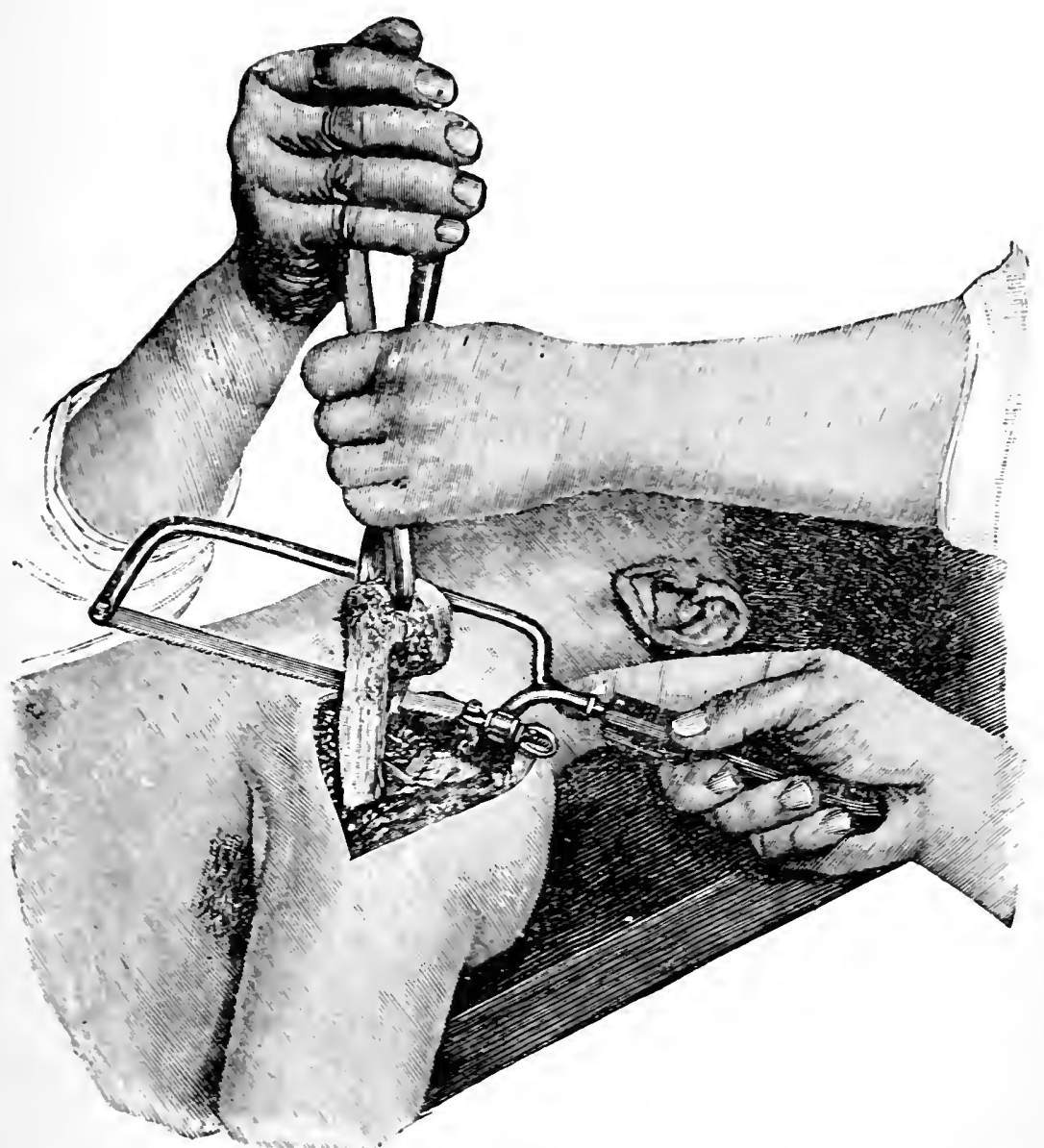

FIG. 120.-Resection of the shoulder: dirision of the head of the humerus with the saw; the head of the bone is fixed by means of Langenbeck's forceps. 

of the biceps the separation of the capsule from the bone is undertaken. With the aid of a hook introduced into the slit in the eapsule the latter is elevated and dissected free from the humerus close to the bone. The operator progresses step by step, while the arm is rotated toward the knife. After the capsule has been divided throughout half of its circumference the remainder is similarly detached from the bone, the operator proceeding from the original slit in the eapsule in the opposite direction. In connection with the eapsule, into whose formation enter fibers of the shonlder-muscles (supraspinatus, infraspinatus, subscapularis), the latter are at the same time detached from the bone at their insertion. When the head of the bone has been thus freed it is lifted out of the wound (Fig. 119), and divided at the level of the surgical neek of the humerus by means of the chain-saw, or, after being fixed with Langenbeck's foreeps, with the arched saw (Fig. 120). The tendon of the biceps muscle is by this procedure preserved intact. After removal of the head of the humerus the glenoid cavity, as well as the whole interior of the eapsule, is sufficiently exposed for whatever further operative procedures may be necessary. Tessels of considerable size are not injured in performing resection of the shoulder through a longitudinal incision.

\section{Resection of the Elbow-joint through a Dorsal Longi-} tudinal Incision.-The arm is flexed at a right angle at the elbow-joint and thrown orer the thorax in such a manner that the extensor aspect of the joint is turned upward. The operator stands upon the side of the thorax that corresponds with the healthy member. The incision is made upon the dorsal aspect of the joint through the lower extremity of the triceps muscle over the olecranon (Fig. 122). Langenbeck makes this longitudinal incision rather nearer the inner border, while Chassaignae makes it upon the outer border of the olecranon, although it may also be made satisfactorily in the middle line (Park). The incision passes through the triceps muscle down to 
the bone. While the lips of the wound in the muscle are energetically separated by means of hooks, the posterior wall of the capsule of the elbow-joint bulges into riew and is divided in the direction of the cutaneous incision. With strokes of the knife directed vertically toward the bone the tendon of the triceps is detached from the olecranon close to the bone. At the same time the muscles

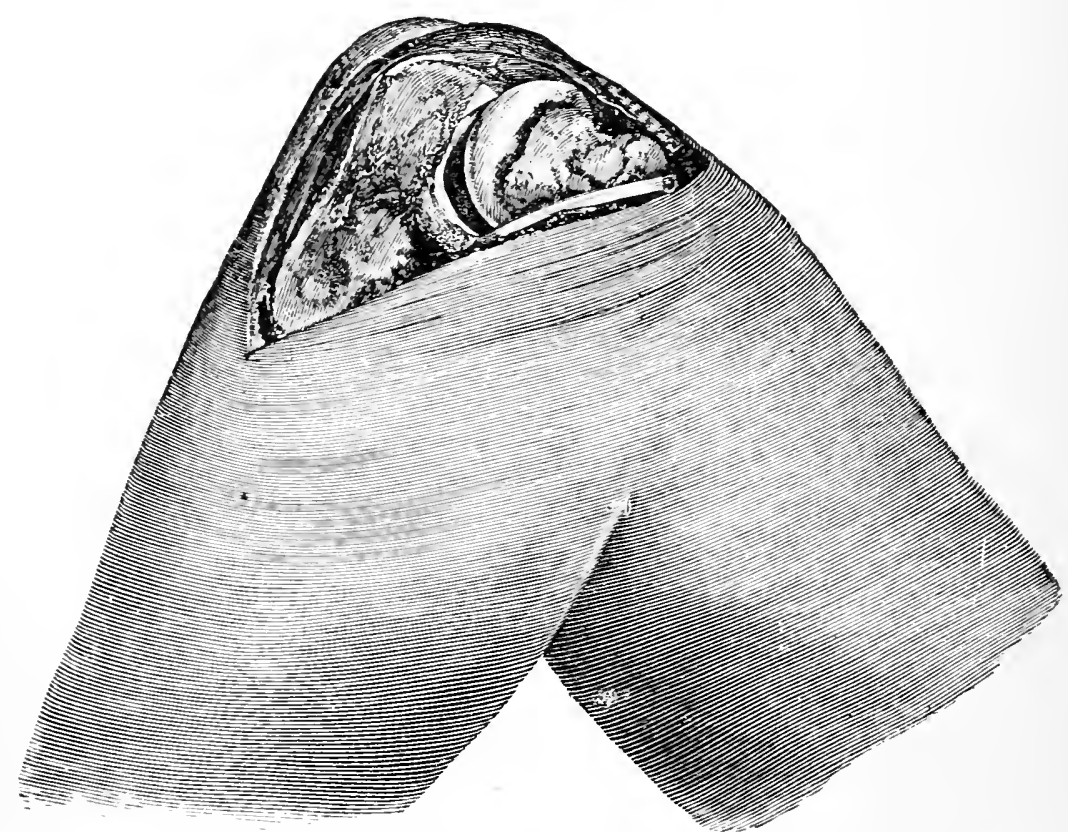

Fig. 121.-Resection of the elbow-joint: exposure of the elbow-joint on its inner aspect; the olecranon and the inner extremity of the trochlea come into view; the ulnar nerve has slipped from the inner epicondyle after retraction of the margin of the wound.

inserted upon the dorsal aspect of the upper extremity of the ulna are detached in conjunction with the periosteum. Upon the outer side the radial head of the humerus and the hear of the radius come into view, and the strong fibrous lateral ligament is to be freed close to the bone. On the inner side the detachment of the tendon of the triceps from the olecranon is begmn. At the same time the muscles are dissected free also from the upper ex- 
RESECTIONS AT JOLNTS OF THE EXTREMITIES. 201

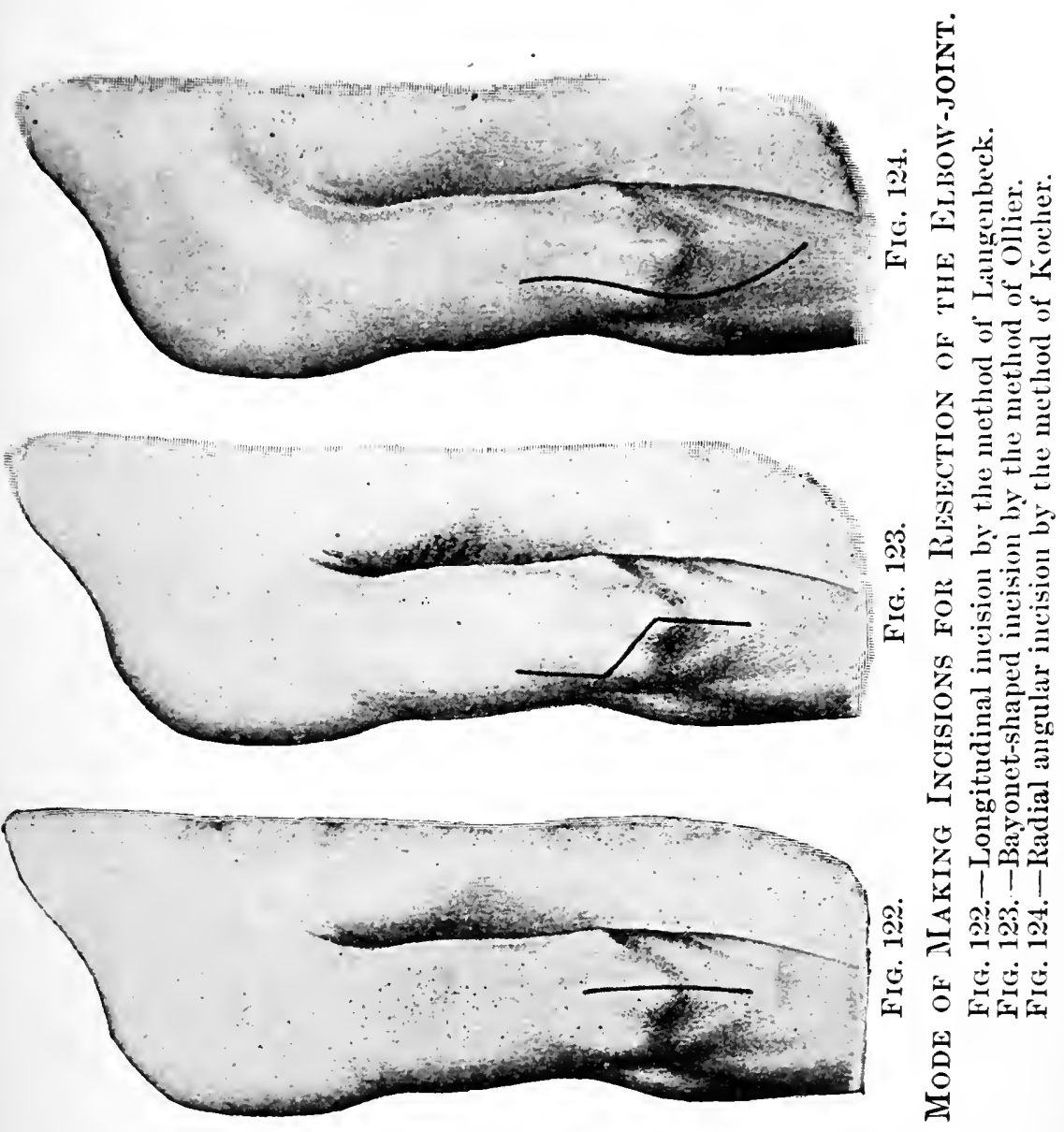



tremity of the ulna. While the free margin of the wound is drawn, through marked flexion at the elbow-joint, inward and toward the flexor aspect, the inner epicondyle of the humerus appears within the field of operation. The muscles attached to this process (pronator radii teres and flexors of the hand and of the fingers) should also be detached close to the bone. In order not to injure the ulnar nerve dislocated from its place on the trochlea by

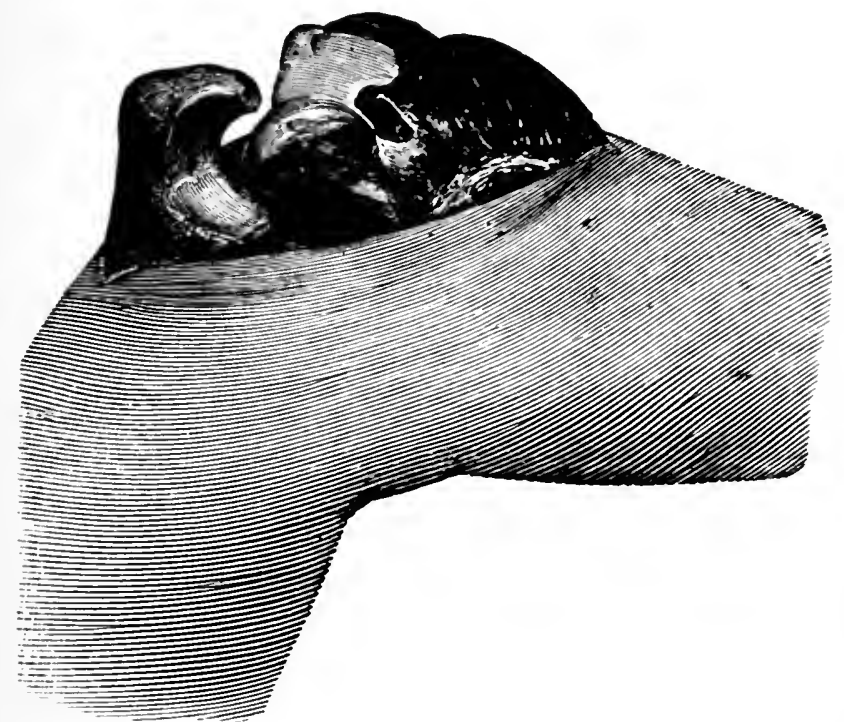

Fig. 125.-Resection of the elbow-joint: the constituent parts of the elbow-joint are completely exposed and pushed out of the longitudinal incision.

the side of the inner epicondyle, the detachment of the museles from the inner epicondyle is carried out in such a manner that the semicireular incisions are made to pass close together from the apex of the epicondyle to the base of this bone (Fig. 121). In this manner the ulnar nerve is kept entirely out of reach of the knife. By further dissection the inner margin of the trochlea and the sigmoid eavity of the ulna are freed. When the separation of the capsule has extended beyond the attachments of the lat- 
eral ligaments the articular extremity of the humerus, as well as those of the bones of the forcarm, can be forced out of the wound (Fig. 125). The bones are grasped with Langenbeck's forceps and sawed through, the humerus above the trochlea, the hones of the forearm simultaneously upon the distal side of the head of the radius and the coronoid process.

In describing the steps of the operation its performance upon the dead subject has been kept in view. In actual clinical experience the mode of procedure is subjected to

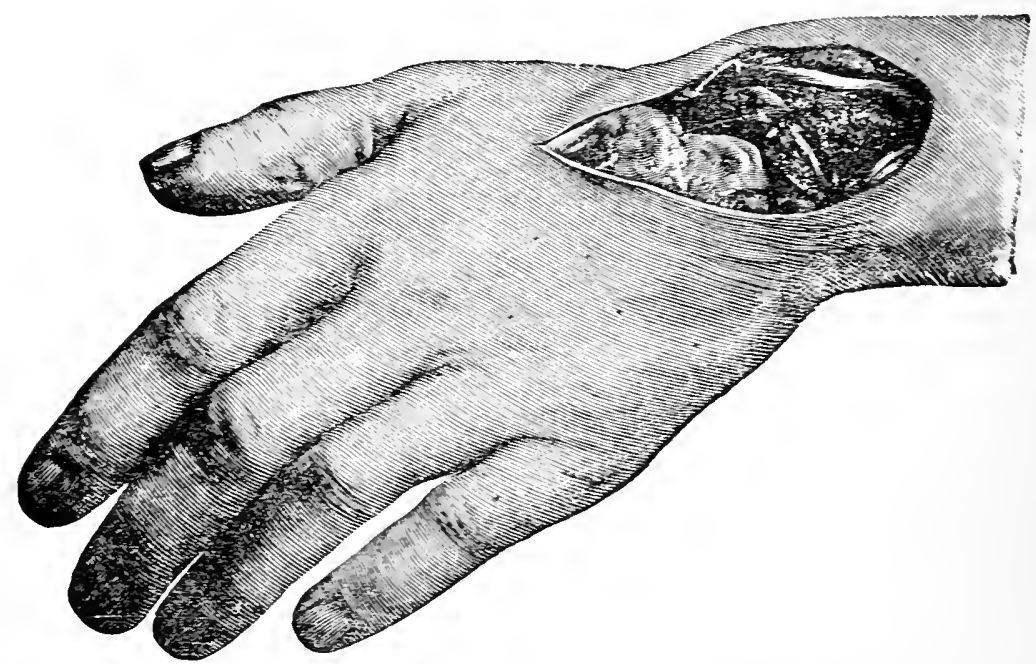

FIG. 126.- Resection of the wrist-joint: dorsoradial incision ; the extensor tendons are displaced to one side; the lower extremity of the radius and the two rows of carpal bones are exposed to riew.

certain modifications. Thus, the disarticulation and the isolated division of the bones with the saw may be omitted in the treatment of cases of ankylosis of the joint.

Other Varieties of Resection of the Elbow-joint.-A longitudiual incision is made on either side of the joint (Vogt, Hneter); or an $\mathrm{H}$-shaped incision is made (Moreau), the transverse portion dividing the tendon of the triceps above its attachment; or a transverse incision is made upon the extensor aspect of the joint over the olecranon process, with temporary transverse division of the olecranon with a saw (Szymanowsky, Bruns); or a baronet-incision is made (Ollier) (Fig. 123). 
Kocher's angular incision passes from the onter aspect of the lower extremity of the humerus, pialled with the axis of the humerus, to the laead of the radius. From this point it contiunes along the outer border of the anconeus muscle to the horder of the ulua from 4 to di com. below the apex of the olecranon (Fig. 1:2). 'The olecranon is broken off with the ehisel and removed. or the muscles are sepanatid from that process. Kocher saws through the bones in an arched manner.

Resection of the Wrist-joint.-Dorsoiradial Incision by Langenbeck's. Method.-A cutaneous incision is made upon

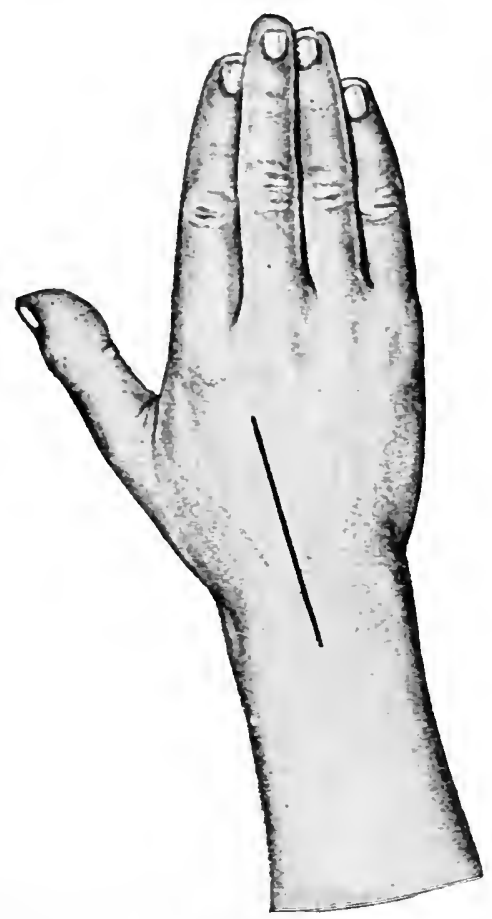

FiG. 127.-Resection of the wrist-joint: Langenbeck's dorsoradial incision.

the dorsal aspect from the middle of the ulnar border of the metacarpal bone of the index-finger to the middle of the lower extremity of the radius (Fig. 127). The incision passes between the tendons of the extensor digitorum communis and the extensor hallueis longus, and divides the dorsal transverse ligament of the carpus. The attach- 
ments of the radial extensor muscles are separated from the base of the metacarpal bones and the radiocarpal joint is opened. By means of a knife or a sharp raspatory the capsule of the joint is freed from the dorsal aspect of the bones at the root of the hand, the individual small joints opened, and the bones extirpated singly (Fig. 126).

The dorso-ulnar incision (Lister) for resection of the wrist-joint is made, with slight radial flexion of the hand, from the middle of the fifth metacarpal bone, over the middle of the wrist-joint, beyond the radial epiphysis. The dorsal transverse ligament of the carpus is divided, when, by the side of the extensor tendons of the finger retracted toward the radial aspect, access to the joint is gained. At the base of the fifth metacarpal bone the tendon of the extensor carpi nlnaris must be detached from the bone. Now the attachment of the capsule may be freed, beginning at the ulna, when the carpal bones are exposed and removed individually. If it be necessary to remove also the epiphysis of the radius, this is forced out of the wound, grasped by its styloid process with Langenbeck's forceps, and divided transversely with the saw.

Another method of resection of the wrist-joint consists in making a longitudinal incision on either side, or an $\mathrm{H}$-shaped incision, according to Gritty. The transverse incision upon the dorsum of the hand severs the tendons.

Resection of the Fingers.-Resection at the metacarpophalangeal joints of the thumb, the index and the little fingers, as well as at the interphalangeal joints, is effected through a lateral incision, thus completely protecting the extensor as well as the flexor tendons. The incision opens the joint laterally. The capsular attachments are separated upon the dorsal and palmar aspects of the bone, and the articular extremities of the bones are forced out of the wound and divided with the saw. After removal of the bones the synovial surface of the joint is completely exposed. The metacarpophalangeal joint of the thumb becomes accessible only after separation of the muscles of the thenar eminence. The metacarpophalangeal joints of 
the middle and ring-fingers are exposed by means of dorsal longitudinal incisions, the opening of the joint and the removil of the articular extremities being effected in the typieal manner. Resection of an entire metacarpal bone is effected through a dorsal longitudinal incision extending from the base to the head of the bone, which is exposed by subperiosteal detachment of the interosseous museles on either side, and is raised from its surroundings: after the joint at its base and that at it: heald have been opened (Fig. 104). The resection of entire phalanges is effected through lateral longritudinal incisions.

Resection of the Joints of the Lower Extremities.-Resection of the Hip-joint.-External Longitudinal Incision by the Wethor of Langenbeck.-The patient to be operated upon lies upon the unaffected side. The diseased extremity is flexed at an obtuse angle and held in a position of slight adduction. The incision passes from the posterior superior iliac spine parallel with the long axis of the extremity over the greater trochanter, and continues at once throngh the gluteal muscles down to the iliae bone and the articular capsule. The tendons inserted into the trochanter are detached close to the bone on both sides, the capsule is divided, and the articular cartilage at the margin of the acetabulum incised. The knife is introduced into the opening into the joint and the round ligament divided while the member is held in a position of forced flexion, adduction, and internal rotation. In this way the head of the femur is made to lie upon the iliac bone. The chain-saw is passed around the neck of the femur and the head of the bone is thus divided.

External Arched Incision by the Method of Velpeau.The patient occupies the same position as in Langenbeck's operation. The resection-knife is introduced at a point midway between the anterior superior iliac spine and the apex of the trochanter, vertically, down to the concarity of the ilium. The incision surrounds the anterior threefourth of the periphery of the trochanter and at all points extends down to the bone (Fig. 128). Care should be 
taken that the ghteal muscles are divided in a vertical direction. If the margins of the wound are separated at its depth, the fibrous capsule of the joint becomes visible. Over the highest prominence of the head of the femur the capsule is divided by an arched incision corresponding with the cutancous incision. The articular cartilage is incised and after division of the ligamentum teres the head of the femur is luxated upon the ilium and removed.

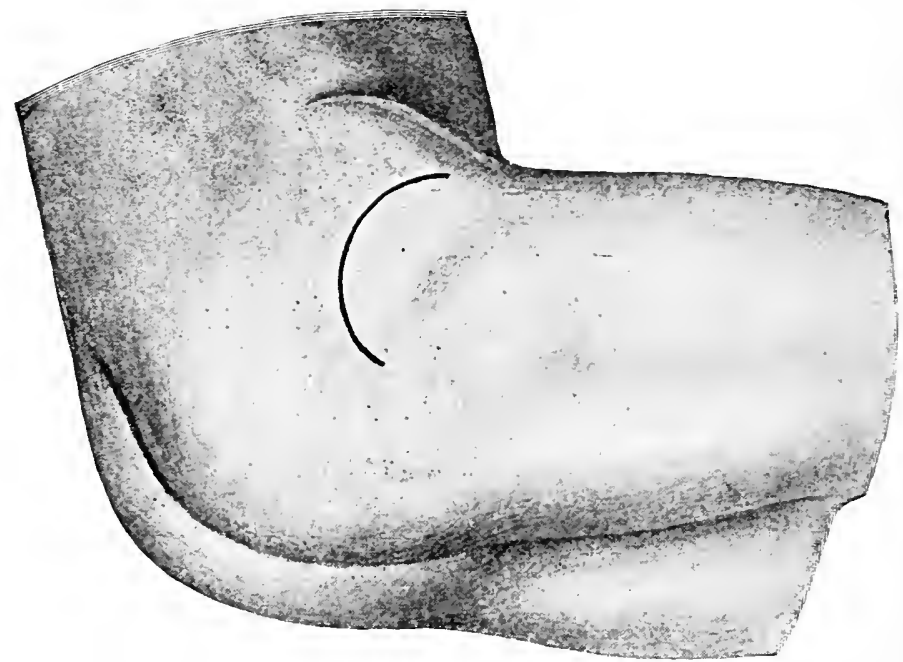

FrG. 128.-Resection of the hip: external arched incision by the method of Velpeau.

If the removal of the head is to be effected at a higher point between the trochanters, or on the shaft of the femur, the tendons inserted into the trochanters must be detached from the bone with the knife. After the head of the bone has been sawed off the acetabulum is exposed for snch further surgical interference as may be neeessary. The extremity is placed in an extended position and a drainage-tube is passed into the depths of the acetabulum.

König has modified Langenbeck's operation hy renoving with a chisel the hearl of the femur $m$ situ before it is luxated. Further, the attachments of the muscles to the greater trochanter are not separated from the bone, but are removed, in conjunction with the cortical structure of 
the trochanter upon its anterior and posterior sides, with chisel and mallet.

Lïcke's and Sehede's anterior longitudinal incision passes downward from the anterior superion iliae spine. The joint is entered to the outer side of the erural nerve.

The anterior transverse incision of Roser is attended with the disadvantage that the fibers of numerous museles are divided transversely.

Resection of the Knee-joint through an Anterior Transverse Incision.-The operator grasps the leg of the ex-

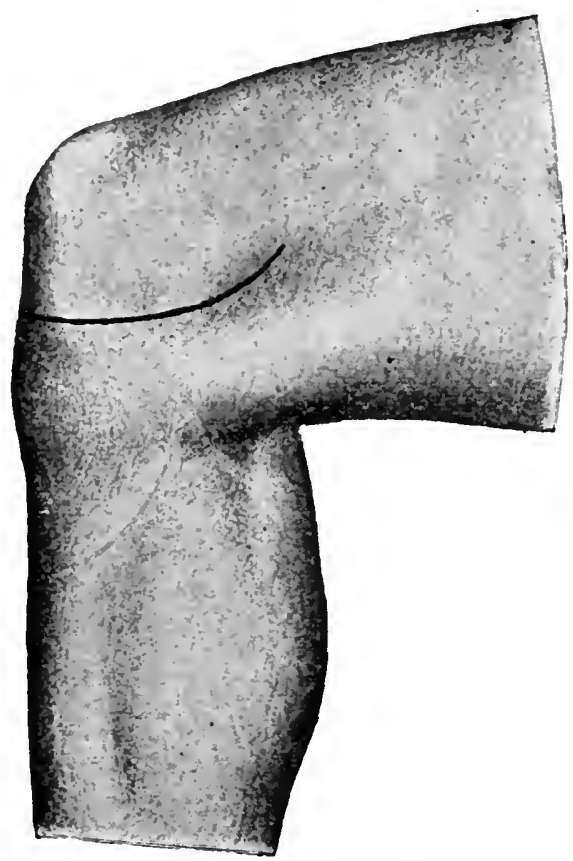

Fig. 129.-Resection of the knee-joint: anterior arched incision by the method of Textor.

tremity flexed at the knee-joint and unites the most prominent points on the lateral aspects of the condyles of the femur by an anterior arched incision passing from left to right and dividing the patellar ligament (Textor, Fig. 129). The incision enters the joint, which is opened adequately upon its anterior aspect.(Fig. 130). The thumb of the left hand is passed into the articular interval between the patella and the femur, and separates the at- 
tachments of the capsule laterally to the condyles of the femur, when by reflecting the patella the sacculated diverticulum of the capsule is visible from above. The crucial ligaments and the accessory lateral ligaments are now divider. The lower extremity of the femur is thus exposed and, after the periosteum has been incised cir-

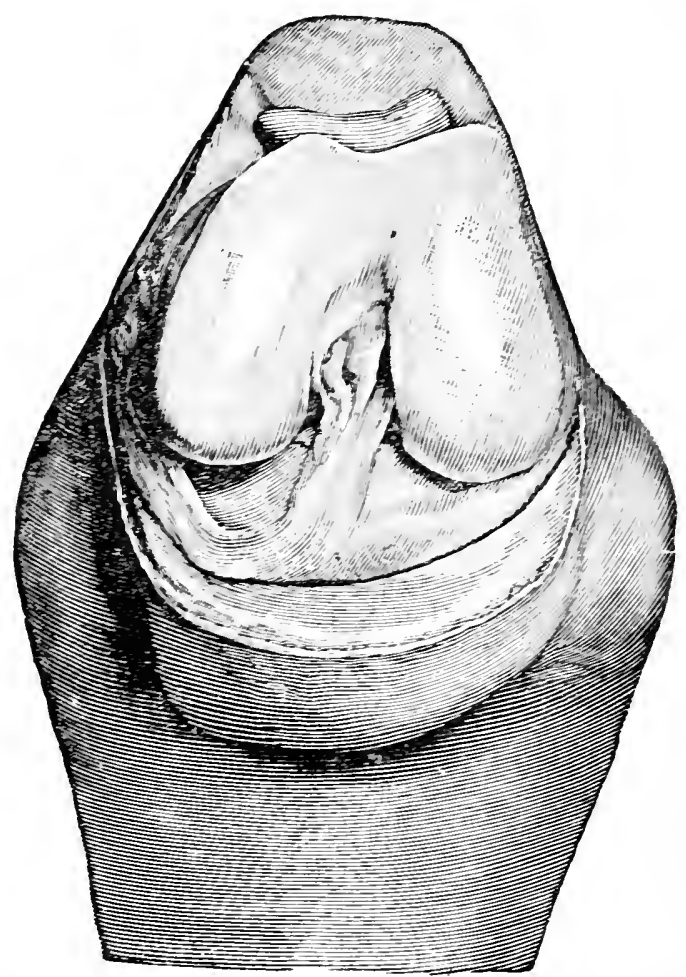

FIG. 130.-Knee-joint opened through the anterior arched incision.

cularly above the condrle;, the bone is fixed with Langenbeck's forceps and divided transrersely. König makes the sawed surfaces in such a plane that the joint is placed in a position of slight flexion. If it be necessary to remove also the apper articular surface of the tibia, this is brought out of the wound, surrounded by a circular incision, and removed in the form of a plate. In sawing 
the bone it is grasped with the foreeps at the intercondyloid eminence and fixed. If the patella is to be removed, it is surrounded by an incision and fieed with strokes of the knife passed close to the bone. The sawed surfaces of the bones are approximated and fixed in apposition by means of sutures, clamps, or pegs.

According to the method of Folkmann, the anterior transverse incision passes from one condyle to the other over the middle of the patella. The periosteum is incised transversely and the patella is sawed through on a line with the cutaneous incision. In the prolongation of the incision through the patella the capsule is incised to the right and the left and the joint is widely opened. The knee is now strongly flexed at an acute angle, so that the incision into the joint is made to gape widely and the lateral ligaments, as well as the lateral attachments of the capsule, are divided. The crucial ligaments are divided from behind forward. The upper segment of the patella is reflected by traction, and the interior of the capsule becomes accessible. The lower extremity of the femur is surrounded by a circular incision, grasped with forceps at the inner condyle, and divided transversely with a saw.

In Hahu's modification the transverse incision passes above the patella (Fig. 131).

Kocher recommends a lateral hooked incision for arthrotomy and resection of the knee-joint. The incision begins at the vastus externus muscle, a hand's breadth above the patella, two fingers' breadth from whose outer border it passes downward, to terminate upon the inner aspect of the tibia below its spine. The articular capsule is divided on its outer aspect and the patellar ligament removed out of the way by separation of the spine of the tibia. The patella with its ligament is made so movable that it can be reflected inward. Division of the crucial ligaments permits a satisfactory view of the joint when placed in a position of flexion. Adequate access to the joint is had also for the performance of resection of the articular elements.

Resection of the Ankle-joint.-Bilateral Longitudinal Incision after Langenbeck.-The longitudinal incisions begin on either side, a hand's breadth above the malleoli, and pass along the tibia and fibula beyond their lower extremities. The incisions pass through skin and periosteum. 
With the foot lying upon its inner border, the fibula is dissected free beneath the periosteum with a knife or a raspatory on its outer and inner aspects, and is divided in a linear clirection above the malleoli with chisel and mallet or with the chain-saw. The peripheral fragment of the bone is reflected outward and separated from its attachments. In an analogous manner the lower extremity of the tibia is excised through the internal longitudinal in-

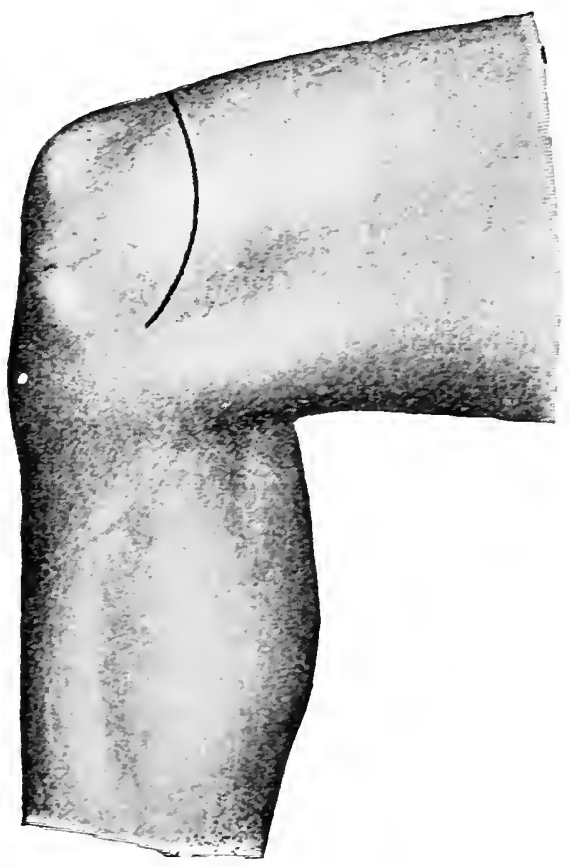

FIG. 131.-Hahn's suprapatellar incision for resection of the knee-joint.

cision. By the removal of the malleoli a view can be had of the interior of the joint, and the trochlear surface of the astragalus, as well as the walls of the capsule, is rendered accessible for further operative procedures.

Langenbeck has obtained the most admirable results with this conservative method of resection following gunshot-injuries of the ankle-joint, and especially transverse 
wounds with destruetion of both malleoli. The operation is less well adapted for the modern procedure of arthreetomy, as the lower extremities of the fibula and tibia are sacrifieed, at any rate, and, besides, the opportunity for inspection of the joint afforded through the incisions is not adequate to meet the needs of extirpation of the capsule.

König's Bilateral Longitudinal Incision.-The incision on the inner aspeet begins 3 or $4 \mathrm{~cm}$. above the level of

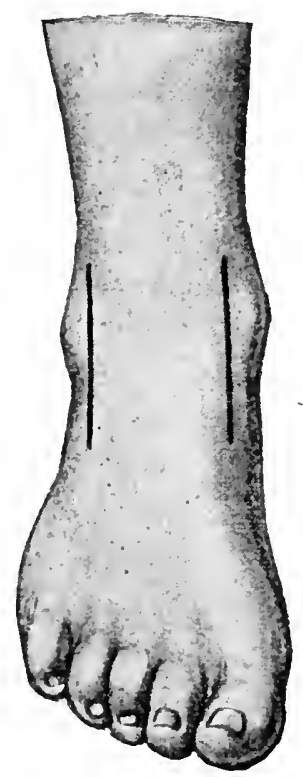

FIG. 132.-Resection of the ankle-joint by König's bilaterai longitudinal incision.

the articulation upon the tibia, somewhat internal to the extensor tendons, and it opens the joint close to the anterior boundary of the inner malleolus. It passes over the body and neek of the astragalus, to terminate at the inner border of the foot at a point corresponding with the tuberosity of the seaphoid bone. The outer ineision runs parallel with the inner, along the anterior surface of the 
tibia, opens the joint at the malleolus, and terminates at the level of the astragaloscaphoid articulation (Fig. 132). The anterior bridge of skin, which contains the extensor tendons, the ressels, and the nerves, is dissected from the subjacent tissues, the insertion of the capsule being at the same time detached transversely from the trochlea of the astragalus and the border of the tibia, and if necessary the anterior portion of the srnovial membrane is excised. By lifting up the bridge-like flap, with dorsal flexion of the foot, the individual portions of the joint may be made accessible to the eye and to instrumental manipulation. The removal of the astragalus is readily effected through the inner incision, when the articular surface of the tibia and the posterior wall of the capsule become visible.

Reverdin-Kocher Method of Luxation through an External Transverse Arched Incision.-The incision begins at the tendo Achillis about a hand's breadth above the malleolus, passing downward, surrounding the external malleolus, and terminates on the outer border of the foot along the outer margin of the extensor tendons (Fig. 133). After division of the skin and exposure of the external malleolus the accessory ligaments of the capsule inserted in this situation are divided. The attachment of the capsule is freed, with displacement of the extensor tendons, and, if necessary, division of the peroneal tendons upon the anterior and posterior aspects of the tibia, when the foot is flexed upward in such a manner over the internal malleolus that its inner border is brought in contact with the inner aspect of the tibia (Figs. 134 and 135). The joint is thus made accessible to inspection, and necessary operative procedures upon the articular extremities, as well as upon the capsule, may be undertaken.

Resection of the Foot by the Method of Wladimiroff and Mikulicz.-Indications :

(1) Caries of the foot localized in the calcaneum, the astragalus, and the astragalocrural articulation.

(2) Extensive loss of substance about the heel.

(3) Injuries of the heel, especially gunshot-wounds. 


\section{RESECTIONS AT JOINTS OF THE EXTREMITIES. 215}

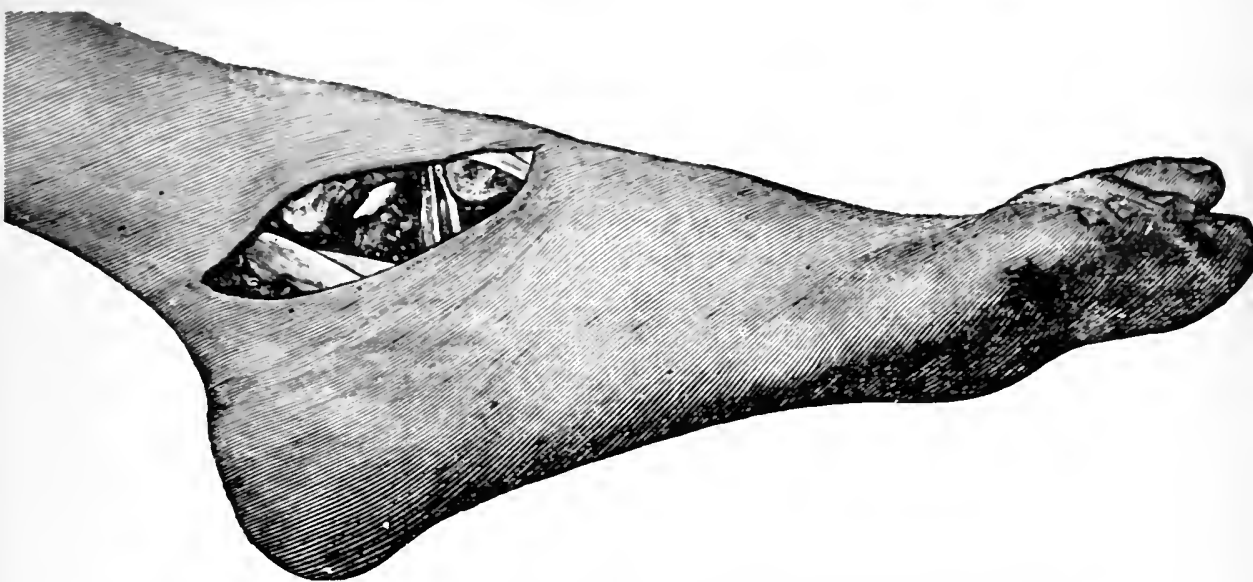

FIG. 133.-Resection of the ankle-joint by the method of ReverdinKocher: cutaneous incision; exposure of the ankle-joint from its outer aspect.

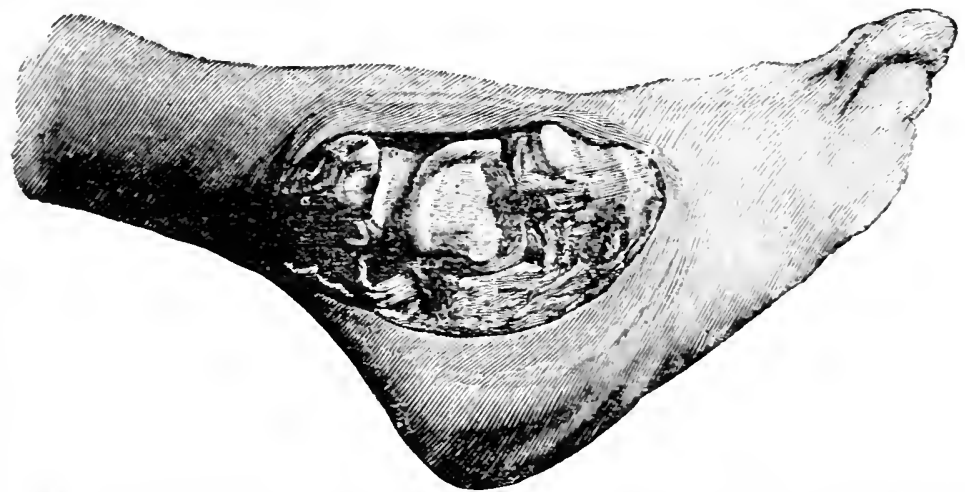

Frg. 134.-First stage of rotation of the font at the ankle-joint about the inner malleolus.

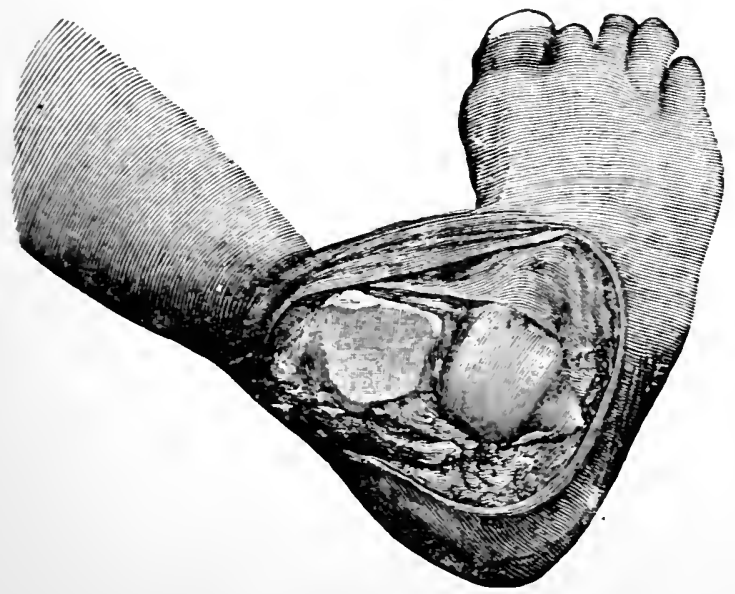

Fir. 135.-Completed rotation: the lower extremities of the tihia and the fibula, as well as the trochlea of the astragalus, are completely exposed. 
.

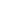


(4) Malignant tumor's about the heel (osteosarcoma, melanosarcoma) (Bruns).

(5) Shortening of the extremity, following luxations of the hip-joint (Caselli); after resections of the knee-joint (Rydygier).

(6) Paralytic club-foot (Bruns).

The parts removed in the resection include the lower extremities of the tibia and the fibula, the astragalus, the calcaneum, and a portion of the cuboid and scaphoid bones in conjunction with the skin of the heel. The anterior portion of the foot is maintained in relation with the leg by means of a dorsal bridge containing the tendons and ressels.

Mode of Procedure.-A transverse incision is made through the sole of the foot corresponding to the extremities of Lisfrane's line, and a second transverse incision is made transversely above the malleoli on the posterior aspect of the leg; the extremities of both being united by additional lateral incisions (Fig. 136). 'The astragalocrural joint is opened from the dorsal aspect and stretched widely, and the bones of the leg are divided transversely above the malleoli. The root of the foot is grasped at the trochlea of the astragalus, and it is freed close to the bone from the dorsal soft parts, with maximum dorsal flexion of the foot. In accordance with the extent of tissue to be removed the tarsus is sawed through in the region of the cuboid and scaphoid bones, or further to the distal (at the base of the metatarsal bones) or to the proximal side. When the operation is performed for orthopedic reasons, only the lower extremities of the tibia and the fibula and the tuberosity of the calcaneum are removed, together with the trochlea of the astragalus. Inasmuch as after the resection has been effected the sawed surfaces of the bones of the leg and the tarsus are approximated and united by bone-suture, there results an artificial club-foot to such a degree that the dorsum of the foot lies in the same plane as the anterior aspect of the leg (Figs. 137 and 138). 
In performing tibiocalcaneal resection by the method of Bruns, the ankle-joint is opened through an arched dorsal incision, the astragalus freed, and the lower extremities of the tibia and the fibula, as well as the upper surface of the calcaneum, sawed through transversely, when the sawed surfaces are nailed together.

Osteotomy.-Osteotomy consists in linear division by bloody means of the long bones. Originally performed through an open wound, the operation has since the time of Langenbeck been performed, like tenotomy, through a small incision in the skin, in a measure subcutaneously. The division of the bone is effected with the aid of sculptor's chisels.

The extremity is placed upon a board or upon a sandbag, the Esmarch apparatus is applied, and the operation of osteotomy is undertaken. A short incision through the soft structures penetrates down to the bone. With slight blows of the mallet the chisel is driven into the bone. After it has penetrated it is removed, and a similar process is gone through in a neighboring situation. In this manner the cortical structure of the bone is successively divided transversely throughout almost its entire circumference. The remainder is fractured by forcible bending, and the extremity, after the cutaneous wound has been properly united, is fixed in an appropriate position in a plaster-of-Paris dressing.

In cases in which simple linear osteotomy will no longer suffice, wedge-shaped excisions are undertaken for the correction of ankylosis, or curvatures of high degree. The base of the wedge corresponds always with the convexity of the currature to be corrected. After adequate exposure of the bone and division and detachment of the periosteum, the wedge is removed with the chisel or the saw, when the correction of the deformity may be readily effected.

A special form of osteotomy employed for the correction of marked arcuate curvature of the long bones consists in longitudinal division, the bone being divided in a direction parallel with its long axis. The displacement 
Resection of the Foot by the Method of WhadimoffMiKulicz.

FIG. 136.

Cutaneous incisions.

Fig. 137.

Configuration of the foot after resection has been effected. The sawed surfaces of the bones of the leg, as well as those of the cuboid and scaphoid bones, are exposed.

FIg. 138.

Appearance of the stump.

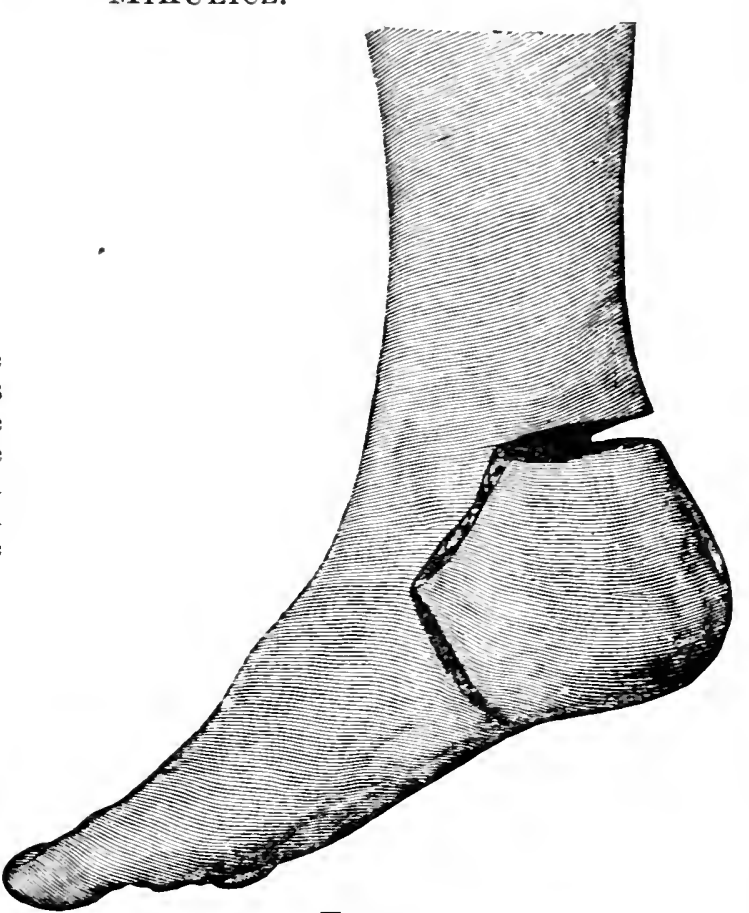

FIG. 136.

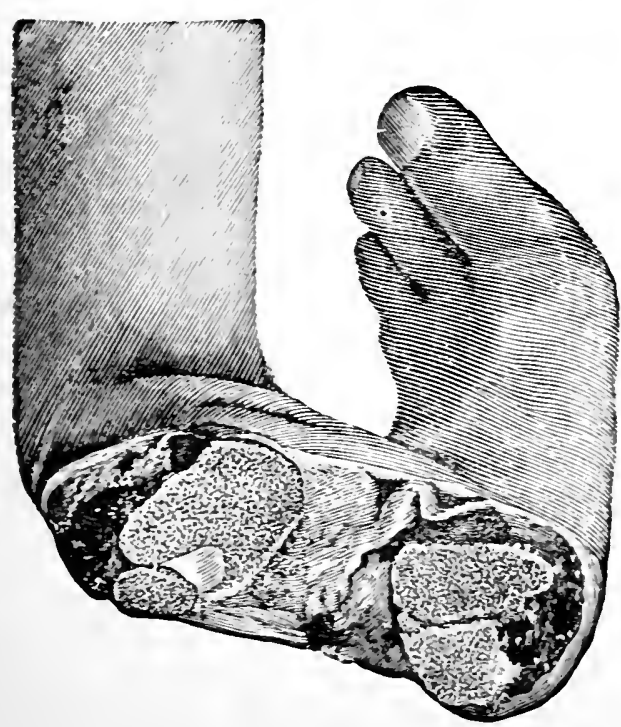

FIG. 137.

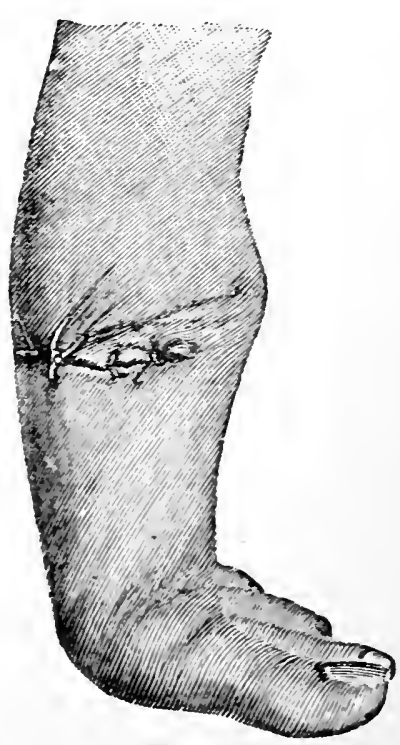

FIG. 138. 
of the segments of bone in the axis of the part renders possible to a certain degree correction of deformity.

Osteotomy of the femur at the upper extremity of the bone is undertaken in cases of contracture of the thigh, if the correction of the faulty position is attended with difficulty after division of the contractured soft parts. Linear osteotomy under these circumstances is undertaken either at the neck of the femur (osteotomia colli femoris), or at a point between the two trochanters (osteotomia intertrochanterica). For the exposure of the upper extremity of the femur a longitudinal incision over the trochanter is made upon the postero-external aspect of the joint. In this situation the neck of the femur can be exposed for osteotomy, as well as a decper portion of the bone after separation of the muscular attachments to the greater trochanter.

Supracondylar osteotomy of the femur has been recommended by Macewen as a routine procedure in the treatment of genu valgum. According to Macewen's recommendation, the short cutaneous incision on the inner aspect of the lower extremity of the femur is made at a point corresponding to the intersection of two lines, of which one passes a finger's breadth above the upper border of the external condyle, and the other in the longitudinal axis of the bone two fingers' breadth in advance of the tendon of the adductor magnus. At the point of intersection of these two lines a short longitudinal incision is made down to the bone, the chisel introduced through the wound, applied transversely, and the cortical structure of the bone successively divided throughout two-thirds of its circumference. The remainder of the bone is severed by manual means. Supracondylar osteotony of the femur may also be undertaken from the outer side of the bone in a corresponding situation.

Linear osteotomy, as well as excision of wedge-shaped portions, may be undertaken upon the bones of the leg for the correction of deformities at the knee-joint, or of excessive curvature. The tibia is exposed at its upper 
extremity, from 4 to $6 \mathrm{~cm}$. below the articular line, by means of a transverse incision around the inner circumference of the bone (Kocher). After detachment of the periosteum the bone is divided with the chisel in the direction of the cutaneous incision.

Excision of a wedge-shaped portion of the tibia may also be effected through the same incision. 


\section{OPERATIONS ON THE HEAD AND NECK.}

Trephining.-Trephining consists in resection of the bones of the skull in their continuity. The term is applied equally to the excision of small circular segments and the establishment of a penetrating defect in the skull, to the temporary removal of a portion of the bone in conjunction with the periosteum and the skin, as well as to the removal of loose depressed splinters, the elevation of the indented calvarium, and the correction of irregularities in wounds following injuries of the skull.

\section{Indications :}

(1) Injuries.-Open, or subcutaneous fractures of the skull ; if the bones exhibit depression ; if local or general symptoms referable to the brain are present (extravasation of blood in cases in which the middle meningeal artery is injured).

(2) Tumors of the cranial bones, of the dura, and of the brain.

(3) Cerebral abscess.

(4) Epilepsy (for the extirpation of cortical centers or for the removal of cicatrices and foreign bodies).

(5) Caries and necrosis of the cranial bones.

Finally, trephining has been recommended for the relief of chronic increase of intracranial pressure, and in cases of progressive paralysis of the insane. ${ }^{1}$

In general, the operation is performed in such a way that after division of the scalp, the aponeurosis of the occipitofrontal muscle, and the pericranium, a suitable segment of bone is removed with the crown of the trephine, the chisel and mallet, or the circular saw. The exposed dura is either opened with a crucial incision or is reflected back as a flap, and after the operation has been

${ }^{1}$ In insanity of traumatic origin, in which the seat of initial trouble is made manifest by a scar, a persistent headache, or muscular phenomena of a local character, it may be proper to trephine. The operation is, however, rarely justifiable in insanity, and will not often be productive of benefit.-ED. 
finished it is closed with catgut-sutures. The deficiency in the bone established either remains open or it is closed. Under the condition first named, the skin being utilized to cover the defect in the bone, the opening becomes closed by connective tissue, a result that is attended with certain disadvantages. It has therefore become the rule, whenever the nature of the case renders it permissible, to close the trephine-opening by means of bone. This may be effected:

1. By reimplantation of the piece of bone trephined;

2. By autoplasty or heteroplasty ;

3. By temporary resection of the cranial bones, employed from the outset as a substitute for typical trephining.

The restored button of bone should at the present time, under aseptic conditions, heal in place in all cases; but reimplantation has been successfully undertaken by $\mathrm{Ph}$. v. Walther.

Autoplasty, an ingenious procedure devised by König, consists in the transplantation upon the defect of a pedunculated flap consisting of shin, periosteum, and a portion of the cortical structure separated with a chisel. The defect resulting from the formation of the flap is covered with a pedunculated cutaneous flap removed from the adjacent region.

Covering in the defect in the bone with foreign bodiesmetallic plates, bone, celluloid plates-is designated heteroplasty.

Steps of the Operation of Trephining.-A linear,' semicircular, or crucial cutaneous incision is made down to the bone. ${ }^{2}$ The periosteum is removed with a raspatory. If the removal of the bone is to be effected with a circular saw, or with mallet and chisel, the extent of tissue to be

1 The observations of Barker indicate that after a piece of liring bone has been transplanted it undergoes anemic necrosis. New, living tissue takes its place, but the transplanted piece does not live. In fact, it seems probable that dead bone is as valuable in filling a defect as is living bone.-ED.

${ }^{2}$ In most cases, a U-shaped flap, the base of which is the dura, gives the best exposure and is followed by the most rapid union.-ED. 
TEMPORARY RHEGTHON OF THE S̈KILL.

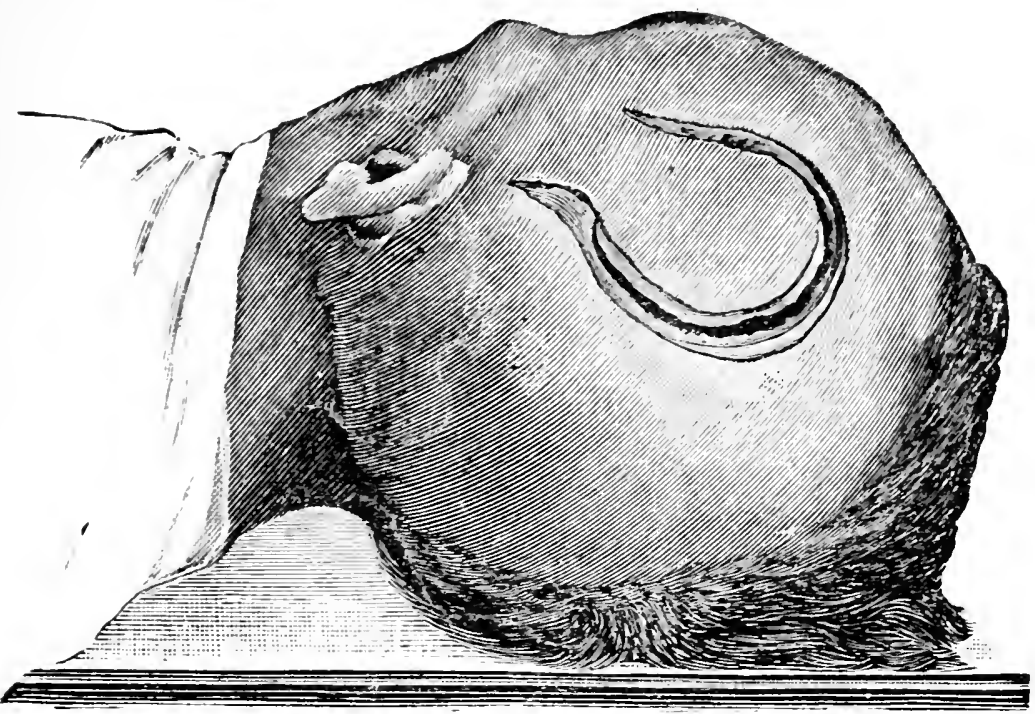

Fig. 139.-Form of the cutaneous flap: the portion of bone to be removed hats been outlined with the chisel.

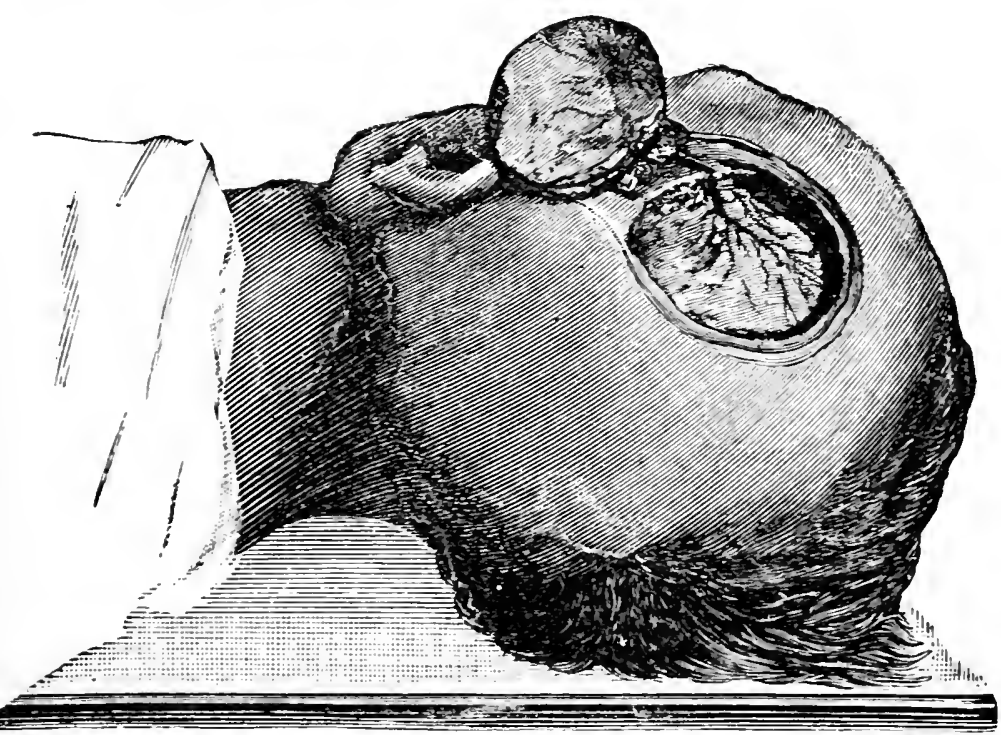

Fig. 140.-The flap of bone, in conjunction with the skin, has been reflected and the dura is exposed. 

removed is first outlined, and the incision through the bone is deepened equally at all parts. In the groove thus made the operator ean determine with the aid of the probe when the vitreous plate has been passed. As soon as the plate of bone is freed throughout its circumference it is raised with an elevator and removed from its place. The circular saw and the chisel mar be adrantageously used together, the boundary of the part to be renoved being outlined with the saw, and the groove being deepened down to the dura with the chisel.

By means of a trephine a button of bone is removed from the skull as large as the opening in the crown of the instrument. The erown is evenly and firmly applied upon the bone with its teeth while the head is fixed. After the teeth of the instrument have entered the bone the pressure and the rotation are continued in eren, though slighter, degree. The groove made by the saw is frequently cleaned and examined as to its deptli. As som as the fragment of bone is loosened it is grasped with the tirefond, a gimlet-like instrument, and is removed. ${ }^{1} \mathrm{By}$ means of a special knife, known as the lenticular, it was customary in the past to smooth the margins of the opening. The mode of procedure does not follow this typical course in cases of fracture of the skull. Completely separated splinters that have been forced into the brain are to be removed, depressed portions of bone are to be raised, and sharp margins are to be cut off, etc. For elevating and removing fragments of bone rongeur-forceps are employed ; for enlarging fissures in bones the chisel and mallet are employed exclusively.

Temporary resection of the skull (Wagner, Wolff, Ollier) has of late almost entirely replaced the classic mode of trephining. Wagner incises the skin in the shape of a lyre or of an omega ( $Q$-shaped) (Fig. 139), the incision passing down to the bone at all points. A furrow is cut

1 Instead of employing a special instrument to lift out the button, the bit of bone can be forced out by means of a periosteum-elevator or a blunt dissector used as a lerer.-ED. 
into the bone with the circular saw corresponding with the cutaneous incision, and the groove is gradually deepened by means of chisel and mallet until the dura is reached. At the base, corresponding with the narrowest portion of the flap, the bone is diviled with a single stroke upon the chisel, when the flap of integument, periosteum, and bone can be reflected (Fig. 140). After the intracranial manipulation has been completed (opening of an abscess, resection of a cortical center, removal of a foreign borly, ligation of the middle meningeal artery, etc.), the bone is replaced in the artificial opening and the cutaneous wound is closed br suture.

To facilitate the localization of the anterior and posterior hranches of the middle meningeal artery steiner has suggested the following anatomic guides: a line is drawn from the middle of the glabella to the apex of the mastoid process. Upon the middle of this line another, vertical line is erected. Where the latter intersects a third line passing horizontally throngh the glabellat the crown of the trephine is applied, and on removal of the button of bone the trunk of the anterior branch of the middle meningeal artery will be reached (Fig. 141).

At the point where a vertical line passing in front of the mastoid process intersects the horizontal line alreadr spoken of a trephine-opening will reach the posterior branch of the middle meningeal artery.

Since the introduction of temporarr resection of the skull br means of the chisel, the making of a number of isolated trephine-openings for the exposure of the two branches of the midrle meuingeal artery is obviated. Br the formation of a flap of suitable size, with its base above the malar bone (Krause's flap for intracranial exposure of the Gasserian gauglion), it has become possible to expose the branches of the middle meningeal artery throughout a sufficient extent (Fig. 141). The length and width of the flap spoken of are about $6 \mathrm{~cm}$.; the former measured from the zygomatic process, the latter a thumb's breadth external to the margin of the orbit.

The upper extremity of the Rolandic fissure lies in an anteroposterior plane, $1.2 \mathrm{~cm}$. behind the middle of a line uniting the root of the nose with the occipital protuberance. ${ }^{1}$

1 In the making of an osteoplastic flap the bone can be sectioned with great neatness and considerable rapidity by the use of the frigli wire-saw after the plan of Obalinski. Such a saw consists of lough steel wire with a loop at each end. The handles of a chain-saw fit the loops. Two or more small trephine-openings are made, the dura between the openings is separated from the skull, a piece of silk is carried from opening to opening br means of a probe, the saw is pulled through by means of the silk, the handles are attached, and the bone is sawed from within outward.-ED. 
Resections of the Jaws. - Resection of the Upper Jaw. -The upper jaw is removed partially or wholly when the seat of malignant disease.

Temporary rexection of the upper jaw may be undertaken to expose the nasopharyx or the sphenomaxillary fossi, the temporal fossis, for purposes of operative intervention. The body of the upper jaw presents three processes through

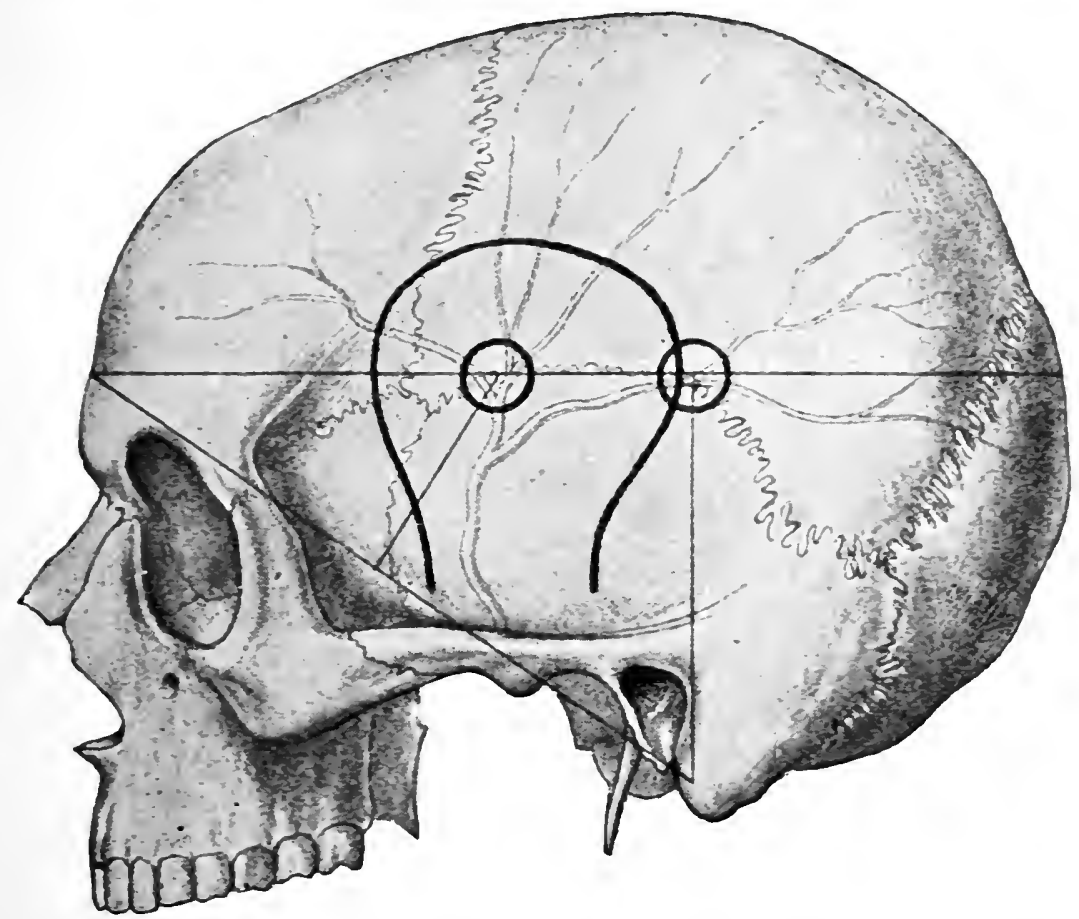

FIG. 141.-Diagrammatic representation of the method of finding the upper and middle branches of the middle meningeal artery.

which it articulates with neighboring bones. The palatal process unites in the middle line with a similar process of the bone of the opposite side. The frontal or nasal process unites the upper jaw with the frontal bone, and the zygomatic process unites it with the malar bone. The posterior surfice of the body of the upper jaw is united with the descending wing of the sphenoid and with the 
pramidal process of the palatine bone. These processes must all be severed if the upper jaw is to be separated from its attachments.

Steps of the Operation.-The head of the patient is placed on a lower level than the trunk. Preliminary tracheotomy and the introduction of a tampon-cannula are not neeessary. The cutancous incision ( $\mathrm{T}^{\mathrm{e}} \mathrm{eber}$ ) is immediately made at all points down to the bone. It begins at the middle of the upper lip, which it divides vertically; after reaching the septum it surrounds the nasal ala on the side to be operated upon to its upper extremity ; it then continues vertically upward to the internal eanthus of the eve, and thence at an acute angle it passes ontwarl in a curved direction along the lower margin of the orbit to end at the extemal canthus of the eye (Fig. 142). The flap thus formed from the soft tissues of the check is dissected from the upper jaw so that the eanine fossa, as well as the malar process, is exposed. S The inferior tarso-orbital membrane is incised along the infra-orbital margin, so that the orbital fat protrudes. The entire contents of the orbit are carefully raised from the floor of the orloit, from which the chain-saw or the wire-saw is passed around the malar proeess through the infra-orbital fissure (Fig. 143) and the process is thus divided. The connection between the nasal proeess of the uper jaw and the frontal bone is divided transversely with the chisel. The division of the palate and of the alreolar process must ret be effected. To this end the mueous-periosteal covering of the palate is incised at the alreolar process and detached from the bone to the median line of the arch of the palate. The chain-saw is introduced through the pyriform aperture, and bronght into the mouth at the junction of the hard and the soft palate. Before the palatal plate is sawed through the middle incisor tooth of the corresponding side should be remored. The jaw is now attached posteriorly only to the pterygoid process and the pyramidal process of the palate bone, and above to the ethmoid bone. It is freed from these con- 
nections, the alveolar process being grasped with I atngenbeck's bone-forceps and remored with slightly rocking movements.

In the large wound exposed the severed infia-prbital artery must be eanght and ligaterl. The margins of the cutaneous wound areaceurately approximated and united

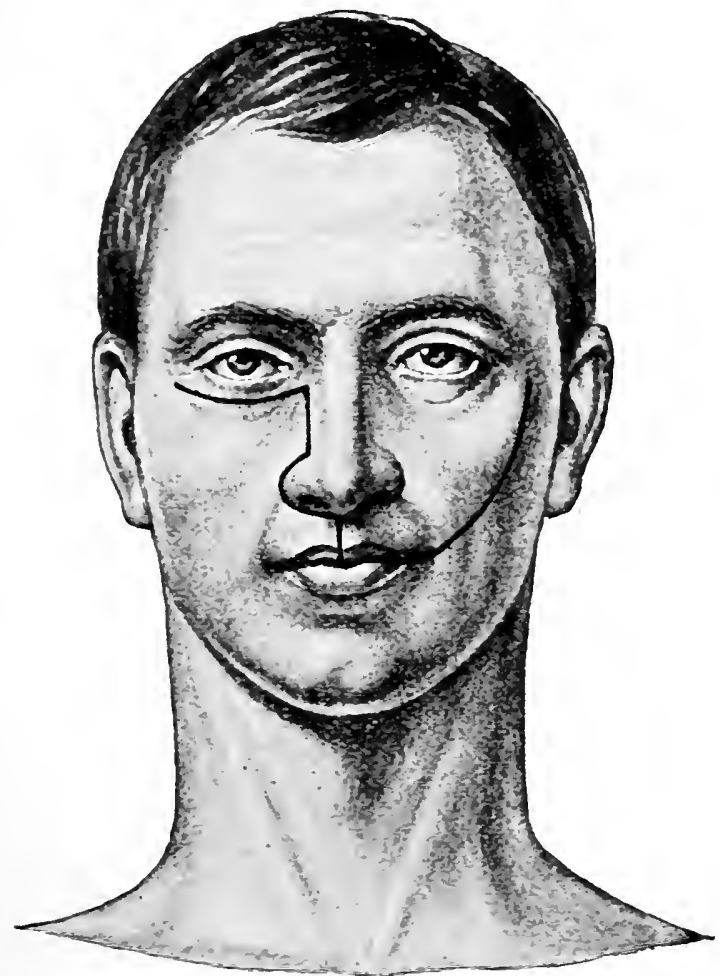

Fig. 142.-Incisions for resection of the upper jaw: $a$, by Weber's method; $b$, by Velpeau's method.

by suture. If it has been possible to preserve the mucous covering of the hard palate, this is united to the mucous membrane of the cheek after the jaw has been completely extirpated. The wound-cavity is in all cases tamponed with gauze. If the cavity is separated from that of the mouth by the preservation of the mucous covering of the La ain palate, the ends of the gauze are brought out of the nose. 
The various methods of resection of the upper jaw differ from one another only in the form of the cutaneous incision, the procedure upon the bone being always the same. Among various forms of cutaneous incision may be mentioned Dieffenbach's median incision, a vertical incision from the root of the nose, over the roof of the nose,

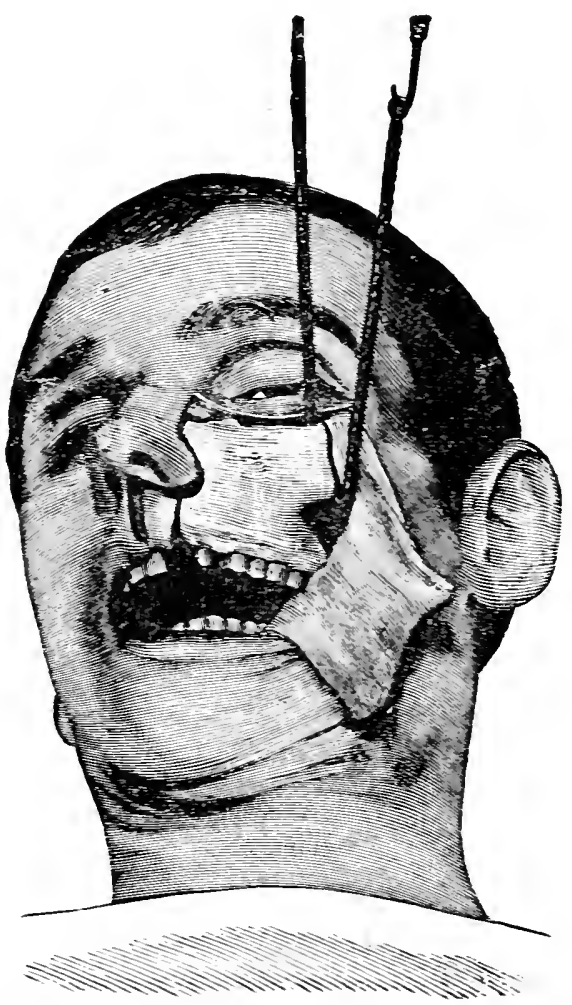

FIG. 143.-The anterior surface of the upper jaw exposed for resection: the maxillary processes are alreads dirided.

through the middle of the upper lip, passing down to the bone and dividing the cartilage of the nose and the upper lip. From the upper extremity of this incision a second, short incision passes to the inner canthus of the eye (Fig. 144). Velpeau divides the check in the form of an arch from the angle of the mouth (Fig. 142, $b$ ). Malgaigne 
combines with Velpeau's incision median division of the upper lip. The incisions should afford convenient access to the jaw, with conservation of the nerves, the vessels, and of Stenon's duct, and, finally, they should yield tavor-

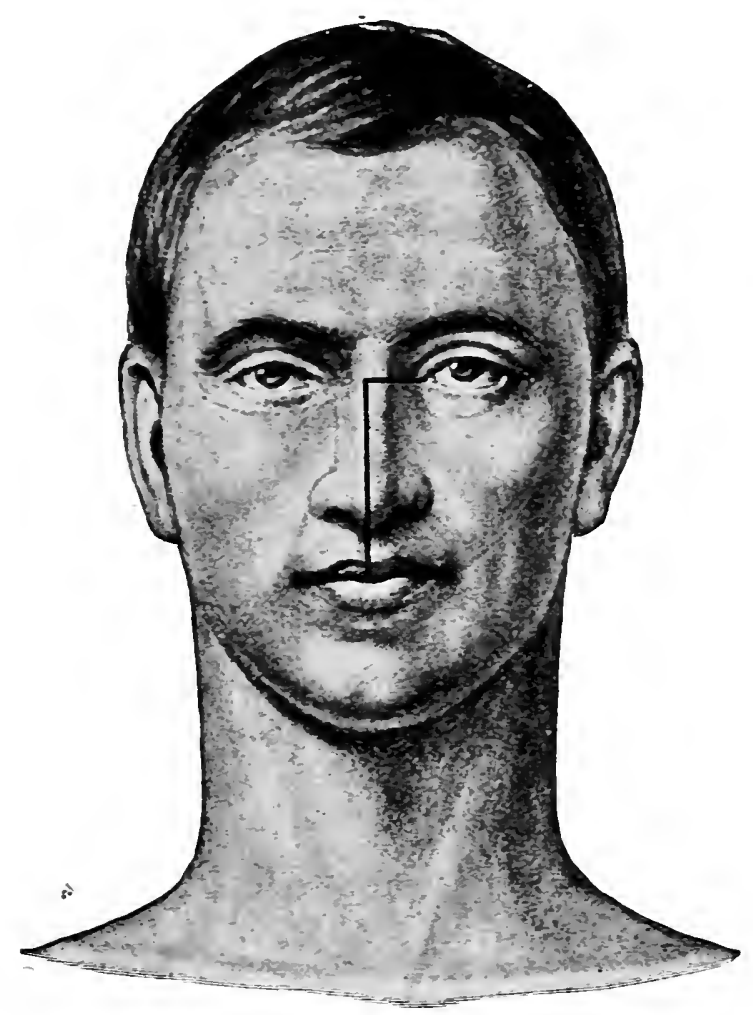

FIG. 144.-Incision for resection of the upper jaw by the method of Dieffenbach.

able cosmetic results. All of these requirements are best met by Weber's incision.

Temporary resection of the upper jaw (Langenbeck's) is made through a tongue-shaped flap whose base corresponds with a line uniting the root of the nose and the lower extremity of the nasal ala of the same side. The flap extends externally beyond the maxillary process. 
The following structures are divided: the upper jaw horizontally above the alveolar process, from the upper incision the frontal process of the upper jaw, the lachrymal bone, the floor of the orbit transversely into the inferior orbital fissure, and further from this fissure the frontal process of the malar bone and transversely the malar arch. The upper jaw may now be removed in conjunction with the overlying skin and be reflected upon the nose.

Resection of the Lower Jaw.-Upon either side of the body of the lower jaw there passes upward a ramus which articulates through its upper extremity with the glenoid cavity of the temporal bone. A second process arising from the upper extremity of the ramus is the coronoid, which serves for the attachment of the tendon of the temporal muscle. The external surface of the ramus of the lower jaw is covered by the masseter muscle, the inner by the internal pterygoid. Both of these muscles are attached at the angle of the jaw. The entrance into the inferior dental canal is marked upon the inner aspect of the ramus of the lower jaw by a bony process. A depression passing from this opening on the imner aspect of the jaw obliquely forward and downward to the chin serves for the attachment of the mylohyoid muscle.

Usually resection of only one-half the lower jaw is necessary. To this end the bone is divided with the saw vertically in the middle line, its body denuded upon its outer and inner aspects, and, after division of the tendon of the temporal muscle, freed at its articulation with the temporal bone. In performing total resection of the lower jaw the bone is likewise first divided in the median line, when the two halves are separately detached. Tumors, as well as necrosis, furnish the indication for the performance of resection of the lower jaw.

Steps of the Operation.- The patient is placed upon the table with the upper portion of the body elevated. A cutaneous incision is made vertically through the middle of the lower lip down to the chin, and from here at an 


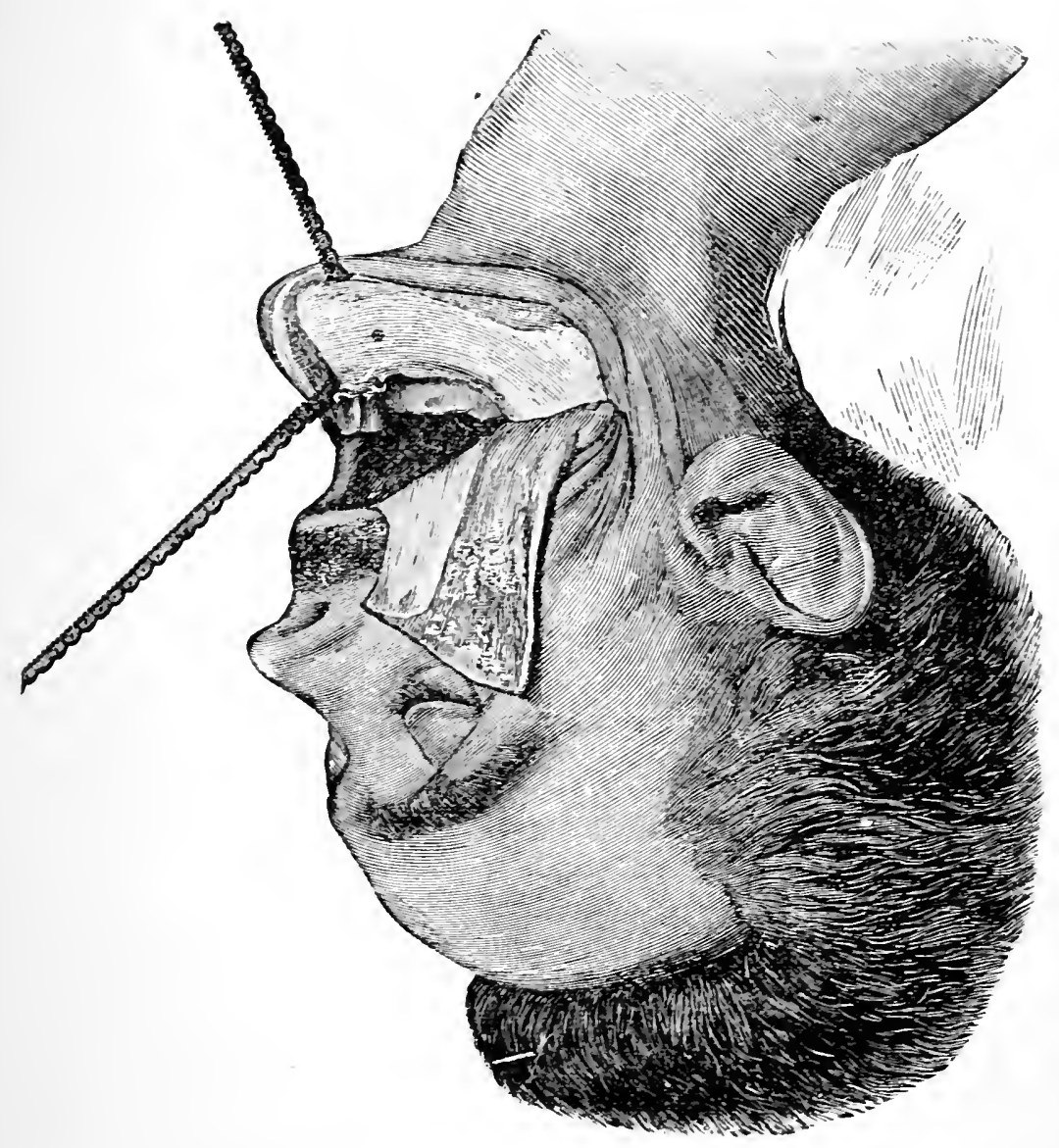

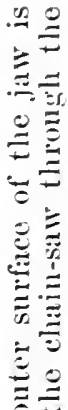

$\cong$

$\because=$

菃

is

$\stackrel{\varrho}{Ð}$

$\overline{\mathrm{c}}$

필

$\div 5$

光=

$4 \cong$

$\therefore \varrho$

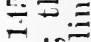

蓄 



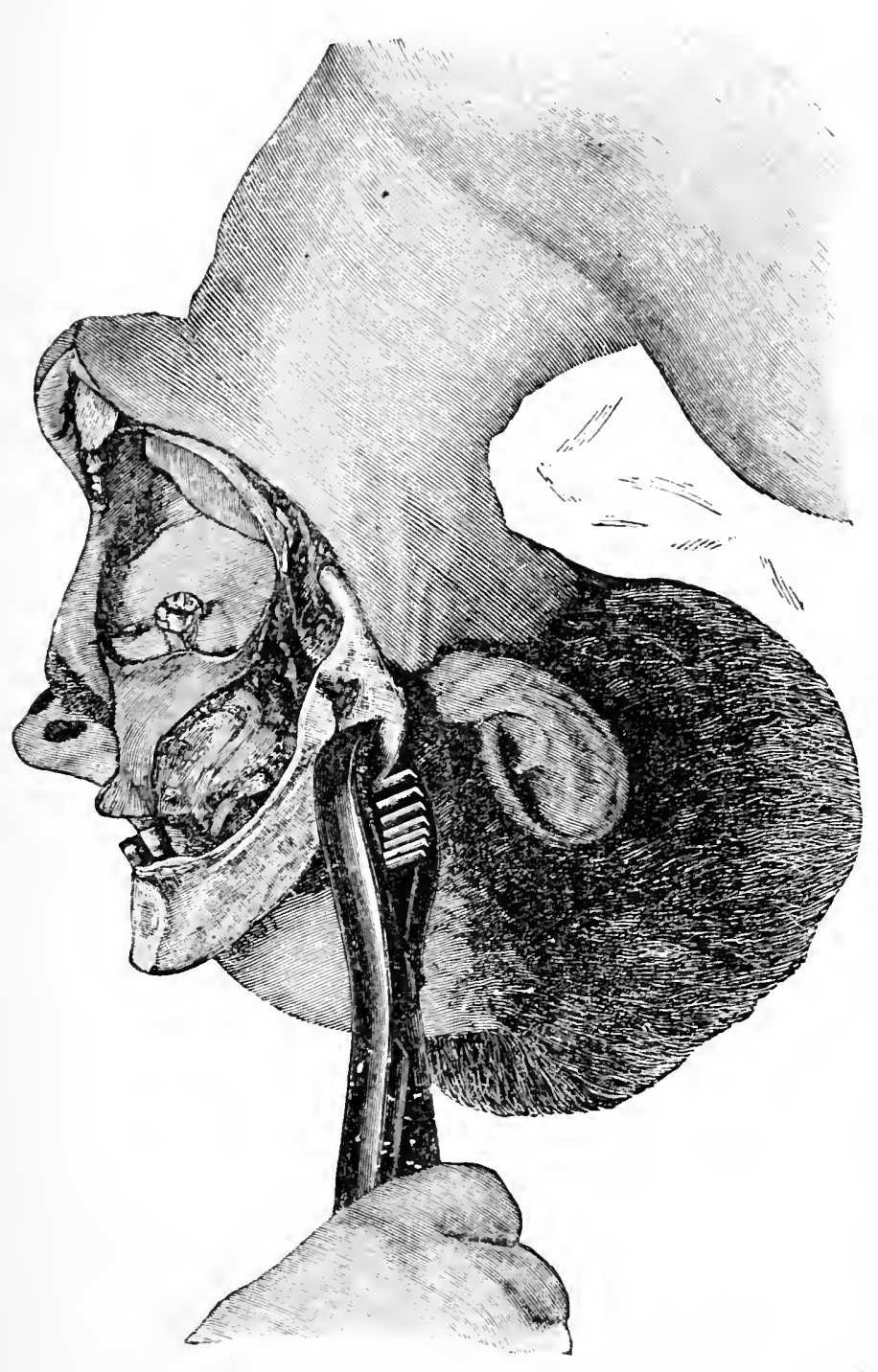

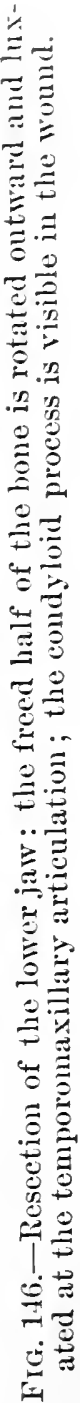


oblique angle it is directed ontward in the line of the border of the lower jaw to its angle. The incision at all points penetrates to the bone. The soft parts are dissected from the external surface of the bone, and the mucous membrane of the cheek is incised along the alveolar process of the jaw at the point where the flap is to be reflected. Through the eutaneous incision the soft parts are to be freed also from the posterior surface of the body of the jaw. After division of the mylohyoid muscle the mucous membrane is incised and the buccal cavity is opened. From the inner side of the chin the origin of the genioglossus and the geniohyoid muscles of the corresponding side are freed with the knife close to the bone. After extraction of the inner incisor tooth the chin-saw or the wire-saw may be passed around the exposed bone, and the division is to be effeeted near the middle line in a vertical direction (Fig. 145). The operator grasps the denuded half of the jaw and while he dislocates the bone in abduction divides with scissors the attachments of the masseter and pterygoid muscles at the angle of the jaw. It is now an easy matter to detach by blunt dissection the soft parts on the inner and outer sides of the ramus of the jaw. While the abducted half of the bone is rotated outward, the operator divides with scissors the vessels and nerves that enter the inferior dental canal, as well as the external pterygoid muscle at its attachment to the articular process (Fig. 146). After the tendon of the temporal muscle also has been divided, and after the head of the lower jaw is forced out of the glenoid fossa, the capsule of the joint is detached from the lower jaw, when the bone is freed from all its attachments. The mucous membrane of the cheek is united by suture with that of the floor of the mouth, and the wound in the skin also is closed by suture.

Temporary Resection of the Lower Jaw.-Simple division of the body of the lower jaw is undertaken when it is desired to render accessible the floor of the mouth, the palatine arch, the tongue, and the base of the brain (re- 
section of the third division of the trigeminus by the operation of Mikulicz for purposes of surgical intervention). The division is made either in the middle line through the chin (Sédillot), or laterally (Langenbeck). The bone is sawed through or is divided with the chisel either in a linear manner or in the form of a step.

Mode of Operation.-The lower lip is divided in the median line to a point below the chin. From the lower extremity of the incision a sharp-pointed knife is passed along the posterior surface of the bone and brought out at the frenum of the tongue. After extraction of the middle incisor tooth a chain-saw or a wire-saw is passed around the bone, which is divided in a linear direction parallel with the cutaneous incision. Br means of tenacula the cut surfaces of the bone are separated widely, when the structures of the floor of the mouth, the sublingual gland, and the tongue itself are rendered sufficiently accessible (Fig. 147).

The posterior portion of the tongue, the palatoglossal arch, the pillars of the fauces, and the wall of the pharynx are better reached through lateral temporary division of the ramus of the jan', as recommended by Langenbeck (Plate 12). The cutaneous incision passes vertically downward from the angle of the mouth to the level of the larynx. The general course of the operation corresponds with that of median division. In both instances, after completion of the operation, the divided halves of the jaw are reunited with metallic sutures. The cutaneous wound is closed with knotted sutures.

Resection of the Louer Jaw in its Continuity.-For the removal of portions of the lower jaw in its continuity the cutaneous incision is made down to the bone at its margin. The soft tissues are detached from the outer and inner surfaces of the jaw, until the mucous membrane of the lips, as well as that of the floor of the mouth, is divided close to the bone. At the two points through which the jaw is to be sawed a tooth is withdrawn, and the segment of bone of determined size is removed with 


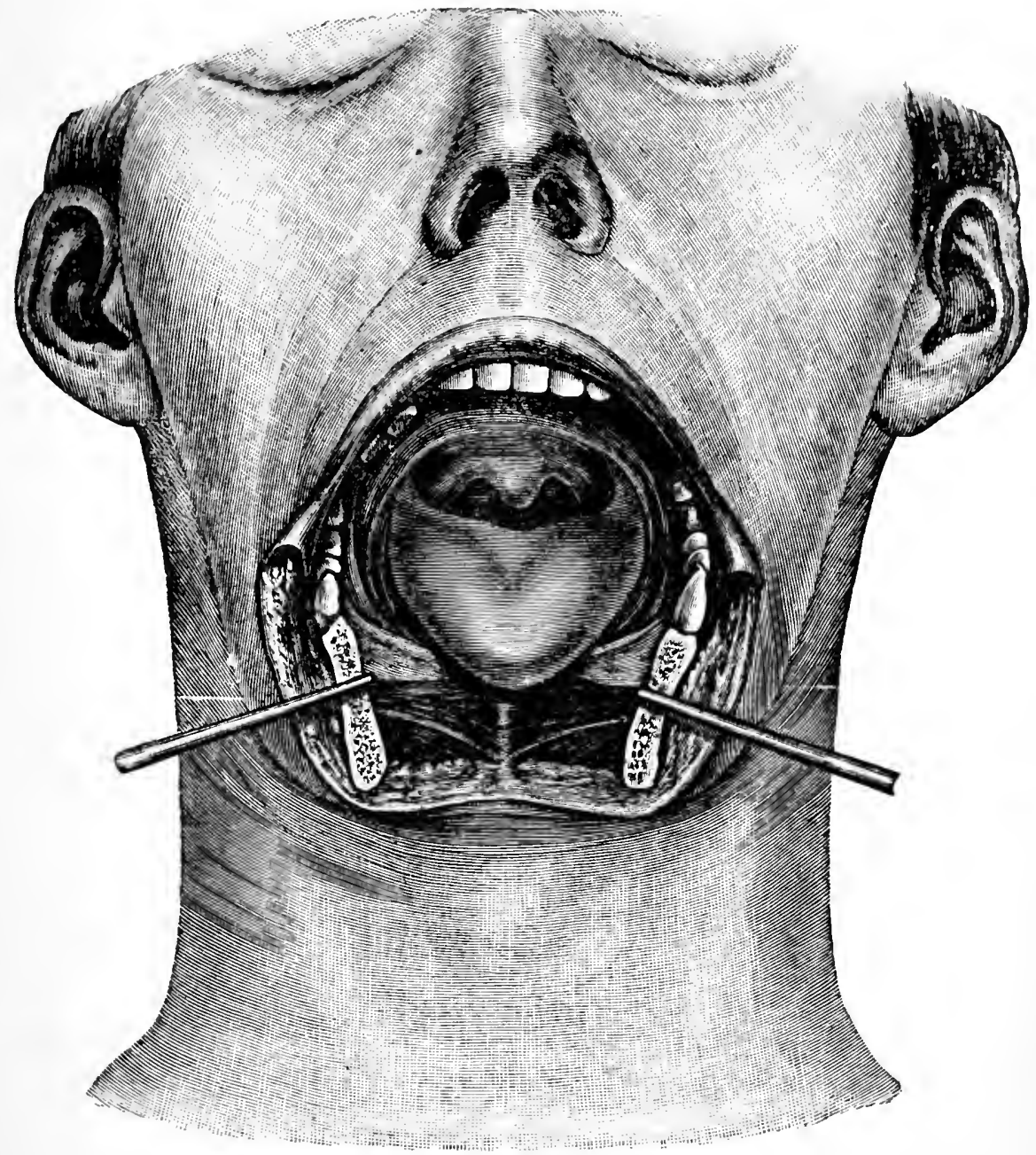

FIG. 147.-Median temporary division of the lower jaw by the method of Sédillot. 

the chain-saw. In similar manner the middle portion of the jaw is removed. In addition to the incision at the margin of the bone, it is recommended that the lower lip in this situation be divided vertically in the middle line in such a mamner that an incision of the following form results: $\frac{1}{1}$. After resection of the middle portion the stumps of the genioglossus muscles, separated from the chin, must be fixed in the entaneous wound by suture, so that the tongue thus deprived of its attichment to the chin does not fall backward, a contingeney not unattended with danger.

Operations on the Tongue. - Extirpation of tumors of the tongue are atypical procedures that are not carried out according to generally applicable rules. It is of the greatest importance that the removal of the tumor be effected through healthy tissue, and that the wound-defect be so made that union through suture or closure of the wound with healthr tongue-substance is possible. The preliminary operations performed for the purpose of facilitating total extirpation of the tongue and rendering the floor of the mouth more convenient of access have already been considered in part (temporary resection of the lower jaw).

In performing operations upon the tongue the patient is placed upon the table with the upper part of the body elevated. 'The neck is stretched and the head is fixed in this position. The mouth is held open with a suitable speculum or gag. The tongue is grasped with a strong silk ligature passed through its structure and drawn forward.

Circumscribed tumors at the margin of the tongue are excised in the form of a wedge through the mouth from healthy tissue with the sealpel or with scissors. The wound can be closed by linear approximation through deep and superficial sutures.

In advance of extirpation of half or the whole of the tongue ligation of the lingual artery upon one or both sides is undertaken to prevent hemorrhage.

If the extirpation is to be effected through the poste- 


\section{Plate 12.-Lateral Temporary Division of the Ramus of the Jaw by the Method of Langenbeck.}

The wound is made to gape by separation of the segments of the jaw: $M$, sawed surfaces of the ramus of the jaw ; Oh, hyoid bone; $B v$, digastric muscle, with its tendon divided; $M h$, mplohyoid muscle: $H g$, hyoglossus muscle; $H$, hypoglossal nerve; $L$, lingual nerve; $S m$, submaxillary gland; Sl, sublingual gland.

rior portion of the tongue, or if together with the whole tongue the floor of the mouth and the sublingual glands are also to be removed, the field of operation is rendered more conveniently accessible by preliminary procedures.

These preliminary operations consist in :

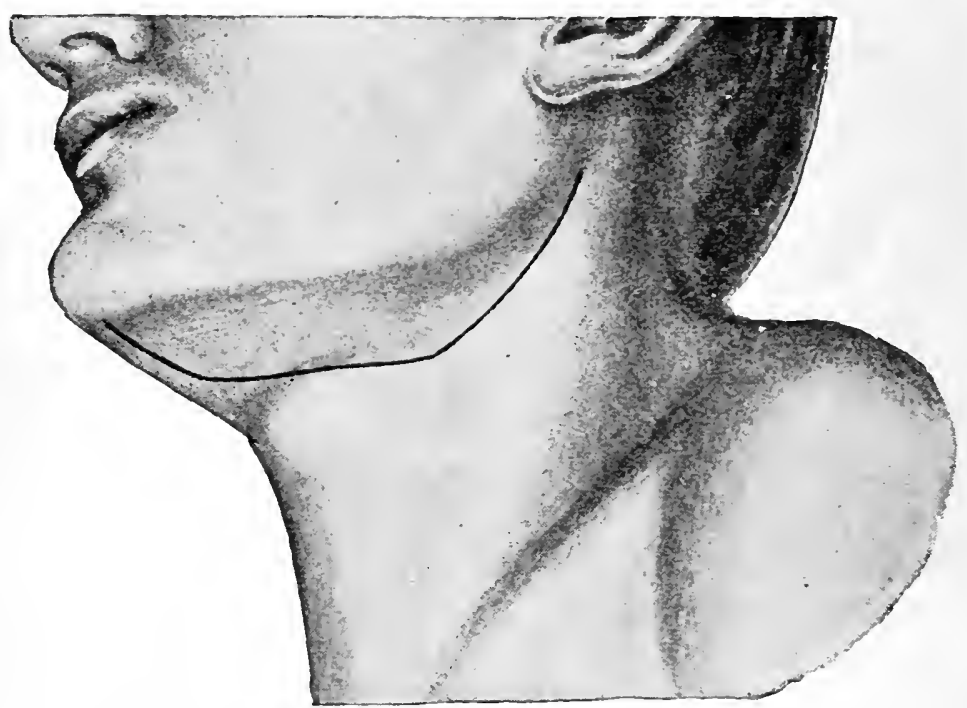

FIG. 148.-Incision for extirpation of the tongue by the method of Kocher.

1. Dirision of the cheek from the angle of the jaw ;

2. Submental incision (Regnoli-Billroth);

3. Temporary division of the lower jaw :

(a) In the middle line (Sédillot-Syme);

(b) Through the ramus of the jaw at a point corresponding with the first molar tooth (B. v. Langenbeck). 
Tab. 12.

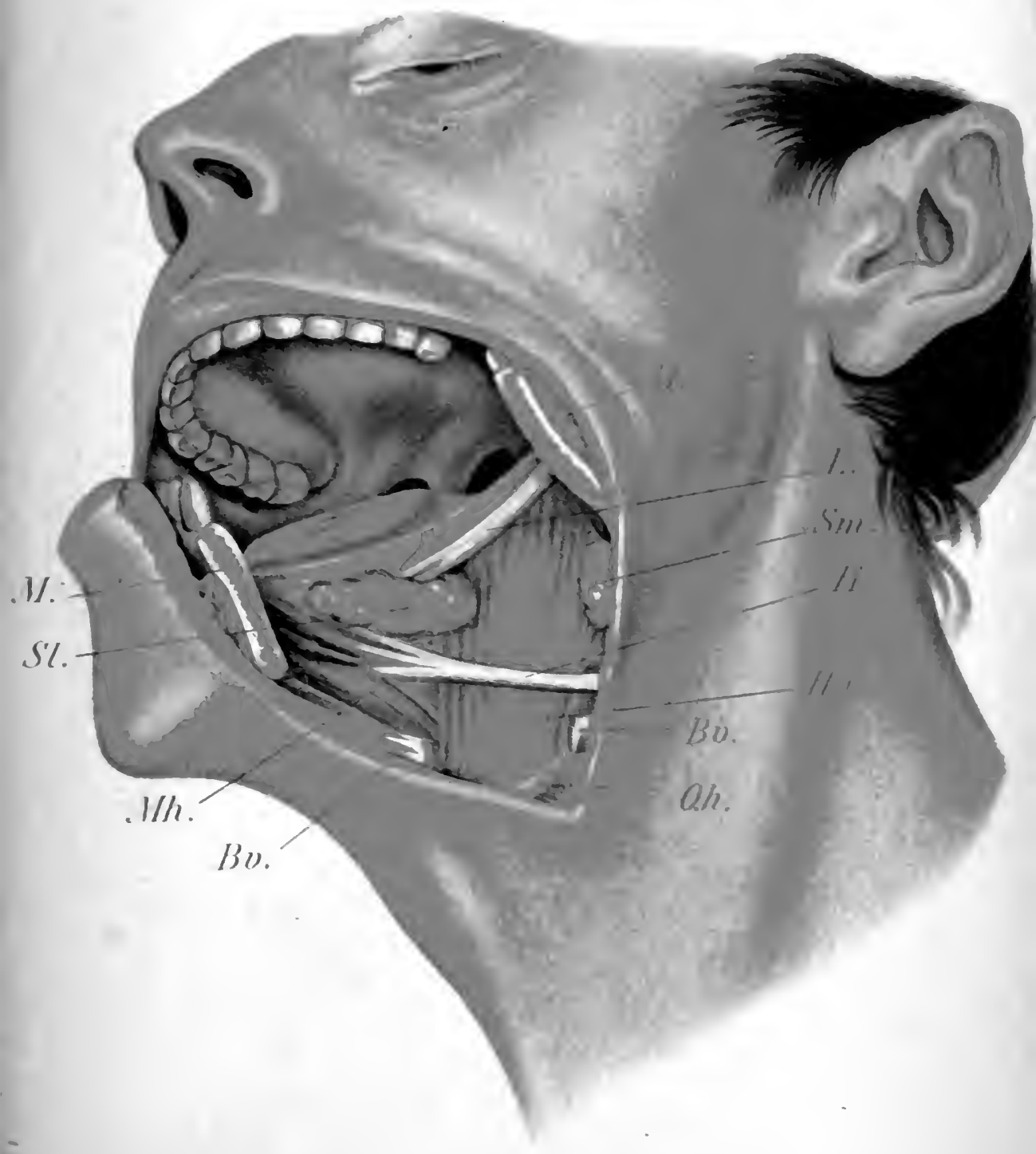





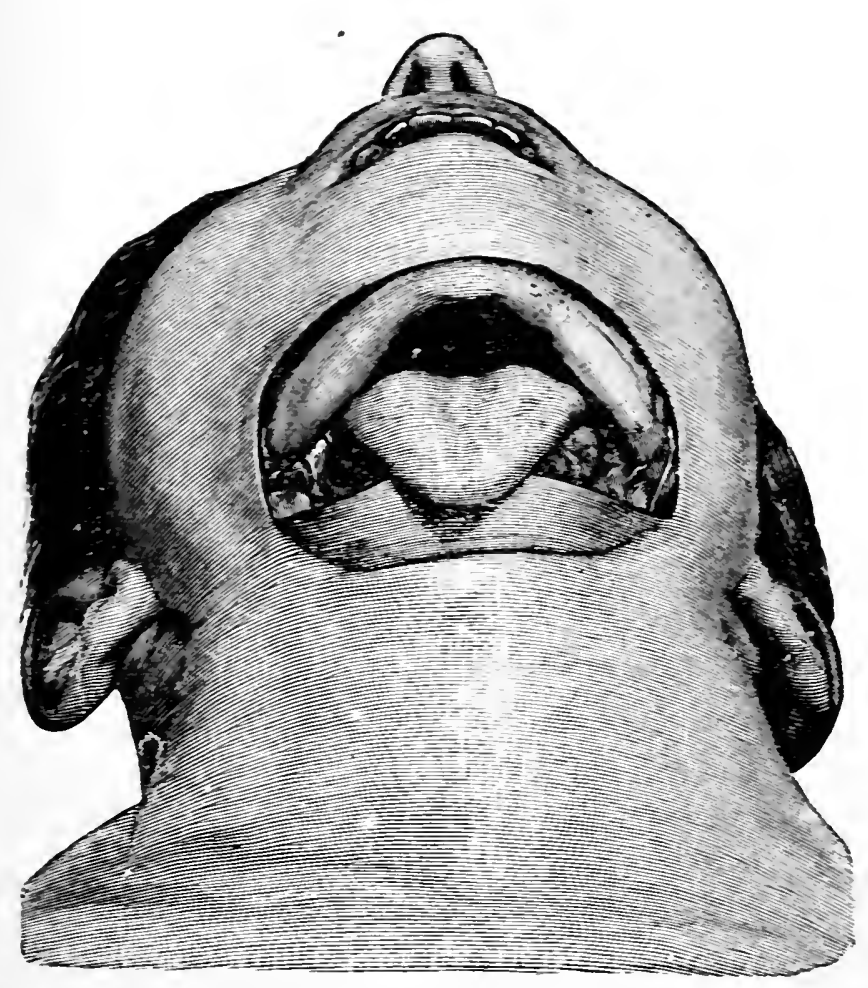

Fig. 149.-Submental exposure of the tongue by the method of Billroth. 
Wedge-shaped Incision of the Lower Lip. Linear UNION.

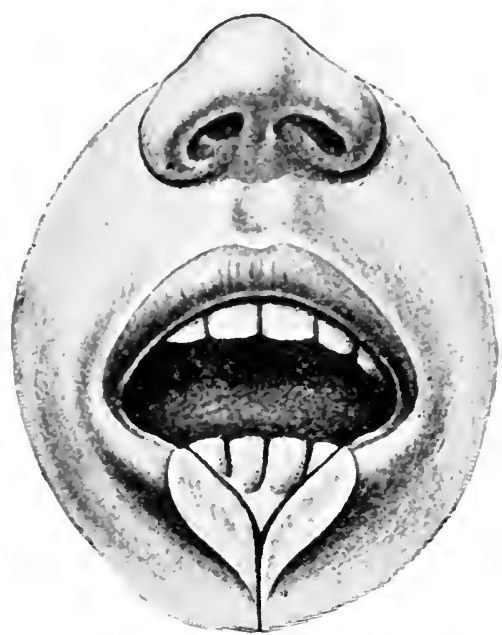

FIG. 150.-Showing the defect in the soft parts.

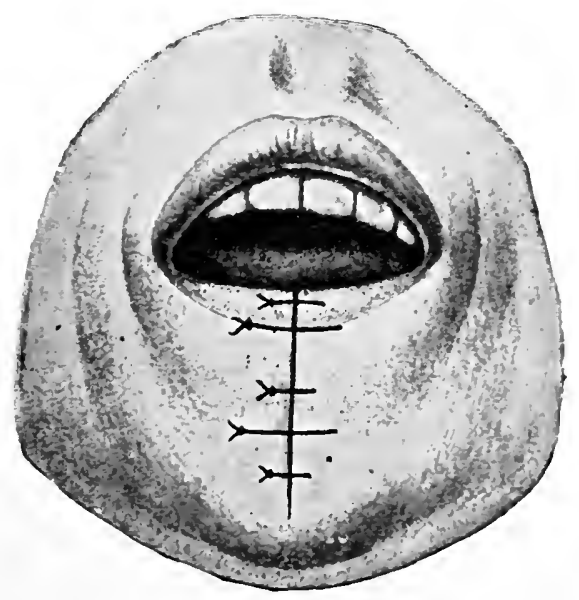

FIG. 151.-Showing the defect united by linear suture. 


\section{Cheiloplasty (Dimfenbach).}

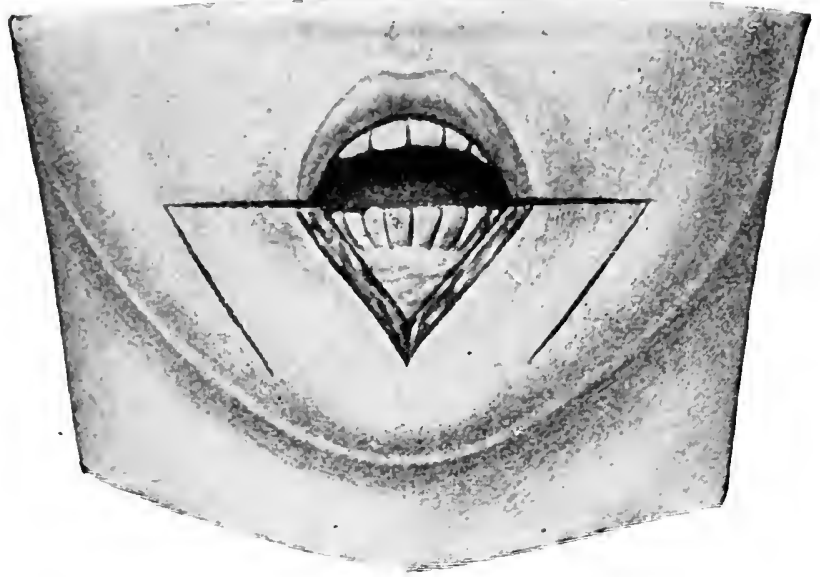

FIG. 152.-Triangular defect in the lower lip with contiguous rhomboid flaps.

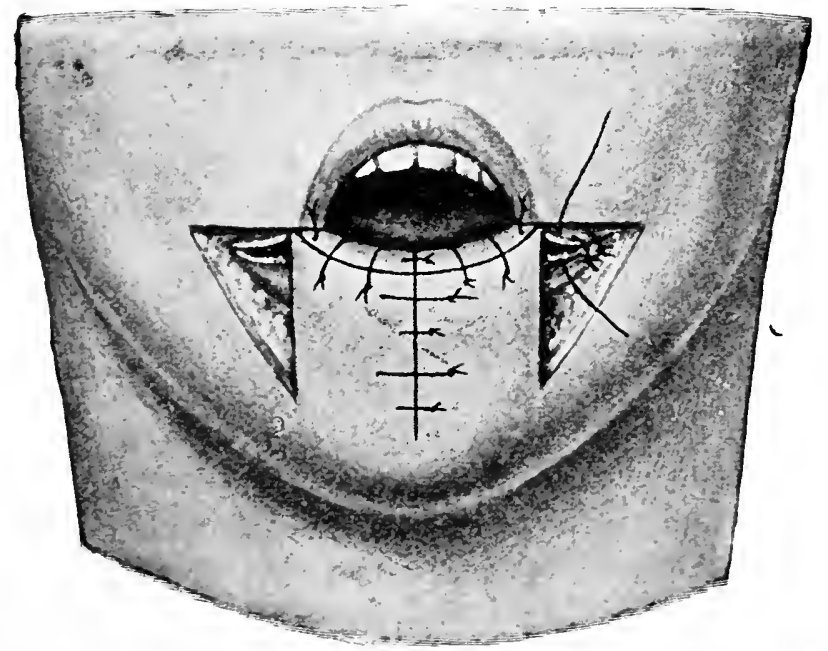

FIG, 153.-The flaps approximated by displacement toward the middle line: suture. 
Division of the check in a horizontal direction from the angle of the month renders the operation more convenient, inasmuch as the field of operation is male roomier, and it can also be better illuminated. After the operation on the tongue has been completed the incision in the cheek can be united by suture.

Submental remocal of the tongue, first performed by Regnoli of Pisa, is effeceted through a semilunar incision made upon the neck along the ramus of the lower jaw. Regnoli conjoined with this arehed incision a second, vertical incision passing from the chin to the middle of the hyoid bone. Billroth made only the simple arched incision. Access is gained to the inner side of the ramus of the jaw, the attachments of the mylohyoid muscle are separated laterally, and those of the genioglossus, geniohyoid, and digastric muscles in the middle, and the mueous membrane of the buceal cavity is opened throughout the entire extent of the incision. The tip of the tongue is caught with a thread and drawn throngh the wound. By these means the structures of the floor of the mouth, as well as the tongne down to its base, are conveniently accessible for operative attack (Fig. 149).

Temporary resection of the lower jaw is effected in accordance with the rules laid down on page 234 . The divided portions of the lower jaw are held apart by means of sharp hooks, in consequence of which the field of operation is rendered more extensive. The division of the lower jaw may be linear, or, to facilitate approximation in suturing the bone, it may be made in steps.

In the presence of extensive disease of the tongue Kocher effects extirpation of the organ from the base. He first performs preliminary tracheotomy. The eutaneous incision passes from the mastoid process along the anterior border of the sternomastoid muscle to the level of the hyoid bone, and from this point, in the furrow between the floor of the mouth and the neck, forward, to end in the median line at the chin (Fig. 148). The flap thus outlined is reflected back, when, after ligation of 
the external maxillary and lingual arteries, the submaxillary glands are removed. The buccal cavity is opened through the mylohyoid muscle and the mucous membrane detached from the lower jaw. The tongue must yet be separated from the hyoid bone, after which the whole organ can be brought forward and divided through healthy structure.

P1astic Operations. - Plastic operations include those accessory operations by means of which existing wounddefects are covered with integument, as well as such procedures as are intended for the correction of congenital or acquired deformity. In the first category belongs, for instance, the formation of pedunculated flaps for the closure of defects left by wounds; in the latter, operations for harelip, rhinoplasty, blepharoplasty, etc.

In covering wound-defects the adjacent skin is drawn over either directly or after being freed by incisions and attached in place. In other cases flaps taken from neighboring structures must be separated from the subjacent tissues, and either displaced laterally or rotated about their base, in order that they may be brought in apposition with the defect, and fixed in place.

A triangular defect that is not too large is covered directly by means of deep sutures parallel to the base, and linear mion is thus effected. (Linear union after wedge-shaped excision of the lower lip is illustrated in Figs. 150 and 151.) If the defect be greater, rhomboid flaps symmetrically situated on either side may be drawn toward the middle line to cover the defect (Dieffenbach, Figs. 15:2 and 153). In place of the rhomboid flap an arched incision passing from the base of the defect on either side may outline a portion of adjacent skin, which is brought over the defect and attached in place. Quadrilateral or oval defects may be covered by one or two symmetrically formed flaps from the immediate neighborhood (cheiloplasty by the nethod of Bruns, or by that of Langerbeck, Figs. 154 and 155).

The flap is made to correspond in shape with that of the defect, though somewhat larger. These methods, in accordance with which the flaps are obtained from the immediate neighborhood of the defect, stand in contradistinction with that in which a pedunculated flap belonging to a remote portion of the body remains attached in. 
its original situation through the pedicle until the flap has healed in the defect (rhinoplasty by means of a flap removed from the arm, according to the method of Tagliacozzi).

Another method for covering in deficiencies by means of skin removed from remote portions of the borly con-

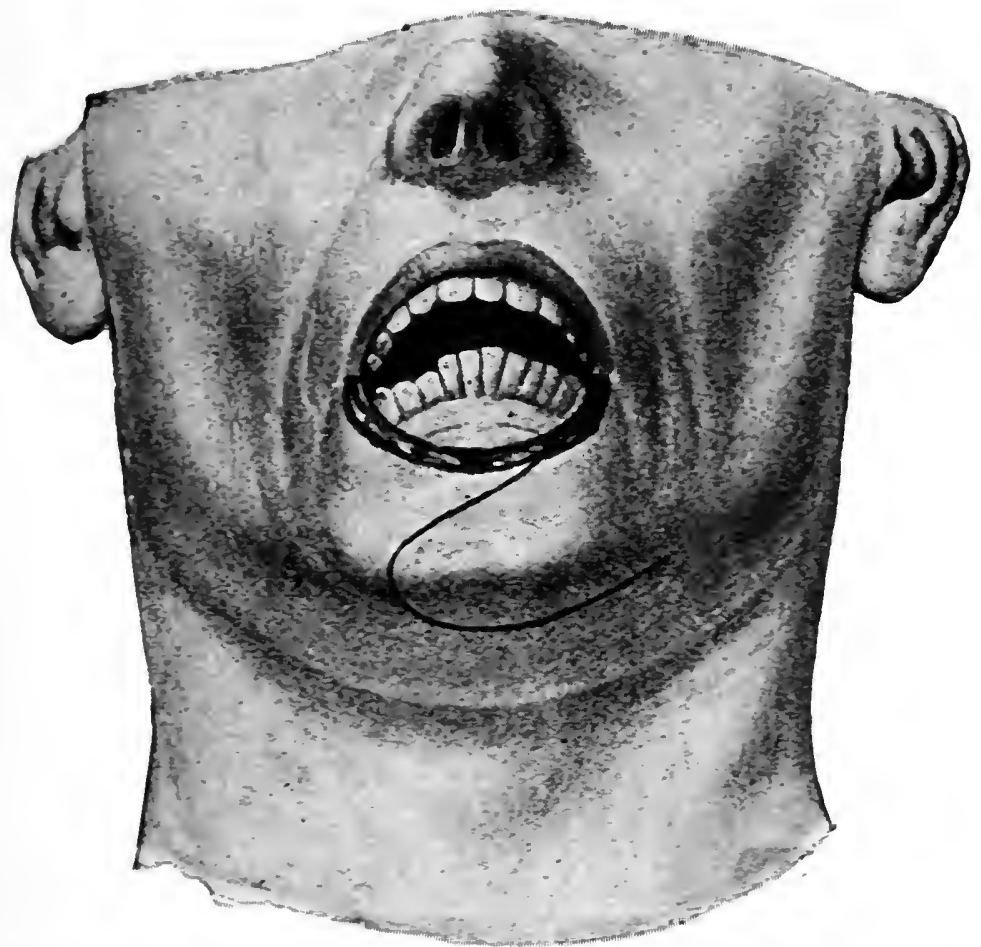

FIG. 154.-Oral defect in the lower lip: outline of the flap (br the method of Langenbeck).

sists in the formation of a bridge-shaped flap, beneath which the part to be covered is pushed and fixed, so that wound-surface comes to lie in apposition with woundsurface. After the flap has united it is detached from the subjacent structures by transverse division of its extremities. Finally, flaps completely separated from their attachments, that are not pedunculated, may be implanted and 
made to heal upon wound-surfaces (Reverdin, Thiersch). Thiersch cuts with a broad knife latrge and small flaps of epidermis which are placed close together upon a freshened wound-surface, preferably like shingles upon a roof. Krause has recently applied large mpechnculated flaps, removed from the entire thickness of the skin, upon recent wound-surfaces free from hemorrhage.

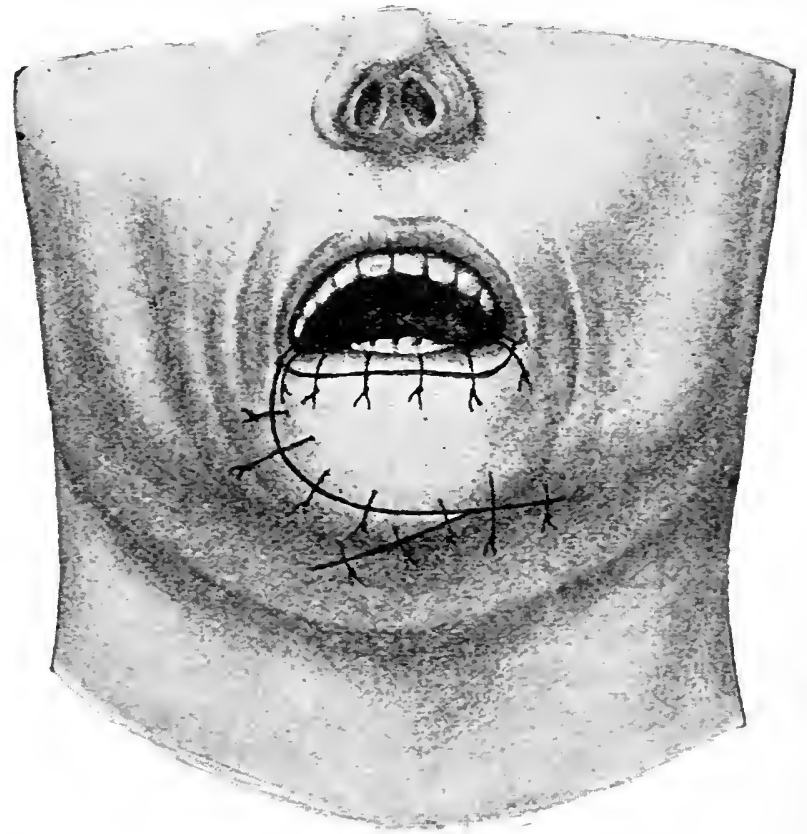

FIG. 155.-The detached flap is placed in the defect and there sutured : the spur is united with the lower border: the free border of the lip is formed from the mucous membrane of the cheek and what is left of the lower lip.

Special Forms of Plastic Operations.-Rhinoplasty.Plastic restoration of the nose. Total or partial rhinoplasty may be undertaken, accordingly as total or partial restoration of the nose may be required.

Formation of a Nose from the skin of the Forehead.The operation consists essentially in grafting upon the freshened margins of the defect in the nose an approxi- 
mately triangular flap from the forehead, from whose base the nasal ala and the septum are formed. Through its apex the flap retains its connection with the skin at the root of the nowe. After the defeet has bern freshened the size of the flap to be formed is determined by masurement, a tentative flap being first made of adhesive plaster or of leather and fitted to the deficiency. After the proper shape and size have been determined the model is spread upon the forehead and the actual flap is ontlined about this. The triangular flap thus formed lies obliquely upon the forehead, with its apex directed toward the supra-orbital margin. The one side of the frontal flap is

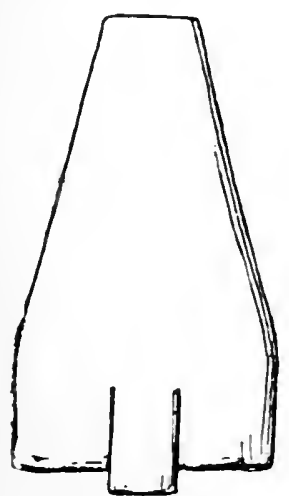

FIG. 156.

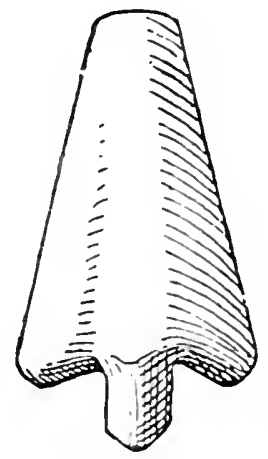

Fig. 157

continuous with one side of the freshened surface of the triangular defect in the nose. The flap, together with the periosteum, is detached from the subjacent structures, rotated at an angle of about $180^{\circ}$, and placed over the defect. The middle segment of the base of the flap, folded longitudinally and fixed by sutures, forms the septum. The lateral portions of the flap are reflected so that wound-surfaces are brought in apposition and nasal alæ are formed on either side (Figs. 156 and 157). The nose thus formed is fitted into the defect and fixed in place by sutures. The posterior extremities of the nasal alie and the septum are to be attached to the freshened surface of 
the cheek and the upper lip, when the lateral margins are to be united with the lateral portions of the defect. The defect in the forehead is reduced br means of silk sutures before the formation of the nasal alæ and the septum. The defect still remaining in the middle is permitted to close by granulation, or it is covered with Thiersch flaps. In the newly formed nasal orifices small rubber tubes are

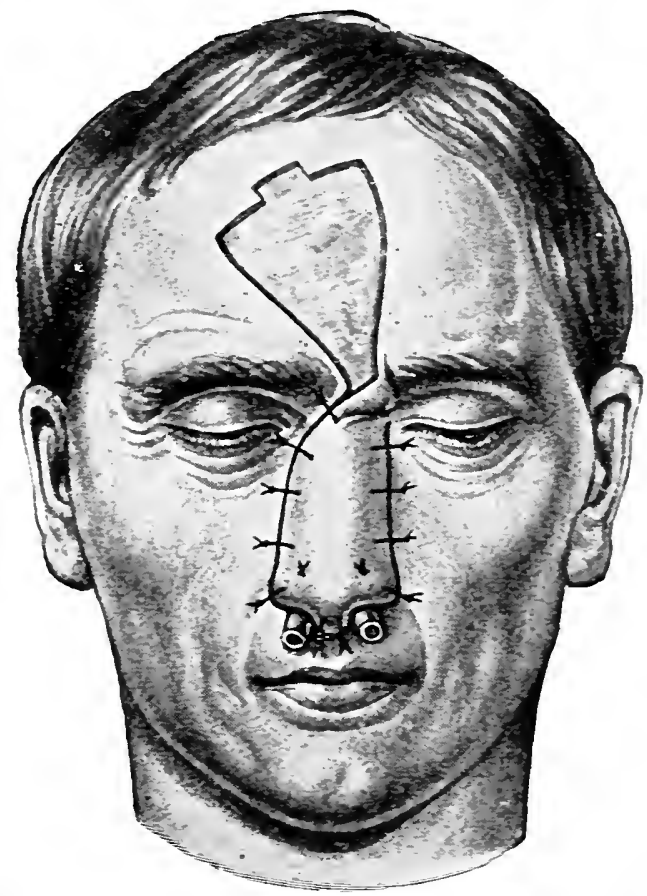

Fig. 158.-Total rhinoplasty from the skin of the forehead.

introduced (Fig. 158). The disfiguring fold at the root of the nose corresponding to the point of reflection of the flap can be removed by excision at a second sitting after healing has taken place.

In operating for the correction of saddle-nose, König utilizes a flap made from the soft tissues and bone of the forehead in the form of a vertical strip with its base at the root of the nose. The flap thus formed is freed, re- 
Formation of a Nasal Ala from the Skin of the Cinele.

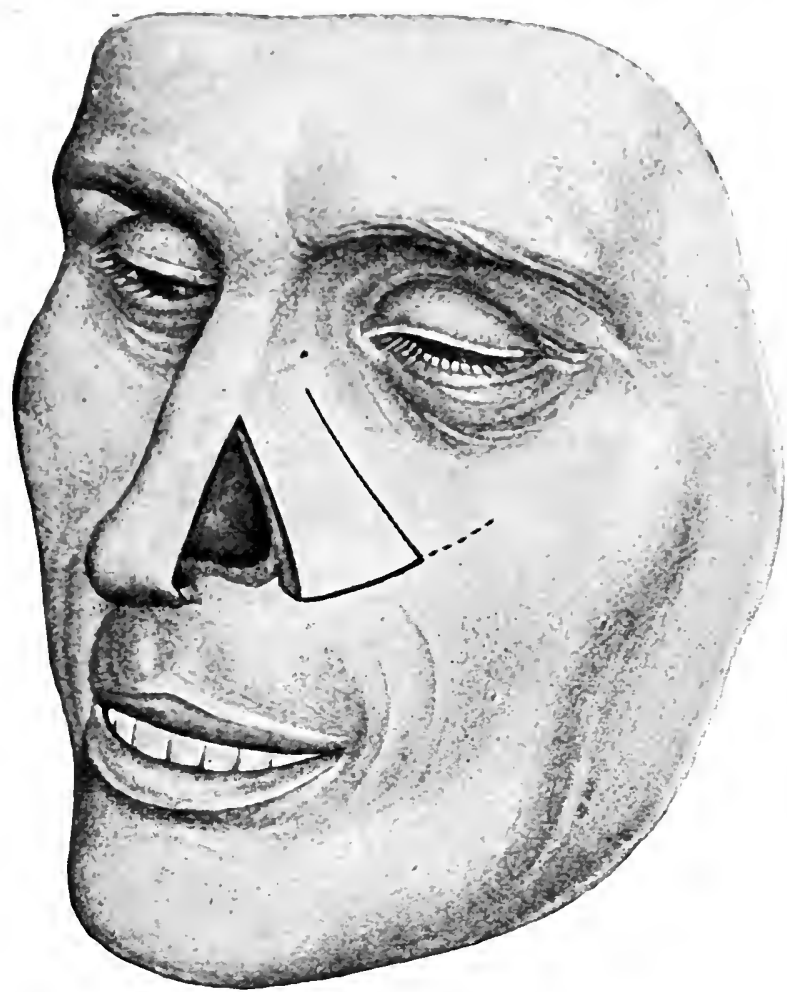

FrG. 159.-Defect and outlines of the flap.

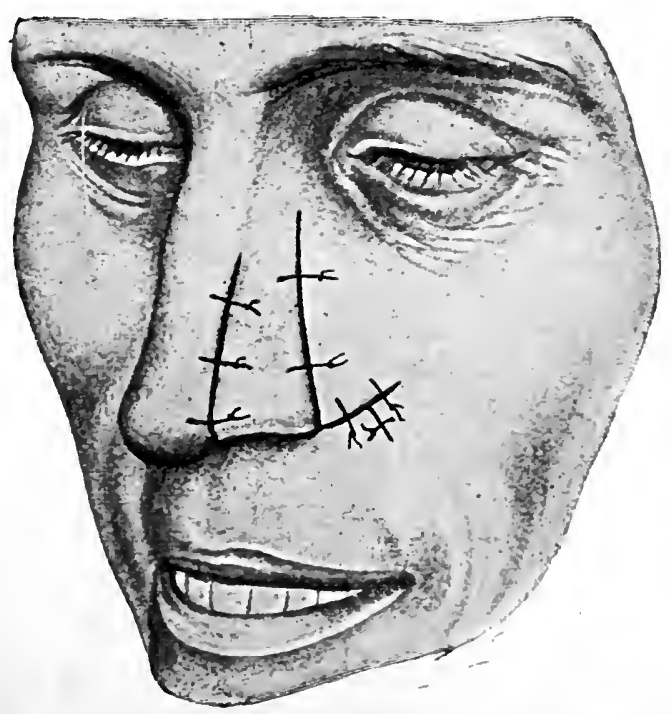

FIG. 160.-The flap is placed in the defect and there sutured. 

flected downward, and fastened in the roof of the mobile and elevated nose. Over this flap, forming the support of the nose, the organ proper is formed from the skin of the forchead in the usual manner. ${ }^{1}$

For partial plastic operations upon the nose strict rules cannot be formulated. Suitable procedures will have to be devised for the individual calse from the elements for the plastic closure of -defects. In the absence of the lateral wall of the nose also the flap to cover in the defect may be taken from the forehead. In the formation of a nasal ala a pedunculated flap is formed from the dorsum of the nose, and from the opposite side of the nose, or from the skin of the cheek. The pedicle of the flap is at the upper portion of the back of the nose (Figs. 159 and 160). A pedunculated flap formed from the substance of the upper lip may, by rotation, be introduced into the freshened defect of the nasal ala and there be fixed by suture. To replace an absent septum the tissues may be advantageously obtained from the upper lip.

Plastic closure of defects in the integument of the cheek involving its entire thickness requires special operations. The flap engrafted in the defect must be covered upon its inner surface with skin or mucous membrane to prevent cicatricial contraction. Usually cutaneous flaps are so sutured in place that their epidermal aspect is directed toward the eavity of the mouth. Gersuny forms a flap from the tissues immediately adjacent to the defect, whose pedicle consists only of subcutaneous tissues. Czerny employs a long flap made from the integument of the neck, which is folded and lies in the defect like a double flap. The flap is twice as long as the defect to be covered.

Israel forms also a long flap from the integument of the neck, whose extremity is sutured in the defect in such a manner that the cutaneous surface is directed toward the buccal cavity. After the flap has healed in place it is

I In saddle-nose an artificial bridge can be made of gold or platinum, and this bridge is slipped in place through an incision which is subsequently sutured.-ED. 


\title{
Operation for Harelip.
}

\author{
NÉlatox's Method.
}

FIG. 161.-Freshening.

FIG. 162.- Wound after adjustment of the lip.

FIG. 163.--Suture.

MALGAIGNE'S JIETHOD.

FIG. 164.-Freshening.

FIG. 165.-The flap on either side is dislocated downward.

FiG. 166.-Suture.

\section{Operation for Harelip.}

\section{Mirallt-LANGeNbeck's Method.}

FIG. 167.-Freshening.

Fig. 168.- Wound.

FIG. 169.-Suture.

\section{Operation for Bilateral Harelip.}

FIG. 170.-Freshening.

Fig. 171.-Wound.

FIG. 172.-Suture.

severed at its point of insertion and the free posterior portion is folded, and, with wound-surface applied to woundsurface, placed upon the already healed portion of the flap. The flap closing in the defect is thus double, with its cutaneous surfaces directed on the one side toward the mouth, and on the other side toward the exterior.

Operation for Harelip.-Wélaton's procedure, intended for the correction of incomplete harelip, consists in division of the upper lip above and parallel with the defect. The margin of the defect is drawn downward and the rhombic wound is uniter in a linear manner at right angles to the transverse fissure of the mouth (Figs. 161, 162, and 16\%). Malgaigne forms a flap upon either side from the free border of the lip bounding the defect and remores the angle of the defect. The flaps are deflected downward and united with one another (Fig. 164). A modification of this method constitutes the operation of Mirault-Langenbeck. From one of the margins of the defect a Malgaigne flap is formed in typical manner. The 


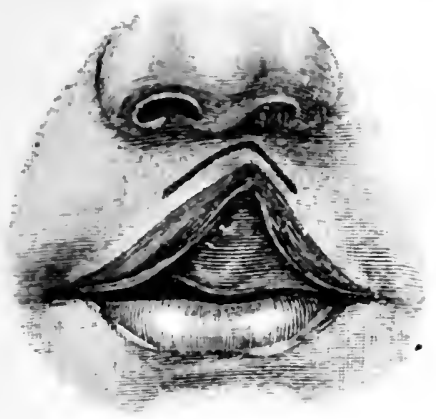

FIG. 161.

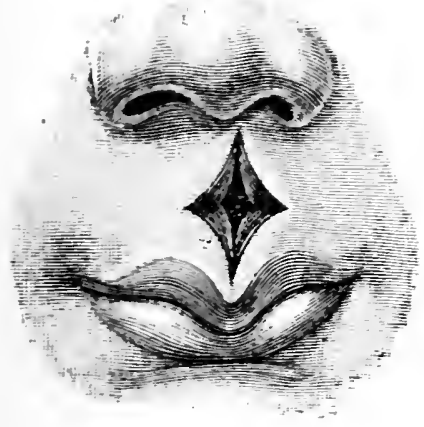

FIG. 162.

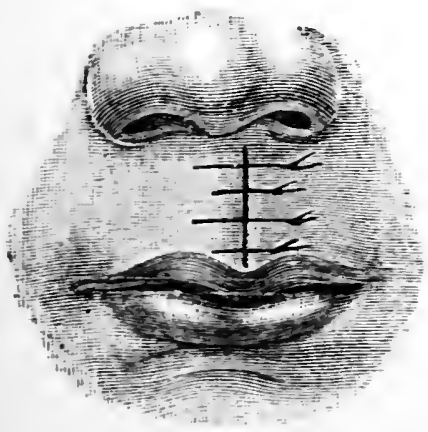

FIG. 163.

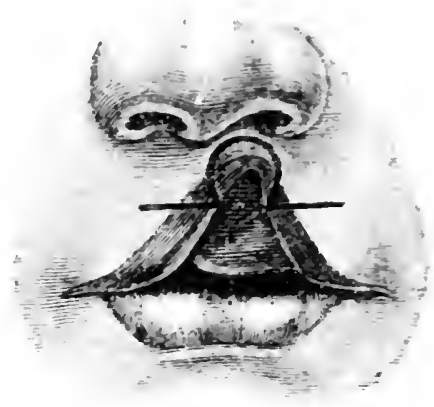

FIG. 1jt.

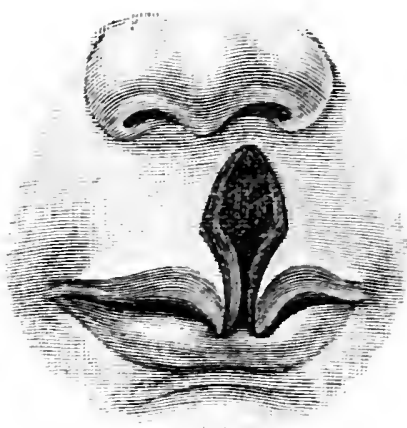

FIG. 165.

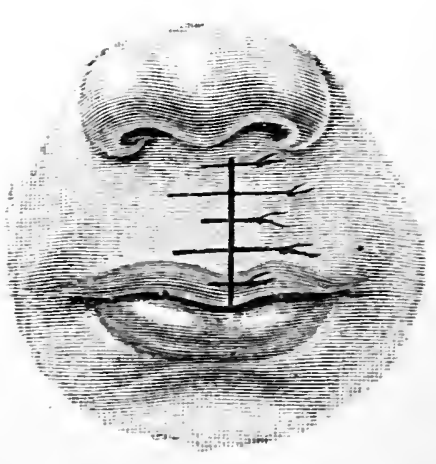

FIG. 166. 



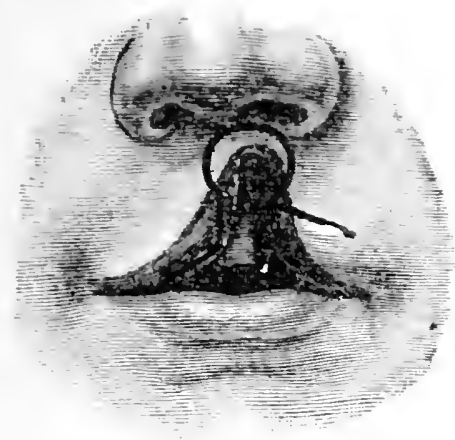

FIG. 167.

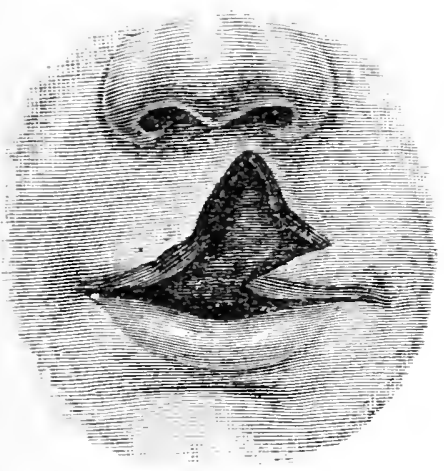

FIG. 168.

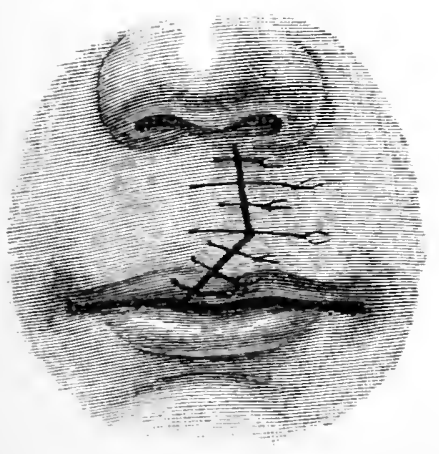

FIG. 169.

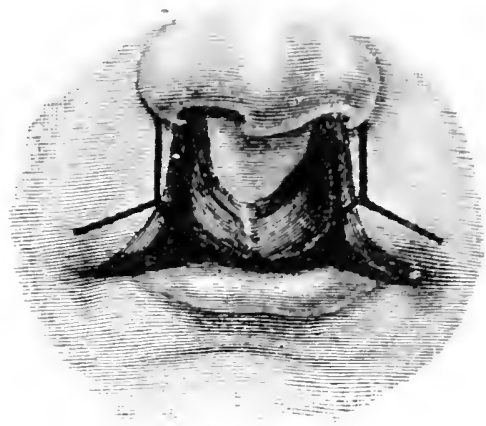

FIG. 170.

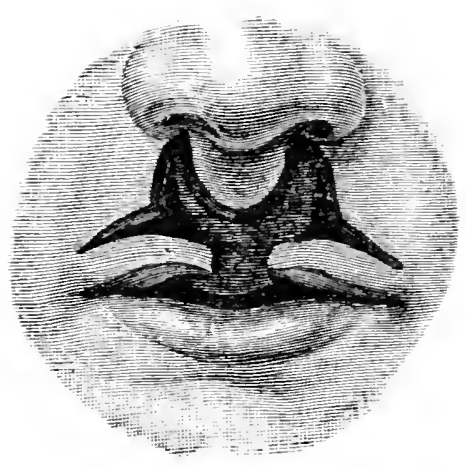

FIG. 171.

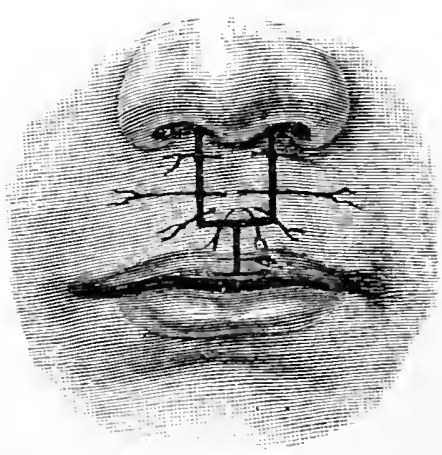

Fig. 172. 

second margin of the defect is freshened in a bevelled mamner and the red border of the lip is removed at the angle of the defect. After union has been effected the flap constitutes the free border of the lip (Figss. 167, 168, and 169$)$.

In cases of bilateral harelip the middle segment, as well as both lateral margins of the defect, must be freshened. A Malgaigne flap is formed on either side (Fig. 170), which is ent through at its apex. The flaps are displaced downward and united with one another below the middle segment. The margin of the defect on either side is bronght in apposition with the corresponding freshened edge of the middle segment, with which it is unised (Figs. 171 and 172).

If the fissure in the lip gapes so widely that its margins cannot be brought in apposition, free incisions are made through the nasal alæ to give mobility to the margins of the fissure (Fig. 173).

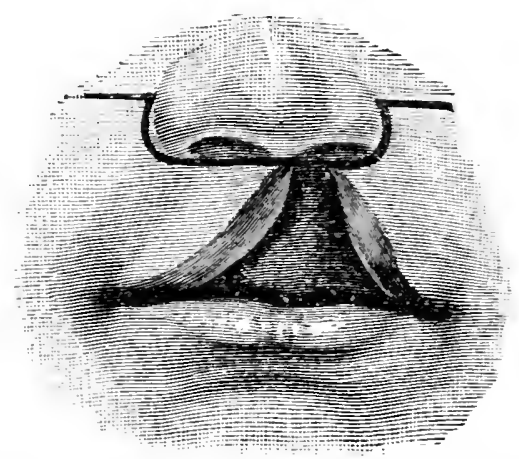

FIG. 173.-Freeing incisions when the fissure in the lip is a large one.

If the middle segment is especially prominent, plastie closure of the double fissure in the lip is to be effected after depression of the intermaxillary bone. To this end the mucous nuembrane at the lower free border of the septum is divided and separated from the vomer on either side. The vomer is divided throughout its entire height with a single stroke of the scissors, when the intermaxillary bone ean be pushed into place by pressure from before backward.

Staphylorrhaphy and Uranoplasty.-Staphylorrhaphy, plastic closure of congenital defects of the soft palate, consists in freshening of the margins of the defect and 
their union by suture. The operation is performed with the patient either in the upright posture or with the head dependent. The margins of the defect are grasped individually with forceps and the freshening is effected with a small sharp-pointed knife, and union is established by means of small, strongly curved needles. If the margins of the defect can be brought together only with great tension, it is recommended that freeing incisions be made on either side of the veil of the palate.

Plastic elosure of defects of the hard palate (uranoplasty) is effected in three stages. The first consists in freshening the margins of the defect and the formation of a bridge-like flap on either side in such a manner that an incision is made through the mucous membrane on each side down to the bone, parallel with the margin of the defect, at the alveolar border of the palate, reaching from the last molar to the outer incisor tooth. In the second stage of the operation the flap thus outlined is rendered mobile, the mucous-periosteal covering of the palate being separated from the bone by means of a small raspatory introduced through the outer incision. To render mobile the soft palate its attachment to the palate bone is freed by means of a knife curved on the flat, so that the flap at all points can be moved toward the middle line without tension. The last stage of the operation, which consists in suture, is carried out in the middle line in the same way as in staphylorrhaphy.

To avoid undue tension upon the approximated flaps semilunar lateral freeing incisions through the veil of the palate have been recommended (Dieffenbach). Billroth dispenses with the incisions through the velum, and prefers chiselling the median plate of the pterygoid process of the sphenoid bone, by displacement of which inward relief of tension is effected. Wolff effects sufficient mobilization of the soft palate by adequate detachment of the coverings of the hard palate, so that accessory operations are unnecessary.

Operations on Nerves.-If in cases of trigeminal neuralgia conservative methods of treatment have been unattended with success, operative intervention becomes allowable. Simple division of the nerve (neurotomy) 
is unjustifiable, as experience has shown that the transverse sections of the divided nerve shortly reunite. The exsection of a portion of the nerve (neurectomy) is more promising in this connection, although the nerve is regenerated after a somewhat longer time. Large segments of nerves, together with their smallest branches, may le removed by means of the proeedure of Thiersch (extraction of nerves, mencexciresis). The nerve in question is exposed in a given situation, grasped transversely with a forceps-like instrument, and removed throughout a considerable extent of both its distal and its proximal course by slowly rotatory movements (a half rotation in the second, 'Thierseh).

First (Ophthalmic) Division of the Fifth Nerve.-The smallest of the three divisions of the trigeminal nerve enters the orbit through the sphenoidal fissure. Of the branches of the ophthalmic, only the frontal nerve is of surgical importance. It runs along the roof of the orbit, and divides into two branches: the supratrochlear, which leaves the orbit above the pulley of the superior oblique musele to supply the skin of the upper eyelid and the forehead ; and the supra-orbital, which passes through the supra-orbital notch to the forehead.

To expose the frontal nerve an incision is made below the eyebrow parallel with the supra-orbital margin, dividing the skin and the tarso-orbital fascia. The contents of the orbit are carefully detached, when the trunk of the nerve becomes risible on the roof of the cavity. The branches of the nerve are looked for, grasped with the forceps, and torn out individually (Fig. 174).

Second (Superior Maxillary) Division of the Fifth Nerve. - The supramaxillary division of the fifth nerve leaves the cranial cavity through the foramen rotundum, enters the sphenomaxillary foramen, and passes without ehange of course through the infra-orbital fissure into the infraorbital canal on the floor of the orbit, leaving the latter through the infra-orbital foramen, to break up upon the face in a fan-shaped distribution into its various branches. 
The infra-orbital foramen is situated in the canine fossa about $0.5 \mathrm{~cm}$. below the middle of the infra-orbital margin.

To expose the infra-orbital nerre at its point of exit in the canine fossa the incision is made about $0.5 \mathrm{~cm}$. below and parallel with the infra-orbital margin throughout an extent of about $4 \mathrm{~cm}$. The lower margin of the wound is retracted by means of hooks. The incision through the elevator muscles of the upper lip passes down to the bone, and the periostemm is reflected downward, when the

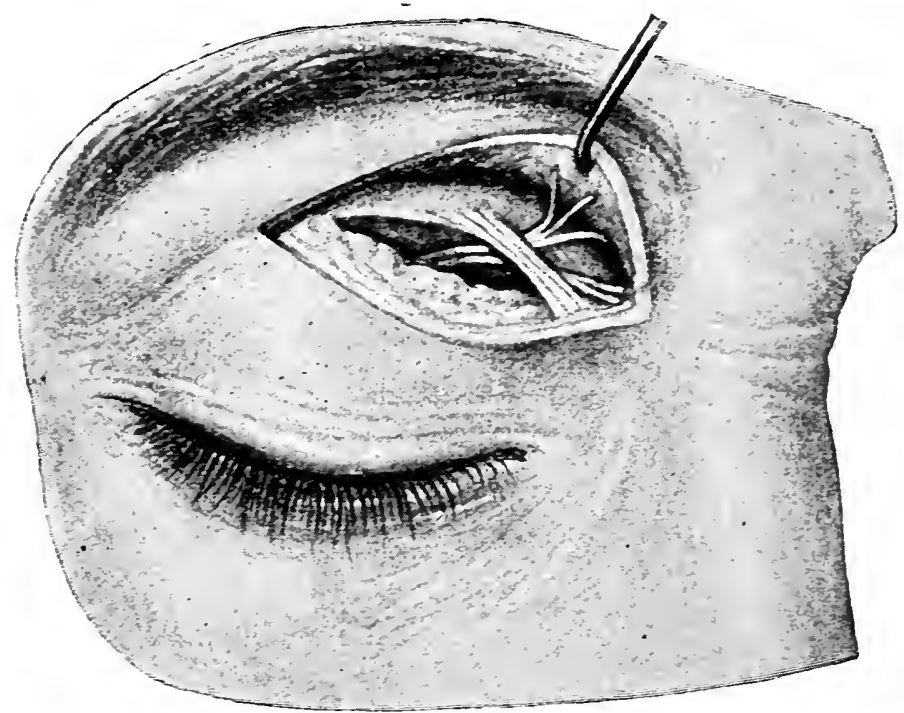

FIG. 174.-Exposure of the frontal nerve.

trunk of the nerve, as it escapes from the infra-orbital foramen, as well as its radiation upon the face, comes into view. After division of the inferior tarso-orbital membrane the contents of the orbit are raised upward, when the infra-orbital canal, covered by a thin lamella of bone on the floor of the orbit, is exposed. This lamella is removed as deeply as possible, the nerve grasped, and its central extremity gradually withdrawn (Fig. 175). The peripheral portion of the nerve can be removed to its finest branches by being rolled up upon Thiersch's forceps. 
If the infra-orbital nerve is to be exposed at the base of the skull, aceess to it will be gained by temporary resection of the malar bone (Lossen, Braun). An incision through the integument is made as in exposing the infraorbital nerve, and it is extended outward over the malar prominenee. The malar bone, together with the malar process of the upper jaw and the zygomatic ridge of the sphenoid bone, is resected in such a manner that a large portion of the outer wall of the orbit is removed. The malar bone is drawn outward, if it is divided also through its temporal process, and the nerve is grasped at the foramen rotundum and extracted with Thiersch forceps.

Third (Inferior Maxillary) Division of the Fifth Nerve. - The third division of the trigeminal nerve leaves the skull through the foramen ovale. Of the upper group of its branches only the buceinator nerve is subjected to surgieal attack. This nerve burrows through the external pterygoid muscle and passes along the outer surface of the buccinator muscle to the angle of the mouth. The nerve is at times the seat of isolited neuralgia. Of the larger branches of the inferior maxillary nerve, the inferior dental and the lingual are of surgical importance. The inferior dental nerve, the largest branch of the inferior maxillary, passes downward between the internal and external pterygoid muscles. On the inner aspect of the ramus of the lower jaw it enters, with the artery of the same name, into the dental foramen and passes through the dental canal, to make its exit at the mental foramen as the mental nerve. The lingual nerve in the first part of its course passes downward with the dental nerve. At the anterior border of the internal pterygoid muscle it turns forward, and passing over the mylohyoid muscle reaches the lateral border of the tongue.

Extrabuccal Exposure of the Buccinator. Nerve (E. Zuckerkandl).-A cutaneous incision is made in the direction of a line passing from the tragus to the middle of the nasolabial fold. The duet of Stenon appears in the wound and is drawn downward. After division of the 
masseter fascia the buceal pad of fat comes into riew, and is freed from its attachments and removed. In the wound there are now visible the coronoid process of the lower jaw, with the prominent lower portion of the tendon of the temporal muscle. At the inner border of this tendon, surrounded by loose cellular tissue, lies the trunk of the buccinator nerve.

The inferior dental nerve may be exposed before its entrance into the dental canal, within the canal, and after its exit at the mental foramen. Prior to its entrance into the canal, at the lingula, the nerve is accessible from without (Sonnenburg-Lücker) as well as from within the carity of the mouth (Paravicini). Sonnenburg makes an incision around the angle of the lower jaw, separates the insertion of the internal pterygoid muscle from the bone, and advances along the inner surface of the ramus of the jaw to the lingula, where the nerre is grasped with a blunt hook, brought out and resected, or extracted with forceps. This method is attended with difficulties in so far as it is necessary to operate at a considerable depth; even operating upon the dependent head simplifies the procedure only in inconsiderable degree. Paravicini has recommended exposure of the nerve from the buccal cavity by separation of the internal pterygoid muscle from the inner surface of the ramus of the jaw at the lingula.

Exposure of the Inferior Dental Nerve within the Dental Canal.-The nerve is most conveniently reached by chiselling ont a piece of the outer plate of the bone at the point of junction between the body and the ramus, and in this manner exposing the dental canal. An arch-shaped cutaneous incision is made at the angle of the jaw. The attachment of the masseter muscle is freed and separated from the bone in the neighborhood of the angle of the jaw by means of a raspatory. In the middle of a line uniting the angle of the jaw with the last molar tooth a piece of bone as large as a lentil is gouged out of the outer wall of the jaw. After the cortical 
structure has been passed profuse arterial hemorrhage from the injured inferior dental artery will indicate that the canal has been opened. With a blunt hook the nerve can be readily raised from its bed, and it is either resected, or, better, it is extrateted with foreeps.

The lingual nerve is most readily reached from the bucal eavity. An incision is made upon the side of the tongue at the point of reffection of the mucons membrane from the inner side of the lower jaw upon the tongue.

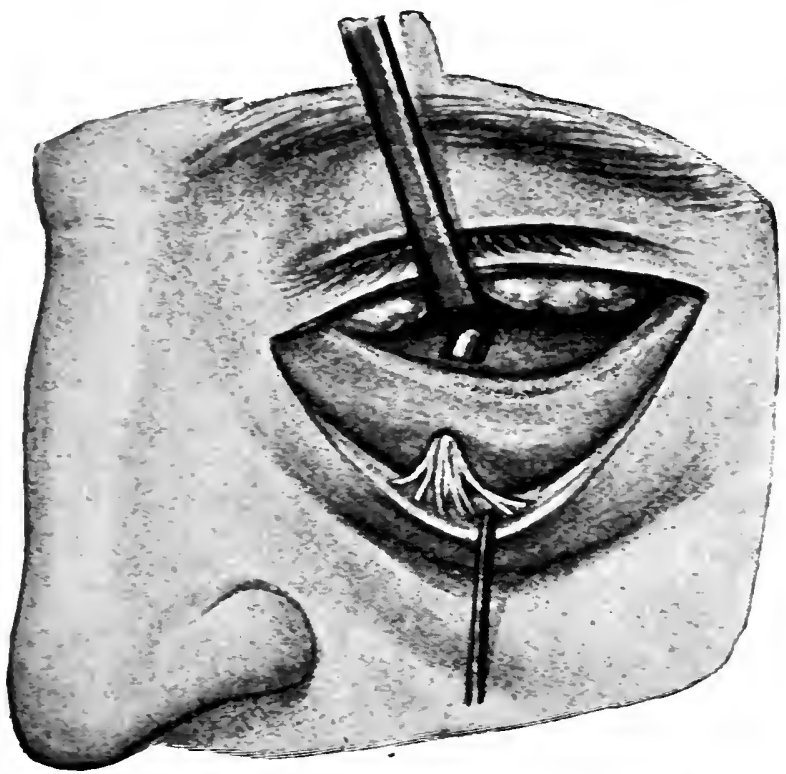

Fig. 175.-Exposure of the infra-orbital nerre.

The large nerve-trunk lies immediately beneath the mucous membrane. The methods of Sonnenburg and Paravicini for exposure of the inferior dental nerve also permit access to the lingual nerve in its upper part.

The mental neive can be made accessible at its point of exit from the lower jaw, from either within or without the buccal cavity. Extrabuccal exposure is accomplished by means of a cutancous incision through the chin at the level of, and several centimeters external to, the incisor 
tooth of the corresponding side. The incision reaches down to the bone, and the soft parts are detached from the jaw, when the nerve can be seen making its exit as a tense cord from the mental foramen. To gain access to the nerve from within the cavity of the mouth an analogous procedure is followed. The incision is made at the point of reflection of the mucous membrane from the

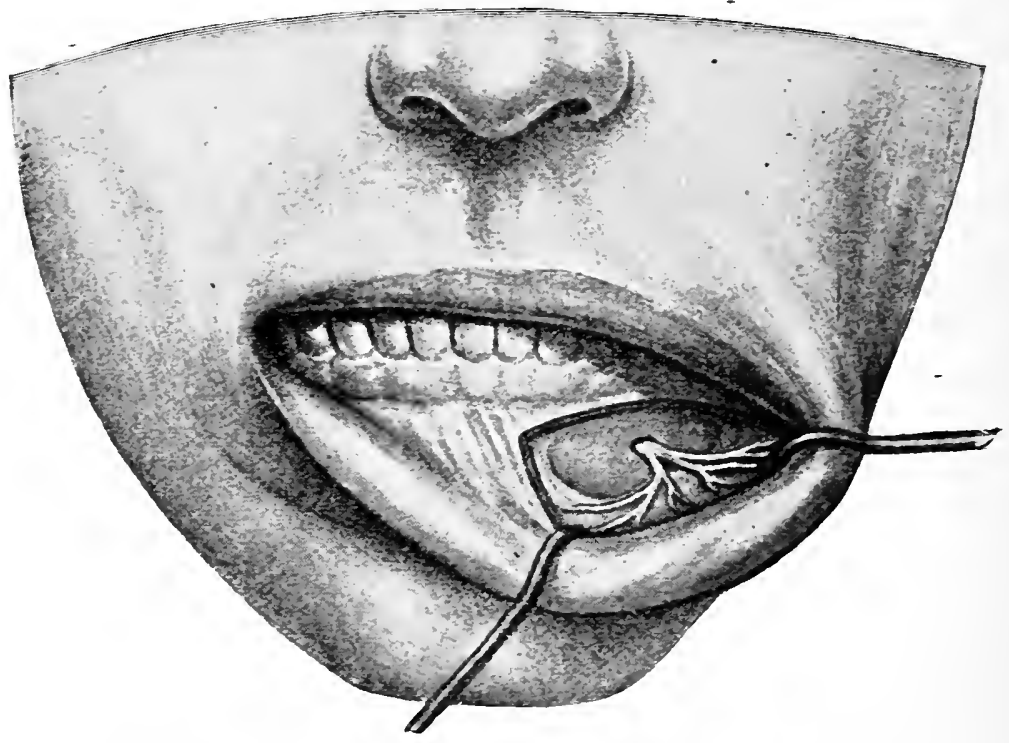

FIG. 176.--Intrabuccal exposure of the mental nerve.

inner surface of the lower lip upon the lower jaw (Fig. $176)$.

Operation for Exposure of the Second and Third Divisions of the Fifth Nerve at the Base of the Skull by the Method of Krönlein.-A semicircular flap, with its convexity downward, is formed from the tissues of the cheek, its base corresponding to the upper boundary of the zygomatic arch. The cutaneous flap is dissected upward, the temporal fascia divided transversely over the malar bone, then the zygomatic arch sawed through in advance of the articular tubercle and through the body of the bone and, with the attachment of the masseter muscle, reflected 
downward. The exposed eoronoid process of the lower jaw is broken through and, together with the tendon of the temporal muscle, is displaced upward. The third division of the fifth nerve is reateded by passing fiom the upper border of the external pterygoid muscle, toward the base of the skull, just behind the root of the pterygoid process, where the formen rotundum is situated.

To reach the supramaxillary nerve progress must be made forward through the wound to the pterygopalatine fossa, where in the depth of the depression the nerve can be grasped with a hook just after its exit from the round canal and drawn forward. In closing the wound the detached segments of bone are fixed in their original situations by means of bone-sutures and the margins of the cutaneous wound are approximated.

Exposure of the Third Division of the Fifth Nerve at the Base of the Skull.-Retrobuceal Method of Krönlein.-A cutaneous incision is made on the eheek from the angle of the mouth to the lobule of the ear. The buccal pad of fat is lifted out of its bed and removed completely, when the coronoid process is divided and dislocated upward. The lingual and inferior dental nerves are exposed and isolated on the inner aspect of the ramus of the lower jaw and followed upward to the base of the brain, while the external pterygoid muscle is drawn to one side. Salzer forms a flap from the tissues of the cheek and the temporal region, with its base downward and extending a finger's breadth beyond the zygomatic areh. The latter is resected and, together with the skin, is reflected downward. The temporal musele, beginning at its insertion, is freed from the bone. Progressing from the outer surface of the temporal bone toward the under surface of the sphenoid bone the foramen ovale is reached, where the nerve is divided with a tenotome having a concave edge. By the method of Mikuliez access to the trunk of the nerve is gained by temporary resection of the lower jaw just in advance of the insertion of the masseter muscle. The cutaneous ineision passes from the mastoid pro- 
cess along the sternomastoid muscle to the cornu of the hyoid bone, and from this point in a curved direction to the anterior border of the masseter muscle.

Intracranial Operations on the Trigeminus. - Extippation of the Gasserian Ganglion by the Method of Krause.-A uterus-shaped flap is formed from the structures in the temporal region (Fig. 141), with its base over the zygomatic arch. The flap is $6.5 \mathrm{~cm}$. long and its base measures 3 or $3.5 \mathrm{~cm}$. Its greatest width is 5 or $5.5 \mathrm{~cm}$. The incision passes down to the bone. The cranium is divided with the circular saw or with chisel and mallet in the shape and size of the cutaneous flap and the skin with the bone attached is reflected downward. The surgeon advances between the bony floor of the skull and the dura in the middle cerebral fossa to the foramen spinosum, where the trunk of the middle meningeal artery is ligated and divided. To this end the brain enclosed in dura must be carefully raised upward with a broad-bladed spatula. After extensive separation of the dura from the bone and elevation of the brain the Gasserian ganglion comes into view, in the depth of the middle cerebral fossa, external to the dura. The ganglion is grasped transversely with Thiersch's forceps at the point where it becomes the trunk of the trigeminus, when the second and third divisions of the nerve are divided in the intracranial openings of the respective canals. The first division of the nerve will have been previonsly divided close to its origin from the ganglion. By rotation of the forceps the ganglion, together with a proximal portion of the trigeminus trunk, will be extracted.

Operations on the Air-passages. - The air-passages may be opened through the larynx (laryngotomy), or through the trachea (tracheotomy). The incisions for opening the respiratory tract are longitudinal, and are made on the anterior aspect of the neck in the middle line. The upper and lower hyoid muscles, which cover the respiratory tract, are arranged symmetrically and meet in the middle line of the neck. Between the inner 
borders of these muscles (white line of the neck) access may be gained to the laryox or the trachea by merely passing through the layers of fisceia and the connectivetissue space.

Opening of the Larynx (Laryngotomy).-The situation of the cartilaginous constituents of the larynx (an be accurately determined from the surface of the neck by palpation. The laryngeal prominenee, the edge formed by the approximation of the lateral halyes of the thy roid calrtilage, the arch of the cricoid cartilage, are accessible to palpation, as woll as the ericothyroid ligament, which appears as a less resistant area between the thyroid and ericoid cartilages. The larynx is opened in the middle line either through the thyroid cartilage (thyiotomy) or through the cricothyroid ligament (cricothyrotomy); finally, the larynx may be divided by a median incision throughout its entire length down to the first tracheal ring (laryngofissure). Thyrotony or laryngofissure is always preceded by tracheotomy and the introduction of a tracheal cammula.

Thyrotomy is undertaken :

(1) In the presence of foreign bodies in the larynx which have become impacted and cannot be removed by endolaryngeal procedures;

(2) In the presence of cicatricial narrowing of the larynx;

(3) In the presence of tumors of the larynx;

(4) As a preliminary operation in the performance of total or unilateral extirpation of the larynx.

Laryngofissure may be necessary in the extirpation of tumors when for their complete removal freer access to the interior of the larynx is required.

In the performance of thyrotomy or laryngofissure the patient is placed in the dorsal decubitus, with the upper portion of the body elevated, and the cervical portion of the spinal column extended. The incision is made accurately in the middle line from the hyoid bone to the ericoid cartilage. The cervical fascia is divided, the sternohyoid muscles are separated, and the thyroid cartilage is 


\section{Plate 13.}

The thyrohyoid membrane, the thyroid cartilage, the cricotbyroid membrane, and the cricoid cartilage are divided in the middle line.

The lateral halves of the thyroid cartilage are held apart by tenacula : the interior of the larsnx, the arytenoid cartilages, the rentricular bands, and the rocal bands are exposed to riew.

exposed. The last is fixed at its lower border with a sharp hook, and a sharp-pointed knife is introduced through the cricothyroid ligament into the lumen of the laryns. Through this opening the thyroid cartilage is divided with scissors, with a blunt-pointed knife, or, if necessary, with bone-scissors, accurately in the middle line upward to the thyrohyoid ligament. The lateral halves of the divided thyroid cartilage are held apart by means of sharp tenacula, exposing the interior of the larvnx to operative attack (Plate 13). In accordance with the nature of the conditions leading to operation, the larynx may be kept open for subsequent treatment. Under other conditions the halves of the thyroid cartilage and the overlying skin are closed by knotted suture. For the first days after the operation, during the process of healing, the entrance of air into the lungs is insured through a cannula introduced into the trachea.

Cricothyrotomy, opening the laryx through the cricothyroid ligament, can if necessary be executed in the shortest possible time by reason of the superficial situation of the ericothyroid ligament. On this account cricothyrotomy is the operation preferred to prevent acute suffocation following occlusion of the larynx by a foreign body, in cases of laryngospasm, etc. A short cutaneous incision is made from the middle of the prominence of the thyroid cartilage downward beyond the cricoid cartilage (Fig. $177, d)$. If delay be dangerous, the lower extremity of the cricoid cartilage may, after division of the skin, be grasped and held securely with a sharp tenaculum passed through the ligament. If the operator has convinced himself by touch with the finger as to the position of the 
(ial). 13.

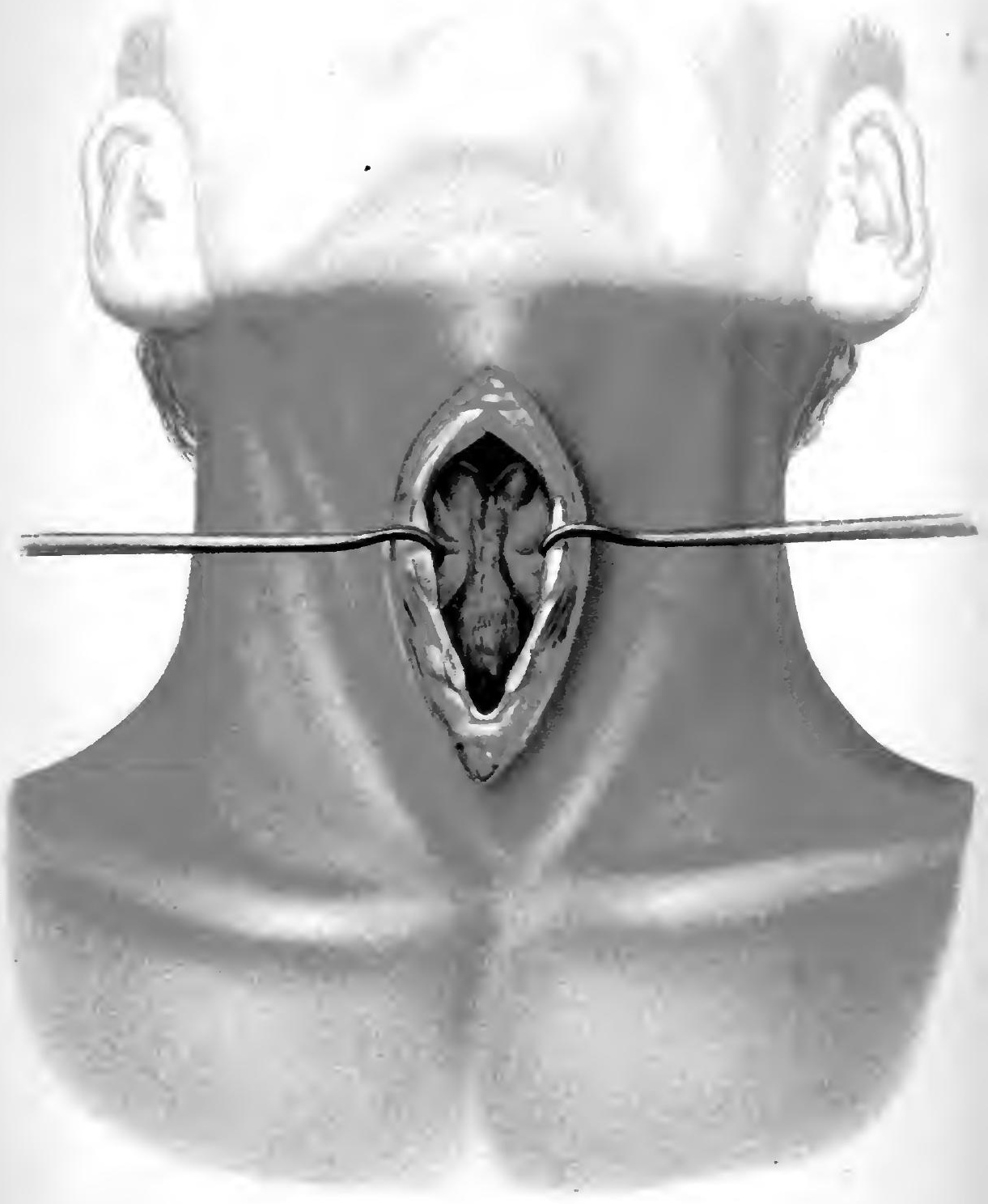



ligament, this is cautionsly ineised in the middle line with a sharp-pointed knife until a sensition is conveyed through the instrument of having entered the lumen of the larvin. At once the opening in the ligament is made to gape by means of a hook inserted on either side, and if necessary the ligament is, further, notehed. The camnula is introduced into the opening thus made and the hooks are removed from the wound. By this mode of procedure there is no loss of blood and the camnula lies so snugly within the wound as to constitute a sort of tampon. If there be sufficient time for the performance of the operation the cutaneous incision is made longer and the ericothroid ligament is laid bare by careful dissection. After the subeutaneous connective tissue has been passed the cerrieal fascia is divided and the cricothrroid ligament is exposed. The lower border of the cricoid cartilage is raised up by means of a simple sharp tenaculum, which is introduced into the middle line, when the ligament is divided vertically and a cannula is introdueed. If necessary, the longitudinal incision in the ligament is notched on the right and the left. Through the crucial incision thus formed the camnula is readily introduced. The latter is held in place in the wound by means of linen tapes attached to the shield of the cannula, and tied at the nape of the neck.

Extirpation of the Larynx.-If removal of the larrnx is to be conjoined with laryngofissure, to the longitudinal incision a transverse incision is added at the level of the hroid bone, when, after division of the muscular attachments to the lower surface of the hyoid bone, the thyrohyoid membrane is divided in correspondence with the superfieial transverse incision. The soft parts on the outer side are to be separated close to the laryngeal cartilages. Upon the posterior aspect the cricoid cartilage is fieed from its loose attachment to the anterior wall of the esophagus. If the upper and lower boundaries of the larrnx also have been incised upon the mucous surface, the lirrnx is completely freed after transverse division of the trachea 
below the larynx. The deficiency left in the anterior portion of the pharynx and esophagus is reduced to a minimum by suture. The cannula is left in the trachea. The wound remains open and is tamponed.

Tracheotomy.-Tracheotomy consists in properly opening the trachea through incision. The procedure is in all cases attended with the introduction of a cannula through the opening into the trachea.

Indications:

(1) Injuries of the larynx (punctured, incised, and gunshot-wounds, fractures of the laryngeal cartilages with dislocation of the fragments).

(2) The presence of foreign bodies in the trachea which cannot be removed by endolaryngeal procedures.

(3) Stenosis of the larynx and the trachea:

(a) Compression-stenosis (goitrous tumors, aneurysms);

(b) Occlusion-stenosis (obstruction of the lumen of the larynx or the trachea, swelling of the laryngeal mucous membrane-diffuse submucous laryngitis; tuberculous, syphilitic, and typhoid disease of the larynx). Narrowing of the lumen of the larynx, or of the trachea, through exudates (diphtheric croup), through neoplasms (carcinoma, papilloma, granulation-tumors);

(c) Cicatricial narrowing of the larynx (after healing of ulcerative processes; after operative procedures upon the larynx.

(4) As a preliminary operation, or in conjunction with. other operations upon the larynx and the pharynx, tracheotomy is performed:

(a) To prevent the entrance of blood into the bronchi (tampon-cannula);

(b) Following operations upon the larynx, without leaving an opening or with closure by tampon, in order to supply the patient with air.

(5) Asphyxia or intoxication, to render possible and to institute artificial respiration.

The trachea is the direct continuation of the larynx, passing in the middle line of the neck toward the upper 
aperture of the thomx. The upper portion of the trachea lies immediately bencath the superfieial structures of the neck. The suprasternal portion is scparated from the skin, in addition to the two layers of cervical fascial, by a considerable layer of cellular tissue containing numerous veins. The thyroid gland overlies the trachea between the thire and sixth cartilaginous rings with its lateral lobes connected by the isthmus. Often a pyramiclal lobe of the thyroid gland covers also the upper portion of the trachea. The anterior surface of the trachea and of the thyroid gland is covered by the museles passing from the sternum to the hyoid bone and the thyroid cartilage (sternohyoid, sternothyroid). In the middle line, between the museles, a strip of trachea is covered only by the cervical fascia. It is through this "white line of the neck" that the trachea is attacked. The isthmus of the thyroid gland divides the trachea into two parts, a suprathyroid and an infrathyroid. The opening through the former constitutes superior tracheotomy; that through the latter, inferior tracheotomy.

Superior Tracheotomy. - The patient lies in the dorsal decubitus, with the neck over-extended, and a cylindrie pillow is placed beneath the shoulders. The operator stands upon the right side of the patient and his assistant upon the opposite side. The cutaneous incision is made aecurately in the median line of the neck from the middle of the thyroid cartilage to below the thyroid gland. After the skin and the subcutaneous connective tissue have been passed the tense fascia of the neek is divided upon a grooved director. The inner borders of the sternohyoid muscles come into view, and are retracted symmetrieally with blunt hooks. The situation of the trachea is retermined by palpation with the finger, and its first cartilaginous ring is exposed by detaching the cellular tissues from the trachea by means of two pairs of anatomic forceps. The field of operation is extended throughout a sufficient extent by incising the layer of fascia stretehed between the upper border of the thyroid gland 


\section{Plate 14.-Inferior Tracheotomy.}

The wound is bounded laterally by the sternohyoid muscles. The trachea is exposed and opened upon its anterior aspect for the introduction of tho cannula. Venous branches (middle thyroid reins) are seen passing cownward from the thyroid gland. Lying close to the right of the trachea in the depth of the wound is the innominate artery.

and the trachea, and disloeating the gland downward by means of blunt hooks. Before proceeding with the opening of the trachea the upper rings must be thoroughly exposed by dissection. Then the trachea is grasped jusi below the cricoid eartilaginous ring accurately in the middle line with a simple sharp tenacuium, raised somcwhat and held fixed in this position. The trachea is then incised accurately in the middle line from below upward for a distance of about $1 \mathrm{~cm}$. with a sharp-pointed knife. The opening thus made is distended by means of sharp tenacula and possibly nicked on either side. While the three tenacula are held, undisturbed, in place, the ope- rator introduces the cannula into the trachea. The eutaneous wound is reduced in size br knotted sutures and the cannula is firmly fixed in place by means of tapes.

Inferior Tiacheotomy.-The patient is placed in the same position as in the performance of superior tracheotomy and a cutaneous incision is made from the lower border of the thyroid gland to below the suprasternal fossa (Fig. 177, c). After the skin and the subcutaneous connective tissue have been penetrated the superficial layer of the cervieal fascia is exposed and divided upon a grooved director in the direetion of the cutaneous incision. A considerable layer of loose connective tissue is passed through by means of two pairs of anatomic foreeps, while the inner border of the sternohyoid muscle on either side is retracted. In the dense laver of connective tissue the middle thyroid veins pass vertically downward to the left innominate vein, and must be aroided or possibly ligated in two places and divided between. During the progress of the blunt dissection the situation of the trachea, toward 
Tab. 14.

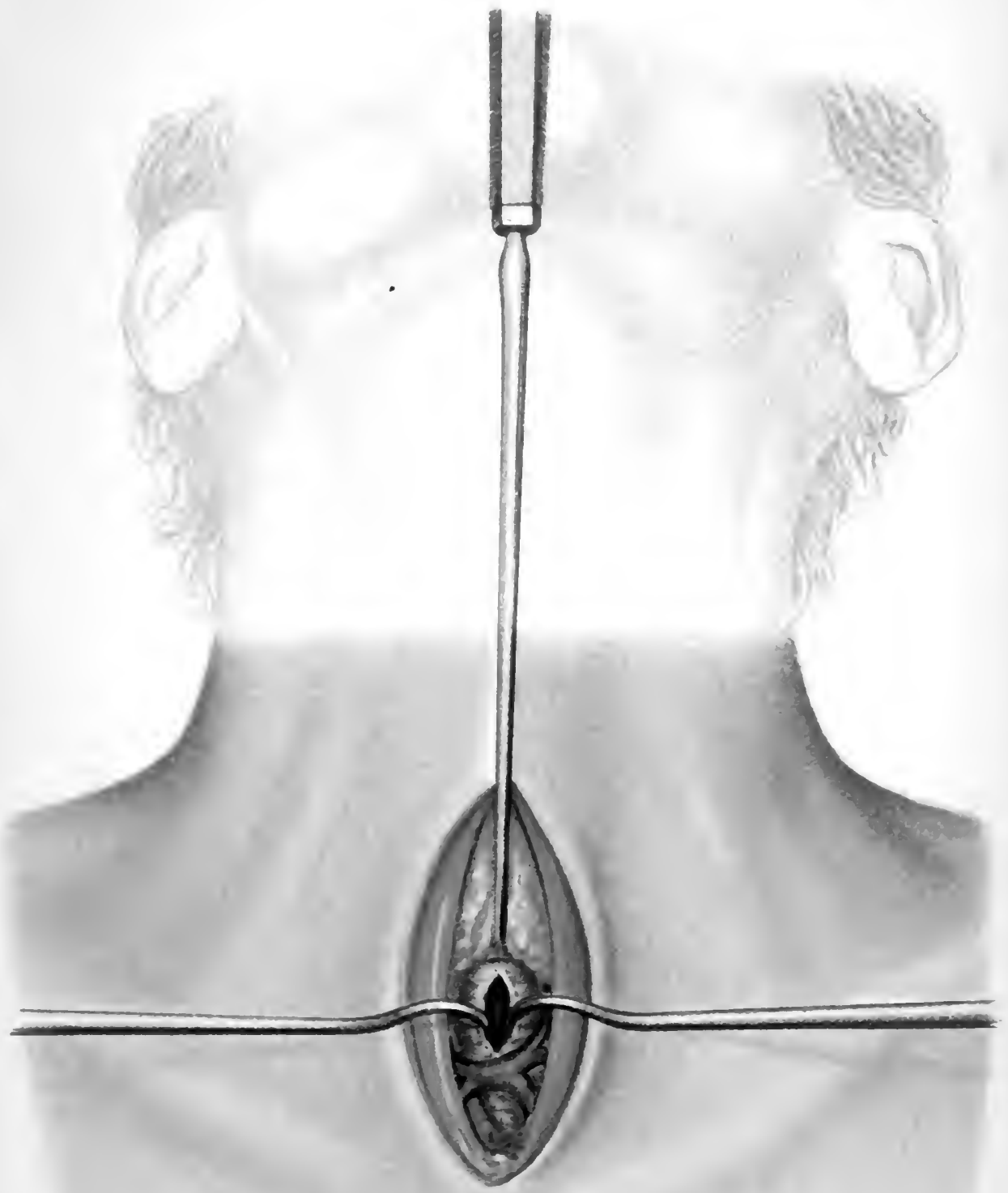


the convex aspect of which the operation proceeds, should be constantly kept in mind by palpation with the indexfinger. Before the trachea is reached the deep layer of the cervical fiscia is divided upon a grooved director. Only after this has been done is it possible to isolate the treachea adequately. Before the lamen of the tube is opened the trachea is grasped with a simple sharp tenacu-

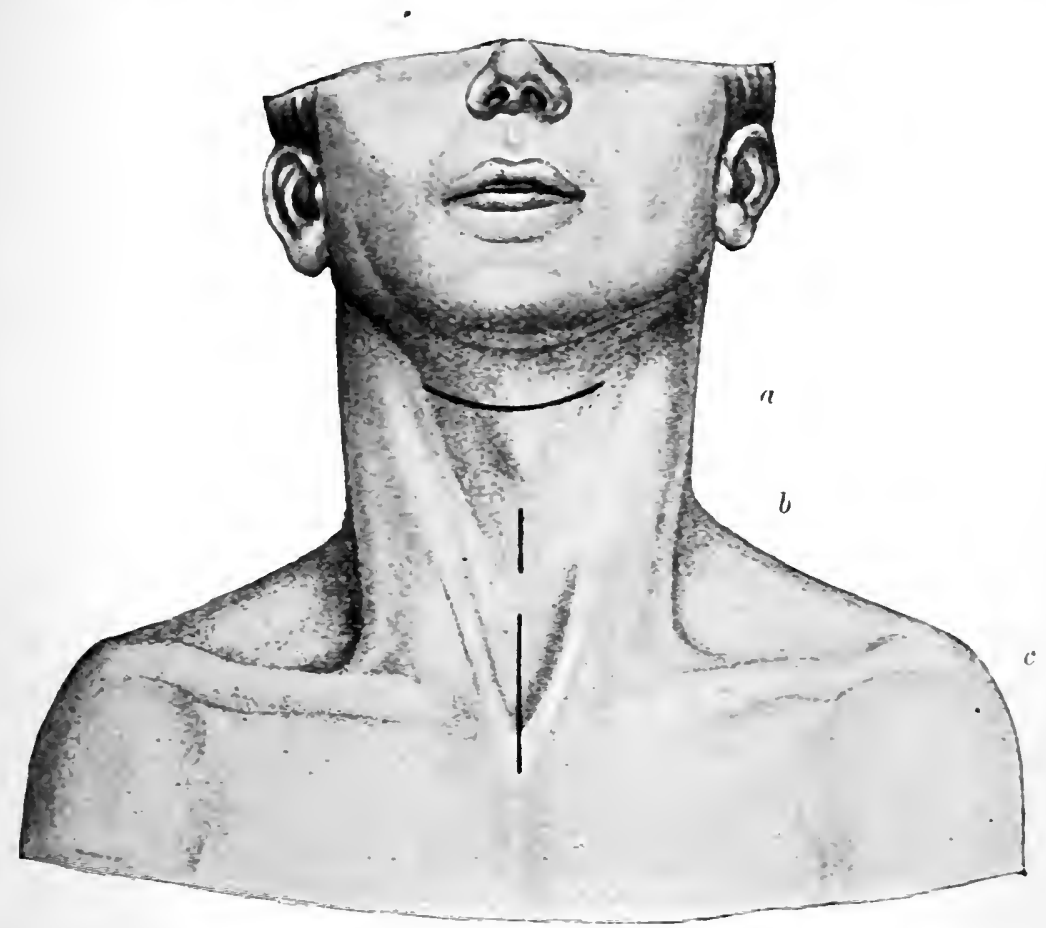

FIG. 17\%.-Cutaneous incisions on the neck: a, infrahyoid pharyngotoms; $b$, cricothyrotoms; $c$, inferior tracheotomy.

lum and raised and fixed at the level of the skin. White the trachea is incised from below upward the index-finger of the left hand is placed in the lower angle of the wound behind the suprasternal noteh, so that the left innominate vein, which passes transversely across the trachea behind the manubrium of the sternum, as well as the innominate artery, which is in elose relation with the trachea, is suf- 
vieiently protected. The tracheal wound is held widely open by means of sharp tenaeula, possibly ineised to right and left, when the introduction of the cannula is undertaken (Plate 14).

After the cannula has been introduced into the trachea the tenacula are removed. The cannula is fixed by means of tapes and the cutaneous wound is reduced by suture. If the tracheotomy can be performed at leisure and under favorable conditions, the typical mode of procedure is unattended with difficulty. The reverse is the case, however, if the operation must be undertaken in the presence of threatened danger to life or of serere dyspnea. Under these cireumstances all of the presence of mind of the operator will be required to maintain the mastery of the situation, which is often a critical one. The smallest veins of the neck are dilated and distended with blood. In the presence of conditions like these the cutaneous ineision is enlarged, as by this means the isolation and ligation of the veins are considerably facilitated. The thin walls of the distended veins are not readily recognizable. Veins that interfere with deep dissection are ligated in two places and divided between. At successive stages of the dissection the position of the trachea is constantly kept in mind. Neglect of this precaution may lead to overlooking the trachea. Before the trachea, previously exposed sufficiently, is opened, all bleeding ressels. are closed by ligature. A tenaeulum is introduced into the trachea for the purpose of placing the organ at rest at the level of the wound, as it would otherwise rise and fall with the respiratory movements, especially in the presence of dyspnea. The opening into the trachea should be made exactly in the middle line, eare being taken that the incision enters the lumen of the tube and does not pass beyond. If the opening is incomplete, it may happen that the tracheal cannula makes a false passage for itself beneath the mucous membrane. A careless incision may, further, injure the posterior wall of the trachea or even the esophagus. After the trachea has been opened the 
incision is dilated by means of tenacula, while at the same time as the cannula is introduced the trachea is held steadily. The latter precaution is important, as through its neglect the opening may be lost to view in consequence of the movements of the trachea. Apart from the fact that such an event may render impossible the proper introduction of the camnula, subcutaneous emphysema may result and extend from the wound to the cellular tissue of the neck.

The cannula (Fig. 178) in aceordance with its curvalture is introduced in an arched manner. The whistling sound

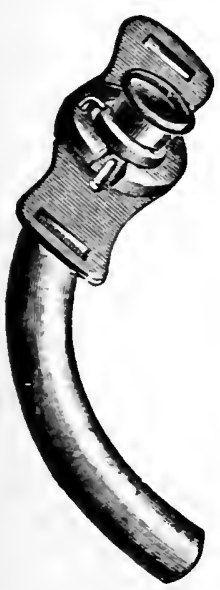

FIG. 178.-Tracheal cannula.

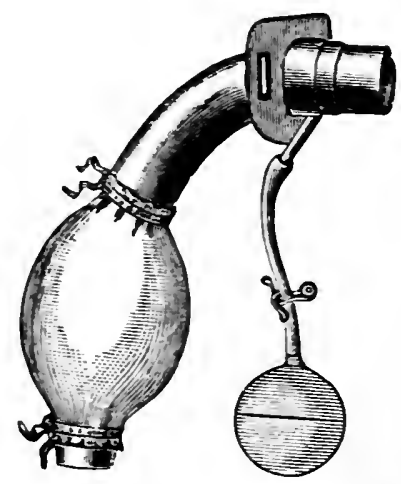

FIG. 179.-Trendelenburg's tamponcannula.

with which the air, after a short period of apnea, escapes from the tube is the indication that the camnula is properly placed. In fixing the cannula by means of the tapes the tube must be held firmly in the wound.

Tracheotomy for the purpose of tamponade of the trachea, with simultaneous insurance of access of air, is sometimes practised as a preliminary procedure in operations upon the mouth, the larynx, and the pharynx. The tampon-cannula is intended to prevent the entrance of blood in the course of operations and the aspiration of 
secretion from wounds in the further progress of the case. The so-called tampon-cannula employed for this purpose is surrounded with compressed sponge (Hahn), which swells in the trachea and completely occupies its lumen; or, the tube is surrounded by a small rubber bag (Trendelenburg), which can be filled with air by means of bellows (Fig. 179). The bag is distended with air after the cannula has been introduced, and adapts itself accurately to the interior of the trachea, occluding its lumen as a stopper does the neck of a flask.

Intubation.-Intubation is a bloodless procedure intended to render the larrnx patulous in the presence of respiratory obstruction by the introduction of a rigid tube. The operation was recommended a number of years ago as a substitute for tracheotomy in cases of laryngeal stenosis from croup, and it has in the course of time secured more and more supporters.

The most important indication for intubation consists in laryngeal stenosis such as is observed in conjunction with laryngeal croup. Further indications are afforded by the various forms of chronic stenosis of the larrnx observed in adults. Under these conditions intubation is a substitute for tubage. Intubation has been recommended also as a palliative measure in cases of whooping-cough and of laryngeal spasm. The procedure is contraindicated :

(a) In the presence of complete occlusion of the nasopharyngeal space ;

(b) In the presence of intense edema of the glottis;

(c) In cases of diphtheria complicated by retropharyngeal abscess.

The original outfit of O'Dwrer is still the best, in spite of numerous modifications. This consists of :

(1) A mouth-gag (Fig. 180).

(2) A series of metallic tubes of varying size (Figs. 181 and 182). Each tube presents at its upper extremity a shoulder resembling the rim of a hat, by means of which it rests upon the vocal bands. Upon the left side of this shoulder is a small opening for the attachment of a thread. 
Fach tube is further provided with a conductor intended to facilitate the gruidance of the rigid tube.

(3) An intubitor (Fig. 18:3), to which the conductor spoken of is attached by means of a serew. Tube and conductor should fit accurately. By means of a lever the tube ean be detached from the eonductor at the proper moment.

(4) An extubator (Fig. 18t). The extremity of this instrument, which is constructed similarly to the intubattor, can be introduced into the lumen of the tube, and be impacted there, and thus serve for the removal of the tube.

O'DWYER'S UUTFIT FOR INTtBATION.

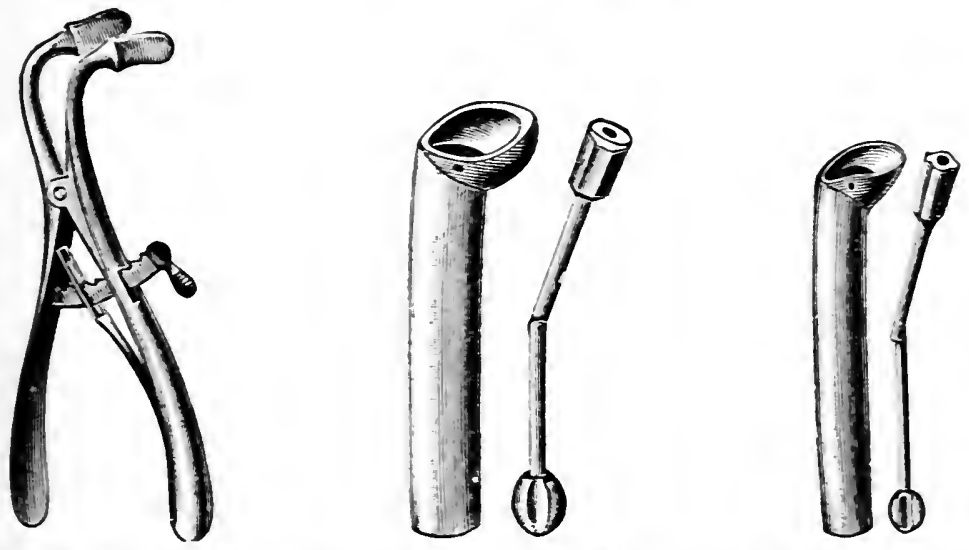

FIr. 1S0.-Mouth-gag. FIG. 181 and Fig. 152.-Tubes with conductors.

The operation is performed as follows:

A nurse takes the ehild to be intubated upon her lap, grasps its lower extremities between her knees, and with her right hand holds its head, and with her left, its hands. An assistant holds the mouth open by means of the gag, while the operator grasps the epiglottis with the indexfinger of his left hand and draws it forward so that the entrance to the larynx is elear. The intubator, adapted to the corresponding tube, is now introduced alongside the finger. If after a slight movement upwarl it is certain that the tube has entered the larynx, the former is then 


\section{Plate 15.-Infrahyoid Pharyngotomy.}

Preliminars inferior tracheotomy has been performed and a cannula introduced. In the pharyngotomy-wound can be seen the stumps of the divided hroid muscles, as well as the hyoid bone $(H)$.

The ejiglottis $(E)$ is drawn out of the wound and the aryepiglottic folds $(A e)$ are made tense. The floor of the wound is constituted by the posterior wall of the pharsux.

pushed gently onward, detached from the intubator, either with the finger of the left hand or by means of slight pressure forward upon a sliding arrangement comnected with the handle of the instrument, and with the indexfinger of the left hand forced deeply into the larrnx. If the child breathes freely, the thread attached to the tube may be permitted to remain, being brought out of the

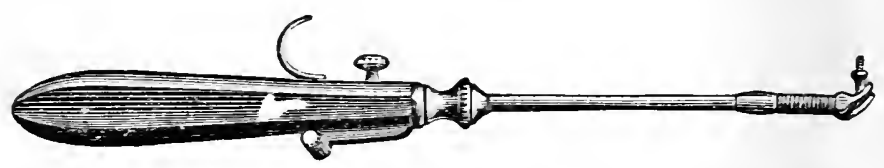

Fig. 183.-Intubator.

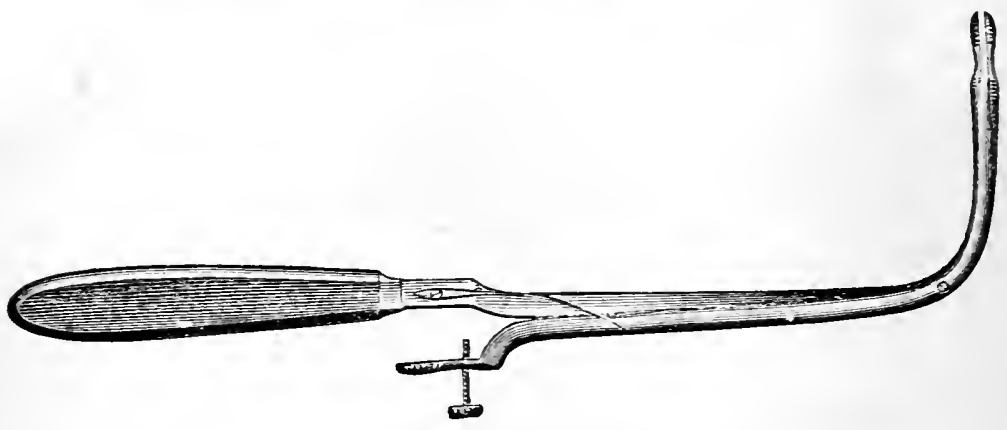

FiG. 184.-Extubator.

mouth and attached to the cheek by means of adhesive plaster, or the index-finger is again introduced into the mouth, the tube held in place, and the divided thread slowly removed.

Extubation is effected in much the same manner. Under the guidance of the index-finger of the left hand the extubator is introduced into the mouth and its closed 
I'ab. 15.

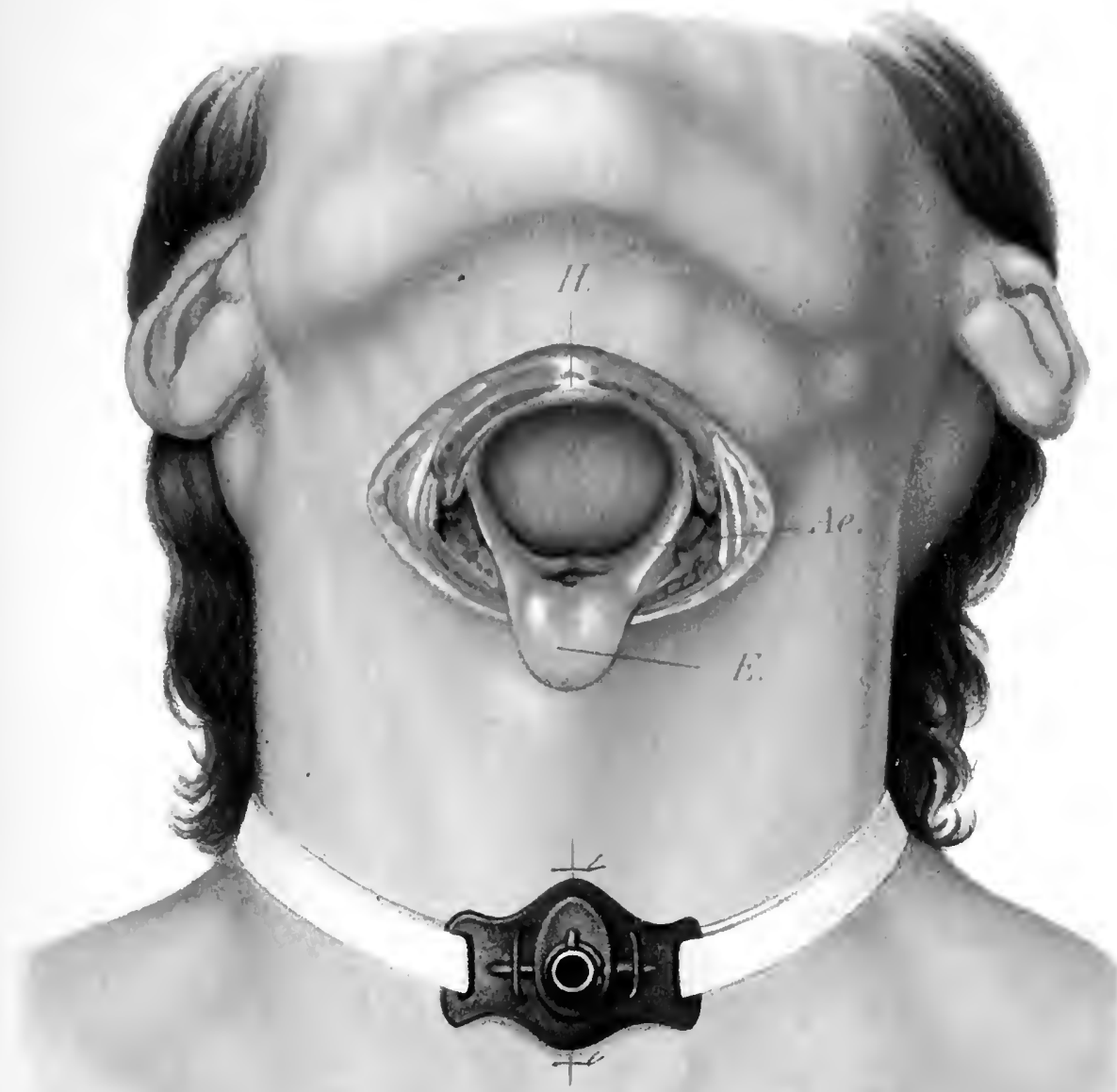



blades are passod into the lumen of the tube. By pressure upon the upper portion of the instrument its two blades are separated and the tube ean thus be carefully removed.

In most cases of intubation the use of the mouth-gag can be dispensed with. Under such eireminstances the index-finger of the left hand must be protected by a metallic band.

Pharyngotomy.-The pharynx ean be opened in its laryngeal portion by transverse division of the thyrohyoid ligament. By this means the pharyngolaryngeal space is exposed to view. This mode of opening the pharynx and the larynx (Malgaigne's infrahyoid laryngotomy) is suitable for the performance of surgical operations upon the epiglottis, in the larynx, and upon the pharynx.

Infrahyoid pharyngotomy is performed:

For the removal of foreign bodies from the larynx and the pharynx;

For the extirpation of tumors of the epiglottis, the larynx, and the pharynx;

In the treatment of cicatricial strictures of the esophagus.

Steps of the Operation.-The patient is placed in the dorsal decubitus with the cervical portion of the spinal column overextended, and preliminary tracheotomy is performed. By means of palpation with the finger the position of the hyoid bone and of the thyroid cartilage is determined. The cutaneous incision is made transversely over the thyrohyoid ligament parallel with and close to the body of the hyoid bone (Fig. 177, a). After the cervical fascia has been opened and the stemohyoid and thyrohyoid muscles have been divided transversely the membrane is exposed to view. It is detached from the posterior surface of the hyoid bone and the pharynx is opened in the middle line at the upper border of this bone. By this method only is the epiglottis protected, falling downward after division of the hyo-epiglottic ligament, when also the lateral portions of the thyrohyoid ligament may be 
divided with a single stroke of the scissors. The epiglottis, the aryepiglottic folds, the vocal bands, the interior of the larynx, as well as the laryngeal portion of the pharynx, are exposed for possible therapentic intervention (Plate $15)$.

When tumors are seated in the deeper portions of the pharynx Langenbeck recommends an incision from the middle of the ramus of the jaw over the greater cornu of the hyoid bone to the cricoid cartilage. The lingual and superior thyroid arteries, as well as the facial vein, are exposed and divided between two ligatures. The digastric and stylohyoid muscles are detached from the hyoid bone, when the pharynx is opened in the direction of the cutaneous incision.

External Esophagotomy.-Opening of the esophagus in the neck is indicated:

(1) In the presence of foreign bodies in the esophagus;

(2) In the presence of impermeable high stenoses of the esophagus, from carcinoma or cicatricial stricture, for the formation of a mutritive fistula beyond the narrowed situation ;

(3) In the presence of cicatricial strictures more deeply seated:

(a) For the performance of dilatation through a fistula established;

(b) For the performance of internal esophagotomy from the wound (Gussenbaner's combined esophagotomy).

The esophagus is opened just below the pharynx, beyond the cricoid cartilage, where it lies behind the trachea and projects upon its left side. The inferior laryngeal nerve, which passes from below upward between the trachea and the esophagus, must be protected. The patient is placed in the dorsal decubitus, with the cervical spine overextended and the head directed toward the right. The cutancous incision is made along the anterior border of the sternomastoid muscle as in the operation for ligation of the common carotid artery. The sheath of this muscle is opened and the muscle itself retracted out- 

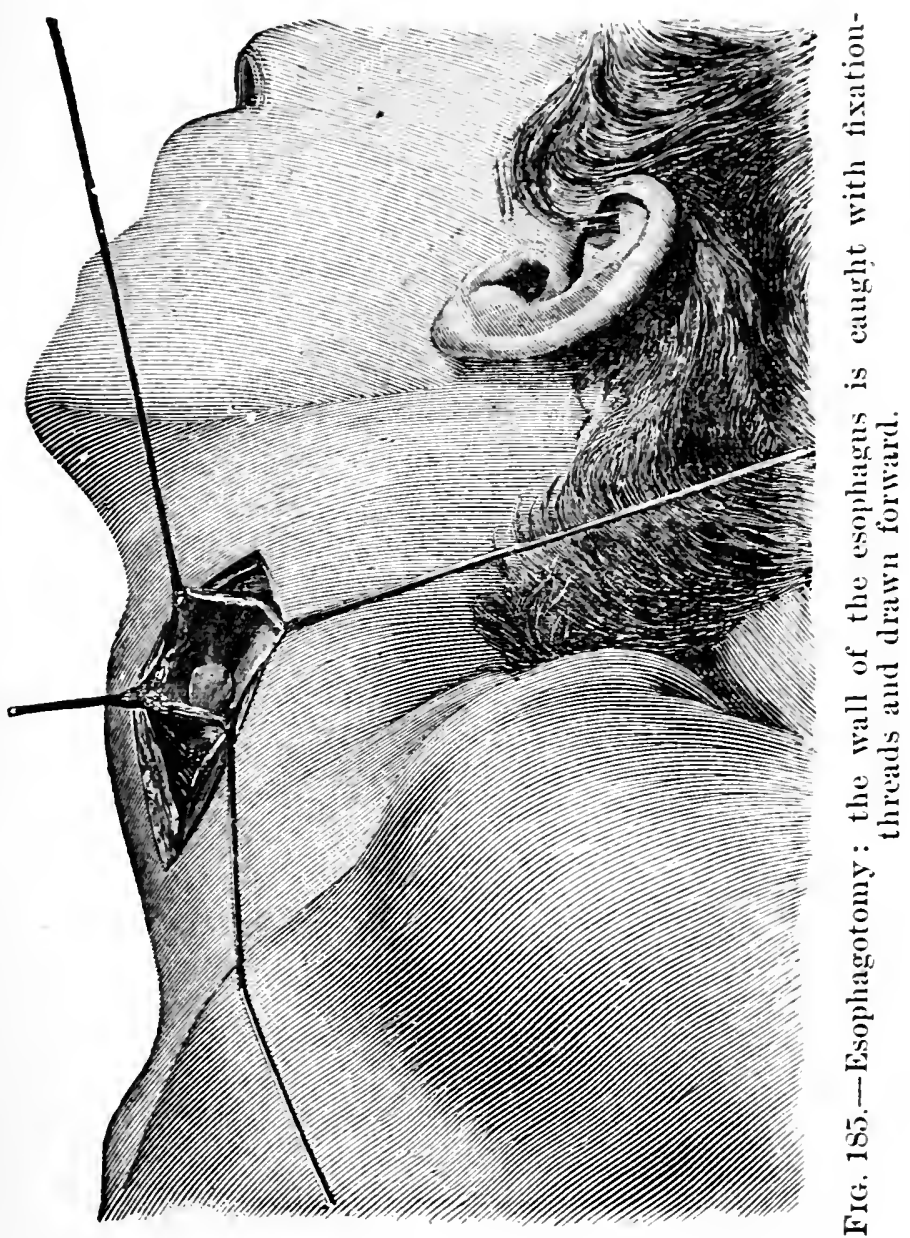

ward. The deep layer of the cervical fiscia is divided upon a grooved dircetor and the whole mass of ressels and nerves is likewise displaced ontward with blunt hooks. If the thyroid gland is at the same time dislocated inward, the trachea comes into view and, behind it, projecting somewhat beyond its left border, the esophagnes, which is to be recognized by its different color and the longitudinal arrangement of its fibers. The gullet is grasped with fixation-threads, drawn to the level of the wound and opened in correspondence with the cutaneons incision (Fig. 185). If the operation has been so performed that the wound in the esophagus can be closed for primary union-for instance, after extraction of foreign bodiesthe wall of the esophagus is approximated with knotted sutures in two tier's. The first row of sutures approximates the mucous membrane and the second the muscular layer. If the operation has been performed for the purpose of establishing an esophageal fistula-for instance, for dilatation of a stricture of the esophagus - the margins of the mucous membrane are united to the skin by means of knotted sutures.

\section{Ligation of Vesse1s in the Cervical Region.-}

Innominate Artery.-The innominate artery, the common trunk of the carotid and the right subclavian artery, arises from the arch of the aorta. Lying against the trachea, the large ressel passes to the right and upward, dividing into the two ressels named at the level of the sternoclavicular articulation. Covered by the manubrium of the sternum, the imnominate artery is accessible from the suprasternal fossa behind the free border of the sternohyoid or of the sternothyroid muscle. The trunk of the vessel is crossed by the left innominate rein as it passes transversely. The recurrent laryngeal nerve winds around the innominate artery. In ligating the ressel the patient occupies the dorsal decubitus, with the neck extended. According to Graefe, the cutaneous incision is made along the anterior border of the sternomastoid muscle in such a way that its lower extremity extends beyond the sternal 
attachment of the musele. The sheath of the muscle is opened and the median faseia of the neek divided, when the inner border of the sternohyoid beeomes visible and below this that of the sternothyroid. These museles are retracted with blunt hooks. Along the right side of the traehea progress is made downward, the common earotid artery being first reached and further on the innominate, lying by the side of the trachea. The artery can be isolated from the surrounding loose cellular tissues by blunt dissection and it is then ligated.

The same plan of procedure is followed in looking for the vessel through an ineision made vertically in the middle line of the neck over the suprasternal noteh, instead of the incision of Graefe.

Carotid Artery.-The carotid artery on the right is a branch of the innominate, while upon the left it arises directly from the areh of the aorta. The common carotid artery on either side passes along the side of the trachea and the larrnx almost vertically upward upon the neck to the level of the thyroid eartilage, where it divides into its primary branches, the internal and the external carotid. In its eourse the earotid artery holds such relations with the jugular vein, the vagus nerve, and the descending branch of the hypoglossal nerve in the loose cellular tissue that the vein lies to the outer side of the artery. The vessels are eovered by fibrous fascia that also constitutes the posterior wall of the sheath of the sternomastoid musele. To render the artery accessible it will thus be necessary to expose and retract the fibers of the sternomastoid muscle and to divide earefully the posterior wall of its sheath.

Ligation of the Common Carotid Artery.-The patient occupies the dorsal decubitus, with the neck stretehed and the head rotated toward the healthy side. By palpation the situation of the larynx and the course of the sternomastoid muscle are determined. The artery is best exposed at the level of the erieoid cartilage just above the point where it is erossed by the omohyoid musele. The cutaneous incision is made along the anterior border of the 
sternomastoid from the thrroid cartilage for a distance downward of 8 or $10 \mathrm{~cm}$. (Fig. 186,b). After the skin and the platrema muscle are divided the sternomastoid muscle, covered by the fascia, comes into view. The fascia is divided in the direction of the cutaneons incision and the inner border of the exposed musele is amrefinly retracted outward, when the so-called middle fascia of the

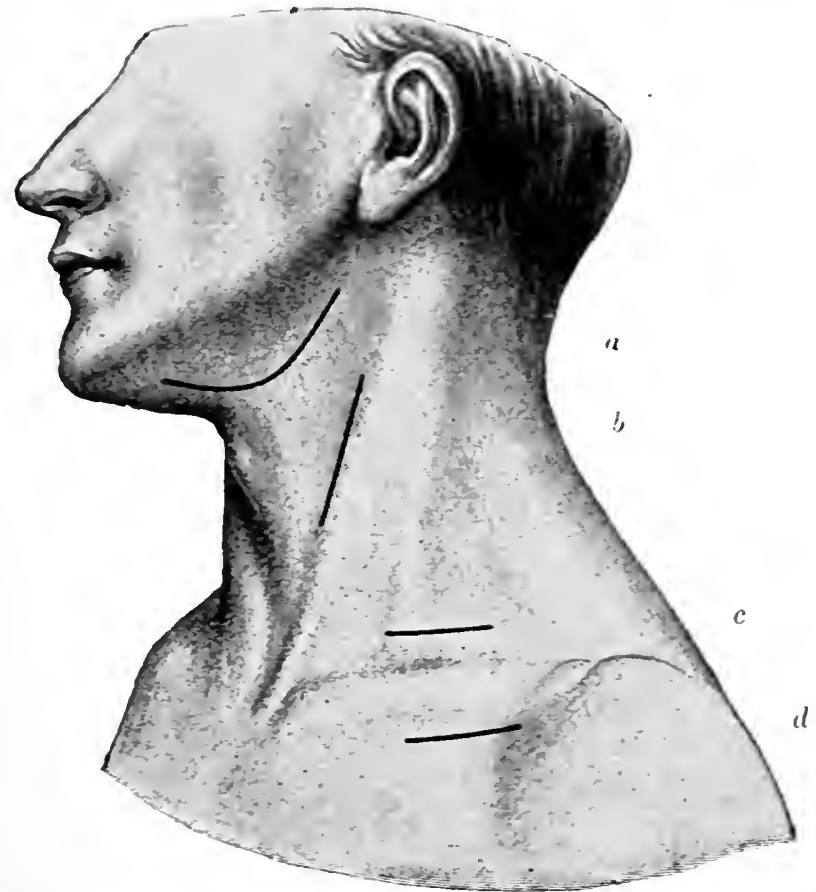

FIG. 1S6.-Ligation of the vessels of the neck: cutaneous incision : $a$, lingual artery ; $b$, common carotid artery ; $c$, subclavian artery above the clavicle; $d$, subclavian artery below the clavicle.

neck and the omohyoid muscle come into view. Above this musele the fascia is divided carefully upon the grooved director and the artery isolated by blunt dissection and raised ont of its bed. To the outer side of the artery lies the internal jugular vein, and between the two vessels the vagus nerve.

Ligation of the External Carotid Artery.-The patient 


\section{Plate 16.}

(1) Exposure of the carotid artery in the neck. The sternomastoid muscle $\left(K^{*}\right)$ is retracted outward; the deep layer of the cerrical fascia $(F . c$.$) is divided, and the common carotid artery (C)$, the jugular vein $(J)$, the vagus nerve, and the descending branch of the hypoglossal nerveare thus bronght into view. The bifurcation of the common carotid into the internal and external carotid is also discernible. The origin of the thyroid from the external carotid, which in the illustration is situated abnormally far ontward, has been freed by dissection.

(2) Exposure of the subclavian artery below the clavicle. The pectoralis major muscle $(P)$ is divided in the direction of the cutaneous incision to the deltoid muscle $(D)$. Below the former the subclavian artery $(A)$ is visible between the vein $(V)$ and the branchial plexus of nerves $(P)$.

is placed in the same position as in ligation of the common carotid artery, and the incision through the skin is made along the anterior border of the sternomastoid muscle, with its upper extremity at the level of the hyoid bone. After the skin, the platysma muscle, and the superficial fascia have been divided the common facial vein comes into view and is retracted toward the middle line by means of blunt hooks. The trunk and the branches of the external carotid artery are exposed by blunt dissection in front of the greater cornu of the hyoid bone between the omohyoid muscle and the posterior belly of the digastric muscle.

An incision like that described suffices also for the exposure of the large branches of the external carotid artery, the external maxillary (facial), the lingual, and the superior thyroid, at their points of origin, for purposes of

- ligation. Of the branches of the external carotid, the superior thyroid and the lingual artery are of surgical importance.

The superior thyroid artery arises from the trunk of the external carotid, just above the bifurcation, at the level of the hyoid bone, and it passes in an arched direction downward to the thyroid gland. To ligate the ressel a cutaneous incision is made along the anterior border of the 


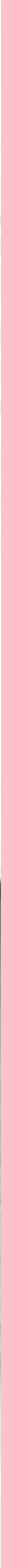




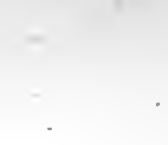

\section{$\sqrt{2}+x^{2}$}


sternomastoid muscle from the hroid hone to the thrroid rartilage. After division of the skin, the platysma miscle, and the cervical filcial the ressel, passing downward in an arched direction, is foumd at the greater cornu of the hroid bone.

The limgunl artery arises from the extromal carotid at about the level of the hyoid bone, and it passes in a slightly curved direction forward and upward, entering the substance of the tongue under corer of the hyoglossus muscle.

Ligation of the Lingual Artery. An arched incision is made from the angle of the jaw to the hyoid bone, thence ascending from this point almost to the chin (Fig. $186, a)$. After division of the skin and the platyma muscle the superticial laver of the cervical fascia comes into view and beneath this the submaxillary gland is visible. The fascia is slit open at a point corresponding to the lower marerin of the gland, which is raised from its bed by blunt dissection and retracted upward. Corered by the deep laver of the cervical fascia may be seen the shining tendon of the digastric muscle curring in an arehed manner, and, above, the hypoglossal nerve, accompanied by a rein, passes horizontally. The free border of the mylohyoid muscle forms with the tendon of the digastric miscle and the hypoglossal nerve a triangle whose floor is constituted by the fibers of the hyoglossus muscle. To expose the lingual artery the fascia covering this lingual trigone is first divided. Then the fibers of the hyoglossus muscle ascending vertically from the hroid bone to the tongue and forming the floor of the triangle referred to must be separated by blunt dissection, when, if the laver of muscle has been passed, the artery becomes visible on the floor of the triangle, and can be ligated.

Ligation of the Subclavian Artery.-The subclavian artery, on the right a branch of the innominate artery and on the left arising directly from the arch of the aorta, lies at its origin at the apex of the pleural cavity. It leaves this cavity through the upper aperture of the thorax, and 


\section{Plate 17.-Exposure of the Lingual Artery.}

The submaxillary gland ( $\mathrm{m}$ ) is raised from its bed after division of the skin and the fascia; the lingual trigone is thus rendered risible. It is bounded by the tendon of the digastric muscle ( $B$, the outer border of the mylohroid muscle ( $M h)$, and the lispoglossal nerve $(H)$, which is accompanied by a rein. The floor of the triangle is formed by the hyoglossus muscle ( $H g$ ), the fibers of which are separated within the triangle, and the artery $(L)$ is thus rendered visible.

reaches the anterior surface at the fir:t rib in the interval between the scalenus anticus and medius muscles (posterior scalene interval, Fig. 187). From this situation it de-

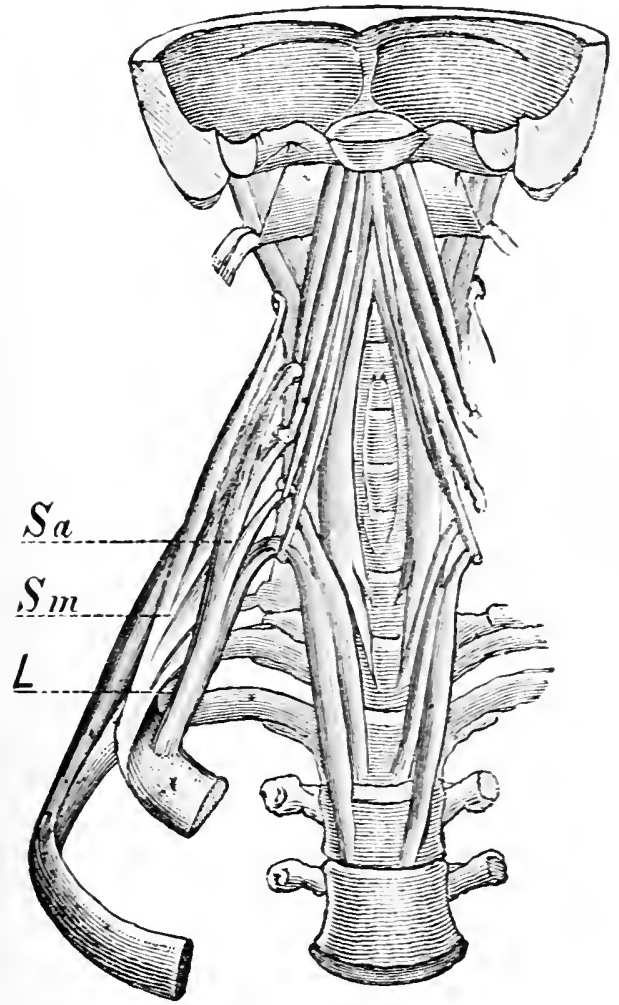

Fig. 157.-Posterior scalene interval $(L)$, between the scalenus anticus ( $(S a)$ and the scalenus medius (Sin) muscle.

scends toward the arm. The point at which the artery crosses the first rib is marked by a slight elevation, the 


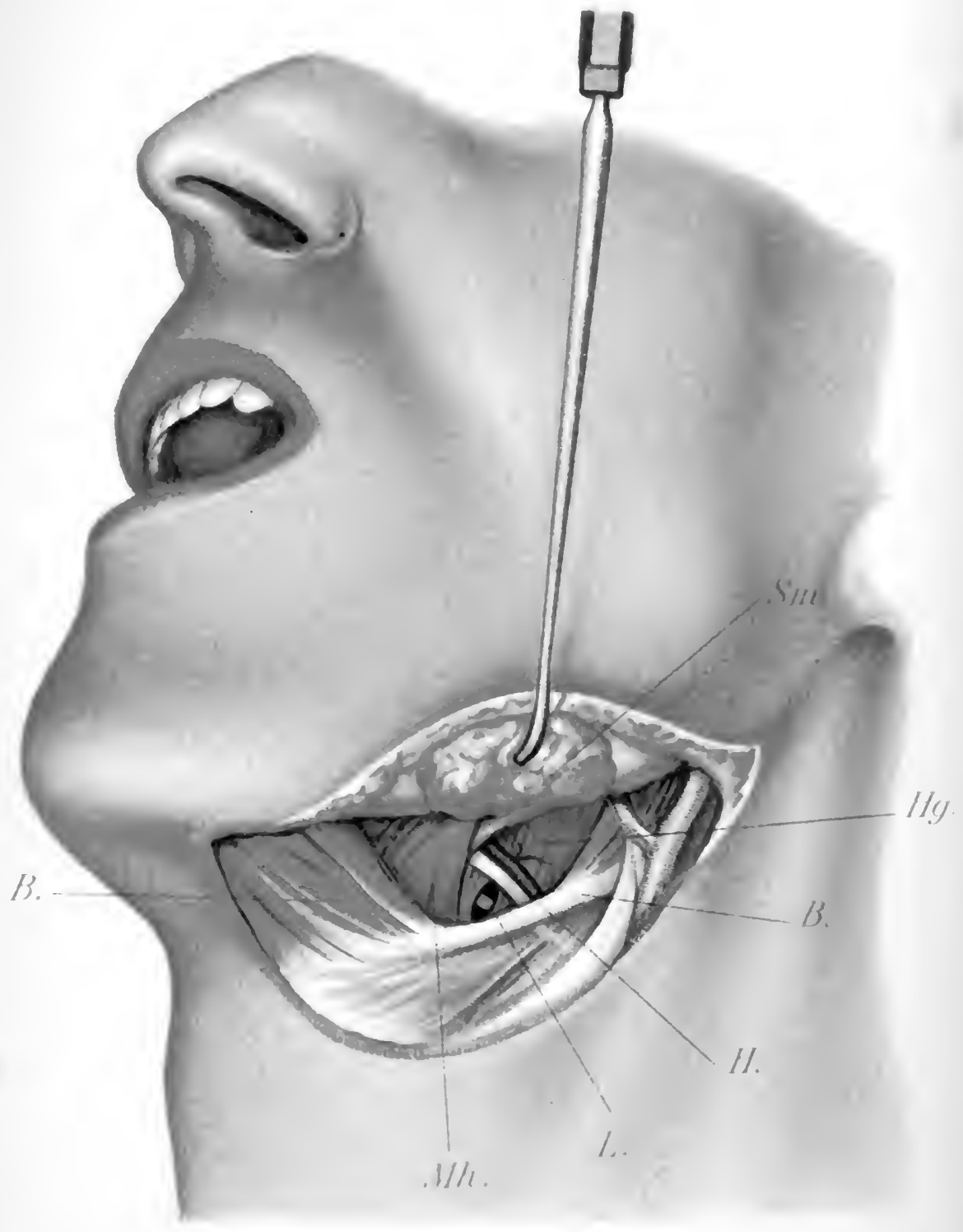


tuberele of Lisfranc or the scalene tubercle. The cords of the brachial plexus also reach the amm through the interval between the two salene muscles. The nerves lie above and to the outer side of the artery (Plate 18).

The subclavian vein passes in the interval between the sternomastoid and the sealenus anticus (anterior scalene interval), to unite with the internal jugular vein. The subelavian vein is thus separated from the subclavian artery by the scalenus anticus muscle.

The subclavian artery is exposed for ligation aljove and below the clavicle; in the supraclavicular fossa just at the point where it lies upon the first rib after energing from the scalene interval; below the clavicle at a point corresponding with the lower margin of the first rib.

Ligation of the Subclavian Artery Above the Clavicle.The patient lies with the upper portion of the trunk elevated and the head rotated toward the opposite side. The arm lies against the trunk. Gentle traction on the arm brings into view the boundaries of the supraclavieular fossa. By this means the clavicle can be seen, forming the base of the triangular space, whose anterior boundary is formed by the onter border of the sternomastoid muscle and the posterior boundary by the anterior border of the trapezius. The plane of the supraclavieular fossa is more or less depressed.

A transverse cutaneous incision is made parallel with and a finger's breadth above the elavicle, from the outer border of the sternomastoid muscle to the anterior border of the trapezius (Fig. 186), dividing the skin, the platysma muscle, and the supraclavicular nerves. By blunt dissection a passage is made through the loose connective tissue of the supraclavicular fossa to the deep layer of fascia that covers the scalenus muscles, the brachial plexus, and the subelavian artery. After the fascia has been divided, the position of the posterior sealene interval is made out, and the situation of the artery is determined by palpation with the finger just behind the attachment of the scalenus anticus to the first rib, to the outer side 


\section{Plate 18.--Situation of the Subclavian Artery in the Supraclavicular Fossa.}

The anterior scalene interval is visible between the sternomastoid $(K)$ and the sealenus anticus $(S a)$; also the posterior scalene interval between the sealenus anticus and the scalenus medius (Sm). Through the latter space pass the nerves of the brachial plexus $(N)$, and to the inner sice of the nerves, lying upon the first rib, the artery.

of the scalene tubercle. The artery lies upon the first rib at the deepest point of the interval, to the inner side of the nerves of the brachial plexus, and can be isolated for ligation between two pairs of anatomic forceps.

Ligation of the Subclarian Artery Below the C'lavicle.The patient occupies the same position as in the operation just described. The line of separation of the clavicular portions of the deltoid and pectoralis major muscles is indicated below the clavicle by a triangular depression (Mohrenheim's triangle). By palpation with the finger the situation of the coracoid process of the scapula is carefully determined, and a cutaneous incision is made from a finger's breadth below the clavicle to above the apex of the coracoid process. The clavicular portion of the pectoralis major muscle is divided in the line of the cutaneous incision and after division of the loose coracoclavicular fascia the upper border of the pectoralis minor is exposed and is retracted downward with blunt hooks. In the loose connective tissue below the clavicle there appear in the direction toward the anterior scalene interval above, the readily accessible subclavian vein, and to its outer side the great mass constituted by the brachial plexus. The artery lies between the vein and the nerves, closer to the wall of the thorax, and can be separated from the loose cellular tisstie by blunt dissection (Plate 16). Following another method, entrance is gaineo to Mohrenheim's triangle, after making the same cutaneous incision, and the artery is exposed without division of the pectoralis major. The superficial fascia is divided and after separation of the margins of the pectoralis major and deltoid muscles the 
Tab. 18.

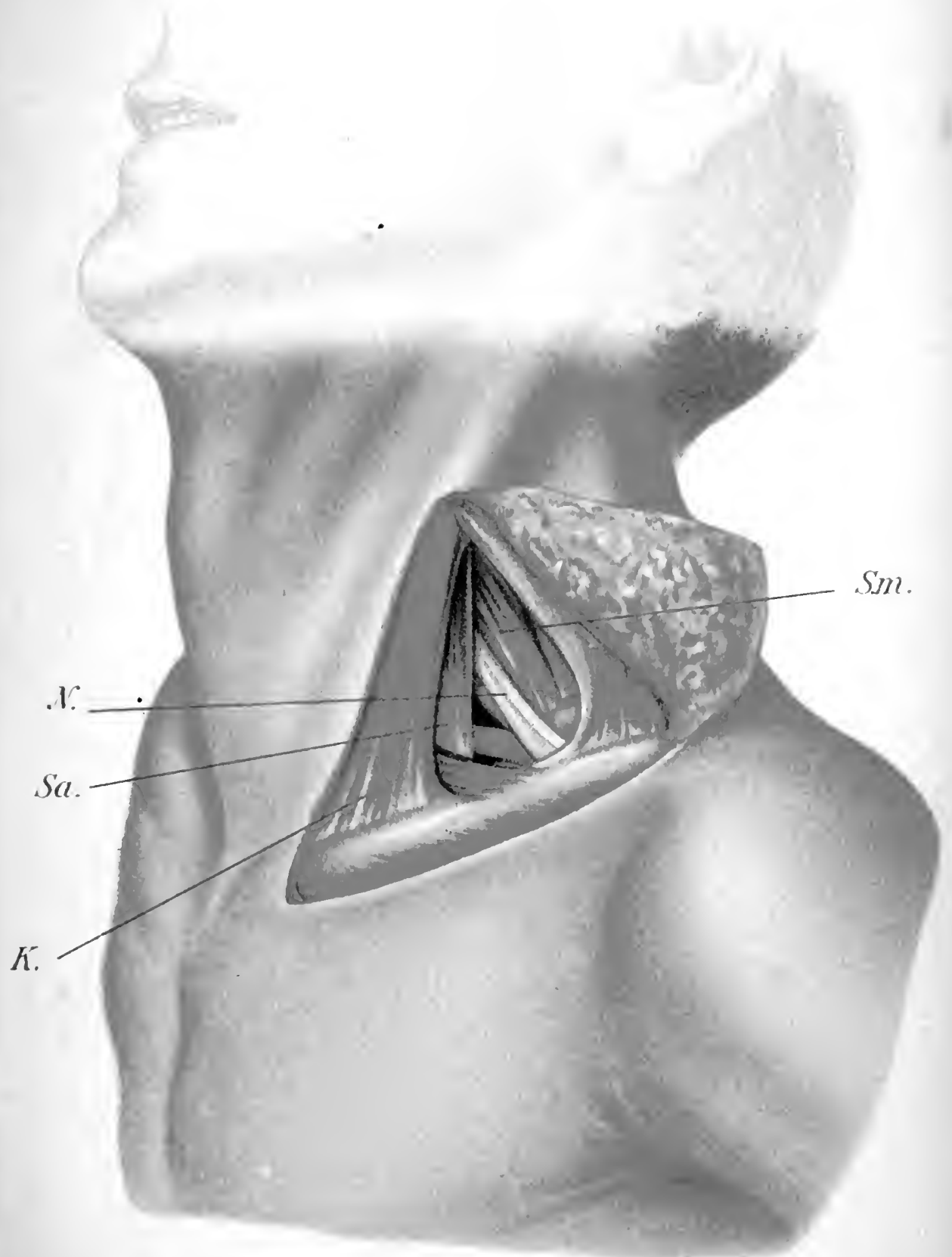


fossa of Mohrenheim is rendered acessible. In the depth of this fossa the mass of vessels and nerves is visible below the elariele after division of the coracoclavicular finscia.

Of the branches of the subclatian artery the following are of surerieal importance: the inferior thyroid artery, which is ligated to bring about atrophy of tumors of the thyroid gland; the vertebral artery, whose ligation has been recommended in the treatment of epilepsy ; the internal mammary artery, whose ligation may becone necessary in connection with contused or punctured wounds of the anterior wall of the thorax.

Ligution of the Inferior. Thyroid Artery.-The artery is exposed for ligation at the point where it lies upon the vertebral column (or rather the longus eolli muscle) and passes behind the common carotid artery in a curved direction upward and inward to the thyroid gland. Kocher has recommended an oblique incision extending upward and outward from above the suprasternal notch along the anterior margin of the sternomastoid. The common carotid artery is exposed and drawn outward. At the inner side of the vessel progress is made between the artery and the thyroid gland drawn toward the middle line down to the spinal column, where the artery will be found pursuing its characteristic arched course. In ligating the vessel care must be taken not to include the inferior laryngeal nerve, the motor nerve of the larynx, which lies in close relation with the vessel.

Operation for Goiter.-As a result of the symptoms developed after total extirpation of the thyroid gland it has been learned that this gland is an indispensable organ of vital importance in the bodily economy. 'Total removal of the diseased thyroid gland, a procedure formerly practised rather commonly, is no longer justifiable in view of recent experiences. The conservative methods that may be employed in the surgical treatment of goiter consist in intraglandular enucleation of the nodule out of the thyroid gland, resection of the gland, and unilateral extirpation of the gland. 
Intraglandular enucleation (Porta, Socin) may be practised in the treatment of cystic goiter, as well as in that of circumscribed, well-limited goitrous nodules. The cutaneous incision is made over the greatest prominence of the tumor, and in accordance with its situation either longitudinally in the middle line of the neck, or transversely in an arched direction with its concarity upward (Kocher). After division of the skin and the platysma muscle the several lower hyoid muscles spread out over the thy roid swelling come into view and are either divided or retracted with hooks, in accordance with the direction of the cutaneous incision. The capsule of the goiter, as well as the healthy parenchyma of the thyroid gland overlying the goitrous nodule, is to be divided, when the nodule or the eyst is removed from its bed by blunt dissection and extirpated. Division of the nodule and isolated removal of its two halves constitute a modification of enucleation recommended by Kocher.

Unilateral Strumectomy.-A longitudinal cutaneous incision is made either in the middle line of the neck, or along the anterior border of the sternomastoid muscle; or an angular incision (Kocher) is begun at the level of the larynx upon the prominence of the sternomastoid muscle, passing transversely to the middle line of the neck, and thence downwarl to the suprasternal notch. The transverse arched incision of Kocher's follows the line of the folds of the neck. After the superficial layer of the cervical fascia has been divided the anterior border of the sternomastoid muscle is isolated and retracted outward. The fascia further is divided in the median line, when the margins of the lower hyoid muscles are freed in the neighborhood of the larynx and divided transversely, so that the goiter is exposed thronghont a large extent of its anterior surface. The capsule is divided and detached from the goiter from within outward, when the latter, if its lateral attachment has been freed, is raised out of its bed and displaced from without inward. After the upper and lower poles of the tumor also have been isolated, the 
prineipal vessels of the thyroid gland, the superior and inferior thyroid arteries, and at the lower pole the middle thyroid vein also, are grasped and divided each between two ligatures. In ligating the inferior thyoid artery care must be taken to avoid the inferior laryngeal nerve, which lies in immediate proximity with the trunk of the artery. The thyroid isthmus, which is rendered accesible by lifting up the lower pole of the goitrons tumor, is freed from the anterior wall of the tracheal by blunt dissection and seeured with two ligatures, between which the isthmus is divided. In order to avoid injuring the reeurrent laryngeal nerve in the process of detaching the thyroid gland from the lateral wall of the trachea Kocher divides the structure of the goiter parallel with the trachea and in this way leaves behind a portion of the capsule of the goiter as a protection against injury of the nerve (resection of goiter). 


\section{OPERATIONS ON THE TRUNK AND THE PELVIS.}

Paracentesis Thoracis, Thoracotomy. - The thorax is opened by puneture or by incision when the presence of accumulations of fluid in the pleural cavity gives rise to threatening symptoms by reason of either their quantity or their character. In general the statement may be accepted that serous and hemorrhagic effusions are to be treated br puneture, and purulent exudates on the other hand by ineision. ${ }^{1}$ Either operation is therefore always preeeded by exploratory aspiration of the pleural contents by means of a hypodermie or similar syringe. The operation of thoracocentesis is performed by the introduction of a trocar and cannula between two ribs into the pleural space, either permitting the fluid simply to escape, or aiding its removal by means of aspiration. If the cannula is so eonstructed that the aspiration of air can be avoided during the removal of the troear, the first method of procedure meets all requirements. Billroth's camnula is provided with a lateral branch for the escape of the fluid, which ean be controlled by a coek. To this branch a rubber tube of suitable length is attached. The branch of the eannula in which the stilet is introduced is also provided with a eock, which is closed after the stilet has been removed. The patient is placed in the sitting posture with the trunk bent somewhat forward. The trocar is introduced, except in the presence of a sacculated effusion, at the most marked convexity of the ribs in the fourth, fifth, or sixth intereostal space, and close to the upper border of the lower rib. The operator marks accurately the point of introduction with the index-finger of his left

I If a hemorrhagic collection is very exteusive and the life of the patient is seriously threatened, it is proper to open the thorax after rib-resection and endeavor to arrest the hemorrhage bs ligatures, br sutureligatures, by packing a small pulmonars wound, or by filling the pleura with sterile gauze, to secure a point of counter-pressure, and packing iodoform-gauze directly against the bleeding lung.-ED. 
hand. The branch of the cammula for the escape of the fluid is closed. Trocar and anmula are introduced vertieally until disappearance of the resistance of the thoracic wall indicates that the point of the instrument has entered the pleural cavity. The operator now grasps the instrument with his left hamd, removes the trocar, and permits the fluid to escape through the lateral branch of the canmula. The extremity of the rubber tube dips into a vessel containing aseptic fluid. The flow shonld take place steadily and slowly. By this mode of procedure the entrance of air is avoided with certainty.

If the discharge of fluid ceases suddenlr, the flow can be facilitated by changing the position of the cannula, if the obstruction be due to approximation of the lung. Oeclusion of the tube by coagula may be overcome by the introduction of a blunt probe.

The evacuation of the fluid can be better controlled when with puncture is conjoined aspiration of the pleural exudate. In place of the trocar and cannula a sharp hollow needle is employed, which is connected by means of a tube with the neck of an airtight bottle from whose interior the air is exhausted with the aid of a suitable pump (Dieulafoy's aspirator). Fluid can thus be evacuated by negative pressure when it would fail to flow spontaneously. from the pressure within the pleural cavity.

Thoracotomy, opening of the pleural cavity by incision, is indicated when the pleural exudate is purulent in character. Unless the exudate be sacculated or circumscribed, the incision is made in the fifth or sixth intercostal space over the greatest convexity of the ribs. To avoid injury of the intercostal ressels the knife is introduced close to the upper border of the rib, dividing the two layers of intercostal muscles, the endothoracic fascia, and the pleura throughout the entire extent of the incision. By the introduction of a rubber tube into the wound drainage of the pleural cavity will be established. To permit of more convenient access and to render possible adequate drainage resection of from 3 to $4 \mathrm{~cm}$. of a rib in its continuity is recommended. Under these circumstances the incision 
is made directly over the rib, dividing its periostenm throughout a distance of 5 or $6 \mathrm{~cm}$. The periosteum is reflected upward and downward by means of a raspatory from the anterior surface of the rib, and then with especial care from its posterior surface. The portion of the rib thus exposed is resected throughout the given extent by means of bone-shears. The uninjured pleura is incised, the purulent contents permitted to escape, and drainage established. If in the presence of a pleural fistula the emprema cannot be made to close on account of the rigidity of the thoracic wall, resection of a series of ribs is a suitable procedure in order to render the wall of the chest more yielding. The possibility thus established of approximating the parietal and visceral layers of the pleura renders the conditions favorable for cessation of the longcontinued and tedious suppurative process. A long, vertical incision exposes the scries of ribs, which are to be subjected individually to subperiosteal resection throughout an extent of from 3 to $10 \mathrm{~cm}$.

Ligation of the Internal Mammary Artery.-The cutaneous incision is made in the third or fourth intercostal space from the border of the sternum outward for a distance of 4 or $5 \mathrm{~cm}$. The skin, the subcutaneous connective tissue, the pectoralis major, and the internal intercostal muscle are divided throughout the extent of the incision. Lying in front of the pleura, in the angle between the rib and the sternum, is the internal mammary artery, which follows the axis of the body and is accompanied by two veins. The ressel can readily be isolated from the loose connective tissue. Esmarch makes a longitudinal incision alongside the sternum and enlarges the field of operation by resection of a costal cartilage.

Removal of the Mammary Gland.-The mammary gland is removed completely when the seat of a malignant neoplasm. With the gland arc also removed en masse the chain of lymph-glands extending from it to the axillary cavity and the mass of axillary lymphatic glands in conjunction with the fat by which they are 
surrounded. The patient acenpies the dersal derubitus, with the upper part of the hody chevated and the arm on the atfected side alducted somewhat abme the horizontal line. Two incisons, formine an wall with its longitudi-

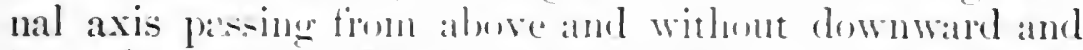
inward, are manle firm the free horeler of the pectrobalis major musele to the cosiform cartilatere inchuliner the mam-

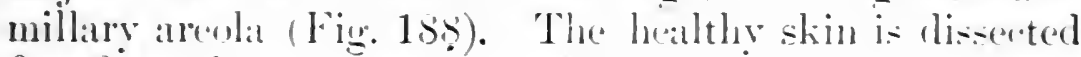
free from the -uljacent structures and when the madroin

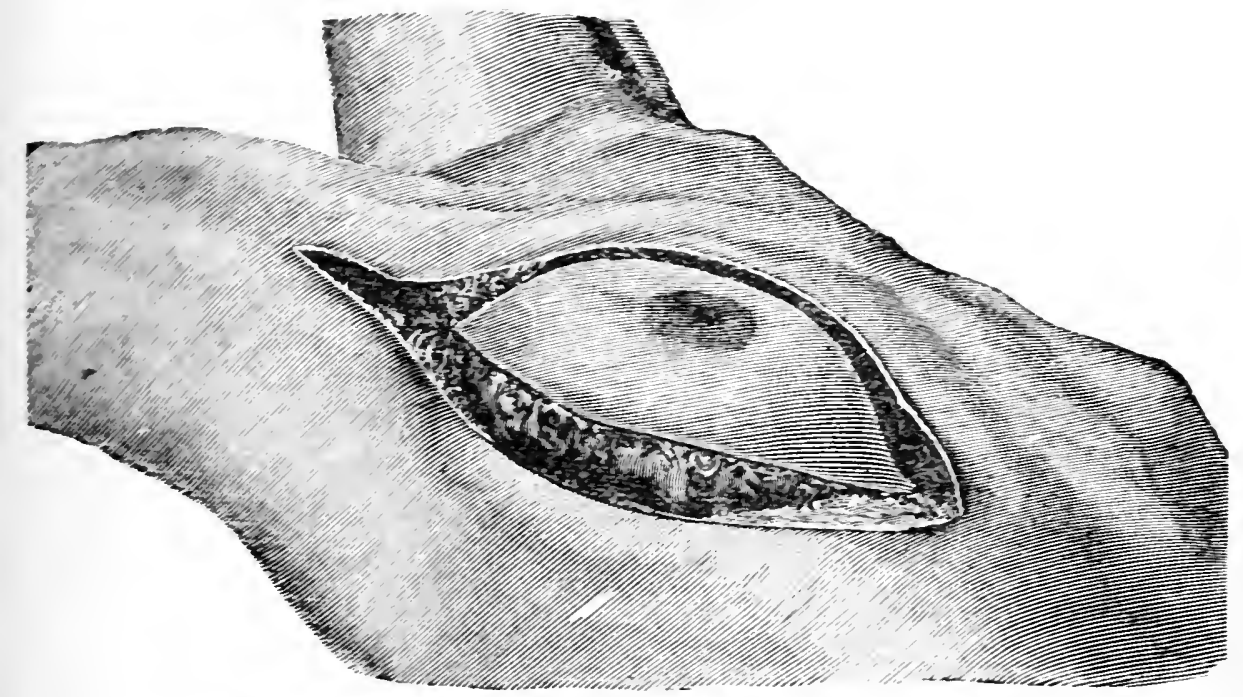

FIG. 1:S.-Amputation of the breast : cutaneous incision.

of the gland has been reached this is remored from the thoracic wall with the ipper layers of the pectoralis major, or in conjunction with the whole muscle. The separation is coffected throughout the entire extent of the mammary gland with the exception of the pole directed toward the axilla. Then the axillary fat and the contained lymphatic glands are removed en masse. The group of glands remains in connection with the breast. From the upper pole of the oval, which is directed toward the axillary eavity, an incision is made along the free 
border of and down to the pectoralis major muscle. The lower margin of the wound is retracted downward, and the pectoralis major upward. Begiming at the pectoralis major, the mass of fat is detached by means of anatomic forceps from the group of large vessels and nerves. Of especial importance in this connection is the large axillary vein, which lies uppermost and whose separation is to be effected with especial care. As the dissection progresses it will become necessary to divide between two ligatures the trunks of arteries and veins passing between the groups of glands drawn downward and the large vessels. After the glands have thus been separated from the large vessels, the subscapularis and latissimus dorsi muscles are yet to be dissected. The connections between the group of glands and these muscles are quickly divided with the knife, when the entire mass of axillary fat nay be removed en masse in conjunction with the breast. Under some circumstances the subscapular artery and vein, the posterior circumflex artery and vein, or the long thoracic artery and vein may require ligation. Küster has called attention to the importance of protecting the long thoracic nerve from injury. If it prove impossible to free the axillary vein, it often becomes necessary to sacrifice a portion of this vessel. After the application of ligatures the vein is resected throughout the necessary extent and removed together with the glands. For the removal of infiltrated glands from the infraclavicular and supraclavicular fossæ accessory operations are necessary. Transverse division of the pectoralis major and minor muscles will render the infraclavicular fossa conveniently accessible. For the removal of supraclavicular glands either an incision is made as in ligation of the subclavian artery above the clavicle, or the clavicle is divided temporarily at the junction of its middle and outer thirds. ${ }^{1}$

1 The operation of Halsted is extensively employed in the United States. In this operation the surgeon removes the entire breast and the skin over it, the axillary glands and fat, and the pectoral muscles. The mass is removed in one piece. In many cases the subclavicular glands and fat are also removed.-ED. 
Abdominal Puncture, Paracentesis Abdominalis.-CThe abdominal eavity may be opened by puneture to effect evacuation of fluid acemmulations, either free within the peritoneal eavity, or saceulated, or contained within cysts. If the Huid be free, the point of Monro, that is, a point midway between the umbilicus and the left anterior iliac spine, is as a rule selected as the situation for puncture. 'Trzebizky has demonstrated that in a small proportion of cases the epigastric artery or one of its branches may be injured in performing puncture by this method. If, however, the trocar is introduced into the outer half of the line between the umbilicus and the superior iliae spine, the possibility of this mpleasant occurrence is safely aroided. The recommendation to make the puncture upon the left side of the abdomen is not of primary importance. If the liver be enlarged, the puncture will be preferably made upon the left side. Enlargement of the spleen of any considerable degree will justify making the puncture upon the right side. The puncture may further be made in the linea alba, midway between the umbilicus and the symphysis pubis. The selection of the point of puncture in the presence of cysts and of sacculated exudates will be governed by the situation of the accumulation of fluid.

In performing puncture of the abdomen a straight trocar and camnula with a lateral branch are employed. The patient occupies a partial lateral position or the upper portion of the body is elevated. Before the trocar is introduced it shonld be determined by careful percussion that the intestine is not adherent to the abdominal wali at the point where the puncture is to be made. The indextinger of the left hand is placed at the point of puncture, and the trocar is introduced vertically through the ab. dominal walls, then grasped with the left hand, while the right removes the trocar. By means of a tube attached to the lateral branch of the cannula the fluid is permitted to escape slowly into a suitable receptacle. If the intraabdominal pressure falls, the escape of the fluid is favored 
by compression of the abdomen with the hand or by tightening a many-tailed bandage around the abdomen. It is an old rule never to permit the escape of all of the fluid contained within the abdominal cavity. The trocar is therefore removed at a time when a certain amount of fluid is yet present, and the wound is closed with a suitable dressing.

Celiotomy.-Opening of the abdominal carity through ineision of the abdominal walls is designated celiotomy. This procedure is a preliminary one in the performance of intraperitoneal operations of all kinds. The abdominal incisions are sometimes made longitudinally, sometimes more or less obliquely, and sometimes even transversely. Longitudinal incisions are made either in the linea alba, or along the outer border of the rectus abdominis muscle. In the epigastrium and the hypogastrium, both oblique incisions parallel with the costal margin, or with Poupart's ligament, and longitudinal and transverse incisions are employed. The incision into the linea alba is indicated in the presence of large formations occupying the abdominal cavity. The incision is made below the umbilieus when the pelvic organs are the object of attack. Through the epigastrium access is gained to the stomach, or upon the right side to the liver and the gall-bladder. An incision is made into the hypogastrium when it is intended to reach upon the right the cecum or the vermiform appendix, and upon the left the descending colon or the sigmoid flexure (Fig. 190). In the performance of intraperitoneal operations the patient is either placed horizontally or the body is placed upon an inclined plane with the head at the lowest and the pelvis at the highest level (Trendelenburg's position, Fig. 189). This position affords a clear view of the arrangement of the pelvic organs after the abdominal cavity has been opened, the intestines sinking down toward the epigastrium in the concavity of the diaphragm. The position therefore permits careful inspection of the abdominal viscera and protects the intestines from extrusion during the course of the operation. 
Mode of Making the Incision thromgh the Abrtominal Walls.-Throngh the lineal albat, as in other portions of the abdominal wall, dissection is effected layer by layer with the sealpel. The skin and the subentaneous conneetive tissue are divided and aceess is gained to the dense fibrons upper layer of the sheath of the reetus muscle or between the two rectus muscles. As a rule, the modian borders of the recti museles are exposed within the wound. After division of the posterior layer of the sheath of the rectus a layer of loose comnective tissue comes into view, and in obese persons a liyer of fit often of considerable

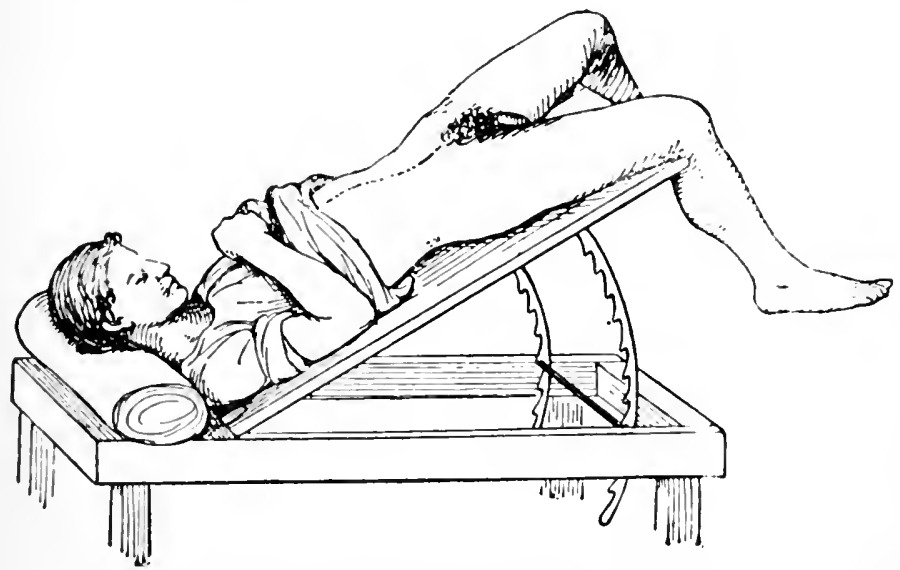

Fir. 189.-Trendelenburg's position.

extent lying directly upon the peritoneum. All of the tissues are earefully divided by blunt dissection with two pairs of forceps. A fold of parictal peritonem is picked up and opened at one point and the incision is enlarged above and below throughout the extent of the superficial wound by means of scissors or a blunt-pointed knife. Longitudinal, ollique, or transverse incisions in the epigastrium extend also, like those in the hypogastrium, successively through the layers of the abdonimal muscles to the subserous fat and the peritoneum. A fold of the parietal peritoneum is picked up with two pairs of forceps 
and snipped with scissors, and the incision is enlarged in the manner already described. The closure of the abdominal wall should be firm and resistant; the resulting cicatrix should display no tendency to ectasis and the

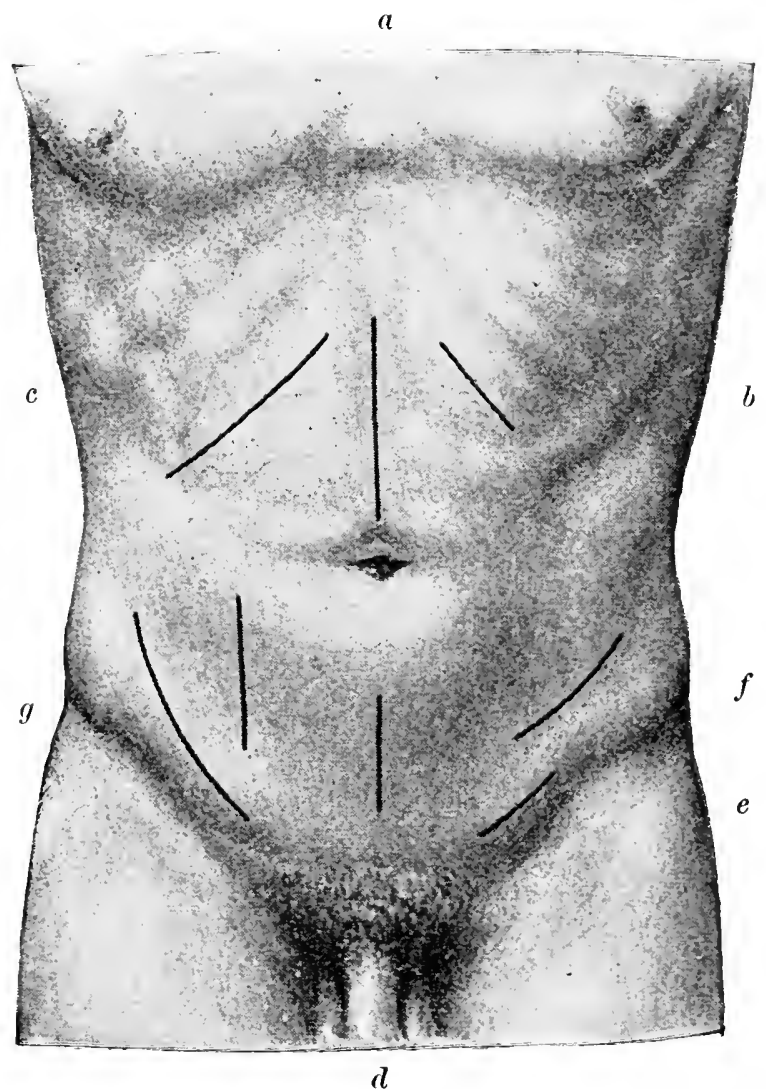

Fra. 190.-Ablominal incisions: a, longitudinal incision for operations on the stomach; $b$, incesion for gist rostomy; $c$, incision for operations on the gall bladler; $d$, incision for epicystotomy; $e$, incision for liation of the external iliac artery; $f$, incision for colotomy ; $g$, incisions for expostre of the cecum and the vermiform appendix.

formation of ventral hernia. Suture of the wound should be effected with silk or absorbable material introduced in tiers. In the linea alba the decpest row of sutures includes the peritoneum only, care being taken that smooth 
serous surfaces are brought in approximation. The second row of sutures includes the reetus musele together with its anterior fibrous sheath; several decp sutures secure the approximation of the muscles; more superficial ones passing thromgh the anterior sheath approximate accurately the aponeuroses. 'The most superficial layer of sutures mnites the skin in the customary manner. In the same way abdominal wounds in other situations are closed by three tiers of sutures. The deepest row unites the peritonemm, the middle the muscle and the aponeurosis, and the upper the skin.

\section{Operations on the Stomach and the Intestines.} -The Establishment of Gastric and Intestinal Fistulæ.In general the operation consists in bringing the selected portion of stomach or bowel ont of the wound after celiotomy and miting the parietal peritonem at the margins of the ablominal wound throughout a suffieient extent. with the visceral peritonem of the stomach or intestine by moans of interrupted or continuous sutures. The stonach or bowel is opened either at once, the gastrie or intestinal mucous membrane being united to the skin, or in the course of several days, after the abdominal eavity has been closed through the formation of adhesions throughout the extent of the wound. The method of operation is subject to various modifications at different portions of the gastro-intestinal tract.

The Formation of a Gastric Fistula; Gastrostomy.-The formation of a gastric fistula is indicated in the presence of impermable constriction of the esophagus :

(a) In consequence of the presence of neoplasms;

(b) In consequence of cicatrieial stricture; as well as for the introduction of nourishment into the stomach and for purposes of dilating deep-seated strictures through the wound.

The stomach is reached by division of the abdominal wall in the left epigastric region. The cutaneous incision is made vither parallel with the left costal margin or vertically through the rectus abdominis muscle close to 


\section{Plate 19.}

Gastrostomy.- Suturing one portion of the anterior wall of the stomach into the round in the abdominal wall. The serous margin of the wound is united with the serous larer of the stomach br means of a continued suture.

Colostomy. - A loop of the sigmoid flexure has been drawn forward and fired in the wound.

its onter border. In the first instance the incision begins a thumb's breadth to the left of the apex of the ensiform cartilage and passes outward and downward for a distance of 6 or $8 \mathrm{~cm}$. some $2 \mathrm{~cm}$. from the costal margin. The peritoneal cavity is opened throughout the extent and in the line of the cutaneons incision, and a small portion of the stomach is brought into the wound.

The stomach may be recornized by the characteristic radiation of the ressels from the greater and lesser curvatures. The walls of the stomach are thicker than those of the small intestine: and the organ is to be distinguished from the large intestine by the absence of sacculation. The stomach is most readily reached by grasping a portion of the great omentum and following it from the periphery toward the greater currature.

The portion of stomach brought into the wound is suspended in position by means of two fixation-sutures that do not penetrate its lumen. The parietal peritoneum is then elosely united to this portion of the stomach with a continued suture, including not only the thin serous layer, but also the subserous tissues of both the abdominal wall and the stomach, thus increasing the firmmess of closure (Plate 19).

The opening into the stomach can be made at once or after an interval of several days. If made at once, an incision is matle into the wall of the stomach with scissors in the line of the cutaneous wound, and the mucous menbrane is mited with the skin. If an interval be permitted to elapse between the making of the cutaneous incision and the opening of the stomach, the organ is entered with the Paquelin cautcry and a drainage-tube is introduced into the opening thius made. The gastric 
Tab. 19.

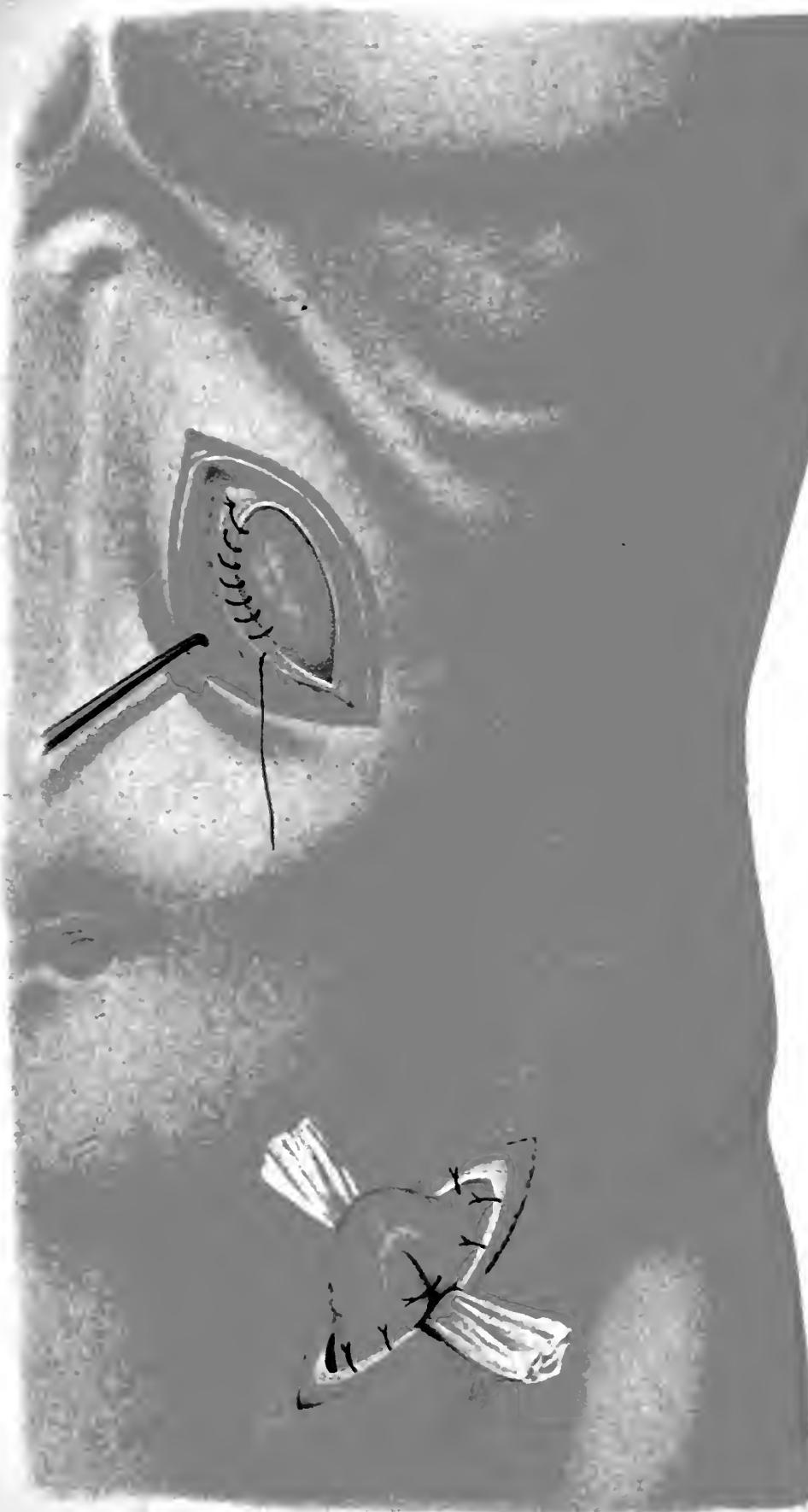

Lith. Anst F: Reichhold, München. 
fistula thus formed is attended with certain objections, as, opening directly upon the skin, it possesses not the slightest degree of continence, the mitritive fluids introduced escaping in the erect posture, while the asid gastric juice exeoriates the surronding skin. Among numerous suggestions to overeome these disadvantages some of the most useful are herewith deseribed.

Witzel forms a canal by uniting with suture two parallel longitudinal folds of the portion of stomach withdrawn. A suitably large portion of the wall of the stomach is brought forward and fixed in the wound. 'Two folds of the stomach are raised and mited by suture over a rubber tube after the lower extremity of the tube has been pushed into the stomach through as small an opening as possible in the gastric parictes. The canal can be prolonged for any distance by the introduction of additional sutures. Witzel makes it from 4 to $8 \mathrm{~cm}$. long. The rubber tube thus enters the stomach in the same way as the lower extremity of the ureter enters the bladder and the stomach remains continent throngh the establishment of this oblique fistula, the canal formed being subjected to a certain amount of compression from the stomach.

Frank makes the eutaneous incision parallel with the eostal areh, and after dividing the peritonem withdraws the stomach for a distance of 3 or $4 \mathrm{~cm}$. and sutures it into the wound, a thread being introduced into its summit. A second incision, $1.5 \mathrm{~cm}$. long, is made through the skin along the costal areh about $3 \mathrm{~cm}$. albove the first. Between the two incisions the skin is freed and the portion of stomach withdrawn is passed beneath the bridge of skin thus formed and fixed into the upper wound and its summit incised. The first incision is then closed by suture. The fistulous opening lies above the level of the stomach and can be readily compressed by the overlying looplike bridge of skin.

Enterostomy (Formation of an Intestinal Fistula) and Preternatural Anus. - A fistulous opening into the intestine through the abdominal wall is formed when a provisional 
means of escape for the intestinal contents is desired in the presence of intestinal obstruction without a definite knowledge as to the seat thereof. A short incision is made through the skin over and in the course of Poupart's ligament. The muscles are next divided and the peritoneum incised. If distended large intestine comes into view, it is drawn forward, or, if on the contrary, a loop of distended small intestine present in the wound, it is sutured through a small extent to the parietal peritoneum. If possible, the opening into the intestine should constitute a second stage of the operation, after the peritoneal cavity has been walled off by means of adhesions.

The formation of a preternatural anus becomes necessary when it is desired to furnish a constant channel of discharge for the intestinal contents through a fistula. The artificial anus is most commonly formed in the descending rolon or the sigmoid flexure (colostomy) when the lumen of the rectum is olliterated by a neoplasm, or by cicatricial narrowing. The descending colon is exposed through the peritoneal cavity. The entaneous ineision is made in the left hypogastric region three or four fingers' breadth above and in the direction of Poupart's ligament. After the peritoneal carity has been opened a loop of the sigmoid flexure, recognizable by its longitudinal bands, as well as its sacculation, is brought forward. At about the middle of the loop, close to the intestinal attachment of the mesentery, a strip of sterilized gauze is passed through a slit in the mesentery, so that the loop of intestine in a masure rides upon the ganze. To prevent the falling back of the bowel the serous layer of the abdominal wound may be united by several sutures with the serous laver of the intestinal loop (Plate 19). At a second sitting the loop of eolon is divided transversely upon its convexity by means of the Paquelin cautery at a point opposite its mesenteric attachment. This incision is progressively enlarged in the course of eight or ten days, until it reaches the mesenteric attachment, so that finally both portions of intestinal lumen lie side by side. 
Jejunostomy, the formation of a fistula through the jejunum, is indieated when nourishment through the stomach has become impossible; for instance, after erosion or corrosion of the stomach; also when the stomach is the seat of a new-formation, whose extent forbids either its removal or gastro-enterostomy. The incision is made in the linea alba, between the umbilieus and the symphysis pubis. After the peritoneum has been divided the transverse eolon is brought forward at the attachment of the greater omentum and reflected upward. The dnodenojejunal flexure appears at the root of the mesentery. One of the uppermost loops of jejunum that can be readily drawn into the wound is brought forward and fixed in the wound by means of seroserous sutures. The intestinal loop is opened either after the lapse of several days, or, better, at once, with the establishment of an oblique fistula by the method of Witzel. The nourishment of the patient can be effected without difficulty through the rubber tube. As the oblique fistula closes accurately bile and pancreatic secretion are not lost.

Resection of the Bowel.-It has been established clinically that considerable portions of the intestine (two meters and more, Kocher) can be removed by resection without detriment. After the resection has been effected the rontinuity of the bowel can be restored by means of circular suture of the stumps, or these may be brought out of the abdominal wound and an intestinal fistula or preternatural anus established. Resection of the bowel is undertaken :

(1) In the presence of injuries of the intestine;

(2) In the presence of gangrene of the bowel;

(3) In the presence of neoplasms ;

(4) In the presence of stenosis of the bowel ;

(5) For the cure of intestinal fistulie.

The portion of intestine intended for resection must be detached from its surroundings, so that it can be brought ont of the aldominal wound. The intestine is closed by means of either clamps or pressure with the fingers or 
strips of sterilized gauze tightened and tied. The contents of the bowel should have been forced backward and forwald before the intestine is incised. The division of the bowel is effected with scissors. The plane of division should be so made, according to the suggestion of Kocher, that a greater portion of bowel is removed from the convexity than from the mesenteric attachment, as by this means the circular ressels of the bowel are less exposed to injury. The mesentery is ligated in successive portions and divided transversely at its attachment to the bowel, or excised in the form of a wedge whose base is formed by the resected bowel and united in a linear direction. After the mucous membrane projecting from the divided surfaces has been dried with sterile gauze circular union of the lumen of the two portions of bowel may be proceeded with. If the lumen of the two portions of bowel is unequal, the smaller is divided obliquely, so that the cut surface is elliptic in shape.

For the union of transversely divided bowel after resection, as well as in the formation of anatomoses, either the intestinal suture or Murphr's anastomotic button may be emplored. 'The method of applying the intestinal suture has alreadr been described at page 53. Murphy's button renders possible rapid effectuation of accurate union of divided intestinal lumen, as well as the establishment of anastomoses between portions of the intestinal tract. This ingenious derice consists of two capsules made of light sheet-iron and nickel-plated and provided with a hollow crlinder internally and a slight shoulder externally (Plate 20), which can be readily pushed the one into the other with the fingers, when by reason of a clamp-like arrangement ther remain thus in secure apposition. The transversely divided portions of intestine, or the slits made in the establishment of anastomoses, are picked up by continued sutures passing through all of the layers of the intestinal wall, which are tied after the intestine has been brought orer the respective half of the button. After the second portion of the intestine has been 
similarly treated the two halves of the button are pushed one into the other and clamped between the fingers (Plate 20). In approximating the parts of the button the prolapse of small portions of the mucous membrane in the interval between the segments is to be avoided. Such portions of intestine may require approximation by means of Lembert's seroserous sutures. The intestine is thus adjusted with broad surfices of serous membrane in apposition. The small openings in the sides of the button serve as an outlet for the escape of secretion from the included portion of bowel, while intestinal contents and gas can escape through the larger central opening. When the clamped portions of the intestine are necrosed, at the end of one or two weeks, firm union of the bowel will have taken place. The button is then freed and escapes with the intestinal contents. The technic of this mode of intestinal union is exceedingly simple and the results, according to the statistics of American surgeons, are arlmirable.

Murphy's reports with regard to the success of his method are, as W. Mayer stittes, almost incredible, but they are in strict aceord with the far.ts. The union of the bowel, after resection, or after the performance of gastro-enternstomy or cholecystenterostoms, can be accurately effected in the course of a few minutes. The experience of German surgeons also testifies to the utility of Murphy's method.

Exclusion of Intestine; Entero-anastomosis.-If in the presence of obstructive tumors or of tuberculosis of the intestine extirpation of the diseased portion is not practicable, the patulousness of the bowel may be reestablished by excluding the diseased portion, by bringing in communication a loop of intestine to the distal and one to the proximal side of the obstruction. The procedure is designated incomplete exclusion of the bovel (Wölfler) if the excluded portion of intestine is not removed; while in complete exclusion of the bouel the diseased portion is entirely separated from its connection with the bowel. In the first mode of procedure the two loops of intestine are placed side by side and union effected throughout the 


\section{Plate 20.-Union of Circularly Divided Intestine by Means of the Murphy Button.}

Fig. 1.- $a, b$, the two halves of the button; $c$, the two halves of the button clamped togetlier; $d$, the mode of introducing the sutures for holding each half of the button in place.

FIG. 2.-Completed union of the intestine by means of the Murphy button. The slit in the mesentery has been closed by linear union.

extent of the two lateral openings made by means of two rows of sutures or of Murphy's button. In case of obstruction of the pylorus continuity of the digestive tract may be reëstablished by making a communication between the stomach and a loop of jejunum (gastro-enterostomy). If the cecum be the seat of an obstructive, inoperable tumor, the continuity of the bowel may be reesstablished by uniting a portion of the ileum with the adjacent ascending colon (ileocolostomy).

Salzer has shown experimentally that the retention within the abdomen of the excluded portion of bowel is not without danger, and he has modified the operation accordingly so that the bowel is divided transversely upon either side of the obstruction and circular union is effected. The ends of the excluded portion of bowel are sewed in the abdominal wound (complete exclusion of the bowel).

Hacker has enlarged the indications for entero-anastomosis and recommends the operation, in addition to cases of inoperable neoplasms, also in cases of penetrating injuries of adjacent portions of bowel, as well as in the treatment of fecal fistulæ. Communications between the bladder and the bowel might also be successfully treated by means of exclusion of the bowel.

Mode of Performing Gastro-enterostomy.-An incision is made in the linea alba between the umbilicus and the ensiform eartilage. The transverse colon, together with the great omentum, is drawn out of the wound and reflected upward. In the transverse mesocolon, at a point where it is in relation with the stomach, is made a slit whose margins are attached by suture to the serous layer of the stomach so as to include an area perhaps as large as a silver dollar. A loop of the upper portion of the 


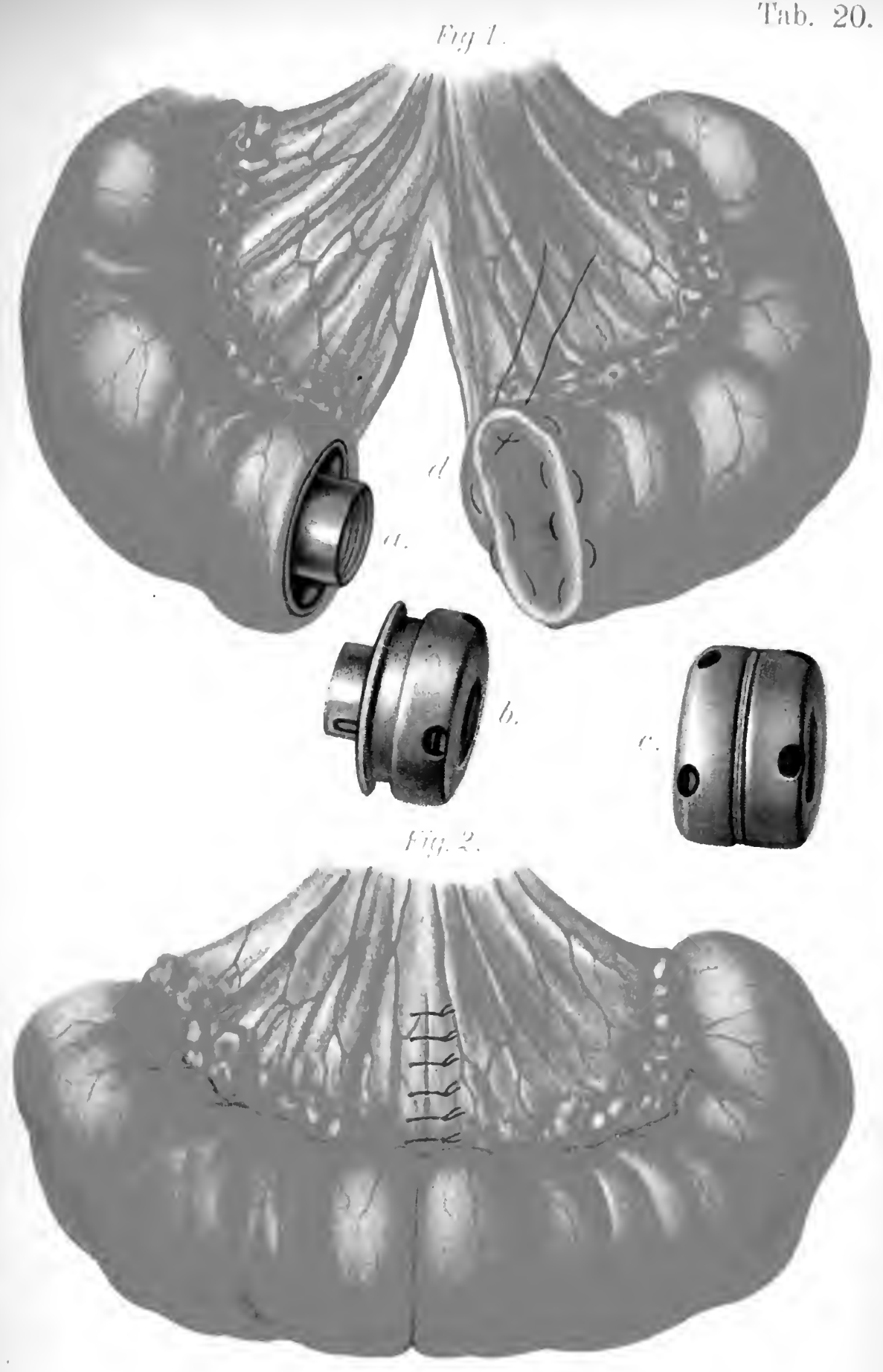

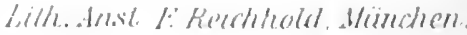




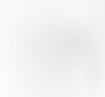
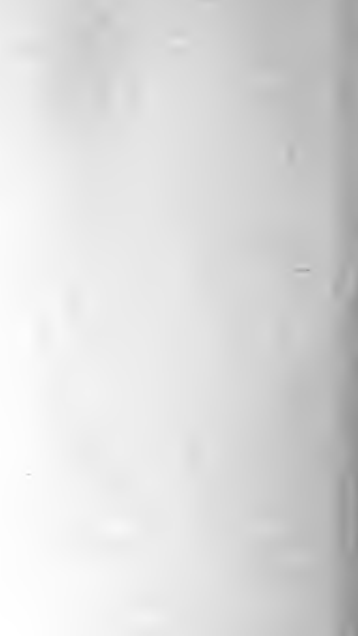
jejunum that can be approximated with the stomach without tension is incisen, tongether with the stomach at its point of attachment to the mesocolon, and mion of the opposed parts is etfected either by suture or with the aid of the Murphy button. The first mo of sutures includes the entire thickness of the intestinal and gatric walls, at the margins of the respective opening: A row of Lembert serous sutures is applied over the first row.

Operations upon the Biliary Apparatus.-Beside abscesses and rosts of the liver, for whose operative treatment no especial rules can be laid down, attacks upon the biliary apparatus are directed especially toward the removal of ealculi and their sequele. The surger of the biliary apparatus, the youngest department of abdominal surerery, has been sytematically practised only since the beginning of the eighties. The cutaneous incisions throurh which the transverse fissure of the liver is reached are raried. At times it is made in the linea alba between the umbilicus and the srmphrsis pubis. At other times it is made along the outer border of the rectus abdominis muscle. C'zerny makes an angular incision, whose rertical arm passes along the linea alba, and is joined below the umbilicus by a horizontal incision passing toward the right and outward. In all cases after opening the peritoneal carity the right lobe of the liver is reflected upward so that its under surface is exposed to view, together with the gall-bladder and the transerse fissure. Between the last and the lesser curvature of the stomach there extends a duplication of the peritoneum (the lesser onentum) in whose right free extremity pass the large biliary ducts, the portal rein, and the hepatic artery in the order given.

Operations on the Gall-bladder.-The gall-bladder is opened by incision (cholecystotomy) for the removal of gallstones, and the evacuation of emprema or dropsy of the gall-bladder. Incision of the gall-bladder may be made in one stage, or, after suture of a portion of the viscus into the abdominal wound, in two stages. In operating 
at a single sitting, after removal of calculi present, the wound in the gall-bladder may be united by suture and the abdominal wound be closed (ideal cholecystotomy, cholecystendysis), or the sutured gall-bladder may be suspended at the level of the round. If primary suture be not undertaken, a fistula of the gall-bladder through the abdominal wall may be established according to the rules that govern intestinal operations (cholecystotomy). With regard to the performance of cholecystotomy it need only be said that after making the cutaneous incision and opening the peritonem the gall-bladder should be brought as fully as possible to the level of the wound, where it is attached and held in position with sutures. After puncture of the viscus it is incised and under guidance of the finger calculi present are removed with the aid of forceps or a spoon, attention being given to the possible presence also of stones in the cystic duct. The wound in the gallbladder $\mathrm{i}$ : united with two tiers of sutures in the same way as wounds in the intestines. The first row of sutures includes the entire thickness of the gall-bladder and approximates opposed wound-surfaces. The sceond row consists of Lembert's serous sutures, and is intended to insure perfect closure of the wound by approximation of broad surfaces of peritoneum. In performing crstotomy in two stages, as well as erstostomy, a portion of the gall-bladder brought into the wound is attached to the parietal peritoneum, as in the operation of gastrostomy, and the exposed portion is opened with the Paquelin cautery after the lapse of several days.

Extirpation of the gall-bladder (cholecystectomy) is indieated in the presence of neoplasms of this viscus, as well as of gall-stones attended with inflammatory processes in the walls of the gall-bladder. The trpical procedure consists after transverse division of the erstic duct between two ligatures, in separating the gall-bladder by blunt dissection from its attachments to the liver, which under favorable conditions can be readily effected after division of its serous covering. 
Opening of the eholedoch duct by incision (choledochotomy) may be neeessary for the removal of impacted calculi, the wound made being closed by suture. In the presence of irremediable obstrution of the choledoch duct, in conseguence of impartion of a calculus or of an oceluding tumor, or of compression by lymphatic glands, a suitable channel for the escape of the bile can be provided only by establishing an artificial communication between the gall-bladder and the bowel (cholecystenterostomy, Winiwarter). The anastomosis is effected in accordance with the rules given in the description of entero-anastomosis (page 317), with the aid of either suture or the Murphy button. The communication is established between the gall-bladder and the jejunum (cholecystojejunostomy), or if possible between the gall-bladder and the duodenum (cholecystoduodenostomy). Artificial communication between the intestine and a dilated choleodoch duct on the proximal side of an obstruction also has been recommended (choledochoduodenostomy).

\section{Opening of Pericecal Abscesses.-Resection} of the Vermiform Appendix.-If the abscess canses bulging of the slin above Poupart's ligament, the incision is marle over the most prominent part of the swelling, parallel with the course of the fibers of the external oblique muscle, pretty much as in ligation of the iliac artery. The muscles of the abdominal wall and the transwersalis fascia are divided layer by layer and the abscess is opened freely. ${ }^{1}$ If in a case of appendicitis it seems probable that the removal of the appendix will be necessary, a vertical incision upon the right side along the outer border of the rectus abdominis muscle is recommended. This incision permits free access and renders possible exploration and removal of the appendix under

1 The incision emplored by MeBurney is very useful. The incision is perpendicular to a line drawn from the anterior superuor illac spine to the umbilicus, and crosses this line tro inches to the inner side of the spinc. The nuscles are scparated by blunt dissection. The fact that muscle-fibcrs are not cut is a great safeguard against hernia, because the muscles do not degeverate.-ED. 
favorable conditions. After the abdominal cavity has been opened the peritnneal cavity is walled off from the cecum by means of compresses, and, after separation of the adhesions of the cecum to neighboring structures, the exact seat of the disease is located. If an accumulation of pus has been found and is eracuated, the rermiform appendix is carefully separated from its surroundings and freed for removal. After ligation of the meso-appendix a circular incision is made around the appendix to the peripheral side of the point of removal through the serous membrane and the muscular layer down to the mucosa, which is ligated and divided transversely to the distal side of the ligature. The short cuff formed is reflected upon the stump, which is grasped and inverted into the lumen of the appendix. The margins of the shallow depression thus made are united by a row of Lembert's sutures over the inverted stump of the appendix (Fowler). ${ }^{1}$

\section{Operations upon the Genito-urinary Organs.-} Catheterization.-From its external orifice to the entrance of the bladder the male urethra consists of three anatomically and functionally different parts, viz., the penile portion, the membranous portion, and the prostatic portion. The penile portion is surrounded by the corpus spongiosum, which presents an enlargement at its anterior and posterior extremities, known respectively as the glans penis and the bulb of the urethra. The membranous portion, around which a sphincter of striated muscular fibers is arranged, penetrates the deep transverse perineal muscle stretched beneath the pubic arch, so that its proximal division, together with the prostatic portion, represents the intrapelvic part of the urethra. The membranous portion may be looked upon as the most fixed segment of the urethria. In front of it is the movable pendulous portion and behind it the prostatic portion movable within slight limits. That portion of the urethra in-

${ }^{1}$ Instead of forming a stump and treatmg it by inversion, some surgeons prefer to remove with the appendix an elliptical puece of the colon, and then to close the colon-wound by means of inversion-sutures.-Ed. 


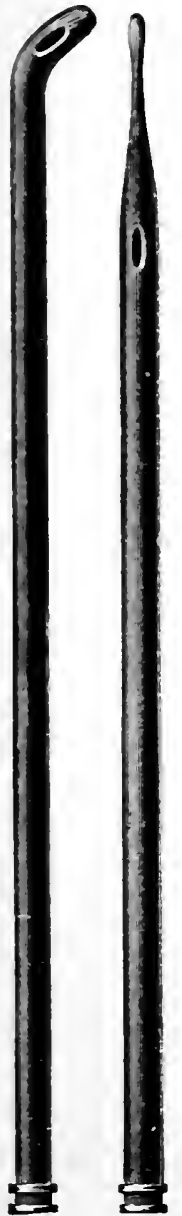

191. 192.
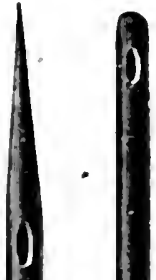

191.
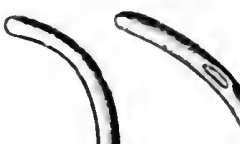

1
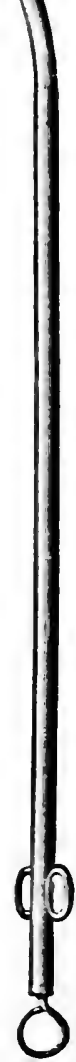

196.

Figs. 191-194.-Soft catheters of varying form.

FIG. 191.-Catheter coudé.

FIG. 192.-Conical and bulb-tipped catheter.

FIr. 193.-Conical and pointed catheter.

EIG. 194.-Cylindrical eatheter.

Fir. 195.-MIassive steel sound (stone-sound) for exploration of the bladder.

FIG. 196.-Metallic catheter. 

cluded between the external orifice and the sphincter of the membranous portion is known as the anterior urethra, that portion posterior to this as the posterior urethra, a distinction especially of elinical significance. The entire fixed portion of the urethra, from the mouth of the bladder to the bend of the penis forms a large arch with its convexity backward. 'The base of the arch corresponds with the point at which the membranous urethra passes through the urogenital diaphragm.

Catheterization consists in proper instrumental evacliation of the contents of the bladder through the urethra. To effect this purpose tubular instruments are employed, which are made either of soft material or of metal, and are of varied shape. The choice of instruments will depend upon the conditions present in the individual ease.

The physician in performing eatheterization will, in aceordance with the symptoms present and the results of external and rectal examination, form an idea of the condition of the urethra and determine accordingly the selection of a suitable instrument. Soft instruments, of rulcanized rubber or of unpigmented woven silk, are made of varying thickness and are either straight or bent slightly at their vesical extremity. The former are either cylindrical, conical, or nodular at their vesical extremity.

Catheters bent at their extremity at an angle (eatheter conde) are, notwithstanding their softness, well adapted by reason of their shape to overeome certain kinds of obstruction, principally dependent upon changed conditions of the prostate.

Rigid instruments made of metal are shaped in accordance with the configuration of the fixed portions of the urethra. The straight distąl portion or shaft passes over into a proximal portion bent in conformity with the curvature of the posterior urethra. In the introduction of a soft flexible catheter the instrument accommorlates itself to the shape and the course of the urethra and readily glides into the bladder. The rigid instrument is shaped 
in accordanee with the eurve of the urethra, although the correspondenee is never complete. By delicacy in the manipulation of the instrument unavoidable distortion of the fixed portions of the urethra will be reduced to a minimum.

Mode of Introrlucing a Soft Catheter through the Urethra into the Bladder.-The patient lies upon his back, with the pelvis somewhat elevated, and the operator stands upon the left side. The penis is grasped with the left hand and

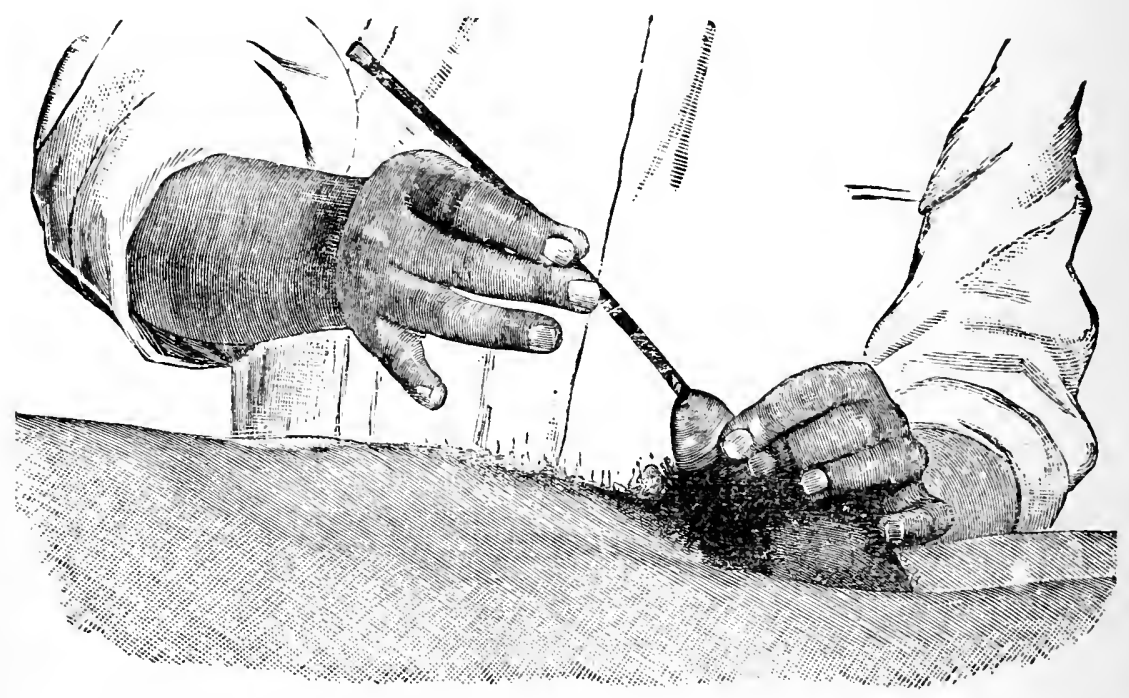

FIG. 197.-Method of introducing a partially rigid catheter.

raised in such a way that the pendulous portion of the urethra is rendered tense, and the eatheter is introduced into the urethra and gently pushed forward. The eatheter condé is so introduced that its beak is directed toward the upper wall of the urethra. In passing the membranous portion a sense of slight resistance is appreeiated, which is overeome by gentle pressure. From this point the beak of the instrument slips into the bladder without further obstruetion. In the presence of hypertrophy of the prostate and of elongation of the prostatic portion of the 
urethra the watheter must be introduced for a greater distance, often "p to its hilt, before urine flows. After the flow of urine has ceased the residual urine in the bladder will arape after slight withdrawal of the soft catheter.

The introdurtion of a rigid catheter into the bladder is a much more difficult procedure, and its sate and proper

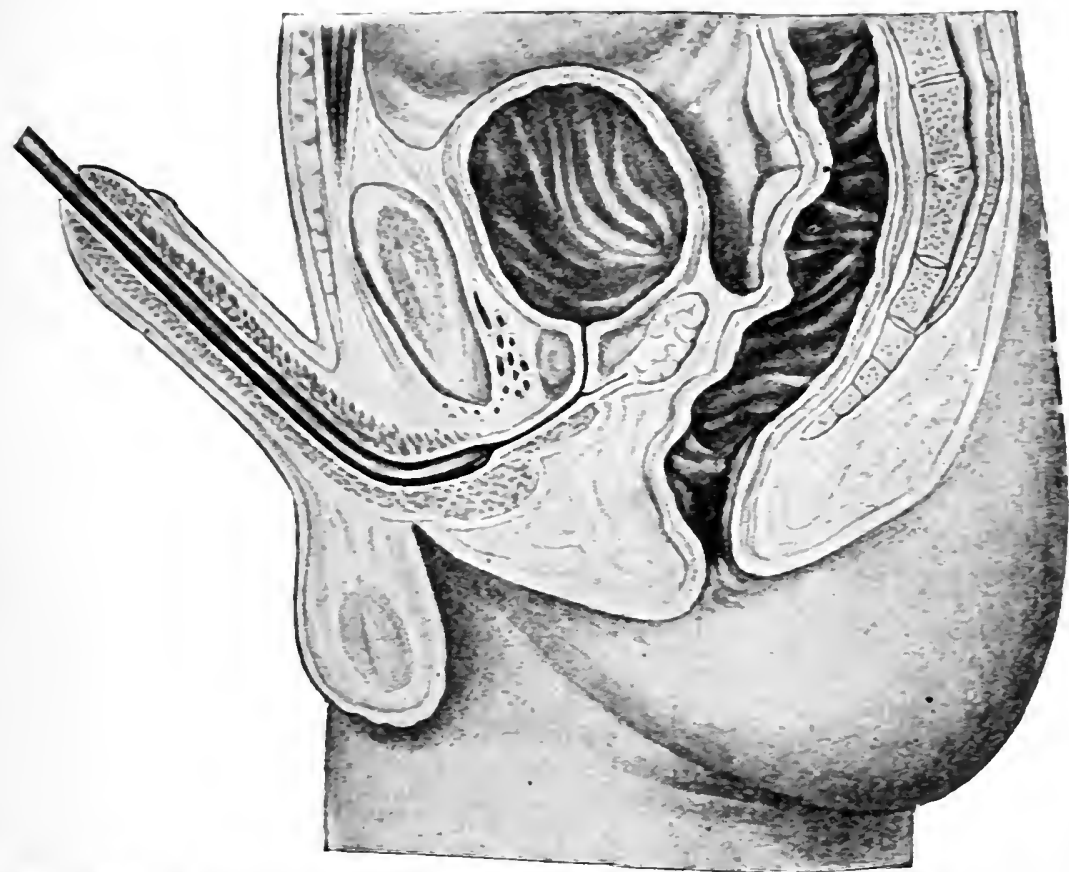

FIG. 193.-Introduction of a riginl instrument into the bladder, the tip of the catheter obstructed at the bulbous portion.

execution requires a certain amount of skill. In general the rule is to be observed that the beak of the instrument be riade to pass along the upper wall of the urethra. If the urethra be normal, no difficulty is experienced in the operation until the membranous portion is reached, but at the junction of the movable with the fixed membranous portion a slight obstruction is eneountered by the beak of the eatheter (Fig. 198). Care must now be taken to pre- 
vent the tip of the instrument engaging in the mucous membrane. The beak must therefore not deviate from the median line, and with eautious movements the instrument should be passed into the membranous portion, naturally without any violence. After the resistance has been overcome the instrument will be felt to enter the membranous portion and pass through the urogenital diaphragm.

From this point the catheter in a normal urethra encounters no further obstruction, and on depressing the

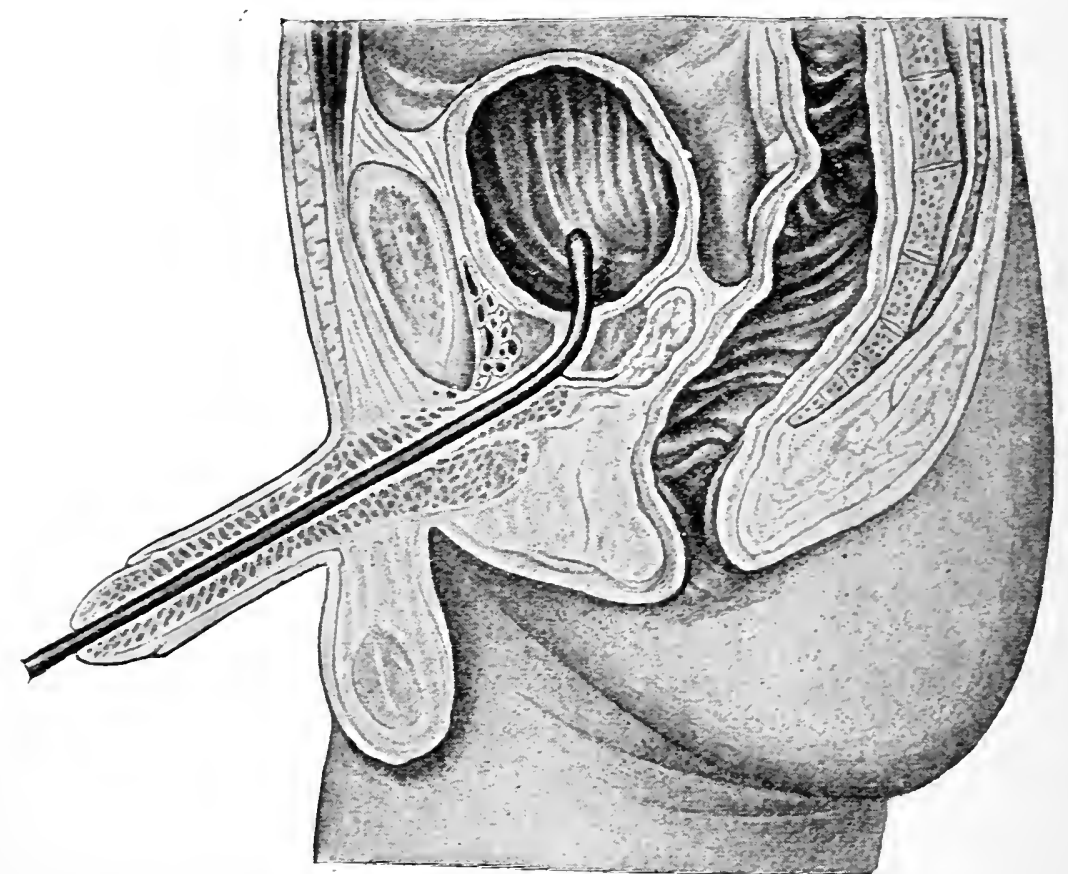

FrG. 199.- Introduction of a rigid instrument into the bladder: by depressing the shaft of the catheter its beak is forced into the bladder.

handle of the instrument the tip enters the bladder without further hindrance (Fig. 199).

Catheterization with a Metallic Instrument.-The patient is placed horizontally upon his back, with the pelvis somewhat elevated, and the operator standing upon his left 
side. The penis is gratpenl with three fingers of the left hand and the lips of the urethra are held apart he means of the thumb and the inlex-fingers. The catheter or the solid sound is grateped at its di-tal extremity with the first three fingers of the right haml, its palmar aspect turned upward, and resting with the little finger upon the middle line of the body (Fig. 200 ).

The operator permits the beak of the instrument to enter the urethra and draws the penis, with a certain degree of

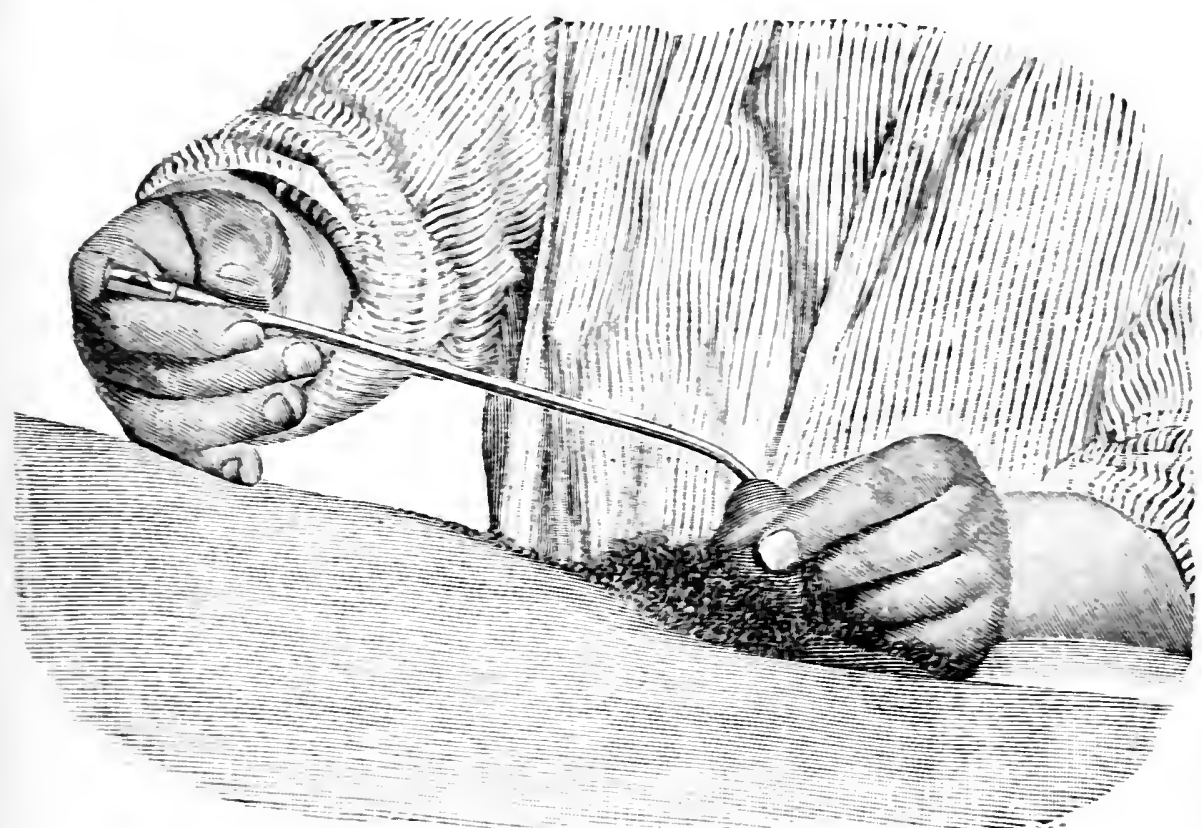

FIG. 200.-First position in the intruanction of the catheter.

tension, over the curve of the catheter. which is, at the same time being steadily kept in the midlle line, cradually raised until it reaches a vertical position. With a slight jerk the tip of the instrument passes the bulbous portion and it ret remains to enter the membranous portion and pass the urogenital diaphragm (Fig. 201).

In all cases a sense of obstruction is encountered at this point which is readily overcome by gentle pressure, while 
the catheter is held accurately in the median line, and is gradually depressed from the vertical to the horizontal toward the lower extremities (Fig. 202).

If the instrument is at the same time pushed forward slightly, its tip enters the bladder. At this moment urine will escape from the catheter. With the rigid instrument, as soon as the prostate has been passed and the bladder has been entered, free movements can be made with the tip of the instrument.

The method of introducing the catheter described is attended with difficulty in obese individuals and in the presence of meteorism and ascites. Under such circumstances it seems desirable to enter the catheter at right angles to the axis of the body, with the penis raised vertically. In this position the instrument is pushed forward and at the same time rotated in an are to the median line and elevated to a position until the tip is grasped by the bulbous portion (Fig. 20:3). In another mode of procedure the operator sits before the patient, who is placed in the position for the operation of cutting for stone. The catheter is introduced into the orifice of the urethra from between the extremities of the patient, with its convexity directed upward. The penis, raised vertically, is drawn over the curve of the catheter and the instrument is rotated through an are of $180^{\circ}$ toward the right until it reaches the median line. During the progress of these manipulations the beak of the instrument enters the urethra to the bulbous portion. Now, the handle of the catheter is elevater and pushed forward in the median line until its beak has passed the posterior urethra and entered the bladder.

In a normal urethra an instrument of cousiderable weight, as for instance a rigid sound of large caliber (lithotriptor), overcomes readily the obstruction encountered on the distal side of the bulbous urethra and glides easily, by reason of its own weight, into the bladder, without further guidance. The guiding hand need only prevent the departure of the instrument from the median line. In the presence of narrowing, however, a certain amount of pressure in the direction of the urethra is necessary, in order to urge the instrument onward through the rigid cicatricial tissue. If the urethra is narrowed in its deeper portion, or, if 
OPERATIONS UPON GENTTO-CRINARY ORGANS. 331

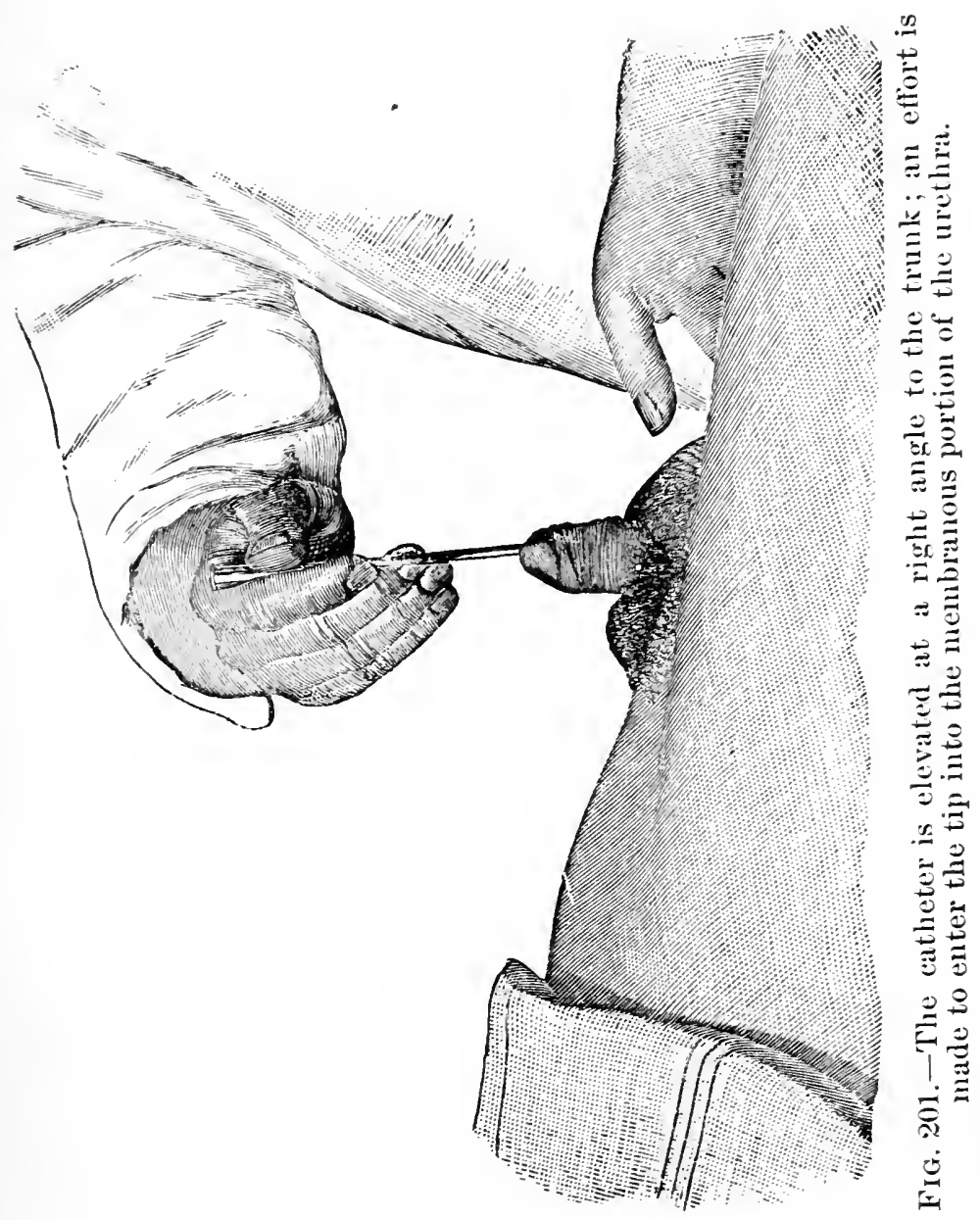



the prostate is enlarged it will of ten be necessary to int roduce the indexfinger of the left hand into the rectum as a guide. In the presence of lypertrophy of the prostate, on account of elongation of the prostatic portion of the urethra, and on aceount of elevation of the orifice of the hadder, the catheter or the sound nust be introduced for a greater distance and be more greatly depressed, in order that the beak may reach into the bladder.

Catheters for introduction into the female urethra correspond with the shortness of this canal, and are either

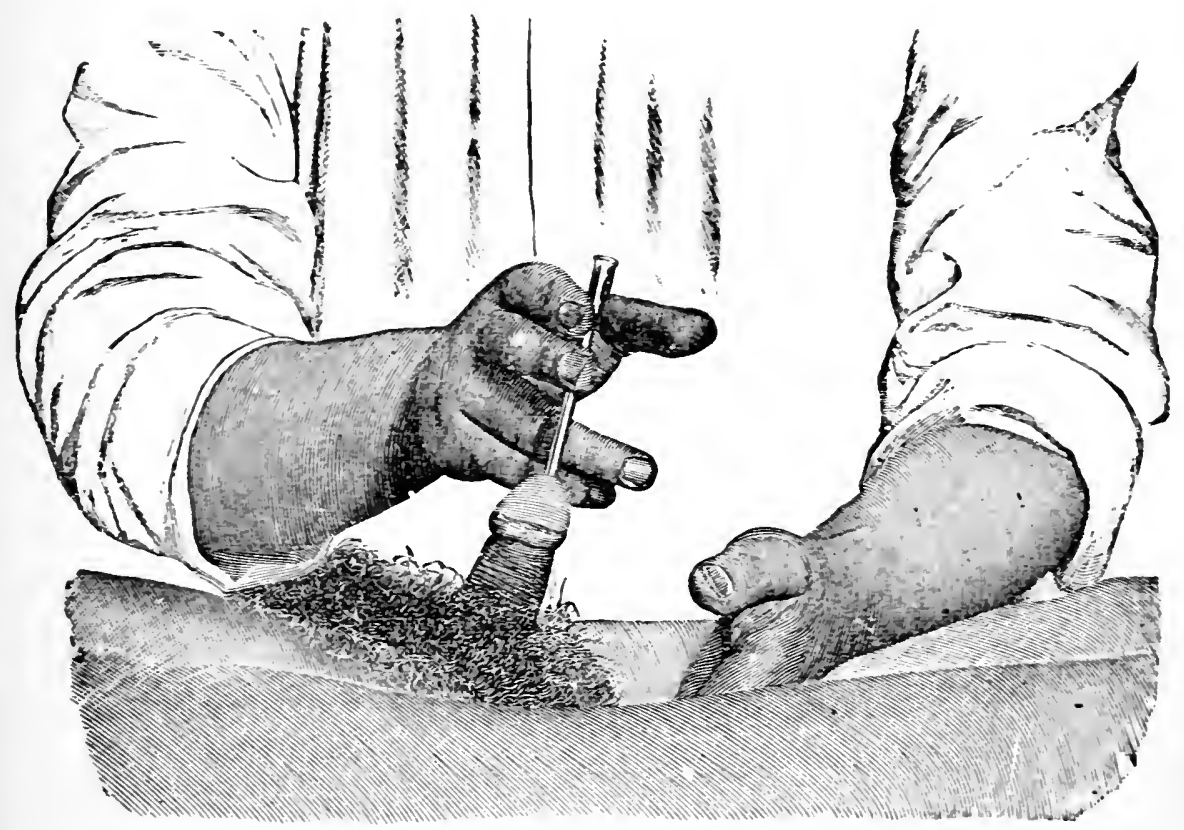

FIG. 202.-Catheterization: the catheter has entered the membranous portion and has passed the urogenital diaphragm; by pressing the handle the tip enters the bladder.

straight, or slightly curved at their extremity. In introducing the instrument the labia are separated and the handle is depressed while the catheter is pushed forward. Only in the presence of pregnancy or of tumors of the genital organs may the female urethra be elongated or distorted. The resulting difficulty in the introduction of a catheter is to be overcome by the employment of partially rigid instruments, as in males. 
Retention-catheter.-A catheter may be permitted to remain within the urethra for days or even weeks. In order to serve its purpose permanently it must be suitably fixed in position. 'The introduction of a retention-eatheter into the urethra permits constant eseape of urine, which, beside, does not eome in contact with the wall of the urethra. Such a form of eatheter is therefore employed when it is desired to place the bladder at rest, to secure a

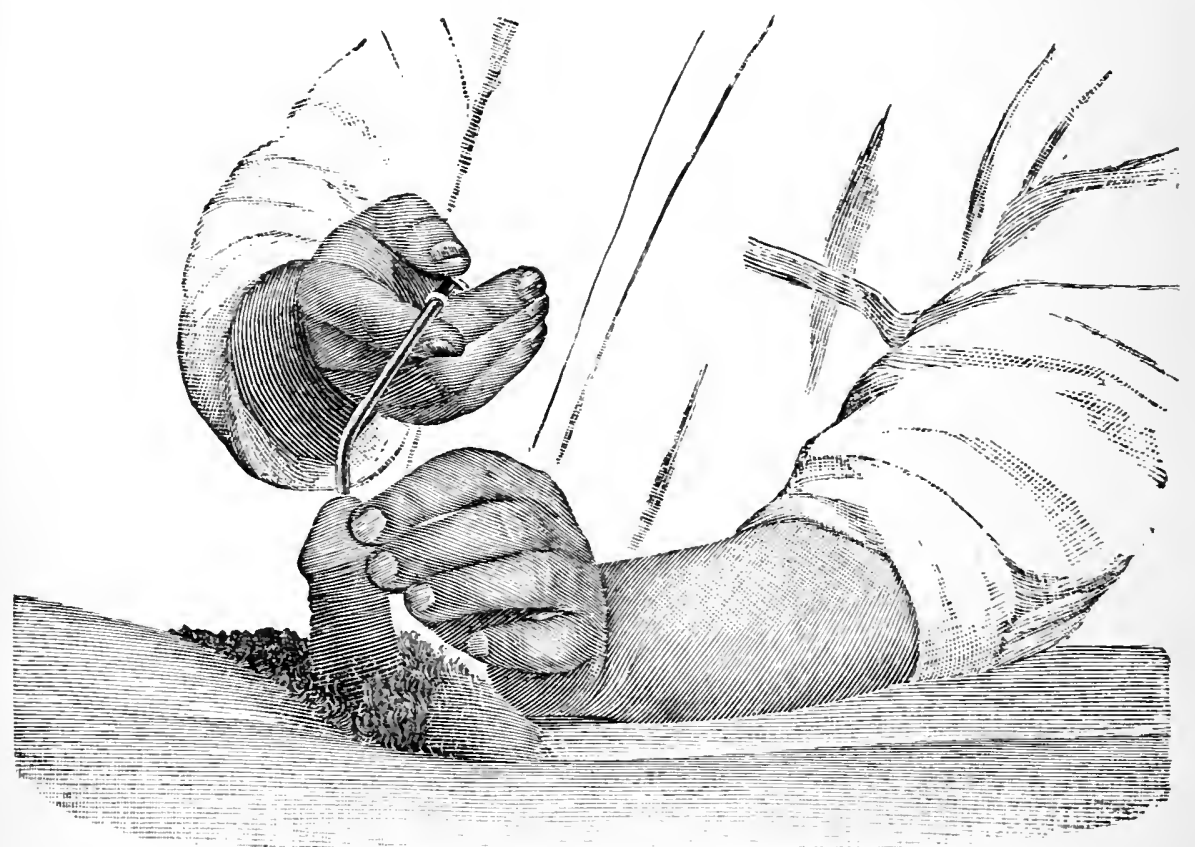

FIG. 203.-Mode of introducing the catheter from the side.

permanent channel of eseape for the urine and finally when the urethra is to bespared irritation. The moderate but constant pressure of the retention-aatheter softens cicatrices of the urethra and exerts a dilating influence upon circular strictures, and for this reason is applicable with advantage in the presenee of eallows and cicatricial strictures of the urethra. Finally, the retention-catheter is to be recommended when catheterization that must be fre- 
longer strip slit in the middle is drawn over the catheter, and comes to rest upon the needle, being made to adhere to the sides of the penis (Figr. 204, II). The entire arrangement is made secure by circular turns of strips of plaster passing around the organ from the glans to the root (Fig. 20\%, III).

Puncture of the Bladder.-Evacuation of the bladder through suprapubic puncture is undertaken as a palliative measure, and also for the purpose of forming a vesical fistula through the abdominal wall. Palliative puncture is practised in the presence of complete retention of urine in consequence of impermeable stricture of the urethra when it is desired to await a more farorable time for the introduction of a sound or for the performance of a radical operation for the relief of the obstruction. For the purpose of establishing a resical fistula the operation is undertaken (1) in the presence of prostatic enlargement, with complete or incomplete retention of urine, when atheterization is attended with difficulty or is followed br hemorrhage; (2) when the mouth of the bladder is obstructed by a tumor that cannot be removed by operative measures: (3) to effect drainage of the bladder in cases of serere purulent crstitis. If the object of the procedure is only evacuation of the bladder, the puncture is made with a thin, so-called exploratory trocar. The operation is in itself of little significance and, if necessary, can be frequently repeated. The patient lies upon his back, with the pelvis somewhat elevated. As the puncture is alwars undertaken by reason of retention of urine, the bladder is distended to the maximum, and is readily palpable as a tumor above the symphrsis pubis. The operator stands to the right of the patient, and marks with the tip of his left index-finger a point in the middle line just above the symphrsis where the puncture is to be made. The trocar is pushed rertically through the abdominat wall, disappearance of the sense of resistance indicating that the point of the instrument has entered the bladder. The cannula is grasped with the thumb and 
the index-finger of the left hand and the stilet is remored with the right. After the mine has escaped, the camnula is removed, its extremity being elosed with the tip of the thumb in order that the wound be not contaminated. The wound of puncture invariably heals without complication.

If in conjunction with puncture a vesical fistula is to be established in the abdominal wall the operation is to be performed with the aid of the semieircularly curved trocar of Fleurant (Fig. 205). The position of the patient and of the operator is the same as that just described. The index-finger of the left hand marks the point accurately in the middle line just above the symphysis, where the puncture is to be made. The instrument

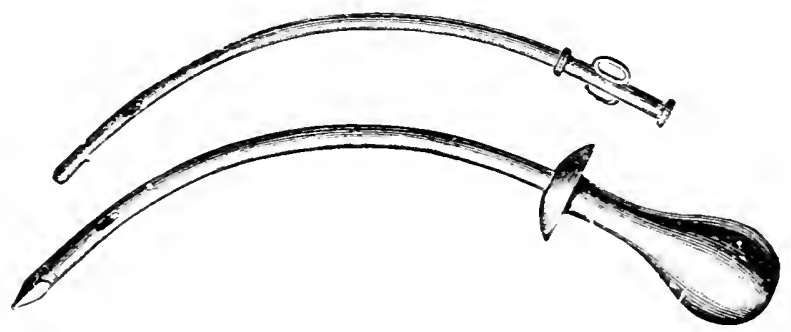

FIG. 205.-Trocar for puncture of the bladder, after Fleurant.

is applied rertically and pushed forcibly through the abdominal wall. When the disappearance of resistance indicates that the abdominal wall has been passed the instrument is pushed onward and its handle is raised so that its extremity is directed toward the fundus of the bladder. The stilet is now removed (Fig. 207) and a suitable tube passed through the cannula. The camnula remains in place for about a week, after which a Nélaton catheter is introduced into the fistula and fixed in the wound.

External Urethrotomy.-External urethrotomy consists in entering the urethra through an incision in the abdominal wall. The operation is undertaken (1) in the presence of calculi and foreign bodies in the urethra, whose removal 
camnot be effected through the natural passages; $(2)$ in the presence of injuries of the urethra ; (3) in the presence of strictures of the urethra which are either inpermeable or not accessible to treatment br dilatation for various reasons; (4) for the establishment of a urethral fistula ; (5) as a preliminary operation to median section for stone.

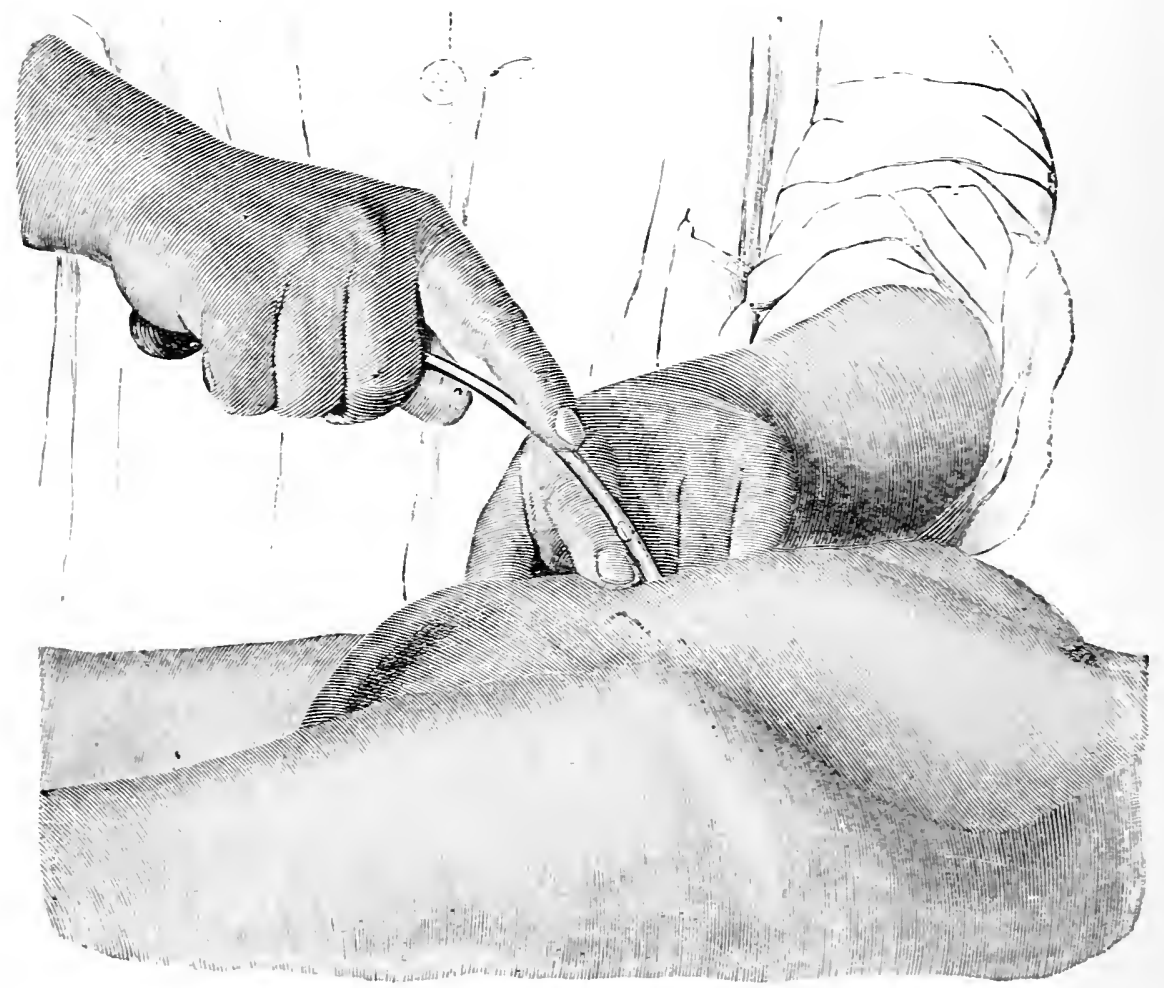

FIG. 206.-Puncture of the bladder: position for making the puncture.

The mucous membrane of the urethra is readily reached with the knife in its pendulous portion after division of the skin, the dense fiscia, and the corpus spongiosum. The bulb of the urethra is accessible in the midclle line through an incision in the perineal raphe, after division of the skin, the tunica dartos, the superficial perineal fascia, and the bulbocavernosus muscle. The corpus 
which is reflected toward the sacrum, the proximal portions of the urethra, the membranous portion and the prostate are rendered visible and accessible to surgical intervention.

The performance of urethrotomy is subject to various modifications in accordance with the indications for the operation. The patient lies upon his back with the lower extremities flexed at the knee and the hip (position for cutting for stone). The operator is seated in front of the patient. The urethra is invariably opened in the median line through the raphe of the perineum.

Urethrotomy with a Guide.-A metallic sound grooved upon its convexity is introduced into the urethra to a point beyond the constriction that is to be divided. The guide is held by an assistant accurately in the middle line. If the incision is to be made into the perineum, the scrotum is lifted up. The operator determines by touch with the finger the position of the resistant portion of the urethra, which is perhaps a stricture surrounded by callus, and makes an incision over it in the middle line. If the narrowing be at the junction between the bulbous and membranous portions, the incision passes from the root of the scrotum almost to the anus. By dissecting layer by layer in the median line the callous and narrowed portion of the urethra is reached and an incision is made in the line of the cutaneous wound until the groove in the guide is exposed. The callus is divided up to a point where the urethra is of normal caliber. The introduction of a retention-catheter concludes the operation.

Urethrotomy without a guide is an incomparably more difficult operation than that just described. The operator occupies the same position as in cutting for stone. The sound can be introduced only to the anterior portion of the stricture if this be impermeable. The cutaneous incision is made as in the operation just described, in the median line through the perineal raphe. The portion of the urethra lying to the distal side of the constriction is incised and the margins of the wound are separated by 
means of small tenacula. An effort is made to find the lumen of the constriction and to gain entrance by means of a thin bulbous instrument. If this can be done, the eicatricial tissne is divided in the middle line on its under surface to the somnd, and the incision is contimued beyond the narrowing of the urethra.

As a rule, the detection of the eanal of the urethra at the distal extremity of the stricture is attended with difficulty. The tissues are changed from the presence of cieatrices, and the hemorrhage from the cavernous bodies and from the bulb is often considerable, so that it can be readily understood that the small lumen of the urethra may escape detection in the limited field of operation. Indiscriminate incision into the callus is not to be commended. By means of manual expression of the bladder it may be possible under circumstancess to cause the escape of a few drops of urine into the wound and in this way to gain an idea as to the situation of the orifice of the stricttire. If it has been possible by this means, under guidance of the eye, to introduce a bulbous bougie into the orifice of the stricture, the division of the narrowed portion of the urethra will be unattended with any further difficulty.

After division of the stricture a catheter of considerable caliber can alwars be introduced with aid from the wound through the entire uretlira into the bladder. In the event of failure to divide the stricture through the wound there remains yet the resource of retrograde sounding of the stricture, posterior catheterization. This may be undertaken :

(1) Through the urethra, after exposure and incision of the urethra to the proximal side of the stricture;

(2) Through the bladder, after this has been opened by means of a suprapubic incision.

In performing retrograde catheterization through the urethra the deeper portions of this canal (the membranous portion) are exposed by detaching the lowermost extremity of the rectum. "To this end the perineal longi- 
tudinal incision is either prolonged to the anus or the detachment of the rectum is undertaken through a prerectal curved incision. After division of the skin the connections between the sphincter ani and the bulbocavernosus muscles are divided transversely and then the anterior wall of the rectum is freed by blunt dissection from the cutaneous covering. If the bulb of the urethra is retracted upward and the rectum downward, the membranous portion can be dissected in the upper angle of the wound. The membranous portion, which is readily palpable as a rounded prominence, is incised longitudinally for a distance of about $1 \mathrm{~cm}$., and retrograde sounding can be practised through the narrowed portion, which is then dirided.

Retrograde sounding of the stricture can be practised also from the bladder, after this has been opened through a suprapubic incision. The patient liss upon his back, with the pelvis elevated, and the bladder is opened in the usual manner above the symphysis pubis. The incision in the bladder is held open by tenacula and an English catheter of small caliber is pushed forward under the guidance of a finger through the neck of the bladder into the urethra to the point of obstruction. The patient may be placed in the position as for the operation for stone, the wound in the perincum held open by tenacula and the stricture is passed or merely entered by pushing the catheter forward from the bladder. In the first event the stricture is divided down to the catheter; in the second, the portion of the urethra lying to the proximal side of the stricture is opened and the stricture itself is successively divided with scissors from behind forward. The last step is, as a rule, effected without difficulty.

Urethrotomy is indicated after traumatic rupture of the urethra, complete or incomplete, when catheterization is attended with difficulty, or urinary infiltration is threatened. The incision is made through the perineum in the raphe, over the greatest prominence of the perineal bulging that is always present. After division of the skin 
and the superficial fatscia, entrance is gained to the woundcavity filled with bloesl-elots. The wound is earefinlly explored in all it: parts; the peripheral stump is alwars readily found, while the central extremity of the urethra is frequently retracted, thongh often enough risible, free in the wounil.

Primary suture is only rarely practised on account of the contused state of the extremities of the urethria. A soft catheter is introduced through the orifice of the urethra into the wound and passed through the central stump into the bladder, and fixed in place, the woundeavity being tamponed.

The simplest form of urethrotomy is practised in cases in which a stone or foreign body in the urethra is to be remored through an incision from without. The incision is made upon the under surface of the urethra, directly upon the palpable foreign body. The mucous membrane is opened by a linear incision, the stone extracted, and the wound in the urethra closed br suture. The fascia and the skin are united at a second sitting. A retentioncatheter is introduced.

Internal Urethrotomy.-Internal urethrotomy, division of a stricture from the lumen of the urethra, is indicated in the presence of:

(1) A contracting stricture.

(2) Narrowing, with ennsecutive disease of the urinary passages, if dilatation is always attended with fever, exacerbations of an existing eytitis, ete.

(3) Complete retention of urine in consequence of stricture, especially in all cases in which rapid restoration of a large caliber for the urethra is desired.

Mode of Performing Internal Liethrotomy:

(1) Introduction of a filiform bougie, to whose extremity is attached the rigid sound provided with a groove for the knife.

(2) The cannulated sound is passed through the stricture according to the rules for catheterization with rigid instruments. 
(3) The blade of the urethrotome is introduced into the anal of the guiding somd and pushed forward to the stricture; by pressure upon the end of the blade the stricture is divided through the upper wall of the urethra.

(4) After remoral of the entire instrument, a retentioncatheter (caliber 16) is introduced into the methra.

Lateral Incision for Stone.-The patient is placed in the usual position for stone-operations, with the operator sitting in front. A convex grooved sound is introduced into the urethra and held rertically br an assistant, with the groove directed toward the left. The incision passes from the middle of the perineal raphe to a point midway between the anus and the left ischial tuberosity. Deep dissection is proceeded with, the position of the groove in the guide bring constantly kept in mind. After the deeper layers of the perineum (superficial and deep perineal fascia, superficial transverse perineal muscle) have been passed and the urethra has been opened upon one side (aroiding the bulb) the groove of the instrument will come into view in the round. The operator grasps the guide with his left hand and introdnces into the wound a blunt-pointed knife, with its edge directed downward, in such a manner that it lies with its back directly against the guide. While the handle of the guide is depressed, the operator divides the membranous portion up to the prostatic portion with the blunt-pointed knife (lithotome), which he pushes forward in the direction of the urethra. At this moment the contents of the bladder stream into the wound by the side of the guide. The wound-canal is enlarged instrumentally or with the finger, which is permitted to enter the bladder, and the calculus is extracted with stone-forceps or a stone-spoon. In the aftertreatment a catheter is permitted to remain in the urethra and the wound is tamponed.

Median Section for Stone.-The details for the operation are precisely like those just described. The guide is held accurately in the median line, with its groove directed 
forward. The cutaneons incision passes through the perineal raphe, beginning just behind the attachment of the scrotum and extending for a distance of 5 or $6 \mathrm{~cm}$. towarrl the anus. The knife is introduced deeply in a vertical direction, an encleavor being made to reach the membranous portion of the urethra, with aroidance of the bulb. The urethra is opened in the median line and the incision is prolonged, as in the operation just deseribed, by means of the blunt-pointed knife to the prostatic portion. The stone is now removed in the typieal manner.

The lateral and median incisions for stone, which in the past were the customary operitions, possess to-day but a limited field of application, having been almost completely displaced by the suprapubic incision. [Displaced particularly by litholapaxy.-ED.] The median incision, the more recent of the two, was chosen in order to avoid the division of the ejaculatory ducts that his heen observed repeatedly as a result of the lateral incision. Both methods are attended with the disadvantage that the removal of large stones through the narrow wound-canal can be effected only with difliculty, so that the wound itself is distorted and lacerated in the efforts at extraction and dilatation and the conditions for recovery are rendered less favorable than otherwise they would be. Finally, a typical form of true incontinence, permanent dribbling of urine, is not rarely observed after the median or the lateral incision, even when union has proceeded smoothly.

Urethrostomy.-In cases of incurable stricture Poncet excludes entirely the narrowed portion of the urethra by means of perineal urethrostomy, dividing the urethra on the proximal side of the strieture and permitting it to open upon the perineum. The stricture is exposed by means of the usual incision through the perineal raphe, when the urethra is divided transversely on the proximal side of the stricture, and is sutured in the lower angle of the eutaneous wound. Poncet divides the central stump throughout a slight extent on its under side before suturing it in the wound. The peripheral stump of the urethra is sutured and dropped into the wound, when the cutaneous wound is closed by suture up to the opening of the fistula.

Litholapaxy.-Instrumental endovesical crushing of stone in the bladder, followed inmediately by evacuation of the fragments, is designated litholapaxy. The instrument for destroying the stone is made of steel, 
shaped like a catheter, and consists of two blades fitting one into the other and the tip of one of which is serrated. The instrument grasps the stone between its two blades, which can be secured firmly and are brought together by means of a screw-mechanism; the stone, thus grasped, is crushed between them. To attain good results with the operation of litholapaxy a careful sclection of cases, as well as skill in the use of the instrument, is required.

Dode of Procedure.-The patient oceupies the dorsal decubitus, with the pelvis somewhat elevated. The bladder is filled moderately with sterile solution of boric acid. The lithotrite is introduced according to the rules laid down for catheterization, the operator standing upon the right side of the patient. An attempt is made to touch the stone with the tip of the closed instrument, when the blades are separated to grasp the stone. The blades of the instrument are fixed by means of a sliding arrangement on its handle, and the stone is crushed by means of the screw-mechanism. Now, the individual fragments of the broken calculus are grasped separately and are crushed. Finally, the residue is converted into a fine powder by crushing. A rigid catheter is introduced and the bladder is irrigated, with the escape of sand. With the bladder moderately full the evacuation-catheter is connected with a pump, whose activity is continued as long as fragments of stone are present in the bladder. If a rather large fragment of stone can be felt, this must be reduced further in size br means of the lithotrite. The crstoscope permits confirmation by ocular inspection of the fact that complete evacuation of the fragments has been effected. With a proper selection of the cases the results of litholapaxy may be admirable.

Operations on the Bladder.-The bladder, situated in the true pelvis just behind its anterior wall, is attached to the pubic arch through the prostate gland and the pubovesical ligament. Beside, the organ is held in place within certain limits by the visceral layer of the pelvic fascia, by the resico-umbilical ligaments and by the peri- 
toneum. The peritoneum passes from the anterior abdominal wall and from the lateral walls of the pelvis upon the bladder, whose findus and posterior and lateral walls it covers. When empty, the bladder is concealed bohind the symphysis. When filled, the upper portion rises above the pelvic brim, so that the anterior wall of the bladeler not covered by peritoneum comes to lie in immediate juxtaposition with the abdominal wall. The bladder can thus be opened above the s.mphysis without injury to the peritoneum if filled to the maximum.

Suprapubic Cystotomy.-Opening of the bladder above the symphysis pubis.

The operation is indicated :

(1) In the presence of calculi and other foreign bodies in the bladder ;

(2) In the presence of tumors of the bladder;

(3) In the presence of tuberculosis of the bladder;

(4) In cases of resical hematuria ;

(5) In cases of rupture of the bladder;

(6) For the removal of hypertrophied lobes of the prostate gland;

(7) For the purpose of forming a fistula;

(8) In cases of serere cystitis;

(9) As a preliminary operation in the performance of posterior catheterization.

The mode of procedure is subject to various modifications in accordance with the indications for its performance. Three types of operation are distinguished:

(1) Simple opening of the bladder for the removal of calculi and other foreign bodies;

(2) Opening of the bladder for the purpose of undertaking endovesical manipulations (extirpation of tumors, etc.) ;

(3) Opening of the bladder for the purpose of establishing a fistula.

In all cases the bladder is distended to the maximum by the injection of fluid into its cavity, so that it rises above the level of the symphysis. If it is impossible 
thus to fill the bladder, the anterior wall of the viscus is forced into the wound by means of a concave grooved guide and incised.

1. Suprepubic Cystotomy for Stone.-. The patient occupies the dorsal decubitus, with the pelvis somewhat raised by means of a pillow, and the operator stands to his right side. After the abdominal wall has been cleansed and shaved, a catheter is introduced and the bladder is irrigated until the escaping fluid is clear. Sterile fluid

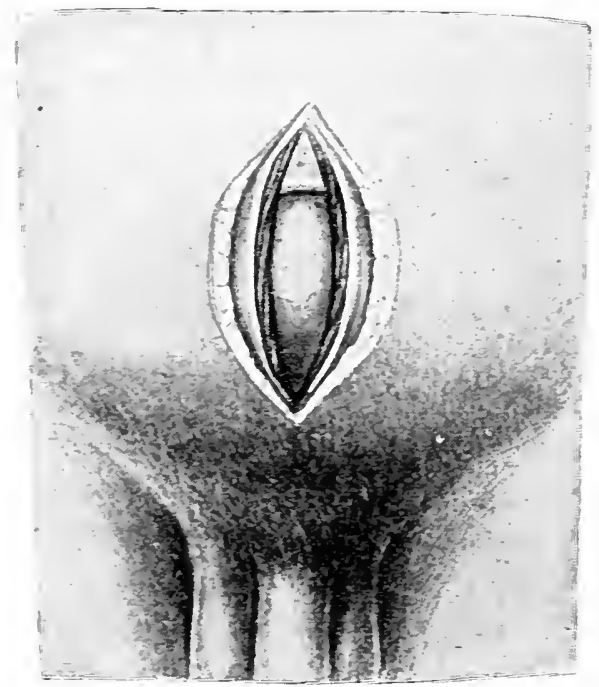

FIG. 208. - Suprapubic erstotoms: the anterior wall of the bladder is exposed; near its summit is the point of reflection of the peritoneum.

is now permitted to flow into the bladder through an irrigator or a sufficient quantity is injected to cause the bladder to become palpable as a tense swelling above the symphrsis pubis. The catheter is removed and the penis is surrounded with a strip of ganze. The incision is made in the linea alba just above the srmphrsis, and is from 5 to $7 \mathrm{~cm}$. long. Passing directly to the depth of the wound the fatty laver is traversed and the anterior rectus sheath or the fibrous linea alba is divided. The rectus muscles are retracted, and in the space of Retzius 
thus exposed the bladder is palpable as a tense mass. The prevesical fat is displaced from the bladder by blunt dissection by means of two pairs of forceps, until the anterior wall of the viscus, recognizable by the difference in conr and by the bundles of muscles and reins upon its surficee, is exposed (Fig. 208).

Just below the transverse line of reflection of the peritoneum a simple pointed tenaculum is introduced into the wall of the bladder, which is divided in the median line with a sharp-pointed knife, in the direction of the symphysis. The margins of the wound are held apart by means of two retractors. The operator introduces the index-finger of his left hand into the bladder, touches the stone or the foreign body, and permits the stone-foreeps to follow the palmar aspect of the finger to the calculus. The blades of the forceps are now separated, the stone grasped and removed from the wound. The wound in the wall of the bladder can be closed at once by suture. Various complicated methods of suture of the bladder have been abandoned. The wound in the viscus is closed by a series of interrupted catgut-sutures, including the entire thickness of the wall of the bladder, with the exception of the mucous membrane.

Fixation of the sutured bladder to the abdominal wall (cystopery) is not without advantage. Suture of the wall of the bladder may be omitted and the wound in the viscus be permitted to remain open. The urine is removed by suction and the bladder is by this means kept perfectly at rest.

2. Sumapubic Cystotomy for the Purpose of Undertaking Intracesical Manipulations.-If opening of the bladder is effected as a preliminary procedure to facilitate intraresical manipulations, it is best to raise the pelvis as high as possible. The preparations for the operation, and the opening of the bladder, are marle in the manner already described. After the bladder has been opened a view of its interior should be possible. To this end the margins of the wound in the bladder are held apart by means of 


\section{Plate 21,-Suprapubic Cystotomy with the Pelvis Elevated.}

The round is enlarged br the introduction of retractors and the interior of the bladder is rendered visible. The wall of the viscus has been provisionally attached to the skin by suture. There may be observed the mouth of the urethra, the trigone, and the entrances of the ureters. The wall of the summit of the bladder appears as a prominence above the broad speculum.

retractors resembling vaginal specula, while a broad Simon speculum is introduced in the upper angle of the wound. If, beside, the interior of the viscus is illuminated by means of an incandescent lamp, inspection is readily possible and the operator may undertake a variety of manipulations within the cavity of the bladder (excision of tumors, control of hemorrhage, suture of deficiencies in the mucous membrane, excochleations, etc.) (Plate 21).

It after extirpation of resical tumors bleeding has been completely controlled, the bladder may be closed by suture. After excochleation of malignant tumors, and after operations upon the prostate, drainage of the bladder is the preferable mode of procedure.

Br making a transverse incision through the skin and opening the bladder in the same manner, after division of the recti muscles, general access to the interior of the viscus is possible.

Suggestions have been made by a number of operators to expose the bladder throughout a greater extent by means of operations upon the bony parts. To this end Helferich resects a triangular portion of the symphysis pubis, while Bramann recommended temporary partial resection of the symphysis, and Niehans lateral resection of the pelvis.

3. Section of the Bladder for the Purpose of Establishing a Fistula._-Cystostomy.-In the performance of crstostomy a short longitudinal incision is made above the symphysis pubis according to the method of Poncet, and the bladder is opened in the usual manner. The wall of the bladder is brought to the level of the skin and fixed in place by 
Tab. 21

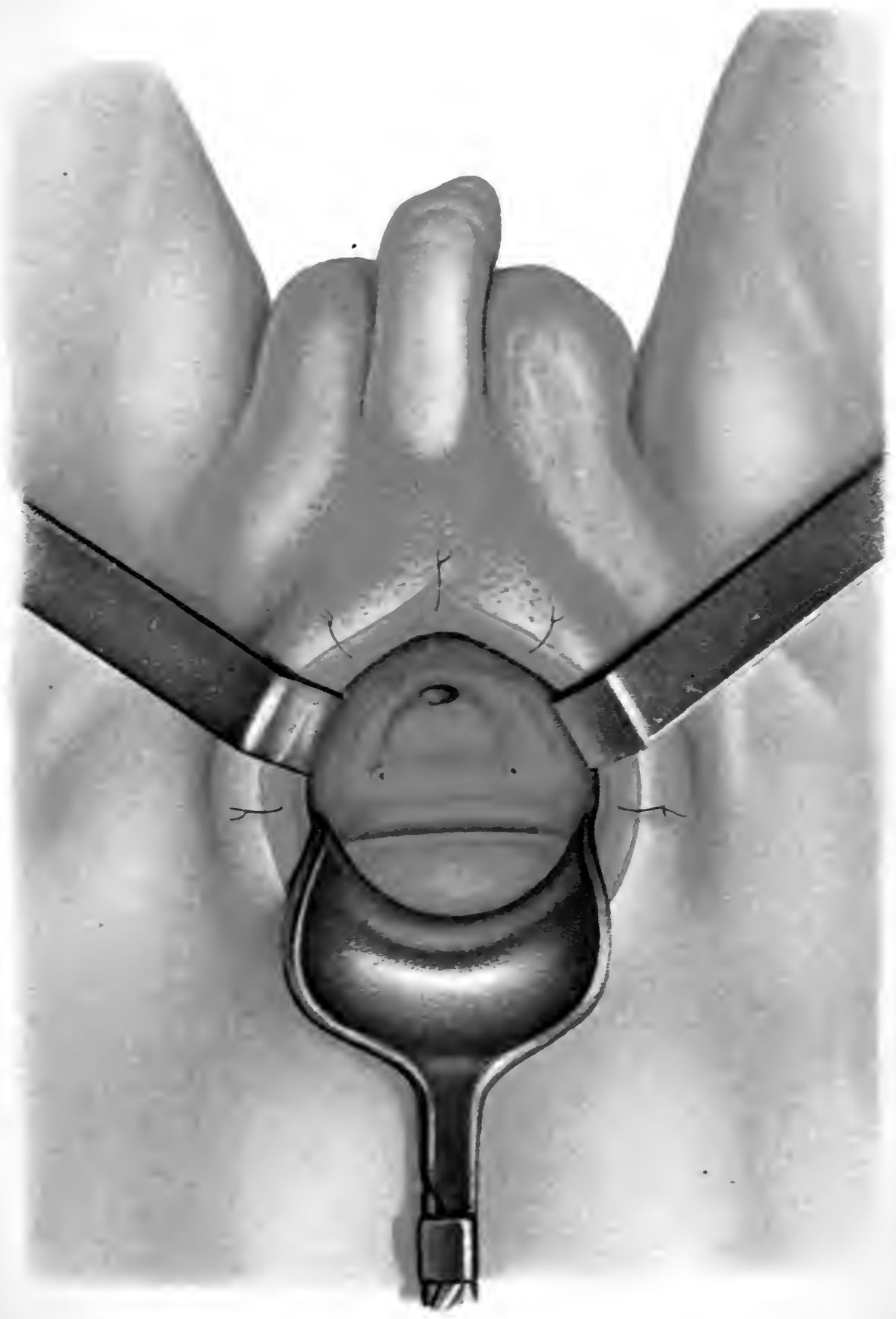

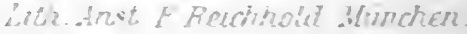


I

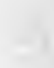


OPERATUAS UTON THE PROSTATE GLAND, ETC. 30̃Y

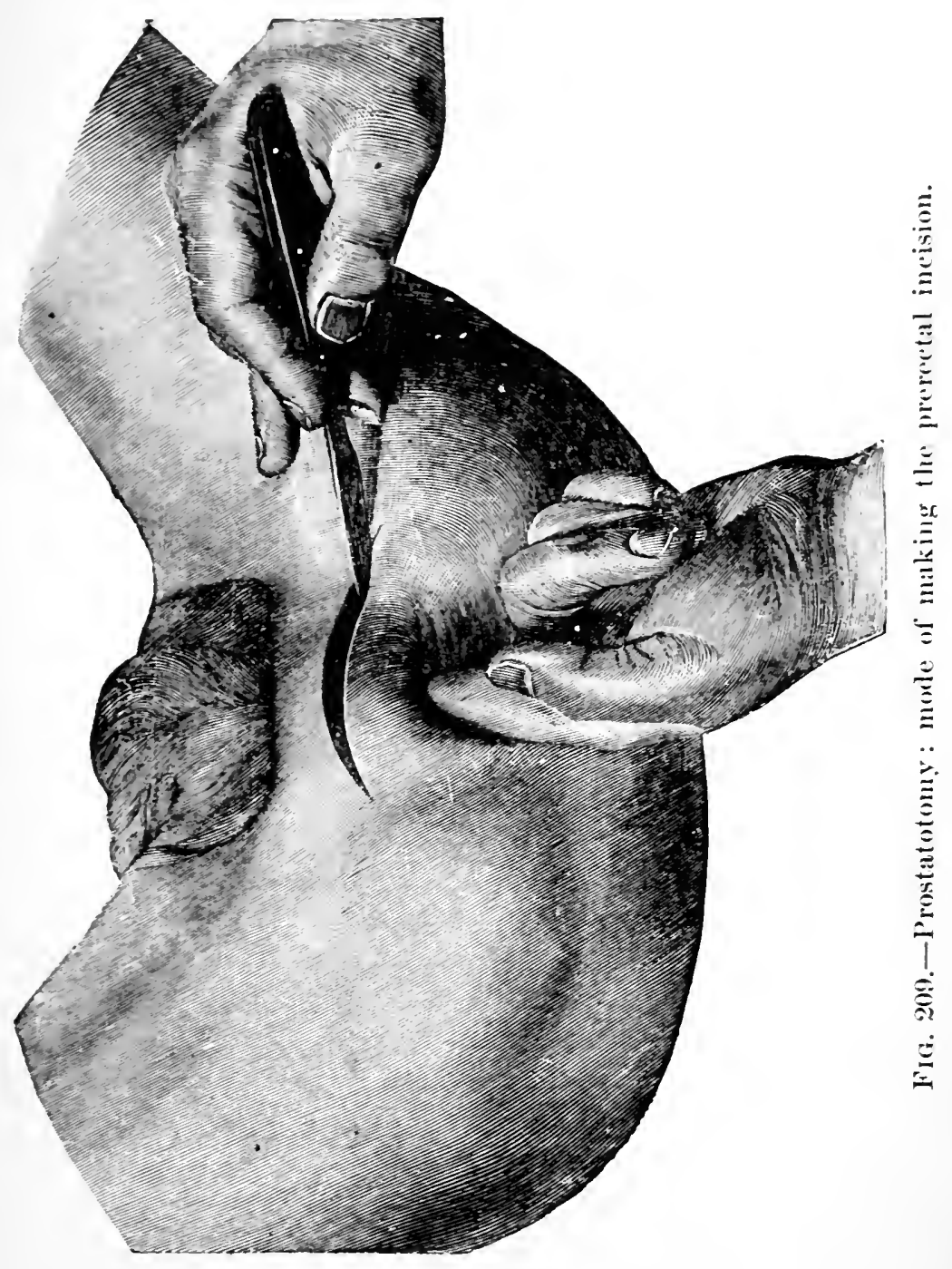





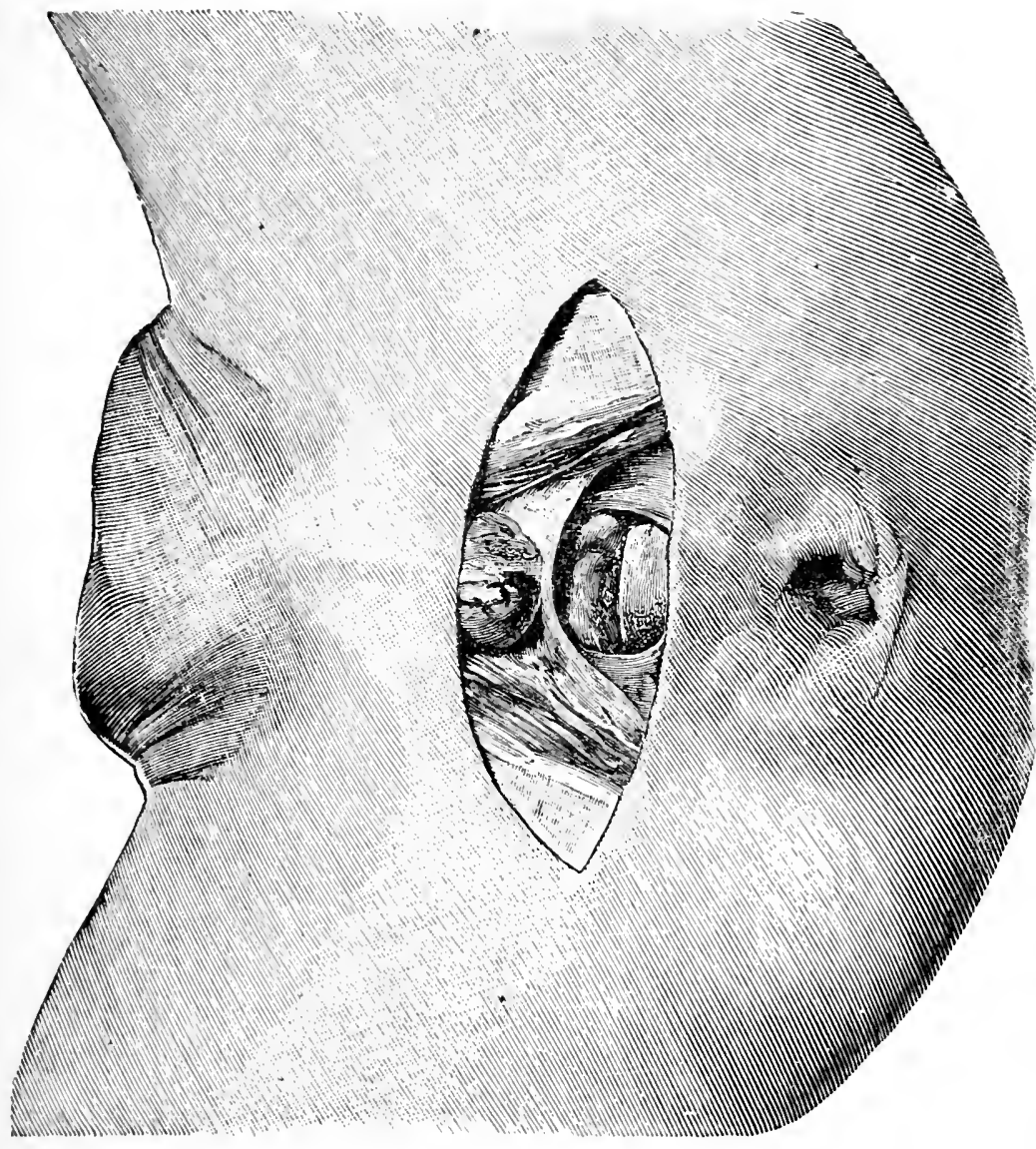

三

$\cong$

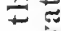

范

일

ํํㅇ

$\Xi$

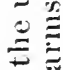

$\Xi \cong$

$\Xi \%$

苟焉

$\stackrel{Ð}{\Xi}$

范.

ธ

$\because \Xi$

$\stackrel{20}{2}$

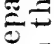

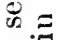

$+\cdots$

$\stackrel{\Xi}{\mathscr{2}}$

昰永

寻苛

อั

$\cong$

绝

크

承

है

ำ

牙

बह

..

․․․

武

$\stackrel{0}{0} \Xi$

$\approx \equiv$

○。

$4 \approx 0$

范芯

व)

$5 \equiv$

$=\stackrel{0}{0}$ ॠ 

sutures. The cutaneous wound is closed in its upper portion, while the resical nucous membrane is sutured in its lower portion. 'The establishment of a fistula may be effected also without suture of the resical mucous membrane, by the emplorment of simple siphon-drainage. The curved tube may in the further course of the case be replaced by a soft eatheter, which is introduced into the bladder through the wound and is permitted to remain.

\section{Operations upon the Prostate Gland, the Semi-} nal Vesicles, and the Vas Deferens.-Prostatotomy. -Opening of the prostate through an incision in the perineum is indicated in the presence of abscesses and for the excochleation of tuberculous masses in the gland. After the root of the penis and the superficial transverse perineal muscle are exposed by an ineision through the perineum and the connections between the sphincter ani and bulbocavernosus muscles are divided transversely, the anterior wall of the rectum can be separated by blunt dissection from the prostate gland and reflected toward the sacrum. Between the triangular ligament of the urethra and the rectum thus displaced the slightly convex posterior aspect of the prostate lies exposed throughout its entire extent (Fig. 210). Connected with the base of the prostate are the seminal resicles, and by continued detachment of the rectum from the bladder the fundus of the latter will be exposed, when the seminal resicles and the rasa deferentia will be visible in the wound.

The performance of prostatotomy in the presence of an abscess is performed as follows: the patient occupies the same position as in the operation for stone, and an English catheter of large caliber is introduced into the urethra. The operator sits in front of the patient and guides the knife with his right hand, while the index-finger of the left hand is introduced into the rectum, in order that in the progress of the deep dissection the anterior wall of the rectum shall be avoided (Fig. 209). A curved incision 4 or $5 \mathrm{~cm}$. long is made through the prerectal tissues. After division of the skin and the subcutaneous 
connective tissue the perineal septum is divided transversely and blunt dissection is made between the rectum and the urethra upward toward the prostate. When the lower pole of this organ or a portion of its posterior wall is exposed in the wound, a grooved director or a pair of forceps with closed blades is introduced into the fluctuating portion, when the pus escapes through the wound. The opening is now suitably enlarged and the abscesscarity is tamponed. If a communication exists between the abscess-cavity and the urethra, the retention of a catheter is necessary in the after-treatment.

Extirpation of the Seminal Vesicles.-In extirpation of the seminal vesicles the perineal route just described appears the most desirable. The patient and the operator occupy the same positions as in the operation just considered. A large perineal flap-incision is made whose posterior extremity on either side extends to the tuberosities of the ischium and whose anterior boundary lies in front of the rectum. The mode of procedure in the depth of the wound is analogous to that pursued in the performance of prostatotomy. The detachment of the rectum is undertaken through a considerable extent until the seminal resicles and the fundus of the bladder become visible on displacement of the rectum toward the sacrum. The altered seminal vesicles are peeled out of their surroundings, dissected from the fundus of the bladder, and removed. At the same time morbid collections in the prostate gland can be excised.

Excisions of the Prostate; Prostatectomy.-In cases of hypertrophy of the prostate gland removal of the enlarged middle and lateral lobes has been recommended for the relief of the difficulty in urination and has been practised by numerous surgeons with varying success. The question whether cure is effected by removal of the prostatic obstruction is not as ret decided. At any rate, advanced cases in which secondary changes in the wall of the bladder and of the upper urinary passages have taken place are not adapted to the operation. 
The enlarged middle lobe of the prostate, which often attains the size of a walnut and more, may be extirpated through the suprapubic ineision into the bladder (II. C. Gill). The bladder is opened above the symphysis pubis in the usual manner and the prominent tumor is removed with the Paquelin cautery, or the galvanocaustic loop, or with curved scissors. . The base of the wound is canterized for the control of hemorrhage, and, if necessary, the bladder is tamponed.

The enlarged lateral lobes of the prostate can be only partially removed or excised, care being taken to aroid opening the urethra. In the performance of partial resection adequate exposure of the body of the prostate is essential. The gland is exposed either with the aid of the perineal prerectal incision or through a sacral incision. The details of the first method are given on page 355. The sacral method of exposing the prostate has been recommended by Dittel (lateral prostatectomy). In this mode of procedure an incision is made in the folds of the anus, the rectum is displaced laterally and in this way the posterior surface of the prostate is bronght into view. The patient occupies the right lateral decubitus and an English catheter is introduced into the urethra. The incision begins at the apex of the cocerx, passes in the middle line to the posterior margin of the anus, which it surrounds upon the right side, and terminates in the perineal raphe in front of the anus. The operator gains entrance into the ischiorectal fossa, and separates the rectum by blunt dissection from the prostate, so that the right lateral lobe of the latter and, if the dissection be continued, the entire posterior aspect are exposed to view. Wedge-shaped portions of the gland are excised on either side. Dittel recommends that so much of the gland be removed that only sufficient remains to surround the urethra. The removal of the coccyx is calculated to enlarge the field of operation.

Resection and Extirpation of the Vas Deferens.-Resection of the vas deferens in its continuity has been recommended recently in the treatment of hypertrophy of the 
prostate gland, and has been performed in numerous cases. The vas deferens is palpable through the skin as a round, firm strand, and it may thus be separated from the remaining structures of the spermatic cord. The cutaneous incision for the isolation of the vas deferens, 3 or $4 \mathrm{~cm}$. long, may be made either in front of the external inguinal ring, or at the neck of the scrotum. The structures forming the spermatic cord are forced out of the wound, the vas deferens is isolated by touch and a portion from 2 to $4 \mathrm{~cm}$. long is excised with scissors. Removal of the vas deferens in connection with the testicle becomes necessary when in the presence of tuberculosis of the epididrmis the vas deferens also is involved in the disease. Under these conditions the incision for exposure of the testicle, which passes longitudinally over the scrotum, is extender upward and outward over the inguinal canal. Throughout the range of the incision the skin and the anterior boundary of the inguinal canal are divided so that the vas deferens is exposed in its course through this canal, and is thus rendered accessible to surgical removal. The pelvic portion of the vas deferens would be accessible by this means only after extended detachment of the peritoneum, entailing injury of disproportionate degree. This portion of the duct is therefore to be reached by the perineal route or with the aid of an incision such as Dittel has recommended in the performance of lateral prostatectomy.

Büngner recommended, in place of extirpation, divulsion of the vas deferens. The dnct is isolated and exposed throughont a considerable extent by gradially increased traction. With careful manipulation of this kind fourfifths of the entire duct may be removed.

Extirpation of the Testicle. Castration.-The indications for this operation consist in the presence of neoplasms of the testicle and tuberculosis of the epididymis. A new indication for castration is afforded by hypertrophy of the prostate. The cutaneous incision is always made longitudinally over the greatest convexity of the tumor. In making this incision the operator grasps the scrotum 
with his left hand in such a manner that the overlying skin is made tonse. If the skin is involved in the diseaseprocess thronghout a circumseribed area (from extension of a neoplasm, or the formation of a tubereulous fistula), the diseased structure is included between the incisions and is removed in eonjunction with the testicle. The incisions are made threngh the skin and the dartos down to the tunica vaginalis and the testicle, with its coverings, is freed from its bed by blunt dissection, so that it remains in connection with the body only through the intermediation of the spermatic cord. By traction on the cord its constituent structures are more clearly brought into view. The vas deferens is isolated and ligated. The remaining structures of the spermatic cord are ligated en masse in two or three segments. The cord is then divided transversely on the distal side of the ligatures, which are cut short. The stump of the cord retracts into the depth of the wound, and the cutaneous wound is closed by suture.

Operation for Hydrocele.-Hydrocele is treated in a palliative way by simple puncture and in a radical way by laying open the tunica vaginalis and excising it. Puncture of a hydrocele is made in accordance with the rules that govern the making of punctures in general. The operator must, however, be assured of the position of the testicle, in order to avoid injury of this organ in introducing the trocar. The scrotum is grasped firmly with the supinated left hand and made tense. The introduction of the trocar is made upward through the anterior wall close to the fundus of the scrotum at a point where no vein is visible through the skin. At first the fluid is expelled in a continuous stream. Later the escape must be facilitated by alteration in the position of the cannula and by kneading movements of the scrotum. The injection through the cannula of from 5 to $10 \mathrm{gm}$. of Lugol's solution, in eonjunction with the puncture, is a favorite mode of radical operation for hydrocele. The procedure, however, is extremely painful and with regard to the certainty of the result stands behind radical incision. 
Radical Incision by the Method of Vollimamn.-The scrotum is grasped firmly and made tense with the left hand and an incision is made longitudinally over the greatest convexity of the tumor almost up to the fundus. II ith careful dissection the incision is carried down to the tunica vaginalis, which is divided in the direction and throughout the extent of the cutaneous incision. After the fluid has escaped, the tunica raginalis is united to the skin by a row of sutures and a strip of gauze is introduced into the cavity that remains. The process of healing often occupies a considerable period of time.

Radical Operation by the Method of Bergmann.-Radical operation by the method of Bergmann is followed by recovery within a short time, by reason of the fact that the wound-conditions render possible union by primary intention. A cutaneous incision is made in the manner just described. Before the sac of the hydrocele is opened, an endeavor is made to free it from the overlying skin throughout a considerable extent. After this has been adequately effected the sac is opened as in the operation of Volkmann. After the fluid has escaped the operator grasps the margins of the incision through the tunica vaginalis and separates this from the testicle on either side, almost to the point of reflection. After this detachment has been thoroughly effected the freed parietal layer of the tunica raginalis is excised. The margins of the incision in the skin are accurately approximated by suture over the testicle, which is dropped back into the wound.

Operation for Phimosis.-Operations for phimosis include surgical procedures of various kinds by means of which congenital or acquired narrowing of the prepuce is removed. In performing the operation either the prepuce is divided longitudinally from its orifice to the glans (incision) or the entire prepuce is removed (circumcision). $\mathrm{By}$ the first of these methods the incision is made in the middle line of the dorsal surface, a grooved director, with its concavity directed upward, being introduced into the orifice between the prepuce and the glans. The two 
layers of the prepuce are divided over the director with a single stroke of the scissors almost up to the eorona glandis. After the division has been effected the prepuce must be readily retractable. Over the glans throughout the extent of the wound the mucous membrane of the prepuce is united with the skin by means of a series of interrupted sutures, or of a continuous suture.

Cireumeision may be effected in various ways. The prepuce may be drawn forward as far as possible and be

Operation fOR SHORTENED Frenum.

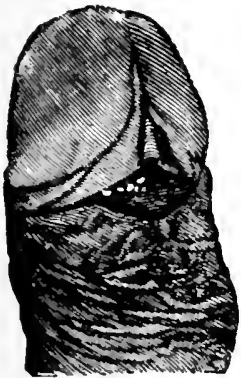

FIG. 211.

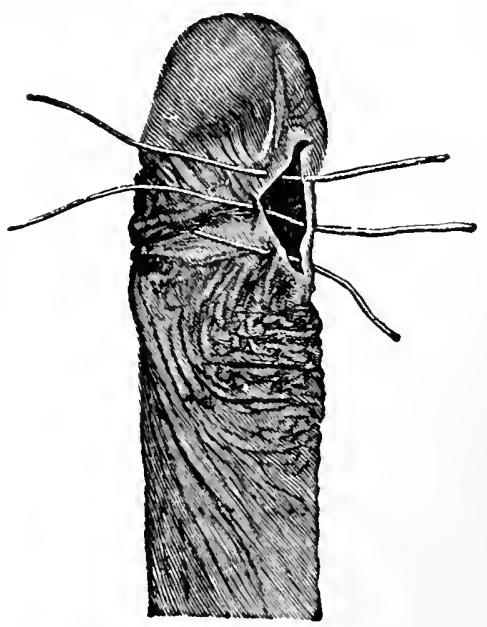

FIG. 212.

FIG. 211.-Transverse division of the frenum.

Fig. 212.-Union at right angles to the direction of the division.

divided just in front of the glans, after which the margins of the outer and inner layers of the prepuce are united by suture. In another method of circumcision the usual dorsal incision is first made, after which portions of the prepuce on either side of the incision are removed with scissors close to the point of reflection on the glans. Throughout the entire extent of the wound the skin is united with the inner layer of the prepuce.

Operation for Shortened Frenum.-Congenital shortness of the frenum, with a normal caliber of the prepuce, is 
attended with numerous discomforts (pain in coitus, frequent laceration, hemorrhage). Simple transverse division of the band is not to be recommended on account of the hemorrhage that follows. Division with the Paquelin cantery secures immunity from the hemorrhage, but a considerable time is occupied in the healing of the wound. Functionally good results, with the possibility of securing union by primary intention, are yielded by the following minor plastic operation.

The frenum is divided with a single stroke of the scissors to such a depth that the prepuce can be retracted to a maximum degree without tension. The small wound thus made is united at a right angle to the direction of the incision (Figs. 211 and 212).

Amputation of the Penis.-Malignant neoplasms constitute the exclusive indication for amputation of the penis. This may be practised through the pendulous portion at a selected level by means of a circular incision. Under certain conditions the deeper portions of the member, the roots of the cavernous bodies, must be removed by operation. In all cases, after ablation of the parts, the urethra must be suitably situated and fixed in the wound.

In amputating the penis through the pendulous portion digital compression is exercised, while a circular incision is made transversely. The skin is, after division, retracted, when the operator divides the cavernous bodies transversely with an amputation-knife, cutting from the dorsal aspect toward the urethra. When the urethra is reached it is dissected free for a short distance toward the periphery and is divided transversely $2 \mathrm{~cm}$. in advance of the line of incision through the cavernous bodies. The urethra is snipped through its inferior surface with a single stroke of the scissors, spread upon the wound and united by its free border with the margin of the skin by means of a series of sutures.

In amputating the penis in conjunction with its perineal connections the scotum is divided in a sagittal direction. In the gaping wound the roots of the cavern- 
ous bodies, with their attachments to the pubie bones, are readily exposed. The urethra is divided transversely through healthy structure, snipped on its lower surface, and sutured in the posterior angle of the wound (perineal urethrostomy).

The avornoms bodies of the penis are detached and reflected upward in conjunction with the peripheral portion of the urethra. If the detachment has been extended to the pendulous portion, the penis is after circular incision of the skin divided transversely at its scrotal attateliment and removed. The scrotal wound is closed by suture up to the newly established orifice of the urethria.

Operation for Urethral Fistula._-As long as the fistulous passage is not covered over by skin spontaneous cure can be effected by local applications conjoined with dilatation of the urethra. Under the reverse conditions, and if the mucons membrane of the urethra is adherent to the skin, freshening of the margins of the wound, with suture, becomes necessary to effect a cure. If the fistula be small, an elliptic area is freshened and the defect is closed by transverse deep and superficial sutures (Fig. 213). In freshening an oval area the formation is recommended of lateral flaps by means of ineisions made on either side close to the upper and low extremities of the oval. These flaps are detached from the subjacent structures and after the introduction of huried sutures are brought togetiner and united over the defect (Fig. 214). It is a useful procedure further, after freshening the margins of the fistula, to separate the skin from the mucous membrane throughout the extent of the refect by means of horizontal incisions, so that the margins of the mucous membrane can be approximated without tension. The nucous membrane is united over the defect by means of catgut sutures, and finally the wound in the external integument is closed. In the presence of an extensive defect in the urethra a tegumentary flap taken from the penis is brought over the freshened defect in such a way that its cutaneous aspect 
is turned toward the lumen of the urethra. The second step consists in union of the wound in the skin.

Under the best of circumstances the success of any operation for urethral fistula will be rendered disappointing by reason of the discharge of urine, as well as through erections, with tearing ont of the sutures. The evacuation of urine interferes with the process of healing whether a catheter is retained or spontaneous micturition takes place.

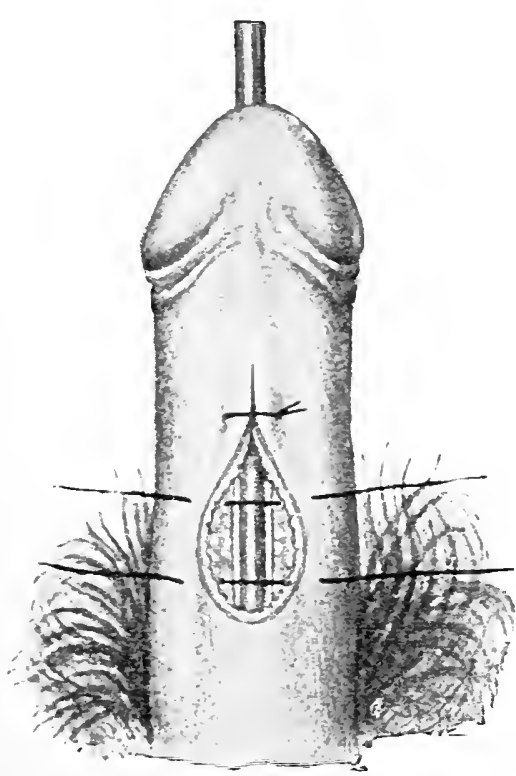

FIG. 213.--Urethral fistula : elliptic freshening; suture.

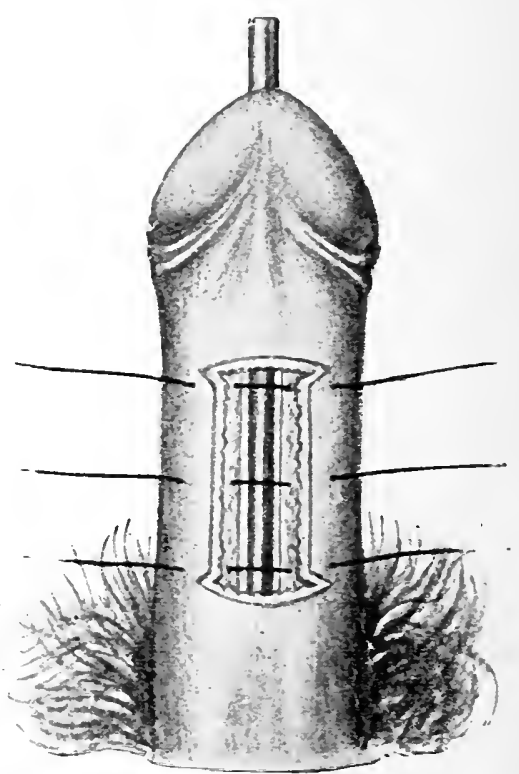

Fig. 214. - Urethral fistula : freshening, with the formation of lateral flaps; suture.

In the performance of extensive plastic operations upon the penis, therefore, the formation of a provisional vesical or deep urethral fistula during the time of healing is to be recommended.

Operations for Hernia.-Bloody operations for hernia are undertaken for the purpose of either releasing an existing strangulation or removing a free or adherent hernia (radical operation). The first form of operation 
(herniotomy) consists in division of the coverings of the herniat, opening of the hernial sac, and division of the constricting ring. The further steps of the operation (reposition of the intestine, formation of a preternatural anus, resection of the bowel) will be governed by the conditions present in the individual case. The cutaneous incision is made in the longitudinal axis of the hernial tumor; in the presence of inguinal hernia in such a manner that the inguinal canal, as well as both inguinal rings, is included within its range. In the presence of femoral hernia the rertical incision passes orer the greatest convexity of the hernial tumor. By careful disection, laver by laver, after division of the subcutineous connertive tissue and the so-called proper fascia of the hernia, the hernial sac is reached. This presents a dull appearance, is in places the seat of small masses of fat, and is often so delicate that the hernial fluil can be scen through it. In the presence of inguinal hornia it will be necessary to divide in the upper portion of the hernial tumor the anterior wall of the inguinal canal, constituted by the aponeurosis of the external oblique muscle and fibers of the internal oblique and transversalis muscles, before the actual coverings of the hernial swelling are reached. The sac of the hernia is incised in the direction of the cutaneous incision, and, after the hernial fluid has been permitted to escape, the remoral of the constriction is undertaken. In the presence of an inguinal hernia it will be possible always to expose the constricting ring by division of the anterior wall of the inguinal wall throughout its entire extent (Fig. 215). This is dons carefull - from without inward until all tension has disappeared.

In the case of femoral hernia division of the constricting band is effected from the carity of the hernia by means of a blunt-pointed knife, or herniotome, under guidance of the finger, and alwavs directed inward. The sharp margin of Gimbernat's ligament is incised and the constriction is thus removed. After the division of the constricting band has been effected the involved loop of 
intestine is brought forward so that the point of strangulation, as well as the proximal intestine, may be serutinized. If the serous layer is smooth and lustrous, the intestinal loop of bowel is restored to the abdominal cavity. To effeet reposition the loop of the intestine is

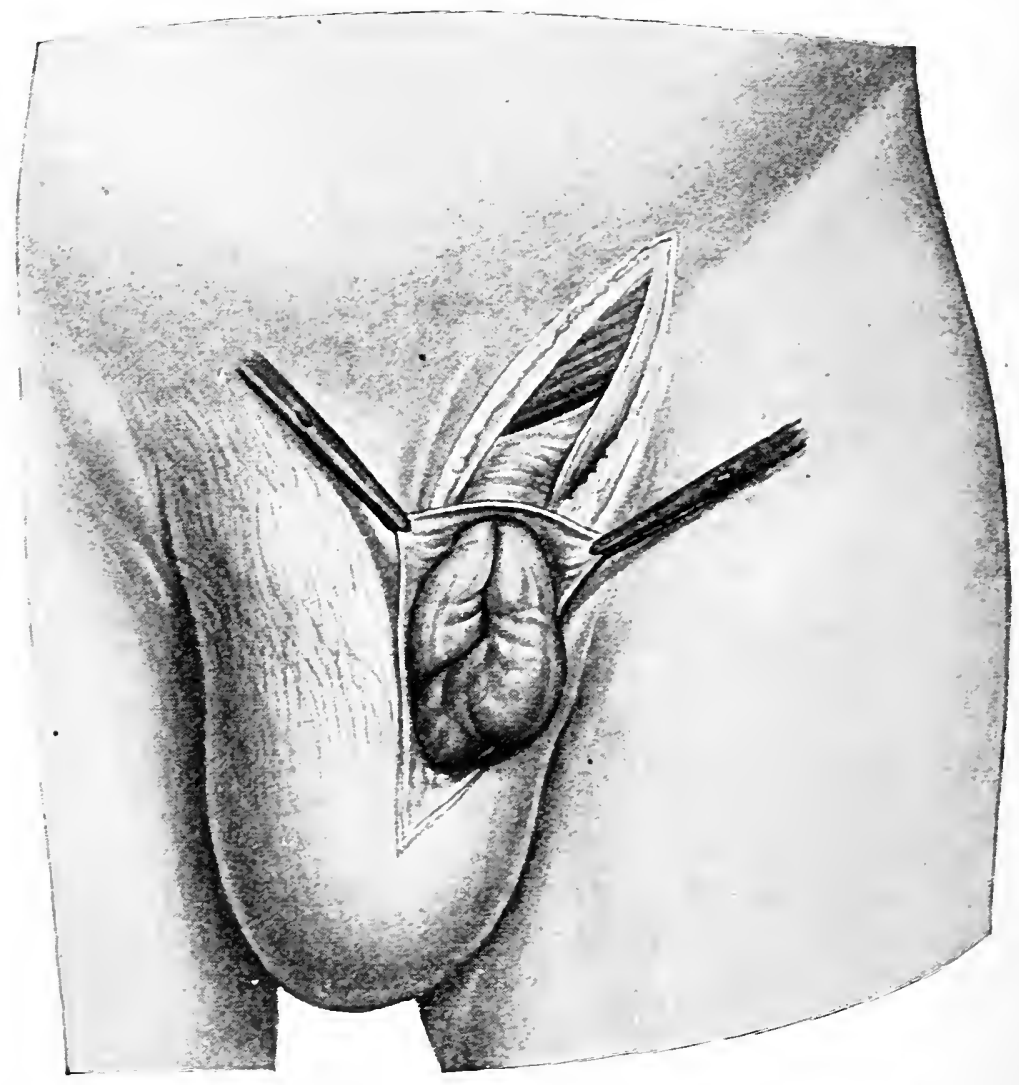

FIG. 215.-Inguinal hernia: the hernial sac is opened; the outer portion of the constricting ring is exposed.

gently compressed, so that any contents that may be present may escape. By means of pushing movements with the fingers the loop is gradually returned to the abdominal cavity through the hernial opening. During the process of reposition the hernial sac is held tense. 
To convince one's self that the bowel has been actually replaced in the abdominal cavity it is recommended after reposition has been effected that a finger be introduced through the hernial ring into the abdominal cavity and be swept around the intemal opening of the hernial canal. In this way defective reposition or apparent reduction will be aroided.

Prolapsed omentum is either simply replaced, or, if thickened, is ligated in segments and removed. If the contents of the hernia are adherent to the inner surface of the hernial sac (adherent hernia), reposition must be preeeded hy detachment of the adhesions.

If the serous membrine is dull at the line of strangulation, the loop of intestine is, after division of the constrieting band, left in the wound. In accordanee with the further course of the ease the loop may be replaced after the lapse of several days or a preternatural anus may be established.

If the loop of intestine prove to be gangrenous at the time of operation, a preternatural anus is at once established, the bowel being sutured in the wound. Another procedure in the presence of gangrene of the bowel consists in primary resection and union by circular suture.

If a fecal abscess have already formed, it is opened widely and the presenting loops of intestine are secured from slipping back into the abdomen by suture.

The last step of the operation, elosure of the wound, demands exact approximation of the hernial canal, with the object of preventing renewed formation of a hernia.

Radical Operation for Hernia.-Radical operation for hernia may be performed in conjunction with herniotomy, and is besides indicated in the presence of adherent hernias or of free hernias that give rise to discomfort and cannot be retained in place with the aid of trusses.

Radical Operation for Inguinal Hernia.-The radical operation for inguinal hernia has for its object closure of the hernial eanal and strengthening of the inguinal canal, which after the operation continues to serve as a channel for the spermatic cord, so that it will offer adequate resistance to the intra-abdominal pressure.

The inguinal canal comprises the interval between the internal and the external inguinal ring and traverses the abdominal wall in an oblique direction, down ward and inward. The intermal opening of this canal, the internal inguinal ring, represents the entrance of the process of transversalis fascia extending into the scrotum. The external inguinal ring is formed by an opening in the aponeurosis of the external 


\section{Plate 22.-Bassini's Operation for Inguinal Hernia.}

I. Exposure of the aponeurosis of the external oblique muscle and the external inguinal ring.

II. The aponeurosis of the external oblique muscle is divided, as well as the internal oblique and transversalis muscles; the spermatic cord is retracted and at the bottom of the wound upon the peritoneum the epigastric vessels can be seen; the layer of tissue with the sharply defined border is the transversalis fascia.

oblique muscle just above Poupart's ligament to the outer side of the tubercle of the pubic bone. The anterior wall of the inguinal canal is formed by the aponeurosis of the external oblique muscle and the fibers of the internal oblique and transversalis muscles. The posterior wall is formed mainly by the transversalis fascia and is fortified at the level of the external inguinal ring by muscular bundles from the internal oblique and transversalis.

Steps of Bassini's Radical Operation for Hernia.-A cutaneous incision is made in the direction of the inguinal canal, from the neighborhood of the internal inguinal ring and passing downward beyond the external inguinal ring. After division of the skin and the subcutaneous connective tissue the aponeurosis of the external oblique muscle is divided in the direction of the cutaneous incision down to the external inguinal ring (Plate 22). After the thin muscular laver of the internal oblique and transversalis is divided in the same direction and throughout the same extent, the structures of the spermatic cord, which are adherent to the hernial sac, come into view. At the neck of the hernial sac the vas deferens, the artery and the vein of the spermatic cord are detached from the sac and they are isolated to a point beyond the hernial ring. The hernial sac is now opened at its fundus and its contents are restored to the abdominal cavity. While the hernial sac is brought forward and is twisted at its neck through an are of $180^{\circ}$ the operator passes a ligature around the neck of the sac and tightens it as closely as possible to the trunk. The hernial sac is divided transversely with a single stroke of the scissors at a point to the periphery of the ligature and is removed. There remains to be effected the re-establishment of a new 
Tab. 22.

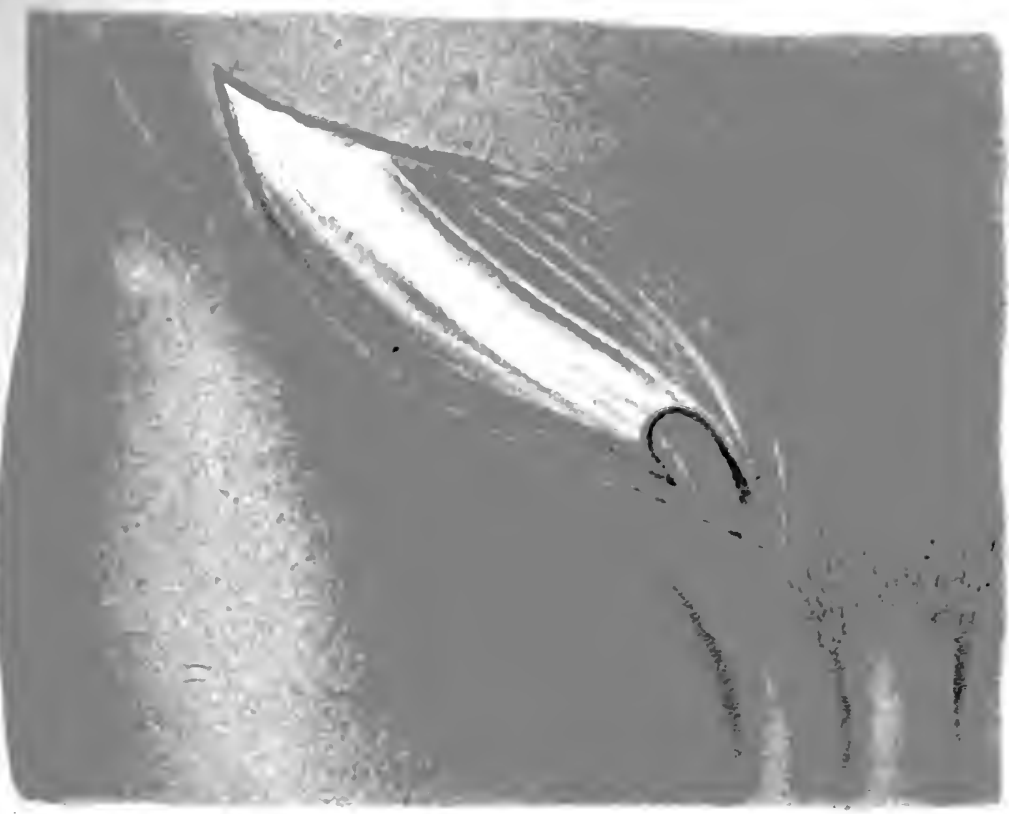

1 .

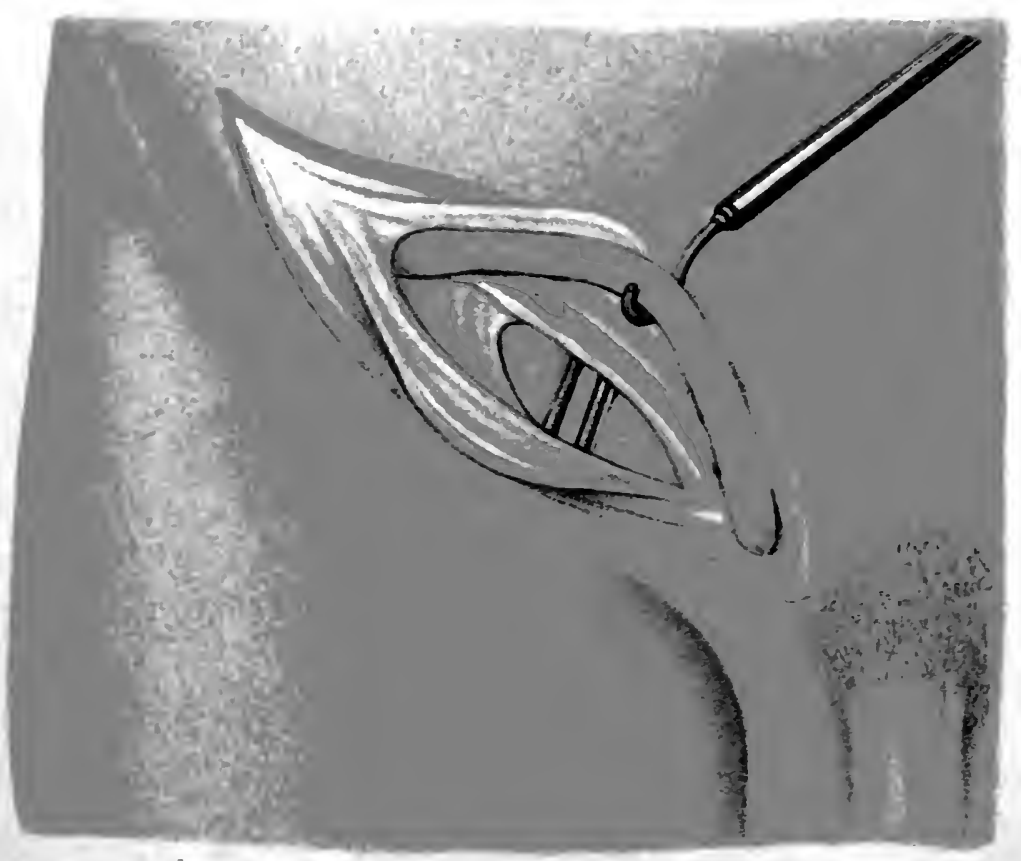

II.

Lith. Anst F. Rechhold. Müncher. 


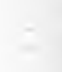

4

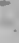


inguinal canal with thoroughly resistant walls. If the spermatic cord has been retrated toward the median line, and if the two margins of the divided aponemrosis of the external oblique musele have been displated upward and downward, there will appear in the wound npon one side the groove of Poupart's ligament and upon the other side the outer border of the rectus musele, together with the muscular plate formed by the divided internal oblique and transversalis muscles. This muscular layer is separated by blunt dissection from the subserous tissnes, as well as from the aponeurosis of the external oblique, so that it is rendered sufficiently movable to be brought down to Poupart's ligament. 'The muscular plate named is attached to the posterior border of Poupart's ligament by sutures (Plate 23, III). The sutures on the pubic bone include also the external border of the rectus muscle. In this way a posterior muscular wall of sufficient resistance is formed, in which lies the newly created narrow internal inguinal ring. The spermatic eord is placed upon this muscular layer, and over it the aponeurosis of the external oblique is elosed by suture except at its lower angle, which constitutes the new external inguinal ring (Plate 23, IV). The inguinal canal thus formed is, after healing has taken place, so resistant that the use of a supporting truss can be dispensed with.

In Kocher's radical operation for inguinal hernia (displacementmethod) the eutaneous ineision is made as in Bassini's operation, altlough the aponeurosis of the external oblique is not divided, but slit to the onter side of the internal inguinal ring. Through this opening a pair of forceps is introdueed and passed through the inguinal canal to the external inguinal ring. The apex of the isolated hernial sac is grasped, and drawn outward through the small opening. The hernial sac is drawn so that it appears sharply bent back ward at the internal inguinal ring. The portion of the sac lying within the abdominal wall is surrounded and strongly ligated. The base of the sac thus folded together is attached to the outer surface of the anoneurosis of the external oblique by means of deep sutures, and the remainder is removed.

\section{Radical Operation for Femoral Heria.}

The Femoral Chual.-The interval between the internal and the exterual femoral ring, which constitutes the path for certain varicties of heruia, is known as the femoral canal. Under normal conditions, 


\section{Plate 23.-Bassini's Operation for Inguinal Hernia.}

III. 'The muscular layer of the internal oblique and transversalis is attached by suture to the inner border of Poupart's ligament. In this was the internal inguinal ring and the posterior wall of the inguinal canal are formed anew.

IV. The aponeurosis of the exterual oblique is united over the spermatic cord except in the situation of the new external inguinal ring.

however, this canal is not present as such. The inner orifice of the canal (internal crural ring) lies at the inner angle of the opening for the femoral ressels, between Poupart's ligament and the horizontal ramus of the pubis. Thespace is bounded within by the free border of Gimbernat's ligament (fan-shaped attachment of Poupart's ligament to the tubercle of the pubic bone) and withont by the large vessels and the crural vein. The outer orifice of the crural canal corresponds with the loose connective tissue of the fascia lata (foramen ovale) through which the saphenous vein passes to enter into the femoral. The tendinous boundary of this opening has its concavity directed toward the middle line and is known as the falciform process.

A femoral hernia, after passing the internal crural ring, enters a space whose floor is formed by the pectineal fascia, which is bounded internally by Gimbernat's ligament, externally by the crural vessels, and in which for a short distance the upper continuation of the falciform process forms a resistant cover. If the free border of this be passed, the hernia can push before it the less resistant lamina cribrosa and in this way it reaches the exterior through the foramen ovale. The coverings of a femoral hernia are thus fewer and thimner than those of an inguinal hernia. The hernial sac may under circumstances, in emaciated subjects, lie just beneath the subcutaneous connective tissue. For this reason, in making the cutaneous incision, especially in further dissection at a depth, great care will be required. In general the radical operation consists, after making a cutaneous incision, in isolation and opening of the hernial sac. Then follow reposition of the hernial contents and ligature of the neck of the sac; with or without torsion. By closure of the hernial opening through suture the recurrence of the disorder is to be prevented. Radical operation for the relief of femoral hernia is rendered difficult by the fact that the 
Tab. 23.

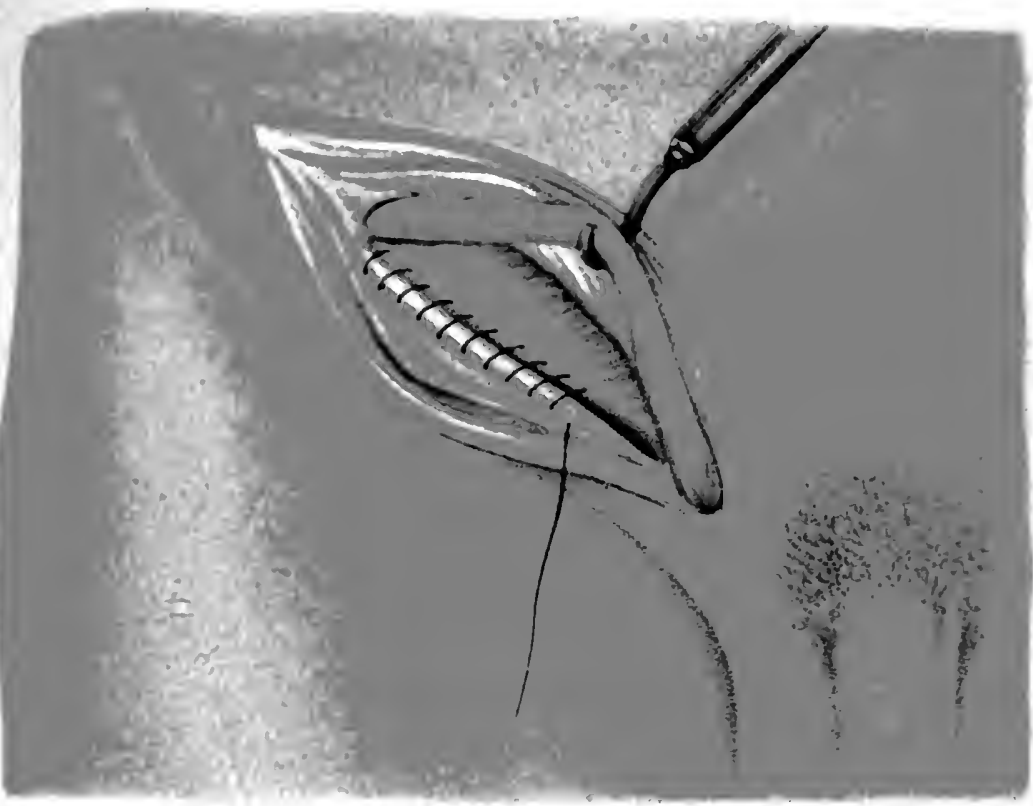

III.

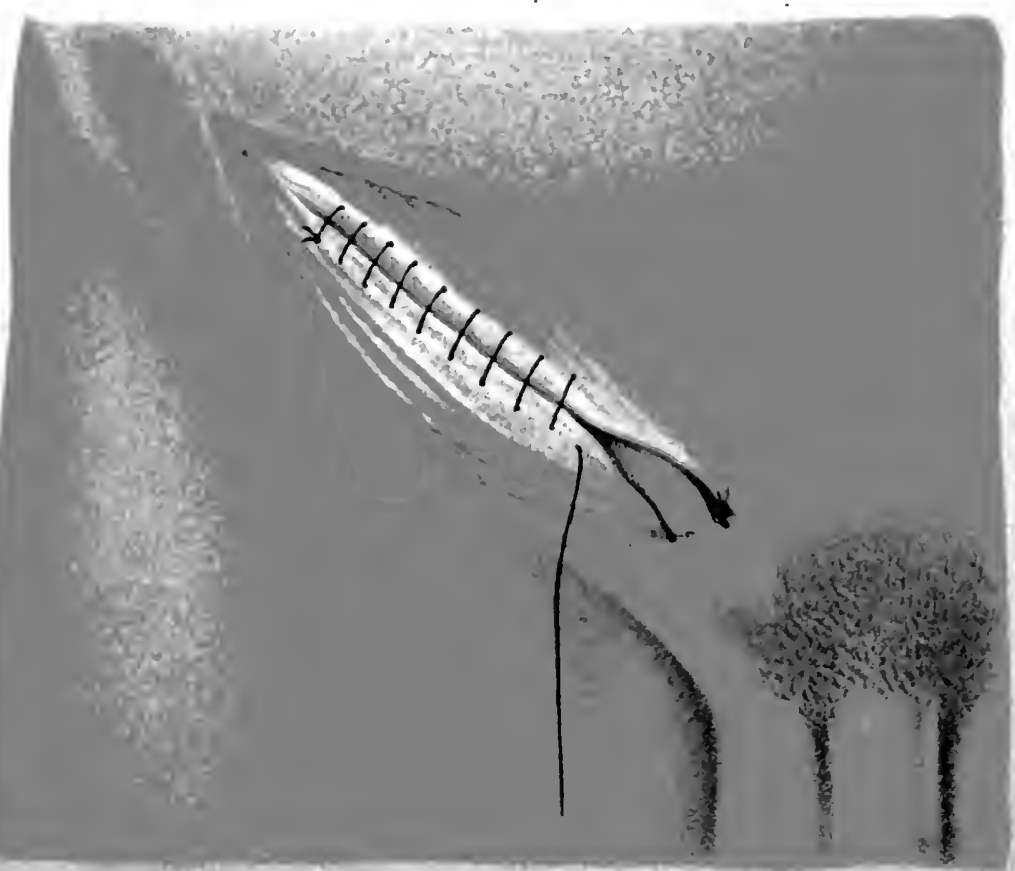

IV.

Lith. Anst F: Reichhold München 
internal opening of the eanal is formed for three-fourths of its extent of tissues (Poupart's ligament, Gimbernat's ligament, horizonal ramus of the pubis) whose resistance would interfere with closure of the hernial opening.

Iode of Performing the Radical Operation for Femoral Hernia.-A critaneons incision is mate by the method of Bassini, rertically orer the greatest convexity of the tumor. 'The outer surfice of the hernial sac and the fasciat to the peripheral side of the swelling are exposed. The body and the neek of the sae are isolated to a point above the level of the internal erural ring. Isolation of the hernial sac upon its outer side, where it is in close relation with the femoral vein, must be undertaken with great care. The hernial sae is opened and its contents replaced after separation of adhesions. The body of the hernial sale is elevated, twisted, and ligated with a thread passed around its neck. The sae is divided transversely beyond the ligature. After replacement of the stump the hernial opening, the pliea faleiformis, the internal opening, with Gimbernat's ligament, and the aponeurosis over the peetineal erest are sufficiently exposed. Suture begins elose to the pubie tubercle and the sutures include the pectineal aponeurosis and the posterior inferior portion of the internal opening. In the further course of the operation the border of the falciform process is united with the peetineal fascia. After all of the sutures have been introduced they are tied, beginning with the innermost and uppermost. The line of suture pursues the course of an oblique C.

Fabricius eloses the hernial opening by suture in cases of femoral hernia after the fat as well as the lymphatic glands present in the femoral canal are removed in such a way that Poupart's ligament is relaxed and is attached by periosteal sutures to the horizontal branch of the pubic bone.

Umbilical Hernia, Radical Operation. Operation for Incarcerated Omphalocele.-The usual procedure consisted until recently in closure of the hernial opening by suture after opening the hernial tumor and replacement of the 
intestine. Greater security against the recurrence of the hernia and a more thorough inspection during the operation are afforded by excision of the umbilical ring (omphalectomy, Condamin, Bruns). The umbilical region is surrounded by two elliptic incisions, each of which extends to the inner border of the rectus muscle and opens the abdominal carity on either side of the hernial ring, so that the entire hernial tumor, together with the neck of the hernial sac, is remored. If, in addition, a portion of the sac is divided from the margin of the wound through the hernial opening, the entire contents of the hernia are exposed to view. In this way satisfactory scrutiny of the conditions present is possible, inasmuch as the abdominal viscera are rendered visible, both prior to the entrance into the hernia, as well as within the hernial sac. Adhesions that may be present are separated, and any existing strangulation can be freed in the open wound. "After replacement of the intestines the abdominal wound is carefully approximated by interrupted sutures in three layers, the first including the serous membrane, the second the recti muscles and sheaths, and the third the skin.

Ligation of the Iliac Artery.-At the level of the fourth lumbar vertebra the aorta divides into the two iliac arteries, each of which in turn divides at the sacro-iliac articulation into two branches, the external and internal iliac arteries. The external iliac artery, the abdominal portion of the femoral, passes along the outer side of the corresponding vein along the psoas muscle to the opening beneath Poupart's ligament for the ressels. The internal iliac artery, also known as the hypogastric, passes from the sacro-iliac symphysis down into the pelvis, to supply the organs of this carity, as well as the gluteal muscles and the genitalia, with blood.

Ligation of the External Iliac Artery.-The artery is exposed in the subserous space just prior to its entrance into the opening for the vessels. The cutaneous incision is made parallel with, and over the niddle of, Poupart's ligament, and the fascia of the external oblique muscle, 
the fibers of the internal oblique and transversalis are divided in the direction, and throughont the extent, of this incision. After division of the transversalis fatscia the subserous fat and the peritonem are exposed to view. The peritonem is separated by blunt dissection from Poupart's ligament and the pelvic margin, after which the external iliac vessels surrounded by loose connective tissue become visible upon the floor of the wound. The artery (the vein lies to its inner side) is isolated by blunt dissection with the aid of two anatomic forceps (Plate 24).

In ligating the internal iliac artery the cutaneous incision passes from the apex of the last rib vertically downward to the crest of the ilium and along this almost to the anterior superior iliac spine. The layers of the abdominal wall and the transversalis fascia are divided, the peritonem separated by blunt dissection from the iliac fossa and displaced toward the median line by means of broad spatule or the palm of the hand. Between the iliac and psoas muscles the external iliac artery is visible and can be followed in a proximal direction as far as the sacro-iliae symphysis, where the internal iliae artery is accessible as it branches off toward the pelvis and can be isolated for ligature. The vein lies to the inner side of the artery. The mode of procedure just described serves also for exposing the commion iliac artery.

The manner of exposing the iliae ressels constitutes in general the mode of procedure in accordance with which the structures of the subserous space are reached. The incision for ligation of the internal iliac artery exposes the kidney and the ureter in its course. In the same way it is possible, with conservation of the peritoneum, to evacuate accumulations of pus in the subserous space (psoas abscess, paratyphlitic abscess, parametric abscess).

If after opening the abdominal cavity by celiotomy the parietal peritoneum upon the posterior wall of the abdomen is divided and in this way the retroperitoneal space is exposed, the procedure is designated transperitoneal exposure of the iliac artery, of the kidney, of the ureter, 


\section{Plate 24.-Exposure of the External Iliac Artery.}

There are divided the aponeurosis of the external oblique muscle (Q.e.), the fibers of the internal oblique (Q.i.), and the transrersalis fascia (F.t.); the peritoneum $(P)$ is separated by blunt dissection and raised up; the iliac artery and vein are exposed in the subserous space.

etc. Under these conditions the peritoneum must be divided at two corresponding points on the anterior and the posterior abdominal wall.

Operations on the Kidneys.-Operations on the kidners may be undertaken :

1. For the purpose of opening the kidney by incision -nephrotomy;

2. For the removal of the totally diseased kidneynephiectomy;

3. For fixation of a movable kidney-nephropexy;

4. For the exsection of portions of the kidney-resection of the kidney.

Nephrotomy and Nephrectomy.-Nephrotomy is indicated in the presence of -1 , simple pyoncphrosis; 2, stones in the pelvis of the kidney, if sufficiently functionally active parenchyma remain; $\dot{3}$, severe renal hematuria; 4, hydronephrosis.

Nephrectomy is indicated in the presence of: 1 , severe pyonephrosis, if the kidney is transformed into a series of pus-carities (calculosis, tuberculosis of the kidney); 2 , injuries of the kidney (rupture, laceration); 3, tumors of the kidney ; 4, incurable ureteral fistulæ.

For exposure of the kidney the patient is placed upon the healthy side of his body over a pillow. The cutaneous incision begins at the twelfth rib and passes thence vertically downward toward the crest of the ilium and along this almost to the anterior superior spine. Skin, fat, lumbodorsal fascia, the fibers of the latissimus dorsi are divided, in order that, after division of the deep layer of the fascia, the quadratus lumborum and, in the anterior portion of the wound, the triplicate layer of the abdominal muscles, may be divided. After the transversalis muscle 
Tab. 24.

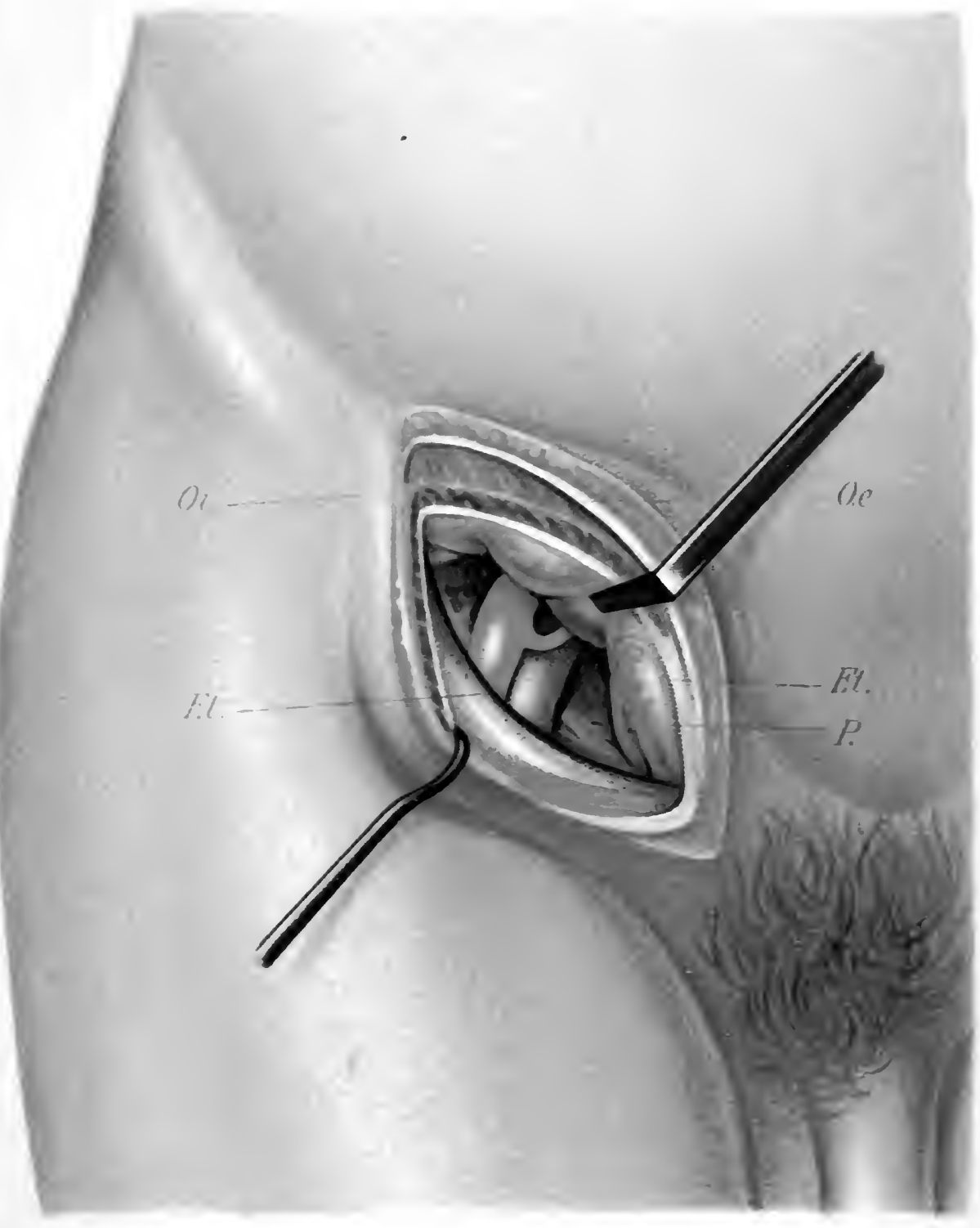



also hats been passed the fitty eapsule of the kidney is exposed throughout a sufficient extent. This capsule is divided and the kidney is removed from its bed by blunt dissection with the finger until the organ, completely freed at all points with the exception of its hilus, ean be brought by traction to the level of the wound.

For exploration of the pelvis of the kidney, for the removal of stones from the pelvis, ete., the kidney is opened from its eonvex border. An incision is made upon the convexity, through the renal parenchyma, down to the pelvis of the kidney, large enough to permit the introduction of the index-finger, with which the pelvis is examined. If necessary, this incision may be extended toward the poles of the organ to a maximum degree until the kidney an be separated in two halves. This procedure is carried out with digital compression of the large vessels at the liilus of the kidney. If the conditions are so constituted that primary union can take place, the wound in the kidney is closed by deep and superficial interrupted catgut sutures, the organ dropped into place, and the cutaneous wound elosed, except for a small opening for a drainage-tube. Provisional suture of the wound in the kidney for the control of hemorrhage may also be undertaken if in inmediate conjunction with an exploratory incision the removal of the entire organ is determined upon. After extirpation of the kidney the large vessels at its hilus must be exposed and carefully ligated. If possible, the kidney is drawn forward and the artery and the vein are isolated at the hilus (Fig. 216). If this is not possible, the operator grasps the hilus of the organ with the thumb and index-finger of the left hand and with the guidance of this hand applies a clamp-forceps around the entire pedicle. The pediele is divided beyond the grasp of the forceps with scissors and ligated en masse upon the proximal side. The large vessels exposed in the transverse incision are further isolated and ligated separately.

The large wound-cavity is closed after perfect control 
of hemorrhage, drained, and the wound closed by sutures in tiers (muceles, fiscia, skin).

To effect operative fixation of a morable lidney (nephropexy, nephrorrhaphy) the organ is exposed in the usual manner, the sutures (ten or twelve) for the fixation of the organ being passed deeply through its parenchyma and placed in the upper angle of the cutaneons incision on

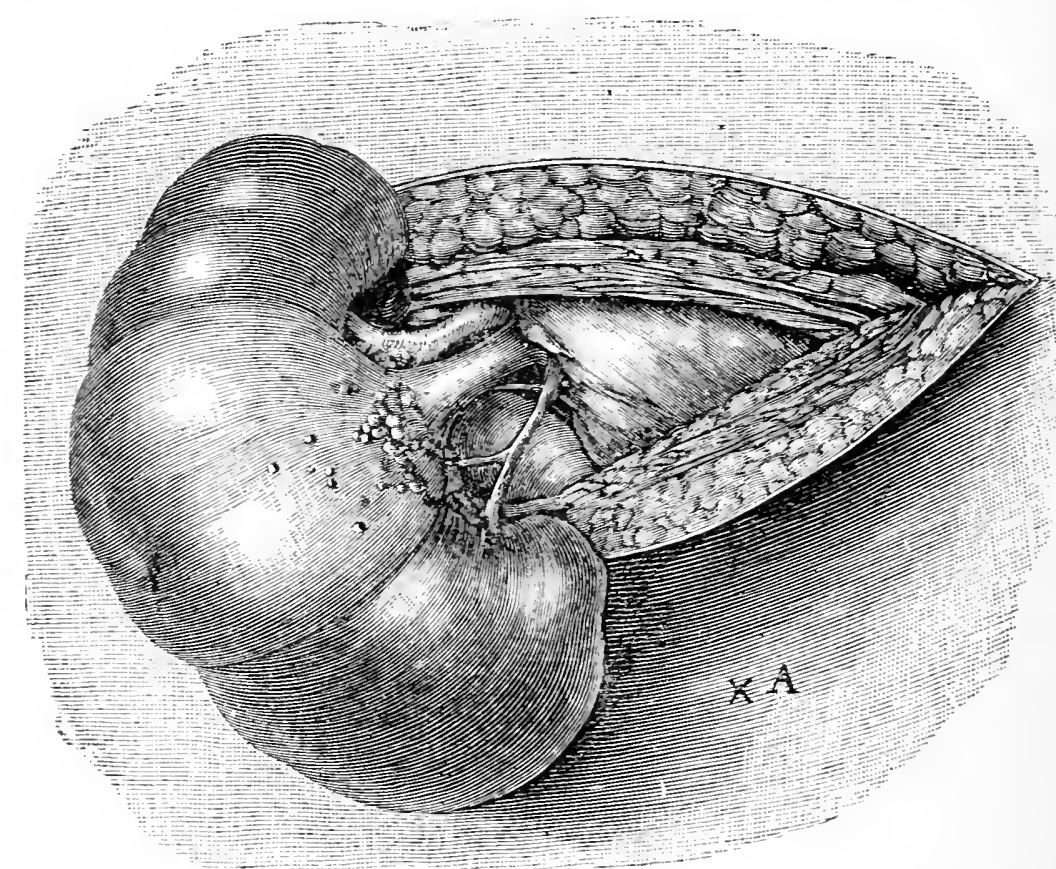

FIG. 216.-Lumbar incision: the kidney is brought out of the wound in the abdominal wall and the structures of the hilus of the organ are isolated and exposed for ligature.

either side and tied. In this manner the kidney is suitably located and fastened. The method of retroperitoneal exposure of the kidney described affords as a rule sufficient access to the organ. In the presence, however, of large diffusely adherent tumors of the kidney, or in the case of adipose individuals, it may be necessary, to afford greater accessibility, to make from the middle of the lumbar incision a transverse incision passing toward the umbilicus. 
Bardenheuer recommends the so-callod trap-door ineision. From the upper and lower extrenities of the vertical longitudinal incision passing from the costal arch to the middle of the crest of the ilimm transverse incisions are made along the rib and the iliac erest. Bardenhener makes three forms of trap-eloor incision, an anterior, $\square$, a posterior, $\square$, and a two-sided one, $\perp$.

In contradistinetion from the retroperitoneal method described is the transperitoncal method for exposing the kidney. In this latter operation the abdominal avity is opened in the usual manner in the linea alba, the peritoneum over the kidney is divided, and the organ is enueleated out of its bed. The retroperitoneal method has, on the other hand, the advantage of permitting, in conjunction with an exploratory procedure, of the establishment of a renal fistula, of the drainage of an abscess of the kidner under favorable conditions, as well as total removal of the entire organ.

Operations on the Ureters. - The ureter passes from the kidney on either side in the subserous space just behind the peritoneum to the fundus of the bladder. In its upper portion it lies upon the psoas muscle, crossing at its entrance into the pelvis the point of division of the common iliac artery and entering the pelvis in a direction forward and inwaird to reach the base of the bladder. Most commonly injuries of the ureter in the course of operations furnish the indication for operations upon this structure; less commonly impaction of stones in the ureter, ocelusion of the lower extremity of the ureter from the presence of a neoplasm, or kinling of the ureter in eases of hydronephrosis.

Of operations there have been performed : linear opening of the ureter for delivery of a stone, with subseguent suture of the incision (ureterolithotomy); the displacement of a stone present along the ureter into the pelvis of the kidney ; and, finally, digital attrition of soft stones without opening the ureter. In the presence of injuries of the ureter, restoration of the lumen of the tube by suture of the stumps or grafting of the central stump of the ureter into a neighboring organ may be undertaken to 
effect closure of ureteral fistulie. With this end in view the ureter has been mited with the bowel (uretero-enterostomy), with the ureter of the opposite sicle (ureteroureterostomy), and with a new portion of the bladder (uretero-neocystostomy). Anastomosis of the ureter with

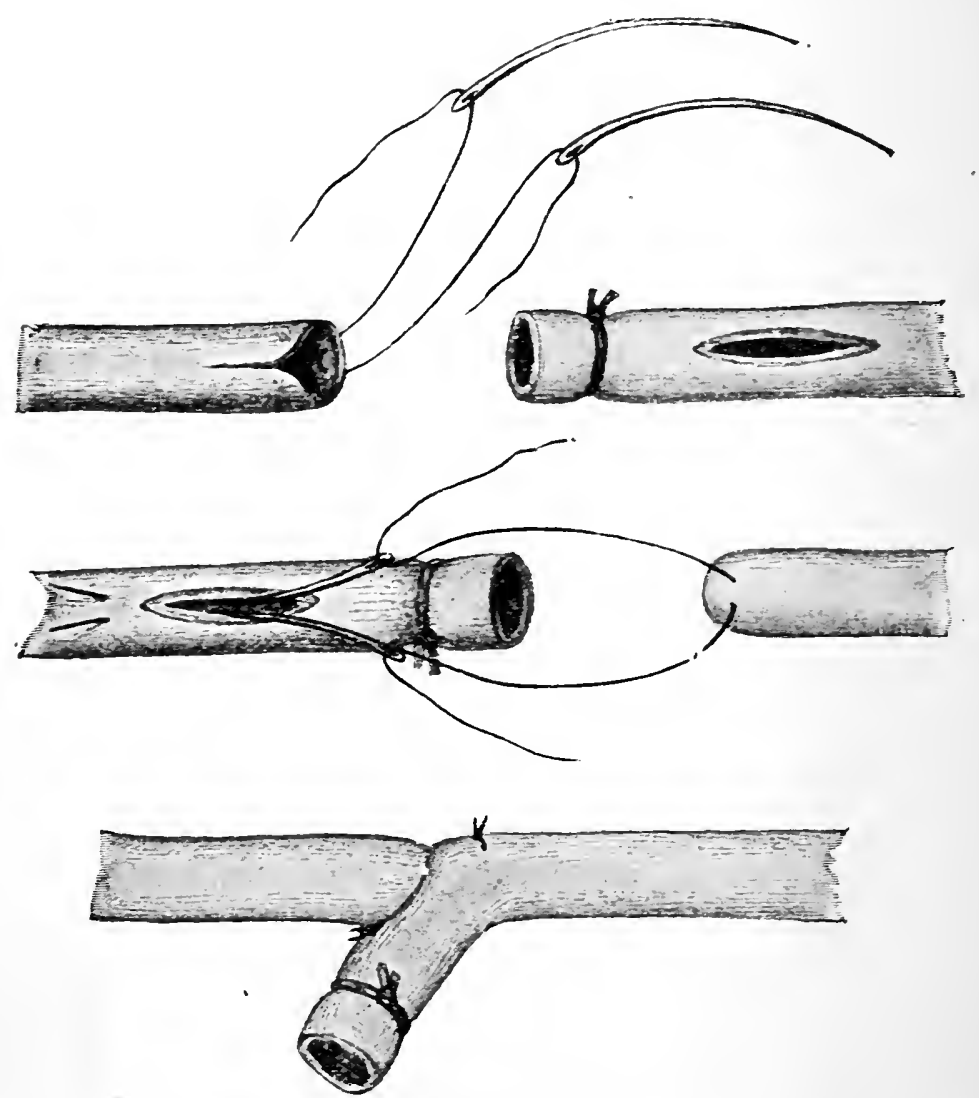

FIG. 217.-Invagination-suture of the stumps of the divided ureter.

the bowel has also been undertaken for the correction of ectopy of the bladder (Maydl). Circular union of the transversely or obliquely divided stumps of the ureter has the disadvantage, in view of the narrow caliber of the ureter, of being followed by such contraction of the cicatrix as to result in narrowing of the lumen of the tube. 
For this reason the invagination-suture of Van Hook is to be preferred. The free end of the peripheral stump is closed by a ligature, 2.5 mm. below which a longitudinal incision is made throngh the thickness of the wall of the ureter. The central stump is caught with a caterut suture, the ends of which are earried by means of a needle through the longitudinal incision in the poripheral stump, the needle being further-passed through the opposite wall of this stump. By gentle traction on the suture the central stump of the ureter is drawn through the slit in the peripheral portion, and fastened in position by knotting the thread in this situation. A few alditional sutures on the outer side insure contiguity of the stumps (Fig. 217).

In the practice of implantation of the ureter by the method of Büdinger and Witzel a normal canal is formed in which the ureter is contained (oblique fistula), thus most nearly imitating the natural mode of entrance of the ureter. The divided ureter is implanted in the wall of the selected organ in the same manner as is the rubber tube in Witzel's operation of gastrostomy, and is fixed in position by suture. For exposure of the upper portions of the ureter the lumbar incision, as for nephrectomy, may be made advantageonsly. The pelvic portion of the ureter in the male is accossible through the sacral route after enucleation of the coccy $x$ and avoidance of the rectum.

\section{Operations upon the Rectum and the Anus.-} Amputation and Resection of the Rectum.-Operations for the removal of tumors of the rectum will vary with the seat and the extent of the morbid process. Circumseribed or pedunculated tumors are surrounded by incisions at their base, and severed through healthy tissue, the wound created being closed by suture. It is difficult in the treatment of cases of this kind to expose sufficiently the field of operation. For more deeply seated tumors, in the region of the anus, it is sufficient to distend this portion of the bowel by means of retractors or suitable specula. 
Tumors seated high up require as a preliminary operation linear division of the sphincter, which is practised on the anterior and posterior aspect- along the line of the raphe, with marked retraction of the margins of the womnd, and renders aceessible to the knife the portions of the rectal mucous membrane above the sphincter. The anterior wall of the rectum can be reached with the aid of a prerectal incision by separation of the rectum from the urethra (see Prostatotomy). By this means the entire thickness of a cireumscribed portion of the wall of the rectum can be resected and the defect be closed by suture. If the neoplasm involve the entire periphery of the lowest portion of the rectum a circular incision is made around the anus and the lower extremity of the rectum is freed from its surroundings. The rectum is then rlivided transversely upon the proximal side of the neoplasm. The wound is so adjusted that the stump of the rectum, brought to the level of the surface, is fixed to the skin by sutures passing through all the layers of the wall of the bowel. This method of amputation has a limited field of application. If the upper border of the tumor can be reached with the palpating finger, its removal by the method detailed can be technically carried ont; but isolation of the rectum in its upper portions and access to the sigmoid flexure are quite impossible by this mode of procedure. The cutaneons wound allows of limited aceess, so that certainty in operation, especially control of hemorrhage in the higher portions of the wound, encounters irremovable obstacles.

Resection of the rectum, with union of the stumps of the intestine, by the method described, is difficult even if access is afforded by anterior and posterior incisions through the raphe, also when the tumor is deeply seater, and entirely impossible if this be situated in the upper portion of the rectum.

The limits of operability of tumors of the rectum were enlarged materially with attempts, on Kraske's suggestion, to expose the rectum sufficiently and also in its 
higher portion through the sacral route. It is possible by this mode of procedure to isolate the rectum even to its intraperitencal portion and to practise rescet tons of this portion of the bowel in its continnity', with an anlepplate field of operation. The rectum is reached from the posterior aspect after division of the sacrotuberous and sacrospinous ligaments through the wide interval on either side between the margin of the salerum, and the tuberosity of the ischium. The acecisibility is increased by removal of a portion of the margin of the sacrum with a chisel.

Mode of Effecting suceral Expositre of the Rectum (Hochenegg).-The patient oecupies the left lateral deenbitus with the lower extremities flexed at the hips and the knees and the operator standing back of the patient. The eutaneous incision begins at the middle of the left sacro-iliac symphysis and passes orer the middle line in an are whose convexity is directed toward the right and terminates below the apex of the coceyx; or, if the anal portion also is to be removed, it surrounds the anns elliptically. The incision is deepened down to the bone. 'The soft parts are retained in connection with the skin and are dissected from the bone so that the left half of the sacrum and the cocer $x$ are exposed in the wound. After enucleation of the coceyx aceess to the rectum will be already relatively free and it becomes considerably greater after division of the attachments of the sacrotuberous and sacrospinous ligaments. The extensive field of operation thus exposed permits of careful scrutiny with regard to the extent and limits of the tumor, and even of the higher portions of the rectum, not aceessible through the usual modes of procedure. Further extension of the field of operation can be effected by chiselling the left margin of the sacrum.

After exposure of the rectum the second step of the operation-that is, isolation of the tumor beyond its limits -is undertaken. The rectum is separated from its surroundings by blunt dissection and the visible vessels are ligated in the wound. If high amputation of the rectum 
is to be performed, the stump of the bowel is brought down and fastened to the skin in the upper angle of the wound (sacral preternatural anus). This procedure is indicated when the anal portion is involved in the newgrowth and must be removed in comnection therewith. If, on the other hand, the anal portion is healthy, the tumor being seated in the middle portion of the rectum, the bowel on either side of the morbid process is isolated by blunt dissection into healthy tissue, ligated and removed by resection. The two stumps of the intestine are united either primarily throughout their entire extent, or sutured only partially, so that a provisional artificial anus is formed. This forms a mural fistula, which may either close spontaneous ly or be closed after a time by a plastic operation. In introducing the sutures, both stumps must be approximated without any tension. In the isolation of tumors seated high up it is often necessary to open the peritoneum of the resicorectal cul-de-sac. The proximal stump of the rectum is brought into the wound for the formation of an artificial anus or for suture and the anterior lip of the peritoncal wound is united at a suitable level with the serous larer of the intestine, so that the abdominal cavity is walled off from the wound. After the introduction of circular intestinal sutures a large drainage-tube is introduced through the anus into the rectum beyond the line of suture.

Operations for Rectal Fistula.-Rectal fistula can be made to heal by division of the fistulous tract and the conversion of the tubular ulcer into an open wound. In the operation for complete fistula a slender probe is introduced through the external fistulous opening, while the index-finger of the left hand is applied to the internal opening, which often is appreciable as a loss of substance. The probe is thus passed through the tract and enters the lumen of the bowel. A grooved director may be readily passed through the fistuli by the side of the probe into the rectum and its extremity brought out through the anus. The soft parts covering the fistula thus come to lie 
upon the director, mpon which they are divided with the knife. By the introduction of tenacula after division of the tissues the character of the lining of the fistuloms tract can be rendered visible. Is a mole, the wound is permitted to heal by gramulation, althomgh after extirpation of the entire tistulous passage the wound cam be closed completely by suture.

Incomplete fistulae noust be converted into complete fistulie before being divided. In the presence of an incomplete external fistula the grooved director is introduced and pushed into the rectum thromgh the deepest portion of the fistula. Division of the fistula thus made complete is effocted in the mamner describul. In the presence of an incomplete internal fistula, with its opening upon the mucous membrane of the rectum the sound or the grooved director is introduced from the rectum toward the skin. When the head of the probe is felt beneath the skin an incision is made down upon it and the complete fistula thus established is divided in the manner clescribed. In the presence of extensive fistulous formations it becomes necessary to follow the manifold ramifying passiages often present and to open them arlequately.

Operation for Hemorrhoids.-Dilatations of the external hemorrhoidal veins do not require operative treatment. Operation is indicated only in those cases of dilatation of the internal hemorrhoidal veins, with consecutive changes in the mueous membrane, in which prolapse of the mucous membrane of the rectum has taken place, which makes itself apparent either only upon increased abdominal pressure or habitually as a result of this influence. The prolapsed masses of mucous membrane are either destroyed with the actual cautery, or subjected to atrophy through the elastic ligature, or excised by a bloody operation.

Canterization.--The patient oceupies the position as in the operation for stone, or the lateral decubitus. By means of digital dilatation of the anus the hemorrhoidal masses are exposed to view. They are grasped in seg- 
ments with a clanmp-foreeps and their base is surrounded by Langenbeek's flat forceps. The tumor lying upon the broad ivory plate of the forceps is totally destroyed with the tip of the Paquelin cautery, after which the forceps is earefully removed. In the same manner the swellings throughiout the entire circumference of the rectum are destroved.

Elristic Ligature.-The patient oecupies the lateral decubitus. By means of a clamp polyp-forceps the extruded mass of mucous membrane is grasped at its base and brought forward. The elastic ligature is passed around the neck of the nodule behind the forceps and tightened and the nodule fixed by means of a silk thread tied around it. In this war the whole series of folds is included in three or four parts and ligated. The necrotic nodules are thrown off in the eourse of a week.

Excision may be practised upon each nodule individually, or a circular incision is made through the skin around the anus and also through the mucous membrane of the rectum above the level of the nodules. The eylinder of mucous membrane, together with the dilated veins, is dissected free from the sphincter and the margin of the mucous membrane is united by suture with the skin at the anus.

Operation for Atresia of the Anus.-The incision is made in the perineal raphe from the apex of the coccyx to the root of the scrotum (posterior commissure). The operator advances into the depth layer by layer, always keeping strictly in the middle line. As a rule, the bluishcolorerl cul-de-sac of the rectum is soon reached, and it is incised in the direction of the cutaneous incision. After the meeonium has been discharged the bowel is united throughout its entire periphery to the skin by sutures passing through the entire thiekness of the wall of the intestine. If the cul-cle-sae be situated high up, an efrort should be made to reach the rectum by the sacral route. In the presence of atresia ani vesicalis, vaginalis, an attempt is made to dissect free the lower end of the intes- 
tine by means of the same incision. The abnormal communication is divided with scissors and the rectum is fixed in the wound by sutures in the manner described. The defect in the vagina or the bladder made by the separation of the rectum must have been closed previously by suture. 



\section{N D E X.}

Abdominal puncture, 30 \%

Abscesses, periueal, opening of, 321 Achillotenotomy, 132

Actial cautery, 39

Air-passages, operations on, 274

Aniputation, 86

by circular incision, 85

by tlap-incision, 96, Figs. 59-62

by oval incision, $10 \mathrm{~s}$

circular, 83

control of hemorrhage after, 109

control of hemorrhage in, 87

division of the bone in, 103

flap, 96

steps of, 83

indications for, 86

knives, 18

of $\operatorname{arm}, 181, \mathrm{Pl} .11$

of finger through metacarpus, 174

of foot at tarsometatarsal joint, 139

intertaræal, 146

Pirogoff's method, 120

of forearm, 181

of leg, 112

Bier's method, 117

by anterior long terumentary periosteal flap, with a posterior short musculotegumentary flap, 116;

by single lateral flap, $11 \%$

by two lateral musculotegumentary flaps, 115

by two lateral tegumentar; flaps of equal size, 114

circular, 11:3, $\mathrm{Pl} .8$

cireular method, Figs. 55-58

flap, 114

Heine's method, 116

supramalleolar, by Syme's method, 118

of penis, 362
Amputation of rectum, 379

of toe through metatarsal bone, 136

of toes in the interphalangeal joint, 132

(all) through the metatarsus, 137

of thigh, 154

by flaps, 156

by Gritty's method, 161

by means of circular incision, $15 \overline{5}$

osteoplastic supracondylar, 161 oral, 108

position of operator in, $8 \boldsymbol{r}$ position of patient in, 87

wound, care of, 109

Aneurysms, ligatiou of vessels for, 58

Anus, atresia of, opelation for, 334 operations upon, 379

preternatural, formation of, 314

Ankle-joint, resection of, $: 211$

Arched saw, 42

Arm, amputation of, 184, Pl. 11

Artery divided between two ligatures, 61, Fig. 39

Arthrectomy, 192

Artlirotomy, 192

Atresia of allus, operation for, 384

Autoplasty after trephining, 22!

Axillary artery, ligation of, $63, \mathrm{Pl}$. 2

location of, 62

BARDENHEUER's trap-door incision for exposing the kidney, 377

Bassini's operation for inguinal hernia, 368

Bellied scalpel, 17

Bicipital artery, ligation of, 68 
Bergmann's operation for hydrocele, 360

Bier's method of amputating leg, $11 \tau$

Biliars apparatus, operations upon, 319

Billroth's caunula, 302 method of submental removal of tollgue, 251

method of uranoplasty, 266

Bladder, operations on, 346 puncture of, 336 suture of, 55

Blood, transfusion of, 69

Bloodless approximation of wounds, 44

methods of dividing tissues, 39

Bloody suture, 44

Blunt dissection, 33

Blunt-pointed knife, 1T

method of nsing, 34

Boue brace. Fig. $3 \pi$

division of, 41

Bone-forceps, 43

Bone-shears, 43

Bones. dirision of. $1 \tau$ percutaneous nailing of, 53 suture of, 5 ?

Borrel, resection of, 315 suture of, $53, \mathrm{Pl} .1$

Brachial arters, ligation of, $65, \mathrm{Pl}$. 2

Bramanu's operation for exposure of bladder. 3.50

Bruns on malignant tumors as an indication for resection of foot, $21 \%$

on paralrtic club-foot as an indication for resection of foot. 217

Brauns's cheiloplasty, 252, Fig. 155

incision for resection of elbow, 204

method of tibiocalcaneal resection of foot. 215

modification of Pirogoff's amputation, 131

Buccinator nerve, extrabuccal exposure of, $: 269$

Büdinger and Witzel's method of implantation of ureter, $3 \approx 9$

Büngner, divulsion of vas deferens, $35 \mathrm{~s}$

Butcher's saw, 42
CAxivel for tracheotomy, 282, 283

Carotid artery, ligation of, 292

Caselli on shortening of leg after luxations of lip as an indication for resection of foot, $21 \pi$

Castration, 358

Catheter coudé, 323,325 metallic, introduction of, 328 retention, 334

rigil, mode of introducing, 327 soft, mode of introducing, 326

Catheterization, 322 posterior, :341

Catheters, varieties of, 323,325

Cautery, actual, 39

Celiotomr, $30 \mathrm{~s}$ mode of making incision in, 309

Chain-saw, 42

Chassaignac's incision for resection of elbow-joint, 199

Cheek, plastic operations on, 259

Cheiloplastr, Bruns's, 252, Fig. 155 Dieffenbach s, 252, Figs. 152, 153

Langenbeck's, 25\%, Fig. 154

Chisel and mallet, 43

Cholecrstectomy, 320

Cholecrstendrsis, 320

Cholecrstenterostoms, 321

Cholecrstoduodenostoms, 321

Cholecrstojejunostomy, 321

Cholecrstotomy, 319

Choledochoduodenostoms, 321

Choledoch otomy, 3:1

Chopart's joint. 149 operation, 149

Circular saw, 43

Circumcision, 360

Colostoms, 314, Pl. 19

Continuity, iigation in, 57

Cricothy rotomy. $255,2 \pi 6$

Cubital artery, ligation of, 66, PI. 3

Cutaneous incisions, 25 forms of, 25

Crstopexr, 349

Cistotomr, 350

suprapubic, 347

for intravesical manipulations, 349

for stone, 348

Czerny's incision for reaching the transverse fissure of the liver, 319

method of plastic operation on cheek, 257 
DEEP dissection, 26

Dieffenbach's cheiloplastr, 25:2, Figs. 152, 153

method of uranoplasty, 266

Dieulafor's aspirator, 303

Dissection between two furceps, 33 ,

blunt, 33

$$
\text { Fig. 35 }
$$

deep, 26

free. 33

with aid of groored director. 33. Fig. 14

Dittel on lateral prostatectomy, 357

Division of bone, 41

of tissues. 17

bloodless methods, 39

by puncture, $3 s$

with scissors, 34, Fig. 17

Dorsalis pedis artery, ligation of, S.)

ÉCraseMEat, 41

Écraseur, 41, Fig. 21

Elastic ligature, 41

Elbow-joint. restetion of. 199

Elephantiasis. ligation of vessels for, $\overline{\text { it }}$

Entero-anastomosis, 317

Enterostomy, 313

Enucleation. 56

at elbow-joint. 1:2

at knee-joint, 153

of all four fingers through metacarpal bone, 175

of toot. subastraqaloid. 150

of hand br circular incision, 175

by flap-incisions, $1 \leq 1$

of thumb at carpometacarpal joint. 173

Epilepss, ligation of vertebral artery for. $5 s$

Esmarch's method of exarticulating the humerus. 153

of exarticulation, 112 of femur, 163

Esophagotomy, external, 233

Exarticulatio pedis sub talo, 151

Exarticulation. 110

at the wrist. 175

of femur at hip-joint br method of Esmarch, 163

of fingers at interphalangeal joints and at metatarsophalangeal joints, $16 \%$

of foot, intertarsal, 144
Exarticulation of great toe, together with metatarsal bone, 138 with formation of anterior and posteriur flaps, 163

of huwerus. 1s:

by a circular incision and with longitudinal incision by Esmarch's method, 183

by a deltoid flap. 1 1 ;

of leg at knee-joint. 153

of little finger. 174

of little toe. together with meta. tarsal bone. 139

of toe in interphalangeal joint, 132

Excision of prostate. 356

Exclusion of intestine. 317

Extirpation of hip. $16 \%$

of seminal resicles. 356

of testicle, 35

Extremities. operations on, 57

Extubator, 2:5

FABRICITS's wethod of operating for femoral heruia. 3i1

Femoral artery. ligation of, at junction of niddle and upper thirds of thigh. 75. Pl. 4

below Poupart's ligament, 75 , $\mathrm{Pl} .4$

in adductor canal, $\%, \mathrm{Pl} .5$

location of. it

hernia. operation for. 365

radical operation for. 369

Femur. exarticulation of, 163 . See Exarticulation.

asteotomy of. 221

Fifth nerve. exposure of second and third divisions br method of Krönlein. 2\%:

exposure of third division at base of skull, 273

Finger, amputation of, 174

Fingers, exarticulation of. 167. See Esarticulation.

resection of, 206

Fistula, gastric. formation of, 311

intestinal, formation of. 313

rectal, operation for. $3=2$

urethral, operation for, 363

Fleurant, trocar of, 337

Foot, amputation of. See Amputation.

resection of. 214

subastragaloid euucleation of, 150 
Forearm, amputation of, 181

Fowler on appendicitis, 322

Frank's method of gastrotomr, 313

Free dissection, 33

Froutal nerve, exposure aud extraction of, $26 \pi$

GALL-BLADDER, operations ow, 319 extirpation of, 320

nature of, 56

Galvanocaustic suare, 41

Galvanocauters, 41

Gasserian ganglion, extirpation of, br method of Krause, 274

Gastric fistula, formation of, 311

Gastroenterostomy, 318

Gastrostom 5 , 311. Pl. 19

Genito-urinary orgaus, operations ou, 32.2

Gersuur's metliod of plastic operation on cheek, 259

Gigli wire saw, 223

Gill (JI. C.) on extirpation of enlarged middle lobe of prostate, $35 \%$

Goiter, operation for, 299

resection of, 301

Graefe's incision for ligation of innominate artery, 291

Granny's kuot, 6?

Great toe, exarticulation of. See Exarticulation.

Gritty's incision for resection of wrist, 206

operation, 161

Grooved director, dissection with aid of, 33, Fig. 14

Günther's modification of Pirogoff's amputation, 131, Figs. 76, 79

operation, 150

Gussenbauer's clamp, 5\%

HAHX's method of resection of kinee-joint, 211

Ealsted's operation for removal of breast, 306

Hand, enucleation of, 176

Harelip, operations for, 260

Heine's method of amputation of leg, 116

Helfrich's operation for exposure of bladder, 350

Hemorrliage after operation, control of, 109
Hemorrhoids, cauterization of, 383

excision of, 334

operation for, 383

remoral by elastic ligature, 384

Hernia, adherent, treatment of, 367 femoral, operation for, 365

radical operation for, 369

inguinal, operation for, 365

radical operation for, 367

operations for, 364

radical operation for, 367

umbilical, radical operation for, $3 \pi 1$

Herniotome, 34

Herniotoms, 365

Heteroplasty after trephining, 224

Hip-joint, resection of, $20 \pi$

Hueter's incision for resection of elbow, 204

Humerus, exarticulation of, 185. See Exarticulation.

Hüter's tenoplast 5,51

Hydrocele, operation for, 359

ILEOCOLOSTOMY, 318

Iliac artery, external, ligation of, $3 \dot{2} 2$

internal, ligation of, $3 \pi 3$

ligation of, 372

Incisions of the skin, 25

Inferior dental nerre, exposure of, $2 \% 0$

exposure of, rithin dental canal, $2 \% 0$

Infrahyoid pharyngotoms, 23\%, Pl. 15

Infra-orbital nerre, exposure and extraction of, 268

Ingniual hernia, operation for, 365

radical operation for, $36 \pi$

Injections, parenchymatous, 39

subcutaneous, 39

Innominate arters, ligation of, 291

Intertarsal amputation of foot, $\mathbf{1 4 6}$ exarticulation of foot, 149

Intestinal fistula, formation of, 313

Intestine, exclusion of, 317

Intestines, operations on, 311

Intraglandular enucleation of thyroid gland, 300

Intubation of larrix, 234

indications for, 234

Intubator, 285

Israel's nethod of plastic operation on cheek, 259 

Ivory pegs, union of bones by, 52,

JAw, lower, reseetion of, 2:34 resection in its continuity, 240 temporary reseetion of, 239 upper, resection of, 229

Jejunostomy, 315

Joints, resection of, 191

KIDNEY, excision of, 374 movable, fixation of, 376 operations on, 374

Knee-joint, resection of, 209

Knife, blunt-pointed, method of using, 31

division of tissues with, 18

methods of using, 18

varieties of, $17,18,19$

Knots, 62

Kocher on reseetion of bowel, 315, 316

Kocher's angular ineision for resection of elbow, 205

incision ror enueleation of thy roid gland, 300

for unilateral strumeetomy, 300 method of extirpation of tongue, 251

of resection of knee-joint, 211 operation for inguinal hernia, 369

König's antoplasty after trephining, 224

incision for resection of anklejoint, 213

method of resecting knee-joint, 213

modifieation of Langenbeck's resection of hip, 208

operation for saddle-nose, 256

Krause's flap for exposure of Gasserian ganglioin, 228

method of extirpation of Gasserian ganglion, 274

skin-grafting, 254

Krönlein's method of exposing second and third divisions of fifth nerve, 272

Küster on protection of thoracic nerve in removal of mammary gland, 306

LANGENBECK's cheiloplasty, 252, Fig. 154
Laugenbeck's dorsoradial incision incision, 107 for resection of wrist, 205

for infrahyoid pharyngotomy, 289

for resection of ankle-joint, 211

for reseetion of elbow-joint, 199

for resection of lower-jaw, 240, Pl. 12

method of excision of hip-joint, 207

of resection of shoulder-joint, 193

of resection of upper jaw, 233

Laryngofissure, 275

Laryugotomy, 275

Larynx, extirpation or removal of, 277

intubation of, 284

opening of, 275

Lefort's modification of Pirogoff's amputation, 131, Fig. 80

Leg, amputation of, 112. See Amputation of leg.

exarticulation of. See Exarticulation.

Lembert's suture, Pl. 1, Fig. 1, $b$

Ligation, cutaneous incision for, 58 in continuity, indications for, 57 method of, 58

in upper extremity, 62

loeation and identifieation of artery in, 59

of axillary artery, 83, Pl. 2

of bicipital artery, 68

of brachial artery, 65, Pl. 2

of carotid artery, 292

of eubital artery, $66, \mathrm{Pl} .3$

of dorsalis pedis artery, 85

of femoral artery at junction of middle and npper thirds of thigh, 75

below Poupart's ligament, $7 \overline{5}$, Pl. 4

cutaneous incision for, Fig. 49 in adduetor canal, 77, Pl. 5

of iliae artery, 372

of innominate artery, 291

of internal mammary artery, 304

of internal saphenous vein for varicose veins, 77

of lingual artery, 295, Pl. 17

of popliteal artery, $77, \mathrm{Pl} .6$ eutaueous incision for, Fig. 51 
Ligation of radial and uluar arteries, $70 . \mathrm{Pl}$. 3

of subclavian arters, 295

above the clavicle, 297

below the clavicle, 298

of thrroid arterr, 294

inferior, $299^{\circ}$

of tibial arteries, $80, \mathrm{Pl} .7$

of ressels in continuity, 57

Ligature, elastic, 41

kut, method of tieing, 61

Lingrual arters, ligation of, 295, Pl. 17

nerve, exposure of, $2 \pi 1$

Lisfranc's articular line, 140, 141 operation, 139

Lister's dorso-ulnar incision for resection of wrist, 206

lead-plate suture, 49

Litholapax 5,345

Little toe, exarticulation of. See Exarticulation.

Iücke's and Schede's incision for resection of hip, 209

Maceires on supracoudrlar osteotomy of femur, 221

Malgaigne's incision for resection of upper jaw, 232

infrahyoid pharyngotoms, 2s\%

operation, 151

for harelip, 260

Mammary arters, internal, ligation of, 304

gland, removal of, 304

Mardl on uretero-enterostoms for ectops of bladder, 378

McBuruer's incision for resection of vermiform appendix. 321

Mental nerve, exposure of, $2 ; 1$

Mikulicz's method of exposing third division of fifth nerve, $2 \pi \%$

Mirault-Langenbeck's operation for harelip, 260

Mohrenheim's triangle, 298

Monro, point of, for abdominal puncture, $30 \%$

Moreau's incision for resection of elhow, 204

Murphy's anastomotic button, 316

Muscles, divided, suture of, 49

of leg, anterior, arrangement of, 81
Muscles of popliteal space and calf, Fig. 50

of thigh, arrangement of, Fig. 47

of upper extremity, arrangement of, Fig. 44

NAILs for uniting severed bones, 52 , 53

Teedles, varieties of, Fig. 29

Nélaton's operation for harelip, 260

Nephrectomy, 374

Nephropexr, 376

Nephrorrhaph 5,376

Nephrotoms, 3\%4

Nerre-suture, 51

Terves, extraction of, 267 operations on, $₹ 66$

Neurectoms, 267

Neurexairesis, $26 \tau$

Neuroplasty, 51

Neurotomy, 266

Niehans's operation for exposure of bladder, 350

Nose, plastic restoration of, 254

O'DWYER's outfit for intubation of larvux, 264, 285

Ollier's baỹonet-incision for resection of elbow, 204

Omphalectomy, 3\%2

Omphalocele, incarcerated, operation for, $3 \pi 1$

Osseous arthrectoms, 192

Osteoclasis, 44

Osteoclasts, 44

Osteotomia colli femoris, 221

intertrochanterica, 221

Osteotomy, 218

of femur, 221

of tibia, 221

supracondylar, of femur, 221

PALATE, hard, plastic operations on, 266

soft, plastic operations on, 265

Paquelin, thermo-cautery of, 41, Fig. 19

Paracentesis abdominalis, 307

thoracis, 302

Paraneural suture, 51

Paratendinous suture, 50

Paravicini's method of exposing inferior dental nerve, 270

Parenchymatous injections, 39 
Park's incision for resection of elbow-joint, 199

Penis, amputation of, 362

Percutaneous nailing of bones, 53

Pericecal abscesses, opening of, 321

Perineural suture, 51

Pharyngotomy, 28:'

infrahyoid, 237, Pl. 15

Phimosis, operation for, 360

Plilebotomy, 69, Fig. 46

Pirogoff's amputation of foot, 120

Plastic operations, 25:2

Point of Mouro, 307

Polloson's method of exarticulating leg at knee-joint, 154

Poncet's operation of urethrostomy, 345

Popliteal artery, ligation of, 77, Pl. 6

Preteruatural anus, formation of, 314

Primary suture, 44

Prostate, excision of, 356

Prostate gland, operation upon, 355

Prostatectomy, 356

lateral, 35\%

Prostatotomy, 355

Puneture, exploratory, 38

mode of performing, 38

of abdomen, 307

of bladder, 336

RACKET incision, 112

Radial artery, ligation of, 70, Pl. 3

Reamputation, indications for, 86

Rectal fistula, operations for, 382

Rectum, operations upon, 379 resection of, 380

sacral exposure of, 381

Reef knot, Fig. 40

Regnoli's method of submental re-

Resection knives, 18 moval of tongue, 251

knife, mode of using, 18, Fig. 11 of ankle-joint, 211

by König's incision, 213

by Langenbeck's incision, 211

by Reverdin-Kocher method, 214

of bowel, 315

of elbow-joint through a dorsal longitudinal incision, 199

of fingers, 206
Resection of foot by method of Wladimiroff and Mikulicz, 214

of goiter: 301

of hip-joint, $20 \%$

of joints, indications for, 192

of the extremities, 191

of knee-joint, 209

of lower jaw, 234

in its continuity, 240

temporary, 239

of rectum, 380

of shoulder-joint by method of Langenbeck, 193

of skull, 2:7

of upper jaw, 229

of vas deferens, 357

of vermiform appendix, 321

of wrist-joint, 205

subperiosteal, 191

Retention-catheter, 334

Retractors, use of, in dissection, 26

Reunion of tissues, 44

Reverdin-Kocher method of resection of aukle-joint, 214

Rhinoplasty, 254

Rorer's incision for resection of hipjoint, 209

Rydygier on shortening of leg after resection of knee as an indication for resection of foot, 217

SADDLE-Nose, operation for, 256

Sailor's knot, 62

Salzer on danger of excluded portion of bowel in exclusion, 318

Salzer's method of exposing third division of fifth nerve, 273

Sartorius muscle, course of, 48

Saw, Butcher's arched, 42

chain, 42

circular, 43

wire, 43

Scalpel, bellied, 17

Scissors, division with, 34, Fig. 17

Scoutetten's method of oral amputation, 108

Secondary suture, 44

Sédillot's incision for resection of lower jaw, 240

Seminal vesicles, 356

Sharp-pointed knife, 17 
Shoulder-joint, resection of, 193. See Resection.

Skin, circular incision of, 25 division of, from within outward, 25

method of holding knife in, 18

Skin-grafting, 54

Skull, temporary resection of, 227

Snare, gal ranocaustic, 41

wire, 41, Fig. :20

Soft parts, division of. 17

Sonnenburg's method of exposing inferior dental nerve, 270

Ssabanajeff's operation, $16 ?$

Staphylor rhaphy. 265

Steiner on localization of branches of middle meningeal arters, 228

Stirrup incision, 118

Stomach, operations on. 311

Stone. lateral incision for, 344 mediau section for. 344

Strumectoms, unilateral, 300

Subastragaloid enucleation of foot, 150

Subclarian artery, ligation of, 295 above the clavicle, 297 below the cla vicle, 295

Subcutaneous injections, method of making. 39

Subperiosteal resection, 191

Supracondrlar shortening of femur. 221

Suprapubic crstotoms, $34 \tilde{\tau}$

Surgical knot. 62

Suture, bloods 44 contimuons, 49, Fig. 26 deep, 49

gauze-pad. 49, Fig. 30

glorer's, 49

interrupted. 49, Fig. 25

Lister's lead-plate, 49

mattress, 49

of bladder. $5 \overline{5}$

of bones, 52

of bowel, 53, P]. 1.

of dirided muscles, 49

of gall-bladder, 56

of nerres, 51

of patella with wire, Fig. 36.

of tendons, 49

paraneural, 51

paratendinous, 50

perineural, 51

primars, 44
Suture, secondars, 44

simple knotted, 44, Fig. 25

introduction of, 45 , Fig. 31

Srme's method of supramalleolar amputation, 118

Srnovial arthrectomy, 192

Szymanowskry incision for resection of elbow, 204

TAMPOX-CANXLLA in tracheotomy, 283

Tenacula, use of, in surgical dissection, 26

Tendons, suture of, 49

Tenoplastr, 51

Tenotome, mode of using, 18, Figs. 12,13

Tenotomes. 18

Testicle, extirpation of, 358

Thermocautery of Paquelin, 41, Fig. 19

Thiersch's extraction of nerves, 267 skin-grafting, 254

Thigh. amputation of, 254 . See Amputation.

Thoracotomr. 303

Thorax, paracentesis of, 302

Thumb. enucleation of, 173

Thyroid arters, inferior, ligation of, 299

superior, ligation of, 294

gland. intraglandular enucleation. 300

Thrrotomy, 275

Tibia. osteotoms of, 221

Tibial arteries, ligation of, $80, \mathrm{Pl}$. $\tau$

Tissues, division of, 1 ז

br puncture, 33

reunion of. 44

Toes, amputation of. See Amputation of toes.

exarticulation of, in interphalangeal joint, 13?

Tongue, operations on, 243

submental remoral of, 251

Tracheal cannula, 283

Tracheotoms. 278

indications for, 273

inferior. 280, Pl. 14

superior, 279

Transfixion, $10 \tau$

Transfusion of blood, 69

Trendelenburg's tampon-cannula, 283 
Trephining, 22:3

indications for, :2:3

Trigeminal neuralgia, ligation of carotid artery for, is

Trigeminus, operations ou, 274

Trocar, mode of using, 35

Trzetizky on injury of vessels in abdomiual puncture, $30 \pi$

Tumors of tongue, excision of, 243

UlNar ARTery, ligation of, 70, Pl. 3

Umbilical hernia, radical operation for, $3 \pi 1$

Unilateral strumectomy, 300

Uranoplast 5,266

Ureter, inflammation of, 379 operations on, 377

Uretero-enterostoms, 378

Ureterolithotomy, $3 \%$

Ureteroneocrstostomy, 378

Urethral fistula, operation for, 3C:

Urethrostomy, 345

Urethrotoms, external, 337

indications for, 342

internal, 343

with a guide, 340

without a guide, 340

VAx Hook's invagination suture, 379

Vas deferens, divulsion of, 358 extirpation of, 358

resection and extirpation of, 357
Velpeau's incision for resection of upper jaw, 23:

method of resection of hip-joint, 207

Venous infusion of saline solution, 69

Vermiform appendix, 321

Verneuil's operation, 164

Vogt's incision for resection of elbow, 204

Volkmann's method of resection of knee-joint, 211

operation for livelrocele, 360

Von Walther on reimplantation of bone after trephining, 224

WALTHER's method of exarticulating little finger, 174

Weber on cutaneous incision for resection of upper jaw, 230

Wire loop for division of tissues, saw, 43 46, Fig. 20

Witzel's metlod of cystostoms, 313

Wladimiroff and Mikulicz method of resection of foot, 214

Wolff's method of uranoplasty, 266

Wölfler, incomplete exclusion of the bowel, $31 \%$

Wölfler's gauze-pad suture, 49

Wrist-joint, resection of, 205

ZUCKERKANDL's operation for exposure of buccinator nerve, 269 


\section{Medical and Surgical Works}

PUBLISHED BY

\section{W. B. SAUNDERS, 925 Walnut Street, Philadelphia, Pa.}

* American Text-Book of Applied 'Therapeutics

*American 'Text-Book of Dis. of Children . 9

* An American Text-Book of Diseases of the Eye, Ear, Nose, and 'Throalt . . . . . $3^{1}$

* An Ámerican 'Text-Book of Genito-Urjnary and Skin Diseases. . . . . . 3 I

*American 'lext-Book of Gynecology . . . 8

* American 'l'ext-Book of Obstetrics

*American Text-Book of Physiology

*American Text-Book of Practice

*American Text-Book of Surgery

Anders'Theory and Practice of Iledicine."

Ashton's Obstetrics .. . . . . . . 28

Atlas of Skin Diseases . . . . . . . . . 12

Ball's Bacteriology .. . . . . . . . . 28

Bastin's Laboratory Exercises in Botany . 22

Beck's Surgical Asepsis . . . . . . . . 26

Boisliniere's Obstetric Accidents . . . . . 23

Brockway's Physics . . . . . . . . . . 28

Burr's Nervous Diseases . . . . . . . 26

Butler's Materia Medica and Therapeutics 29

Cerna's Notes on the Newer Remedies. I 8

Chapin's Compendium of Insanity . . . . 3I

Chapman's Medical Jurisprudence . . . . 26

Church and Peterson's Nervous and Mental Diseases.

Clarkson's Histology . . . . . . . . . . 14

Colien and Eshner's Diagnosis . . . . . 28

Corwin's Diagnosis of the Thorax . . . . 29

Cragin's Gynæcology . . . . . . . . . . 28

Crookshank's Text-Book of Bacteriology . I 3

DaCosta's Manual of Surgery . . . . . 26

De Schweinitz's Diseases of the Eye . . . I5

Dorland's Obstetrics . . . . . . . . . 26

Frothingham's Bacteriological Guide . . . 16

Garrigues' Diseases of IVomen . . . . . 20

Gleason's Diseases of the Ear . . . . 28

*Gould and Pyle's Anomalies and Curiosities of Medicine

Griffin's Materia Medica and Therapeutics 26

Griffith's Care of the Baby . . . . . . . 24

Gross's Autobiography . . . . . . . . Io

Hampton's Nursing . . . . . . . . . 23

Hare's Physiology . . . . . . . . . 28

Hart's Diet in Sickness and in Health . . 22

Haynes' Manual of Anatomy . . . . . . 26

Heisler's Embryology ........ . . 31

Hirst's Obstetrics . . . . . . . . . . . 3I

Hyde's Syphilis and Venereal Diseases . . 26

Jackson and Gleason's Diseases of the Eye,

Nose, and Throat .

Keating's Pronouncing Dictionary . . . . 10

Keating's Life Insurance . . . . . . . . . 23

Keen's Operation Blanks . . . . . . . . 22

Keen's Surgery of Typhoid Fever . . . . $3^{\mathbf{I}}$

Kyle's Diseases of Nose and Throat . . . 26

Laine's l'emperature Charts .
Lockwood's Practice of Medicine PAGE

Long's Syllabus of Gyuecology • • • 26

Macdonald's Surgical Diagnosis and l'reatnent

McFarland's Pathogenic Bacteria . . . I6

Mallory and Wright's Pathological 'I'ech-

nique . . . . . . . . . . . . 31

Martin's Surgery . . . . . . . . . . . . 28

Martiu's Minor Surgery, liandaging, and

Venereal Diseases. . . . . . . . 28

Mejgs' Feeding in Early Infancy . . . . . I6

Moore's Orthopedic Surgery . . . . . : . 31

Morris' Materia Medica and 'Therapeutics 28

Morris' Practice of Medicine . . . . . . 28

Morten's Nurses' Dictionary' . . . . . . 24

Nancrede's Anatomy and Dissection. . . I7

Nancrede's Anatomy . . . . . . . . . 28

Norris' Syllabus of Obstetrical Lectures . 2 I

Penrose's Gynecology . . . . . . . . . 3r

Powell's Diseases of Children . . . . . . 28

Pye's Bandaging and Surgical Dressing . 29

Raymond's Physiology . . . . . . . 26

Rowland's Clinıcal Skiagraphy . . . . 14

Saundby's Renal and Urinary Diseases . . 29

*Sannders' American Year-book of Medi-

cine and Surgery

Saunders' Medical Hand-Atlases $\cdot 33,32$

Saunders' Pocket Medıcal Fornulary . 19

Situnders' Pocket Medical I exicon . . . . 32

Saunders' New Series of Manuals . . . 25, 26

Saunders' Series of Question Compends 27, 28

Sayre's Practice of Pharmacy . . . . . . 28

Semple's Pathology and Morbid Anatomy 28

Semple's Legal Medicine, Toxicology, and

Hygiene

Senn's Genito-Urinary "luberculosis : - .

Senn's 'I'umors . . . . . . . . . . . . . I I

Senn's Syllabus of Lectures on Surgery . . 2 I

Shaw's Nervous Diseases and Insanity . . 28

Starr's Diet-Lists for Children . . . . . . 24

Stelwagon's Diseases of the Skin . . . . . 28

Stengel's M:anual of Pathology . . . . . . . 26

Stevens' Materia Medica and 'I'herapeutics 18

Stevens' Practice of Medicıne . . . . I 7

Stewart's Manual of Physiology . . . . . 2 I

Stewart and Lawrance's Medical Elec-

tricity ............. . . 28

Stoney's Practical Points in Nimsing . . . I3

Sutton and Giles' Diseases of Women . . . 26

Thomas's Diet-List and Sick-Room Dietary 24

Thornton's Dose-Book and Manual of Pre-

scription-Writing ....... . . 26

Van Valzah and Nisbet's Diseases of the

Stomach ........... 3 I

Vierordt and Stuart's Medical Diagnosis . I2

Warren's Surgical Pathology . . . . . I I

Wolff's Chemistry . . . . . . . . . 28

Wolff's Examination of Urine . . . . . 28

The works indicated thus (*) are sold by subscktption (not by booksellers), usually through travelling solicitors, but they can be obtained direct from the office of publication (charges of shipment prepaid) by remitting the quoted prices. Full descriptive circulars of such works will be sent to any address upon application.

All the other Books advertised in this catalogue are commonly for sale by booksellers in all parts of the United States; but any book will be sent by the publisher to any address (post-paid) on receipt of the price herein given. 


\section{GENERAL INFORMATION.}

One Price. One price absolutely without deviation. No discounts allowed, regardless of the number of books purchased at one time. Prices on all works have been fixed extremely low, with the view to selling them strictly net and for cash.

Orders.

An order accompanied by remittance will receive prompt attention, books being sent to any address in the United States, by mail or express, all charges prepaid. We prefer to send books by express when possible, and if sent C. O. D. we pay all charges for returning the money. Small orders of three dollars or less must invariably be accompanied by remit. tance.

How to Send There are four ways by which money can be sent at our Money by risk, namely: a post-office money order, an express money Mail. order, a bank-check (draft), and in a registered letter. Money sent in any other way is at the sender's risk. Silver should not be sent through the mail.

Shipments. All books, being packed in patent metal-edged boxés, necessarily reach our patrons by mail or express in excellent condition.

Subscription Books in our catalogue marked "For sale by subscription Books. only" may be secured by ordering them through any of our authorized travelling salesmen, or direct from the Philadelphia office; they are not for sale by booksellers. All other books in our catalogue can be procured of any bookseller at the advertised prices, or directly from us. We handle only our own publications, and cannot supply second-hand books nor the publications of other houses.

Latest

Editions.

Bindings.

Descriptive

Circulars.
In every instance the latest revised edition is sent.

In ordering, be careful to state the style of binding desiredCloth, Sheep, or Half-Morocco.

A complete descriptive circular, giving table of contents, etc. of any book sold by subscription only, will be sent free on application. 


\section{For Sale by Subscription.}

AN AMERICAN TEXT-BOOK OF PHYSIOLOGY. Edited by William H. Howell, PH. D., M. D., Professor of Physiology in the Johns Hopkins University, Baltimore, Md. One handsome octavo volume of 1052 pages, fully illustrated. Prices: Cloth, $\$ 6.00$ net; Sheep or HalfMorocco, $\$ 7.00$ net.

This work is the most notable attempt yet made in America to combine in nne volume the entire subject of Human Physiology by well-known teachers who have given especial study to that part of the subject upon which they write. The completed work represents the present status of the science of Physiology, particularly from the standpoint of the student of medicine and of the medical practitioner.

The collaboration of several teachers in the preparation of an elementary textbook of physiology is unusual, the almost invariable rule heretofore having been for a single author to write the entire book. One of the advantages to be derived from this collaboration method is that the more limited literature necessary for consultation by each author has enabled him to base his elementary account upon a comprehensive knowledge of the subject assigned to him; another, and perhaps the most impurtant, advantage is that the student gains the point of view of a number of teachers. In a measure he reaps the same benefit as would be obtained by following courses of instruction under different teachers. The different standpoints assumed, and the differences in emplasis laid upon the various lines of procerlure, chemical, physical, and anatomical, should give the student a better insight into the methods of the science as it exists to-day. The work will also be found useful to many medical practitioners who may wish to keep in touch with the development of modern physiology.

The main divi-ions of the subject-matter are as follows: General Physiology of Muscle and Nerve - Secretion - Chemistry of Digestion and NutritionMovements of the Alimentary Canal, Bladier, and Ureter-Blood and Lymph -Circulation-Respiration-Animal Heat-Central Nervous System-Special Senses - Special Muscular Mechanisms - Reproduction - Chemistry of the Animal Body.

\section{CONTRIBUTORS :}

HENRY P. BOWDITCH, M. D.,

Professor of Physiology, Harvard Medical School.

\section{JOHN G. CURTIS, M. D.,}

Professor of Physiology, Columbia University, N. Y. (Cullege of Physicians and Surgeons).

ITENRY H. DONALDSON, Ph. D.,

Head-Professor of Neurology, University of Chicago.

W. H. HOWELL, Ph. D., M. D.,

Professor of Physiology, Johns Hopkins University.

FREDERIC S. LEE, Ph. D.,

Adjunct Professor of Physiólogy, Colımbia University, N. Y. (College of Pliysicians and Surgeons).
WARREN P. LOMBARD, M. D.,

Professor of Physiology, University of Michigan.

GKAHAM LUSK, Ph. D.,

Professor of Physiology, Yale Medica' School.

W. T. PORTER, M. D.,

Assistant Professor of Physiology, Har. vard Medical School.

EDWARD T. REICHERT, M. D.,

Professor of Physiology, University of Pennsylvania.

HENRY SEWALL, Ph. D., M. D.,

Professor of Physiology, Medical Depart ment, University of Denver. 


\section{For Sale by Subscription.}

\section{AN AMERICAN TEXT-BOOK OF APPLIED THERAPEU-} TICS. For the Use of Practitioners and Students. Edited by JAMES C. Wilson, M. D., Professor of the Practice of Medicine and of Clinical Medicine in the Jefferson Medical College. One handsome octavo volume of I 326 pages. Illustrated. Prices: Cloth, $\$ 7.00$ net; Sheep or Half-Morocco, \$8.0o net.

The arrangement of this volume has been based, so far as possible, upon modern pathologic doctrines, beginning with the intoxications, and following with infections, diseases due to internal parasites, diseases of undetermined origin, and finally the disorders of the several bodily systems-digestive, respiratory, circulatory, renal, nervous, and cutaneous. It was thought proper to include also a consideration of the disorders of pregnancy.

The list of contributors comprises the names of many who have acquired distinction as practitioners and teachers of practice, of clinical medicine, and of the speciaities.

\section{CONTRIBUTORS :}

Dr. I. E. Atkinson, Baltimore, Md.

Sanger Brown, Chicago, iil.

John B. Chapin, Philadelphia, $\mathrm{Pa}$.

William C. Dabney, Charlottesville, Va.

John Chalmers DaCosta, Philada., Pa.

I. N. Danforth, Chicago, Ill.

John L. Dawson, Jr., Charleston, S. C

F. X. Dercum, Philadelphia, Pa.

George Dock, Ann Arbor, Mich.

Robert T. Edes, Jamaica Plain, Mass. Augustus A. Eshner, Philadelphia, Pa.

J. T. Eskridge, Denver, Col.

F. Forchheimer, Cincinnari, $\mathrm{O}$.

Carl Frese. Philadelphia, Pa.

Edwin E. Graham, Philadelphia, Pa.

John Guitéras. Philadelphia. Pa.

Frederick P. Henry, Philadelphia, Pa.

Guy Hinsdale, Philadelphia, Pa.

Orville Horwitz, Philadelphia, Pa.

W. WV. Johnston, Washington, D. C.

Ernest Laplace, Philadelphia, Pa.

A. Laveran, Parss, France.
Dr. James Hendrie Lloyd, Philadelphia, Pa. John Noland Mackenzie, Baltimore, Md. J. IV. McLiughlin, Austin, Texas.

A. Lawrence Mason, Boston, Mass. Charles K. Mills, Philadelphia, $\mathrm{Pa}$. John K. Mitchell, Phıladelphia, Pa. IV. P. Northrup. New York City.

William Osler, Baltimore, Md. Frederick A. Packard, Philadelphia, Pa. Theophilus Parvin, Philadelphia, $\mathbf{P a}$. Beaven Rake, London, England.

E. O. Shakespeare, Philadelphia, Pa. Wharton Sinkler, Philadelphia. Pa. Louis Starr, Philadelphia, Pa.

Henry W. Stelwagon, Philadelphia, Pa. James Stewart, Montreal, Canada. Charles G. Stockton, Buffalo, N. Y. James Tyson, Philadelphia, $\mathbf{P a}$.

Victor C. Vaughan, Ann Arbor, Mich. James T. Whittaker, Cincinnati, $\mathrm{O}$. J. C. Wilson, Philadelphia, Pa.

The articles, with two exceptions, are the contributions of American writers. Written from the standpoint of the practitioner, the aim of the work is to facilitate the application of knowledge to the prevention, the cure, and the alieviation of disease. The endeavor throughout has been to conform to the title of the book-Applied Therapeutics-to indicate the course of treatment to be pursued at the bedside, rather than to name a list of drugs that have been used at one time or another.

While the scientific superiority and the practical desirability of the metric system of weights and measures is admitt $\epsilon \mathrm{d}$, it has not been deeined best to discard entirely the older system of figures, so that both sets have been given where occasion demanded. 


\section{For Sale by Subscription.}

\section{AN AMERICAN TEXT-BOOK OF OBSTETRICS. Edited by} Richard C. Norris, M. D. ; Art Editor, Robert L. Dickisson, M. D. One handsome octavo volume of over I 000 pagres, with nearly 900 colored and half-tone illustrations. Prices: Cloth, $\$ 7.00$; Sheep or Half-Morocco, $\$$ \$. OO.

The advent of each successive volume of the series of the A.MERICAN TEXTBooks has been signalized by the most flattering comment from both the Press and the Profession. The high consideration received by these text-books, and their attainment to an authoritative position in current medical literature, have been matters of deep international interest, which finds its fullest expression in the demand for these publications from all parts of the civilized world.

In the preparation of the "AMERICAN TEXT-BOOK OF OBSTETRICS" the editor has called to his aid proficient collaborators whose professional prominence entitles them to recognition, and whose disquisitions exemplify Practical Obstetrics. While these writers were each assigned special themes for discussion, the correlation of the subject-matter is, nevertheless, such as ensures logical connection in treatment, the deductions of which thoroughly represent the latest advances in the science, and which elucidate the best modern methods of procedure.

The more conspicuous feature of the treatise is its wealth of illustrative matter. The production of the illustrations had been in progress for several years, under the personal supervision of Robert L. Dickinson, .I. D., to whose artistic judgment and professional experience is due the most sumptuously illustrated work of the period. By means of the photographic art, combined with the skill of the artist and draughtsman, conventional illustration is superseded by rational methods of delineation.

Furthermore, the volume is a revelation as to the possibilities that may be reached in mechanical execution, through the unsparing hand of its publisher.

\section{CONTRIBUTORS :}

Dr. James C. Cameron.

Edward P. Davis.

Robert L. Dickinson.

Charles Warrington Earle.

James H. Etheridge.

Henry J. Garrigues.

Barton Cooke Hirst.

Charles Jeweti.
Dr. Howard A. Kelly.

Richard C. Norris.

Chauncey D. Palmer.

Thenphilus Parvin.

George A. Piersol.

Edward Reynolds.

Henry Schwarz.

"At first glance we are overwhelmed by the magnitude of this work in several respects, viz.: First, by the size of the volume, then by the array of eminent teachers in this department who have taken part in its production, then by the profuseness and character of the illustrations, and last, but not least, the conciseness and clearness with which the text is rendered. This is an entirely new composition, embodying the highest knowledge of the art as it stands to-day by authors who occupy the front rank in their specialty, and there are many of them. We cannot turn over these pages without being struck by the superb illustrations which adorn so many of them. We are confident that this must practical work will find instant appreciation by practitioners as well as students." -New York Medical Times.

Permit me to say that your American Text-Book of Obstetrics is the most magnificent medical work that 1 have ever seen. I congratulate you and thank yoll for this superb work, which alone is sufficient to place you first in the ranks of medical publishers.

With profound respect I am sincerely yours, ALEX. J. C. SKene. 


\section{For Sale by Subscription.}

\section{AN AMERICAN TEXT-BOOK ON THE THEORY AND PRACTICE OF MEDICINE. By American Teachers. Edited} by William Pepper, M. D., LL.D., Provost and Professor of the Theory and Practice of Medicine and of Clinical Medicine in the University of Pennsylvania. Complete in two handsome royal-octavo volumes of about IOo0 pages each, with illustrations to elucidate the text wherever necessary. Price per Volume: Cloth, $\$ 5.00$ net; Sheep or Half-Morocco, $\$ 6.00$ net.

\section{VOLUME I. CONTAINS:}

Hygiene.-Fevers (Ephemeral, Simple Continued, Typhus, Typhoid, Epidem ic Cerebrospinal Meningitis, and Relapsing).-Scarla. tina, Measles, Rötheln, Variola, Varioloid, Viccinia, Varicella, Mumps. Whooping-cough, Anthrax, Hydrophobia, Trichinosis, Actinn- mycosis, Glanders, and Tetanus.-Tuberculosis, Scrofula, Syphilis, Diphtheria, Erysipelas, Maiaria, Cholera, and Yellow Fever.Nervous, Muscular, and Mental Diseases etc.

\section{VOLUME IL. CONTAINS :}

Urine (Chemistry and Microscnpy).-Kid- I - Peritoneum, Liver, and Pancreas.-Diathetney and Lungs.-Air-passages (Larynx and ic Diseases (Rheumatism, Rheumatoid ArBronchi) and Pleura.-Pharynx, Esophagus, thritis, Gout, Lithæmia, and Diabetes.)Stomach and Intestines (including Intestinal Blood and Spleen.-Inflammation, Embolism, Parasites), Heart, Aorta, Arteries and Veins. Thrombosis, Fever, and Bacteriology.

The articles are not written as though addressed to students in lectures, but are exhaustive descriptions of diseases, with the newest facts as regards Causation, Symptomatology, Diagnosis, Prognosis, and Treatment, including a large number of approved formulie. The recent advances made in the study of the bacterial origin of various diseases are fully described, as well as the bearing of the knowledge so gained upon prevention and cure. The subjects of Bacteriology as a whole and of Immunity are fully considered in a separaie section.

Methods of diagnosis are given the most minute and careful attention, thus enabling the reader to learn the very latest methods of investigation without consulting works specially devoted to the subject.

\section{CONTRIBUTORS :}

Dr. J. S. Billings, Philadelphia.

Francis Delafield, New York.

Reginald H. Fitz, Boston.

James W. Holland, Philadelphia.

Henry M. Lyman, Chicago.

William Osler, Baltimore.
Dr. William Pepper, Philadelphia.

W. Gilman Thompson, New York.

W. H. Welch, Baltimore.

James T. Whittaker, Cincinnati.

James C. Wilson, Philadelphia.

Horatio C. Wood, Philadelphia.

"We reviewed the first volume of this work, and said: 'It is undoubtedly one of the best text-books on the practice of medicine which we possess.' A consideration of the second and last volume leads us to modify that verdict and to say that the completed work is, in our opinion, THE BEST of its kind it has ever been our fortune to see. It is complete, thorough, accurate, and clear. Ii is well written, well arranged, well printed, well illustrated, and well bound. It is a model of what the modern text-book should be." - New York Medical Fournal.

"A libiary upon modern medical art. The work must promote the wider diffusion of scund knowledge." - American Lancet.

"A trusty counsellor for the practitioner or seniur student, on which he may implicitly rely."-Edinburgh Medical .7ournal. 


\section{For Sale by Subscription.}

AN AMERICAN TEXT-BOOK OF SURGERY. Edited by WILLiaM W. KeeN, M.D., LL.D., and J. Wili.iaM White, M. D., Ph. D. Forming one l:andsome royal-octavo volume of 1250 pages (IO 77 inches), with 500 wood-cuts in text, and 37 colored and half-tone plates, many of them engraved from original photographs and drawings furnished by the authors. Prices: Cloth, $\$ 7.00$ net; Sheep or Half-Morocco, $\$ 8.00$ net.

\section{SECOND EDITION, REVISED AND ENLARGED,}

With a Section devoted to "The Use of the Röntgen Rays in Surgery."

The want of a text-book which could be used by the practitioner and at the same time be recommended to the medical student has been deeply felt, especially by teachers of surgery; hence, when it was suggested to a number of these that it would be well to unite in preparing a text-book of this description, great unanimity of opinion was found to exist, and the gentlemen below named gladly consented to join in its production. While there is no distinctive American Surgery, yet America has contributed very largely to the progress of modern surgery, and among the foremost of those who have aided in developing this art and science will be found the authors of the present volume. All of them are teachers of surgery in leading medical schools and hospitals in the United States and Canada.

Especial prominence has been given to Surgical Bacteriology, a feature which is believed to be unique in a surgical text-book in the English language. Asepsis and Antisepsis have received particular attention. The text is brought well up to date in such important branches as cerebral, spinal, intestinal, and pelvic surgery, the most important and newest operations in these departments being described and illustrated.

The text of the entire book has been submitted to all the authors for their mutual criticism and revision-an idea in book-making that is entirely new and original. The book as a whole, therefore, expresses on all the important surgical topics of the day the consensus of opinion of the eminent surgeons who have joined in its preparation.

One of the most attractive features of the book is its illustrations. Very many of them are original and faithful reproductions of photographs taken directly from patients or from specimens.

\section{CONTHIBUTORS:}

Dr. Charles H. Burnett, Philadelphia. Phineas S. Conner, Cincinnati. Frederic S. Dennis, New York. William W. Keen, Philadelphia. Charles B. Nancrede, Ann Arbor, Mich. Roswell Park, Buffalo, N. Y.

Lewis S. Pilcher, New York.
Dr. Nicholas Senn, ('hicago.

Francis J. Shepherd. Montreal, Canada. Lewis A. Stimson, New York.

William Thomson, Philadelphia.

J. Collins Warren, Boston. J. William White, Philadel phia.

"If this text-book is a fair reflex of the present position of American surgery, we must admit it is of a very high order of merit, and that English surgeons will have to look very carefully to their laurels if they are to preserve a position in the van of surgical practice." London Lancet. 


\section{For Sale by Subscription.}

\section{AN AMERICAN TEXT-BOOK OF GYNECOLOGY, MEDICAL AND SURGICAL, for the use of Students and Practitioners. Edited by J. M. BALDY, M. D. Forming a handsome royal-octavo volume, with $34 \mathrm{I}$ illustrations in text and 38 colored and half-tone plates. Prices: Cloth, $\$ 6.00$ net; Sheep or Half-Morocco, $\$ 7.00$ net.}

\section{SECOND EDITION, THOROUGHLY REVISED.}

In this volume all anatomical descriptions, excepting those essential to a clear understanding of the text, have been omitted, the illustrations being largely depended upon to elucidate the anatomy of the parts. This work, which is thoroughly practical in its teachings, is intended, as its title implies, to be a working text-book for physicians and students. A clear line of treatment has been laid down in every case, and although no attempt has been made to discuss mooted points, still the most important of these have been noted and explained. The operations recommended are fully illustrated, so that the reader, having a picture of the procedure described in the text under his eye, cannot fail to grasp the idea. All extraneous matter and discussions have been carefully excluded, the attempt being made to allow no unnecessary details to cumber the text. The subject-matter is brought up to date at every point, and the work is as nearly as possible the combined opinions of the ten specialists who figure as the authors.

\section{CONTRIBUTORS :}

Dr. Henry T. Byford.

John M. Baldy.

Edwin Cragin.

1. H. Etheridge.

villiam Goodell.
Dr. Howard A. Kelly.

Florian Krug.

E. E. Montgomery.

William R. Pryor.

George M. Tuttle.

"The most notable contribution to gynecological literature since $1887, \ldots$ and the most complete exponent of gynecology which we have. No subject seems to have been neglected, .... and the gynecologist and surgeon, and the general practitioner who has any desire to practise diseases of women, will find it of practical value. In the matter of illustrations and plates the book surpasses anything we have seen."-Boston Medical and Surgical fournal.

"A valuable addition to the literature of Gynecology. The writers are progressive, aggressive, and earnest in their convictions."-Medical Nerws, Philadelphia.

"A thoroughly modern text-book, and gives reliable and well-tempered advice and in. struction." - Edinburgh Medical Journal.

"The harmony of its conclusions and the homogeneity of its style give it an individuality which suggests a single rather than a multiple authorship."-Annals of Surgery.

"It must command attention and respect as a worthy representation of our advanced clinical teaching."-American Fournal of Medical Sciences. 


\section{For Sale by Subseription.}

AN AMERICAN TEXT-BOOK OF THE DISEASES OF CHIL. DREN. By American Teachers. Edited by Louis STARr, M. D., assisted by Thompson S. WestcotT, M. D. In one handsome royal-8vo volume of $125^{\circ}$ pages, profusely illustrated with wood-cuts, half-tone and colored plates. Net Prices: Cloth, \$7.00; Sheep or Half-Morocco, \$8.00.

\section{SECOND EDITION, REVISED AND ENLARGED.}

The plan of this work embraces a serics of original articles written by some sixty well-known prediatrists, representing collectively the teachings of the most prominent medical schools and colleges of America. The work is intended to be a P'RACTICAL book, suitable for constant and handy reference by the practitioner and the advanced student.

Especial attention has been given to the latest accepted teachings upon the etiology, symptoms, pathology, diagnosis, and treatment of the disorders of children, with the introduction of many special formulæ and therapeutic procedures.

Special chapters embrace at unusial length the Diseases of the Eye, Ear, Nose and 'Throat, and the Skin; while the introductory chapters cover fully the important subjects of Diet, Hygiene, Exercise, Bathing, and the Chemistry of Food. Tracheotomy, Intubation, Circumcision, and such minor surgical procedures coming within the province of the medical practitioner are carefully considered.

\section{CONTRIBUTORS:}

Dr. S. S. Adams, Washington. John Ashhurst, Jr., Philadelphia. A. D. Blackader, Montreal, Canada. David Bovaird, New York.

Dillon Brown, New York.

Edward M. Buckingham, Boston.

Charles W. Burr, Philadelphia.

W. E. Casselberry, Chicago.

Henry Dwight Chapin, New York.

W. S. Christopher, Chicago.

Archibald Church, Chicago.

Floyd M. Crandall, New York.

Andrew F. Currier, New York.

Roland G. Curtin, Philadelphia

J. M. DaCosta, Philadelphia.

I. N. Danforth, Chicago.

Edward P. Davis, Philadelphia.

John B. Deaver, Philadelphia.

G. E. de Schweinitz, Philadelphia.

John Dorning, New York.

Charles Warrington Earle, Chicago.

Wm. A. Edwards, San Diego, Cal.

F. Forchheimer, Cincinnati.

J. Henry Fruitnight, New York.

f. P. Crozer Griffith, Philadelphia.

W. A. Hardiway. St. Louis.

M. P Hatfield, Chicago.

Barton Cooke Hirst, Philadelphia.

H. Illoway, Cincinnati.

Henry Jackson, Boston.

Charles G. Jennings, Detroit.

Henry Koplik, New York.
Dr. Thomas S. Latimer, Baltimore.

Albert R. Leeds, Hoboken, N. J.

J. Hendrie Lloyd, Philadelphia. George Roe Lockwood, New York.

Henry M. Lyman, Chicago.

Francis '1'. Mliles, Baltimore.

Charles K Mills, Philadelphia.

James E. Moore, Minneapolis.

F. Gordon Morrill, Boston.

John H. Musser, Philadelphia.

Thomas R. Neilson, Philadelphia.

W. P. Northrup, New York.

William Osler, Baltimore.

Frederick A. Packard, Philadelphia.

William Pepper, Philadelphia.

Frederick Peterson, New York.

WV. T. Plant, Syracuse, New York.

William M. Powell, Atlantic City.

B. Alexander Randall, Philadelphia.

Edward O. Shakespeare, Philadelphia

F. C. Shattuck, Boston.

J. Lewis Smith, New York.

Louis Starr, Philadelphia.

M. Allen Starr, New York.

Charles W. Townsend, Boston.

James Tyson, Philadelphia.

iN. S. Thayer, Baltimore.

Victor C. Vaughan, Ann Arbor, Micn Thompson S. Westcott, Philadelphia. Henry R. Wharton, Philadelphia.

J. William White, Philadelphia.

J. C. Wilson, Philadelphia. 
A NEW PRONOUNCING DICTIONARY OF MEDICINE, with Phonetic Pronunciation, Accentuation, Etymology, etc. By JoHN M. Keating, M. D., LL.D., Fellow of the College of Physicians of Philadelphia; Vice-President of the American Pædiatric Society; Ex-President of the Association of Life Insurance Medical Directors; Editor "Cyclopædia of the Diseases of Children," etc.; and Henry Hamilton, author of "A New Translation of Virgil's Æneid into English Rhyme;" coauthor of "Saunders' Medical Lexicon," etc.; with the Collaboration of J. Chalmers DaCosta, M. D., and Frederick A. Packard, M. D. With an Appendix containing important Tables of Bacilli, Micrococci, Leucomaines, Ptomaines, Drugs and Materials used in Antiseptic Surgery, Poisons and their Antidotes, Weights and Measures, Thermometric Scales, New Official and Unofficial Drugs, etc. One very attractive volume of over 800 pagres. Second Revised Edition. Prices: Cloth, $\$ 5.00$ net; Sheep or Half-Morocco, $\$ 6.00$ net; with Denison's Patent Ready-Reference Index; without patent index, Cloth, $\$ 4.00$ net; Sheep or HalfMorocco, $\$ 5.00$ net.

\section{PROFESSIONAL OPINIONS.}

"I am much pleased with Keating's Dictionary, and shall take pleasure in recommending it to my classes."

Professor of Principles and Practice of Medicine, Rush Medical College, Chicago, Ill.

"I am convinced that it will be a very" valuable adjunct to my sludy-table, convenient in size and sufficiently full for ordinary use."

C. A. Lindsley, M. D.,

Professor of Theory and Practice of Medicine, Medical Dept. Vale University: Secretary Connecticut State Board of Health, Nezu Haven, Conn,

AUTOBIOgRAPHY OF SAMUEL D. GROSS, M. D., Emeritus Professor of Surgery in the Jefferson Medical College of Philadelphia, with Reminiscences of His Times and Contemporaries. Edited by his sons, Samuel W. Gross, M. D., LL.D., late Professor of Principles of Surgery and of Clinical Surgery in the Jefferson Medical College, and A. Haller Gross, A. M., of the Philadelphia Bar. Preceded by a Memoir of Dr. Gross, by the late Austin Flint, M. D., LL.D. In two handsome volumes, each containing over 400 pages, demy 8 ro, extra cloth, gilt tops, with fine Frontispiece engraved on steel. Price per Volume, $\$ 2.50$ net.

This autobiography, which was continued by the late eminent surgeon until within three months of his death, contains a full and accurate history of his early struggles, trials, and subsequent successes, told in a singularly interesting and charming manner, and embraces short and graphic pen-portraits of many of the most distinguished men-surgeons, physicians, divines, lawyers, statesmen, scientists, etc. - with whom he was brought in contact in America and in Europe ; the whole forming a retrospect of more than three-quarters of a century. 
SURGICAL PATHOLOGY AND THERAPEUTICS. By JOHN Collins Warrex, M. D., LI..D., Professor of Surgery, Medical Department Harvari University; Surgeon to the Massachuse!ts General Hospital, etc. A handsome octavo volume of 832 pages, with 136 relief and lithographic illustrations, 33 of which are printed in colors, and all of which were drawn by William J. Kaula from original specimens. Prices: Cloth, $\$ 6.00$ net; Half-Morocco, \$7.00 net.

"The volume is for the bedside, the amphitheatre, and the ward. It deals with things not as we see them through the microscope alone, but as the practitioner sees their effect in his patients; not only as they appear in and affect culture-media, but also as they influence the human body; and, following up the demonstrations of the nature of diseases, the author yoints out their logical treatment." (New Lork Medical foumal). "It is the handsomest specimen of book-making *** that has ever been issued from the American medical press" (American Foumal of the Medical Sciences, Philadelphia).

\section{Without Exception, the Illustrations are the Best ever Seen in a Work of this Kind.}

"A most striking and very excellent feature of this book is its illustrations. Without exception, from the point of accuracy and artistic merit, they are the best ever seen in a work of this kind. *** Many of those representing microscopic pictures are so perfect in their coloring and detail as almost to give the beholder the impression that he is looking down the barrel of a microscope at a well-mounted section." - Annals of Sicrgery", Philadelphia.

\section{PATHOLOGY AND SURGICAL TREATMENT OF TUMORS.}

By N. Sexx, M. D., Ph. D., LL. D., Professor of Practice of Surgery and of Clinical Surgery, Rush Medical College; Professor of Surgery, Chicago Polyclinic; Attending Surgeon to Presbyterian Hospital; Surgeon-in-Chief, St. Joseph's Hospital, Chicago. One volume of $710^{\circ}$ pages, with 515 engravings, including full-page colored plates. Prices: Cloth, $\$ 6 . \infty 0$ net: Half-Morocco, $\$ 7.00$ net.

Books specially devoted to this subject are few, and in our text-books and systems of surgery this part of surgical pathology is usually condensed to a degree incompatible with its scientific and clinical importance. The author spent many years in collecting the material for this work, and has taken great pains to present it in a manner that should prove useful as a text-book for the student, a work of reference for the busy practitioner, and a reliable, safe guide for the surgeon. The more difficult operations are fully described and illustrated. More than one hundred of the illustrations are original, while the remainder were selected from books and medical journals not readily accessible.

"The most exhaustive of any recent book in English on this subject. It" is well illustrated, and will doubtless remain as the principal monograph on the subject in our language for some years. The book is handsomely illustrated and printed, . . . . and the author has given a notable and lasting contribution to surgery." - Fournal of American Medical A:sa ciation, Chicago. 
MEDICAL DIAGNOSIS. By Dr. Oswald Vierordt, Professor of Medicine at the University of Heidelberg. Translated, with additions, from the Fifth Enlarged German Edition, with the author's permission, by Fraicis H. Stuart, A. M., M. D. In one handsome royal-octavo volume of 600 pages. 194 fine wood-cuts in the text, many of them in colors. Prices: Cloth, $\$ 4.00$ net; Sheep or Half-Morocco, $\$ 5.00$ net.

\section{FOURTH AMERICAN EDITION, FROM THE FIFTH REVISED AND ENLARGED GERMAN EDITION.}

In this work, as in no other hitherto published, are given full and accurate explanations of the phenomena observed at the bedside. It is distinctly a clinical work by a master teacher, characterized by thoroughness, fulness, and accuracy. It is a mine of information upon the points that are so often passed over without explanation. Especial attention has been given to the germ-theory as a factor in the origin of disease.

This valuable work is now published in German, English, Russian, and Italian. The issue of a third American edition within two years indicates the favor with which it has been received by the profession.

\section{THE PICTORIAL ATLAS OF SKIN DISEASES AND SYPHI- LITIC AFFECTIONS. (American Edition.) Translation from} the French. Edited by J. J. Pringle, M. B., F. R. C. P., Assistant Physician to, and Physician to the department for Diseases of the Skin at, the Middlesex Hospital, London. Photo-lithochromes from the famous models of dermatological and syphilitic cases in the Museum of the Saint-Louis Hospital, Paris, with explanatory wood-cuts and letter-press. In 12 Parts, at $\$ 3.00$ per Part. Parts I to 8 now ready.

“The plates are beautifully executed."-Jonathan Hutchinson, M. D. (London Hospital).

"The plates in this Atlas are remarkably accurate and artistic reproductions of typical examples of skin disease. The work will be of great value to the practitioner and student." -William Anderson, M. D. (St. Thomas Hospital).

"If the succeeding parts of this Atlas are to be similar to Part I, now before us, we have no hesitation in cordially recommending it to the favorable notice of our readers as one of the finest dermatological atlases with which we are acquainted."-Glasgow Medical fournal, Aug., 1895 .

"Of all the atlases of skin diseases which have been published in recent years, the present one promises to be of greatest interest and value, especially from the standpoint of the general practitioner."-American Medico-Surgical Bulletin, Fẻb. 22, 1896.

"The introduction of explanatory wood-cuts in the text is a novel and most important feature which greatly furthers the easier understanding of the excellent plates, than which nothing, we venture to say, has been seen better in point of correctness, beally, and general merit." - New York. Medical Journal, Feb. 15, 1896.

"An interesting feature of the Atlas is the descriptive text, which is written for each picture by the physician who treated the case or at whose instigation the models have been made. We predict for this truly beautiful work a large circulation in all parts of the medical world where the names St. Louis and Baretta have preceded it." -Medical Record, N. Y., Feb. I, ז896. 
PRACTICAL POINTS IN NURSING. For Nurses in Private Practice. By EMILy A. M. Stoney, Graduate of the Training-School for Nurses, Lawrence, Mass.; Superintendent of the Training-School for Nurses, Carney Hospital, South Boston, Mass. 456 pages, handsomely illustrated with 73 engravings in the text, and 9 colored and balf-tone plates. Cloth. Price, $\$ \mathbf{I} .75$ net.

\section{SECOND EDITION, THOROUGHLY REVISED.}

In this volume the author explains, in popular language and in the shortest possible form, the entire range of private nursing as distinguished from hospital nursing, and the nurse is instructed how best to meet the various emergencies of medical and surgical cases when distant from medical or surgical aid or when thrown on her own resources.

An especially valuable feature of the work will be found in the directions to the nurse how to improvise everything ordinarily needed in the sick-room, where the embarrassment of the nurse, owing to the want of proper appliances, is frequently extreme.

The work has been logically divided into the following sections:

I. The Nurse: her responsibilities, qualifications, equipment, etc.

II. The Sick-Room : its selection, preparation, and management.

III. The Patient : duties of the nurse in medical, surgical, obstetric, and synecologic cases.

IV. Nursing in Accidents and Emergencies.

V. Nursing in Special Medical Cases.

VI. Nursing of the New-born and Sick Children.

VII. Physiology and Descriptive Anatomy.

The APPENDIX contains much information in compact form that will be found of great value to the nurse, including Rules for Feeding the Sick; Recipes for Invalid Foods and Beverages; Tables of Weights and Measures; Table for Computing the Date of Labor; List of Abbreviations; Dose-List; and a full and complete Glossary of Medical Terms and Nursing Treatment.

"This is a well-written, eminently practical volume, which covers the entire range of private nursing as distinguished from hospital nursing, and instructs the nurse how best to meet the various emergencies which may arise and how to prepare everything ordinarily needed in the illness of her patient." - American Journal of Obstetrics and Diseases of Women and Children, Aug., 1896.

A TEXT-BOOK OF BACTERIOLOGY, including the Etiology and Prevention of Infective Diseases and an account of Yeasts and Moulds, Hæmatozoa, and Psorosperms. By Edgar M. Crookshask, M. B., Professor of Comparative Pathology and Bacteriology, King's College, London. A handsome octavo volume of 700 pages, with 273 engravings in the text, and 22 original and colored plates. Price, $\$ 6.50$ net.

This book, though nominally a Fourth Edition of Professor Crookshank's "MANUAL OF Bacteriology," is practically a new work, the old one having been reconstructed, greatly enlarged, revised throughout, and largely rewritten, forming a text-book for the Bacteriological Laboratory, for Medical Ofncers of Health, and for Veterinary Inspectors. 
A TEXT-BOOK OF HISTOLOGY, DESCRIPTIVE AND PRACTICAL. For the Use of Students. By ARThUR Clarkson, M. B., C. M., Edin., formerly Demonstrator of Physiology in the Owen's College, Manchester; late Demonstrator of Physiology in the Yorkshire College, Leeds. Large Sro, 554 pages, with 22 engrarings in the text, and $\mathbf{1 7 4}$ beautifully colored original illustrations. Price, strongly bound in Cloth, $\$ 6.00$ nei.

The purpose of the writer in this work has been to furnish the student of Histology, in one volume, with both the descriptive and the practical part of the science. The first two chapters are deroted to the consideration of the general methods of Histology; subsequently, in each chapter, the structure of the tissue or organ is first systematically descrited, the student is then taken tutorially over the specimens illustrating it, and, finally, an appendix affords a short note of the methods of preparation.

"We would most cordially recommend it to all students of histology."-Dublin Medical Fournal.

"It is pleasant 10 zire unqualified praise to the colored illustrations; . . the standard is high, and many of them are not coly extremely beautiful. but very clear and demonstrative... The plan of the book is excellent."-Liverpool Medical fournal.

ARCHIVES OF CLINICAL SKIAGRAPHY. By SydNey RowlaNd, B. A., Camb. A series of collotype jllustrations, with descriptive text, illustrating the applications of the New Photography to Medicine and Surgery. Price, per Part, \$1.00. Farts I. to V. now ready.

The object of this publication is to put on reccrd in permanent form some of the most striking applications of the new photography to the needs of Medicine and Surgery.

The progress of this new art has been so rapid that, although Prof. Ronntgen's discovery is only a thing of yesterday, it has already taken its place among the approved and accepted aids to diagnosis.

WATER AND WATER SUPPLIES. By Johs C. Thresh, D. Sc., M. B., D. P. H., Lecturer un Public Health, King's College, London; Editor of the "Journal of State Medicine," etc. 1 $2 \mathrm{mo}, 43^{8}$ pages, illustrated. Handsomely bound in Cloth, with gold sice and back stamps. Price, $\$ 2.25$ net.

This work will furnish any one interested in public health the information reguisite for forming an opinion as to whether any supply or proposed supply is sufficiently wholesome and abundant, and whether the cost can be considered reasonable.

The work does not pretend to be a treatise on Engineering, yet it contains sufficient detail to enable any one who has studied it to consider intelligently any schem which may be submitted for supplying a community with water. 


\section{DISEASES OF THE EYE. A Hand-Book of Ophthalmic Prac-} tice. By G. E. 1): SchweiniT\%, M. D., Professor of Ophthalmology in the Jefferson Mélical College, l'hiladelphia, etc. A handsome royaloctavo volume of 679 pages, with 256 fine iilustrations, many of which are original, and 2 chromo-lithographic plates. I'rices: Cloth, $\$ 4.00$ net; Sheep or Half-.Moroccu, $\$ 5.00$ net.

The object of this work is to present to the student, and to the practitioner who is beginning work in the fields of ophthalmology, a plain description of the optical defects and diseases of the eye. To this end special attention has been paid to the clinical side of the question; and the method of examination, the symptomatolugy leading to a diagnosis, and the treatment of the various ocular defects have been brought into prominence.

\section{THIRD EDITION, THOROUGHLY REVISED.}

The entire book has been horoughly revised. In addition to this general revision, special paragraphs on the following new matter have been introduced: Filamentous Keratitis, Blood-staining of the Cornea, Essential Phthisis Bulbi, Foreign Bodies in the Lens, Circinate Retinitis, Synmetrical Changes at the Macula Lutea in Infancy, Hyaline Bodies in the Papilla, Monocular Diplopia, Subconjunctival Injections of Gernicides, Infiltration-Anæsthesia, and Sterilization of Collyria. Brief mention of Ophthalmia Nodosa, Electric Ophthalmia, and Angioid Streaks in the Retina also finds place. An Appendix has been added, containing a full clescription of the method of determining the corneal astigmatism with the ophthalmometer of Javal and Schiötz, and the rotations of the eyes with the tropometer of Stevens. The chapter on Operations has been enlarged and rewritten.

"A clearly written, comprehensive manual. . . One which we can comınd to students as a reliable text-book, written with an evident knowledge of the wants of those entering upon the study of this special branch of medical science."-British Mledical Fournal.

"The work is characterized by a lucidity of expression which leaves the reader in no doubt as to the meaning of the language employed. . . We know of no work in which these diseases are dealt with more satisfactorily, and indications for treatment more clearly given, and in harmony with the practice of the most advanced ophthalmologists."-Maritime Medical Neaus.

"It is hardly too much to say that for the student and practitioner beginning the study of Ophthalmology, it is the best single volume at present published." - Medical Nezus.

"The latest and one of the best books on Ophthalmology. The book is thoroughly up to date, and is certainly a work which not only commends itself to tlie student, but is a ready reference for the busy practitioner." -International Medical Review.

\section{PROFESSIONAL OPINIONS.}

"A work that will meet the requirements not only of the specialist, but of the general practitioner in a rare degree. I am satisfied that unusual success awaits it."

Willuam Pepper, M. D.

Provost and Professor of Theory and Practice of Medicine and Clinical Medicine in the University of Pennsylvan:a.

"Contains in concise and reliable form the accepted views of Ophthalmic Science."

William Thomson, M. D., Professor of Ophthalmology, Gefferson Medical College, Philadelphia, Fa. 
TEXT-BOOK UPON THE PATHOGENIC BACTERIA. Specially written for Students of Medicine. By Joseph McFarLavd, M. D., Profess or of Parhology and Bacteriology in the Medico-Chirurgical College of Philadelphia, etc. 497 pages. finely illustrated. Price, Cloth, $\$ 2.50$ net.

\section{SECOND EDITION, REVISED AND GREATLY ENLARGED.}

The work is iniended to be a text-book for the medical student and for the practitioner who has had no recent laboratory training in this department of medical science. The instructions given as to needed apparatus, cultures, stainings, microscopic examinations, etc. are ample for the siudent's needs, and will afford to the physician much information that will interest and profit him relative to a subject which modern science shuws to go far in explaining the etiology of many diseased conditions.

In this second edition the work has been brought up to date in all departments of the subject, and numerous additions have been made to the technique in the endearor 10 make the book fulinl the double purpose of a systematic work upon bacteria and a laboratory guide.

"It is excellenily a dapted ior the medical students and practitioners for whom it is avowedly written. ... The descriptions given are accurate and readable, and the book should prove useful to those for whon it is writen. - Lontan Lancet, Aug. 29. 13̂;5.

"The author has succeded admirably in presenting the essential details of bacteriological technics, tzgether with a judiciously chosen summary of our presen: knowledge of pathogenic bacteria. .. The work. we think, should have a wide circulation among English-speaking

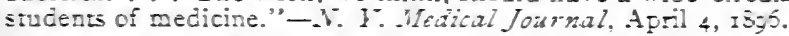

"The book will be found of considerable use by medical men who have not had a special bacteriological training. and who desire to understand this impartant branch of medical

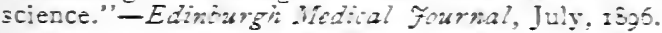

\section{LABORATORY GUIDE FOR THE BACTERIOLOGIST. By}

LANGDON FROThINGH.AM, M. D. V., Assistant in Bacteriology and Veterinary Science, Sheffield Scientific School, Yale University. Illustrated. Price, Cloth, 75 cents.

The technical methods involved in bacteria-culture, methods of staining, and microscopical study are fully described and arranged as simply and concisely as possible. The book is especially intended for use in laboratory work

"It is a convenient and useful little work, and will more than repay the outlay necessary for its purchase in the saving of time which would otherwise be consumed in looking up the various points of technique so cleasly and concisely laid down in its pages." -American Med.. Surg. Bullotin.

FEEdiNg in EARLy infancy. By Arthle V. Meigs, M. D. Bound in limp cloth, flush edges. Price, 25 cents net.

Srropsis: Analyses of Milk-Importance of the Subject of Feeding in Early Infancy-Proportion of Casein and Sugar in Human Milk-Time to Begin Artificial Feeding of Infants-Amount of Food to be Administered at Each Feeding-Intervals between Feedings-Increase in Amount of Food at Different Periods of Infant Development-Unsuitableness of Condensed Milk as a Substitute for Mother"s Milk-Objections to Sterilization or "Pasteurization" of Milk-Adrances made in the Method of Artificial Feeding of Infants. 


\section{ESSENTIALS OF ANATOMY AND MANUAL OF PRACTI-} CAL DISSECTION, containing "Hints on Dissection" By Charles B. Nancrede, M. D., Professor of Surgery and Clinical Surgery in the University of Michigan, Ann Arbor; Corresponding Member of the Royal Academy of Medicine, Rome, Italy; late Surgeon Jefferson Medical College, etc. Fourth and revised edition. Fost 8 vo, over 500 pages, with handsome full-page lithographic plates in co.ors, and over 200 illustrations. Price : Extra Cloth or Oilcloth for the dissection-room, \$2.00 net.

Neither pains nor expense has leen spared to make this work the most exhaustive yet concise Student's Manual of Anatomy and Dissection ever published, either in America or in Europe.

The colored plates are designed to aid the student in dissecting the muscles, arteries, veins, and nerves. The wood-cuts have all been specially drawn and engraved, and an Appendix added containing 60 illustrations representing the structure of the entire human skeleton, the whole being based on the eleventh edition of Gray's Anatomy.

"The plates are of more than ordinary excellence, and are of especial value to students in their work in the dissecling-room." -Journal of American Medical Association.

"Should be in the hands of every medical student."-Cleveland Medical Gazette.

"A concise and judicious work."-Buffalo Medical and Surgical Journal.

A MANUAL OF PRACTICE OF MEdicine. By A. A. Stevens, A. M., M. D., Instructor of Physical Diagnosis in the University of Pennsylvania, and Demonstrator of Pathology in the Woman's Medical College of Philadelphia. Speciaily intended for students preparing for graduation and hospital examinations, and includes the following sections: General Diseases, Diseases of the Digestive Organs, Diseases of the Respiratory System, Diseases of the Circulatory System, Diseases of the Nervous System, Diseases of the Blood, Diseases of the Kidneys, and Diseases of the Skin. Each section is prefaced by a chapter on General Symptomatology. Post 8vo, 520 pages. Numerous illustrations and selected formulæ. Price, $\$ 2.50$, bound in flexible leather.

\section{FIFTH EDITION, REVISED AND ENLARGED.}

Contributions to the science of medicine have poured in so rapidly during the last quarter of a century that it is well-nigh impossible for the student, with the limited time at his disposal, to master elaborate treatises or to cull from them that knowledge which is absolutely essential. From an extended experience in teaching, the author has been enabled, by classification, to group allied symptoms, and by the judicious elimination of theories and redundant explanations to bring within a comparatively small compass a complete outline of the prac. tice of medicine. 
MANUAL OF MATERIA MEDICA AND THERAPEUTICS. By A. A. Srevess, A. M., M. D., Instructor of Physical Diagnosis in the University of Pennsylvania, and Demonstrator of Pathology in the Woman's Medical College of Philadelphia. 445 pages. Price, Cloth, \$2.25.

\section{SECOND EDITION, REVISED.}

This wholly new volume, which is based on the last edition of the Pharmacopaia, comprehends the following sections: Physiological Action of Drugs; Drugs; Remedial Measures other than Drugs; Applied Therapeutics; Incompatibility in Prescriptions; Table of Doses; Index of Drugs; and Index of Diseases; the treatment being elucidated by more than two hundred formulæ.

"The author is to be congratulated upon having presented the medical student with as accurate a manual of therapeutics as it is possible to prepare."-Therapeutic Gazette.

"Far superior to most of its class; in fact, it is very good. Moreover, the book is reliable and accurate."-New York Medical Journal.

"The author has faithfully presented modern therapeutics in a comprehensive work, . . . and it will be found a reliable guide."-University Medical Magazine.

NOTES ON THE NEWER REMEDIES: their Therapeutic Applications and Modes of Administration. By DAvid CERNA, M. D., PH. D., Demonstrator of and Lecturer on Experimental Therapeutics in the University of Pennsylvania. Post-octavo, 253 pages. Price, \$I.25.

\section{SECOND EDITION, RE-WRITTEN AND GREATLY ENLARGED.}

The work takes up in alphabetical order all the newer remedies, giving their physical properties, solubility, therapeutic applications, administration, and chemical formula.

It thus forms a very valuable addition to the various works on therapeutics now in existence.

Chemists are so multiplying compounds, that, if each compound is to be thoroughly studied, investigations must be carried far enough to determine the practical importance of the new agents.

"Especially valuable because of its completeness, its accuracy, its systematic consideration of the properties and therapy of many remedies of which doctors generally know but little, expressed in a brief yet terse manner."-Chicago Clinical Review.

TEMPERATURE CHART. Prepared by D. T. LAINì, M. D. Size $8 \times 131 / 2$ inches. Price, per pad of 25 charts, 50 cents.

A conveniently arranged chart for recording Temperature, with columns for daily amounts of Urinary and Fecal Excretions, Food, Remarks, etc. On the back of each chart is given in full the method of Brand in the treatment of Typhoid Fever. 
SAUNDERS' POCKET MEDICAL LEXICON; or, Dictionary of Terms and Words used in Medicine and Surgery. By Jom $M$. Keating, M. D., editor of "Cyclopedia of Diseases of Children," etc.; author of the "New Pronouncing Dictionary of Medicine;" and HEYRY Hamilton, author of "A New Translation of Virgil's Eneid into English Verse;" co-author of a "New Pronouncing Dictionary of Medicine." A new and revised edition. 32mo, 282 pages. Prices: Cloth, 75 cents: Leather Tucks, \$r.oo.

This new and comprehensive work of reference is the outcome of a demand for a more modern handbook of its class than those at present on the market, which, dating as they do from I 855 to $\mathbf{I} 884$, are of but trifling use to the student by their not containing the hundreds of new words now used in current literature, especially those relating to Electricity and Bacteriology.

"Remarkably accurate in terminology, accentuation, and definition."-Journal of American Medical Association.

"Brief, yet complete... . it contains the very latest nomenclature in even the newest departments of medicine." -New York Hedical Record.

SAUNDERS' POCKET MEDICAL FORMULARY. By WILLIAM M. Powell, M. D., Attending Physician to the Mercer House for Invalid Women at Atlantic City. Containing 1800 Formulæ, selected from several hundred of the best-known authorities. Forming a handsome and convenient pocket companion of nearly 300 printed pages, with blank leaves for Additions; with an Appendix containing Posological Table, Formulæ and Doses for Hypodermatic Medication, Poisons and their Antidotes, Diameters of the Pemale Pelvis and Fœtal Head, Obstetrical Table, Diet List for Various Diseases, Materials and Drugs used in Antiseptic Surgery, Treatment of Asphyxia from Drowning, Surgical Remembrancer, Tables of Incompatibles, Eruptive Fevers, Weights and Measures, etc. Fourth edition, revised and greatly enlarged. Handsomely bound in morocco, with side index, wallet, and flap. Price, \$1.75 net.

$\Lambda$ concise, clear, and correct record of the many hundreds of famous formulæ which are found scattered through the works of the most eminent physicians and surgeons of the world. The work is helpful to the student and practitioner alike, as through it they become acquainted with numerous formula which are not found in text-books, but have been collected from among the rising generation of the profession, college professors, and hospital physicians and surgeons.

"This little book, that can be conveniently carried in the pocket, contains an immense amount of material. It is very useful, and as the name of the author of each prescription is given is unusually reliable."-New York Medical Record.

"Designed to be of immense help to the general practitioner in the exercise of his daily calling."-Boston Mfedical and Surgical fournal. 
Diseases OF Women. By Henry J. Garrigurs, A. M., M. D., Professor of Gynecology and Obstetrics in the New York School of Clinical Medicine; Gynecologist to St. Mark's Hospital and to the German Dispensary, New York City. In one handsome octavo volume of 728 pages, illustrated by 335 engravings and colored plates. Prices: Cloth, $\$ 4.00$ net; Sheep or Half Morocco, $\$ 5.00$ net.

A PRACTICAL work on gynecology for the use of students and practitioners, written in a terse and concise manner. The importance of a thorough knowledge of the anatomy of the female pelvic organs has been fully recognized by the author, and considerable space has been devoted to the subject. The chapters on Operations and on Treatment are thoroughly modern, and are based upon the large hospital and private practice of the author. The text is elucidated by a large number of illustrations and colored plates, many of them being original, and forming a complete atlas for studying embryology and the anatomy of the female genitalia, besides exemplifying, whenever needed, morbid conditions, instruments, apparatus, and operations.

\section{Second Edition, Thoroughly Revised.}

The first edition of this work rnet with a most appreciative reception by the medical press and profession both in this country and abroad, and was adopted as a text-book or recommended as a book of reference by nearly one hundred colleges in the United States and Canada. The author has availed himself of the opportunity afforded by this revision to embody the latest approved advances in the treatment employed in this important branch of Medicine. He has also more extensively expressed his own opinion on the comparative value of the different methods of treatment employed.

"One of the best text-books for students and practitioners which has been published in the English language; it is condensed, clear, and comprehensive. The profound learning and great clinical experience of the distinguished anthor find expression in this book in a most attractive and instructive form. Young practitioners, to whom experienced consultants may not be available, will find in this book invaluable counsel and help."

ThaD. A. Reamy, M. D., LL.D.,

Professor of Clinical Gynecology, Medical College of Ohio: Gynecologist to the Good Samaritan and Cincinenati Hospitals.

A SYLLABUS OF GYNECOLOGY, arranged in conformity with "An American Text-Book of Gynecology." By J. W. Long, M. D., Professor of Diseases of Women and Children, Medical College of Virginia, etc. Price, Cloth (interleaved), \$1.00 net.

Based upon the teaching and methods laid down in the larger work, this will not only be useful as a supplementary volume, but to those who do not already possess the text-book it will also have an independent value as an aic to the practitioner in gynecological work, and to the student as a guide in the lectureroom, as the subject is yresented in a manner at once systematic, clear, succinct, and practical. 
A MANUAL OF PHYSIOLOGY, with Practical Exercises. For Students and Practitioners. By (i. N. STEWART, .I. A., M. I)., D. Sc., lately Examiner in Physiology, University of Aberdeen, and of the New Museums, Cambrilge University; I'rofessor of I'hysiology in the Western Reserve University, Cleveland, Ohio. Handsome octavo volume of 800 pages, with 278 illustrations in the text, and 5 colored plates. Price, Cloth, $\$ 3.50$ net.

"It will make its way by sheer force of merit, and amply deserves to do so. It is one of the a'ery best English text-books on the subject."-London Lancet.

"Of the many text-books of physiology published, we do not know of one that so nearly comes up to the ideal as does Professor Stewart's volume." - B'ritish Medical Journal.

\section{ESSENTIALS OF PHYSICAL DIAGNOSIS OF THE THORAX.}

By Arthur M. Corwin, A. M., M. D., Demonstrator of Physical Diagnosis in the Rush Medical College, Chicago; Attending Physician to the Central Free Dispensary, Department of Rhinology, Laryngology, and Diseases of the Chest. 200 pages. Illustrated. Cloth, flexible covers. Price, \$1.25 net.

\section{SYLLABUS OF OBSTETRICAL LECTURES in the Medical} Department, University of Pennsylvania. By RICHARD C. NORRIS, A. M., M. D., I ecturer on Clinical and Operative Obstetrics, University of Pennsylvania. Third edition, thoroughly revised and enlarged. Crown 8vo. Price, Cloth, interleaved for notes, \$2.00 net.

"This work is so far superior to others on the same subject that we take pleasure in call. ing attention briefly to its excellent features. It covers the subject thoroughly, and will prove invaluable both to the student and the practitioner. The author has introduced a number of valuable hints which would only occur to one who was himself an experienced teacher of obstetrics. The subject-matter is clear, forcible, and modern. We are especially pleased with the portion devoted to the practical duties of the accoucheur, care of the child, etc. The paragraphs on antiseptics are admirable; there is no doubtful tone in the directions given. No details are regarded as unimportant; no minor matters omitted. We venture to say that even the old practitioner will find useful hints in this direction which he cannot afford to despise."-Neze York Medical Record.

\section{A SYLLABUS OF LECTURES ON THE PRACTICE OF SUR- GERY, arranged in conformity with "An American Text-Book of Surgery." By N. SENN, M.D., PH. D., Professor of Surgery in Rush Medical College, Chicago, and in the Chicago Polyclinic. Price, $\$ 2.00$.}

This, the latest work of its eminent author, himself one of the contributors to "An American Text-Book of Surgery," will prove of exceptional value to the advanced student who has adopted that work as his text-book. It is not only the syllabus of an unrivalled course of surgical practice, but it is also an epitome of or supplement to the larger work.

"The author has evidently spared no pains in making his Syllabus thoroughly comprehen. sive, and has added new matter and alluded to the most recent authors and operations. F will references are also given to all requisite details of surgical anatomy and pathology." - British
Medical Journal, London. 
AN OPERATION BLANK, with Lists of Instruments, etc. required in Various Operations. Prepared by W. W. KEEN, M. D., LL.D., Professor of Principles of Surgery in the Jefferson Medical College, Philadelphia. Price per Pad, containing Blanks for fifty operations, 50 cents net.

\section{SECOND EDITION, REVISED FORM.}

A convenient blank, suitable for all operations, giving complete instructions regarding necessary preparation of patient, etc., with a full list of dressings and medicines to be employed.

On the back of each blank is a list of instruments used-viz. general instru ments, etc., required for all operations; and special instruments for surgery of the brain and spine, mouth and throat, abdomen, rectum, male and female genito-urinary organs, the bones, etc.

The whole forming a neat pad, arranged for hanging on the wall of a surgeon's office or in the hospital operating-room.

"Will serve a useful purpose for the surgeon in reminding him of the details of preparation for the patient and the room as well as for the instruments, dressings, and antiseptics needed "-New York Medical Record

"Covers about all that can be needed in any operation."-American Lancet.

"The plan is a capital one."-Boston Medical and Surgical Fournal.

LABORATORY EXERCISES IN BOTANY. By EDSON S. BASTIN, M. A., Professor of Materia Medica and Botany in the Philadelphia College of Pharmacy. Octavo volume of 536 pages, 87 full-page plates. Price, Cloth, \$2.50.

This work is intended for the beginner and the advanced student, and it fully covers the structure of flowering plants, roots, ordinary stems, rhizomes, tubers, bulbs, leaves, flowers, fruits, and seeds. Particular attention is given to the gross and microscopical structure of plants, and to those used in medicine. Illustrations have freely been used to elucidate the text, and a complete index to facilitate reference has been added.

"There is no work like it in the pharmaceutical or botanical literature of this country, and we predict for it a wide circulation."-American Fournal of Pharmacy.

DIET IN SICKNESS AND IN HEALTH. By Mrs. ERnest Hart, formerly Student of the Faculty of Medicine of Paris and of the London School of Medicine for Women; with an Introduction by Sir Henry Thompson, F. R. C. S., M. D., London. 220 pages; illustrated. Price, Cloth, \$1.50.

Useful to those who have to nurse, feed, and prescribe for the sick. In each case the accepted causation of the disease and the reasons for the special diet prescribed are briefly described. Medical men will find the dietaries and recipes practically useful, and likely to save them trouble in directing the dietetic treatment of patients. 
HOW TO EXAMINE FOR LIFE INSURANCE. By JOHN M. Keating, M. D., Fellow of the College of Physicians and Surgeons of Philadelphia; Vice-President of the American Pædiatric Suciety; ExPresident of the Association of Life Insurance Medical Directors. Royal 8vo, 211 pages, with two large half-tone illustrations, and a plate prepared by Dr. McClellan from special dissections; also, numerous cuts to elucidate the text. Second edition. Price, Cloth, $\$ 2.00$ net.

"This is by far the most useful boof which has yet appeared on insurance examination, a subject of growing interest and importance. Not the least valuable portion of the volume is Part II., which consists of instructions issued to their examining physicians by twenty-four representative companies of this country. As the proofs of these instructions were corrected by the directors of the companies, they form the latest instructions obtainable. If for these alone, the book should be at the right hand of every physician interested in this special branch of medical science." - The Medical Nerws, Philadel phia.

NURSING: ITS PRINCIPLES AND PRACTICE. By ISABEL Adams Hampton, Graduate of the New York Training School for Nurses attached to Bellevue Hospital; Superintendent of Nurses and Principal of the Training School for Nurses, Johns Hopkins Hospital, Baltimore, Md.; late Superintendent of Nurses, Illinois Training School for Nurses, Chicago, Ill. In one very handsome 12 mo volume of 484 pages, profusely illustrated. Price, Cloth, $\$ 2.00$ net.

This original work on the important subject of nursing is at once comprehensive and systematic. It is written in a clear, accurate, and readable style, suitable alike to the student and the lay reader. Such a work has long been a desideratum with those entrusted with the management of hospitals and the instruction of nurses in training-schools. It is also of especial value to the graduated nurse who desires to acquire a practical working knowledge of the care of the sick and the hygiene of the sick-room.

\section{OBSTETRIC ACCIDENTS, EMERGENCIES, AND OPERA-}

TIONS. By L. Ch. Boisliniere, M. D., late Emeritus Professor of Obstetrics in the St. Louis Medical College. $38 \mathrm{I}$ pages, handsomely illustrated. Price, $\$ 2.00$ net.

"For the use of the practitioner who, when away from home, has not the opportunity of consulting a library or of calling a friend in consultation. $\mathrm{He}$ then, being thrown upon his own resources, will find this book of benefit in guiding and assisting him in emergencies."

INFANT'S WEIGHT CHART. Designed by J. P. CROzer Griffith, M. D., Clinical Professor of Diseases of Children in the University of Pennsylvania. 25 charts in each pad. Price per pad, 50 cents net.

A convenient blank for keeping a record of the child's weight during the first two years of life. Printed on each chart is a curve representing the average weight of a healthy infant, so that any deviation from the normal can readily be detected. 
THE CARE OF THE BABY. By J. P. Crozer Griffith, M. D., Clinical Professor of Diseases of Children, University of Pennsylvania; Physician to the Chıldren's Hospital, Philadelphia, etc. 404 pages, with 67 illustrations in the text, and 5 plates. I 2 mo. Price, $\$ \mathbf{I} .50$.

\section{SECOND EDITION, REVISED.}

A reliable guide not only for mothers, but also for medical students and practitioners whose opportunities for observing children have been limited.

"The whole book is characterized by rare good sense, and is evidently written by a mas. ter hand. It can be read with benefit not only by mothers, but by medical students and by :ny practitioners who have not had large opportunities for observing children." -American Yuurnal of Obstetrics.

\section{THE NURSE'S DICTIONARY of Medical Terms and Nursing} Treatment, containing Definitions of the Principal Medical and Nursing Terms, Abbreviations, and Physiological Names, and Descriptions of the Instruments, Drugs, Diseases, Accidents, Treatments, Operations, Foods, Appliances, etc. encountered in the ward or the sick-room. By HonNor Morten, author of "How to Become a Nurse," "Sketches of Hospital Life," etc. I6mo, I 40 pages. Price, Cloth, \$1.00.

This little volume is intended for use merely as a small reference-book which can be consulted at the bedside or in the ward. It gives sufficient explanation to the nurse to enable her to comprehend a case until she has leisure to look up larger and fuller works on the subject.

DIET LISTS AND SICK-ROOM DIETARY. By JEROME B. ThomAS, M. D., Visiting Physician to the Home for Friendless Women and Children and to the Newsboys' Home; Assistant Visiting Physician to the Kings County Hospital ; Assistant Bacteriologist, Brooklyn Health Department. Price, Cloth, $\$ \mathbf{I} .5^{\circ}$ (Send for specimen List.)

One hundred and sixty detachable (perforated) diet lists for Albuminuria, Anæmia and Debility, Constipation, Diabetes, Diarrhœa, Dyspepsia, Fevers, Gout or Uric-Acid Diathesis, Obesity, and Tuberculosis. Also forty detachable sheets of Sick-Room Dietary, containing full instructions for preparation of easily-digested foods necessary for invalids. Each list is numbered only, the disease for which it is to be used in no case being mentioned, an index key being reserved for the physician's private use.

\section{DIETS FOR INFANTS AND CHILDREN IN HEALTH AND}

IN DISEASE. By Louis Starr, M. D., Editor of "An American

Text-Book of the Diseases of Children." 230 blanks (pocket-book size): perforated and neatly bound in flexible morocco. Price, $\$ \mathbf{I} .25$ net.

The first series of blanks are prepared for the first seven months of infant life; each blank indicates the ingredients, but not the quantities, of the food: the latter directions being left for the physician. After the seventh month, modifications being less necessary, the diet lists are printed in full. Formulo for the preparation of diluents and foods are appended. 


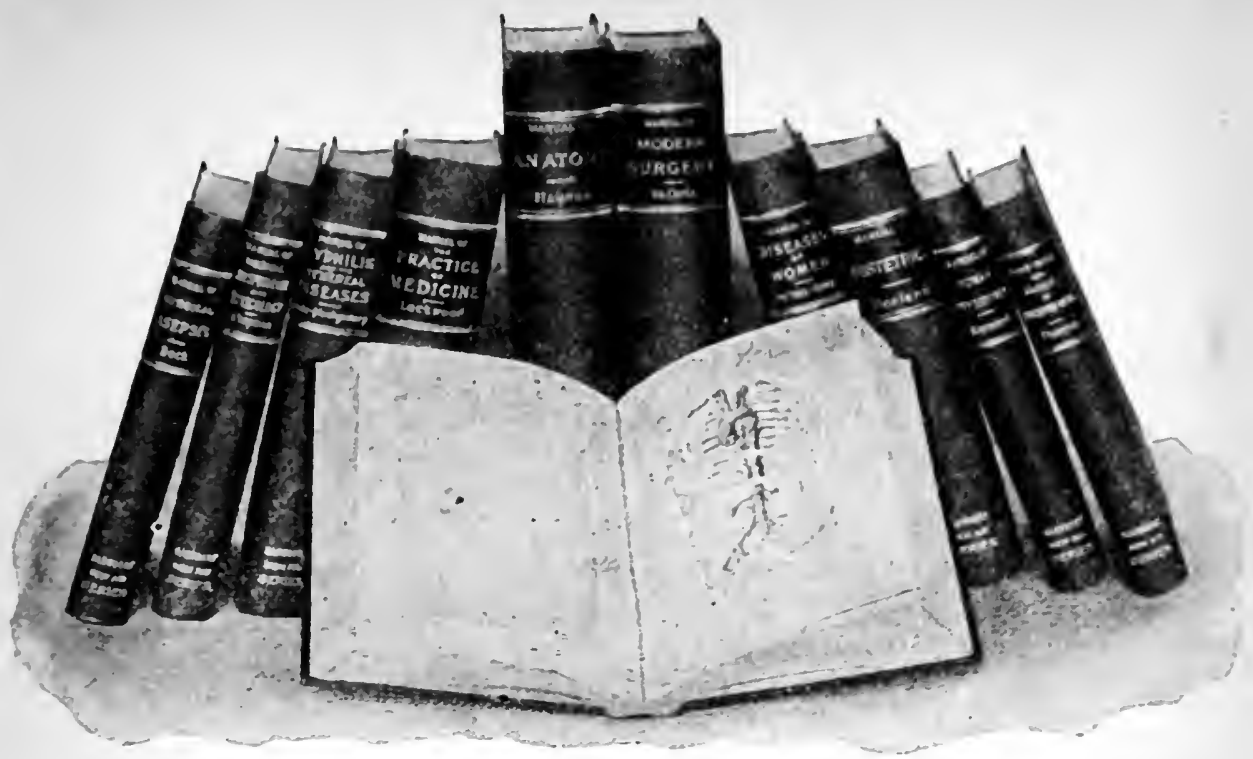

\section{SAUNDERS}

NEW SERIES

\section{of MANUALS}

for Students

and

Practitioners.

$\Gamma^{\text {HAT }}$ there exists a need for thoroughly reliable hand-books on the leading

1 branches of Medicine and Surgery is a fact amply demonstrated by the favor with which the SAUNDERS NEW SERIES OF MANUALS have been received by medical students and practitioners and by the Medical Press. These manuals are not merely condensations from present literature, but are ably written by well-known authors and practitioners, most of them being teachers in representative American colleges. Each volume is concisely and authoritatively. written and exhaustive in detail, without being encumbered with the introduction of "cases," which so largely expand the ordinary textbook. These manuals will therefore form an admirable collection of advanced lectures, useful alike to the medical student and the practitioner: to the latter, too busy to search through page after page of elaborate treatises for what he wants to know, they will prove of inestimable value; to the former they will afford safe guides to the essential points of study.

The SAUNDERS NEW SERIES OF MANUALS are conceded to be superior to any similar books now on the market. No other manuals afford so much information in such a concise and available form. A liberal expenditure has enabled the publisher to render the mechanical portion of the work worthy of the high literary standard attained by these books.

Any of these Manuals will be mailed on receipt of price (see next page for List).

W. B. SAUNDERS, Publisher, 


\section{SAUNDERS' NEW SERIES OF MANUALS.}

\section{VOLUMES PUBLISHED.}

PHYSiology. By Joseph Howard Raymond, A. M., M. D., Professor of Physiology and Hygiene and Lecturer on Gynecology in the Long Island College Hospital, etc. Price, \$1.25 net.

SURGERY, General and Operative. By John Chalmers DaCosta, M. D,, Demonstrator of Surgery, Jefferson Medical College, Philadelphia, etc. Octavo, 9II pages, 386 illustrations. Cloth, \$4.00 net; HalfMorocco, \$5.00 net.

DOSE-BOOK AND MANUAL OF PRESCRIPTION-WRITING. By E. Q. ThORNTON, M. D., Demonstrator of Therapeutics, Jefferson Medical College, Philadelphia. Price, \$1.25 net.

MEdical JURisprudence. By Henry C. Chapman, M. D., Professor of Institutes of Medicine and Medical Jurisprudence in the Jefferson Medical College of Philadelphia, etc. Price, \$1.50 net.

SURGICAL ASEPSIS. By CARL BeCK, M.D., Surgeon to St. Mark's Hospital and to the German Poliklinik; Instructor in Surgery, New York Post-Graduate Medical School, etc. Price, $\$ 1.25$ net.

Manual of anatomy. By Irving S. Haynes, M. D., Adjunct Professor of Anatomy and Demonstrator of Anatomy, Medical Department of the New York University, etc. (Double number.) Price, \$2.50 net.

SYPHILIS AND THE VENEREAL DISEASES. By JAMES Nevins Hyde, M.D., Professor of Skin and Venereal Diseases, and Frank H. Montgomery, M. D., Lecturer on Dermatology and Genitourinary Diseases, in Rush Medical College, Chicago. (Double number.) Price, $\$ 2.50$ net.

PRACTICE OF MEDICINE. By George Roe Lockwood, M. D., Professor of Practice in the Woman's Medical College of the New York Infirmary, etc. (Double number.) Price, \$2.50 net.

obstetrics. By W. A. Newman Dorland, M. D., Asst. Demonstrator of Obstetrics, University of Pennsylvania; Chief of Gynecological Dispensary, Pennsylvania Hospital. (Double number.) Price, \$2.50 net.

Diseases of Women. By J. Bland Sutton, F. R. C. S., Assistant Surgeon to the Middlesex Hospital, and Surgeon to the Chelsea Hospital for Women, London; and Arthur E. Giles, M. D., B. Sc. Lond., F. R. C. S. Edin., Assistant Surgeon to the Chelsea Hespital for Women, London. $43^{6}$ pages, handsomely illustrated. (Double number.) Price, $\$ 2.50$ net.

\section{VOLUMES IN PREPARATION.}

NERVous DISEASES. By Charles W. Burr, M. D., Clinical Professor of Nervous Diseases, Medico-Chirurgical College, Philadelphia, etc.

NOSE AND ThroAT. By D. Braden Kyle, M. D., Clinical Professor of Laryngology and Rhinology, Jefferson Medical College, Philadelphia.

*** There will be published in the same series, at short intervals, carefully prepared works on various subjects, by prominent specialists. 


\section{SAUNDERS' QUESTION COMPENDS.}

\section{Arranged in Question and Answer For'm.}

\section{THE LATEST, MOST COMPLETE, and BEST ILIUSTRATED SERIES OF.COMPENDS EVER ISSUED.}

\section{Now the Standard Authorities in Medical Literature}

WITH

Students and Practitioners in every City of the United States and Canada.

\section{THE REASON WHY.}

They are the advance guard of "Student's Helps"-that Do HELP; they are the leaders in their special line, well and authoritatively written by able men, who, as teachers in the large colleges, know exactly what is wanted by a student preparing for his examinations. The judgment exercised in the selection of authors is fully demonstrated by their professional elevation. Chosen from the ranks of Demonstrators, Quiz-masters, and Assistants, most of them have become Professors and Lecturers in their respective colleges.

Each book is of convenient size ( $5 \times 7$ inches), containing on an average 250 pages, profusely illustrated, and elegantly printed in clear, readable type, on fine paper.

The entire series, numbering twenty.four subjects, has been kept thoronghly revised and enlarged when necessary, many of them being in their fourth and fifth editions.

\section{TO SUM UP.}

Although there are numerous other Quizzes, Manuals, Aids, etc. in the market, none of them approach the "Blue Series of Question Compends;" and the claim is made for the following points of excellence :

1. Professional distinction and reputation of authors.

2. Conciseness, clearness, and soundness of treatment.

3. Size of type and quality of paper and binding.

*** Any of these Compends will be mailed on receipt of price (see over for List). 


\section{SAUNDERS' QUESTION-COMPEND SERIES.}

Price, Cloth, $\$ 1.00$ per copy, except when otherwise noted.

I. ESSENTIALS OF PHYSIOLOGY. 3d edition. Illustrated. Revised and enlarged by H. A. HARE, M. D (Price, \$1.00 net.)

2. ESSENTIALS OF SURGERY. 6th edition, with an Appendix on Antiseptic Surgery. 90 illustrations. By Edwakd Maktin, M. D.

3. ESSENTIALS OF ANATOMY. 5th edition, with an Appendix. I 80 illustrations. By Charles B. NANCREDE, M. D.

4. ESSENTIALS OF MEDICAL CHEMISTRY, ORGANIC AND INORGANIC. 4th edition, revised, with an Appendix. By LAw. REXCE WOLFF, M. D.

5. ESSENTIALS OF OBSTETRICS. 4th edition, revised and en. larged. 75 illustrations. By W. EAsterly Ashton, M. D.

6. ESSENTIALS OF PATHOLOGY AND MORBID ANATOMY. 7 th thousand. 46 illustrations. By C. E. ARMAND SEMPLE, M. D.

7. ESSENTIALS OF MATERIA MEDICA, THERAPEUTICS, AND PRESCRIPTION-WRITING. 4th edition. By HENRY MORRIS, MI. D.

8, 9. ESSENTIALS OF PRACTICE OF MEDICINE. By HENRY Morris, M. D. An Appendix on URine Examination. Illustrated. By LAwrexce WolfF, M. D. 3d edition, enlarged by some 300 Essential Formulx, selected from eminent authorities, by WM. M. PowelL,

M. D. (Double number, price \$2.00.)

10. ESSENTIALS OF GYN ECOLOGY. 4th edition, revised. With ó2 illustrations. By Edwix B. CRAgIN, M. D.

II. ESSENTIALS OF DISEASES OF THE SKIN. 3d edition, rerised and enlarged. 7 I letter-press cuts and I $_{5}$ half-tone illustrations. By HENRY W. STELWAgox, M. D. (Price, \$I.OO net.)

12. ESSENTIALS OF MINOR SURGERY, BANDAGING, AND VENEREAL DISEASES. 2d edition, revised and enlarged. 78 illustration:. By EDWARD MARTIN, M. D.

13. ESSENTIALS OF LEGAL MEDICINE, TOXICOLOGY, AND HYGIENE. I jo illustrations. By C. E. Armand Semple, M. D.

14. ESSENTIALS OF DISEASES OF THE EYE, NOSE, AND THROAT. I 24 illustrations. 2d edition, revised. By EDWARD IACKSON, M. D., and E. BALUWix Gleason, M. D.

15. ESSENTIALS OF DISEASES OF CHILDREN. Second edi. tion. By William H. Powell, M. D.

6. ESSENTIALS OF EXAMINATION OF URINE. Colored "Vogel SCAle," and numerous illustrations. By LAwrexce WolfF, Ii. D. (Price, 75 cents.)

17. ESSENTIALS OF DIAGNOSIS. By S. SOLIS-CoHEN, M. D., and A. A. Eshser, M. D. 55 illustrations, some in colors. (Price, \$I.50 net.)

18. ESSENTIALS OF PRACTICE OF PHARMACY. By L. E. SAIRE. 2d edition, revised.

20. ESSENTIALS OF BACTERIOLOGY. 3 d edition. 82 illustrations. By M. Y. BALL, M. D.

21. ESSENTIALS OF NERVOUS DISEASES AND INSANITY 48 illustrations. $3^{\mathrm{d}}$ edition, revised. By Johx C. Shaw, M. D.

22. ESSENTIALS OF MEDICAL PHYSICS. I55 illustrations. 2d edition, revised. By FreD J. BROCKWAY, M. D. (Price, \$1.00 net.)

23. ESSENTIALS OF MEDICAL ELECTRICITY. 65 illustrations. By David D. Stewart, M. D., and Edward S. Lawrance, M. D.

24. ESSENTIALS OF DISEASES OF THE EAR. By E. B. Gleasox, M. D. I I 4 illustrations. Second edition, revised and enlarged. 


\section{RECENT PUBLICATIONS}

\section{PENROSE'S DISEASES OF WOMEN}

A Text-Book of Diseases of Women. By CHARILS B. PliNROSL, M.D, PH.D., Professor of Gynecology in the University of Penusylvania; Surgeon to the Gyncectin Hospital, Pluiladelphia. Octavo volume of 529 pages, handsomely illustrated. Cloth, $\$ 3.50$ net.

"I shall value very highly the copy of Penrose's "Diseases of Women" received. I have already recommended it 10 my class as THL BLST book." - HOWARD A. KELLY, Professor of Gynecology and Obstetrics, Johns Hopkins Lniversity, Battimore, ifl.

\section{SENN'S GENITO=URINARY TUBERCULOSIS}

Tuberculosis of the Genito-Urinary Organs, Male and Female. By Nichol.AS SENN, M.D., PH.D., LL.D., Professor of the Practice of Surgery and of Clinical Surgery, Rush Medical College, Chicago. Handsome octavo volume of 320 pages, illustrated. Cloth, $\$ 3.00$ net.

\section{SUTTON AND GILES' DISEASES OF WOMEN}

Diseases of Women. By J. Bland Sutron, F.R.C.S., Assistant Surgeon to Middlesex Hospital, and Surgeon to Chelsea Hospital, London; and ARTHLR E. Gnlfs, M.D., B.Sc. Lond., F.R.C.S. Edin., Assistant Surgeon to Chelsea Hospital, London. 436 pages, handsomely illustrated. Cloth, $\$ 2.50$ net.

\section{BUTLER'S MATERIA MEDICA, THERAPEUTICS, AND PHAR= MACOLOGY. Second Edition, Revised}

A Text=Book of Materia Medica, Therapeutics, and Pharmacology. By GEORGE F. Butler, PH.G., M.D., Professor of Materia Medica and of Clinical Medicine in the College of Physicians and Surgeons, Chicago; Professor of Materia Medica and Therapeutics, Northwestern University, Woman's Medical Sclrool, etc. Octavo, 858 pages, illustrated. Cloth, $\$ 4.00$ net; Sheep, $\$ 5.00$ net.

\section{SAUNDBY'S RENAL AND URINARY DISEASES}

Lectures on Renal and Urinary Diseases. By ROBERT SAUNDBY, M.D. Edin., Fellow of the Royal College of Physicians, London, and of the Royal Medico-Chirurgical Society; Physician to the General Hospital; Consulting Physician to the Eye Hospital and to the Hospital for Diseases of Women; Professor of Medicine in Mason College, Birmingham, etc. Octavo volume of 434 pages, with numerous illustrations and 4 colored plates. Cloth, $\$ 2.50$ net.

\section{PYE'S BANDAGING}

Elementary Bandaging and Surgical Dressing, with Directions Concerning the Immediate Treatment of Cases of Emergency. For the Use of Dressers and Nurses. By WALTER PyE, F.R.C.S., Late Surgeon to St. Mary's Hospital, London. Small i2mo, with over 80 illustrations. Cloth, flexible covers. Price, 75 cents net.

\section{MALLORY AND WRIGHT'S PATHOLOGICAL TECHNIQUE}

Pathological Technique. By FrANK B. MALLORY, A.M., M.D., Assistant Professor of Pathology, Harvard University Medical School; and JAstes H. WRight, A.M., M.D.. Instructor in Pathology, Harvard University Medical School. Octavo volume of 396 pages, handsomely illustrated. Cloth, $\$ 2.50$ net.

"I have been looking forward to the publication of this book, and I am glad to say" that I find it to be a most useful laboratory and post-mortem guide, full of practical information, and well up to date."-IVIlliam H. IVELch, Professor of Pathology, Johns Hopkins University, Baltimore, Md.

\section{ANDERS' PRACTICE OF MEDICINE. Second Edition}

A Text=Book of the Practice of Medicine. By JAMEs M. ANders, M.D., Ph.D., LL.D., Professor of the Practice of Medicine and of Clinical Medicine, MedicoChirurgical College, Philadelphia. In one handsome octavo volume of $\mathrm{I}_{2} \mathrm{~S} 7$ pages. fully illustrated. Cloth, $\$ 5.50$ net; Sheep or Half Morocco, $\$ 6.50$ net. 


\section{ANOMALIES}

\section{AND \\ CURIOSITIES OF MEDICINE.}

BY

GEORGE M. GOULD, M. D., AxD WALTER L. PYLE, M. D.

Several years of exhaustive research have been spent by the authors in the great medical libraries of the Lnited States and Europe in collecting the material for this work. Medical literature of all ages and all languages has been carefully searched, as a glance at the Bibliographic Index will show. The facts, which will be of extreme value to the author and lecturer, have been arranged and annotated, and full -reference footnotes given, indicating whence they have been obtained.

In view of the persistent and dominant interest in the anomalous and curious, a thorough and systematic collection of this kind (the first of which the authors have knowledge) must have its own peculiar sphere of usefulness.

As a complete and authoritative Book of Reference it will be of value. not only to members of the medical profession, but to all persons interested in general scientific, sociologic, and medico-legal topics; in fact, the general interest of the subject and the dearth of any complete work upon it make this volume one of the most important literary innovations of the day.

An especially valuable feature of the book consists of the Indexing. Besides a complete and comprehensive General Index, containing numerous cross-references to the subjects discussed, and the names of the authors of the more important reports, there is a convenient Bibliographic Index and a Table of Contents.

The plan has been adopted of printing the topical headings in bold= face type, the reader being thereby enabled to tell at a glance the subjectmatter of any particular paragraph or page.

Illustrations have been freely employed throughout the work, there being 165 relief cuts and 130 half-tones in the text, and 12 colored and half-tone fullpage plates-a total of over 320 separate figures.

The careful rendering of the text and references, the wealth of illustrations, the mechanical skill represented in the typography, the printing, and the binding, combine to make this book one of the most attractive medical publications ever issued.

Handsome Imperial Octavo Volume of 968 Pages. PRICES: Cloth, $\$ 6.00$ net; Half Morocco, $\$ 7.00$ net. 30 


\section{JUST ISSUED}

\section{AN AMERICAN TEXT=BOOK OF GENITO=URINARY AND SKIN DISEASES}

Edited by I. Bolton Baxis, M.D., Iate Professor of Genito-Crimary and Venereal Diseases, New York I'ost-Graduate Medical School aud Hospital; and WIt.1AM A. HARDAWAy, M.D., Professor of Distases of the Skil, Missouri Medical Crillege. Octavo volume of over 1200 pages, with 300 ilhstrations in the text, and 20 full-page colored plates. Prices: Cloth, \$7.00 net; Sheep or Half Morocco, \$8.00 net.

\section{MOORE'S ORTHOPEDIC SURGERY}

A Manual of Orthopedic Surgery. By JAmLs E. MOORF, M1.D., Profussor of Orthopedics and Adjunct Professor of Clinical Surgery, University of Minnesnta, College of Medicine and Surgery. Svo, 356 pages, handsomely illustrated. Cloth, $\$ 2.5011$ tet.

\section{MACDONALD'S SURGICAL DIAGNOSIS AND TREATMENT}

Surgical Diagnosis and Treatment. By J. W. MACDONAI.D. M.D. Edin., L..R.C.S Edin., Professor of the Practice of Surgery and of Clinica! Surgery in Hamline Lniversity; Visiting Surgeon to St. Barnabas' Hospital, Mimmeapolis, etc. Octavo volume of Soo pages, handsomely illustrated. Cloth, \$5.00 net; Half Morocco, \$6.co net.

\section{CHAPIN ON INSANITY}

A Compendium of Insanitz' Py. Johs B. ChAPIN, M.D., L.L.D., Physician-in-Chier, Pennsylvania Hospital for the hisane; late Physician-Superintendent of the Willard State Hospital, New York, etc. I2mo., 234 pages, illustrated. Cloth, \$1.25 net.

\section{KEEN ON THE SURGERY OF TYPHOID FEVER}

The Surgical Complications and Sequels of Typhoid Fever. By. IVM. W. KEEN, M.D., LL.D., Professor of the Principles of Surgery and of Clinical Surgery, Jefferson Medical College, Philada. Octavo volume of 400 pages. Cloth, $\$ 3.00$ net.

\section{VAN VALZAH AND NISBET'S DISEASES OF THE STOMACH}

Diseases of the Stomach. By WILlam W. VAN VALZAH, M.D., Professor of General Medicine and Diseases of the Digestive System and the Blood. New York Polyclinic; and J. Douglas Nisbet, M.D., Adjunct Professor of Gencral Medicine and Diseases of the Digestive System and the Blood, New York Polyclinic. Octavo volume of 674 pages, illustrated. Cloth, $\$ 3.50$ net.

\section{IN PREPARATION}

\section{AN AMERICAN TEXT=BOOK OF DISEASES OF THE EYE, EAR,} NOSE, AND THROAT

Edited by G. E. DE Schweinitz, M.D., Professor of Ophthalmology in the Jefferson Medical College; and B. Alexander RANDAL, M.D., Professor of Diseases of the Ear in the University of Pennsylvania and in the Philadelphia Polyclinic.

CHURCH AND PETERSON'S NERVOUS AND MENTAL DISEASES

Nervous and Mental Diseases. By ARchibald Church, M.D., Professor of Mental Diseases and Medical Jurisprudence, Northwestern Lniversity Medical School, Chicago; and Frederick PETERSoN, M.D., Clinical Professor of Mental Diseases, Woman's Medical College, New York, etc.

\section{KYLE ON THE NOSE AND THROAT}

Diseases of the Nose and Throat. By D. BrADEN KYLE, M.D., Clinical Professor of Laryngology and Rhinology, Jefferson Medical College, Philadelphia; Consulting Laryngologist, Rhinologist, and Otologist, St. Agnes' Hospital, etc.

\section{STENGEL'S PATHOLOGY}

A Manual of Pathology. By Alfred Stengel, M.D. Physician to the Pliiladel. phia Hospital; Professor of Clinical Medicine in the Woman's Medical College; Physician to the Children's Hospital, etc.

\section{HIRST'S OBSTETRICS}

A Text-Book of Obstetrics. By BARTon CoOkE HIRst, M.D., Professor of Ob stetrics, University of Pennsylvania.

\section{HEISLER'S EMBRYOLOGY}

A Text-Book of Embryology. By John C. Hfisler, M.D., Professor of Anatomy, Medico-Chirurgical College, Philadelphia. 


\section{NOW READY, VOLUMES FOR 1896,189\%, 1898.}

\section{SAUNDERS'}

\section{AMERICAN YEAR-BOOK OF MEDICINE and SURGERY.}

Edited by GEORGE M. GOULD, A. M., M.D.

\section{Assisted by Eminent American Specialists and Teachers.}

Notwithstandivg the rapid multiplication of medical and surgical works, still these publications fail to meet fully the requirements of the general physician, inasmuch as he feels the need of something more than mere text-books of wellknown principles of medical science. Mr. Saunders has long been impressed with this fact, which is confirmed by the unanimity of expression from the profession at large, as indicated by advices from his large corps of canvassers.

This deiciency would best be met by current journalistic literature, but most practitioners have scant access to this almost unlimited source of information, and the busy practiser has but little time to search out in periodicals the many interesting cases whose study would coubtless be of inestimable value in his practice. Therefore, a work which places before the physician in convenient form an stitomization of this literature by persons competent to fronounce upon

The Value of a Discovery or of a Method of Treatment cannot but command his highest appreciation. It is this critical and judicial function that will be assumed by the Editorial staff of the "American YearBook of Medicine and Surgery."

It is the special purpose of the Editor, whose experience peculiarly qualifies him for the preparation of this work, not only to review the contributions to American journals, but also the methods and discoveries reported in the leading medical journals of Europe, thus enlarging the survey and making the work characteristically international. These reviews will not simply be a series of undigested abstracts indiscriminately run together, nor will they be retrospective of "news" one or two yecrs old, but the treatment presented will be synthetic and dogmatic, and will include only what is new. Moreover, through expert condensation by experienced writers these discussions will be

\section{Comprised in a Single Volume of about 1200 Pages.}

The work will be replete with original and selected illustrations skilfully reproduced, for the most part in Mr. Saunders" own studios established for the purpose, thus ensuring accuracy in delineation, affording efficient aids to a right comprehension of the text, and adding to the attractireness of the rolume. Prices: Cloth, $\$ 6.50$ net; Half Morocco, \$7.50 net. 





\section{COLUMBIA UNIVERSITY}

This book is due on the date indicated below, or at the expiration of a definite period after the date of borrowing, as provided by the rules of the Library or by special arrangement with the Librarian in charge.

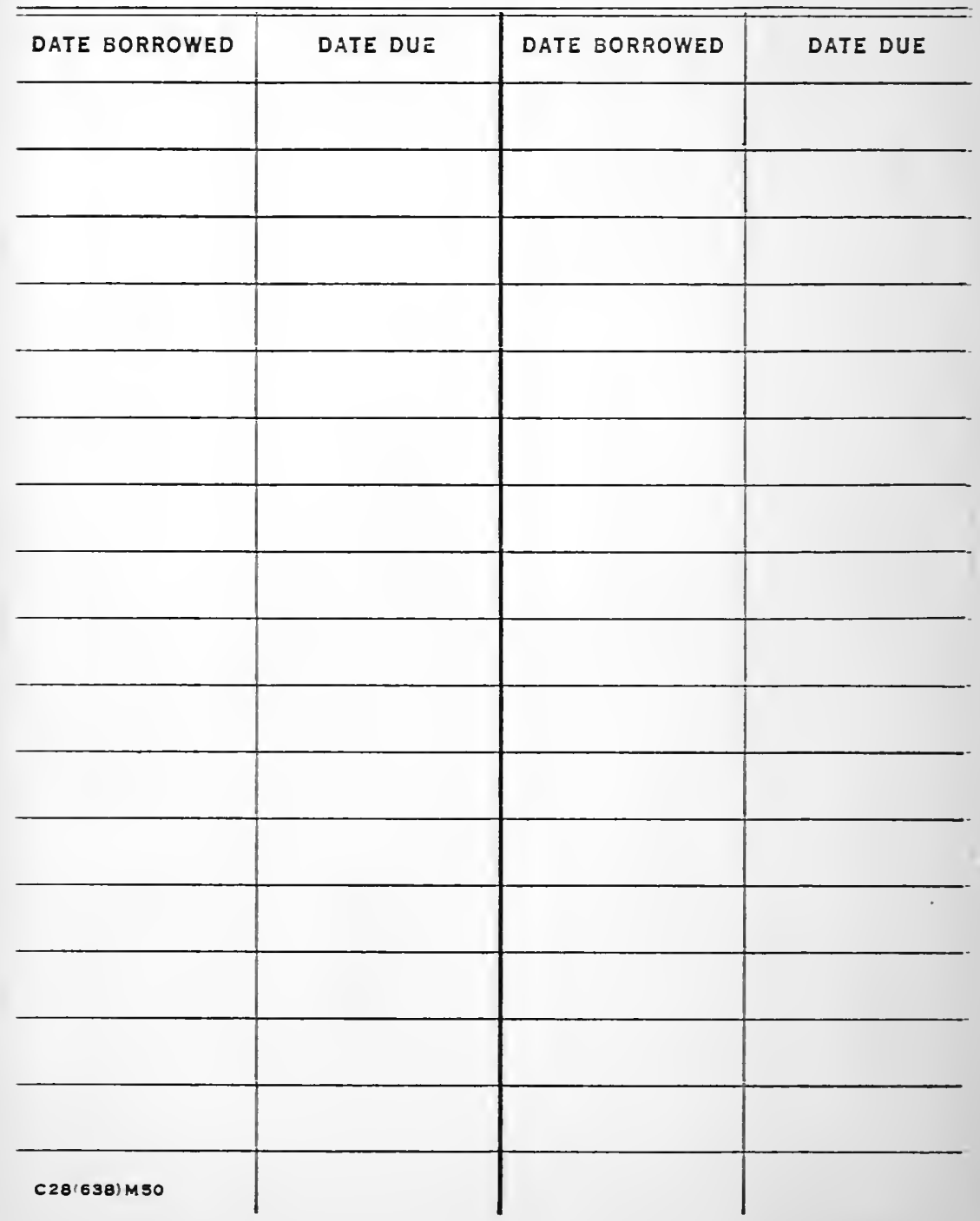




\section{VOLUMES NOW READY.}

Atlas of Internal Medicine and Clinical Diagnosis. By DR. CHк. JAKOn, of Erlangen. Edited by AUcustus A. Eshner, M.D., Professor Clinical Medicine in the Philadelphia Polyclinic; Attending Physician to the Philadelphia Hospital

Atlas of

Vienna. Prof-

Atlas and epitome of operative suroery

Professor of Dermatology: Jefferson Medical College, Philadelphia. With So colored plates from original water-colors.

Atlas of Pathological Histology. Atlas of Orthopedic Surgery. Atlas of General Surgery.
Atlas of Operative Gynecology. Atlas of Psychiatry. Atlas of Diseases of the Ear. 
